
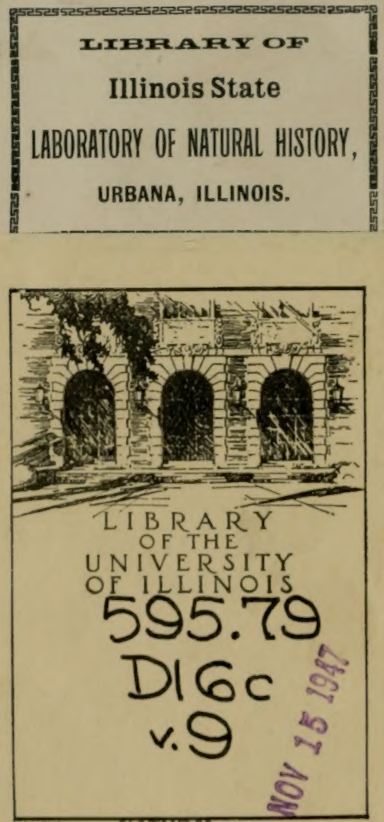

MATURAL HISTORY 
 \\ \begin{tabular}{c}
3 \\
701.9 \\
\hline
\end{tabular}

$$
\begin{gathered}
3 \\
7.91 .9 \\
\hline
\end{gathered}
$$
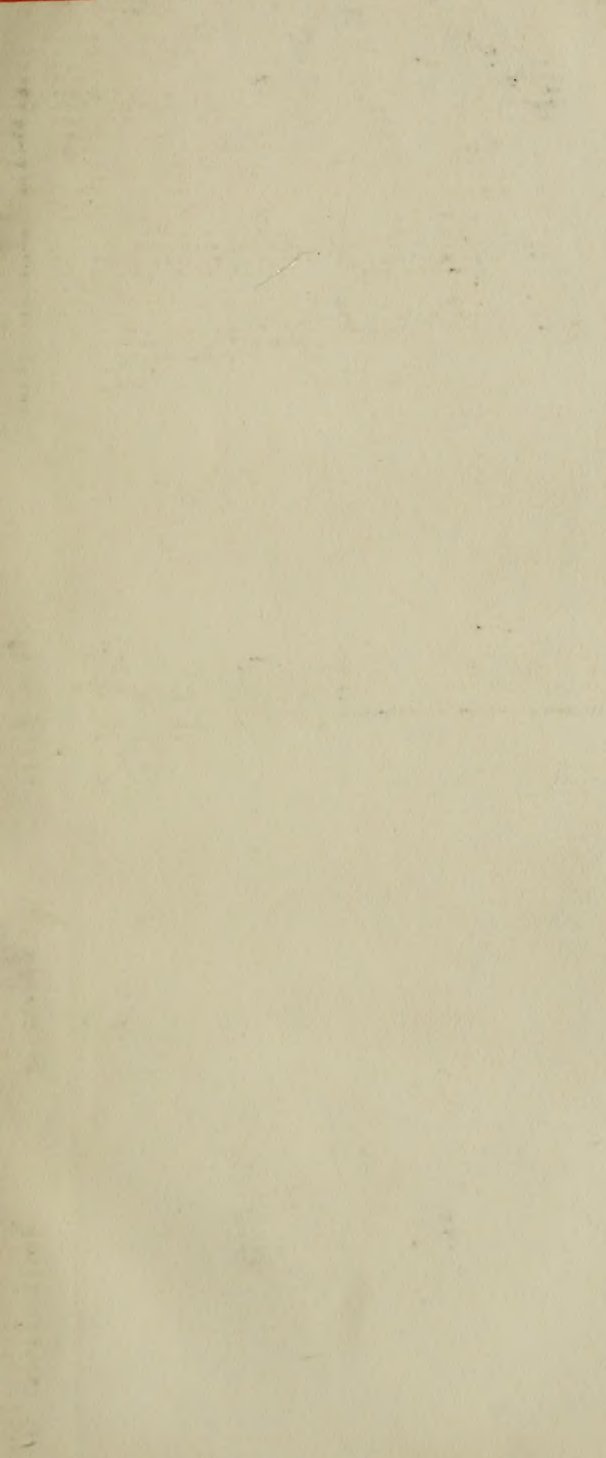





\section{CATALOGUS HYMENOPTERORUM}

hucusque descriptorum

systematicus et synonymicus

Auctore

DR. C. G. DE DALLA TORRE

Professore Oenipontano.

Volumen IX:

VESPIDAE (DIPLOPTERA)

LIPSIAE

Sumptibus Guilelmi Engelmann MDCCCXCIV. 
Abgeschlossen mit Vol. XXIX. (1892) des Zoological Record und Jahrg. XVI. (1893) des Zoologischen Anzeigers. 


\section{VORWOR'T.}

Indem ich hiermit das Werk einer fast 20 jährigen Thätigkeit der Öffentlichkeit übergebe, scheint mir mehr als ein Grund dafür zu sprechen, dasselbe mit einigen erläuternden Worten einzuleiten.

Das vorliegende Werk, ,Catalogus Hymenopterorum hucusque descriptorum systematicus et synonymicus" hat den Zweck, zum ersten Male einen alle (bis zum Schlusse des Jahres 1890) bekannt gewordenen Hymenopteren-Arten aller Länder enthaltenden Catalog dem sich dafür Interessirenden zu bieten, und hat gewissermaßen einen Vorläufer, ja Doppelgänger in dem von Br. E. Harold und Dr. E. Gemminger herausgegebenen "Catalogus Coleopterorum", der zur vorliegenden Arbeit auch die Anregung gegeben hat. Mit ihm hat er außer dem Zwecke, ein Bild des gegenwärtigen Wissensstandes dieser Tiergruppe zu bieten, auch die systematisch-alphabetische Anlage, die Citate und die Angaben über die geographische Verbreitung mutatis mutandis gemeinsam - und doch unterscheidet er sich von demselben in einigen ganz wesentlichen Punkten, ich glaube zu seinen Gunsten.

Ein solcher Hauptunterschied liegt darin, dass in dem „Münchener Cataloge" gewisse Gattungen bloß nach den vorhandenen Monographien behandelt, resp. verzeichnet wurden, während in der vorliegenden Arbeit ausnahmslos aus erster Quelle geschöpft und somit Citat für Citat selbst verglichen wurde. Liegt nun hierin schon eine sehr wertvolle Garantie für die Richtigkeit der Citate, so wächst der Wert dieser selbständigen Forschungs- und Studienmethode noch mehr durch die dadurch erzielte Consequenz bezüglich der Nomenclatur. Ein weiterer wesentlicher Unterschied liegt darin, dass in den aufgeführten Citaten keinerlei Auswahl erfolgte; es sind vielmehr alle Beschreibungen, alle biologischen, anatomischen und physiologischen Angaben, alle Abbildungen mit und ohne Metamorphose, sowie alle Versetzungen in verschiedene Genera ersichtlich 
gemacht, wodurch der wissenschaftliche Apparat zwar größer und complicirter, zugleich aber auch bis incl. 1890 abgeschlossen erscheint; der kleine Mangel, dass da und dort, wo es sich um eine ältere namentlich vorlinnéische Angabe handelt, das eine oder andere Citat vielleicht etwas unsicher geworden ist, steht wohl in keinem Verhältnisse zu dem klaren Bilde, welches man in Bezug auf die Geschichte der Kenntnisnahme derselben erhält. - Ein weiterer Unterschied endlich liegt in der formalen Ausführung des Werkes, in der Art der Citate und ihrer Wiedergabe, in den geographischen Verbreitungsangaben, in der Aufnahme der fossilen Arten, und in der Aufnahme der Nahrungspflanzen bei den Phytophagen und der Nahrungstiere bei den parasitischen Arten, wodurch das Werk ein sehr wertvolles Nachschlagebuch über den heutigen Wissensstand geworden ist, wie ein ähnliches nicht existirt. Auch die Beigabe der gesamten hymenopterologischen Literatur möge als ein weiterer wertvoller Unterschied zu Gunsten dieser Arbeit angesehen werden.

Haben wir damit gewissermaßen von oben her das vorliegende Werk in seiner Grundanlage kennen gelernt, so erscheint es immerhin noch nötig, auch auf die Einzelheiten desselben einzugehen, und ich beginne hierbei mit dem Literaturschatze der Hymenopteren. Es erscheint auf den ersten Blick, namentlich in Rücksicht auf die grundlegende Arbeit Dr. A. Hagen's und auf eine vor einem Decennium erschienene Arbeit A. Mocsáry's, sowie auf die beiden erscheinenden Jahresberichte (im Archiv für Naturgeschichte Bd. II. und im Zoological Record) und Carus-Engelmann und Taschenberg's Bibliotheca zoologica (Leipzig, Engelmann $1887 \mathrm{ff}$.) ein nicht sehr schwieriges, vielleicht sogar ein überflüssiges Unternehmen, eine derartige Zusammenstellung zu veröffentlichen. Ein vergleichender Blick auf alle diese Publikationen im $\mathrm{Zu}$ sammenhalte mit dieser meiner Bibliographie genügt wohl vollständig, die Existenzberechtigung derselben vollauf anzuerkennen. Die wenigen nicht persönlich verglichenen Aufsätze sind mit einem Sternchen (*) bezeichnet; Auszüge, Übersetzungen und Kritiken sind stets beim Originale aufgeführt; im Übrigen erleichtern zahlreiche Hinweise den Gebrauch und ergänzen die Citate gegeneinander. Eine schwierige Frage war die Bestimmung der Grenzlinie zwischen den rein wissenschaftlichen, den sog. populärwissenschaftlichen und den rein populären Zeitschriften und Vereinsorganen; ich habe alle in den Jahresberichten aufgeführten Aufsätze verglichen, doch prinzipiell die beiden letzteren Kategorieen yon Journalen auf die hymenopterologischen Artikel nicht durchgesehen. Ebenso glaubte ich die rein forst- und landwirtschaftlichen, sowie die 
Jagrlzeitungen und endlich die russischen und ungarischen Zeitschriften (ohne Revue) mit Ausnahme der rein zoologischen resp). rein entomologischen, dann die Bienenliteratur, sowie die liologische (Blumen und Insekten), forstliche und cecidologische Literatur (Gallen' so kurz als thunlich behandeln zu sollen.

Was den Catalog selbst anlangt, so muss ich vor Allem hervorhehen, dass derselbe lediglich nur die Aufgabe hat, den Wissensstand der bis heute beschriebenen Ilymenopteren-Arten zu bieten. Sellsst beim besten Willen und beim objectivsten Studium war es nur in wenigen Fällen möglich und statthaft, für diesen Zweek zunïchst Kritik zu üben und dadurch gewissermaßen einer Monographie vorzugreifen. Wo es aher nütig erschien, trotzdem kritische Bemerkungen zu machen, erschien es mir zweckilienlich, diesellsen separat unter dem Titel „Hymenopterologische Notizen" in der Wiener entomologischen Zeitung klarzulegen. Für die richtige Begrenzung der Gattungen, Rassen (stirps) und Arten, für die Stellung derselben im Systeme, für die richtige Rinordnung gewisser Arten in eine bestimmte Gattung, für die Auslassung oder Zuziehung gewisser Synonyma u. s. w. darf und muss ich die vor mir arbeitenden Forseher verantwortlich machen, denn für gewisse Fragen entziehen sich deren Beschreibungen und Abbildungen ebenso, wie deren „Typen" unzugänglich oder unzulänglich sind. Dass man aber trotzdem die Arbeiten der älteren Autoren (Linné, Fabricius, Schrank u.s.w.) und die mit ihnen zusammenhängende Tradition etwas freicr beurteilen muss, als jene der neueren, steht mir fest, um so mehr, als ja sonst die ganze Arbeitsleistung der ,V̈̈ter der Entomologie" in tausende von Zweifeln und schlieBlich in Nichts zusammensinken würde. Die Familien, Sul,familien und Genera sind systematisch und zwar in aufsteigender Reihenfolge angeordnet; bei den Tenthrediniden mit Cimbex beginnend, steigen sie, soweit dieser Ausdruck benützt werden darf, zu den Apiden resp. zur Gattung Apis hinan; die neu aufgestellten Genera wurden nach Thunlichkeit conform den dort gemachten Angaben eingereiht; wo aher in der Originalbeschreibung Angaben über ihre Stellung fehlen, dürfte freilich manchesmal ohne Verschulden ein Verstoß in der Einreihung untergelaufen sein.

Die Genera wurden nach dem Priorititsgesetz in der Weise gestellt, dass ein im Gesamtgebiete der Zoologie einmal vorkommender angewandter Name jede zweite Anwendung ausschließt: anderseits aber reicht jede, auch die geringste Formverschiedenheit des Namens im An- oder Auslaute hin, den ähnlichlautenden Genusnamen benutzbar zu machen. Auf den Gienusnamen, der, wo nütig, ausnahmslos etymologisch richtig 
grestellt wurde, was auch stets bemerkt ist (z. B. bei Eriades), folgt das Citat der ersten Anwendung derselben, wobei freilich auf dessen Originalumfang keine Rücksicht genommen werden konnte. Ein zweites ('itat gibt, wenn nötig, den Autor des heutigen G'attungsumfanges: Was Alles verstand Linné, um nur ein Beispiel zu bringen, unter seiner Gattung Apis, die heute doch nur einen ganz kleinen Formenkreis umschließt! Weiter folgt dann fast ausnahmlos die etymologische Ableitung, und ich benütze mit Freuden diese Gelegenheit, um meinem Freunde, Prof. M. Hech fellner, hier den herzlichsten Dank zu erstatten für die collegiale Teilnahme, mit welcher derselbe meine dieshezüglichen Notizen revidirte und mehrfach corrigirte; einige Namen mussten freilich trotz alledem unaufgeklärt bleiben. Da es mir im Allgemeinen richtiger scheint, den Genusbegriff eher im weiteren als im engeren Lmfange aufzufassen, so erscheinen als Erlïuterungen desselben die Synonyma, sowie die Sulgenera, und nur dort, wo neue Monographieen einzelner Gruppen vorlagen, welche einer weiten Gattungstrennung huldigen, machte auch ich von diesem meinem Principe eine Ausnahme, dem Auctor allein die Verantwortung überlassend. Endlich folgen noch jene Gattungsnamen, unter denen die heute einer Gattung substituirten Arten im Laufe der Zeit beschrielien wurden, mit Angabe der hetreffenden Auctoren; dit dieselben Namen meist an anderer Stelle als noch in Verwendung stehend aufgeführt sind, wurde daselbst von der Anführung der Citate und der etymologischen Ableitung Umgang genommen, um nicht das Werk durch verhältnismäßig wenig wichtige Angalen all zu sehr zu vergrößern und zu belasten.

Innerhalb der einzelnen Gattungen folgt das alphabetische Verzeichnis der Arten. Hat bereits schon Br. Harold nachgewiesen, dass bei dessen Catalogus Coleopterorum von einer systematischen Anordnung abgesehen und nur die alphabetische Anordnung gewählt werden musste, so sind alle dort aufgefïhrten Grïnde für diesen Vorgang noch viel zutreffender fïr die IIymenopteren, hei denen die Anzahl unsicher und unzureichend heschrielsener Formen bedeutend größer ist, als bei den ('oleopteren. Natürlich sind auch diese mangelhaft heschriebenen der alphalietischen Aufaihlung einverleibt; um sie aber sofort als der Erklïrung und brliiuterung hedürftig zu kennzeichnen, ist denselhen ein Rufzcichen ! beigesetzt, und es mögen daher künftige Forscher diesen Namen, lieschreibungen und Exemplaren ihre besondere Aufmerksamkeit zuwenden oder sie endlich endgiltig ausscheiden. Wo es thunlich ersehien, wurden auch die Species-Namen etymologisch richtig gestellt; es sind dies zwar nur wenige Fälle, doch erscheint dies geradezu eine Forderung des wissen- 
schaftlichen Anstandes. So ist es z. B. wissenschaftlich gewiss nicht gerechtfertigt, wenn jeder Elementarlateiner hefihigt und - berechtigt ist, Wörter, wie laevis, coelestis, sylvaticus zu corrigiren, ohne dass man in wissenschaftlichen Kreisen je davon Notiz genommen hat, und ihnlich verhält es sich auch mit den Geschlechtsendungen, wo auch alle erdenklichen grammatikalischen Fehler gemacht und fortwähnend colportirt werden. Möge diese Anregung wenigstens auf dem Gebiete der IIymenopterologie nachhaltig einwirken! Ich habe auch für diese formale Seite der Arbeit keine Mühe gescheut und tausende von lexicographischen Angaben verglichen. Auf diese endgiltig nach dem Prioritätsgesetz fixirten Namen folgt die Angabe, welche Sexualformen bis jetzt beschrieben sincl, wolsei natürlich ab und zu noch Mïnnchen und Weibchen einer und dersollien Art unter verschiedenen Namen erscheinen, weil deren sexuelle Zusammengehörigkeit bis jetzt noch nicht erkannt worden ist. Bei den Tenthrediniden und Siriciden, bei welch beiden Familien die Aufzucht der Larven cinen wichtigen Zweig des biologischen Studiums bildet, wurde durch ein Ringelchen $\left(^{\circ}\right)$ auch die gesicherte Kenntnis der Larve angedeutet; ebenso wurde für die Gallen ein hesonderes Zeichen ( $\oplus$ ) heigesetzt. In derselben Zeile folgt weiter noch das geographische Verbreitungsareal der betreffenden Art, eine Angalie, deren Eruirung mir sehr viele Arbeit und Mühe gekostet hat; selbstverständlich wurden jegliche irgendwie Zweifel erregende Notizen von vorneherein ausgeschlossen. Dass ich hierbei die Bezeichnung der ('ontinente beibehielt und dimn, wo es möglich und wertroll war, die politisch alggegrenzten Ländereomplexe von Nord gegen Süd rorsehreitend, aufführte, hat seinen Grund darin, dass mir die Sclater-Wallace'sche Kinteilung der Erdoherflïche dem praktischen Bedlïfnisse weniger entsprechend und auch wissenschaftlich noch nicht vollstïndig und allseitig gesichert und erhürtet scheint. Freilich wïre es ron großem Werte gewesen, die geographischen Angahen, soweit als möglich, durch Autoren zu belegen, allein auch hier musste Maß im liaum gehalten werden; zudem liegen die Quellen dem mit der hymenopterologischen Literatur Vertrauten doch nicht so ferne, wie dies in anderen Disciplinen, z. B. bei den Vügeln der Fall ist. liemerkenswert erscheint nur, dass die Namen der Länder meist lateinisch oder latinisirt, wo dies zu Schwierigkeiten geführt hïtte, in der Originalsprache der Bewohner oder Colonisten angeführt sind.

Die folgenden Zeilen bieten den ('itaten- und synonymenschatz, in welchem eigentlich der Schwerpunkt der ganzen Arbeit liegt. Derselhe citirt, wie schon oben angedeutet, in chronologischer folge ron der ersten 
Beschreilung oder Ablildung oder sellsst namenlosen Anführung der lietreffenden Art alle Beschreibungen derselben, alle Abbildungen, alle auf die Anatomie, Physiologie oder Biologie bezüglichen Stellen, natïrlich mit Ausschluss aller jener, wo dieselbe blos dem Namen nach erwïhnt wircl, wie dies \%. 13. hei faunistischen Listen der Fall ist; wenn jedoch üher das isolirte Vorkommen oder auffallend häufige Auftreten, über Nutzen oder schaden u. s. w. weitliufigere Angalen vorgebracht wurden, wurde auch dieses Citat mit aufgenommen, ebenso wie die älteste auf die betreffende Art mit Sicherheit zu deutende Stelle stets citirt erscheint, sellsst wenn für dieselle noch kein Name oder nur eine Paraphrase beigebracht worden ist; desgleichen sind alle Versetzungen von einem Genus in das andere ersichtlich gemacht. lis gibt somit dieser Citatenschatz wirklich das historische 13ild der Kenntnisnahme der Art und zwar in einer Form, welche durch die zeilenweise Anordnung und durch die Ausführlichkeit ıles Citates sell,st wohl nichts zu wïnschen übrig lässt. Beilüufig sei hier auch erwähnt, dass bei gleichzeitig erschienenen Hinweisen die selbständigen Werke den Zeitschriftenaufsützen vorangehen und dass die Zeitschriften nach dem IIauptschlagworte im 'Titel geordnet sind. Zur leichteren Orientirung wurden die Initialen der germanischen Sprachen groß, jene der romanischen klein geschrieben; bei den im Buchhandel erschienenen und erscheinenden Journalen blieb der Verlagsort und der Redacteur des leichten Wechsels halber gänzlich weg; bei den Gesellschaftsschriften erscheint der Vereinssitz stets vor der Band- resp. Jahreszahl. Die Bandzahl wird stets in römischen, die Ileftzahl in arabischen Ziffern angeführt, letytere jedoch nur dort, wo sie wegen der unregelmäßigen lirscheinungszeit alisolut notwendig ist; ebenso sind auch IIefte, Fascikel, Partes, Livraisons, No. des Aufsatzes u.s.w. nur dann speciell angeführt, wenn dies ron chronologischer Bedeutung ist. Bruchstriche der Bände oder IIcfte deuten auf das gleichzeitige Frscheinen derselhen hin; die Serieszahlen sind in Klammern eingeschlossen; wo aber möglich, wurde ohne Serien fortlaufend nummerirt. Auf die Band-oder Ileftzahl folgt die Jahreszahl und hier sei bemerkt, dass der Jahrgang stets organisch genommen ist, so dass jedes Ileft eines Jahrbuches dem hetreffenden Jahre zugeschrielen wurle, sellist wenn es nach Jahresschluss ausgegehen wurde. $/ \mathrm{u}$ diesem Vorgange Jeitete mich die lirfahrung, dass wenigstens die Separata meistens noch im entsprechenden Jahre ausgegehen werden und dass das verspätete Irscheinen der betreffenden Ilefte meist nur rom '/ufalle abhängig ist; die wissenschaftliche Conception entspricht daher wohl immer dem betreffenden Jahrgange. line Ausuahme musste natürlich dann gemacht werden, wenn 
zwischen dem Datum der Abfassung und dem des Erscheinens ein mehrjühriger Zeitraum verflossen ist, wie dies bei einigen italienischen Journalen z. 13. Atti accad. sc. fis. Napoli) statt hat. In diesem Falle ist die Zeit der wissenschaftlichen Conception resp. Mitteilung durch Vorlage in Klammern, die Zeit des Erscheinens außerhalb derselben gesetzt. Dem gegenüber ist es selbstverständlich, dass bei Zeitschriften, welche heftweise erscheinen und deren Beginn der Bände nicht mit dem Kalenderjahr zusammenfällt, ohne Weiteres dasjenige Jahr angesetzt wird, in welchem der betreffende Aufsatz wirklich erschienen ist. Nach der Jahreszahl wird mit p. die Seitenzahl und mit n. die Nummer der betreffenden Species angegeben; dann folgt noch die event. Angabe des Geschlechtes und wo nötig die Tafel (T.j und die Figur (F.) der Abbildung, oder der bloße Hinweis auf die Nummer der Abbildung im 'Text (Fig.): bei nicht nummerirten Tafeln ist dies durch ein vorgesetztes Tab., bei mit Buchstaben, nicht mit Zahlen bezeichneten Figuren, durch ein beigesetztes Fig. angedeutet. Die sexuellen und agamen Formen der Cynipiden wurden gleich den Rassen (stirpes) der Formiciden je für sich liehandelt und durch Ilinweise als zusammengehörig bezeichnet.

Da das rorliegende Werk den Charakter eines ausgesprochenen Nachschlagebuches für Hymenopterologen hat, so glaubte ich noch ein Besonderes zu thun, indem ich anhangsweise in Fußnoten bei den Phytophagen die Nahrungspflanze der Larven, bei den Parasiten die Wirtstiere, soweit es bekannt geworden, anführte. Da hierbei die Verlïsslichkeit des Auctors von ganz besonderer Bedeutung ist, und auch nur dieser für die betreffende Angabe verantwortlich gemacht werden muss, so wurde stets auch dessen Name in Kilammern aufgeführt; die betreffenden Pflanzen- oder Tiernamen erscheinen aber ohne Berïcksichtigung jeglicher Synonymie mit oder ohne Auctor, genau wie in der Originalmitteilung, um deren Charakter müglichst zu wahren; zu allgemeine Angaben, wie Gattungen von Pflanzen (auf "Weiden") oder Ordnungen von Insekten ',Dipteren“, wurden principiell ausgeschlossen.

Den Schluss eines jeden Bandes bildet ein alphabetisches Register der Genus- und Speciesnamen; bei ersteren wurden auch die Synonyma berücksichtigt, die letzteren sind der Einfachheit halber ohne Rücksichtnahme auf die Gattungen angeführt.

Endlich sei noch hervorgehoben, dass das gesamte Werk mit dem Jahre 1590 abgeschlossen wurde, dass jedoch auch die folgenden Jahresberichte (Zoological Record, Zoologischer Anzeiger u. s. w.) his zur 1)rucklegung für die Feststellung der Synonymic berücksichtigt wurien; 
das Datum des Abschlusses ist bei jedem Bande speciell angegeben. Daturch ist manche mir sehr unangenehme, nichts desto weniger aber unvermeidliche Inconseyuenz zu erklïren und wohl zu entschuldigen. Ehenso dïrfte auch die eine oder andere aufstokende Inconsequenz in der Behandlung dureh die lange Zeitlauer der Abfassung ihre Entschuldigung finden, withrend welcher ich rom Jïngling zum Manne gereift und ab und zu auch meine Ansicht und deren Durchführung zu ändern mich veranlasst sah.

Schließlich erfïlle ich noch die angenehme Pflicht, allen Instituten. an denen ich arbeitete und die mich durch die Vermittelung ihrer reichen Schüize unterstïtzten, inshesondere dem k. k. naturhistorischen IIofmuseum in Wien, der k. k. Universitütslibliothek in Wien, der k. b. IIof- und Statsbiblinthek in München und der k. k. Universititsbibliothek in Innsbruck, sowie meinen zahlreichen Freunden, welche einzelne Partieen des (atalogs zu revidiren die Freundlichkeit hatten, den wärmsten Dank auszusprechen. lihenso sei iler Verlagshuchhandlung. für ihr coulantes Entgegenkommen in jeder Bezichung und für ihre schïne, zweckmilßige typographische Ausstattung auf das Freundlichste gedankt.

Und so hahe ich die leruhigung, mit klarem Blick die Arbeit am lieginne uberschaut, mit gröliter Gewissenhaftigkeit sie durchgeführt und mit hegeisterter Freude sie algeschlossen zu hahen: möge sie nun auch der Wissenschaft jenen Dienst erweisen, für wolchen sie geschaffen wurle!

Innsbruck, im Juli 1591 .

Prof. Dr. K. W. v. Dalla T'orre. 
Herrn

\section{DR. FERDINAND MORAWITZ,}

wirklicher Staatsrath, Vice-Präsident

der russischen entomologischen Gesellschaft in St. Petersburg

gervidmet

vom Verfasser. 



\section{Fam. Vespidae Latr.}

\section{Subfam. Masaridinae}

Smith, Catal. Hymen. Brit. Mus. V. 1857 p. 1.

\section{PARAGIA}

Shuckard, Trans. Entom. Soc. London II. 1. 1837 p. 81. $\pi \ll \varrho r ́ y \omega$, induco.

australis Sauss. - 우 $\tilde{o}$ - Austr.: 'Tasmania.

Paragia australis Saussure, Ann. soc. entom. France (3) I. 15.53 Bull. p. XXI.

Paragia australis Saussure, Étud. fam. Vespid. III. Masar. 1556 p. 57 n. 4. f j ; T. 2 F.2. bicolor Sauss. - o of - Austr.

Paragia bicolor Saussure, Ann. soc. entom. France 3) I. 15533 Bull. p. XXI.

Paragia bicolor Saussure. Etud. fam. Vespid. III. Masar. 1556 p. 55 n. $5, \subseteq$ s; T. 2 F.3. bidens Sauss. - ฮै - Austr.

Paragia bidens Saussure. Étud. fam. Vespid. III. Masar. 1856 p. 59 n. $6, \hat{\jmath}$. calida Smith - ơ - Austr.

Parargia calida Smith, Trans. Entom. Soc. London (3) II. 5.1565 p. 392 n. 11, ô. concinna Smith - 우 - Austr.

Paragia concinna Smith, Trans. Entom. Soc. London 1868 p. 251 n. 3, ㅇ․ deceptor Smith - Austr.

Paragia deceptor Smith, Trans. Entom. Soc. London (3) I. 2.1862 p. 56. decipiens Shuck. $-\subseteq \widehat{o}-$ Austr.

Paracia decipiens Shuckard. Traus. Entom. Soe. I.ondon II. 1. 1537 p. \$2; T. \& F. 3.

Paragia decipiens Saussure, Etud. fam. Vespid. III. Masar. 15.iti p. 5.4 n. 1, S.

Paragia decipiens Smith. Trans. Intom. Soc. Iondon 3, II. 5.1465 p. 391 n. 1, $f \hat{j}$. Paragia decipiens Waterhouse, Trans. Entom. Soc. London 3: V. 1567 Proc. p. I.XXXVIII. Paragia decipiens Kriechbaumer, Stettin. entom. Zeitg. XXXV. 1574 p. 254, f j. excellens Smith - 우 0 - Austr.

Paragia excellens Smith, Trans. Entom. Soc. London $1 \$ 69$ p. 309 n. 1, S oै. morosa Smith $-\&-$ Austr.

Paragia morosa Smith, Trans. Entom. Soc. London 1868 p. 251 n. 2, ㅇ․ nasuta Smith - \& - Austr.

Paragia nasuta Smith, Trans. Entom. Soc. London 1868 p. 252 n. 4, \&. odyueroides Smith - ot - Austr.

Paragia odỵneroides Smith. Trans. Entom. Soc. London 2: I. 2.1 h.50 p. 41 n. 2, 3ै; T.5 F.2. Paragia odỵneroides Saussure. Kitud. fam. Vespid. IlI. Masar. 14.56 p. 56 n. 4.3. pietifrons Smith $-\rho-$ Austr.

Paragia pictifrons Smith, Catal. Hỵmen Brit. Mus. V. 1557 p. 2 n. 9. S.

Catalogus Hymenopterornm. IX. 
praedator Sauss. $-\circ-$ Austr.

Paragia praclator Saussure, Ftud. fam. Vespid. III. Masar. 15.56 p. 59 n. $7 . \subseteq$. saussurei Smith - 우 - Austr.

Paragia tricolor Smith. Trans. Fintom. Soc. London 2) I. 2. 15.50 p.41 n.1. .; T.5 F.1 excl. j.

Paragia tricolor Saussure, Etud. fam. Vespid. III. Masar. 1456 p. 54 n. 2. ᄃ.

Paragia Saussurii Smith, Catal. Hymen. Brit. Mus. V. 1857 p. 2 n. 3.

P’aragia Saussurii Smith, Trans. Fintom. Soc. London 3 II. 5.156 .5 p. 392 n. 3.

solvrina Smith - 으- Austr.

Paragia sobrina Smith, Trans. Entom. Soc. London 1869 p. 309, ㅇ․

tricolor Smith - 우 ơ - Austr.

Paragia tricolor Smith, Trans. Entom. Sue. Lomdon 2 I. 2. is, 10 p. 41 n.1, Jै; T.5 F.1a excl. $\subseteq$.

Paragia Smithii Saussure, Eitud. fam. Vespid. III. Masar. 1556 p. 55 n. $3 . \subseteq 3$; T. 2 F. 1 I.

Paragia tricolor Smith, Trans. Entom. Soc. Jondon (3, II. 5. 1 S(i5) p. 392 n. 2.

Paragia tricolor Smith, Trans. Entom. Soc. London 1569 Proc. p. XVII.

Paragia tricolor Wilson, Trans. Entom. Soc. London 18699 Proc. p. XVIII.

Paragia trieolor Kricchbaumer, Stettin. entom. Zeitg. XXXV. 14.4 p. $2: 1$.

venusta Smith - 우 - Austr.

Paragia venusta Smith, Trans. Entom. Soc. London "3, II. 5. 15155 p. 393 n. 12. S. respiformis Smith - 우

Paragia respiformis Smith. Trans. Entom. Soc. London 3. II. 5. 156.5 p. 393 n. 13. S.

Paragia respiformis Smith, Trans. Entom. Soc. London 1564 p. 250 n. $1, j$.

Paragia vespiformis Smith, Trans. Entom. Soc. London 1869 p. 310, วै; T. 6 F. 2.

Paragia respiformis Smith, Brenekley: Cruise of the Curaçoa 1573; T. 45 F. 2.

\section{EUPARAGIA}

Cresson, Trans. Amer. Entom. Soc. VII. 1879 Proc. p. VI.

$\varepsilon \tilde{v}$, bene; Paragia, nom. propr. Hymen.

sentellaris Cress. - o ơ - Am.: Nevada.

Euparagia scutellaris Cresson. Trans. Amer. Fintom. Soc. VII. 14i9. Proc. p. VI. $\Omega$;.

\section{CERAMIUS}

Latreille, Consid. génér. 1810 p. 329 n. 499.

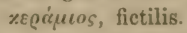

Synon.: Gnatho Klug, Magaz. Ges. naturf. Fr. Berlin IV. 1510 p. 36 mec Illiger $1-4$ i .

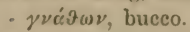

M as aris Blanchard.

Sul?!.: Ceramioides Saussure. Eitud. fam. Vespid. III. Masar. 1456 p. 71.

Ceramius, nom. propr. Hymen; Eiरos, forma.

Paraceramius Saussure, Étud. fam. Vespid. III. Masar. 1856 p. 67.

acẹcé, ad; Ceramius, nom. propr. Hymen.

eaif'er Sauss. - 우 - Afr. : Cap.

Ceramius caffer Saussure, Etud. fam. Vespid. 1II. Masar. Lhiti p. iti n. 11. L.

eapensis Sauss. - - - Afr.: Cap.

Ceramius Capensis Saussure, Ctud. fam. Vespid. III. Masar. 14.51; p. liti n. 3. S: 'T. 3 F. 3. canensicus André - ơ - As.: Caucasus.

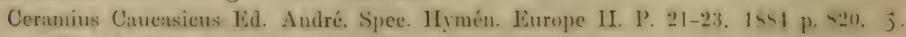
cerceriformis Sauss. - of - Afr.: Cap.

Ceramius eereeriformis Saussure. Ann. see entom. France :3/ I. 1853 IBull. p. XXI. 3.

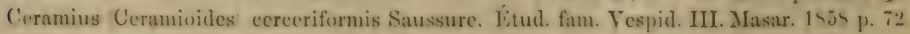
[n. 8, ฮี; 'T. 4 F. .. 
consobrinus Sauss. - - - Afr. : Cap.

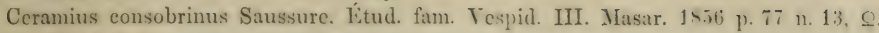
fonseolombei Latr. - 우 ô - Eur. mer.; Afr.: Algeria.

Ceramius Fonscolombei Latreille, Consid. génér. 1810 p. 329 \& 438.

Ceramius Fonscolombii Klug, Entom. Monogr. 1824 p. 229 n. 3.

Ceramius Fonscolombei Fonscolombe. Ann. soc.entom. France IV. 1435 p.421-427; T.10 F. A.

Ceramius Fonscolombei I.epeletier. Hist. nat. Inseet. Hỵmén. II. 15.11 p. 549 . 3 j.

Ceramius Oraniensis Lepeletier, Hist. nat. Inseet. Hymén. II. 14.11 p. 591 , f ; nec Sauss. .

Ceramius Fonscolombei Guérin. Iconogr. règn. anim. VII. Insect. 14.45 p. 446; T. 72 F. 2. C'cramius Oraniensis Lucas, Explor. sc. Algéric. Zool. III. 1846 p. 225 n. 176 . f $\hat{\jmath}$.

Masaris Ceramius) Fonscolombei Blanchard, Cuvier: Règne anim. Ėd.3a Insect. II. 1 \$4!; Ceramius Fonscolombei Saussure. Ann. soc. entom. France (3) I. 1 s53 p.XXI. [T.123 F.11). Ceramius Fonscolombii Saussure, Iitud. fam. Vespid. III. Masar. 18.56 p.1;2 n.1. $\subseteq$ ơ; T.2 F. 1 . Ceramius Fonscolombei Lichtenstein, Ann. soc. entom. France (5; VIII. 18i $\mathrm{s}$ Bull. p. ('VII. Ceramius Fonscolombei Iichtenstein. Ann. soc. entom. Franee (5) IX. 1879 p. 44 n. i. Ceramius Fonscolombei Ed. André, Spec. Hỵmén. Europe II. P. 21-23. 159.1 p. s1 i. S j. koreensis (Rad.) D. T. - $\subseteq-$ As.: Korea.

Paraceramius Koreensis Radoszkowski. Horae soc. entom. Ross. XXI. 1 ssi p. 433. ᄃ Ceramius Koreensis m.

lichtensteinii Klug - - - Afr.: Cap.

Gnatho Lichtensteinii Klug. Magaz. Ges. naturf. Fr. Berlin IV. 1s10 p. 36; T. 1 F. 3! Ceramius Lichtensteinii Klug, Entom. Monogr. 1824 p. 225 n. 1.

C'eramius Lichtensteinii Saussure. Etud. fam. Vespid. III. Masar. 1s5̆ł p. 73 n. 9. $\subseteq$.

linearis Klug - ô - Afr.: Cap.

Ceramius lincaris Klug, Entom. Monogr. 1824 p. 227 n. 2, ô.

('cramius (Paraceramius linearis Saussure. Citud. fam. Yespid. III. Masar. 1 4., 6 p p.71 n. $\mathbf{i}$. j

Iusitanieus Kklug - $\subseteq \hat{\jmath}$ - Eur.: Lusitania. Hispania, Gallia. Austria inf.

Ceramius Lusitanicus Klug, Entom. Monogr. 1824 p. 230 n. 4.

Ceramius Fonseolombii var. Fonscolombe, Ann. soc. entom. France IV. 1535 p. 42خ.

Ceramins tubereulifer Saussure, Ann. soc. entom. Franee 3) I. 1553 p. XXI.

Ceramius (Paraceramius) Lusitanieus Saussure, Ltud. fam. Vespid. III. Masar. 1ৎ.j(i p. 69 [n. 5 , 워; T. 3 F, 6 .

C'eramius Iusitanicus Giraud. Ann. soc. entom. France (j) I. 1871 p. 375-379. 5 s.

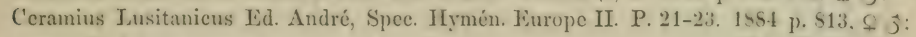
macrocephalns Saussure - - - Afr.: Cap.

['T. 45 F. $5,8,9,10$. Ceramius macrocephalus Saussure. Ftud. fam. Vespid. III. Masar. 1556 p. 76 n.12, S; T.3 F.2. nigripennis Sauss. - ㅇ - Afr.: Cap.

Ceramius Paraceramius nigripennis Saussure. Kitnd. fam. Yespid. III. Masar. 1 sti p.ti! n. fi. oramiensis Sauss. - 우 ơ - Afr.: Algeria.

[Q; T. 3 F.4.

Ceramius Oraniensis Saussure. Kitud. fam. Vespid. III. Masar. 1556 p. 65 n. 2. $\subseteq 3$ [nee Lep.]. Ceramius Doursii Ed. André. Spee. Ifymén. Europe II. P. 21-23. 154 p. 41 4 , -3. rex Sauss. - 우 - Afr.: Cap.

Ceramius rex Saussure, Étud. fam. Vespid. III. Masar. 1856 p. 75 n. 10, S.

spiricornis Sauss. - 워 - Eur.: Hispania, Gallia.

Ceramius spirieornis Saussure. Amn. soe. entom. France 33 I. 14.33 p. XXI. T.3 F... Ceramius Paraceramius spiricornis Saussure, Ritud. fam. Vespid. III. Masar. 1456 p.67 n.4. S:

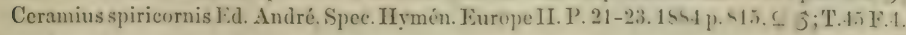

respiformis Sauss. - ㅇ - Afr.: Cap.

Ceramius respiformis Saussure. Litud. fam. Tespid. III. Masar. 1596 p. 7! n. 11. ‥ 


\section{MASARIS}

Fabricius, Entom. system. II. 1793 p. 194 \& 283 n. 152.

Masaris, nom. propr.

Synon.: Ceramius Klug.

Eurynnis Saussure.

edwardsii Cress. - 우 - Am. : Californien.

Masaris Edwardsi Cresson. Trans. Amer. Entom. Soc. IV. 1572 p. $57, \subseteq j$.

marginslis Cress. - $q-$ Am.: Colorado.

Masaris marginalis Cresson. Proe. Entom. Soc. Philadelphia III. 156.4 p. 67\%. C.

Masaris marginalis Saussure, Smithson. Miscell. Collect. XVI. P. 25.1. 1575 p. 10 n. 3. $\subseteq$. oceidentalis Cress. - 우 $\hat{o}-$ Am.: Texas.

Masaris occidentalis Cresson. Trans. Amer. Entom. Soc. III. 1871 p. 345 . C.

Masaris vecidentalis Cresson. Trans. Amer. Entom. Soc. IV. 1572 p. 231. $\mathfrak{\jmath}$.

spinolae Sauss. - 우 - Afr.: Cap.

Masaris Spinolae Saussurc, litud. fam. Vespid. III. Masar. 1956 p. 95 n. 2. ᄃ.

texanus Cress. - 우 0 - Am.: Texas.

Masaris Texanus Cresson. Trans. Amer. Entom. Soc. III. $1 \varsigma ; 1$ p. $34 \subseteq, \subseteq j$.

respiformis Fabr. - $\hat{o}$ ㅇ - Afr.: Aegyptus, Algeria.

Masaris vespiformis Fabricius, Entom. system II. 1793 p. 283 n. 1 !

Masaris respiformis Coquebert. Illustr. iconogr. Insect. II. $15(11$ p. 62 ; T. 15 F. $4 / \vec{J}$.

Masaris vespiformis Latreille, Hist. nat. Crust. \& Insect. III. 1502 p. 368.

Masaris vespiformis Fabricius, Syst. Piez. 1804 p. 292 n. 1.

Masaris vespiformis Latreille, Hist. nat. Crust. SInsect. XIII. 1S0; ], 35:3; T. 102 F. 5.

Masaris respiformis Klug, Magaz. Ges. naturf. Fr. Berlin II. 1507 p. 52 n. 61.

Masaris vespiformis Latreille, Gen. Crust. \& Insect. IV. 1809 p. 144.

- Savigny, Descr. de l'Égypte. Hymén. 1812 ; T. 9 F. 18.

Masaris vespiformis Lamarek, Hist. nat. anim. s. vert. IV. 1817 p. 81 n. 1.

Ceramius hylaciformis Klug, Entom. Monogr. 1824 p. 230 n. 5.

Masaris vespiformis Iamarck. Hist. nat. anim. s. vert. Ed. $2^{2}$ IV. 1435 p. 295 n. 1.

Masaris respiformis Blanchard, Hist. nat. Insect. III. 1840 p. 387.

Masaris respiformis Lepeletier. Hist. nat. Insect. IIymén. II. $1 \varsigma 41$ p. $5 \$ 9$ n. $1.3 \hat{\jmath}$ !

Masaris respiformis Lucas. Explor. se. Algérie. Zool. III. 1 4.46 p. 225 n. 175.

Masaris vespiformis Blanchard, Cuvier: Regne anin. Ed. $3^{3}$ Insect. II. 1549 'T. 123 F. `.

Masaris respiformis Romand, Ann. soc. entom. France (2) IX. 1551 Bull. p. I.II, $\Omega$ (exel. 3 i.

Masaris respiformis Dufour, Ann. soc. entom. France $2 ;$ X. 1852 Bull. p. I.XXXVI.

Masaris respiformis Sehaum, Ann. soc. entum. France 2) X. 1552 Bull. p. I.XXXVI-

[LXXXVIII, ð̊(ด).

Masaris respiformis Lucas, Amn. soc. entom. France 2) X. 14.52 Bull. p. X('IV.

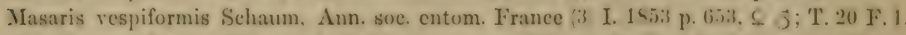

Masaris vespiformis Blanchard, Ann. soc. entom. France (3) I. 1 s53 Bull. p. VI-VII.

Masaris vespiformis Fairmaire, Ann. soe. entom. France ;3] I. 16.53 Bull. p. XVI-XVII.

Masaris respiformis Saussure, Ann. soe. chtom. France (3) I. 1553 13ull. p. XIX.

Erymis Romandi Saussure, Ann. soc. cntom. France 3 I. hais 13ull. p. XIX n. 1, c.

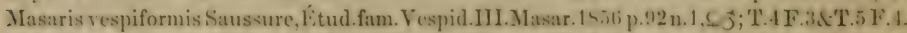

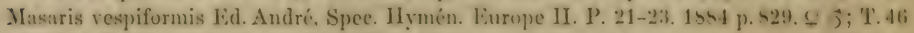
vespoides Cress. - $\rho$ of - Am.: Colorado.

[F. $2-7$.

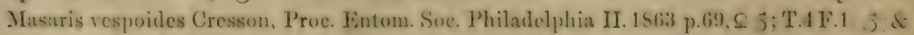

Masaris respuides ('resson, Proc. Fintom. Soe. Philalelphia III. 1561 p. 673. \& 3. 2 S.

Masaris vespoides Saussure. Smithson. Miscell. Collect. XIV. P. 2.4. 1475 p.5 n.1.5.3. 
zomalis Cress. - 오 $\widehat{0}-$ Am.: Colorado.

Masaris zonalis Cresson. Proc. Entom. Soc. Philadelphia III. 1564 p. $674 . \subseteq j$.

Masaris zonalis Saussure. Smithson. Miscell. Collect. XIV. P. 254. 1575 p. 5 n. $2, \subseteq 3$.

\section{TRIMERIA}

Saussure, Étud. fam. Vespid. III. Masar. 1854 p. 81.

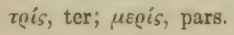

Synon.: Erỵnn is Saussure, Ann. soc. entom. France (3) I. 1953 Bull. p. XXII. [nee Sehrank Nom. propr.

[1801].

americana Sauss. - - Am.: Brasilia.

Masaris respiformis Romand, Ann. soc. entom. France (2) IX. 1 S51 Bull. p. LII.

Erynnis Americana Saussure, Ann. soc. entom. France (3) I. 1553 Bull. p. XX n. 2, s. Trimeria Amerieana Saussure, Étud. fam. Tespid. III. Masar. 1856 p. \$1. \&; T. 4 F. 2.

Trimeria Americana Saussure, Smithson. Miseell. Collect. XIV. P. 254. 1575 p. 4 n. 1.

\section{JUGURTHIA}

Saussure, Étud. fam. Vespid. III. Masar. 1854 p. 83 [Jugurtia].

Jugurtha, nom. propr.

Synon:- Celonites Lepeletier \& auct.

chlorotiea Mor. - 우 - As.: Transcaspia.

Jugurtia chlorotica Morawitz, Horae soc. entom. Ross. XXII. 1958 p. 270, c.

numida Sauss. - ฮૈ - Afr.: Algeria.

Jugurtia Numida Saussure, Étud. fam. Vespid. III. Masar. 1856 p. 85 n. 2, วิ; T. 5 F. 3.

Jugurtia Numida Ed. André, Spec. Hymén. Europe II. P. 21-23. 1854 p. 82.2, วิ .

oraniensis (Lep.) Sauss. - o ơ - Eur.: Hispania; Afr.: Algeria.

Celonites Oraniensis Lepeletier, Hist. nat. Insect. Hymén. II. 15.11 p. 556 n. 2 . $\varsigma$ j!

Celonites Oraniensis Lucas, Explor. sc. Algérie. Zool. III. 1946 p. 227 n. 178, f ơ; 'T.10 F. 12 !

Celonites Oraniensis Dufour, Ann. soc. entom. France (2) IX. 1851 p. 58 n. 4.

C'clonites dispar Dufour, Ann. soc. entom. France 2) IX. 1851 p. 55 n. 3, \& fే; T. 3 F. I!

Celonites dispar Romand, Ann. soc. entom. France 2) IX. $1 \$ 51$ 3ull. p. LII.

Celonites dispar Schaum, Ann. soc. entom. France 2 X. 1452 Bull. p. LXXXYI.

('elonites Oraniensis Saussure, Ann. soc. entom. Franec 3) I. $145 \overline{3}$ Bull. p. XIX.

Jugurtia Oraniensis Saussure, Kitud. fam. Vespid. III. Masar. 1456 p. S4 n. 1, $\leq$ j.

Jugurtia Oraniensis Ed. André, Spec. Hymén. Europe II. P. 21-233.1484 p. $\$ 21$, ऽ 3ै; T.4. F.2

is 6 .

\section{CELONITES}

Latreille, Hist. nat. Crust. \& Insect. UI. 1802 p. 368 [cm.]. *îj.

Synon.: Chrysis Rossi.

Cimbex Olivier.

Masaris Fabricius, Panzer.

Vespa Villers.

abbreviatus (Vill.) Sauss. - o o $\bigcirc$ - Eur. mer.; Afr.: Algeria.

Vespa abbreviata Villers, C. Linnaei Entom. III. 1789 p. $2 \$ 1$ n. 38.

Chrysis dubia Rossi, Fauna Etrusca II. 1790 p. 77 n. 849 ; T. 7 F. 10 \& 11.

Cimbex vespiformis Olivier. Eneyel. method. Inseet. V. 1790 p. 772 n. 16 . 
Masaris apiformis Fabricius, Entom. system II. 1793 p. 284 n. 2.

Masaris apiformis Panzer, Faun. Inseet. German. VII. 1801 P. 76 T. 19.

Celonites apiformis Latreille, Hist. nat. Insect. III. 1802 p. 368.

Celonites apiformis Fabricius, Syst. Piez. 1804 p. 292 n. 1.

C'clonites apiformis Latreille. Hist. nat. Crust. d Insect. XIII. 1 su5 p. 3.44 ; T. 103 F. 2. C'elonites apiformis Panzer, Krit. Revis. II. 1806 p. 161.

Celonites apiformis Spinola, Insect. Ligur. I. 1. 1806 p. 91 n. 1.

Chrysis dubia llliger. Russi: Fauna Etrusea Kid. 2 II. 1407 p. 124 n. 54 !! ; T. i F. 10 \& 11.

Masaris apiformis Jurine. Nour. méth. class. Hymén. 1507 p. 151, O 3; T. 10 F.17.

C'elonites apiformis Klug. Magaz. Ges. naturf. Fr. Berlin II. 1407 p. 52 n. 62.

Celonites apiformis Latreille, Gen. Crust \& Insect. IV. 1809 p. 145.

C'elonites apiformis Klug. Germar: Reise n. Dalmat. II. 1517 p. 264 n. 372.

Masaris apiformis Lamarck, Hist. nat. anim. s. rert. IV. 1817 p. 81 n. 2.

Celonites vespiformis Lepeletier, Encycl. méthod. Insect. X. 1825 p. 574.

Masaris apiformis Iamarck. Hist. nat. anim. s. vert. Fid. 2 a IT. 1 s.35 p. 29 n n. 2.

Celonites apiformis Blanchard, Hist. nat. Insect. III. 1840 p. 387.

C'elonites apiformis I.epeletier. Hist. nat. Insect. Hymén. II. 1541 p. 55 i n. 1 . C.

Celonites apiformis Guérin. Ieonogr. règn. anim. VII. Insect. 15.45 p. $445 ;$ T. 72 F. 1.

Masaris C'elonites apiformis Blanchard. Cuvier: Rign. anim. Ed. 3 n Insect. II. 14.4!; T. 123

Celonites apiformis Dufour. Ann. soc. entom. France 2 IX. 1551 p. 55 n. 1. T.9.

Celonites apiformis Saussure, Ann. soc. entom. France (3; I. 1453 Bull. p. XIX.

Masaris apiformis Schenck, Jahrb. Ver. Naturk. Nassau IX. 1. 19.93 p. 46. ᄃ.

('elonites abbreriatus Saussure. Eitud. fam. Vespid. III. Masar. 1556 p. «n. 1, $\subseteq$ J; T. 5 F 1.

Celonites abbreviatus Schenck, Jahrb. Yer. Naturk. Nassau XVI. 1561 p. 59 n. 1, $\subseteq 3$.

('elonites abbreriatus Imhoff. Mittheil. schweiz. entom. Ges. I. 4. 14633 p. 91, C.

Masaris apiformis Taschenberg, Hymen. Deutschl. 1866 p. 248, $\Omega$ ô.

C'clonites apiformis Iichtenstein. Ann. soc. entom. France (4) IX. 1Stiy Bull. p. I.XXII. Li.

C'clonites apiformis Iichtenstein. Ann. soc. entom. Franec 4) IX. 1569 Bull. p. XXIX. O.

Celonites apiformis Lichtenstein. Ann. soc. entom. France 5) V. 1 55 p. CCXS.

Celonites abbreviatus Rudow; Arch. Ter. naturf. Fr. Mecklenburer XXX. 14iti p. 2:30.

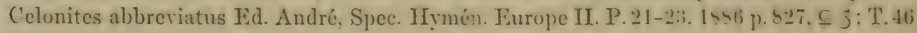

Celonites abbreriatus Rudow, Soc. entom. III. 1889 p. 170.

[F. $1,8,9,10,11$.

var. hungarieus Mocs. - - of - Eur.: Hungaria.

Celonites abbreviatus var. Ifungaricus .Ioesíry. Termész. Füzet. I. 1577 p. 90 n. $6 . \subseteq \vec{j}$.

crenulatus Mor. - 으 - As.: Transcaspia.

Celonites crenulatus Morawitz. Horae soe. entom. Ross. XXII. 1545 p. $26 \%$.. .

eyprins Sauss. - ‡゙-As.: Cypria.

[p. 4 n. 1, తే.

C'elonites Cyprius Saussure. Mém. soc. phṛs. of hist. nat.Geneve XIV.1. 15.44 [Mél. hỵmen. I.]

C'clonites Cyprius Iid. André, Spee. Hymén. Iturope II. 1. 21-2:3. 1444 p. -29. 3.

flscheri Spin. - 오 of - Eur.: Gallia; Afr.: Algeria.

- Savigny, Deser. de l'Égypte 1812 ; T. 19 F. 9.

Celonites Fischeri Spimola, Ann. soe. entem. France VII. 1 -34 p. 50.5 n. XI.IX. S.

Celonites afer Lepeletier, Hist. nat. Insect. Hymén. 1841 p. 555 n. 1, \&.

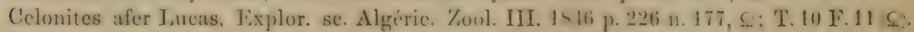

Celonites Fischeri Dufour, Ann. soc. entom. France (2) IX. 1851 p. 58 n. 2.

Celonites afer Dufour, Ann. soc. entom. France (2) IX. 1851 p. 58 n. 5.

Celonites afer Saussure, Ann. soc. entom. France (3) I. 1853 Bull. p. XIX.

('elonites Fischeri Saussure, Citud. fam. Vespid. III. Masar. 145it p. 49 n. 2., 9 .

('elonites Fischeri Lichtenstcin. Ann. soe. entom. France 5) V. 1475 Bull. p. C'CXI.

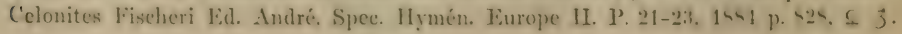


mongoliens Mor. - $ᄋ$ ô - As.: Mongolia.

('elonites Mongolieus Morawitz. Horae soc. entom. Ross. XXIII. 1s4! p. $166 \mathrm{n} .1 \mathrm{~s}, \subseteq \mathrm{\jmath}$.

ossens Mor. - - As.: Transcaspia.

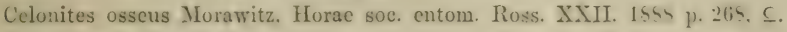

sariguyi Sauss. - 우 0 - Afr.: Aegyptus.

- Sarigny, Deser. de l'Égypte 1812; T. 9 F. 19 (우 ô).

C'elonites Sarignyi Saussure. Etud. fam. Vespid. IJI. Masar. 1576 p. 91 n. 3.

Celonites Savignyi Smith, Catal. Hymen. Brit. Mus. V. 1857 p. 7 n. 3.

\section{QUARTINIA}

Gribodo) Ed. André. Splec. Hymén. Europe II. P. 21-23. 1554 p. 511 \& 422 n. 3; T. 45. Quartinia, nom. propr.

dilecta André - $\underline{o}$ - Afr.: Tunisia.

[T. 45 F. 7.

Quartinia dilecta Gribodo Ed. André. Spec. Hrmén. Europe II. P. 21-23. 1554 p. \$24. \& 5;

\section{Subfam. Eumenidinae}

Westwood, Introd. Classif. Insect. II. 1810 Synops. p. 83.

\section{RHAPHIDOGLOSSA}

S. S. Saunders. Trans. Entom. Soe. I.ondon 2. I. 3. 15.j1) p. 71 (Rhaphiglossa .

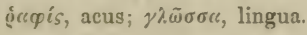

eumenoides Saund. - $-\widehat{\jmath}-$ Eur.: Albania.

[\& 72 n. 1, 우후 T. 5 \& 6 F. 4 .

Rhaphiglossa eumenoides S. S. Saunders. Trans. Fntom. Soc. London (2 I. 3. 1 s.51) Proce p. 17 Rhaphiglossa eumenoides Saussure. Étud. fam. Vespid. I. Eumén. 15.52 p. 2 n. 1. $\subseteq$ j. Rhaphiglossa eumenoides S. S. Saunders. Trans. Entom. Soe. London 1573 p. 40 T.

Jhaphiglossa eumenoides Ed. André. Spec. Hymén. Europe II. P. 20. 1544 p. 610 n. 1. S j;

[T. 41) F. 17.

flliformis Sauss. - ô - Afr.: Algeria.

[T. 8 F. 1 (@).

Rhaphlighlossa filiformis Saussure. Ftud. fam. Vespid. I. Fumén. 1552 p. 3 n. $3 . \subseteq$ exel. t)

Rhaphiglossa filiformis Ed. André. Sprec. IIymén. Europe II. P. $20.1<54$ p. 612 n. 3. ऽ. natalensis Smith - $0-$ Afr.: Port Natal.

Rhaphiglossa Natalensis Smith. ('atal. Hymen. Brit. Mus. V. $14 . \overline{7}$ p. \& n. 5.6 .

symmorpha Sauss. - 우 - Afr: Algeria.

Rhaphiglossa filiformis Saussure, Ktud. fam. Vespid. I. Gumén. 15.5 p. 3 n. 3, Q nee. 3 . Rhaphiglossa symmorpha Saussure. Eitud. fam. Vespid. I. Eumén. 14;2 p. 279, ऽ; T.4 I: 2. Rhaphighossa simmorpha Ed. André. Spee. Hymén. Europe II. P. 20. 1594 p. 612 n. 4. c. Rhaphiglossa symmorpha Magretti, Ann. mus. civ. Genova XXI. 14ab p. likn n. 111.

zethoides Sauss. \& - Afr.. Algeria.

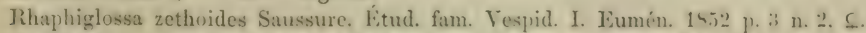

Rhaphiglossa zethoides E.d. André, Spee. Hỵmén. Furnepe II. Y. 20. 1 not p. 511 n. 2. L. 


\section{PSILOGLOSSA}

S. S. Saunders, Trans. Entom. Soc. London 1873 p. 405 (Psiliglossa:

$\psi \iota \lambda . o ́ s$ nudus; $\gamma \lambda \tilde{\omega} \sigma \sigma r e$ lingua.

Siynom.: Stenoglossa Saussure. Fitud. fam. Vespid. I. Eumén. 1552 p. 4 ; T. 2 I. 2.

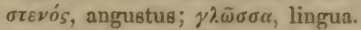

Rhaphigloss a S. S. Saunders.

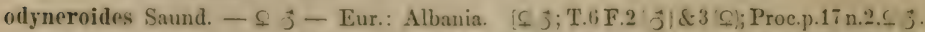
Rhaphiglossa odyneroides S. S. Saunders. Trans. Entom. Soe. London (2) L.3. 15.50 p. 72 n. 2. Stenoglossa odyneroides Saussure, Etud. fam. Vespid. I. Eumén. 1552 p. 5 n. 1. ㅇ.

Psiliglossa odyneroides S. S. Saunders. Trans. Entom. Soc. London 1573 p. 407.

Psiliglossa odyneroides Ed. André. Spec. Hymén. Europe II. P. 20.1854 p. 1515 n. 1.50.

\section{GAYELLA}

Spinola, Gay: Hist. fis. Chile. Zool. VI. 1851 p. 328 n. 3.

Gay, nom. propr.

eumenoides Spin. - 우 ơ - Am.: Chile.

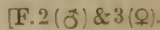

Gayella eumenoides Spinola, Gar: Hist. fis. Chile. Zool. VI. 1551 p. 333 n. 1, e 3; T. 2

Gayella eumenoides Saussure, Kitud. fam. Vespid. I. Eumén. 1 552 p. 6, \&5; T. S F. 4 is.

Gayella eumenoides Saussure. Smithson. Misecll. C'ollect. XIV. P. 254. 1575 p. 13 n. 1. mutilloides Sauss. - - - Am.: Chile.

Gayella mutilloides Saussure, Étud. fam. Vespid. III. Masar. 1856 p. 114 n. 1. ؟. pulchella Smith - 우 - As.: Borneo.

Gayella pulchella Smith, Journ. of Proc. Linn. Soc. Zool. II. 1857 p. 1 US n.1.S;T.2 F.9-11.

\section{ELIMUS}

Saussure, Iitud. fam. Vespid. I. Eumén. 1852 p. 1 n. 4 ; T. 3 F. 1.

Nom. propr.

anstralis Sauss. - ఫิ - Austr.

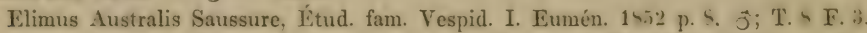

Elimus Australis Maindron, Ann. soc. entom. France (6; II. 1592 p. 267 n. 1.3.

\section{LABUS}

Saussure, Reise d. Novara. Zool. II. 1. 1867 Hymen. p. 3.

Nom. propr.

floricola - - - Afr.: Madagascar.

Labus floricola Saussure. Grandidier: Hist. Madagascar XX. P.1. 1 591 p.151.S:T. 2.2 F.:. humbertianus Sauss. $-q$ ơ - As.: Ceylon.

J.abus Humbertiamus Saussure, Reise d. Novara. Zool. II.1. 1 s67 Hymen. p.4n.2. $\subseteq$ j; 'T.1 F.2. sicheliauns Saurs. - Q - Am.: Chile.

['T. $4 \mathrm{~T}, 20 \mathrm{~d}: 20^{3}$.

Tabus Sichelianus Saussure, Smithson. Misecll. Collect. XIV. P. 254. 1475 p.57 n. 1. L : spiniger Sauss. - ఫै- As.: Java.

I.abus spliniger Saussure, Reise d. Fovara. Zool. II. 1. 1 sti7 Hỵmen. p. 1 n. 1. 3: T. 1 F. 1.

\section{SMITHIA}

Saussure, Rer. \& mag. zool. (2) VII. 1555 p. 371.

Fr. Smith, nom. propr.

natalensis Sauss. - $\delta-\Lambda f r .:$ Port Natal.

Smithia Natalensis Saussure, Rev. \& mag. zool. (2) VII. 1555 p. 371, ô. 


\section{ZETHUS}

Fabricius, Syst. Piez. 1804 p. 2 S2 n. 51.

Zethus, nom. proprr.

Synon.: A p is Linné, Olivier, Christ.

Epipona Spinola.

Eumenes Latreille.

Polistes Fabricius.

Vespa Fabricius \& auct. ant.

Suby.: Calligaster Saussure, Etud. fam. Vesplid. I. Eumén. 1552 p. 22.2.

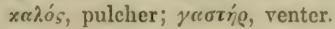

Didymogastra Perty, Delect. anim. artic. Brasil. 1833 p. 145. Sisvuos, duplex: $\gamma \alpha \sigma \tau \dot{r} \varrho$, venter.

Heros Saussure, Étud. fam. Vespid. III. Masar. 1856 p. 15. n’oos.

Zethuseulus Saussure, Étud. fam. Vespid. I. Eumén. 1 5.52 p. 15 \& III. Masar. $14.51 ;$ Zethus, nom. propr. Hymen. dimin.

[p. 118.

albopictus Smith - 우 - Am.: San Domingo.

Zethus albopictus Smith, Catal. Hymen. Brit. Mus. V. 1957 p. 15 n. 32, f.

Zethus albopictus Saussure, Smithson. Miscell. Collect. XIV. P. 25.4. 1475 p. 43 n. 32, c.

arictis (Fabr.) Klug - of 우 - Am. mer., Brasilia.

Vespa arietis Fabricius, Syst. entom. 1775 p. 373 n. 47.

Vespa arietis Fabricius, Spec. Insect. I. 1781 p. 469 n. 66.

Vespa arietis Fabricius, Mant. Insect. I. 1787 p. 294 n. 80.

Vespa arietis Gmelin, Linné: Syst. nat. Ed. 13a I. 5. 1790 p. 2755 n. 64.

Vespa arietis Olivier, Encycl. méthod. Insect. VI. 1791 p. 676 n. 32.

Vespa arietis Fabricius, Entom. system. II. 1793 p. 282 n. 101.

Polistes arietis Fabricius, Syst. Piez. 1804 p. 280 n. 50.

Vespa arietis Jurine, Nouv. méth. class. Hymén. 1807 p. 170, đ.

Zethus arietis Klug, Magaz. Ges. naturf. Fr. Berlin II. 1807 p. 52 n. 58.

Zethus arietis Saussure, Kitud. fam. Vespid. I. Eumén. 1552 p. 14 n. 11.0 ô.

Zethus arietis Saussure, Rev. \& mag. zool. (2) X. 1858 p. 64, ơ. [n. 6, § ô.

Zethus Zethusculus, arietis Saussure, Smithson. Miseell. Collect. XIV. P. 254. 14i5 p. 19

aurulens Sauss. - $\widehat{\sigma}-\mathrm{Am}$. Brasilia.

[T.6 F.5.

Zethus Didymogastra aurulens Sausiure, litud. fam. Vespid. III. Masar. 1556 p. 121 n.s, 3; ;

Zethus (Didymogastra, aurulentus Saussure, Smithson. Miscell. Collect. XIV. P. 254. 1575

aztecus Sauss. - - - Am.: Mexico.

[p. $48 \mathrm{n} .41$.

Zethus Zethuseulus Aztecus Saussure, Rer. \& mag. zool. 2. IX. 1557 p. 270.

Zethus (Zethuseulus) Aztecus Saussure, Smithson. Misecll. Colleet. XIV. P. 254. 1575 p.36

biglumis Spin. - 우 - Am.: Cayenne.

[n. 22, \&; 'T. 1 F. 1 \& 1 .

Zethus biglumis Spinola, Ann. soc. entom. France X. 1841 p. 135 n. 83 , ㅇ․

Zethus (Didymogastra) biglumis Saus`ure, litud. fam. Vespid. I. Eumén. 14.52 p. 19 n. 20. ธ.

Zcthus (Zethusculus biglumis Saussure. Stud. fam. Tespid. III. Masar. 1556 p. 11 . n.27.

Zethus Z Zethusculus biglumis, Saussure, Smithson. Miseell. Collect. XIV. P. 251. 1975 p.3!

binodis (Fabr.) Sauss. - 우 $\hat{o}-$ Am.: Cayenne.

Vespa binodis Fabricius, Suppl. entom. system. 1795 p. 264 n. 100-101.

Vespa binodis Coquebert, Illustr. iconogr. Insect. II. 1401 p. 62: 'T. 15 F. 2.

Eumenes binodis Fabricius, Syst. Piez. 1504 p. 287 n. 8.

Zethus Didỹnogastra) binodis Saussure, Kitud. fam. Vespid. I. Fumen. 14.52 p. 20 n. 23. C. 


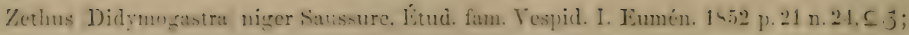
['I'. 5 F. $9($ (f).

Yethus 1)idymogastra himotiis Saussure. Smithson. Miscell. Collect. XIY. 1'.251. 1475 p.54 caernleopemis Fabr. - 요 ô - Am. mer.

[n. 50.

Vespa cocruleopennis Fabricius, Suppl. entom. system. 1795 p. 263 n. 86-87.

Vespa coeruleopennis Coquebert, lllustr. iconogr. Insect. I. 1799; 'T. 6 F. 4.

Zethus coeruleopennis Fabricius, Syst. Piez. 1801 p. 282 n. 1.

[F. 12\&13.

Eumenes Zetlus, eocruleopenni Iatreille. Gen. C'rust. d Insect. IV. 14019 p. 1:45; T. 3933

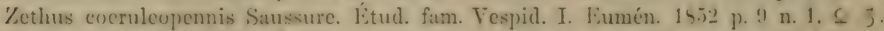

Zcthus magnus Saussure. Etud. fam. Tespid. I. Eumén. 15.2 p. 11 n. 4. 3; T. S F.s di.

Zethus coeruleopennis Saussure, Rev. \& mag. zool. (2) X. 1858 p. 64.

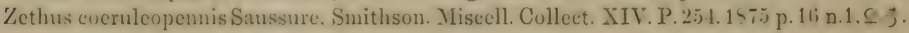
var. brasiliensis Sauss. - 우 ô - Am. : Brasilia.

Zethus Brasiliensis Saussure, Ktud. fam. Vespid. I. Jumen. 15.52 p. 10 n. 2. ᄃ.

Zethus Brasiliensis Saussure, litud. fam. Vespid. III. Masar. 14.jti p. 115. jo.

Zethus cocruleopennis var. Brasiliensis Saussure. Rev. \& mag. zool. 2 X. 1 s.je p. (i.:. carbonarius Smith $-\Omega-$ Am.: Brasilia.

Zethus carbonarius Smith, Catal. Hymen. Brit. Mus. V. 1857 p. 10 n. 5, ㅇ.

Zethus carbonarius Saussure. Smithson. Miscell. Collect. XIV. P. 254. 1875 1. 17 n. 4. earinatus Smith - ô-Am.: Brasilia.

Zethus carinatus Smith, Catal. Hymen. Brit. Mus. V. 1857 p. 13 n. 20 , oิ.

Zethus carinatus Saussure. Smitlıson. Miscell. Collect. XIV. P. 254. 15i5 p. 41 n. 34. ว. ceylonicus Sauss. - 우 - As.: Cayenne.

Zethus (Calligaster; Ceylonieus Saussure, Reise d. Novara. Zool. II. 1. 1S67 IIymen. p. 5. . . chalybens Sauss. - 우 - Am.: Brasilia.

Zethus chalybeus Saussure. Kitud. fam. Tespid. I. Eumén. 14,52 p. 10 n. 3. ᄃ.

Zethus chalybeus Saussure, Rev. \& mag. zool. (2) X. 1858 p. 63.

Zethus chalybeus Saussure. Smithson. Miseell. Collect. XIV. P. 254. 1475 p. 17 n. 3. C. ehiconteneati Sauss. $-\Omega-$ Am.: Mexico.

Zethus Jidymogastral Chiconteneatl Saussure, Rev. \& mag. zool. (2) IX. 1457 p. 271, C.

Zethus IDidymogastra C'hicontencatl Saussure, Smithson. Miscell. Collect. XIV.P.254.1475 clirysopterus Sauss. - ơ - Am.: Mexico. [p.51 n.46, §; '1.1 F.5\&5.

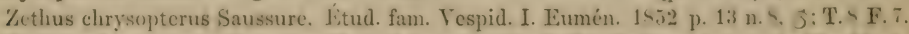
Zethus chrygepterus Saussure, Rev. \& mag. zool. 2) X. 1 s5s p. 1i6. p.34 n.24. 3. Zethus Zethusculus chrysopterus Saussure. Smithson. Misecll. Collect. XIV. P.254. 1 5 is einerascens Sauss. - - - Am.: Brasilia.

Zethus cincrascens Saussure. Titud. fam. Tespid. III. Masar. 1556 p.117n.2. p.34 n.26.C. Zethus Zethuseulus cinerascens Suussure, Smithson. Miseell. Collect. XIY. P. 251. 14 is elypearis Sauss. - of - Am.: Mexico. [p. 34 n.21, $\overrightarrow{0}$.

Zethus (Zethusculus) clypearis Saussure. Smithsom. Miseell. ('olleet. XIV. P. 254. 16i5 eyanopterus Sauss. - $\subsetneq$ $ో$ - As.: Java, Celebes.

['I. 9 F. 7.

Calligatster eyamptera 1 ham Saussure, Citud. fam. Vespid. I. Eumén. 14.22 p. 2.3 n.2.5:

Zethus Calligastor, cyanopterus Saussure, Étud. fam. Vespid. III. Masar. 1856 p. 115.

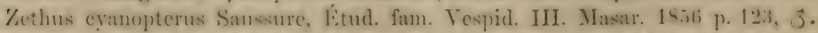

Zethus cyanopterus Maindron, Ann. soc. entom. France fi; II. 14n-2 p. 265 . 5.

Zethus Calligaster cyamopterus Gribode. 13ull. soe. entom. Ital. XXIII. 1491 p. 260, L. dieomboda (Spin.) Sauss. - \& ơ - Am.: Chile.

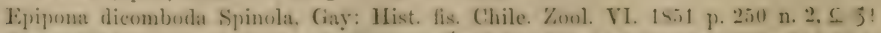

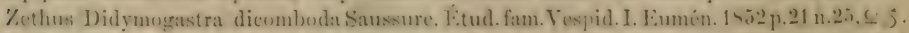
Kethus Jidrmogastra dicombuda Saussure. Smithson. Miseell. ('ollect. XIV. P.251. 1575 
didymogaster Spin. $-\subseteq-$ Am.: Cayenne.

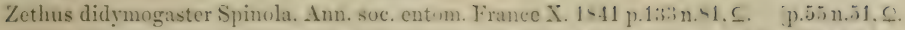

Zethus Didymograstra didymotraster Saussure. Smithson. Miscell. Collect. XIV. P.254. 1675

discoelioides Sauss. - $\subseteq-$ Am. mer., Brasilia.

Zethus discoelioides Saussure. Ftud. fam. Vespid. I. Kumén. 1452 p. 17 n. 17, C.

Zethus Zethusculus discuelioides Saussure. Smithson. Miseell. Cullect. XIV. P.251. 1575

dubius Smith. - $0 \hat{\sigma}-$ Am.: Brasilia.

[p. 39 n. 28, ㅇ․

Zethus dubius Smith, Catal. Hymen. Brit. Mus. V. 1857 p. 13 n. 19, $\subseteq$ ô.

Zethus dubius Sau-sure. Smithson. Miscell. C'ollect. XIV. P. 25.1. 1575 p. 1.1 n. 37.0 3.

elongatus Fabr.! - Am. mer.

Zethus elongatus Fabricius, Syst. Piez. 1804 p. 283 n. 5 .

favillaceus Walk. $-\odot$ ô - Afr.: Aegyptus.

Zetlus farillaceus Walker. List of Hymen. in Eg!pt 1471 p. 25 n. 115.63.

ferrugineus Sauss. - ㅇ - Am. mer., Brasilia.

Zethus ferrugineus Saussure, litud. fam. Tespid. I. Fumén. 1452 p. 14 n. 111. $\subseteq$. p. 3s n. 25.

Zethus Zethusculus ferrugineus Saussure. Smithson. Miscell. Collect. XIV. P. 254. 1<i5

fraternus Sauss. $-\Omega \delta$ - Am.: Brasilia.

Zethus fraternus Saussure. litud. fam. Vespid I. Kumén. 14.52 p. 16 n. 14. j̧.

Zethus fraternus Saussure, Étud. fam. Vespid. III. Masar. 1856 p. 123.

Zethus Zethusculus fratcmus Saussure. Rev. \& mag. zool. 2) X. 1555 p.16i3. [n.9. (4).

Zethus (Zethusculus, fratemus Saussure. Smithsun. Misecll. Collect. XIV. P. 25. 1575 p.211

fusens (Perty) Sauss. $-\subseteq-$ Am.: Brasilia.

Didymograstra fusca Perty. Delect. anim. artic. Brasil. 14;3. p. 145 nee 133 !; T. 24 F. 5.

Zethus Didymogastra fuseus Saussure. Ktud. fam. Tespid. I. Jumen. 1452 p. 19 n. 21. .

Zethus IDidrmogastra fuscus Saussure. Ktud. fam. Yespid. III. Masar. 15.56 p. 120.

Zethus 1Didymogastra! fuscus Saussure, Smithson. Misecll. Cullect. XIV. P. 25. 15i.)

genieulatus (Spin.) Sauss. - 우 ơ - Am.: Brasilia.

[p. 55 n. 52 , 우.

Didymogastra geniculata Spinola. Micm. acad. sc. Torino (2) XIII. 1451 p. $\$ 0$ n. $60 . \vec{\jmath} \mathrm{C}$.

Zethus geniculatus Saussure. Eturl. fam. Tespid. I. Eumén. 1h.j2 p. 22.2.

[n.6. 53 .

Zethus I)idynogastra genieulatus Saussure, Kitud. fam. Vespid. III. Masar. 1556 p. 120

Zethus Didymegastral geniculatus Saussure, Smithson. Misecll. Collect. XIV. P. 254. 14is gigas Spin. - 오 0 - Am. mer., Cayenne.

[p. 50 n. 44 .

Zethus gigas Spinola, Ann. soc. entom. France X. 1841 p. 129 n. 80, f.

Zcthus gigas Saussure, Étud. fam. Vespid. I. Eumén. 1852 p. 12 n. 7 , ‥

Calligaster hero Haan Saussure, Kitud. fam. Yespid. I. Eumén. 1952 p. 23 n. 1. ऽ; T. 9 T. 1.

Zethus Iferos gigas Saussure, Witud. fam. Vespiel. III. Masar. 15.56 p. 115.

Zethus gigas Saussure, Iitud. fam. Vespid. III. Masar. 1856 p. 123.

Zethus Heros, gigas Saussure, Smithson. Miseell. C'ollect. XIV. P.25. 1575 p. 17n. 5.5 j.

Zethus (Ileros gigras Gribodo, Bull. soc. entom. Ital. XXIII. IS!11 p. 25!, $\vec{j}$.

gracilis Smith $-\underline{\delta}$ - Am.: Mexico.

Zethus gracilis Smith, Catal. Hymen. Brit. Mus. V. 1857 p. 17 n. 43, ô.

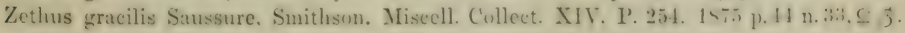

heydenii Sauss. - 으 ơ - Am.: Brasilia.

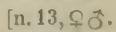

Zethus (Zethusculus) Heydeni Saussure. Smithson. Miscell. Collect. XIV. 1'.251. 1575 p. 23

hilarianus Sauss. $-\Omega$ ơ - Am.: Brasilia, Venezucla.

[; T. 6 F. 6.

Zethus 1)idymogastra) Hilarianus Saussure, Ctud. fam. Vespid. III. Masar. 1 4.j6 p. 120 n. 7. Zethus Didrmogastra, Hilarianus Saussure. Smithson. Misecll. Cullect. XIY. P. 254. 1875

jaranns Grib. - ô-As.: Java.

[p. $50 \mathrm{n} .43, \mathrm{fo}$.

Zethus Calligaster Jarmus Gribodo, Bull. seec. cntom. Ital. XXIII. 1 <91 p. 261. J. 
imitator Sauss. - 우 - Am.: Mexico. $[\mathrm{n} .20$, .

Zethus /\%ethusculus imitator Saussure, Smithson. Misecll. Collect. XIV. P.254. 1575 p. 33 inca Kirsch - of ơ - Am.: Columbia.

Zethus Zethuseulus) inca Kirsch. Mittheil. zool. Mus. Dresden III. 15is p. 375. $\subseteq 3$. jurinei Sauss. - $\hat{o}-$ Am. mer., Antilles.

Ketlus Jurinei Saussure, Etud. fam. Vespid. I. Eumén. 15.52 p. 15 n. $13,3$.

Zethus Jurinei Saussure, Étud. fam. Vespid. III. Masar. 1856 p. 118.

Zethus (Zethusculus, Jurinei Saussure, Rev. \& mag. zool. 2) X. 15.5 p. 162 . [n.11, c ;

Zethus Zethusculus Jurinei Saussure, Smithson. Misecll. Collect. XIV. P.254. 1575 p. 22

levinodus Smith - 0 - Am.: Mexico.

Zethus lacvinodus Smith, Catal. Hymen. Brit. Mus. V. 1857 p. 17 n. 44, ㅇ.

Zethus lacrinodus Saussure, Smithson. Miscell. Collect. XIV. P. 25.4. 1575 p. 44 n. $34 . \subseteq$.

lobulatus Sauss. - - - Am.: Brasilia.

Zethus lobulatus Saussure. Ėtud. fam. Yespid. III. Masar. 1456 p. 116 n. 2. \&; T. 6 F. 4. Zethus Zethuseulus lobulatus Saussure, Smithson. Miscell. Collect. XIV. P.254. 1575 p.27 matzicatzin Sauss. - ơ - Am.: Mexico.

Zethus IDidỵmogastral Matzicatzin Saussure, Rer. \& mag. zool. 2 IX. 1557 p. 271.

Zethus (1)idymogastra) Matzicatzin Saussure, Smithson. Miscell. Collect. XIV. P.254. $15 i 5$

mexieanus (L.) Sauss. - 0 ôt - Am. mer., Cayenne, Brasilia.

[p. 45 n. 42.5 .

? Apis Surinamensis Linné, Syst. nat. Ed. $10^{2}$ I. 1758 p. 575 n. 6.

Apis Mexicana Linné, Syst. nat. Ed. $12^{a}$ I. 2.1767 p. 953 n. 6.

Vespa recurvirostra Degeer, Mém. hist. Insect. III. 1773 p. 579 n. 2; T. 29 F. 4-6.

Apis Mexicana Ph. I. Müller, Linné: Vollst. Naturṣ̣st. Y. 2.1775 p. $\$ 92$ n. 6.

Vespa recurvirostra Göze, Degeer: Abh. Gesch. Insect. III. 1-60 p. 375 n. 2; T. 29 F. 4-ti.

Vespa recurvirostris Retzius, Gen. \& spec. Insect. 1783 p. 63 n. 233.

Apis Mexicana Olivier, Encycl. méthod. Insect. IV. 1789 p. 79 n. 3.

Apis Mexicana Gmelin, Linné: Syst. nat. Ed. 13a I. 5. 1790 p. $27 i 2$ n. 6.

Apis Mexicana Christ, Naturg. d. Insect. 1791 p. 199.

Vespa Mexicana Olivier, Encycl. méthod. Insect. VI. 1791 p. 673 n. 19.

Vespa cyanipennis Fabricius, Entom. system. II. 1793 p. 277 n. 86.

Vespa cyanipennis Coquebert. Illustr. iconogr. Insect. I. 1799 p. 24 ; T. 6 F. 4.

Eumenes cyanipennis Latreille. Hist. nat. Crust. \& Insect. III. 1502 p. 3610.

Vespa Mexicana Tigny, Hist. nat. Insect. IUI. 1802 p. 579.

Polistes eyanipennis Fabricius, Syst. Piez. 1804 p. 275 n. 30.

Tumenes cyanipennis Latreille. Hist. nat. Crust. \& Insect. XIII. 1805 p. 345 n. 1.

Vespa cyanipennis Jurine, Nouv. méth. class. Hymén. 1807 p. 170, \&.

Zethus cyanipennis Klug, Magaz. Ges. naturf. Fr. Berlin II. 1407 p. 52 n. is.

Zethus cyanipennis Latreille, Gen. Crust. \& Insect. IV. 1809 p. 138.

Zethus cyanipennis Lamarck, Hist. nat. anim. s. vert. IV. 1817 p. 84 n. 1.

Polistes eyanipennis Iepeletier. Eneycl. méthod. Insect. X. 1425 p. 171 n. 1 . f j.

Zethus eyanipennis Iamarck. Ilist. nat. anim. s. rert. Fol. 2a IV. 1635 p. 301 n. 1.

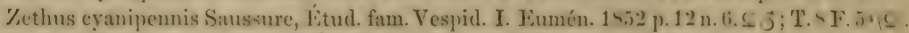

Yethus Mexieanus Saussure, Etud. fam. Vespid. III. Masar. 1456 p. 116.

Zethus cyanipennis Saussure, Rev. \& mag. zool. (2) X. 1858 p. 63.

Zethus eyanipennis J)esmarest, Clemu: Eneyel. hist. nat. Annelles 14 (301 p. 113 ; F. 125.

Zethus reeurvirostris Saussure, Smithson. Miseell. Collect. XIV. P. 254. 1575 p.17 n.2. 5 J.

var. Ingubris Perty - - - Am.: Brasilin.

Zethus lugubris Perty, Deleet. anim. artic. 13rasil. 1833 p. 144; 'T. 28 F. 4.

Zethus lugubris Saussure, Etud. fam. Vespid. I. Eumén. 1952 p. 11 n. 5, S.

Zethus eyanipennis var. lugubris Saussure. Rer. \& mag. mol. 2 X. 1sis p. fi3.

Zethus Mexienus var. lugulris Saussure, Smithson. Miseell.('ollect. XIV. P.254.1575 p.17. 
miniatus Sauss. - $\Omega$ oै - Am.: Brasilia.

Zethus miniatus Saussure. Rev. \& mag. zool. 2, X. 15is p. $61, \subseteq \vec{j}$. [n. $15, \subseteq \vec{j}$.

Zethus (Zethusculus miniatus Saussure. Smithson. Miscell. Collect. XIV. P. 251. 1575 p. 27

miseogaster Sauss. - 우 - Am. mer.

Zethus miscogaster Saussure, Gitud. fam. Vespid. I. Fumén. 1s.i2 p. 14 n. 18. S.

Zethus (Didymogastra, microgaster Saussure, Rev. (dmagr. zool. 2. X.1 4.in p.16i3 [err.typogr.].

Zethus (Didymogastra' miscogaster Saussure, Smithson. Miseell. Collect. XIV. P.25.1. 1575

montezuma Sauss. - of ơ - Am.: Mexico.

[n. 54 n. 49, \&.

Zethus Zethusculus: Montezuma Saussure. Rer. \& mag. zool. 2) IX. $145 \pi$ p. 27 .

Zethus (Zethusculus, Montezuma Saussure, Smithson: Miseell. Collect. XIV. P. 254. 16:5 var. guatemotzin Sauss. - Am.: Mexico.

[p. 40 n. 29, 워 ; T. 1 F. $3 \& 3$.

Zethus Zethuseulus Guatemotzin Saussure, Rer. \& mag. zool. 22. IX. 1857 p. $270 . \quad$ (p. 40.

Zethus .Iontezuma var. Guatemotzin Saussure, Smithson. Collect. Misecll. XIV. P.254. 14.5

nigricornis Sauss. - 우 $\widehat{o}-$ Am.: Mexico.

[p. 22 n. 12, 유.

Zethus Zethusculus! nigricornis Saussure, Smithson. Miscell. Collect. XIV. Y. 2\%4. 147.)

olmecns Sauss. - $ᄋ$ ơ - Am.: Mexico.

[n. 14, 우요.

Zethus Zethusculus, Olmecus Saussure, Smithson. Miscell. Collect. XIV. P.254. 1475 p. 2 .

otomitus Sauss. - ô - Am.: Mexico.

[p. $37 \mathrm{n} .23$, ơ.

Zethus IZethuseulus Otomitus Saussure. Smithson. Misecll. Collect. XIV. P. 254. 14i.) pallidus Smith - 으-Am.: Brasilia.

Zethus pallidus Smith, Catal. Hymen. Brit. Mus. V. 1857 p. 11 n. 10, ㅇ.

Zethus pallidus Saussure, Smithson. Miscell. Collect. XIV. P. 254.1575 p. 44 n. 35.

pamparum Berg - 우 - Am.: Patagonia.

[p. 112 n. $116 ;$ T. 2 F. 20.

Zethus (Didymogastra) Pamparum Berg. Inform. Comic. Exped. Rio Negro. Zool. I’. 1. 1 i 1

Zethus (Didymogastrai Pamparum Berg. Stettin. entom. Zeitg. XLII. 1 ind p. 69 n. 117. . .

parrulus Sauss. - $9-$ Am.: Brasilia.

[T. 6 F. 1.

Zethus (Zethusculus parrulus Saussure, Kitud. fam. Yespid. III. Masar. 1956 p. 119 n. 5. S;

Zethus Zethusculus' parrulus Saussure, Smithson. Miscell. Collect. XIV. P. 2i.4. 1 575 p. 41

piriformis Spin. - $f$ $\sigma$ - Am.: Cayenne.

[n. 30, ㅇ.

Zethus piriformis Spinola, Ann. soc. entom. France X. 1841 p. 134 n. $82, \& \delta$.

Zethus piriformis Saussure. Kitud. fam. Vespid. I. Eumén. 15.2 p. 15 n. 12, S 3; T. \& F. S

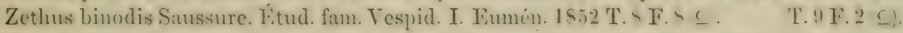

Zethus (Zethusculus piriformis Saussure. Smithson. Miscell. Collect. XIV. P. 254. 15it poeyi Sauss. - $\subseteq \widetilde{o}-$ Am. : Cuba.

[p. 20 n. S, $\mathrm{S}$.

Zethus (J)idymogastra Poeri Sausure, Rer. \& mag. nool. (2 IX. 1457 p. 270.

Zethus (Didymogastrat, Poeyi Saussure. Smithson. Miscell. C'ollect. XIV. P.254. 14 it p. 15

pubeseens Smith - $\&-$ Afr.: Port Natal.

[u. 39, ऽ ô; 'I'. 1 F. $4 \& 4^{\text {a }}$.

Zethus? pubescens Smith, Catal. Hymen. Brit. Mus. V. 1857 p. 9 n. 2, \&.

romandinus Sauss. - ô - Am.: Cayenne.

[T.9F.1.

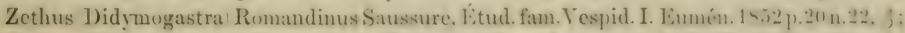
Zethus (Didymogastra Romandinus Saussure, Res \& magr zoul. 2 X. I his p. llil, ;.

Zethus (D)idymogastra Romandinus Saussure. Smithson. Misedl. ('ollect. XIV. P.2.14. 14:5

ruflnodus (Latr.) Lam. $-\subseteq-$ Am.: Antilles.

[p. 47 n. 40, s.

Eumenes rufinoda Latreille, Gen. Crust. \& Insect. I. 1806; 'T. 14 F. 4.

Eumenes rufinoda Latreille, Gen. Crust. \& Insect. IV. 1509 p. 137.

Zethus rufinodis Lamarck, Hist. nat. anim. s. rert. IV. 1817 j. 55 n. 3.

Zethus rufinodis Lamarck, Hist. nat. anim. s. rert. Ed. 2a IV. 1835 p. 301 n. 3.

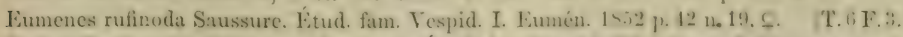

Zethus Zethuseulus' rufinodus Saussure, Litud. fam. Vespid. 111. Masar. 14.iti p. 11- n. 4. L :

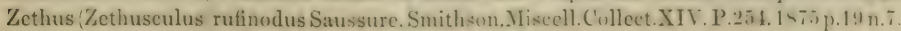


seulpturalis Smith - ơ - Am.: Brasilia.

Zethus sculpturalis Smith, Catal. Hymen. Brit. Mus. V. 1557 p. 11 n. 11, oో.

Zethus sculpturalis Saussure. Smithson. Miscell. Colleet. XIV. 1.254 1575 p.41 n.36, j.

slossonae Fox $-\simeq$ ơ - Am.: Florida.

Zethus Slossonae Fox, Entom. News IIr. 1592 p. 29, 우 0 .

smithii Sauss. - $0-$ Am. mer.

Zethus Jid!mogastra Smithii Saussure. Fitud. fam. Vespid. III. Masar. 14.56 p. 12.2n.4. . .

Zethus Didymogastra Smithii Saussure. Smithson. Misecll. Collect. XIV. P. 254. 14i5

spinipes Say - 오 - Am.: United States.

[p. $55 \mathrm{n}$ n. 53, 오.

Zethus spinipes Say, Boston Journ. Nat. Hist. I. 4. 1837 p. 357, ㅇ.

Jumenes Pennsỵlvanica Ifaldeman. Proe. Acad. Nat. Sc. Philadelphia 1453 p. 36.5. 3.

Zethus spinipes Saussure, Étud. fam. Vespid. III. Masar. 1856 n. 122 n. 10.

Zethus spinipes Ieconte. Writ. of Th. Say Entom. II. 15.99 p. $767 . \quad$ [n. 15.5 j.

Zethus Z'Zethuseulus spinipes Saussure. Smithson. Miscell. C'ollect. XIV. P. 254. 1575 p. 29!

var. snbstrictus (Hald.) Sauss, - Am. bor.

Eumenes substricta Haldeman. Proc. Acad. Nat. Soc. Philadelphia II. 1 s 41 p. 5.

Eumenes substrictus Saussure, Étud. fam. Vespid. III. Masar. 15.56 p. 152 n. 42.

Zethus spinipes var. substrictus Saussure, Smithson. Miseell. Collect. XIV. P.25-1.157.5p.30.

var. variegatus Sauss. - 우 oै-Am.: Mexico.

Zethus rariegatus Saussure, Eitud. fam. Tespid. I. Eumén. 1552 p. 13 n. 9, S.

Zethus bicolor Saussure, Étud. fam. Vespid. I. Eumén. 1852 p. 17 n. 16, ô.

Zethus variegatus Saussure, Rev. \& mag. zool. (2) X. 1858 p. 66.

Zethus spinipes rar. variegatus Saussure. Smithson. Miseell. Collect. XIV. P. 254 . 1575 p.30.

spinosus Sauss. - 우 ơ- Am.: Mexico.

Zethus Zethusculus) spinosus Saussure, Rer. \& mag. zoul. 2 IX. 1457 p. 27ー1.

Zethus Zethusculus spinosus Saussurc. Smithson. Miseell. Collect. XIV. P.254. 1475 p. 31

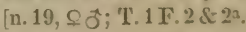

strigosus Sauss. - 오

[n. 31, \& 0 .

Zethus (Zethuseulus) strigosus Saussure. Smithson. Aisecll. Collect. XIV. P. 25.4. 15 is p.42

tolteeus Sauss. - 우 ơ - Am.: Mexico.

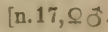

Zethus Zethusculus Toltecus Saussure. Smithson. Miscell. Cullect. XIV. P. 254. 1575 p. 27

tabulifer Sauss. - 우 - Am.: Mexico.

[p. 18 n. 19, 우.

Zethus Didymogastra tubulifer Westwood Saussure, Titud. fam. Vespid. I. Eumen. 15.22

Zethus (Didymogastra tubulifer Saussure, Smithson. Miscell. Colleet. XIY. P. 254. 1675

westwoodii Sauss. - $2 \hat{o}-$ Am: Mexico.

[p. $53 \mathrm{n} .47$.

Kethus Westwondi Saussure, Citud. fam. Vespid. I. Jumén. 1 s.52 p. 16 n. 15 ..

Zethus Westwondi Saussure, Citud. fam. Vespid. III. Masar. 14.5t p. 11s, c; T. 6 F. 2.

Zethus IZethusculus Trestwoodi Saussure, Smithson. Miscell. Collect. XIY. P. 254. is:5

zeudúlus Sauss. $-\subseteq-A$ m.: Mexico. [p. 21 n. 10, $\$$ ơ.

Zethus Didymogastral Kendulus Snussure. Smithson. Misecll. ('ollect. XIV. P. 254. Is i.

(p. 53 n. 4 S, ㅇ.

\section{STROUDIA}

Gribodo, Bull. soc. cntom. Ital. XXIII. 1 S91 p. 262.

1)r. Stroud.

armata Girib. $-\Omega-\Lambda$ fr. austr.

Stroudia armati Gribodo, Bull. soc. entom. Ital. XXIII. 1891 p. 264, f. 


\section{DISCOELIUS}

Latreille, Gen. Crust. \& Insect. IV. 1509 p. 110.

dis, bis; xoinos, carus.

Synon.: Epipona Spinola.

Eumenes Latreille, Audouin.

Vespa Panzer.

Zethus Lamarck.

dufourii Lep. - o f - Eur.: Gallia.

Diseoelius Dufourii Lepeletier. Hist. nat. Insect. Hỵmén. II. 14.11 p. 60.5 n. 2 , ㅇ․

Discoelius I)ufourii Saussure, Etud. fam. Tespid. I. Eumén. 1552 p. 27 n. 5. C; T. 9 F. 5.

Discoelius Dufourii Saussure, Ĺtud. fam. Tespid. III. Masar. 15.96 p. 127, ठ.

I)iscoelius Dufourii Schenck. Jahrb. Yer. Niaturk. Nassau XVI. 1561 p. 3.. ․ s .

Diseoclius Dufourii Ed. André, Spec. Hrmén. Europe II. P. 20. 1 SS1 p. 617 n. 2. C.

elongatas Sauss. - 0 - Austr.

Disenelius elongatus Saussure. Etud. fam. Vespid. III. Masar. 15506 p.12⿺ n. 11. _; T.6 F. .

ephippium Sauss. - ơ - Austr.

Discuelius ephippium Saussure, Fitud. fam. Vespid. III. MIasar. 15.56 p. 12.5 n. 13, j3; 'T. 6 F. ᄂ.

insignis Sauss. 오- Austr.

Discuelius insignis Saussure, Étud. fam. Vespid. III. Masar. 145ti p. 126 n. 14, ؟.

Diserelius insignis Smith. Brencliley: Cruise of the Curacoa 1573; T. 4.7 F. 1.

merula Hal. $-\Omega-$ Am.: Chile.

Discoclius merula ICurtis Haliday, Trans. Limn. Soc. London XVII. 3. 1536 p. 325 n. 36. . .

Epipona Chilensis Spinola, Gay: Hist. fis. Chile. Zool. VI. 15.51 p. 2.4 s n. 1, f.

1)iscoelius Chilensis Saussure. Etud. fam. Vespid. I. Eumén. 1852 p. 2 . n. 1, S; T.9 F.3.

Discoelius merula Saussure, litud. fam. Vespid. III. Masar. 1854 p. 124.

Discoelius merula Saussure, Smithson. Miscell. Collect. XIV. P. 254. 1475 p. 59 n. 1. var: spinolae Sauss. $-\subseteq-$ Am.: Chile.

Disenelius Spinolac Saussure, Etud. fam. Yespid. I. Eumén. 1452 p. 25 n. 2. S.

Disevelius Spinolae Saussure, Etud. fam. Vespid. III. Masar. 15.56 p. $124 . \quad$ p. .49.

Discoelius merula rar. Spinolac Saussure. Smithson. Misecll. C'ollect. XIV. 1'. 25. 1s is

pulehellus Sauss. - 오 ôt Am.: Mexico, Jamaica.

Disenelius pulchellus Sausure, litud. fam. Vespid. III. Masar. 1sint p. 127. f $\vec{j}$.

Discoelius pulchellus Saussure. Smithson. Misecll. Collect. XIV. P. 251 . 1 155 p. 59 n.2, \& j.

spiuosus Sauss. - \& - Austr.: New South Wales.

Discoelius spinosus Saussure. Etud. fam. Vespid. III. Matsar. 15.01; p. 125. S.

verreauxii Sauss. $-\propto \hat{\sigma}-$ Austr.: 'Tasmania.

Discoclius Verreauxii Saussure, Etud. fam. Vespid. I. Fumen. 14.2- p. 26 n. 3. S: T.9 F.4.

Diseoclius Verreauxii Santsure, Litud. fam. Vespid. III. Masar. 1556 1) 125, j.

zoualis (Panz.) Latr. - 0 ơ - Lur. centr. \& mer.

Vespa zonalis Panzer, Faun. Insect. German. VU. 1801 Fasc. 81 T. 18

Vespa (Eumenes) zonalis Panzer, Krit. Revis. II. 1806 p. 159.

Vespa zonalis Jurine, Nouv. méth. class. Hymén. 1507 p. 171, \&.

Fumenes Discoelius zonalis Iatreille, Gen. ('rust. \& Insect. IV. I 4199 p. 1:37.

Zethus zonata Lamarek, Hist. nat. anim. 8. vert. IV. 1817 p. 84 n. 2.

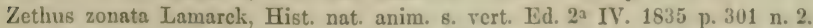

Discoclius zonalis Lepcleticr. Hist. nat. Inseet. Hỵmén. II. $1811 \mathrm{p}$. $610.3 \mathrm{n} .1 .6 \hat{\jmath}$.

Eumenes (Disenelius zonalis Audunin. Hist. nat. Insect. nuis. 1412 p. 191: T. 199 F. 4.

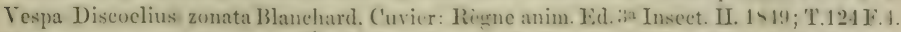

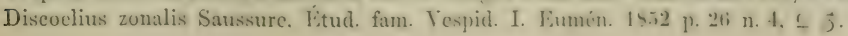


Disenclius zunelis Saussure. Etud. Lam. Vespid. III. Masar. 1 S5r p. 126.

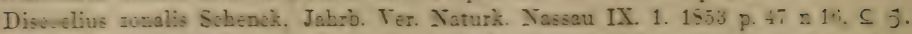

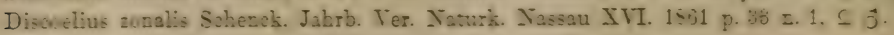

Discoelius zonalis Thomson, Opuse entom. P. 2. 1570 p. $\$ 3$ n. 1.

Discoelius zonalis Thomson, Hrmen. Scandin. III. 1574 p. 32 n. 1 , 으 $\hat{\jmath}$.

Discoslius suaslis Rudow. Areh. Ver. naturf. Fr. Meeklenburg XIX. $18: 6$ p. 212 n. 1.

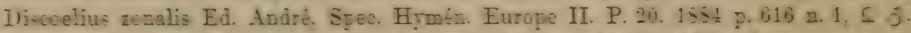
I)

\section{EUMENES}

Latreille, Hist. nat. Crust. \& Insect. III. 1502 p. 360.

ex $\mu \varepsilon v \dot{s}$, , benevolus.

Synon: Polistes Fabricius.

Sphex Christ.

Tespa Linné \& auct. ant.

Zethus Spinola.

Subg.: Alpha Saussure, Etud. fam. Tespid. I. Eumén. 1552 p. $2 \varsigma$.

cs littera.

Beta Saussure, Smithson. Miscell. Collect. XIV. P. 25t. 1575 p. S૬.

3 littera.

Omieron Saussure, Étud. fam. Vespid. I. Eumén. 1552 p. 71.

o littera.

Pechraenes Sawsure. Etud. Sam. Tespid. I. Eumén. 1552 p. 73: T. 5 F. 1

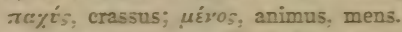

Poreumenes Ssusure. Etud. fom. Verpid. III. Eumen. 1552 s. 155: T. 7 F. 1-2.

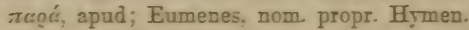

Zeta Saussure, Ettud. fam. Yespid. I. Eumén. 1552 p. 67. ¿ littera.

ahdominalis (Drury) Westw. - $\subseteq \vec{j}-$ Ara.: Antilles.

Sphex abdominalis Drur, Illustr. Nat. Hist. I. 1770 p. 105; T. 45 F. 2.

Tespa attenuata Fabricius. Syst. entom. 1775 p. 372 n. 44.

Tespa attenuata Fabricius, Spec. Insect. I 1751 p. 469 n. 63.

Vespa attenuata Fabricius, Mant. Insect. I 1757 p. 293 n. 77.

Tespa attenuata Gmelin, Linné: Syst. nat. Ed. $13^{3}$ I. 5.1790 p. 2754 n. 60.

Sphex extensa Christ, Naturg. d. Insect. 1791 p. 321 ; T. 32 F. 7.

Yespa attenuats Olivier, Enerel. méthod. Insect. VI 1791 p. $67 t$ n. 21.

Vespa attenuata Fabricius, Entom ststem. II 1793 p. 292 n. 95.

Pólistes attenuata Fabricius, Srst. Piez. 1504 p. 279 n. 44.

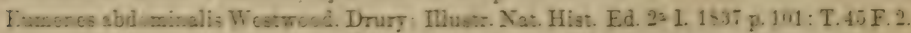

Fumeses abc mindia Sensure. Eud. Cum. Vespid. I. Eumen. 1552 p. Te n. 35. .

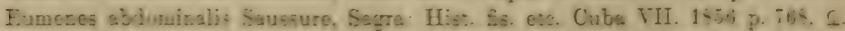

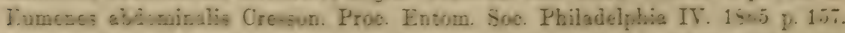

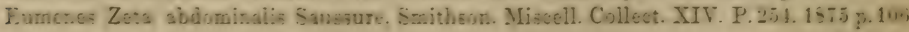

rar. colona Sauss. - - Am: Jamaica, Haiti.

[n. 49, 오 ㅇ․

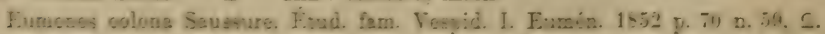

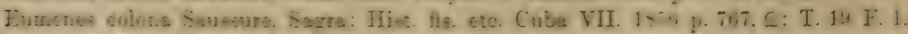

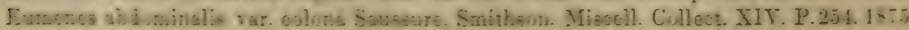

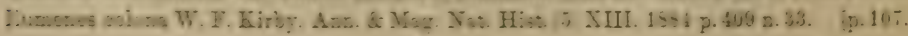


car. orasta Sauss $-\subseteq-A$ : Antilles.

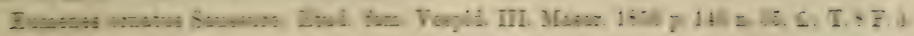

Eumeres ormatus Sansaure. Sagre: Hist fis. Ete. Cube 1556 p. T69, C. ip.107.

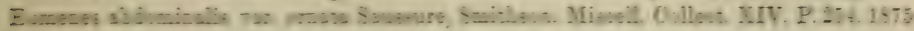

rer. pieta Smith $-\hat{\jmath}-\hat{A}=$ : Sen Domingo.

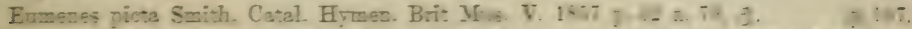

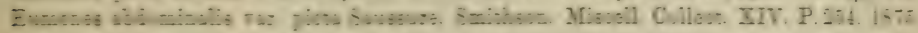

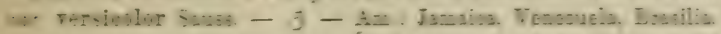

Eumeres remsicolor Saussure, Étud. fam Tespid. I Eumén 1552 p. $71=60$.

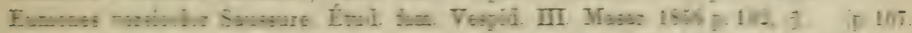

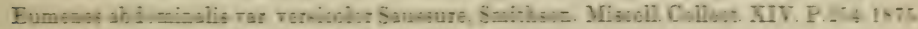

aeaminata Sanss. $-\subseteq \hat{\jmath}-1$ ir: Cap.

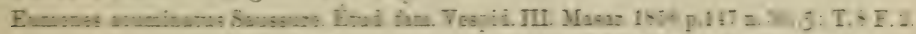

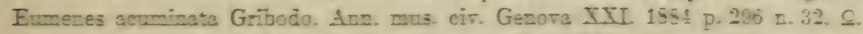

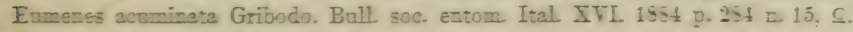

sethiopiea Seass. - $f$ - Aft:: Congo.

Eumenes Aethiopice Saussure, Étrad. fim. Tespid. I. Eumén. 1552 p. 62 I. 4i, c.

agillima D. T. $-\subseteq-$ As: Amboina

Eumenes agilis Smith Jours. of Proe Lim Soe. Zool TV. 1 Saj Suppl p. 127 n. 7. ㅇ

Eumenes agillime $\mathrm{m}$

amerieana Sauss. $-\subseteq j-1 m$ : Mexico.

Eumenes Americase Saussure. Ėud. fam Tespid. I Eumén. 1552 p. 39 n. 13. c

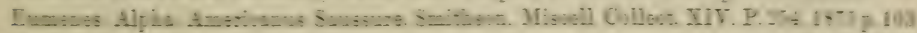

andrei D. T. - $\subseteq-$ Eur: Gellia

[ㄷ. $46, \subseteq \jmath$.

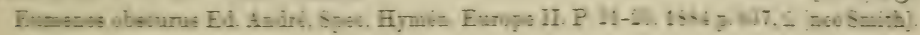

Enmenes Ardirei m

arbustoram $\mathrm{Pann}-\subseteq \hat{j}-$ Eur. mer. \& cencr.

Tespa arbustorum Parter. Faun. Insect German. VI 1799 P. 63 T. 5.

Texpe Eumeres arbustorum Panzer, Krit Reris. II. 1906 p. 159.

Tespe arbustorum Jurine. Nour. méth class. Hỵmén. 1507 p. 1i1, C.

Eumenes Amedei Lepeletier, Hist nat. Insect. Hrmén II ist1 p. $59 s$ c. 2, C o.

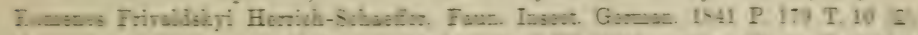

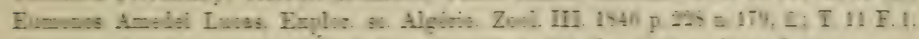

Eumenes tmę̉ei Saussure. Etud. far Tespid. I Eumén. 1952 p. 34 n. 6, c.

Eumenes arbustorum Schenek, Jahrb. Ver. Vaturk Vassan IX. 1. 15.53 p. 79 a. 4. S.

Eumenes pomifornis Fobre, Ann. se. nat. Zool. (i) II. 1554 p. 149, c.

Eumenes arbustorum Tascheaberg. Hymen. Deutachl. 1 se6 p. 215.

Eumenis arbustorum Kriechbaumer. Entom Nachr. V. 1599 p. 5 i n. 1, c o.

Eumenis Frivaldstri Kriechbaumer. Entom Vachr. V. 1579 p. 59 n. 3, c.

Eumenes Amedei Lichtenstein. Pet nour. eztom II. 1579 p. 301.

Eumenes 1medei Febre. Your. sour. entom 15s- p. 57.

CV \& CVI

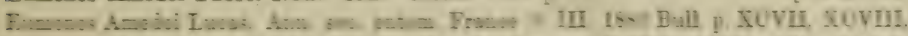

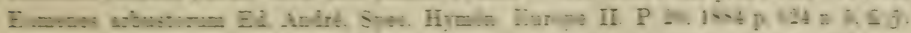

Eumenes arbustcrum EC. André, Spec. Hrmén. Europe II. P. 21-26. 1556 p. 569.

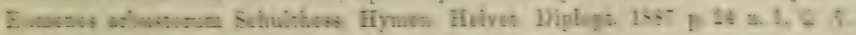

cor. baeri Rac் - $-\hat{j}$ - Eur: reg. Caspica.

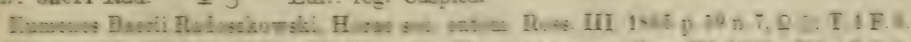

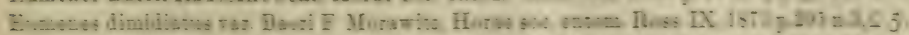

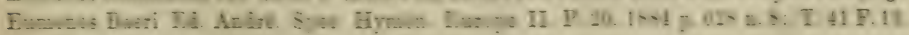
cor. dimidiata Brollé $-\subseteq-$ Eur: Graecia

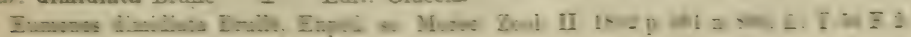

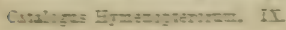


Eumenes dimidiatus Saussure. Kitud. fam. Yespid. III. MInsur. 14.51; p. 129. [T.41 F.6. Eumenes arbusturum var. dimidiatus Ed. Andre. Spee. IIymén. Europe II. P. 20 . 1 is 1 p. 6.20 ; rar. tauriea Sauss. - Eur.: Crimea.

Eumenes Tauricus Saussure, litud. fam. Vespid. III. Masar. 14,56 p. 137 n. 20.

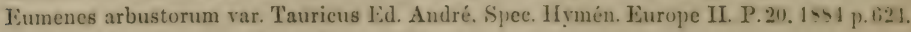
arcuata (Fabr.) L. - 우 0 - Austr.: Neu Guinea.

Vespa areuata Fabricius, Syst. entom. 1775 p. 371 n. 40.

Vespa arcuata Fabricius, Spec. Insect. I. 1781 p. 467 n. 54.

Vespa arcuata Fabricius, Mant. Insect. I. 1787 p. 292 n. 66.

Vespa arcuata Gmelin, Linné: Syst. nat. Ed. $13^{\text {a } ~ I . ~} 5.1790$ p. 2753 n. 49.

Sphex arcuata Christ, Naturg. d. Insect. 1791 p. 312.

Vespa arcuata Olivier, Encycl. méthod. Insect. VI. 1791 p. 670 n. 2.

Vespa arcuata Fabricius, Entom. System. II. 1793 p. 276 n. 83.

Eumenes arcuata Fabricius, Syst. Picz. 1804 p. 287 n. 11.

Vespa arcuata Jurine, Nouv. méth. class. Hymén. 1807 p. 171, ㅇ․

Eumenes arcuata Saussure, Etud. fam. Vespid. I. Eumén. 1552 p. 63 n. $50 . \subseteq$;

Eumenes fulvipennis Smith, Catal. Hymen. Brit. Mus. V. 15.57 p. 24 n. $26 . \subseteq \hat{\jmath}$.

Eumenes areuata Saussure. Stettin. entom. Zeitg. XXIII. 1562 p. 179 n. 16. oै.

var. blanchardii Sauss. - 우 - As.: Archipel. Indic.

Eumenes Blanchardii Saussure, Étud. fam. Vespid. I. Eumén. 14.22 p. 66 n. 53, S; T.12 F. 2.

Eumenes arcuata var. Blanchardii Maindron. Ann. soc. entom. France (i) II. 1552 p. 272 n. 12. var. flaropieta Blanch. - $ᄋ$ ơ - As.: India.

Vespa areuata Donoran, Epit. Nat. Hist. Insects India 1\$00; Tab. 57 nee Fabricius:

Fumenes flavopicta Blanchard, Dict. hist. nat. Hỵmén. II. 1840; T. 11 F. 2.

Eumenes arcuata Donoran. Westwood: Epit. Insect. Ind. $14+2$ p. 90; T. 57 F. 3.

Eumenes flaropicta Saussure, litud. fam. Tespid. I. Eumén. 1552 p. 65 n. 52. c.

Eumenes flaropictus Saussure. Ėtud. fam. Vespid. III. Masar. 1550 p. 132. วิ.

Eumenes arcuata var. flavopicta Maindron, Ann. soc. entom. France 6 II. 1552 p. 272 n. 12. var. praslinia Guér. - $\mathrm{Q}$ - Austr.: New Guinea, New Ireland.

Eumenes Praslinia Guérin, 1)uperry: Voy. Coquille. Zool. II. 2. 1-30 p. 267: T. 9 F. i. Jimenes Praslinia Saussure, Ktud. fam. Vespid. I. Eumén. 1552 p. 64 n. .1. C.

liumenes arcuata var. Praslinia Maindron, Ann. soc. entom. France 6; II. 1552 p. 272 n. 12. artifex Smith - 우 - As.: Celebes.

Eumenes artifex Smith, Journ. of Proc. Linn. Soc. Zool. IV. 1560 Suppl. p. ‘i n. 3. ᄃ. Fumenes artifex Maindron, Ann. soc. entom. France (6 II. 1552 p. $2(19)$ n. 3.5 .

asiua Sauss. - 우 - Afr.: Senegal.

Jumenes Asina Saussure, litud. fam. Vespid. I. Eumén. 1452 p. 59 n. 43. S: T. 11 F. 1. atra Sauss:- - o $\hat{o}-$ Am.: Brasilia.

Pachymenes atra Saussure, Kitud. fam. Tespid. I. Eumén. 1452 p. 75 n. 2. 6 .

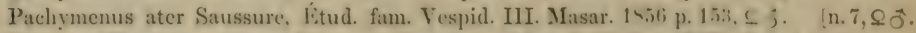

Eumenes Pachymenes ater Saussure, Smithson. Miscell. Collect. XIV. P. 254. is is p. 65 atrata Fabr. - Am. mer.

Eumenes atrata Fabricius, Syst. Piez. 1804 p. 287 n. 12.

linmenes atrata Saussure, Smithson. Miscell. ('olleet. XIY. P. 254. 147. p. 379 n. 1. atricornis Fabr. - $\subsetneq$ † - Eur.: Austria, Succia.

Eumenes atricornis Fabricius, Syst. Piez. 1804 p. 259 n. 17.

Eumenes atricornis Latreille, Gen. Crust. \& Insect. IV. 1809 p. 137.

Eumenes atricornis Curtis, Brit. Entom. I. 1824 p. 13, 요 ơ; F. 13.

Eimenes atricornis W estwood. Introd. mod. Classif. Insect. II. 1840 Synops. p. 239; F.4i, 15;

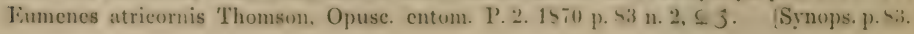


Eumenes atricornis Thomson, Hymen. Scandin. IJI. 1874 p. 37 n. 2 , \& $\delta$.

Eumenes atricornis Kriechbaumer, Entom. Nachr. V. 1879 p. 311.

aurata Sauss. - $\odot-$ Am.: Brasilia.

$[\mathrm{p} .62 \mathrm{n} .2$, ㅇ.

Eumenes (Pachymenes) auratus Saussure, Smithson. Miscell. Collect. XIV. P. 254. 15ij

auropilosa Smith - ơ - Am.: Brasilia.

Eumenes auropilosa Smith, Catal. Hymen. Brit. Mus. V. $155 \bar{i}$ p. 30 n. $71, \hat{j} . \quad$ in 44, j.

Eumenes 'Alpha auropilosa Saussure, Smithson. Miscell. Collect. XIV. P. 254.1575 p.102

avieula Sauss. - 오 oิ - Am.: Mexico.

[n. 13, Q

Eumenes (Omicron; ariculus Saussure, Smithson. Miscell. Collect. XIY. P. 254.1575 p. 73

azteca Sauss. - 으- Am.: Mexico.

Eumenes Aztecus Saussure, Rev. \& mag. zool. (2) IX. 1857 p. 272.

[n. 33, \&.

Iinmenes Alpha Aztecus Saussure, Smithson. Miscell. Collect. XIV. P. 254. 1575 p. 9:3

belfragii Cress. $\left.{ }^{1}\right)-\rho-$ Am.: Texas.

Eumenes Belfragii Cresson, Trans. Amer. Entom. Soc. IV. 1872 p. 232, s.

bicineta Sauss. - 0 o - Austr.

Eumenes bicineta Saussure, Étud. fam. Vespid. I. Eumén. 1852 p. 44 n. 23, f $f$.

bipmetis Sauss. - 우 - Eur.: Gallia.

Eumenes bipunetis Saussure, litud. fam. Vespid. I. Eumén. 1552 p. 33 n. 1, f; T. 11 F. 7.

Eumenes bipunctis Ed. André. Spec. Hymén. Europe II. P. 20. 1584 p. 627 n. 7.

bisignata Walk. - $\mathfrak{o}-$ As.: Arabia.

Eumenes bisignatus Walker. Hist. of Hymen. in Egypt 1571 p. 29 n. 154, $\vec{j}$.

bispinosa Mor. - 오 $\widehat{o}$ - Eur.: Dalmatia.

Eumenes bispinosus F. Morawitz, Horae soc. entom. Ross. XIX. 1455 p. 135 n. 1, $\subseteq$ j.

Eumenes bispinosus Ed. André, Spec. Hymén. Europe II. P. 24-26. 1 s\$6 n. sib.

bollii Cress. - of $\delta$ - Am. : Texas.

Eumenes Bollii Cresson, Trans. Amer. Entom. Soc. IV. 1872 p. 232, 우 ơ.

brasilana Sauss. $-q-$ Am.: Brasilia.

[p. 79 n. 17, \&.

Fumenes Omieron Brasilanus Saussure. Smithson. Miscell. Collect. XIV. P. 254. 15i5

brevirostrata Sauss. - 우 $\hat{o}-$ As.: India.

$[\mathrm{n} .18$, \& $ð$; T. 7 F. 11 (ㅇ).

Jimenes 'Pareumenes) brevirostratus Saussure. Etud. fam. Vespid. III. Masar. 1556 p. $1: 36$

eafrra (L.) Sauss. - $Q-$ Afr.: Cap.

Vespa caffra Linné, Syst. nat. Ed. $12^{3}$ I. 2.1767 p. 951 n. 21.

Vespa caffra Ph. L. Maller, Linné: Vollst. Natursyst. V. 2. 1775 p. 855 n. 21.

Vespa caffra Gmelin, Linné: Syst. nat. Ed. $13^{3}$ I. 5. 1790 p. 2758 n. 21.

Vespa caffra Christ, Naturg. d. Insect. 1791 p. 2149.

Sphex cruciata Christ, Naturg. d. Insect. 1791 p. 317 ; 'T. 32 F. 2.

Vespa caffra Olivier, Eneyel. méthod. Insect. VI. 1791 p. 671 n. 6.

Vespa spec. Lichtenstein, Catal. Mus. Zool. III. 1796 p. 292 n. 162.

Vespa cratiata Weber, Observ, entom. 1801 p. 101 n. 3 [err. typ.].

Fumenes caffra Saussure. Jitud. fam. Vespid. I. Fumén. 1552 p. 45 n. 25. C.

eallimorpha Sauss. - 오 ô - Am.: Brasilia.

? Eumenes campanulata Fabricius, Syst. Piez. 1804 p. 291 n. 22.

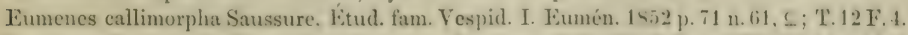

Eumenes Omieron callimorphus Saussure, Smithson. Miscell. Collect. XIV. P. 254. 1675

eampanulata Fabr.! - Am. mer.

(p. $\$ 3$ n. $25, .0 \hat{\sigma}$.

Eumenes campanulata Fabricius, Syst. Piez. 1804 p. 291 n. 22.

1) $=$ ? E. Smithii Sauss. 
canalieulata Oliv. $-\Omega \delta ̊-A m$. mer.

- Degeer, Mém. hist. Insect. III. 1773 p. 579 ; T. 29 F. 3.

Göze, Degeer: Abh. Gesch. Insect. III. 1780 p. $374 ;$ T. 29 F. 3.

Vespa canaliculata Olivier, Encycl. méthod. Insect. VI. 1791 p. 672 n. 12.

Vespa diadema Fabricius, Suppl. entom. system. 1798 p. 263 n. 88-89.

Eumenes diadema Fabricius, Syst. Piez. 1804 p. 285 n. 3.

Vespa diadema Jurine, Nour. méth. class. Hỹmén. 1807 p. 171, ㅇ.

[F. $5($ 우)

Eumenes eanaliculata Saussure. Kitud. fam. Vespid. I. Eumén. 1 \$.52 p. 6s n. 56. $\subseteq 3$; T. 11

Eumenes Zeta canaliculatus Saussure. Smithson. Miscell. C'ollect. XIV. P. 251. 1<75 p. 107

ehalicodomae Sauss. - $ᄋ-$ Am.: Brasilia.

Fumenes Zeta, Chalicodomae Saussure, Smithson. Misecll. Collect. XIY. P. 254. 1675 ehrysothorax Sauss. - 우 - Am.: Brasilia.

[p. $108 \mathrm{n} .52$, 으.

Pachymenes chrysothorax Saussure. litud. fam. Vespid. III. Masar. $1 \varsigma 56$ p. 153 n. 43. C.

Eumenes Pachymenes chrysothorax Saussure. Smithson. Miscell. Collect. XIV. P.254.1575

cincta Prov. - ㅇ - Am.: Trinidad.

[p. 63 n. 3.

Eumenes cinctus Provancher, Addit. faun. Canada. Hymén. 1555 p. 422 n. 4. 5.

eingulata Fabr. - Am.: Cayenne.

Eumenes cingulata Fabricius, Syst. Piez. 1804 p. 287 n. 13.

[p. 82 n. 21.

Eumenes (Omieron) cingulatus Saussure, Smithson. Miscell. Collect. XIT. P. 254. 15i5

cireinalis Fabr. - 오 $\widehat{o}$ - As.: India; Austr.

Eumenes circinalis Fabricius, Syst. Piez. 1804 p. 286 n. 4.

Eumenes circinalis Saussure, Etud. fam. Vespid. I. 1552 p. $4 i$ n. $27, \subseteq \rho$; T. 10 F. $i \subseteq$.

Eumenes circinalis Maindron, Ann. soc. entom. France if II. 1552 p. 269; T. 3 F. $7-10$. coaretata (L.) Latr. - 우 of - Lur. tota.

— Frisch, Beschr. Inseet. 'Teutschl. IX. 1730 p. 16; T. 9.

Vespa spec. Linné, Fauna Suec. 1746 p. ? n. 1002.

Vespa coarctata Linné, Syst. nat. Ed. $10^{\text {a }}$ I. 1758 p. 573 n. 9, ‥

Vespa coarctata Linné, Fauna Suec. Ed. 2a 1761 p. 417 n. 1676.

Vespa coarctata O. F. Müller, Fauna Insect. Fridrichsdal 1764 p. 73 n. 639.

Vespa coarctata Linné, Syst. nat. Ed. $12^{\mathrm{a}}$ I. 2. 1767 p. 950 n. 11.

Vespa IX Schaeffer, Icon. Insect. Ratisbon. I. 2. $176 \overline{7}$; T. 53 F. 10.

Vespa coarctata Berkenhout, Outlin. Nat. Hist. Gr. Britain I. 1769 p. 165 n. 2.

Vespa XXIII Schaeffer, Icon. Insect. Ratisbon. II. 2. 1769; T. 190 F. 7.

? Vespa coarctata Fabricius, Syst. entom. 1775 p. 370 n. 39 , §̋.

Vespa coaretata Ph. L. Müller, Limé: Vollst. Natursyst. V. 2. 1775 p. \$૬:, n. 11.

Vespa coarctata O. F. Müller, Zool. Danic. prodr. 1776 p. 163 n. 1892.

? Vespa coarctata Fabricius, Spec. Insect. I. 1781 p. 467 n. 53.

? Vespa coarctata Fabricius, Mant. Insect. I. 1757 p. 292 n. 65.

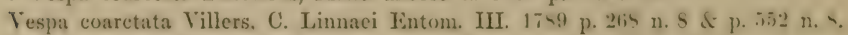

Vespa coarctata Gmelin, Linné: Syst. nat. Ed. $13^{\mathrm{a}}$ I. 5. 1790 p. 2752 n. 11.

Vespa coarctata Olivier, Encycl. méthod. Insect. VI. 1791 p. 671 n. 9.

? Vespa coarctata Fabricius, Entom. system. II. 1793 p. 276 n. 82.

Vespa coarctata Cederhjelm, Faun. Ingric. prodr. 1795 n. 169 n. 524.

Eumenes eoarctata Latreille, Hist. nat. Insect. III. 1802 p. 360.

Vespa coarctata Schrank, Fauna Boica II. 2. 1802 p. 359 n. $2 \cdot 18$.

Vespa coarctata Walckenaer, Fauna Paris. II. 1802 p. 87 n. 1.

? Kumenes coaretata Fabricius, Syst. Piez. 1804 p. 288 n. 16.

? Eumenes atricornis Fabricius, Syst. Piez. 1804 p. 289 n. 17.

Eumenes coarctata Latreille, Hist. nat. Insect. XIII. 1505 p. 345 n. 2.

Vespa (Eumenes) coaretata Panzer, Krit. Reris. II. 1806 p. 159.

Eumenes coaretata Spinola, Insect. Ligur. I. 1806 p. 83 n. 2. 
Vespa coarctata Jurine, Nour. méth. class. Hymén. 1807 p. 171, 우 ő. ? Eumenes atricornis Latreille, Gen. Crust. \& Insect. IV. 1809 p. 137. Eumenes coarctata Latreille, Gen. Crust. \& Insect. IV. 1809 p. 139.

Eumenes coarctata Lamarck, Hist. nat. anim. 8. vert. IV. 1817 p. 83 n. 1.

Eumenes atricornis Curtis, Brit. Entom. I. 1824 p. 13, 우 శే; T. 13.

Eumenes coarctata Brullé. Expéd. sc. Morée. Zool. II. 1832 p. 362 n. \$1)1.

Eumenes coarctatus Hartig, Forstl. Conversationslex, 1834 p. 201.

Eumenes coaretata Lamarck, Hist. nat. anim. s. vert. Ed. 2a IY. 1833 p. 300 n. 1.

Eumenes coaretata Zetterstedt, Insect. Lappon. I. 1838 p. 458 n. 1, 우 ô.

Eumenes coarctata Goureau. Ann. soc. entom. Franee VIII. 1839 p. 531, O; 'T. 1 S III. F.1-3.

? Eumenes atricornis Testwood. Introd. mod. Classif. Insect. II. 1S40 Synops. p.23!; F. S7. 6 ;

Fumenes coarctata Blanchard, Hist. nat. Insect. III. 1840 p. 393 n. 1. [Synops.p.\$3.

Vespa Eumenes coarctatus Ratzeburg, Forstinsect. III. $184 t$ p. 52 n. 3.

Eumenes coaretata Luciani, Ann. soc. entom. France (2) III. 1845 Bull. p. CX. [F.1.

Vespa (Eumenes) coarctata Blanchard, Cuvier: Règne anim. Ed. $3^{3}$. Insect. II. 14.4! T. 124

Eumenes coarctata Vallot. Ann. soc. entom. France (2; VII. 1849 Bull. p. LXXIV.

Eumenes coarctata Saussurc, lítud. fam. Vespid. I. Eumén. 1552 p. 31 n. 2 , $f \hat{\jmath}$.

Eumenes coarctata Schenck, Jahrb. Ver. Naturk. Nassau IX. 1. 15.53 p. is n. 3, $\subseteq$ Jै.

Eumenes coarctata Smith, Catal. Brit. Fossor. Hymen. 15.58 p. 197 n. 1, S J; T. 5 F. 2 \& 3.

Vespa coarctata Duméril, Mém. acad. sc. Paris XXXI. 1860 p. 862 n. 8.

Eumenes eoarctatus Schenck, Jahrb. Ver. Naturk. Nassau XVI. 1561 p. 29, 30, 32 n. 2, $\leq$ j

Eumenes coarctata Radoszkowski, Horae soe. entom. Ross. III. 1565 p. $5 i$ n. 1.

Eumenes coarctatus Smith, Entomol. Anmual f. 1866 p. 134.

Eumenes coarctatus Smith, Trans. Entom. Soc. Jondon (3) V. 1567 Proc. p. CVI.

Eumenes coarctata Thomson, Opusc. entom. P. 2. 1870 p. 83 n. 1.

? Eumenes atricornis Thomson, Opusc. entom. P. 2. 1870 p. 83 n. 2 , \& ô.

Eumenes coarctatus F. Morawitz, Horae soc. entom. Ross. IX. 1873 p. p. 294 n. 2. ᄃ. Eumenes coarctata Thomson, Hymen. Scandin. III. 1874 p. 35 n. 1, 우 ô.

? Eumenes atricornis Thomson, Hymen. Scandin. III. 1874 p. 37 n. $2, \subseteq j$.

Eumenes coarctata Kriechbaumer, Entom. Nachr. V. 1879 p. 309.

Eumenes coarctata P. G., Naturaliste I. 1879 p. 99.

[F.16.

Lumenes coarctata Canestrini \& Berlese, Atti soe.Venet.-Trentin. VII. P.1.14\$1 p. 7; ; T.7

Eumenes coarctatus E. Saunders, Trans. Fintom. Soc. J.ondon 1542 p. $141 \mathrm{n} .1$.

Eumenes conretatus Ed. André, Spee. Hymén. Europe II. P. 21-23. 154 p. (it1.

Eumenes coarctatus Rudow, Soc. entom. I. 1886 p. 84.

Eumenes coarctatus Schulthess. Hymen. Melret. Diplopt. 1 s4i p. 26 n. 3. $\leq \mathbf{j}$.

var. bimaeulatı André. - Eur. mer.

Eumenes bimaculatus Fid. Indré, Spee. IIYmén. Lurope II. P. 21-23. 1444 p. (4t).

Eumenes coarctatus var. bimaculatus Schulthess. Hymen. Melvet. 1)iplopt. 154i p. 25.

var. marginella Herr.-Schaeff. - $f$ f - Eur.: Germania.

Eumenes marginella Herrich-Sehaeffer, Faum. Insect. German. 1441 P. 17! T. \& I 5 :

Eumenes marginella Sehenek, Jahrb. Yer. Naturk. Nassau IX. 1. 1453 p. is n. 2, $\subseteq 3$.

Eumenes coarctata var. marginella Saussure, litud. fam. Vespid. I. Eumén. 1452 p. 31.

var. mongolica Mor. - o ơ - As.: Mongolia.

[p. 161 n. 6.

Fumenes coaretatus var. Mongolicus Morawitz. Iforae soe. entom. Ross. XXIII. 1ss?

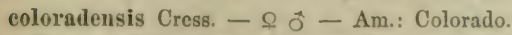

[p. 717 n. 90, 오

Eumenes Coloradensis Cressou. Rep. geogr. of geol. Fixplor. d Surv. 1moth Merid. 157t V.

compressa Sauss. - 우 ô-Am.?

[F. $5($ C)

Eumenes compressus Saussure. Ltud. fam. Yespid. III. Masar. 1 s.ici p. 142 n. 2s, Q j : T. I

Fumenes .1pha compressus Saussure. Smithson. Miscell. Collect. XIV. P. 251.1875 p. 10, 
concinua Sauss. $-f \hat{o}-$ Afr.: Nubia.

Eumenes concinnus Saussure, Litud. fam. Vespicl. III. Masar. 1656 p. 114 n. 30, 3.

Eumenes concinna Gribodo, Amm. mus. civ. Genova XXI. 1 s4 p. 2913 n. 30. 5 .

Eumenes concinna Magretti, Ann. mus. eiv. Genota XXI. 144t p. li11 n. 147, 5 J. conformis Smith - Q - As.: Ceram.

Eumenes conformis Smith, Icon, of Proc. Linn. Soc. Zool. VII. 1s6i3 p. is n. 5 , 5 . conica Fabr. - $f \hat{\sigma}-$ As.: India, China, Philippines.

Vespa conica Fabricius, Syst. entom. 1775 p. 372 n. 46.

Vespa conica Fabricius, Spec. Insect. I. 1781 p. 469 n. 65.

Vespa conica Fabricius, Mant. Insect. I. 1787 p. 293 n. 69.

Vespa conica Gmelin, Linné: Syst. nat. L̇d. $13^{3}$ I. 5.1790 p. 2754 n. 63.

Vespa conica Olivier, Encycl. méthod. Insect. VI. 1791 p. 670 n. 4.

Vespa conica Fabricius, Entom. system. II. 1793 p. 278 n. 88.

Eumenes conica Fabricius, Syst. Piez. 1804 p. 285 n. 2.

Vespa conica Jurine, Nouv. méth. class. Hymén. 1807 p. 171, ㅇ.

Eumenes conica Saussure, Kitud. fam. Vespid. I. Eumén. 1852 p. 52 n. $34 . \subseteq 3$.

Eumenes conica Horne, Trans. Zool. Soc. London VII. 3. 1870 p. 16t;, S; T. 211 F. 2 \& 2 .

consobrina Sauss. - ô- Am.: Brasilia.

lumenes consobrinus Saussure, litud. fam. Vespid. III. Masar. 1556 p. 140 n. 25, j.

Eumenes (Alpha) consobrinus Saussure, Smithson. Miseell. Collect. XIV. P. 25\%. 1575 eressoniana Sauss. - 우 - Am.: Mexico.

[p. $100 \mathrm{n} .40$, ô.

Eumenes Beta Cressonianus Saussure, Smithson. Miscell. Collect. XIV. P. 254. 1575 p.90 erneifera Prov. - + - Am.: California.

[n. 31, ㅇ․

Eumenes crucifera Provancher, Addit. faun. Canada. Hymén. $15 \varsigma 5$ p. 421 n. 2, $\subseteq$.

eubensis Cress. - 으-Am.: Cuba.

Eumenes Cubensis Cresson, Proe. Entom. Soe. Philadelphia IV. 1S6j p.15i. S. ' n.t5. .

Eumenes Alpha. Cubensis Saussure, Smithson. Miscell. Collect. XIV. P. 254. 1-i5 p. 102

curvata Sauss. - 오-As.: Philippines.

Eumenes curvatus Saussure, Kitud. fam. Vespid. III. Masar. 15506 p. 145 n. 32. S; T.S F. 1. eyathiformis Fabr.! - As.: Java.

Eumenes cyathiformis Fabricius, Syst. Piez. 1804 p. 289 n. 18.

decorata Smith - 우 $\widehat{o}-$ As.: China.

Eumenes decoratus Smith, Trans. Entom. Soc. Tondon 2, II. 2. 1952 p. $36 . \subseteq \hat{\jmath}$.

Eumenes decoratus Saussure, Etud. fam. Vespid. III. Masar. 1856 p. 135 n. 22. ᄃ.

depressa Sauss. - - - As.: India.

[n. 17, $\subseteq$.

Eumenes (Pareumenes, depressus Saussure, Kitud. fam. Vespid. III. Masar. 18 it p p. 135 dichroa Maindr. - ot - As.: Tidorc.

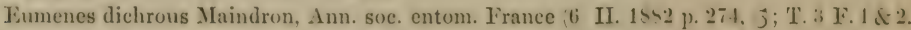
dilectula Walk. - ô- As.: Arabin.

Eumenes dilectula Walker, List of Hymen. in Egypt 1871 p. 30 n. 15i, ô.

diligens Smith - 으-As.: Bouru.

Eumenes diligens Smith, Journ. of Proe. Limn. Soc. Zoul. VII. 1 sti3 p. 391 n. 12. S.

dimidiatipeunis Sauss. $-\rho \hat{\sigma}-$ As. Arabia, India; Afr.: Aegyptus.

- Savigny, Deser. de l'Égypte 1812; T. 8 F. 5.

Eumenes dimidiatipemis Saussure, Etud. fam. Tespid. I. Fumén. 1s.2 p. 51 n. 33, $5 j$.

Lumenes dimidiatipennis Ed. André. Spee. Ilymén. Furop̣e II. 1'.21-23. 14s4 p. 631. \& j.

Eumenes dimidiatipennis Magretti. Ann. mus. civ. Genora XXI. 14 b p. 610 n. 146.

Eumenes dimidiatipennis Saussure, Grandidier: Hist. Madagasear XX. P. 1. 14!91 p. 154

distineta Sauss. - ô - Afr.

$[$ ก. $3, \subseteq 0$.

Eumenes distinctus Saussure, litud. fam. Vespid. 1II. Masar. 1456 p. $14: 3$ n. 29. $\vec{j}$. 
doryens Maindr. - $\mathrm{Q}-$ As.: New Guinea.

Eumenes Dorycus Maindron. Ann. soc. entom. France 6. II. 146? p. 273; T. 3 F. 3-6. dubia Sauss. - of of - Eur.: Gallia.

Fimmenes dubia Saussure. Fitud. fam. Vespid. I. Fumén. 1852 p. 32 n. $3, \subseteq \widehat{s}$.

Eumenes dubius Saussure, Étud. fam. Vespid. III. Masar. 1856 p. 129.

dumetorum Panz. - $\widehat{o}$ - Eur.: Helvetia.

Vespa coarctata Sulzer, Abgek. Geseh. Insect. I. 1776 p. 196; T. 27 F. 12 [nee L.].

Vespa coarctata Römer, Gen. Insect. 1789 p. 124; T. 27 F. 12.

Vespa dumetorum Panzer, Faun. Insect. German. VI. 1799 P. 63 F. 4.

Vespa (Eumenes) dumetorum Panzer, Krit. Revis. II. 1806 p. 159.

Vespa dumetorum Jurine, Nouv. méth. class. Hymén. 1807 p. 171. đ.

dyschera Sauss. - 우 - Afr. trop.

Eumenes Dyschera Saussure, litud. fam. Vespid. I. Eumén. 1552 p. 50 n. 31, \&.

edwardsii Sauss. - 우 - As.: India.

Eumenes Edwardsii Saussure, Étud. fam. Vespid. I. Eumén. 1852 p. 60 n. 45 , f; T. 11 I.4.

Eumenes Edwardsii Horne, Trans. Zool. Soc. Jondon VII. 3.1870 p.167, S; T. 20 F. $4 \& 4^{3}\left\{S_{i}\right.$.

elegans Sauss. - - - As.: Arabia, India.

Eumenes elegans Saussure, Étud. fam. Vespid. I. Eumén. 1552 p. 58 n. 42, ऽ; T. 11 F. 3. elegantula D. T. - ㅇ - As.: Batchian.

Pachymenes elegans Smith, Journ. of Proc. Iimm. Soc. Zool. IV. 1560 Suppl. p. 131 n. 1, C.

Eumenes elegantula $\mathrm{m}$.

esuriens Fabr. - 우 ô - As.: India.

Vespa esuriens Fabricius, Mant. Insect. I. 1787 p. 293 n. 75.

Vespa esuriens Gmelin, Linné: Syst. nat. Ed. $15^{a}$ I. 5.1790 p. 2754 n. 58.

Vespa pediculata Olivier, Encycl. méthod. Insect. VI. 1791 p. 670 n. 7.

Vespa esuriens Olivier, Encycl. méthod. Insect. VI. 1791 p. 673 n. 17.

Vespa esuriens Fabricius, Entom. system. II. 1797 p. 280 n. 94.

Eumenes esuriens Fabricius, Syst. Piez. 1804 p. 286 n. 7.

Vespa esuriens Jurine, Nour. méth. class. Hymén. $180 \tau$ p. 171, $\subseteq$ ô.

$[\mathrm{F} .2 \Omega]$.

Eumenes esuriens Saussure. litud. fam. Vespid. I. Eumén. 1852 p. 56 n. 40, $\subseteq$ ơ; T. 11

Eumenes esuriens Smith, Journ. of Proc. Linn. Soc. Zool. IV. 1560 Suppl. p. S6 n. 1.

Eumenes esuriens Horne. Trans. Zool. Soc. London VII. 3. 1570 p. 166, \&; T.20 F. 6 \& 6.

Fimmenes esuriens Ed. André, Spee. Hrmén. Europe II. P. 20.1854 p. 619 n. $1 . \subseteq \hat{\jmath}$.

Eumenes esuriens Maindron, Ann. soe. entom. France (i) II. 1852 p. 271 n. 9. 5 .

var. campaniformis Fabr. - $\mathrm{Q}-$ Austr.

Vespa campaniformis Fabricius, Syst. entom. 1775 p. 371 n. 41.

Vespa campaniformis Fabricius, Spec. Insect. I. 1781 p. 467 n. 55.

Vespa campaniformis Fabricius, Mant. Insect. I. 1787 p. 292 n. 67.

Tespa campraniformis Gmelin. Linné: Syst. nat. Ed. $13:$ I. 5.1790 p. 2753 n. 50.

Sphex campaniformis Christ, Naturg. d. Insect. 1791 p. 312.

Vespa campaniformis Olivier, Encycl. méthod. Insect. VI. 1791 p. 670 n. 3.

Vespa campaniformis Fabricius, Entom. system. II. 1793 p. 277 n. 84.

Eumenes campaniformis Fabricius, Syst. Picz. 1804 p. 287 n. 10.

Eumenes campaniformis Saussure, litud. fam. Vespid. I. Eumén. 15.52 p. 3.5 n. $34 . \varsigma$. Eumenes esuriens var. eampaniformis Saussure, Ktud. fam. Vespid. III. Masar. 1556 p p. 131.

Eumenes esuriens var. eampaniformis Maindron. Ann. soe. entom. Franee fi II. 1552 p. 271 var. graeilis Sauss. $-\subseteq \hat{\sigma}-$ Afr.: Aegyptus.

$[\mathrm{n} .9$, \&.

- Savigny, Deser. de l'Égypte 1812 ; T. 8 F. 6.

Eumenes gracilis Sanssure. Etud. fam. Vespid. I. Eumén. 14.72 p. 5 i n. 41 . \& j.

Eumenes esuriens rar. graeilis Saussure, litud. fam. Yespid. III. Masar. 15.jt p. 1:31.

Eumenes esuriens var. grateilis Miggretti. Ann. mus. cir. Genova XX1. 144 p. (612 11.14s. 
var. nrvillei Sauss. - 0 of - Austr.: New Guinca.

Eumenes Lrrillei Saussure, Étud. fam. Vespid. I. Eumén. 15.52 p. 59 n. 41. $\leq$ j.

Eumenes esuriens var. Urvillei Maindron, Amn. sue. entom. France b. II. $15-2$ p. 271. exigua Sauss. - $\hat{o}-$ As.: India.

Eumenes exiguus Saussure, Ktud. fam. Vespid. III. Masar. 1456 p. 150 n. 39. 3̈; T. \& F. 4. eximius Smith - ㅇ - As.: Batchian.

Eumenes Pareumenes eximius Smith. Journ. of Proc. Linn. Soc. Zool. IV. 1 (fi) Supprl. fasciata Fabr.1 - As.: Java.

[p. 126 n. 6, ㅇ.

Eumenes fasciata Fabricius, Syst. Piez. 1504 p. 290 n. 19.

fenestralis Sauss. - - ơ - Afr.: Congo, Senegal.

Eumenes fenestralis Saussure, Étud. fam. Vespid. I. Eumén. 1452 p. 53 n. 35, $\subseteq$ jे; T. 10

Eumenes fenestralis Desmarest. Chenu: Encycl. hist. nat. Annelles 1460 p. 1.13; Fig. 123.

Jumenes fenestralis Heyden, Berlin. entom. Zeitschr. XI. 146i p. 395.

ferruginea Cress. - $\widehat{o}-$ Am.: Cuba.

Eumenes ferruginea Cresson, Proe. Entom. Soc. Philadelphia IV.1465 p.154, j. [n. $35, j$

Eumenes (Alpha, ferrugineus Saussure, Smithson. Miscell. Collect. XIV. P.251. 157.5 p.94

fliformis Sauss. $-\Omega-$ Am.: Brasilia.

Eumenes filiformis Saussure, Etud. fam. Vespid. III. Masar. 1 \$5li p.146 n.34, ᄃ. [n. 53. . .

Eumenes Zeta filiformis Saussure. Smithson. Misecll. Collect. XIV. P. 254. 1475 p. 1u!

flavicornis Sauss. - $\hat{\sigma}-$ Am.: Venezuela.

Eumenes flavicornis Saussure, Étud. fam. Vespid. III. Masar. 15566 p. 141 n. 27. ö; T. 7 F.4.

Eumenes (Alpha) flavicornis Saussure, Smithson. Miscell. Collect. XIV. P. 254 . 1475 p. 14

flavopectus Prov. - $ᄋ-$ Am.: Trinidad.

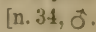

Eumenes flavopectus Provancher, Addit. faun. Canada. Hỵmén. $15 s$ s p. 422 n. J. c.

flnetuaus Sauss. - 우 - Austr.

Eumenes fluctuans Saussure, Etud. fam. Vespid. I. Eumén. 1552 p. 43 n. 22, $\subseteq$.

formicaria Fabr.! - Am. mer.

Eumenes formicaria Fabricius, Syst. Piez. 1804 p. 285 n. 15.

formosa Sauss. - - - Afr.: Congo.

Eumenes formosa Saussure, Étud. fam. Vespid. I. 1852 p. 55 n. 39, 우.

fratereula D. T. - 우 - As.: Japonia.

Eumenes fraterna Smith. Trans. Entom. Soc. I.ondon 1573 p. 195 n. 1, $\subseteq$ [non Say]. Eumenes fratercula $\mathrm{m}$.

fraterua Say - $+\hat{o}-$ Am.: Canada, United States.

Eumenes fraterna Say, Keating's Narrat. Exped. II. 1424 App. p. 344 n. 1, $\leq 3$.

Eumenes fraterna F. W. Harris, Boston Cultiv. X. 1848 p. 225; Fig.

Eumenes fraterna Saussure, litud. fam. Vespid. I. Eumén. 1552 p. 40 n. 16, \& 5 ; T. 11 F.4.

Eumenes fraterna Leconte, Writ. of 'Th. Say Entom. II. 1859 p. 232 n. 1, § §ิ.

Fumenes fraterna Walsh of Riley, Amer. Entomol. I. 1869 p. 135; Tigr. 110.

Limmenes fraterna Riley, 24 Ann. Rep. Insect. Missouri 1870 p. 103; Fig. 71.

Eumenes fraternus Couper, Canad. Entomol. IUI. 1571 p. 62 n. 1.

Eumenes fraterna Smith, Trans. Entom. Soc. London 1873 p. 195 n. 1, 우.

liumenes Alpha fraterna Saussure, Smithson. Miscell. ('ollect. XIV. P. 254. 14 is p. 4.5

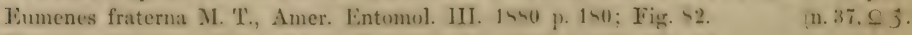

Eumenes fraternus Riley, Amer. Entomol. III. 1550 p. 150; Tig. 82.

Eumenes fraterna S. Saunders, Rep. Fruit Growers Assex. Ontario 1442 p. 241.

dimenes fraterna Prowancher, Natural. Camad. XIII. 14a2 p. 141 if 6 is s s 3 .

Eumenes fratema Riley, $3^{\text {th }}$ Rep. U. St. Entom. Commiss. 1443 p. 117: Fig. 3.

Eumenes fraterna Southwick, Insect Life V. 1892 p. 107. 
var. ferrens Sauss. - 우 ठొ - Am.: Carolina.

Eumenes ferrens Saussure. Kitud. fam. Vespid. I. Eumén. $14.2 \mathrm{2}$ p. 40 n. 15, $\subseteq$ oै.

Eumenes fraterna var. fervens Saussure, litud. fam. Vespid. IlI. Mlasar. 1556 p. 131). var. maerops Sauss. - 으 Am.: Carolina.

Eumenes macrops Saussure, Citud. fam. Vespid. I. Eumén. 15.2 p. 41 n. 1 S, C.

Eumenes fraternus rar. maerops Saussure, Smithson. Miscell. C'ollect. XIV. P. 254. 1575 p.95. var. minuta Sauss. - ô - Am. bor.

Eumenes minuta Saussure, litud. fam. Vespid. I. Eumén. 1552 p. 39 n. 14, $\overrightarrow{3}$ [non Fabricius . Eumenes fraternus var. minuta Saussure, Smithson. Miscell. Collect. XIV. P.254. 157.5 p.95. fulva Ev. - 으 - As. centr.

Eumenes fulva Eversmann, Bull. soc. natural. Moscou XXVII. 3. 1554 p. 199.

Eumenes fulra Radoszkowski, Horae soc. entom. Ross. III. 1565 p. 59 n. 6; T. 1 F. . . Eumenes fulvus Ed. André, Spec. Hymén. Europe II. P. 20.1554 p. 626 n. 6, f. germainii Luc. - $ᄋ$ $\widehat{f}$ - Austr.: New Caledonia.

Eumenes Germaini Lucas, Ann. soe. entom. France (5) V. 1575 Bull. p. LXXVI.

Eumenes Germaini Lueas, Ann. soc. entom. France (5) VI. 1576 p. $296 \& 300$, o ; 'T. 4 I. 1-s. Eumenes Germaini Iucas, Ann. soc. entom. France (5 IX. 1579 Bull. j). LXV.

Eumenes Germaini Maindron, Ann. soc. entom. France 6) II. 18s2 p. 76; T. 4 F. 1-5. ghilianii Spin. - ô - Am.: Brasilia.

Eumenes Ghilianii Spinola. Mem. acad. sc. Torino (2) XIII. 1851 p. 81 n. $61, \hat{\jmath}$.

Fumenes Ghilianii Saussure, itud. fam. Vespid. III. Masar. 1656 p. 140 n. $26 . \vec{\jmath}$.

Eumenes Alpha' Ghilianii Saussure, Smithson. Miscell. Collect. XIV. P.254. 1575 p.95 n.36, globicollis Spin. - - - Am.: Guinea, Brasilia.

Zethus globicollis Spinola. Ann. soc. entom. France X. 1541 p. 136 n. 84, §; T. 3 F. 6. Eimenes globicollis Saussure, Titud. fam. Vespid. III. Masar. 1556 p. 151 n. 40, \&; T. S F.ti. Fumenes Omicron globicollis Saussure, Smithson. Miscell. Collect. XIV. P. 254. 1 i i5 p. 69 globulosa Sauss. - $\subseteq-$ Am.: British America, Illinois, Wisconsin.

[1. 10,. .

Eumenes globulosus Saussure, Kitud. fam. Vespid. III. Masar. 1556 p. 139 n. 23. ᄃ.

Eumenes IAlpha; globulosus Saussure, Smithson. Miscell. Collect. XIV. P. 254. 1875 p. 111 gnerinii Sauss. - ㅇ- - Afr.: Madagascar.

Eumenes Guérini Saussure, litud. fam. Vespid. I. Eumén. 1852 p. 62 n. 4 , ᄃ.

hottentotta Sauss. - 우 - Afr.: Cap.

Eumenes Hottentotta Saussure, Étud. fam. Tespid. I. Eumén. 15.52 p. 63 n. 19. S.

humbertiana Sauss. - 우 ô - As.: Ceylon.

Eumenes Humbertianus Saussure, Reise d. Torara. Zool. II. 1. 1 -67 Ifymén. p. 6. Q.3.

japonica Sauss. $-\propto-$ As.: Japonia.

Eumenes Japonicus Saussure, Rev. \& mag. zool. (2) X. 1858 p. 164, \&.

imperatrix Smith - 우 - As.: China.

Eumenes imperatrix Smith, Catal. Hymen. Brit. Mus. V. 15.37 p. 24 n. 31, ᄃ.

impuncta Prov. - $\&-$ Am. : T'rinidad.

Eumenes impunetus Proraneher, Addit. faun. Canada. Hỵmén. 145s p. 421 n. 3. L.

incerta Sauss. - $Q-$ Am.: Brasilia.

[n. 26, Q.

Fumenes Omicron incertus Saussure, Smithson. Miscell. ('ollect. XIV. 1'.254. 1575 p. 4

indiana Sauss. - 우 - Afr.? Ass?

[n. 19, \&; T. 7 F. 3.

Eumenes Pareumenes Indianus Saussure. litud. fam. Vespid. III. Masar. 1sati p. 136

inferualis Sauss. - 우 - Am.: Brasilia, Guiana.

[p. 56 n. 29, s.

Eumenes IOmieron) infernalis Saussure, Smithson. Misecll. Collect. XIV. P. 251. 14it

inflexa Sauss. - - - (Am.: Cayenne?) As.?

Eumenes inflexus Saussure, Kitud. fam. Vespid. III. Masar. 15.36 p. 145 n. 33. ᄃ. 
insolens Smith - $\subseteq-$ As.: Gilolo.

Eumenes insolens Smith, Journ. Linn. Soc. Zool. VUI. 1864 p. SS n. 7, S.

insularis Smith - 오

liumenes insularis Smith, Catal. Hymen. Brit. Mus. Y. 1657 p. 27 n. $52 . \leq 3$.

iturbile Sauss. - $f$ ô - Am.: Mexico.

Eumenes Iturbide Saussure, Rev. \& mag. zool. 2 IX. 1457 p. $271 . \quad$ [n. $39 . \subseteq 3$.

Eumenes Alpha. Iturbide Saussure, Smithson. Miscell. Collect. XIV. P. 254.1575 p. 95

kohlii Brancs. - $\Omega-$ Afr.: Nossibé.

Eumenes Kohli Branesik, Jahresh, natur. Ver. Trenesin XIII/XIV. 1590991 p. 159. ᄃ.

Iaboriosa Smith - \& - As.: Celebes.

Eumenes laboriosus Smith, Journ. of Proe. Iinn. Soc. Zool. IV. 1 4ijo Suppl. p. 57 n. 4.5.

Eumenes laboriosus Maindron. Ann. soc. entom. France (6) II. 1552 p. 265 n. 4.

Iaminata Kricchb. - ô - Eur.: Dalmatia.

Eumenes arbustorum Herrich-Schaeffer. Faun.Inseet.German.14.41 1.179.T.9 j inee Panz..

Eumenes laminata Kriechbaumer, Entom. Nachr. V. 1879 p. 59 n. 2, $\vec{o}$.

Eumenes laminata Ed. André, Spec. Hymén. Europe II. P. 24-26. 1546 p. s71. j.

latreillei Sauss. - $\mathrm{C}-\mathrm{Austr}$.

Eumenes Iatreillei Saussure, Iitud. fam. Vespid. I. Eumén. 1852 p. 51 n. 32. C; F. 10 F. 5.

Jumenes Latreillii Roth, Journ. Linn. Soc. I.ondon Zool. XVIII. 107. 1455 p p.321; Fig. 2.1, 1 .

lepeleterii Sauss. - 우 ơ - Afr.: Senegal.

Eumenes Lepeleterii Saussure, Étud. fam. Vespid. I. Eumén. 1552 p. 45 n. 24, @; T. 10 F. 1. Eumenes formosa var. Lepeletieri Saussure, Litud. fam. Vespid. III. Masar. 15.56 p. 130. Eumenes I.epeletieri Gerstaecker, v. d. Decken: Reise in Ost-Afrika. Gliederthiere. 1५i3

[p. 322 n. 12, ㅇ․

Eumenes I.epcleticrii Radoszkowski, Horac soc. entom Ross. XII. $15 i 6$ p. 141 n. s, s. Eumenes Lepeletieri Ed. André, Spec. Hymén. Europe II. P. 21-23. 1 th4 p. 632.

Eumenes Iepeletieri Giribodo, Ann. mus. civ. Genova XXI. 1344 p. 292 n. 27 . ᄃ.

Fumenes Iepeletieri Magretti, Ann. mus. civ. Genora XXI. 1554 p. 611 n. $1.15 . \subseteq 3$.

leptogastra Walk. - ô - As.: Arabia.

Eumenes leptogaster Walker. List of Hymen. in Ligypt $15 i 1$ p. $30 \mathrm{n.} 155, \hat{\jmath}$.

levis Sauss. - $\mathrm{Q}-\mathrm{Am}$. Brasilia.

Eumenes cingulatus Saussure, Ktud. fam. Vespid. III. Masar. 1556 p.151 n.41, $\subseteq$ non Fabr. Fumenes Omicron) laevis Saussure. Smithson. Miscell. Collect. XIV. P.254. 15 .5 p. is n. 15. . .

longirostris Gerst. - $‡$ - Afr.: Mosambique.

Eumenes longirostris Gerstaceker, Monatsber. Akad. Wiss. Berlin 1857 p. 462 n. ‥ 3. Eumenes longirostris Gerstaecker. Peters: Reise n. Aossambique. Zool. V. $151 ; 2$ p. 462 . J; Ineasia Sauss. - ơ - Afr.: Abyssinia.

[T. 30 F. 1.

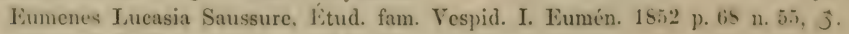

maerocephala Sauss. - ơ - Afr.: Cap.

Eumenes macrocephala Saussure, Etud. fam. Vespid. I. Eumén. 1852 p. 37 n. 11. j.

mainpuriensis Smith $-\rho \tau-A s$.: India.

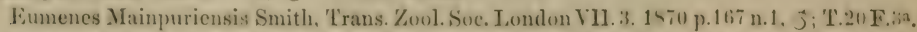

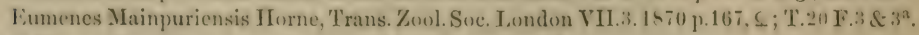
maxillosa (Deg.) Sauss. - o ô - Afr.: Aegyptus, Senegal, Congo.

Vespa maxillosa Degeer, Mém. hist. Insect. III. 1773 p. 577 n. 1; T. 29 F. 1-2.

Vespa maxillosa Giöıe, Degeer: Abh. (iesch. Insect. III. 17-1) p. 373 n. 1; T. 2!9 F. 1-2.

Vespa maxillosa Retzius, Gen. \& spec. Insect. 1783 p. 63 n. 232.

Sphex tinctor Christ, Naturg. d. Insect. 1791 p. 311; T. 31 F. 1.

- Savigny, Deser. de l'égypte 1512 Hymén.; T. 5 F. 4.

Eumenes Sasignyi spinola. Am. soe. entom. France VII. 1934 p. 603 n. XIVIII. 
Eumenes Sarignyi Guérin. Icon. règn. anim. VII. Insect. 1515 p. 416 ; 'I'. 72 F. 4. Eumenes tinetor Saussure, Etud. fam. Vespid. I. Eumén. $14 ; 2$ p. 49 n. $30, \subseteq$ j.

Eumenes tinctor Smith. Trans. Entom. Soc. London 2 III. 15.56 Proc. p. $12 \mathrm{~s}$ n. 3. Eumenes tinctorius Gueinzius, Trans. Entom. Soc. London 2, V. 155 - Proc. p. 10. Eumenes tinctor Gerstaceker, Peters: Reise n. Mossambique. Zool. V. 14i2 p. 462. Eumenes tinctor Heyden, Berl. entom. Zeitschr. XI. 1867 p. $398 . \quad$ n.11. Eumenes tinctor Gerstaecker, v. d. Decken : Reise in Ost-Afrika. Gliederthiere. 15 i3 p. 321 Eumenes tinctor Ed. André, Spec. Hymén. Europe II. P. $21-23.1544$ p. 6i30, $\leq \vec{j}$.

Eumenes tinctor Gribodo, Ann. mus. cir. Genova XXI. 1854 p. 293 n. 29,..

Eumenes tinctor Magretti, Ann. mus. civ. Genora XXI. 1884 p. 609 n. 145.

Eumenes maxillosus Saussure. Grandidier: Hist. Madagascar XX. P. 1. 1591 p. $15.3 \mathrm{n} .1, \mathrm{c} \vec{j}$. mediana Smith - ô - As.: Ceram.

Eumenes medianus Smith, Joum. of Proc. Linn. Soc. Zool. VII. 1563 p. 35 n. $7 ., j$.

mediterranea Kriechb. - $ᄋ \widehat{o}$ - Eur. mer.; As. occ.

Eumenes mediterranea Krichbaumer, Entom. Nachr. V. 1879 p. 85, 오 oิ.

Eumenes mediterraneus Ed. André, Spec. Hymén. Europe II. P. 21-23. 1 ş 1 p. 6.40. ᄃ ô. Eumenes coarctatus var. mediterranca Schulthess, Hrmen. Helvet. Diplopt. 1457 p. 25. var, heerii Kriechb. - 9 ô - Eur. mer.

Eumenes mediterranea var. Heeri Kriechbaumer, Entom. Nachr. V. 1579 p. \&s. \& $\vec{\jmath}$. var. neesii Kricchb. - 0 o - Eur. mer.

Eumenes mediterranea var. Neesii Kricchbaumer, Entom. Nachr. V. 1 is! p. SS, $\subseteq \vec{j}$

melanosoma Sauss. - 0 ơ - As.: Java.

[F. 1 (ㅇ).

Eumenes melanosoma Saussure, litud. fam. Vespid. I. Eumén. 1552 1) 61 n. 4t. ᄃ J; T. T2

Eumenes melanosoma Maindron. Ann. soc. entom. France (6) II. 1552 p. 272 n. 10.

mexicana Sauss. - $f \hat{\jmath}-$ Am.: Mexico.

Eumenes Mexicanus Saussure, Rer. \& mag. zool. (2) IX. 1857 p. 272.

Eumenes Omicron) Mexicanus Saussure, Smithson. Miscell. Collect. XIV. P. 254.1475 p. 7.5 mieroseopiea Sauss. - 우 - Am.: Brasilia. [n. 15, 우우 'T. 1 F. $8 \& 8$ ? Eumenes microscopica Saussure, Étud. fam. Tespid. I. Fumén. 1552 p. 72 n. 62 , $\subseteq$.

Jumenes (Omicron microscopicus Saussure, Smithson. Miscell. Collect. XIV. P. 251.145 miles Sauss. - 우 - Am.: Guiana, Surinam.

[p. 82 n. 24.

Eumenes Omicron miles Saussure, Smithson. Misecll. Collect. XIV. P. 251.1575 p.55 n.27.f. minuta Fabr. - - - Am. mer., Brasilia.

? Eumenes minuta Fabricius, Syst. Piez. 1804 p. 291 n. 23.

[n. 20, 우.

Eumenes Omicron minutus Saussure. Smithson. Miscell. C'ollect. XIV. 1'. 251. 157.5 p.41 multipieta Sauss. - ô - As.: Sumatra.

Eumenes multipictus Saussurc, Rev. \& mag. zool. (2) VII. 1855 p. 372, ơ.

nana Kirsch $-\Omega-$ Am.: Columbia.

Eumenes nanus Kirsch, Mittheil. zool. Mus. Dresden III. 1578 p. 376, f.

nigra Brullé - $\odot$ ot - Eur.; Afr.: Aegyptus.

?- Sarigny, Deser. de l'ligypte 1812 ; T. 8 F. 3.

Eumenes nigra Brullé, Barker- Wl ebb of Berthelot. Hist. nat. iles Canar. II. 1 440 p. S4 n. 11 . Eumenes nigra Saussure, litud. fam. Vespid. I. Eumen. 1452 p. 34 n. 12. $\leq$ j.

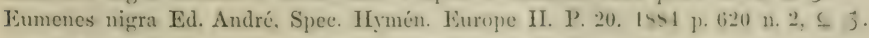

nortoniana Sauss. - - ơ - Am. : Mexico.

[p. $85 \mathrm{n} .30$, 우

Eumenes, Beta Nortonianus Saussure. Smithron. Miseell. ('ollect. XIV. P. 254. 1475 novarne Sauss. - $\Omega-$ Am.: Brasilia.

Eumenes Novarae Saussure, Reise d. Novara. Zevel. II. 1. Isti Hymen. p. (i. S; T. 1 F. 3. Eumenes Omieron Novarae Saussure. Smithson. Miscell. Collect. XIY. P. 254. 1s is p. 1 
olsseura Smith - $\subseteq-$ Am.: Mexico.

Pachymenes obseura Smith, Catal. Hymen. Jrit. Mus. V. 155 p. p. 34 n. 7. . . p. 64 n.6. C. Eumenes Pachymenes) obscurus Saussure. Smithson. Miscell. Collect. XIV. P. 254. 1575 olivacea Sauss. - $Q-$ Am. : Surinam.

[p. 64 n. 5, . .

Eumenes Pachymenes; oliraceus Saussure, Smithson. Miscell. Collect. XIV. P. 254. 15i5

olmecus Sauss. - $q-$ Am. : Mexico.

[n. 28, c.

Eumenes Omieron Olmecus Saussure, Smithson. Miseell. Collect. XIV. P. 254. 1575 p. \5 orbignyi Sauss. - 우 - Am.: Bolivia.

Eumenes Orbignyi Saussure, Etud. fam. Yespid. I. Fumén. 1552 p. 69 n. 5i. c.

Jumenes canaliculatus var. Orbignyi Saussure, Etud. fam. Vespid. III. Masar. 1556 p. 132.

Eumenes (Zeta) Orbignyi Saussure. Smitlıson. Miscell. Collect. XIV. P.25.4. 1575 p.105 n.51.

ovalanensis Sauss. - $Q$ - Austr.: Ovalau.

Eumenes Ovalauensis Saussure, Stettin. entom. Zeitg. XXX. 1569 p. 53 n. 1, C.

pallidipes Oliv. - $\odot$ ơ - Am. mer., Cayenne.

? Vespa pallipes Olivier, Encycl. méthod. Insect. VI. 1791 p. 675 n. 30.

Pachrmenes pallipes Saussure, Étud. fam. Vespid. I. Eumén. 1552 p. 75 n. 3. $\subseteq$ 3.

Pachymenes pallidus Saussure, litud. fam. Vespid. III. Masar. 1556 p. 153 . (p. 63 n. $4 . \subseteq$.

Eumenes Pachymenes; pallipes Saussure, Smithson. Misecll. Collect. XIV. P. 254. 15i5 parvula Sauss. - 우 - Am.: Brasilia.

Eumenes parvulus Saussure, Etud. fam. Vespid. III. Masar. 1556 p. 149 n. 37, ᄃ; T.8 F. 5.

Eumenes Omicron parrulus Saussure, Smithson. Miseell.Collect. XIV.P.254.1575 p.52 n.22.

pennsylvanica Hald. - Am.: Pennsylvania.

Eumenes Pennsylvaniea Haldeman, Proc. Acad. Nat. Sc. Philadelphia VI. 1553 p. 36.5.

pensilis Sauss. - 오 $\widehat{o}$ - Afr.: Madagascar.

[T. 4 F. 10 \& T. 17 F. 7.

Eumenes pensilis Saussure, Grandidier : Hist. Madagasear XX. P. $1.15 .+1$ p. 155 n. 4. $\subseteq$ j; perplexa Smith - \&-Afr.: Bourbon.

Eumenes perplexus Smith, Journ. of Proc. Iinn. Soc. Zool. VII. $1<63$ p. 39 n. 11. S. petiolata Fabr. - $\odot$ oิ - Afr: Cap.

Vespa petiolata Fabricius, Spec. Insect. I. 1781 p. 467 n. 56.

Vespa petiolata Fabricius, Mant. Insect. I. 1787 p. 292 n. 68.

Vespa petiolata Gmelin, Linné: Syst. nat. Ed. $13^{2}$ I. 5.1790 p. 2753 n. 51.

Sphex hesperus Christ, Naturg. d. Insect. 1791 p. 311 ; T. 31 F. 3.

Sphex rubicunda Christ, Naturg. d. Insect. 1791 p. 316 ; T. 32 F. 1.

Sphex turrimarius Christ, Naturg. d. Insect. 1791 p. 321 ; 'T. 32 F. 8.

Sphex thoraciea Christ, Naturg. d. Insect. 1791 p. 324 ; 'T. 32 F. 9.

Vespa petiolata Olivier, Encycl. méthod. Insect. VI. 1791 p. 670 n. 1.

Vespa petiolata Fabricius, Entom. system. II. 1793 p. 278 n. 87.

Vespa petiolata Donovan, Epit. nat. hist. Insect. India 1800 Tab. 5 i.

Eumenes petiolata Fabricius, Syst. Piez. 1804 p. 254 n. 1.

Vespa petiolata Jurine, Nouv. méth. class. Hymén. 1807 p. 171, f.

Eumenes petiolata Saussure, Etud. fam. Vespid. I. Eumen. 1\$52 p. 47 n. 24. o 3.

Eumenes petiolatus Maindron, Ann. soe. entom. France fi V. 1455 p. 219: T. A.

philantes Sauss. $-\rho-$ Austr.

Eumenes Philantes Saussure, Ftud. fum. Vespid. I. Eumén. 14.i2 p. $.4 \mathrm{n}$ n. 37,. .

phithisiea Gerst. - ฮ๋ - Afr.: Mosambique.

Eumenes phthisica Gerstaceker. Monatiber. Akad. Wiss. Berlin 14.57 p. $41.3 \mathrm{n}$ n. 3. 3 .

Eumenes phthisica Gerstaceker. Peters: Reice n. Mussambique. Znol. V. 14ti2 p.463, 3: pietetii Sauss. - $\subsetneq$ o - Eur.: Gallia.

['. 30 T. 2.

Eumenes Pieteti Saussure, Litud. fam. Tespid. I. Jumen. 14.52 p. 67 n. 34, e 3 ; T. 12 F. $3 \mathrm{C}$.

Tinmenes l'ieteti Iid. André, Spee. Ilymen. Furope 11. P. 21-23. 1464 p. 630. 
pietifrons Smith $-\subseteq-$ As.: Celebes.

Eumenes pictifrons Smith, Journ. of Proc. Lim. Soc. Zoul. IV. 1Sito Suppl. p. Sti n. 2, C.

Eumenes pietifrons Maindron, Ann. soc. entom. France fi. II. $155 \geq$ p. 269 n. 2, S.

piriformis Sauss. - $\subseteq-$ As.: Java, Sumatra.

Eumenes piriformis Saussure, Stettin. entom. Zeitg. XXIII. $15(i 2$ p. 177 n. $13, \subseteq$. placida Smith - 우 ô - Am.: Panama.

Eumenes placidus Smith. Trans. Entom. Soc. London :3, I. 1. 1562 p. 3i. j3.

Eumenes Alpha) placidus Saussure, Smithson. Misecll.Collect. XIV. P.2.4. 1 5is p. 101 n. 12.

pomiformis Fabr. - 오

Vespa coaretata Poda, Insect. mus. Graec. 1761 p. 109 n. 5 [nec Linné].

? Vespa spec. Geoffroy, Hist. abr. Insect. II. 1762 p. 375 n. 7.

Vespa spec. Geoffroy, Hist. abr. Insect. II. 1762 p. 376 n. 8.

Vespa spec. Geoffroy, Hist. abr. Insect. II. 1762 p. 377 n. 10.

Vespa coarctata Scopoli, Entom. Carn. 1763 p. 311 n. 830.

Vespa pomiformis Fabricius, Spec. Insect. I. 1781 p. 467 n. 58.

Vespa coarctata Schrank, Enum. Insect. Austr. 1781 p. 391 n. 790.

? Vespa infundibuliformis Fourcroy, Entom. Paris. II. 1785 p. $43 \pm$ n. 7.

Vespa globulosa Fourcroy, Entom. Paris. II. 1785 p. 434 n. 8.

Vespa coarctata Fourcroy, Entom. Paris. II. 1785 p. 435 n. 10.

Vespa pomiformis Fabricius, Mant. Insect. I. 1787 p. 293 n. 71.

Vespa pomiformis Villers, C. Linnaei Entom. III. 1789 p. 276 n. 26.

Vespa histrio Villers, C. Linnaci Entom. III. 1789 p. 282 n. 41 ; T. 8 F. 20.

Vespa lunulata Villers, C. Linnaci Entom. III. 1789 p. 285 n. 48.

Vespa pomiformis Gmelin, Linné: Syst. nat. Ed. 13 a I. 5.1790 p. 2753 n. 54.

Vespa pomiformis Rossi, Fauna Etrusca II. 1790 p. 86 n. 868.

Sphex viatica Christ, Naturg. d. Insect. 1791 p. 315.

Sphex annularis Christ, Naturg. d. Insect. 1791 p. 315 ; T. 31 F. 17.

Sphex papillaria Christ, Naturg. d. Insect. 1791 p. 325 ; T. 32 F. 10.

Vespa pomiformis Olivier, Encycl. méthod. Insect. VI. 1791 p. 671 n. 8.

Vespa histrio Olivier, Encycl. méthod. Insect. VI. 1791 p. 672 n. 11.

Vespa globulosa Olivier, Encycl. méthod. Insect. VI. 1791 p. 676 n. 36.

Vespa pomiformis Fabricius, Entom. system. II. 1793 p. 279 n. 90.

Vespa coarctata Panzer, Faun. Insect. German. VI. 1799 P. 63 F. 6 (ô).

Vespa pomiformis Panzer, Faun. Insect. German. VI. 1799 P. 63 T. 7.

Vespa globosa Walckenaer, Fauna Paris. II. 1802 p. 87 n. 2.

Eumenes pomiformis Fabricius, Syst. Piez. $180 \pm$ p. 287 n. 9.

Vespa (Eumenes) pomiformis Panzer, Krit. Reris. II. 1806 p. 159.

Vespa pomiformis Illiger, Rossi: Fauna Iitrusca Ed. 2a II. $1 \varsigma_{47}$ p. 112 n. $\varsigma 67$.

Vespa pomiformis Jurine, Nouv. méth. class. Hymén. 1807 p. 171, 우 ơ.

Eumenes pomiformis Spinola, Insect. Ligur. II. 1809 p. 189 n. 3.

Eumenes pomiformis Latreille, Gen. Crust. \& Insect. IV. 1809 p. 137.

Eumenes pomiformis Klug. Germar: Ruise n. 1)almat. II. 1517 p. 26.4 n. 36 .5.

Eumenes pomiformis Lamarck, Hist. nat. anim. s. vert. IV. 1817 p. 83 n. 2.

Eumenes marginata v. d. Höven, Bijdr. natuurk. Wet. 1826 p. 441 n. 16.

Eumenes pomiformis Lamarek. Hist. nat. anim. 8. vert. Eel. 2 IV. $15: 35$ p. 301 n. 2.

Eumenes pomiformis Blanchard, Hist. nat. Insect. III. 1840 p. 393 ; T. 5 F. 7.

Eumenes pomiformis I.epeletier. Hist. nat. Inseet. Hymén. II. 1541 p. 601 n. 3, \& j.

Lumenes pomiformis I.ueas. Explor. se. Algéric. Zool. III. 14.46 p. 229 n. 1511.

Jumenes pomiformis Saussure. Etud. fam. Vespid. I. Eumén. 15.i2 p. 29 n. 1. \& j.

Eumenes pomiformis Sehenck. Jahrb. Ver. Xaturk. Xissau IX. 1. 15.13 p. 4 i n. 15, Cs

Vespa pomiformis Duméril, Mém. acad. sc. Paris XXXI. 1960 p. $\$ 62$ n. 7. 
Eumenes pomifurmis Schenck. Jahrb. Yer. Yaturk. Yassau XVI. 1 s61 p. $2 !$ n. 1, $\subseteq j$. limenes pomiformis Radoszkowski, Horae soc. entom. Ross. III. 14ij.; p. 57 n. 2.

Eumenes pomiformis H. Müller, Befrucht. d. Blumen 1873 p. 466.

Eumenes pomiformis Rudow, Arch. Ver. naturf. Fr. Mecklenburg XXX. 1576 p. 210 n. 1, s. Fumenes pomiformis Radoszkowski. Horate soc. entom. Ross. XII. $15 i t i$ p. 141 n. 5.). İumenes pomiformis Radoszkowski, Horae soc. entom. Ross. XIII. Bull. 1sit p.XIII. Eumenes pomiformis Kriechbaumer, Entom. Nachr. V. 1879 p. 309.

Eumenes pomiformis Fabre, Nouv. souv. entom. 1882 p. 57. [p. 315 .

Eumenes pomiformis Letzner, 61. Jahresher. schles. Ges. f. vaterl. Cultur i. J. 14-3. 1sh4 Eumenes pomiformis Ed. André. Spee. Hỵmén. Iurope II. P. 21-23. 1554 p. 642, 83. Eumenes coarctatus Schulthess, Hymen. Helvet. Diplopt. 1887 p. 27.

Eumenes pomiformis Rudow, Soc. entom. I. 1887 p. 84,157 \& 164.

Eumenes pomiformis Palumbo, Natural. Sicil. VII. 1555 p. 162, 144, 207.

Eumenes pomiformis Magretti, Ann. mus. civ. Genora XXIX. 1891 p. 5.36 n. 36. $\subseteq 3$. var. afflnissima Sauss. - $\hat{o}-$ As.: India.

Eumenes affinissima Saussure, Étud. fam. Vespid. I. Eumén. 1852 p. 37 n. 9, j.

Eumenes pomiformis rar. affinissima Maindron. Ann. soc. entom. France (6) II. 1552 p. 265 n.1. var. agilis Sauss. - ơ $-\mathrm{Am}$. bor.

Eumenes agilis Saussure, Kitud. fam. Vespid. I. Eumén. 1552 p. 42 n. $21,3.3$.

Eumenes pomiformis var. agilis Maindron, Amn. soc, entom. France $(6$, II. 1852 p. 265 n. 1. var. arehitecta Smith $-Q \hat{o}-$ As.: Celebes, Java.

Eumenes architectus Smith, Journ. of Proc. Limn. Soc. Zool. III. 1555 p. 20 n. 4. $\subseteq \jmath$.

Eumenes architectus Saussure, Stettin. entum. Zeitg. XXII. 1862 p. 179 n. 15 . C.

Fumenes pomiformis var. architecta Maindron, Ann. soc. entom. France, 6 II. 1852 p. 269. var. blanda Smith - 우 As.: Batchian.

Eumenes blandus Smith, Journ. of Proc. Linn. Soc. Zool. IV. 1560 Supjl. p. 127 n. S. ᄃ.

Iimenes blandus Saussure, Stettin. entom. Zeitg. XXIII. 1462 p. 179 n. 14.

Eumenes pomiformis var. blanda Maindron, Ann. suc. entom. Franee ( 6 , II. 1 s 2 p. 2lis n. 1. var. coronata Panz. - ㅇ - Eur.: Germania.

Vespa VII Schacfier, Icon. Insect. Ratisbon. II. 2. 1767; T. 52 F. 2.

Vespa coronata Panzer, Faun. Insect. German. VI. 1799 P. 64 T. 12 (\&).

Vespa Eumenes pomiformis var. coronata Panzer. Krit. Revis. II. 1 sut p. 159. var. floralis Smith - ô-As.: Celebes.

Eumenes floralis Smith, Journ. of Proc. Iimm. Soc. Zool. III. 14.j4 p. 20 n. .5, of.

Jiumenes pomiformis var. floralis Maindron, Ann. soc. entom. France 6. II. $15 \backsim 2$ p. 265 n. 1. var. inconspieua Smith - - As.: Borneo.

Eumenes inconspicua Smith, Journ. of P'roc. Limn. Soc. Zool. II. 1557 p. 109 n. 7. .

Eumenes pomiformis var. inconspicua Maindron, Aun. soc.entom. France (fi) II. 1 \$ 2 p. 26 n n.1. var. peduneulata Panz. - 우 - Eur.: Germania.

Vespa pedunculata Panzer, Faun. Insect. German. VI. 1799 P. 63 'I. S.

Vespa Eumenes, pomiformis var. pedunculata Panzer, Krit. Revis. II. 14u6 p. 159.

Vespa pedunculata Jurine, Nouv. méth. class. Hymén. 1807 p. 171, \&.

var. piriformis Fabr.! - As.: China.

Vespa pyriformis Fabricius, Syst. entom. 1775 p. 371 n. 42.

Vespa pyriformis Fabricius, Spec. Insect. I. 1781 p. 467 n. 57.

Vespa pyriformis Fabricius, Mant. Insect. I. 1787 p. 293 n. 70.

Vespa pyriformis Gmelin, Linné: Syst. nat. Ed. 13 a I. 5.1790 p. 2753 n. 53.

Vespa pyriformis Olivier, Encycl. méthod. Insect. VI. 1791 p. 670 n. 5.

Vespa pyriformis Fabricius, Entom. system. II. 1793 p. $27 \mathrm{~s}$ n. 59.

Eumenes pyriformis Fabricius, Syst. Piez. 1504 p. 286 n. 5.

Funenes poniformis sar. piriformis Maindron. Ann. soc. entom. France (6) II. 1552 p. 2 tis n.1. 
var. polita Smith - 우 - As.: Batchian.

Eumenes politus Smith. Journ. of Proc. I.imn. Soc. Zoel. IT. 14to Suppl. p. 127 n. 9) C.

Eumenes pomiformis var. politu Maindron. Anm. soc. entom. France 6 II. 1552 p. 26!) n. 1. var. singularis Smith - 우 As.: Borneo.

Eumenes singularis Smith, Journ. of Proc. Linn. Soc. Zool. II. 14.; p. 109 n. S, $\subseteq$.

Eumenes pomiformis rar. singularis Maindron, Ann. soc. entom. France 6; II.1552 p.265 n.1. var. vindex Smith - $\hat{o}-$ As.: Celebes.

Eumenes vindex Smith, Journ, of Proe. Iinn. Soc. Zool. III. $155 \overline{5}$ p. 20 n. 3, jै.

Eumenes pomiformis var. vindex Maindron, Ann. soe. entom. France 60 II. 1552 p. 269. pullata Smith. - $q-$ As.: Ceram.

Eumenes pullatus Smith. Journ. of Proc. Linn. Soc. Zool. VII. 1463 p. 39 n. 11). ᄃ. pumetata Sauss. - 우 ơ - As.: China.

Eumenes punctata Saussure, Étud. fam. Vespid. I. Eumén. 1552 p. 37 n. 10, $\subseteq 3$. pusilla Sauss. - $\odot$ ơ - Am.: Brasilia.

Eumenes pusillus Saussure, Etud. fam. Vespid. III. Masar. 1556 p. 149 n. $35, \subseteq \vec{\jmath}$.

Eumenes Omicron pusillus Saus sure, Smithson. Miseell.Collect. XIV.P. 254.1575 p.42 n.2:3. pusio Kirsch - ô - Am.: Brasilia.

Eumenes pusio Kirsch, Mittheil. zool. Mus. Dresden III. 1878 p. 376, ð.

quadrata Smith - 우 ơ - As.: China.

Eumenes quadratus Smith, Trans. Entom. Soc. London 2) II. 2. 1552 p. 37, $\subseteq \hat{\jmath}$.

Eumenes quadratus Saussure. Etud. fam. Vespid. III. Masar. 1556 p. 144 n. 31 . $\subseteq$ j.

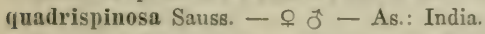

Eumenes Pareumenes quadrispinosus Saussure, Fitud. fam. Vespid. III. Masar. 14.j6 regina Sauss. - 우 $\widehat{o}$ - Afr.: Madagascar.

[p. 134 n. 16, 우

Eumenes regina Saussure, litud. fam. Vespid. I. Eumén. 1552 p. 4! n. 2!) §; 'T. 10 F. ५.

Kumenes regina Saussure. Reise d. Novara. Zool. II. 1. 1467 Hymén. p. 154 n. 2 . $\subseteq \hat{\jmath}$. regulus Sauss. - $0 \hat{\sigma}-$ Am.: Mexico.

Eumenes regulus Saussure, Rev. \& mag. zool. (2) IX. 1857 p. 272.

Eumenes Omicron regulus Saussure, Smithson. Miseell. Collect. XIV. P.254. 1-i5 p. in rethoides Spin. - Am.: Brasilia.

[n. 11, \& ơ; T. 1 F. $7,7^{\mathrm{a}} \& 7^{\mathrm{b}}$.

Eumenes rethoides Spinola, Oseulati: Explor. reg. equat. 1454 p. 203 n. 14 [sine deseript.]. sanguimolenta Gerst. - 우 ô - Afr.: Mosambique.

Eumenes sanguinolenta Gerstaecker. Monatsber. Akad. Wiss. Berlin 145i p. 46.3 11. 4. j.

Fumenes sanguinolenta Gerstaceker, Peters: Reise n. Mossambique. Zool. Y. 1562 p. 161. _; santa-anna Sauss. - 0 ơ - Am.: Mexico.

Pachymenes Santa Anna Saussure, liev. \& mage zool. 2 IX. 1-57 p. 272, of 5 .

Eumenes 'Pachymenes' Santa Anna Saussure, Smithson. Miscell. Collect. XIV. P. 254.1575 sareptana André - 우 - Eur.: Russia.

[p. 66 n. 8, \& ठ; T'. 1 F. $6 \& 6^{a}$.

Eumenes Sareptanus Ed. André, Spec. Hymén. Europe II. P. 21-23. 1544 p. 634, C. sanudersii Nietn. - As.: Ceylon.

W. W. Sauders, 'Trans. İntom. Soc. I. 1834 p. 60-62.

A. Menzel, Mittheil. naturf. Ges. Zürich I. 1818 p. 97.

Limmenes Saundersii /Westwood Nietner, Stettin. entom. Zeitg. XVI. 4.55 p. 22:3-226. sericen Sauss. $-\subseteq-$ Am.: Brasilia.

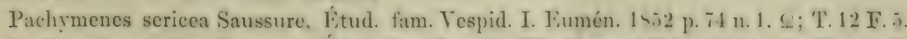

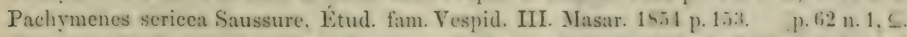
Eumenes |Pachỵmenes sericeus Saussure. Smithson. Misecll. Collect. XIV. 1'. 2i4. 15is sichelii Sauss. - - - Eur. : Albania.

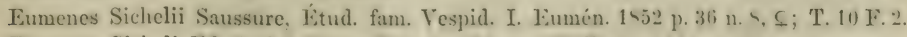

Eumenes Sicheli Ed. André, Spee. Hỵmén. Europe II. P. 20. 1 s54 p. 622 n. 4. c. 
siguaticoruis Walk.! - Afr.: Aegyptus.

Sumenes signaticornis Walker, List of Hymen. in Egypt 1471 p. 30 n. 15 ti.

simulans Sauss. - Q - Am.: Mexico.

[n. 32, ᄋ.

Eumencs Beta simulans Saussure, Smithson. Misecll. Collect. XIV. Y. 251. 15i5 p. 91

sinensis Smith - 우 - As.: China.

Eumenes Sinensis Smith, Brenchley: Cruise of the Curacoa 1573 p. 462. ᄃ; T. 43 F. 3. Eumenes Sinensis Smith, Trans. Entom. Soc. London 1873 p. 195 n. 1.

smithii Sauss. - 오 ơ - Am.: Florida.

Eumenes Smithii Saussure, Etud. fam. Vespid. I. Eumén. 14.52 p. 43 n. $21 . \subseteq$ j; T10 F. 1 j.

Eumenes Alpha Smithii Saussure. Smithson. Miscell. Collect. XIV. P. 254. 1975 p. 104 spinosa Fabr.! - Afr.: Algeria.

Eumenes spinosa Fabricius, Syst. Piez. 1804 p. 290 n. 21.

subleris Smith - $Q-$ As.: Borneo.

Eumenes sublatevis Smith. Catal. Ifymen. Brit. Mus. V. 15.57 p. 23 n. 25 . ᄃ.

suleigastra Grib. - 으 - Afr.

Eumenes sulcigastra Gribodo. Ann. mus. civ. Genova XXI. 1541 p. 295 n. 31, \&

sumichrastii Sauss. - - - Am. : Mexico.

[p. $78 \mathrm{n} .16$, 우.

Eumenes Omicron' Sumichrasti Saussure, Smithson. Miscell. Collect. XIV. P. 254. 1475

tabida Ev. - 우 ơ - Eur.: Russia.

Lumenes tabida Liversmann. Bull. soc. natural. Moscou XXVII. 3. 1454 p. 199.

Eumenes tabida Radoszkowski, Horae soc. entom. Ross. III. 1665 p. 5 s n. $5 ;$ T. 1 F. 4.

Eumenes tabidus Ed. André. Spec. Hymén. Lurope II. P. 20. 1554 p. 621 n. 3, $\leq 5$.

tenuis Mor. - $₫$ - As.: 'Transeaspia.

Lumenes tenuis Morawitz, Horae soc. entom. Ross. XXII. 1sৎs p. 261, $\vec{j}$.

thoracica Sauss. - of 0 - Am.: Mexico.

[p. 74 n. $14, \& \widehat{o}$.

Fumenes Omicron) thoracicus Saussure. Smithson. Miscell. Collect XIV. P. 254. 1575

totonacus Sauss. - o of - Am.: Mexico.

[n. $12,9 \overrightarrow{0}$.

Eumenes Omicron 'Totonacus Saussure, Smithson. Miscell. Collect. XIV. P. 254.1575 p. 72

tricolor Smith $-Q-$ As.: Celebes.

Eumenes tricolor Smith, Journ. of Proc. Linn. Soc. Zool. IV. 1560 Suppl. p. \$7 n.5. ๔.

Eumenes tricolor Maindron, Ann. soc. entom. France 6! II. 1852 p. 269 n. $5 . \varsigma$.

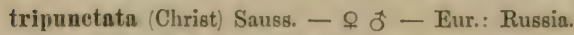

Sphex tripunetata Christ, Naturg. d. Insect. 1791 p. 317 ; T. 32 F. 3.

Sphex tripunctata Lichtenstein, Catal. mus. zool. III. 1796 p. 202 n. 169.

Vespa trimaculata Weber, Observ. entom. 1801 p. 102 n. 6.

$[\&-2(\$)$.

Eumenes renusta Fischer-Waldheim. Magaz. de zool. XIII. 1443 P.122 p. 1; T.122 F. 1 j)

Jumenes tripunctatus Saussure, F.tud. fam. Vespid. III. Masar. 1556 p. 137 n. $21 . \varsigma$.

İumenes tripunctata Radoszkowski. IIorae soc. entom. Ross. III. 156.5 p. is n. 3.

Eumenes tripunctatus Ed. André, Spee. IỴmén. Europe II. P. 21-23. 1 s4 p. 6i36. S 3 .

tropiealis Sauss. - $\subseteq-$ Afr.: Senegal.

lsumenes tropicalis Saussure. Fitud. fam. Vespid. I. Fumén. 1652 p. 54 n. 36. C.

unguieulata (Vill.) - f $\hat{f}-$ Eur. mer.; Afr.: Aegyptus.

Vespa unguiculata Villers, C. Linnaci Eintom. III. 1789 p. 282 n. 40.

Vespa coangustata Rossi, Fruna Etrusca II. 1790 p. 84 n. 863 , \&; T. 4 F. 10.

Vespra infundibuliformis Olivier, Fneyel. mithod. Insect. VI. 1791 p. 6iz' n. 10.

Sphex cursor Christ, Naturg. d. Insect. 1791 p. 314 ; T. 31 F. 6.

Sphex lapicida Christ, Naturg. d. Insect. 1791 p. 318 ; 'T. 32 F. 5.

Sphex coarctata Christ, Naturg. d. Insect. 1791 p. 320 ; T. 32 F. 6.

Vespa conica Rossi, Mant. Insect. 1792 p. 135 n. 299. 
Eumenes coangustatus Spinola, Insect. Ligur. I. 1506 p. 82 n. $1, \Omega \delta^{-}$.

Vespa coangustata Illiger. Rossi: Fauna Etrusca Ld. 2 II. 1 so7 p. 135 n. 8633 ; ' I. 4 F. 10. Eumenes coangustata Latreille, Gen. Crust. \& Insect. IV. 1809 p. 137.

Eumenes Olivieri Lepeletier, Hist. nat. Insect. Iymen. Il. $14+1$ p. 596 n. 1, $\subseteq$ j.

Eumenes eoangustata Herrich-Schaeffer. Faun. Insect. German. 1541 P. 179 T. 7 \& 3 . Eumenes dumetorum Imhof \& Labram. Insect. d. Schweiz III. 1542; T. 36 F.1 Eumenes infundibuliformis Perris, Ann. soc. entom. France (2) VII. 1519 p. 155-19.4; 'T. 72

[F. $1-7 \&$ X. 1852 p. $557-559$.

Eumenes infundibuliformis Vallot. Ann. soc. entom. France (2, VII. 1549 Bull. p. I.XXIV.

Eumenes infundibuliformis Perris, Anm. soc. entom. France :2 X. 14.2 p. 55i-55!.

Bumenes coungustata Saussure, Kitud. fam. Vespid. I. Eumén. 14.52 p. 3.4 n. 7. S. 3 .

Eumenes coangustata Sehenck. Jahrb. Ver. Naturk. Nassau IX. 1. 145: p. in n. $1 \leq$ j.

Eumenes unguicula Smith, Catal. Hymen. Brit. Mus. V. 1857 j. 20 n. 7.

Eumenes coangustatus Sehenck, Jahrb. Yer. Naturk. Nassau XVI. 1 \$661 p. 32, $\subseteq$ j.

Eumenes coangrustata Radoszkowski. Horae soe. entom. Ross. III. 1465 p. is n. 4.

? Eumenes Amedei Fabre, Nouv, souv, entom. 1882 p. $5 \bar{i}$.

Eumenes coangustata Lichtenstein, Ann. soc. entom. France $\{6$. III. 1853 Bull. p. CV-CVI. Eumenes unguiculus Ed. André. Spec. Hymén. Europe II. P. 20-23. 1 ş4 p. 635. \& ș; T. 41

Eumenes unguiculus Schulthess. Hymen. Helvet. Diplopt. 1457 p. 25 n. 2. S.5. F. F. 11.

Eumenes unguiculus Rudow, Soc. entom. I. 1857 p. 157.

var. hubertii Sauss. - of - Eur.: Gallia.

Eumenes Huberti Saussure, litud. fam. Vespid. I. Eumén. 18.52 p. 33 n. .5. Jै; T. 11 1․6.

Eumenes unguieulus var. Huberti Saussure. Citud. fam. Tespid. III. Masar. IS56 p. 129.

nruguyensis Sauss. $-\subseteq-$ Am.: Uruguay.

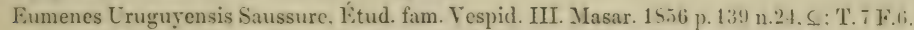

Eumenes Alpha Uruguyensis Saussure, Smithson. Miscell. Collect. XIV. P. 2.1. 15is

ventricosa Sauss. - $\subseteq \tilde{\sigma}-$ Am. mer., Bogota, Venezuela.

(p. 100 n. 41.

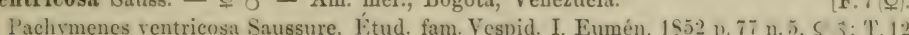

Eumenes Pachymenes rentricosus Saussure. Smithson. Misell. Collect. XIV. P. 25.1. 1s is

verticalis Say!" - Am.: Pennsylvania.

[p. 67 n.9.

Eumenes verticalis Say, Keating's Narrat. Exped. II. 1834 App. p. 346 n. 2.

Eumenes verticalis Saussure. Étud. fam. Vespid. I. Eumén. 15.2 p. 41 n. 17.

Eumenes verticalis Leconte, Writ. of Th. Say Entom. II. 1859 p. 233 n. 2.

viridis Smith - O - As.: Key.

Pachymenes viridis Smith. Journ. of Proc. I.inn. Soc. \%oul. III. 155s p. 163 n. 1. c.

Eumenes viridis $\mathrm{m}$.

volatilis Smith - $\mathrm{C}-$ As.: Mysol.

Eumenes volatilis Smith. Journ. of Proce. I.inn. Sise. \%oul. VII. 1 sii3 p. 34 n. 9. 5.

wagae Rad. - $f$ ơ - As.: Caucasus.

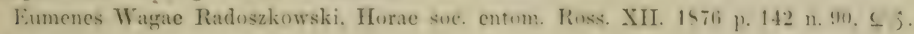

wagneriana Sauss. - $\subseteq-$ Am.

[p. 94 n. 35, , .

Eumenes Alpha Wagneriamus Saussure. Smithsen. Miscell. ('ollect. XIV. P. 251. 15is walkeri Rits. - $\tilde{\sigma}-$ Afr.

Eumenes Walkeri Ritsema. Tijdschr. v. Entum. XVII. IS: p 1919 n. IS. 3; T'.11 F. i j. xanthura Sauss. - $\mathrm{Q}-$ As.: India or.

Eumenes xanthura Saussure, Ftud. fim. Vexpid. I. Fumen. 14.2 p. 46 n. 26. C; T. 10 F. 1.

1) $=$ ? E. fraternus Say. 


\section{SYNAGRIS}

Latreille, Hist. nat. Crust. \& Insect. III. 1502 p. 360.

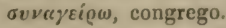

Synon.: A pis Drury.

Vespa Linné \& auct. ant.

Subg.: Antagris Saussure, Mém. soc. phỵs. \& hist. nat. Genère XVII. 1. 1963 n. 1々). cevri, contra; (Syn)agris, nom. propr. Hymen.

Hy pagris Saussure, Étud. fam. Vespid. III. Masar. 1856 p. 157. író, subtus; (Syn)agris, nom. propr. Hymen.

Paragris Saussure, Étud. fam. Vespid. III. Masar. 1856 p. 156. $\pi \propto \varrho \dot{a}$, ad; (Syn)agris, nom. propr. Hymen.

Micragris Saussure, Étud. fam. Vespid. III. Masar. 1856 p. 158. Mexós, parvus; (Syn)agris, nom. propr. Hymen.

abdominalis Sauss. - ơ - Afr.

[T. 8 F. T.

Synagris (Hypagris) abdominalis Saussure, Etud. fam. Vespid. III. Masar. 14.56 p.157 n.47, วँ;

Synagris (Hypagris) abdominalis Saussure, Mém. soc. phys. \& hist. nat. Genève XVII. 1.1563 abyssiniea Guér. - 0 ô-Afr.: Abyssinia.

[p. 202 n. $30, \delta$.

Srnagris Abyssinica Guérin, Lefebure: Voy. Abyssinie VI. P. 4.1848 p. 360, 의.

Symagris Abyssinica Reiche \& Fairmaire, Ferret \& Galliner: Voyage en Abyssinie III. 1949

[p. 452 n. 2 ; T. 29 F. 9-10.

Synagris Abyssinica Saussure, Étud. fam. Tespid. I. Eumén. 15.52 p. 84 n. S. ơ; T. 13 F.6.

Synagris (Paragris) Abỵssinica Saussure, Mém. soc. phys. \& hist. nat. Genève XVII.1.1563

aefuatorialis Sauss. - ô - Afr.: Senegal.

[p. 192 n. 15.

Synagris aequatorialis Saussure, Étud. fam. Vespid. I. Eumén. 1552 p. $\$ 1$ n. 4. ठే; T. 13 F. 4.

Synagris 'Antagris' aequatorialis Saussure, Mém. soc. phỵs. \& hist. nat.Genève XVII. 1. 1563

[p. 183 n. 5, ơ; T. 2 F. 2.

Srnagris Hypagris aequatorialis Saussure, Mém. soc. phỵs. \&- list. nat. Genère XVII. 1. aestuans (Fabr.) Serv. - 우 ơ - Afr.

[1863 p. 198 n. 24, ö.

Vespa aestuans Fabricius, Spec. Insect. II. 1781 p. 462 n. 22.

Vespa aestuans Fabricius, Mant. Insect. I. 1787 p. 289 n. 26.

Vespa aestuans Gmelin, Linné: Syst. nat. Ed. 13a. I. 5.1790 p. 2752 n. 4 S.

Vespa aestuans Olivier, Encycl. méthod. Insect. VI. 1791 p. 683 ฉ. 65.

Vespa aestuans Fabricius, Entom. system. II. 1793 p. 262 n. 32.

Vespa aestuans Fabricius, Syst. Piez. 1804 p. 258 n. 24.

['T. 10 F. 5.

Srmagris aestuans Serville, Palisot-Beaurois: Insect. Afr. \& Amér. 1805 p. 261 Hỵmén.:

Synagris aestuans Lepeletier, Hist. nat. Insect. 11 ymén. II. 14.11 p. $594 \mathrm{n} .3, \mathrm{~S}$.

Synagris aestuans Saussure, litud. fam. Vespid. I. Iiumén. 1552 p. 51 n. 3. o j.

Synagris aestuans Saussure, Eitud. fam. Vespid. III. Masar. Msifi p. 155. (p. 154 n. 111, S. Synagris Paragris) aestuans Saussure, Mém. soe. phỵs. \& hist. nat. Geneve XVII. 1. 1 63

aethiopica Sauss. - \& - Afr.: Natal, Cap.

[1863 p. 200 n. 26, \&; T. 2 F. 14. Synagris HỊpagris' Aethiopiea Saussure, Mém. soe. physs. \& hist. nat. Genere XVII. 1.

afflnis Sauss. - $Q-$ Afr.: Abyssinia.

[p. 186 n. 8, \&; 'T'. 2 F.5.

Synagris Antagris, affinis Saussure, Mem. soe. phỵs. \&f hist. nat. Geneve XVII. 1. 1`63

amplissima Kohl $-\tilde{o}-\Lambda$ fr. occid.

[T. 16 F. 102.

Synagris amplissima Kohl, Ann. naturh. IIofmus. Wien IX. $16 ! 11$ p. 337. Jै; T. 15 F. 93 \& analis Sauss. - ô- Afr.: Abyssinia.

Synagris analis Saussure, Ctud. fam. Vespid. I. Kumén. 1452 p. 46 n. 11 , Jै; T. 13 F. 5. Sỵnagris Paragris analis Saussure. Mém. soc. phỵs. \& hist. nat. Genève XVII. 1. $15+33$ p. 195 
bellicosa Sauss. - $ᄋ$ ơ - Afr.: Senegal.

Synagris bellicosa Saussure, Etud. fam. Vespid. I. Eumén. 1452 p. $\$ 4$ n. $7, \subseteq \hat{\jmath}$.

Sỵnagris (Paragris) bellicosa Saussure, Mém. soc. pliys. S- hist. nat. Genève XVII. 1. 1863

calida (L.) Serv. - \& ơ - Afr.

[p. 191 n. 14; T. 2 F. 7, ઠิ.

Vespa calida Linné, Syst. nat. Ed. $10^{a}$ I. 1758 p. 574 n. 17.

Vespa Capensis Linné, Syst. nat. Ed. $12^{a}$ I. 2.1767 p. 951 n. 22.

Vespa calida Linné, Syst. nat. Ed. 12 a I. 2.1767 p. 952 n. 27.

Vespa calida Fabricius, Syst. entom. 1775 p. 366 n. 18.

Vespa Capensis Ph. L. Müller, Linné: Vollst. Natursyst. V. 2. 1775 p. 896 n. 22.

Vespa calida Ph. L. Müller, Linné: Vollst. Natursyst. V. 2. 1775 p. 887 n. 27.

Vespa carbonaria Degeer, Mém. serv. hist. Insect. VII. 1778 p. 609 n. 7; T. 45 F. 9.

Vespa calida Fabricius, Spec. Insect. I. 1781 p. 462 n. 23.

Vespa carbonaria Güze, Degeer: Abh. Gesch. Insect. VII. 1783 p. 217 n. 7 ; T. 45 F. 9.

Vespa carbonaria Retzius, Gen. \& spec. Insect. 1783 p. 64 n. 241.

Vespa calida Fabricius, Mant. Insect. I. 1787 p. 289 n. 27.

Vespa Capensis Gmelin, Linné: Syst. nat. Ed. 13a I. 5.1790 p. 2758 n. 22.

Vespa calida Gmelin, Linné: Syst. nat. Ed. 13a I. 5. 1790 p. 2759 n. 27.

Vespa Capensis Christ, Naturg. d. Insect. 1791 p. 215.

Vespa microrrhoea Christ, Naturg. d. Insect. 1791 p. 218; T. 18 F. 6.

Vespa calida Christ, Naturg. d. Insect. 1791 p. 225.

Vespa Capensis Olivier, Encycl. méthod. Insect. VI. 1791 p. 676 n. 33.

Vespa calida Olivier, Encycl. méthod. Insect. VI. 1791 p. 683 n. 66.

Vespa calida Fabricius, Entom. system. II. 1793 p. 262 n. 33.

Vespa spiniventris Illiger, Magaz. f. Insectenk. I. 1802 p. 190 n. 20.

Vespa calida Fabricius, Syst. Piez. 1804 p. 259 n. 25.

Synagris calida Serville, Palisot-Beaurois: Insect. Afr. \& Amér. 1805 p. 260 Hymén.; T. 10

Vespa calida Jurine, Nour. méth. class. Hymén. 1507 p. $169, \subseteq \hat{\sigma}$.

Sỵnagris calida Lepeletier. Eneycl. méthod. Insect. X. 1825 p. 509, $\subseteq$ .

$[$ F. $6(\widehat{o})$.

Sỵnagris callida Lepeleticr. Hist. nat. Insect. Hỵmén. II. 1841 p. 594 n. 2, f $\vec{\jmath}$.

Sỵnagris calida Guérin, Iconogr. règn. anim. VII. Insect. 1845 p. 446 ; T. 72 F. 3.

Synagris callida Reiche \& Fairmaire, Ferret \& Galliner: Voyage en Abyssinie III. 1S49

[p. 452 n. 1, ơ; 'T. 29 F. T-8.

Sỵnagris calida Saussure, Gitud. fam. Vespid. I. Eumén. 1552 p. 79 n. 1 , f $\widehat{\jmath}$; T. 13 F. 2 ô).

Synagris calida Smith. Trans. Entom. Soc. London 2. III. 1556 Proc. p. 125 n. 1.

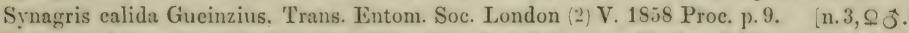

Sỵnagris (Antagris) calida Saussure, Mém. soc. phỵs. \& hist. nat. Genève XVII. 1. 1 \$ 63 p.181

Sỵnagris Vespa calida Tasehenberg. Zeitschr. f. d. ges. Naturw. XXXIX. 1572 p.1-5 n.1.

Syynagris calida Maindron, Quelques mots s. 1. Guêpes maconnes. St. Louis 1579. so.

Synagris calida Anonym, La Nature XIII. 1885 p. 24.

capitata Sauss. - $̋$ - Afr.: Senegal.

[p. 189 n. 12, oิ.

Sỵnagris Paragris; capitata Saussure, Mém. sue. phỵs. \& hist. nat. Genève XVII. 1. 1563

earinata Sauss. - ơ - Austr.: Port Natal.

[p. 203 n. 31, ơ; 'T. 2 F. 18.

Sỵnagris (Pseudagris carinata Saussure: Mém. soc. plỵs. \& hist. nat. Genève XVII. 1. 1863

combusta Sauss. - $\&-$ Afr: Abyssinia, Guinea.

[p. 185 n. 7, \&; T. 2 F. 4.

Synagris /Antagris combusta Saussure, Mém. soe. physs. \& hist. nat. Genere XVII. 1. 1sti3

Synacris combusta Ritsema, Tijdschr. v. Entom. XVII. 157t p. 200 n. 19, S.

Synagris combusta Gribodo, Ann. mus. eir. Genova XXI. 1454 p. 291 n. 24.

cornuta (L.) Latr. - 우 of - Afr.: Cap, Guinea.

Vespa cornuta Linné, Syst. nat. Ed. $10^{3}$ I. 1758 p. 574 n. 13.

Vespa cornuta Linné, Mus. Ludov. Ulric. 1764 p. 409 n. 1. 
Vespa cornuta Linné, Syst. nat. Ed. 12a I. 2.1767 p. 951 n. 20.

A pis cornuta Drury, Illustr. Nat. Hist. II. 1773 p. 85; T. 45 F. 3.

Vespa cornuta Fabricius, Syst. entom. 1775 p. 363 n. 7.

Vespa cornuta Ph. L. Müller, Linné: Vollst. Natursyst. V. 2. 1775 p. $\$ 55$ n. 20.

Vespa cornuta Fabricius, Spec. Insect. I. $17 \$ 1$ p. 459 n. 7.

Vespa cornuta Fabricius, Mant. Insect. I. 1787 p. 287 n. S.

Vespa cornuta Gmelin, Linné: Syst. nat. Ed. $13^{n}$ I. 5.1790 p. 2755 n. 20.

Vespa cornuta Olivier, Encycl. méthod. Insect. VI. 1791 p. 678 n. 45.

Vespa cornuta Fabricius, Entom. system. II. 1793 p. 255 n. 8.

Synagrus cornuta Latreille, Hist. nat. Crust. \& Insect. III. 1802 p. 360.

Synagris cornuta Fabricius, Syst. Piez. 1804 p. 252 n. 1.

Synagris cornuta Latreille, Hist. nat. Insect. XIII. 1805 p. 344.

Vespa cornuta Jurine, Nouv. méth. class. Hymén. 1807 p. 169, \& శึ.

Synagris cornuta Klug, Magaz. Ges. naturf. Fr. Berlin II. 1807 p. 50 n. 55.

Synagris cornuta Latreille, Gen. Crust. \& Insect. IV. 1809 p. 135.

Synagris cornuta Lamarck, Hist. nat. anim. s. vert. IV. 1817 p. 52 n. 1.

Synagris cornuta Lepeletier, Encycl. méthod. Insect. X. 1825 p. 509, ô.

Synagris comuta G. R. Gray. Griffiths: Anim. Kingdom XV. 1532 p. 106; T. 107 F. 1.

Synagris cornuta Lamarek, Hist nat. anim. s. vert. Ed. 2a IV. 1835 p. 299 n. 1.

Srnnagris cornuta Westwood. Drury: Illustr. Nat. Hist. Ed. 2a II. 1437 p. 94; T. 45 F. 3.

Synagris cornuta Blanchard, Hist. nat. Insect. III. 1840 p. 389.

Srnagris cornuta Lepeletier. Hist. nat. Insect. Hymén. II. 1511 p. 593 n. 1. ऽ 3.

Synagris cornuta Saussure, Fitud. fam. Vespid. I. Eumén. 1452 p. 52 n. $6 . \subseteq$ J; T. 13 F. 1.

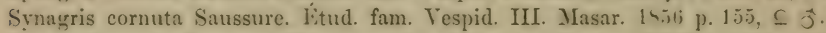

Sỵnagris cornuta Saussure, Mém. soc. phỵs. \& hist. nat. Genère XVII. 1.1463 p. 1 sil n.1.ᄃ J.

erassipes Kohl - ㅇ - Afr. occid.

[F. 112 \& 113 .

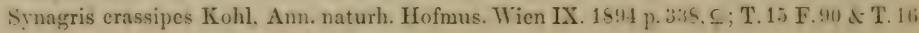

dentata Sauss. - 우 $\widehat{0}-$ Afr.: Senegal.

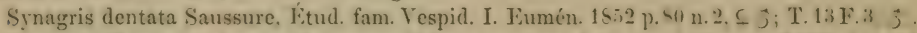

Sỵnagris Mypagris dentata Saussure, Mém. soc. phỵs. \& hist. nat. Genère XYII. 1. 1 1 - i.:

dubia Sauss. - of - Afr.: Abyssinia.

[p. 197 n. 23, ơ; T. 2 F. 12.

Srnagris (Hypagris, dubia Saussure. Mém. soc. phys. (f hist. nat. Genive XVII. 1. 151 i3 p. 2112

emarginata Sauss. - ot - Afr.

[n. 29, ठ̊; T'. 2 F. 17.

Srnagris Paragris emarginata Saussure, litud. fam.Vespid. III. Masar. $14 . j 6$ p. $157 \mathrm{n} .46$, j.

Synagris /Paragris emarginata Saussure, Mém. soe. phỵs. of hist. nat. Genewe XVII. 1. I atia

ferox Sauss. - $\subsetneq-$ Afr.: Senegal.

$[\mathrm{p} .195 \mathrm{n.19}, \mathrm{o}$.

Sỵnagris (Hỵagris) ferox Saussure, Mém. soe. phỵs. c hist. nat. (ienève XVII. 1. 1 ati.3 p.2111

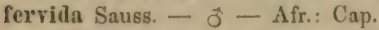

[n. $28, s$.

Synagris IIypagris' fervida Sanssure, Mém. soe. phỵs. A hist. nat. Genèse XVII. 1. Istia

heyleniana Sauss. - of - Afr.: Abyssinia.

[p.199 n. 25, ơ; 'T. 1 F. 13.

Sỵnagris I Paragris Heydeniana Saussure, Mém. soe, phỵs. d hist. nat. Geneve XVII. 1. Ihis

[p. 194 n. 18 , ô; T. 2 F. ?.

lunbertii Sauss. - ơ - Afr. trop.

['T. 8 F. 8.

Synagris Paragris Huberti Saussure, Litud. fam. Vespiel. III. Masar. 14.51 p. 156 n. 45, j;

Synagris Paragris, Inberti Sausture. Mém. soe. phỵs. d hist. nat. Cieneve XVII. I. Istia

mandibularis Sauss. - ô - Afr.: Senegal, Guinea.

$\left[\mathrm{p} .159 \mathrm{n} .11, \delta^{\top}\right.$.

Symagris Antagries mandibularis Saussure, Mem. soe, phys. \& list, nat. Genive XVII. 1.

maxillosa Sauss. - $\subseteq-$ Afr.: Senegal.

[1563 p.184 n. 1, đ;;T. 2 F. 3.

Synugris Paragris maxilhsa Saussure. Mém. sue phys. o hist. nat. Cienéve XVII. 1. 1s 63

[p. 196 n. 2], \&; T. 2 F. 11. 
micelii Grib. - $\widehat{o}$ - Eur.

Sỵnagris Micelii Gribodo. Fid. André: Spee. Hỵmén. Kurope II. 13.24-26. 144lj p. 473. j. minuta Sauss. - ô - Afr.: Cap.

Sỵnagris minuta Saussure. Ktud. fam. Vespid. I. Fumén. 1452 p. 5. n. 9. 3; T. 1:3 F. 7. Sỵnagris Paragris minuta Saussurc. Mém. soc. phỵs. \& hist. nat. Genère XYII. 1. I nti3 mirabilis Guér. - ơ - Afr.: Abyssinia.

[p.192 n. 16, ơ; 'T.2 T.8.

Synagris mirabilis Guérin. Lefebure: Yoy. Abyssinie VI. P. 41845 p. 359; T. \ F. ५.

Sỵnagris mirabilis Reiche of Fairmaire. Ferret \& Galliner: Yoyage en Abyssinie III. 151?

[p. 452 n. 3; 'T. 29 F. 11.

Șrnagris mirabilis Saussure. Kitud. fam. Tespid. I. Tiumén. 15.52 p. $\$ 2$ n. 5. oै.

Synagris mirabilis Smith. Trans. Fintom. Soc. London 2 III. 15.56 Proc. p. 125 n. 2.

Sịnagris 'Antagris miralilis Saussure. Mém. soc. phỵs. \& hist. nat. Genève XVII. 1. 15f;:3

odontophora Schlett. - 우 ơ-Afr.: Congo.

[p. $181 \mathrm{n} .2,07$.

Symagris oduntophora Schletterer, Ann. soc. entom. Belgique XXYT. 1491 p. 21, $\subseteq$ j.

pentameria Sauss. - ô - Afr.: Cap.

Sinnagris pentameria Saussure. Etud. fam. Vespid. I. Eumén. 14.j2 p.\$ i n.12, ja; T.13 F.S.

Sỵnagris Hỵagris pentameria Saussure. Mém. soc. phṛs. d hist. nat. Genère XVII. 1. 1 4ti3

[p. 197 n. 22, ふ઼.

Synagris pentameria Gribodo. Amm. mus. eir. Genova XVI. 1551 p. 240 n. 1.5.

proserpina Grib. - ô - Afr.: Zanzibar.

Synagris Proserpina Gribodo. Bull. soc. entom. Ital. XXIII. 1891 p. 265. 3.

ruppelliana Sauss. - 으 - Afr.

[1863 p. 201 n. 27, , ; T. 2 F. 16.

Sỵnagris Hypagris' Ruppelliana Saussure. Mém. soc. phỵs. I hist. nat. Genève XVII. 1.

Sịnagris Ruppeliana Gribodo, Ann. mus. cir. Genova XVI. 1551 p. 240 n. 2. S.

sericen Spin.! - \& - Afr.

Synagris sericea Spinola, Insect. Ligur. II. 1808 p. 188 note; ㅇ․

sicheliana Sauss. - ot - Afr.: Guinea.

[p. 183 n. 4, ô; T. 2 F. 1.

Sỵnagris Antagris Sicheliana Saussure, Mém. soc. phỵs. of hist. nat. Genèe XVII. 1. 15 ti3

spinolae Sauss. - ô - Eur.: Hispania.

[T. 18 F. 9.

Synagris Micragris Spinolae Saussure. Ktud. fam. Vespid. III. Masar. 1556 p. 15 - n. 14. j);

Sỵnagris Micragris Spinolac. Saussure. Mém. soc. phỵs. (f hist. nat. Genève XVII. 1. 15ti3

[p. 204 n. 32, ठ઼.

Micragris Spinolae I:d. André. Spec. Hymén. Europe II. I. 21-23. 1und p. 6it6. \&; T.42 F.1.

spinosuscula Sauss. - $\odot$ ơ - Afr.: Abyssinia.

Synagris spinosuscula Saussure. Etud. fam. Vespid. I. Eumén. 1552 p. s. n. 10. ( 3 . Synagris Paragris spinosuscula Saussure, Mém. soc. phỵs. d hist. nat. Genève XVII. 1. tarsalis Gerst. - $\subseteq \hat{o}-$ Afr.

[1863 p. 189 n. 13 ; 'T. 2 F. 6 (ठ̋).

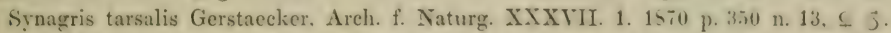

Synagris tarsalis Gerstaecker, r. d. Decken: Reise in Ost-Afrika. Gliederthiere. 1573 p.322

tetrameria Sauss. - - - Afr.: Abyssinia.

[n. 13, fơ.

Sỵnagris Paragris tetrameria Saussure. Mém. soe. phỵs. o hist. nat. Genere XVII. 1. 1 rti.3 tropidia Schlett. - 우 ô - Afr.: Congo.

(p.193 n. 17, \&.

Sỵnagris tropidia Sehletterer, Ann. soe. entom. Belgique XXXV. 1591 p. 20. O. 5.

xanthura Sauss. - $\subseteq-$ Afr.: Senegal.

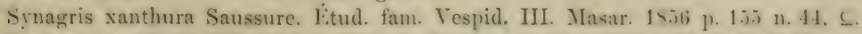

Synagris xanthura Gerstaecker. l'eters: Reise n. Mossambique. Zool. V. 1562 p. 4til. S. Synagris Antagris xanthura Saussure. Mém. soc. plỵs. A hist. nat. Geneve XVII. 1. 1 atjis Synagris xanthura Gribodo, Ann. mus. cir. Genowa XXI. $13-1$ p. 292 n. 25. p. 157 n.9.9. Sịnagris xanthura Magretti. Ann. mus. civ. Genora XXI. 1s-1 p. 613 n. 150. c. 


\section{MONTEZUMTA}

Saussure, Étud. fam. Vespid. I. Eumén. 1852 p. 87 ; T. 5 F. 3.

Nom. propr.

Synon.: Eumenes Fabricius.

Odynerus Spinola.

Subg.: Alpha Saussure, Étud. fam. Vespid. I. Eumén. 1852 p. 88. ¿ littera.

Antezumia Saussure, Smithson. Miscell. Collect. XIV. P. 254. 1875 p. 113.

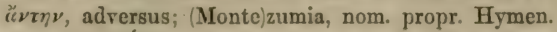

Beta Saussure, Étud. fam. Vespid. I. Eumén. 1852 p. 90. $\beta$ littera.

Metazumia Saussure, Smithson. Miseell. Collect. XIV. P. 25t. 1575 p. 114. $\mu \varepsilon \tau$ ć, circum; (Monte)zumia, nom. propr. Hymen.

Parazumia Saussure, Étud. fam. Vespid. III. Masar. 1856 p. 166.

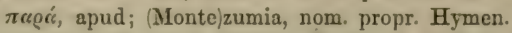

Pseudzumia Saussure, Smithson. Niscell. Collect. XIV. P. 254. 1575 p. 125. $\psi \varepsilon v \delta_{\eta j}$, falsus; (Monte)zumia, nom. propr. Hymen.

analis Sauss. - jo - Am. mer.

Montezumia analis Saussure, Kitud. fam. Vespid. III. Masar. 1556 p. 163 n. 53 , ठే.

Montezumia analis Saussure, Smithson. Miscell. Collect. XIV. P. 254.1575 p. 119 n. 12. anceps Sauss. - $ᄋ-$ Am.: Chile.

Montezumia anceps Saussure, Ėtud. fam. Vespid. I. Eumén. 1852 p. 92 n. 8.

Montezumia anceps Saussure, Smithson. Miscell. Collect. XIV. P. 254. 1875 p. 125 n. 20, C. andeus Smith - ฮै - Am.: Quito.

Montezumia Andeus Smith, 1th Rep. Peabody Acad. Sc. 1869 p. 60, Јे.

azteca Sauss. - of - Am.: Mexico.

[T. 2 F. $10 \& 10^{3}$.

Montezumia Azteca Saussure, Smithson. Miscell. Collect. XIV. P. 254. 1875 p. 125 n. 21, Jै;

azureseens (Spin.) Sauss. - 우 ᄚ - Am. mer.

Odynerus? azurescens Spinola, Mem. accad. sc. Torino 2) XIII. 1851 p. $\$ 2$ n. $62, \vec{o}$.

Montezumia rufipes Saussure, Ltud. fam. Vespid. I. Eumén. 1552 p. \$9 n. 3. \&; T. 15 F.1.

Montezumia rubritarsis Saussure, Étud. fam. Vespid. I. Eumén. 1552 p. 90 n. 4, ऽ.

Montezumia cocrulea Saussure, Étud. fam. Vespid. I. Eumén. 1852 p. 90 n. 5. .; T.12 F. .

Montezumia coerulea Saussure, Étud. fam. Vespid. III. Masar. $1 \$ 56$ p. 160.

Montezumia azurescens Saussure, Kitud. fam. Vespid. III. Masar. 1556 p. 160.

Montezumia coerulea Saussure, Smithson. Miscell. Collect. XIV. P.254.1575 p.11 in.6..$\hat{\jmath}$.

brasiliensis Sauss. - $\approx-$ Am. : Brasilia.

Montezumia Brasiliensis Saussure, litud. fam. Vespid. III. Masar. 18.66 p. 164 n. 51 , j.

MIontezumia Trasiliensis Saussure, Smithson. Miscell. Collect. XIV. P. 254. 1575 p. 127

brunea Sauss. - 0 of - Am. mer., Cayenne.

n. 22,0 .

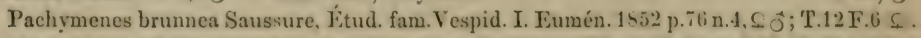

Montezumia Antezumia) brunnea Saussure, Smithson. Micell. Collect. XIV. P. 254. 14i5

earinulata (Spin.) Sauss. - 우 - Am.: Brasilia.

[p. 114 n. $3, \subseteq \sigma$.

Odynerus? carinulata Spinola, Mem. aeead. se. Torino 2 XIII. 1551 p. $\$ 3$ n. 63, s.

Montezumia Parazoumia) carinulata Saussure, Etud. fam. Vespid. III. Masar. 1556 p. 166

[n. 58, \& [non $\hat{o}]$.

Montezumia (Parazumia) carimulata Saussure, Smithson. Miscell. Collect. XIV. P. 254.

chalybea Sauss. - $\subseteq-$ Am.: Brasilia.

[1875 p. 128 n. 24, ㅇ.

Montezumia Chalybea Saussure, Ktud. fam. Vespid. IlI. Masar. 1556 p.161 n.49, S; T. 9 F. 2.

Montezumia Antezumia chalybea Snussure, Smithson. Misecll. Collect. XIV. P. 251. 1575

[p. $113 \mathrm{n} .1$. 
cortesia Sauss. - \&- Am.: Mexico.

Montezumia cortesia Saussure, Étud. fam. Vespid. I. Eumén. 1652 p.92 n.10, 千; T.15 F.2.

Montezumia cortesiana Saussure. Smithson. Miscell. Collect. XIV. P.254. 1575 p.119 n.10, @.

ferruginea Sauss. - 우 of - Am. mer.

Montezumia ferruginea Saussure. Étud. fam. Vespid. I. Eumén. 1852 p. 91 n. $7, \subseteq$ ơ. Montezumia ferruginea Saussure, Smithson. Miscell.Collect. XIV.P.254. 1575 p.123 n.16, S.

ghilianii Sauss. - $t$ - Am.: Brasilia.

Montezumia Ghilianii Saussure, Smithson. Miscell. Collect. XIV.P.254.1575 p.121 n. 14.3ૈ.

huasteca Sauss. - 오 ơ - Am.: Mexico.

Montezumia Huasteca Saussure, Rer. \& mag. zool. IX. 1857 p. 172.

Montezumia Metazumia Huasteca Saussure, Smithson. Miscell. Collect. XIV. P. 25.4. 1575

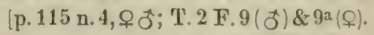

indica Sauss. $-q-\mathrm{Am}$.

[n. 59 , 우; T. 9 F. 4.

Montezumia (Parazoumia) Indica Saussure, Étud. fam. Vespid. III. Masar. 1856 p. 167

Montezumia (Pseudozumia Indica Saussure, Smithson. Miscell. Collect. XIV. P. 254. 15i5

infernalis (Spin.) Sauss. - 우 $\vec{\sigma}-$ Am.: Surinam, Brasilia.

[p. 128.

Odynerus infernalis Spinola, Mem. accad. sc. Torino (2) XIII. 1851 p. 84 n. 64, ơ.

Montezumia Spinolae Saussure. Étud. fam.Vespid. I. Eumén. 1852 p.93 n.12, $\subsetneq$ ơ; T.12 F.9.

Montezumia infernalis Saussure, Etud. fam. Vespid. III. Masar. 1856 p. 162 n. $52 . \zeta$.

Montezumia Spinolae Saussure, Smithson. Miscell. Collect. XIV.P.254.1875 p.119 n.13, f 3.

infundibuliformis (Fabr.) Sauss. - $ᄋ$ ô - Am.: Brasilia.

Eumenes infundibuliformis Fabricius, Syst. Piez. 1804 p. 288 n. 14.

Montezumia dimidiata Saussure, Étud. fam.Vespid. I. Eumén. 1852 p. 94 n. 13, ऽ [non Oliv.].

Montezumia infundibuliformis Saussure, Ktud. fam. Vespid. III. Masar. 1556 p. 160.

Montezumia infundibuliformis Saussure, Smithson. Misccll. Collect. XIV. P. 254. 1575

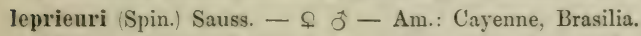

[p. 118 n. $9, \subseteq 3$.

Odynerus Lepricuri Spinola. Ann. soc. entom. France X. 1541 p. 127 n. 7 , $f$.

Montezumia pelagiea Saussure, litud. fam. Vespid. I. Eumén. 1852 p. 93 n. 11, \& ơ; T. 12

Yontezumia Leprieuri Saussure, Étud. fam. Yespid. III. Masar. 1856 p. $160 . \quad$ F. 10 '

Montezumia (Metazumia) I.cpricuri Saussure, Smithson. Miscell. Collect. XIV. P. 251. 1875

liliacen Grib. - $ᄋ$ ơ - Am.: Brasilia.

Montezumia liliacea Gribodo, Bull. soc. entom. Ital. XXIII. 1891 p. 27:3, $\subseteq \hat{\jmath}$.

liliaciosa Grib. - $₫$ - Am.: Peru.

IIontezumia liliaciosa Gribodo, Bull. soc. entom. Ital. XXIII. 1891 p. $2 \overline{5}, \widehat{o}$.

macrocephala Sauss. - 우 ơ - Am.: Brasilia.

['T. 9 F. 3 .

Iontezumia macrocephala Saussure. Fitud. fam. Vespid. III. Masar. 1456 p. 165 n. 57 , 3ै;

Montezumia macrocephala Saussure. Smithson. Miscell. Collect. XIV. P.254. 1975 p. 127

marthae Sauss. - $\subseteq-$ Am.: Antilles.

[n. 23, \& ठै.

IIontezumia Marthae Saussure, Smithson. Miscell. ('ollect. XIV. P. 254. 1575 p. 124 n. 18, C. mexieana Sauss. - - - Am.: Mexico.

Montezumia Mexicana Saussure, litud. fam. Vespid. I. Kumén. 1552 p. 94 n. 14. $f$.

Montezumia Mexicana Saussure. Smithson. Miscell. Collect. XIV. 1'. 254. 1875 p. 12:2

morosa Sauss. $-\rho \sigma-A m$. mer.

[n. 15, 우 T. 2 F. 10.

Montezumia morosa Saussure, litud. fam. Vespid. I. Eumén. 1852 p. 90 n. 6, ऽ §.

Montezumia morosa Saussure, Kitud. fam. Vespid. III. Masar. 1456 p. 160.

Montezumia morosa Saussure, Smithson. Miscell. Collect. XIV. P.254. 1475 p.121 n.17, s j.

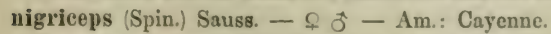

Eumenes nigriceps Spinola, Ann. soc. entom. France X. 1511 p. 125 n. 79. j.

Montezumia nigriceps Saussure, Étud. fam. Vespid. III. Masar. 1 sili p. 162 n. $51, \hat{\jmath}$.

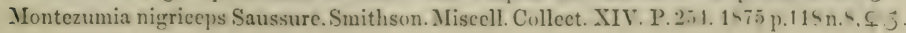


orientalis Grib. $-\Omega-$ As.

Montezumia orientalis Gribodo, Bull. soc. entom. Ital. XXIII. 1991 p. 272. 5. petiolata Sauss. - $\mathrm{Q}-$ Am.: Brasilia.

Montezumia petiolata Saussure. Ktud. fam. Vesprid. III. Masar. 15.56 p. 161 n. 50.C; T. 9 F.1. Iontezumia Antezumia petiolata Saussure. Smithson. Miseell. Collect. XIV. P. 251. 15is platinia Sauss. $-\rho-$ Am.

[p. 113 n. 2.

Montezumia Platinia Saussure, litud. fam. Vespid. I. Fumén. 1552 p.92 n. 9; T. 15 F 3. Montezumia J'latensis Saussure, Smithson. Miscell. Collect. XIY. P. 251. 1475 p.125 n. 13. L. rufldentata Sauss. - $\subseteq$ ô - Am.: Mexico, Brasilia.

Montezumia rufidentata Saussure. Kitud. fam. Vespid. I. Eumén. 1452 p. ss n. 1 . f. Montezumia azureipennis Saussure. Jitud. fam. Vespid. I. Jumén. 1552 p. $>9$ n. 2.9. Montezumia azureipennis Saussure. Etud. fam. Vespid. III. Masar. 14.56 p. 160. 3.

Montezumia azureipennis Saussure, Smithson. Miscell. Collect. XIV. P. 25.4. 15i5 p.117 saussurei Kirsch - 오 of - Eur.: Woodlack.

Montezumia Saussurei Kirsch, Mittheil. zool. Mus. 1)resden II. 1579 p. si: $\leq 3$.

sepulchralis Sauss. - 우 ot-Am.: Brasilia.

Iontezumia sepulchralis Saussure. Kitud. fam. Vespid. III. Masar. 1956 p. 163 n. 54. L. Montezumia mortuorum Saussure. Étud. fam. Vespid. III. Masar. 1956 p. 164 n. 5.5, 3. Montezumia sepulchralis Saussure. Smithson. Miscell. Collect. XIV. P. 254. 1575 p. 119 [n. 11, ठึ.

\section{MONOBIA}

Saussure, Ítud. fam. Vespid. I. Eumén. 1952 p. 9ł; 'T. 6 F. 2. uóvos, solus; $\beta$ เów, vivo.

Synon.: M ontezumia Saussure.

Odynerus Guérin, Say, Lepeletier.

Vespa Linné \& auct. ant.

angulosa Sauss. - $\subsetneq$ ô - Am.: Mexico, Surinam, Brasilia.

Monobia angulosa Saussure, Kitud. fam. Vespid. I. Fumén. 1 52 p. 94 n. 15. . : T. 15 F. .

Monobia angulosa Saussure, Étud. fam. Vespid. III. Masar. 1556 p. 169.

Monobia angulosa Saussure, Smithson. Miseell. Collect. XIV. P. 254.1475 p. 131 n. 6., 5. anomala Sauss. - $\hat{o}-$ Am.: Brasilia.

Monobia anomala Saussure, Etud. fam. Tespid. I. Eumén. 1552 p. 96 n. 3. 3: T. 1: F.4. Monobia anomala Saussure, Smithson. Miscell. Collect. XIV. P. 254. 1575 p. 132 n. 4. apienlipennis Sauss. - 우 - Am.: Brasilia.

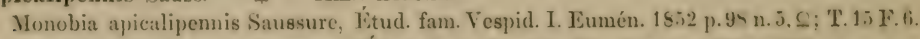
Momobia apiealipennis Saussure, Étud. fam. Vespid. III. Masar. 1456 p. 16is.

Monobia apicalipennis Saussure, Smithsen. Miseell. ('ollect. XIV. P. 254. 1 55 p. 1:5 n 19.4. biangulata Sauss. $-\rho-$ Am.: Mexico.

[T. 2 1: 12 \& $12 \mathrm{a}$

Monobia biangulata Saussure, Smithson. Miscell. ('ollect. XIV. P. 254. 1575 p. 135 n. 7.4; bieolor Prov: $-\Omega-A m$.

Monsbia bicolor Prosancher. Addit. faun. ('anada. Hymén. lans p. 420, L.

californien Sauss. - $\frac{?}{7}-$ Am.: California.

Montezumia Californica Saussure, Rev. \& mag. zool. XV. 1963 p. ?

Ifonobia Californica Suussure, Smithson. Miseell. (G)llect. XIV. P. 254.157 .5 p.129 n.1. S j. eavifrons Grib. - $? \overrightarrow{0}-$ As.

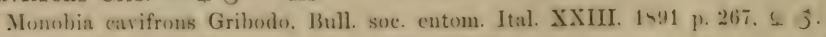

eyanipennis (Guér.) Sauss. - ô - Am. : Chile.

[F.5(す̄).

Odynerus cyanipennis Guérin. 1)uperry: Voy. Coquille. Zowl. II. 2. 14i31 p. 2tit. Ṡ; T. 9

Monohia cranipennis Sanssure, Titud. fam. Vespid. I. Eumén. 15.22 p. 96 n. 2. 3.

Monobia eyanipennis Saussure. Smithson. Miseell. ('ollect. XIV. P.2.4. 15i. p. 131 n. 2. 
egregia Sauss. - $\subseteq-$ Am.: Antilles.

Menobia egregia Saussure. litud. fam. Vespid. III. Masar. 14.56 p. 1lis n. 60. S: T. 4 F. 5.

Monobia egregia Saussure. Smithson. Miscell. ('ollect. XIV. 1'.254. 1 > i. p. 134 11.11. L. funebris Grib. - $ᄋ \hat{o}-$ Am.: Brasilia.

Monobia funebris Gribodo. Bull. soc. entom. Ital. XXIII. 1s:11 p. 2tit; $\leq$ 3.

mina-palumboi Grib. - $\subseteq-$ Am.: Columbia.

Monobia Mina Palumboi Gribodo. Bull. soc. entom Ital. XXIII. 1591 p. 2211. 5.

nigripennis Sauss. - 으-Am.: Mexico.

Monobia nigripennis Saussure. Smithson. Miscell. Cullect. XIV. P. 254. 147, p. 134 n. ๑. . . quadridens (L.) Sauss. - 오

Vespa quadridens Linné, Centur. Insect. rar. 1763 p. 31 n. 92.

Vespa quadridens Linné, Amoen. acad. VI. 1763 p. 413 n. 92.

Vespa quadridens Linné, Syst. nat. Ed. $12^{a}$ I. 2.1767 p. 951 n. 15.

Tespa cincta-nigra Degeer. Mém. hist. Insect. III. 1773 p. $54 \pm$ n. 5: T. 29 F. 12.

Vespa uncinata Fabricius, Syst. entom. 1775 p. 367 n. 22.

Vespa quadridens Ph. L. Müller. Linné : Vollst. Naturș̣st. V. 2. 177.5 p. ss:3 n. 1.i.

Vespa quadridens Gmelin, Onomatol. hist. nat. VII. $177 \overline{\text { p. }} 709$.

Tespa cineta-nigra Güze. Degeer: Abh. Gesch. Insect. III. 17al p. 3T n. s; T. 29 F. 12.

Vespa uncinata Fabricius, Spec. Insect. I. 1781 p. 463 n. 27.

Vespa cincta-nigra Retzius, Gen. \& spec. Insect. 1783 p. 64 n. 239.

Vespa uncinata Fabricius, Mant. Insect. I. 1787 p. 289 n. 32.

Vespa quadridens Gmelin, Linné: Syst. nat. Ed. 13a I. 5.1790 p. 2757 n. 15.

Vespa quadridens Christ, Naturg. d. Insect. 1791 p. 234.

Vespa uncinata Olivier, Eneycl. méthod. Insect. VI. 1791 p. 655 n. 77.

Vespa uncinata Fabricius, Entom. system. II. 1793. p. 264 n. 39.

Vespa incincta Fabricius, Syst. Piez. 1804 p. 259 n. 31.

Odynerus quadridens Say, Boston Journ. Nat. Hist. I. 4. 1837 p. 386 n. 3.

Odỵnerus uneinatus Lepeletier. Hist. nat. Insect. Hymén. II. 15.11 p. 619 n. $9, \leq 3$

Monobia quadridens Saussure. Etud. fam. Tespid. I. Eumén. 1552 p. 97 n. $4, \subseteq$ ș: T. 16 F. 1.

Munobia uneinata Saussure. F́tud. fam. Vespid. I. Eumén. 1552; T. 16 F. 1.

Monobia quadridens Saussure. 1.tud. fam. Vespid. III. Masar. 15.56 p. 16ৎ.

Odynerus quadridens Leconte. Writ. of Th. Say Iintom. II. 1459 p. 766 n. 3.

Monobia quadridens Cresson, Trans. Amer. Entom. Soc. IV. 1872 p. 233.

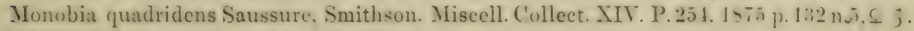
silvatiea Sauss. - $\Omega-$ Am.: Brasilia.

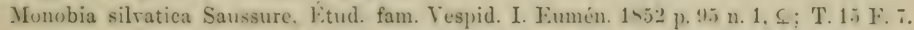

Monobia silvatica Saussure. Smithison. Miscell. Collect. XIV. P. 254. 14i.s p. 132 n. 3. . . variabilis Sauss. $-\varsigma-$ Am.: Mexico.

Monobia rariabilis Saussure. Smithson. Miscell. ('ollect. XIY. P.25. 157. 13.137 n. 10.5.

\section{ABISPA}

Mitehell, Exped. East. Austral. I. 1839 p. 104.

Vox barbara.

Symun.: Monerebia Saussure. Etud. fam. Vespid. I. Eumen. 1952 p. an: T. if F. 2.

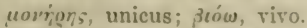

? Oneorrhinu s Shuekard. Fres: Journ. Fxped. King Georeses Sumd II. 1- 11 p.4:1. ŏ y'xos, unguis; ơir', nasus.

Odynerus Guérin.

Vespa Fabricius \& auct. ant.

anstralis Smith - $\subseteq-$ Austr.

Abispa Australis Smith, Catal. Hymen. Brit. Mus. V. 1557 p. 42 n. 2, C. 
ephippinm (Fabr.) Mitch. - $f-$ Austr.: Tasmania.

Vespa ephippium Fabricius, Syst. entom. 1775 p. 362 n. 2.

Vespa ephippium Fabricius, Spec. Insect. I. 1781 p. 458 n. 2.

Vespa ephippium Fabricius, Mant. Insect. I. 1787 p. 287 n. 3.

Vespa ephippium Gmelin, Linné: Syst. nat. Ed. 13 a I. 5.1790 p. 27 is n. 30.

Vespa ephippium Christ, Naturg. d. Inseot. 1791 p. 237.

Tespa ephippium Olivier, Encycl. méthod. Insect. VI. 1791 p. 677 n. 40.

Vespa ephippium Fabricius, Entom. system. II. 1793 p. 254 n. 3.

Vespa ephippium Fabricius, Syst. Piez. 1804 p. $25 \pm$ n. 3.

Abispa ephippium Mitchell, Exped. East. Austral. I. 1838 p. 104.

Abispa ephippium Smith, Trans. Entom. Soc. London 2) I. 7. 1851 p. 180; T. 16 F. 4. MIonerebia ephippium Saussure, Étud. fam. Vespid. I. Eumén. 1552 p. 100 n. 2. \&; T. 15 F.9. Abispa ephippium Saussure, Etud. fam. Vespid. III. Masar. 1856 p. 170.

splendida (Guér.) Sauss. - 오 ơ - Austr.: 'Tasmania.

Odynerus splendidus Guérin, Duperry: Voy. Coquille. Zool II. 2. 1530 p. 265.

Odỵnerus splendidus Guérin \& Percheron, Gen. d. Insect. livr. 6 P. 7. 1538; T. 5.

? Oncorhinus xanthospilus (Shuckard) White, Grey: Journ. Exped. King Georges Sound II.

[1841 p. 471 n. 34, ฮ̃.

Monerebia splendida Saussure, Étud. fam. Vespid. I. Eumén. 1552 p. 99 n.1, ô; T. 15 F. S. Abispa splendida Saussure, Étud. fam. Vespid. III. Masar. 1956 p. 169, 요 $\delta$. [F.15. ? Oncorhinus xanthospilos Smith, Catal. Hymen. Brit. Mus. VII. 1859 p. 65 n. 1, ฮ๋; T. 3 Abispa splendida H. L. Roth, Journ. Linn. Soc. London. Zool. XVIII. P.107. 1555 p. 323;

[Fig. $3 \mathrm{~A}-\mathrm{C}$.

\section{RHYNCHIUM}

Spinola, Insect. Ligur. I. 1806 p. 84 n. 36 \& II. 1508 p. 3 n. 2 [Rhỵgchium]. @́v́y̆os, rostrum.

Synon.: Ancistrocerus Smith.

Eumenes Klug.

Odynerus Guérin.

Polistes Fabricius.

Scolia Cyrillo.

Vespa Fabricius.

Sulıg.: Anterhy nehium Saussure, Mém. soc. phỵs. \& hist. nat. Genève XVII.1. 1863 p. 2115. 'i $\nu^{\prime} i$, contra; Rhynchium, nom. propr. Hymen.

Parrhynchium Saussure, Stettin. entom. Zeitg. XXIII. 1862 p. 1 S3. a๘@ณ́, apud; Rhynchium, nom. propr. Hymen.

Prorhynchium Saussure, Stettin. entom. Zeitg. XXIII. 1862 p. 187. $\pi$, ante; Rhynchium, nom. propr. Hymen.

abflominale (IIl.) Sauss. - 우 $\hat{o}-$ As.: India.

Vespa abdominalis Illiger, Magaz. f. Insectenk. I. 1802 p. 192 n. 21.

Vespa transversa Fabricius, Syst. Piez. 1804 p. 257 n. 16.

Vespa transwersa Jurine, Nouv. méth. elass. Hymén. 1807 p. 169, ðૅ.

Jhygehium transversum Saussure, Étud. fam. Vespid. I. Eumén. 1552 p.117 n.26. \&;T.14 F.i.

Rhynchium abdominale Saussure, Etud. fam. Vespid. III. Masar. 1456 p. 172 . S $\vec{j}$.

Rhynchium abdominale Saussure, Grandidier: Hist. Madagasear XX. P. 1. 1591 p.159 n. 2, var. diehotomum Sauss. - $ᄋ \hat{o}-$ As.: India or.

Rhygehium dichotomum Saussure, Ktud. fam. Vespid. I. Eumén. 18.52 p. 116 n. 25. $\subseteq . j$. Rhynchium abdominale var. dichotomum Saussure, litud. fam. Vespid. III. Masar. Lhiti 
aestuans Sauss. - $f$ - Afr.: Senegal.

[1863 p. 206 n. 34, ㅇ.

Rhỵnchium (Anterhynchium) aestuans Saussure, Mém. soc. phỵs. \& hist. nat. Genère XVII.1.

Rhygghium aestuans Gribodo, Bull. soc. entom. Ital. XXIII. 1591 p. 285, f.

alecto (Lep.) Sauss. - $\rho-$ Austr.

Odynerus Alecto Lepeletier, Hist. nat. Insect. Hymén. II. $1 \$ 41$ p. 617 n. $32,5$.

Rhỵgchium Alecto Saussure, Ktud. fam. Vespid. I. Eumén. 1852 p. 114 n. 20 , S. auceps Grib. - 우 - Afr. occ.

Rhygchium anceps Gribodo, Bull. soc. entom. Ital. XXIII. 1891 p. 250, S.

annuliferum Boisd. - Austr.: New Guinea.

Rhynchium annuliferum Boisduval, Voy: Astrolabe. Zool. II. 6. 1833 p. 654 ; T. 12 F. 3.

Rlỵgchium annuliferum Saussure, Étud. fam. Vespid. I. Eumén. 1552 p. 115.

arilens (Guér.) Sauss. - $ᄋ$ ô - Afr.: Abyssinia.

Odynerus ardens Guérin, Léfébure: Voy. Abyssinie VI. 1918 P. 4. p. 362; T. 8 F. 9.

Rhygchium Abyssinicum Saussure, Étud. fam. Vespid. I. Eumén. 1852 p. 103 n. 2, ‥

Rhygehium ardens Saussure, Étud. fam. Vespid. I. Eumén. 1552 p. 104 n. 3, ठే.

Rhynchium (Anterhynchium) Abyssinicum Saussure, Mém. soc. phys. \& hist. nat. Géèèe

[XVII.1.1863 p.208 n.36,오.

Rhynchium Abyssinicum Saussure, Grandidier: Hist. Madagasear XX. P. 2.1591 p.175 n. 3, ardens Walk.! - $\rho-$ As.: Arabia.

[우 ず.

Rhynchium ardens Walker, List of Hymen. in Egypt 1S71 p. 31 n. 161, ․

argentatum (Fabr.) Sauss. - 우 ô - As.: India, Sumatra.

Vespa argentata Fabricius, Syst. Piez. 1804 p. 260 n. 39.

Vespa argentata Jurine, Nouv. méth. class. Hymén. 1807 p. 170, $\subseteq$ ô.

F. S.

Rhỵgchium metallicum Saussure, Étud. fam. Vespid. I. Eumén. 1552 p. 114 n. 21, ૬ ठే; T. 14

Phynchium argentatum Saussure, Étud. fam. Vespid. I. Eumén. 1552 p. 115 n. 22, $\subsetneq$ ô.

Rhynchium metallicum Saussure, Ĺtud. fam. Vespid. III. Masar. 1556 p. 172.

Rhynchium Prorhynchium) argentatum Saussure, Stettin. entom. Zeitg. XXIII. 1862 p. 1 S7 var. bengalense Sauss. - ot - As.: Bengalia.

[n. 23, 우

Rhỵnchium Bengalense Saussure, Étud. fam.Vespid. III. Masar. 1 \$5 6 p. 176 n. 66, ơ; T. 9 F. 8.

Rhynchium Bengalense Saussure, Stettin. entom. Zeitg. XXIII. 1562 p. 191 n. 25, \& ô.

Rhynchium argentatum var. Bengalense $\mathrm{m}$.

auromaculatum Sauss. - - of - Afr.: Senegal; As.: Java?

[F.1.

Rhỵnchium auromaculatum Saussure, Etud. fam. Vespid. I. Eumén. 1S.52 p.104 n.4, \& ş; T.14

bicoloripenne Grib - - - As.: Paulo Lant (Ind.).

Rhyggehium bicoloripenne Gribodo, Bull. soe. entom. Ital. XXIII. 1591 p. 256, s.

bioculatum Sauss. - ㅇ - Afr. occ.

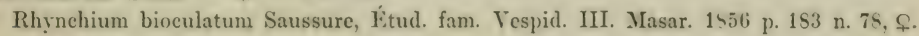
carbonaxium Sauss. $-\subseteq-$ As.: India.

Rhynchium earbonarium Saussure, Amn. soc. entom. France 3; V. 1857 p. 317, $\subseteq$.

Rhỵnchium Parrhynchium, carbonarium Saussure. Stettin. entom. Zeitg. XXIII. 1Sli2 p. 153 ceperoi Med. - Eur.

Rhygchium C'eperoi Medina, Anal. soc. Espan. hist nat. XX. 1 s91 p. 105.

chinense Sauss. $-\rho \hat{\sigma}-$ As.: China. [n. 22, $ᄋ \hat{\sigma}$.
.

[n. 18, ‥

Rhỵnchium Prorhynchium, Chinense Saussure, Stettin. entom. Zeitg. XXIII. 15 ti2 p. 146 clirysomallum Schlett. - - - Afr.: Congo.

Rhỵnchium chrysomallum Schletterer, Ann. soc. entom. Belgique XXXV. 1 s91 p. 23. S. cẙnopterum Sauss. - 우 0 - Afr.: Aegyptus, Senegal.

- Sarigny, Deser. de l'Égypte. Hymén. 1812; T. 9 F. 9.

Rhygchium cyanopterum Saussure, Etud. fam. Vespid. I. Eumén. 1 s.2 p. 105 n. $9 . \subseteq \vec{s}$.

Rhỵnchium cyanopterum Ed. André, Spee. Hymén. Europe II. P. 21-23. 16st p. (it9. C 3 . 
Rhynchium eyanopterum Magretti. Ann. mus. civ. Genova XXI. 144 p.613 n.151. I j. Rhynchium cyanepterum Saussure. Grandidier: Hist. Madagasear XX. P.1 14:11 p. 159 n. 1. llecoratum Sauss. - - - Austr.

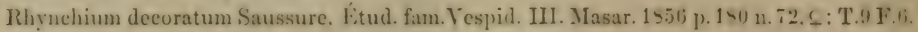
dimiliatum (Spin.) Sauss. - $-\hat{\sigma}-\Lambda$ fr.: Aegyptus.

Odynerus dimidiatus Spinola. Amn. sue. entom. France VII. 1435 p. 502 n. XIVI. \& 5. Rhynchium dimidiatum Saussure, Citud. fam. Vespid. III. Masar. 14.56 p. 142 n. 76. - 3 erythrinum Sauss. - 우 - Afr.: Cap.

Rhynchium erỵthrinum Saussure. Reise d. Novara. Zool. II. 1. 1 4ij7 Ifymen. p. z n. 2. . . fallax Sauss. $-Q-$ Afr. occ.

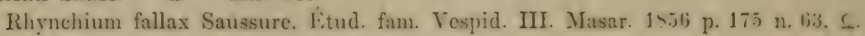

ferrugineum Rad. - ô - Afr.: Angola.

[n. 61, ö.

Rhỵnehium ferrugineum Radoszkowski. Journ. acad. se. math. etc. Lisboa VIII. 1sul p. 20.3 fervens Walk. - $0 \hat{o}-$ As.: Arabia.

Rhynchium fervens Walker. List of Hymen. in Egypt $14 i 1$ p. $\$ 1$ n. 162 . 0 j.

flavomarginatum Smith $-\hat{o}-$ A6.: China.

Rhynchium flaromarginatum Smith. Trans. Entom. Soc. London 2) II. 2. 14.52 p. 35. 3. Rhynchium flavomarginatum Saussure, Kitud. fam. Vespid. III. Masar. 1856 p. 177 n. fis. j.

flaropunctatum (Smith) Sauss. - 우 ơ - As.: China.

Ancistrocerus flavopunctatus Smith. Trans. Entom. Soc. London 2. II. 2. 1552 p. 31. L. Rhynchium flaropunctatum Saussure. Etud. fam. Vespid. III. Masar. 1 s56 p.1 i s n.69;T.9 F. . Rhynchium flavopunctatum Saussure. Stettin. entom. Zeitg. XXIII. 1 S62 p.192 n.26. - j. Rhỵnchium flavopunctatum Saussure. Reise d. Novara. Zool. II. 1.1 167 Hymen, p. n.4.ᄃ.

furax Kohl - 오 ơ - Afr. occid.

Rhynchium furax Kohl. Ann. naturh. Hofmus. Wien IX. 1594 p. 3399 \& 3: T. 15 F. 49. gestroi Magr. - §̋ - Afr. or.

Rhynchium Gestroi Magretti. Ann. mus. cir. Genora XXI. 1s54 p. 614 n. 153. 3. gianellii Grib. - 오 oี - Am.: Brasilia.

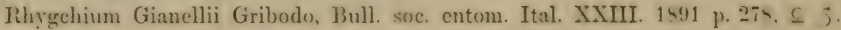

grayi Sauss. - 우 - Afr.: Port Natal.

Rlynchium Grayi Saussure, Fitud. fam. Vespid. III. Masar. 1456 p. 175 n. 61. C.

Rhygchium Grayi Gribodo, 13ull. soc. cntom. Ital. XXIII. 1891 p. 285.

haemorrhoidale (Fabr.) Sauss. - $-\widehat{\sigma}-$ As.: India, Java; Afr.: Cap.

Vespa haemorrhoidalis Fabricius, Syst. entom. 1775 p. 366 n. 19.

Vespa haemorrhoidalis Fabricius, Spec. Insect. I. 1781 p. 462 n. 24.

Vespa haemorrhoidalis Fabricius, Mant. Insect. I. 1787 p. 289 n. 29.

Vespa haemorrhoidalis Gimelin, Limi: Syst. nat. Fid. 18 a I. 5. 1790 p. 27.59 n. 5. .

Vespa hacmorrhoidalis Christ, Naturg. d. Insect. 1791 p. 242.

Vespa haemorrhoidalis Olivier. Inneyel, method. Insect. VI. 1791 p. tis:3 n. 6s.

Vespa haemorrhoidalis Fabricius, Entom. system. II. 1793 p. 263 n. 36.

Vespa haemorrhoidalis Fabricius, Syst. Piez. 1804 p. 259 n. 28.

Odynerus dimidiatus (inérin, Bélanger: Voy. Ind. orient. 1434 p. $503 ;$ T. 4 F. 4.

Rhygrhium haemorhoilale Saussure, Citud. fam. Vespid. I. Eumen. 1552 p. 109 n. 12 s 3 . Rhynchium haenorrhoidale Smith. Journ. of Proce. Limn. Soc. Zoul. IV. 1stio Suppl. p.124 n.1.

Rhynchinm hatemorrhoidale Maindron. Ann. soc. entom. France 6 II. 1452 p. 27:: T. 1 var, atrum Sauss. - $\odot \hat{o}-$ As.: India; Austr.: New Guinea.

[F. 10-11.

lihygchium atrum Sanssure, Kind. fum. Vespid. I. Eumen. 15.52 11. 109 n. 11. S 3. lihỵchium atrum Saussure. Stettin. entom. Zeitg. XXI11. 14ti2 p. 190 n. 21 . \& j.

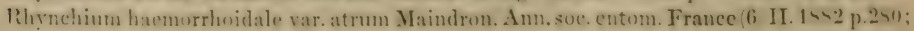


var. caruaticum (Fabr.) Sauss. - $\subseteq$ ô - As.: India.

Vespa carnatica Fabricius, Suppl. entom. system. 1798 p. 261 n. 22-23.

Vespa carnatica Fabricius, Syst. Piez. 1804 p. 258 n. 22.

Rhygchium carnaticum Saussure, Étud. fam. Vespid. I. Eumén. 1 5.52 1. 112 n. 17, $\subseteq$ j.

Rhynchium brunneum var. carnaticum Saussure. Vtud. fam. Vespid. III. Masar. 14.56 p. 17:.

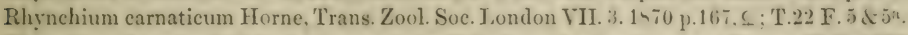

Ihynchium haemorrhoidale var. carnaticum Maindron. Ann. soc. cntom. France 6, II. 18,2

[p. 277.

var. javanum Sauss. $-\subseteq-$ As.: Jara.

[n. 21, \&.

Rhynchium Prorhynchium Javanum Saussure. Stettin. entom. Zeiter. XXIII. 1962 p. 1 4;

Rhỵnchium haemorrhoidale var.Jaranum Maindron, Ann.soc. entom. France 6,II.1442 p. 2801 . var. mellium Maindr. - $\subseteq$ - Austr.: New Guinea.

[p. 278, \&; T. 5 F. $1-5$.

Rhýnchium haemorrhoidale var. medimm Maindron, Ann. soc. entom. Franee 6 II. Int? var. parentissimum Sauss. - 우 $\widehat{\sigma}-$ As.: Java.

Rhy̆gehium parentissimum Saussure, Kitud. fam. Vespid. I. Eumén. 1452 p. 111 n. 14, $\{3$. Rhynehium haemorrhoidale var. parentissimum Saussure. Etud. fam. Vespid. III. Masar.

$[1856$ ]. 172.

Rhynchium haemorrhoidale var. parentissimum Maindron, Ann. soc. entom. France if II.

[1882 p. $80 ;$ T. 4 F. $8-9$.

Rhynchium haemorrhoidale var. parentissimum E. A. Fitch. Trans. Entom. Soe. I.ondon car. quinquecinctum (Fabr.) D. T. $-\subseteq \widehat{f}-$ As.: Bengal.

[1883 Proc. II.

Vespa quinquecineta Fabricius, Mant. Insect. I. 1787 p. 288 n. 24.

Vespa quinquecincta Villers, C. Linnaei Entom. III. 1789 p. 283 n. 42.

Vespa quinqueeincta Gmelin. Linné: Syst. nat. Ed. $13^{3}$ I. 5. 1790 p. 2752 n. 4 ti.

Vespa quinquecincta Olivier. Encycl. méthod. Insect. VI. 1791 p. tiS2 n. 63.

Vespa quinquecincta Fabricius, Entom. system. II. 1793 p. 261 n. 30.

Vespa brunnea Fabricius, Entom. system. II. 1793 p. 264 n. 41.

Vespa quinquecincta Fabricius, Syst. Piez. 1504 p. 258 n. 23.

Vespa brunnea Fabricius, Syst. Piez. $180 t$ p. 260 n. 33.

Odynerus brunneus Latreille, Gen. Crust. \& Insect. I. 1806; T. 14 F. 3.

Rlyynchium? brunneum Spinola, Insect. Ligur. II. 1808 p. 189, nota.

Eumenes brunnea Latreille, Gen. Crust. \& Inscet. IV. 1809 p. 136.

Rhygghium brunneum Saussure. Situd. fam. Tespid. I. Eumén. 14.;2 p. 112 n.16. \& 5; 'T.14

Rhynchium brunneum Saussure. litud. fam. Yespid. III. Masar. 1456 p. 172. F.4.

Rhỵnchium brunneum Iforne. Trans. Zool. Soc. London VII. 3. 1 sio p. 16 .

Rhynchium brumeum Smith. Trans. Fntom. Soc. Iondon 1571 Proc. p. V. [p. 277.

Rhynchium hacmorrhoidale var. brumeum Maindron. Ann. soe. entom. France (6) II. 1552

Rhỵgchium brunneum Gribodo. Ann. mus. civ. Genova XXI. 14-4 p. 357 n. 16.

var. rubropictum Smith $-\subseteq \tilde{\sigma}-$ As.: Amboina.

[f ơ.

Rhynehium rubropictum Smith. Journ. of Proc. I.inn. Soc. Zool. IV. I stio Suppl. p. 12s n 4.

Rhynchium hacmorrhoidale var. mobopictum Matindron. Ann. soe. entom. France 6 II. 1-?

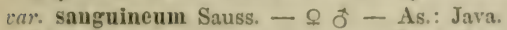

[р.277; 'T'.5 T.10-11.

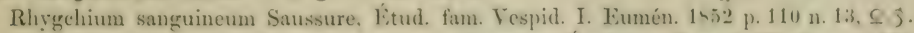

Rhỵnchium haemorrhoidale var, sanguineum Saussure, Ftud. fam. Vespid. III. Masar. Is.ti

[p. 172.

ear. smellenii Sauss. $-\subseteq-$ As.: Borneo.

[n. $20, \Omega$.

Rhynehium Prorhynchium Snelleni Saussure. Stettin. cntom. Zeitg. XXIII. 14ti2 p. 1sis

Rhrnchium haemorrhoidale var. Snelleni Maindron. Ann.soe.entom. France if II. 1452 p. 241 . var, rollenhovenii Sauss. - 오 ô - As: Java.

[p. 184 n. 19, 웅.

Rhrnchium Prorhynchium Vollenhwreni Saussure. Stettin. entom. \%eitg. XXIII. 1662

Rhỵnchium hatemorrhoidale var. Vollenhoveni Maindron. Ann, soce entom. France (6) II. 144:

[p. 280 . 
histrionicum Gerst. - $\&$ - Afr.: Mosambique.

Rhynchimm histrionieum Gerstaecker, Monatsber. Akad. Wiss. Berlin 1557 p. 463 n. 6, S. Rhỵnchium histrionicum Gerstaecker, Peters: Reise n. Mossambique. Zool. V. 1562 p.465, \&;

holosericeum Rad. - 우 - Afr.: Angola.

['T. 30 F. 4 .

Rhynchium holosericeum Radoszkowski, Journ. acad. sc. math. etc. Lisboa VIII. 1551

japonicum D. T. - \& - A8.: Japonia.

[p. 206 n. 62, ㅇ.

Rhynchium ardens Smith, Trans. Entom. Soc. London $15-3$ p. 196 n. 1, $\Omega$ nee Guér., nee Rhynchium Japonicum $m$.

[Walk.].

iridipenue Smith - - - As.: Amboina.

Rhynchium iridipenne Smith, Journ. of Proc. Linn. Soc. Zool. IV. 1860 Suppl. p. 125 n. 3, s.

kiinekelii Maindr. - $t-$ Austr.: New Guinea.

Rhỵnchium Künckeli Maindron, Ann. soc. entom. France (6) II. 1552 p. 279. วิ; T. 5 F. 5-6.

Iaminatum Grib. - ô-As.: Celebes.

Rhygghium laminatum Gribodo, Bull. soc. entom. Ital. XXIII. 1591 p. 2५s, $\overrightarrow{0}$.

laterale (Fabr.) Sauss. - 우 $0^{\star}-$ Afr.

Vespa lateralis Fabricius, Spec. Insect. I. 1781 p. 466 n. 49.

Vespa lateralis Fabricius, Mant. Insect. I. 1787 p. 292 n. 61.

Vespa lateralis Gmelin, Linné: Syst. nat. Ed. 13 a I. 5.1790 p. 2756 n. 74.

Vespa lateralis Olivier, Encycl. méthod. Inseet. VI. 1791 p. 691 n. 105.

Vespa marginella Fabricius, Entom. system. II. 1793 p. 263 n. 34.

Vespa lateralis Fabricius, Entom. system. II. 1793 p. 275 n. 7 i.

Vespa Africana Fabricius, Syst. Piez. 1804 p. 257 n. 19.

Vespa marginella Fabricius, Syst. Piez. 1804 p. 259 n. 26.

Polistes lateralis Fabricius, Syst. Piez. 1804 p. 273 n. 22.

Rhygchium Africanum Saussure, Śtud. fam.Vespid. I. Eumén. 1852 p. 1115 n. 10, \&; T.14 F.3. Rhỵnchium laterale Saussure, Kitud. fam. Vespid. III. Masar. 1856 p. 171.

Rhỵnchium laterale Magretti, Ann. mus. cix. Genora XXI. 1556 p. 614 n. $152, \hat{\jmath}$.

limbatum Sauss. - $Q$ - Afr.: Madagascar.

Rhyggehium limbatum Saussure, litud. fam. Vespid. I. Eumén. 155:2 p.11 i n.27, \&; T.13 F.10.

Inctuosum Gerst. - f oే - Afr.: Mosambique.

Rhỵnchium luctuosum Gerstaecker, Monatsber. Akad. Wiss. Berlin 1557 p. 463 n. $9, \mathcal{f} \hat{j}$. Rhỵnchium luctuosum Gerstaecker, Peters: Reise n. Mossambique. Zool. V. $1 \$ 62$ p. 46i, madecassae Sauss. - + - Afr.: Madagascar.

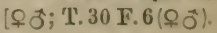

Rhygrehium Madecasse Saussure, lítud. fam. Vespid. I. Eumén. 1552 p. 111 n. 15, S.

Rhỵnchium Madecassum Saussure, Grandidier: Hist. Madagasear XX. P.1. 1591 p. 160

magnifienm Smith $-\rho-$ Austr.

[n. 3, \&; T. 4 F.11.

Rhynchium magnificum Smith, Trans. Entom. Soc. London 1569 p. 310. \&.

Rhỵnchium magnificum Smith, Brenekler: Cruise of the Curaçoa 1573 p. ?; T. 45 F. 4 .

mandarineum Sauss. $-\rho-$ As.: China.

Rhỵnchium mandarineum Saussure, Ĺtud. fam. Vespid. III. Masar. 15.56 p. 150 n. $73, \varsigma$.

mellyi Sauss. - $\rho$ ô - As.: India, China.

Rhygrchium Mellyi Saussure, litud. fam. Yespid. I. Jumén. 1552 p. 116 n. 24, ๔.

Rhỵnchium Mellỵi Saussure, Fitud. fam. Vespid. III. Masar. 15.16 p. 172, $\hat{\jmath}$.

mephisto Grib. - $f-\Delta f r$ : Sierra Leone.

Rhygehium Mephisto Gribodo, Bull. soc. entom. Ital. XXIII. 1491 p. 253. S.

mirabile Sauss. - \& of-As.: India \& insulac; Austr.: Tasmania.

Rhygchium mirabile Saussure. Ktud. fam. Vespid. I. Kumén. 1452 p. 106 n. 6, f; T. 14 F. 5.

?ayyuchium mirabile Smith, Journ. of P'roc. Limm. Soc. Zool. III. 1555 p. 163 n. 1, 3 . 
multispinosum Sauss. - $\subsetneq-$ Afr.: Port Natal.

Rhynchium multispinosum Saussure, Étud. fam. Vespia. III. Masar. 1556 p. 177 n. $67, \mathrm{~S}$.

niloticnm Sauss. - - Afr.: Abyssinia.

Rhynchium Niloticum Saussure, Étud. fam. Vespid. III. Masar. 1 st t p. 141 n.74, S; T.16 F.S.

nitidulum (Fabr.) Sauss. - 우 ot - As.: India or.

Vespa nitidula Fabricius, Suppl. entom. system. 1799 p. 262 n. 44-45.

Vespa nitidula Fabricius, Syst. Piez. 1804 p. 260 n. 35.

Rhygehium nitidulum Saussure, Étud. fam. Vespid. I. Eumén. 18.52 p. 110.j n.5, f j; T. 14

Rhynehium nitidulum Horne, Trans. Zool. Soc. London VII. 3. 1870 p. 16S, \&; T.20 F. 1\& 13.

? Rhynchium nitidulum Maindron, Ann. soc. entom. Franec (6) II. 1852 p. 276.

nyassae Kirsch - ㅇ - Afr.: Nyassa.

Rhỵnchium Nyassae Kirsch, Mittheil. zool. Mus. Dresden III. 187S p. 37S, \&.

obseurum Smith - 우 - As.: Borneo.

Rhỵnchium obscurum Smith, Journ. of Proc. Linn. Soc. Zool. II. 1857 p. 110 n. 5. ᄃ. oculatum (Fabr.) Lep. - 우 0 - Eur. mer.; Afr.: Aegyptus.

Vespa oculata Fabricius, Spec. Insect. I. 1781 p. 463 n. 30.

Scolia mutabilis Cyrillo, Entom. Neap. specim. 1787 p. 3 ; T. 2 F. 8.

Vespa oculata Fabricius, Mant. Insect. I. 1787 p. 289 n. 35.

Vespa oculata Villers, C. Linnaei Entom. III. 1789 p. 275 n. 21.

Vespa oculata Gmelin, Linné: Syst. nat. Ed. $13^{3}$ I. 5. 1790 p. 2760 n. 90.

Vespa oculata Rossi, Fauna Etrusea II. 1790 p. 85 n. 864.

Vespa oculata Olivier, Encycl. méthod. Insect. VI. 1791 p. 685 n. 80.

Vespa oculata Fabricius, Entom. system. II. 1793 p. 264 n. 43.

Vespa oculata Fabricius, Syst. Piez. 1804 p. 260 n. 34.

Rhynchium Europaeum Spinola, Insect. Ligur. I. 1806 p. 86 n. 1, 우 đే.

Vespa oculata Illiger, Rossi: Fauna Etrusea Ed. 2 a II. 1507 p. 140 n. $\$ 64$.

Vespa oculata Jurine, Nouv. méth. class. Hymén. 1807 p. 170, 우 đొ.

Rhỵnehium Europaeum Latreille, Gen. Crust. \& Insect. IV. 1809 p. 139.

- Savigny, Descr. de l'Égypte. Hymen. 1812; T. 9 F. 10.

Eumenes oculata Klug, Germar: Reise n. Dalmat. II. 1817 p. 264 n. 366

Rhygchium oculatum Lepeletier, Encycl. méthod. Insect. X. 1525 p. 317.

Rhynchium oculatum Germar, Fauna Insect. Europe P. 13. 1527; Tab. 22.

Rhỵchium oculatum Lepeletier. Hist. nat. Insect. Hymén. II. 15.11 p. 650 n. $2 . \subseteq 5$. Rhỵgchium oculatum Saussure, 1.tud. fam. Vespid. I. Eumén. 1452 p. $1117 \mathrm{n} . \checkmark, \subseteq 5$. Rhỵnchium oculatum Jichtenstein, Ann. soc. entom. Franec 1 IX. 1569 p. I.XXIII. Rhynchium oculatum Jueas, Ann. soe. entom. France (5 IX. 14-9 Bull. p. CXI.VI. [F.t. Rhỵnehium oenlatum Fid. André. Spec. Hymén. Europe II. P. $21-23.155 .4$ p. 617 , e s. ; T. 42 var. lefebrrei Lep. - $\subseteq-$ Eur.: Sicilia.

Rhynchium 1.efebrrei Lepeletier, Hist. nat. Inseet. Hỵmén. II. 15.11 p. 679 n. 1, S.

Rhỵnchium oculatum var. Lefebrei Saussure, Etud. fam. Vespid. I. Eumén. 1452 p. 105. ornatum Smith - $\odot-$ As.: China.

Rhynchium ornatum Smith. Trans. Entom. Soe. Iondon 2 II. 2. 15.32 p. 36, ธ; T. 4 F. 10. Rhỵnchium (Pararhynchium ornatum Saussure, Etud. fam. Vespid. III. Masar. 15.56 p. 173

[n. 61, 年.

Rhynchium Parrhynchium ornatum Siussure, Stettin. entom. Zeitg. XXIII. 1862 p. 142 pulehellum Gerst. - ô - Afr.: Mosambique.

[n. 17 .

Rhynchium pulchellum Gerstaecker. Monatsber. Akad. Wiss. Berlin 14.37 1). 46.3 n. 5, 3.

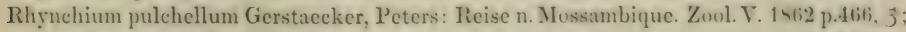

radiale Sauss. - Afr.: Port Natal.

[T. 30 F. 5 .

Rhynchium radiale Saussure. Étud. fam. Vespid. III. Masar. 1456; p. 17! n. 71. 
rothii Kby. $-\subseteq \hat{o}-$ Austr.

Rhynehium Rothi F. W. Kirb!. Journ. Linn. Suc. London Znol. XYIII. 1117. 154.5 p. 324. rubens Sauss. $-\subseteq \hat{o}-$ Afr.: Cap.

Rhynchium rubens Saussure. Jitud. fam. Tespid. III. Masar. 18.66 p. 141 n. $75 . \subseteq j$.

Rhynchium rubens Gerstaecker, Peters: Reise n. Mossambique. Zuol. V. 1462 p. $46 t$. . .

rufipes (Fabr.) Sauss. - $f \widehat{o}-$ Austr.: New Guinea, Otahaiti.

Vespa rufipes Fabricius, Syst. entom. 1775 p. 367 n. 23.

Vespa rufipes Fabricius, Spec. Insect. I. 1781 p. 463 n. 28.

Vespa rufipes Fabricius, Mant. Insect. I. 1787 p. 289 n. 33.

Vespa rufipes Gmelin, Linné: Syst. nat. Ed. $13^{\text {a }}$ I. 5. 1790 p. 2759 n. 88.

Vespa rufipes Christ, Naturg. d. Insect. 1791 p. 240.

Vespa rufipes Olivier, Encycl. méthod. Insect. VI. 1791 p. 685 n. 78.

Vespa rufipes Fabricius, Entom. system. II. 1793 p. 264 n. 40.

Vespa rufipes Fabricius, Syst. Piez. 1804 p. 259 n. 32.

Odynerus rufipes Guérin, Duperry: Voy. Coquille. Zool. II. 2. 1830 p. 265.

Rhygehium rufipes Saussure, Etud. fam. Vespid. I. Fumén. 1452 p. 115 n. 23 . ᄃ.

Rhynchium rufipes Saussure, Reise d. Novara. Zool. II. 1. 1467 Hymen. p. 5 n. 3. $\subseteq$ j.

Rhỵnchium rufipes Maindron, Ann. soc. entom. France 6 II. 1552 p. 241 n. $8 . \subseteq$.

rufiventre Rad. - ㅇ - Afr.: Angola.

[n. 63, ㅇ.

Rhynchium rutiventre Radoszkowski: Jorn. acad. se. math. ete. Jisboa VIII. 1 \&s 1 p. 201 i

sabniosum Sauss. - Afr.: Senegal.

Rhynchium sabulosum Saussure. Ftud. fam. Vespid. III. Masar. $155($ p. 17! n. in.

seulii Rad. - ô-As.: Korea.

Rhynchium Seuli Radoszkowski. Horae soc. cntom. Ross. XXIV. 15910 p. 231. j.

sichelii Sauss. - Afr. trop.

Rhynchium Sichelii Saussure, Citud. fam. Vespid. III. Masar. 1456 p. $176 \mathrm{j}$ n. 6.5.

smithii Sauss. - $ぇ-$ As.: China.

[n. $62, \hat{o}$.

Rhynchium (Prorhynchium Smithii Saussure, litud. fam. Vespid. III. Masar. 14ifi p. 174

superbum Sauss. - $f-$ Austr.

Rhynehium superbum Saussure. litud. fam. Tespid. I. Fumén. 15:5? p. $11: 3$ n. 1 s. ᄃ.

Rhynchium superbum Smith. Journ. of Proc. Linn. Soc. Zool. III. 1S.in p. 16.3 n. 2.

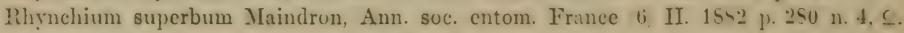

synagroides Sauss. - $\subsetneq$ o - Afr. trop.

[F. 2.

Rhynchium synagroides Saussure. Etud. fam. Tespid. I. Eumén. 1452 p. 103 n. 1. 3; T. 14

Rhỵnchium Anterhỵchium synagroides Saussure. Mém. soe. phỵs. o hist. nat. Geneve

[XVII. 1. 1563 p. 207 n. 35, \&.

Rhynchimm synagroides Saussure. Grandidier: Hist. Madagasear XX. P. 1. 1591 p. 174

tahitense Sauss. - $\subseteq$ oै - Austr.: Tahiti.

[n. 2, \& శึ; 'T. 4 F. 13 \& 14.

Rhynchium Tahitense Saussure. Reise d. Novara. Zoul. II. 1. 1 Gti Wespen p. 7 n. 1. S j;

xanthurum Sauss. $-\rho \neq \tilde{f}-\mathrm{Afr}$ : Cap.

['. 1 F. 4 .

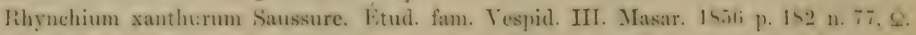
Rhỵchium xantiurum Saussure, Mém. sue phỵs. o hist. nat. Geneve XVII. 1. 186i3 p. 209 [n. $37, \Omega 0$.

Hhyndhum xanthurum Saussure. Grandidier: Hist. Madagasear XX. 1'.1. $15 ! 91$ p. 173 n. 1.

zonale Sauss. $-\subseteq \widehat{f}-$ Austr.: New Guinen.

[옹 'T. 4 F.12.

Rhygchium zonale Saussure, Citud. fam. Vespiel. I. liumén. 14i2 p. $11: 3$ n. 19. \& 3 .

lihynchimm zonale Maindron. Anu. soe. entom. France ti II. 1-52 p. 281 n. 5. j.

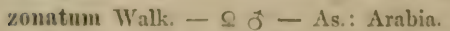

Rhynehium zonatum Walker, List of Hymen. in Egypt 1571 p. 31 n. 163, $\subseteq ~ \widehat{~}$. 


\section{ODYNERUS}

Latreille, Hist. nat. Crust. \& Insect. III. 1802 p. 362.

Ed. André, Spec. Hymén. Europe II. P. 21-23. 1884 p. 649.

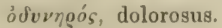

Synon.: Discoelius Brullé.

Epipona Blyon.

Eumenes Latreille \& auct. plur.

Monobia Saussure.

Polistes Fabricius, Brullé.

Pterochilus Herrich-Schaeffer, Lepeletier, Lucas, Schenck.

Rhynchium Spinola, Say, Holmgren (Rhygchium).

Vespa Linné \& auct. ant.

Sulg.: Ancistroceroides Saussure, Étud. fam. Vespid. III. Masar. 18.j4 p. 221.

Ancistrocerus, nom. propr. Hymen; eisos, forma.

Ancistrocerus Wesmael, Bull. acad. sc. Belgique III. 1836 p. 45.

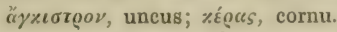

Antepipona Saussure, Étud. fam. Vespid. III. Masar. 1854 p. 244.

¿e $\nu$ ti, contra; Epiponus, nom. propr. Hymen.

Antodyncrus Saussure, Étud. fam. Vespid. III. Masar. 1854 p. 287.

¿ $(\nu \tau i$, contra; Odynerus, nom. propr. Hymen.

Epiponus Saussure, Smithson. Misecll. Collect. XIV. P. 254. 1875 p. 360.

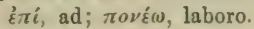

Epsilon Saussure, Etud. fam. Vespid. III. Masar. 1854 p. 229 \& 252.

$\varepsilon$, littera.

Hoplomerus Westwood, Introd. mod. Classif. Insect. II. 1810 Synops. p. $\$ 4$ [Oplo-

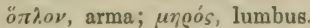

[merus].

Hoplopus Wesmael, Bull. acad. se. Belgique III. 1536 p. 45 [Oplopus].

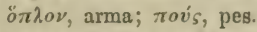

Hy paneistrocerus Saussure, Etud. fam. Vespid. III. Masar. $155 . t$ p. 222. ító, sub; Ancistrocerus, nom. propr. Hymen.

Hypodynerus Saussure, Étud. fam. Vespid. III. Masar. 1854 p. 225. vंтó, sub; Odynerus, nom. propr. Hymen.

Leionotus Saussure, Étud. fam. Vespid. I. Eumén. 1852 p. 151. $\lambda \varepsilon$ ĩos, levis; vั̃นos, notum.

Leptochilus Saussure, Etud. fam. Vespid. I. Eumén. 1552 p. 233; T. 6 F. 3.

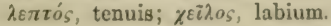

Microdynerus Thomson, Hymen. Scandin. III. 1874 p. 58. uex@ós, parvus; Odynerus, nom. propr. Hymen.

Pachodynerus Saussure, Smithson. Miscell.Collect. XIV. P. 254. 157.5 p.213 d.2.24. rayv́s, crassus; Odynerus, nom. propr. Hymen.

Parodynerus Saussure, Étud. fam. Vespid. III. Masar. 1854 p. 245. tucé, ad; Odynerus, nom. propr. Hymen.

Protodynerus Saussure, Étud. fam. Vespid. III. Masar. 1854 p. 186.

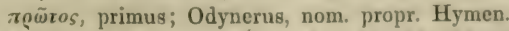

Pseudepipona Saussure, Ktud. fam. Yespid. III. Masar. 18.1 p. 309.

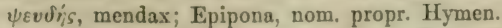

Pseudodynerus Saussure. Stettin. entom. Zeitg. XXIII. 18ti2 p. 193. $\psi \varepsilon v \delta r_{i}$, , mendax; Odynerus, nom. propr. Hymen. 
Suby. : St enancistrocerus Saussure, Mém. soc. phỵs. \& hist. nat. Genève XVII. 1. 1 S1i3

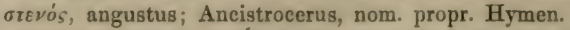

[p. 47.

Stenodynerus Saussure, Étud. fam. Vespid. I. Eumén. 1852 p. 206. бъєขós, angustus; Odynerus, nom. propr. Hymen.

$\mathrm{Subancistrocerus} \mathrm{Saussure.} \mathrm{Ktud.} \mathrm{fam.} \mathrm{Vespid.} \mathrm{III.} \mathrm{Massar.} 18.54$ p. 206.

Sub-Ancistrocerus, nom. propr. Hymen.

Symmorphus Wesmael, Bull. acad. sc. Belgique III. 1836 p. 45.

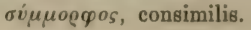

abdelkader Sauss. - 우 of - Eur.: Gallia, Sardinia; Afr.: Algeria.

? Odynerus bivittatus Lepeletier. Hist. nat. Insect. Hymén. II. 1841 p. 617.

Odynerus bivittatus Lucas, Explor. se. Algéric. Zool. III. 1546 p. 231 n.185, 오 đే; T. 11 F. 5 !

Odynerus (Aitodynerus; Abdelkader Saussure, Étud. fam. Vespid. III. Masar. 1554p. 294

[n. 182, 웅.

Odynerus (Microdynerus) Abdelkader Ed. André, Spec. Hymén. Europe II. P. 21-23. 1554

[p. $734 \mathrm{n} .5$, 유.

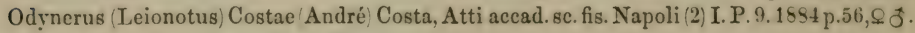
acolhuns Sauss. - ot- Am.: Mexico.

Odynerus Acolhuus Saussure, Rev. \& mag. zool. (2) IX. 1857 p. 280, ฮิ.

Odynerus (Stenodynerus) Acolhuus Saussure, Smithson. Miscell. Collect. XIV. P. 254.1875 acutus Menz.1 - Patria?

[p. 352 n. $149, \Im$.

Odynerus acutus Menzel, Mittheil. naturf. Ges. Zürich I. 2. 1848 p. 97-105.

adiabatus Sauss. - ô-Am.: Carolina.

Odynerus (Ancistrocerus) adiabatus Saussure, Ĺtud. fam. Vespid.I. Eumén.18.52 p.135 n.20, oే .

Odỵnerus 'Aneystrocerus) adialbatus Saussure, Smithson. Miscell. Collect. XIV.P.254.1875

[p. $171 \mathrm{n} .14,0$.

adrena Sauss. $-q-$ Am.: Brasilia.

[n. 117, 우; T. 11 F. $3 \& 4$.

Odynerus (Hypaneistrocerus) advena Saussure, Étud. fam. Vespid. III. Masar. 1556 p. 222

Odynerus (IIypancystrocerus) advena Saussure, Smithson: Miscell. Collect. XIV.P.254. 15.5

aegyptiacus Sauss. - 우-Afr.: Aegyptus.

[p. 211 n. 45 ,우.

- Savigny, Descr. de l'Égypte. Hymén. 1812; T. 18 F. 18.

Odynerus Hypancistrocerus) Aegyptiacus Saussure, Mém.soc.phys. \& hist.nat.Genève XVII.

$[1.1863$ p. 210 n. 3 S, 우; T. 2 F. 19.

Odỵnerus Aegyptiacus Ed. André, Spec. Hymén. Europe II. P. 21-23. 1554 p. 664 n. 2, S.

aequinoetialis Sauss. - 우 $-\mathrm{Afr}$.

[n. 190,우.

Odỵnerus (Antepipone) aequinoctialis Saussure, Étud. fam. Vespid. III. Masar. 1556 p. 360 aestimandus (Mor.) D. 'T. - 우 - As.

Hoplomerus aestimandus F. Morawitz, Horae soc. entom. Ross. XXII. 1 ss p. 264, 5.

Odynerus aestimandus $\mathrm{m}$.

aestunns Sauss. $-\rho$ o - Afr.: Abyssinia.

[T. 2 F. 25.

Odỵnerus aestuans Saussure, Mém. soc. phys. \& hist. nat.Genève XVII.1. 1863 p.222n.45, c. ja; aethiopieus Sauss. - $\vec{\sigma}-$ Afr.: Sierra Leona.

[n. 173, ठี; T. 13 F. 8.

Odynerus Antodynerus Aethiopicus Saussure, Citud. fam. Vespid. III. Masar. 1556 p. 25 i agilis Smith - ô- Ins. Aru.

Odynerus agilis Smith, Journ. of Proc. Linn. Soc. Zool. III. 1555 p. 164 n. 2, $\hat{u}$.

alariformis Sauss. $-q-$ Austr.

()dỵnerus aluriformis Saussure. Ftud. fam. Vespid. III. Masar. 1856 p. 242 n. 165 , \&; T. 14 F. 6.

alaris Sauss. - \& ô - Austr.: 'Tasmania.

T. 18 F. 5.

Odynerus I.cionotus alaris Saussure, litud. fam. Vespid. I. Fumén. 1852 p. 203 n. 106, §oే; alnstoripennis Sauss. - 으 - Austr.: 'Tasmania.

[n. 34, 우; T. 16 F. 5.

()dỵerus (Ancistroecrus) alastoripennis Saussure. Litud. fam. Tespid. I. Eumén. 1552 p. 147 
alastoroides Sauss. - of - Am.: Uruguay.

[n. 33, ơ; T. 16 F. 6.

Odynerus (Ancistrocerus) alastoroides Saussure, Eitud. fam. Vespid. I. Eumén. 1552 p. 147

Odynerus (Ancistroceroides, alastoroides Saussure. Smithson. Miscell. Collect. XIV. P. 254.

albifer Walk. - - - Afr. : Aegyptus.

[1575 p. 211 n. 46, ơ.

Odynerus albifer Walker, List of Hymen. in Egypt 1571 p. 36 n. 179, \&.

albocinctus Puls - O - Am.: Argentinia.

Odynerus albocinctus Puls, Atti soc. Ital. sc. nat. XI. 1868 p. 256, ․

Odynerus albocinctus Puls, Ann. \& Mag. Nat. Hist. (4) IV. 1 \&9 p. 29.5, c. [p.224n.58, c.

Odynerus (Hypodynerus! albocinctus Saussure, Smithson. Miscell. Collect. XIY.P.254.1575

albomarginatus Sauss. - 우 - Am. bor.

[n. 90, \&.

Odynerus (Symmorphus) albomarginatus Saussure, litud. fam. Vespid. III. Masar. $15_{56}$ p.195

Odỵnerus (Symmorphus) albomarginatus Saussure, Smithson. Miscell. C'ollect. XIV. P. 254.

alhophaleratus Sauss. $-\subsetneq \vec{s}-$ Am.: Canada, United States. 1875 p. 154 n. 3 , ㅇ․

[n. $109, \subseteq$ J.

Odynerus (Ancystrocerus) albophaleratus Saussurc, Ktud. fam. Vespid. III. Masar. 1456 p. 217

Odynerus (Ancystrocerus) albophaleratus Saussure, Smithson. Miscell. Collect. XIV. P.254.

$[1875$ p. 167 n. 12, Q ๙

Odynerus albophaleratus Packard, 10th Rep. U. St. Geol. \& Geogr. Surv. f. 15i6. 15is p.?

Odynerus albophaleratus Provancher, Natural. Canad. XIII. 1852 p. 140 n. $6, \Omega$ j.

Odynerus albophaleratus Provancher, Faun. entom. Canada. Hymén. 15533 p. 675 n. 6, s s

albopictus Sauss. - ơ - Eur. mer.

[T. 15 F. 5.

Odynerus (Oplopus) albopictus Saussure. Étud. fam. Vespid. III. Masar. 1456 p p.312n.196, ô;

Odỵnerus albopictus Ed. André, Spee. Hymén. Europe II. P. 21-23. $185+$ p. 767 n. 33, oే.

albotrinetus Zett. 1 - $‡$ - Eur.: Lapponia.

Odynerus albotrinctus Zetterstedt, Insect. Lappon. I. 1838 p. $45 \bar{i}$ n. 5.3 .

aldrichii Fox - 우 ô - Am. : Dakota, Colorado.

Odynerus Aldrichi Fox, Entom. News III. 1892 p. 197, 우 $\widehat{o}$.

alexandrinus Sauss. - 우 ô - Afr.: Aegyptus.

— Savigny, Descr. de l'Égypte. Hymén. 1812; T. 8 F. 10.

Odvnerus Oplopus Alexandrinus Saussure, Etud. fam. Vespid. I. Eumén. 1552 p. 225 n. 133.

Oplopus Alexandrinus Radoszkowski, Horae soc. entom. Ross. XII. 1 si6 p. 141 n. 11 ,. .

Odỹnerus Alexandrinus Fid. André, Sppec. Hỵmén. Europe II. P. 21 -23. 1554 p. 765 \& í2 n. 31.

alienus Walk. - ô-As.: Arabia.

[오

Odynerus alienus Walker, List of Hymen. in Egypt 1471 p. 39 n. 155, $\vec{\jmath}$.

allobrogus Sauss. $-\subseteq \vec{\jmath}-$ Eur.: Gallia. Germania, Suecia. [n. \&4. s; T. 10 F.4. Odynerus (Symmorphus) Allobrogus Saussure, Etud. fam. Vespid. III. Masar. 1556 p. 1911 Odynerus (Symmorphus Allobrogrus Schenek, Jahrb. Ver. Naturk. Nassau XVI. 1561 p. 36,

[39 \& 44 n. $9, £$ oี.

Odỵnerus (Protodỵnerus) Allobrogus Morawitz, Horae soc. entom. Ross. IV. 1 stii p.111, $\mathbf{f} \overrightarrow{\mathrm{J}}$.

Odynerus bifasciatus Thomson, Opuse. entom. P. 2. 1570 p. $89 \mathrm{n}$. is. \& s non Limé,

Odynerus bifasciatus Thomson, IIỵmen. Seandin. III. 147.4 p. 45 n. 4 . \& is non Linné?

Odynerus ISrmmorphus Allobrogus Rudow. Arch. Ver. Fr. Naturg. Mecklenburg XXX.

$(1876$ p. 221 n. $5, \Omega 0$.

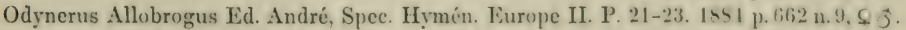

Odynerus Allobrogus Sehulthess, Hymen. Helvet. 1)iplopt. $155 \mathrm{i}$ p. $36 \mathrm{n}$. 3. S $\hat{\jmath}$.

alpestris Sauss. - 우 ô - Eur. centr.

[Fig. b (ô) [non Fabr.].

Odynerus minutus Herrich-Schaeffer, Faun. Insect. German. 1811 P. 176 T. 6 (S) d T. S

Odynerus Hypodỵnerus: alpestris Saussure. Kitud. fam. Vespid. III. Masar. 1856 p. 272

[n. 153, 우후; 'I. 13 F. 3 . 
Odynerus Teionotus alpestris Sehenck, Jalırb. Ver. Naturk. Nassau XVI. 1 \$ 61 p. 65, 67 \& 76 Microdynerus alpestris Thomson, Hymen. Scandin. III. $15 i 4$ p. 55 n. $1 . \& 5$. [n. $10.9 \delta$. Odynerus Leionotus alpestris Rudow, Arch. Ver. Fr. Naturg. Mecklenburg XXX. 1676 [p. 227 n. 5, 워.

Odỵnerus al pestris Ed. André, Spec. Hymén. Europe II. P. $21-23.145 .1$ p. 724 n. 15.5 5; T. 36 alvarado Sauss. - 우 $\hat{o}-$ Am.: Mexico.

Odynerus Alvarado Saussure, Rev. \& mag. zool. (2) IX. 1857 p. 276.

Odỵnerus Alvaradi Saussure. Smithson. Miscell. Collect. XIV. P. 254.1575 p. 265 n. $91 . \subseteq 3$. amatanensis Sauss. - 0 - As.: Persia.

[\&279 n. 105, ठै; T. 12 F. 9.

Odynerus Ancystrocerus, Amadanensis Saussure, Étud. fam. Vespid. III. Masar. 1556 p. 214 ambiguus Spin. - $ᄋ \hat{f}-$ Am.: Chili.

Odynerus ambiguus Spinola, Gay: Hist. fis. Chile. Zool. VI. 1851 p. 264 n. 9. 5. Odynerus Bustillosi Saussure, Gay: Hist. fis. Chile. Zool. VI. 1551 p. 567 n. 10, Jै. Odynerus :Ancistrocerus: ambiguus Saussure, Kitud. fam. Vespid. I. Eumén. 15.52 p. 141 [n. 23, 우 T T. 16 F. 4 .

Odỵnerus Ancistrocerus; Bustillos Saussure, Étud. fam. Vespid. I. Eumén. 1552 p. 141 n.25, Jै Odỵnerus Bustillosi Saussure, Étud. fam. Vespid. III. Masar. 1856 p. 205.

Odynerus 'Ancystrocerus) ambiguus Saussure, Smithson. Miscell. Collect. XIV.P.254.1875 [p. 162 n. 9, 워

andreanus Sauss. - 우 ô - Afr.: Madagascar.

[T. 19 F. 4 .

Odynerus Andreanus Saussure, Grandidier: Hist. Madagascar XX. P. 1. 1591 p.167 n.5, $\odot$ j; andrei Mocs. - + - Eur.: Hispania.

[n. 64, ㅇ․

Odynerus (Leionotus) Andrei Mocsáry, Magy. Akad. Termész. Értek. XIII. P.11. 1553 p. 50 Odỵnerus Andrei Ed. André, Spec. Hỵmén. Europe II. P. 21-23. 1894 p. 710 n. 8, \&.

angolensis Rad. - ㅇ - Afr.: Angola.

Odynerus Angolensis Radoszkowski, Jorn. acad. sc.Math. etc. Lisboa VIII. 1 s\$1 p. 2114 n.54.S. angulatus Sauss. - $\sigma-$ Austr.

Odynerus angulatus Saussure, Etud. fam. Vespid. III. Masar. 1656 p. 294 n. 168, ơ; T. 14 F.7.

angustus Sauss. - 0 - Afr.: Abyssinia.

[p. $228 \mathrm{n} .55$, đే. Odỵnerus Stenodynerus) angustus Saussure, Mém.soc.physs.\&-hist.nat.Genère XVII.1. 1 sti3

anuectens Sauss. - 0 - Am.: Florida.

Odynerus annectens Saussure, Rev. \& mag. zool. 22 XXII. 1471 p. 59 n. 14 ..

()dynerus annectens Saussure, Smithson. Miscell. Collect. XIV.P. 25 ł. 1575 p. 272 n. 93, \&.

annulatus Say - $f$ fึ - Am.: United States.

Odynerus annulatus Say, Keatings Narrat. Exped. II. 1824 App. p. 349 . $\subseteq 3$.

Rhynchium annulatum Say, Boston Journ. Nat. Hist. I. 4.1537 p. 344 n. 4.

Odỵnerus annulatus Saussure, F́tud. fam. Vespid. I. Eumén. 1552 p. 232.

Rhynchium unnulatum Saussure, Kitud. fam. Vespid. III. Masar. 1556 p. 1 s.

Odynerus annulatus Leconte, Writ. of Th. Say Entom. I. 1859 p. 235.

Rhynchium annulatum I.econte, Writ. of 'Th. Say Entom. II. 1859 p. 765 n. 4.

Odymerus annulatus ('resson, Trans. Amer. Entom. Soc. I. 1567 p. 380 n. 21.

Odỵuerus anmulatus Saussure. Smithson. Miscell. Collect. XIV. P. 25.4. 1575 p. 273 n.9.4, C J.

var. bairdii Sauss. $-\hat{\sigma}-\mathrm{Am}$. ' ''exas.

Odynerus Bairdi Saussure, Rev. \& mag. zool. (2) X. 1855 p. 169, §.

Odỵnerus aumulatus var. Bairdi Saussure, Smithson. Miscell.Colleet. XIV. P.254. 1875 p.273. anormis Say - $\&$ ô - Am. bor.

Eumenes anomis Say, Keatings Narrat. Exped. II. 1824 App. p. 346 n. $3, \subseteq[$ exc]. 3 . Odynerus oculatus Say, Boston Journ. Nat. Hist. I. 4. 1837 p. 385 n. 2, ơ.

Odynerus abnormis Say, Boston Journ. Nat. Hist. I. 4. 1837 p. 387 n. 5. 
Odỵnerus (Ancystrocerus, oculatus Say) Saussure. litud. fam. Vespid. I. Kumén. 1852 p. 219 Eumenes anormis Saussure, Étud. fam. Vespid. I. Lumén. 1552 p. 232.

Rhynchium anorme Saussure, Etud. fam. Vespid. III. Masar. 1856 p. $18 \mathrm{~d}$.

Eumenes anormis Saussure, Kitud. fam. Vespid. III. Masur. 1556 p. 340 n. 225.

Eumenes anormis Leconte, Writ. of Th. Say Entom. I. 1559 p. 234 n. $3, \subseteq$ excl. $\hat{3}$ : Odynerus oculatus Leconte, Writ. of Th. Say Entom. II. 1559 p. 766 n. 2, 33.

Odynerus abnormis Leconte, Writ. of Th. Say Entom. II. 1859 p. 767 n. 5.

Odynerus oculatus Cresson, Trans. Amer. Entom. Soc. I. 1867 p. 387 n. 23.

Odynerus anormis Cresson, 'Trans. Amer. Lntom. Soc. IV. 1872 p. 242.

Odynerus (Stenodynerus) anormis Saussure, Smithson. Miscell. Collect. XIV. P. 254. 1875)

[p. 318 n. 119, 우 ठ․

var. persecutor Sauss. - 우 $\widehat{0}$ - Am.: United States.

[우 ơ; T. $14 \mathrm{~F} .1$ (ㅇ).

Odynerus (Hypodynerus) persecutor Saussure, Étud. fam. Tespid.III.Masar.1856 p. 256 n.133,

Odỵnerus anormis var. persecutor Saussure, Smithson. Miscell. Collect. XIV. P. 254. 1875

antillarum Prov. - $ᄋ-$ Am.: Trinidad.

[p. 318 .

Odỵnerus Antillarum Provancher, Addit. faun. Canada. Hỵmén. 18 s. p. 420 n. 12. $\mathrm{f}$

antilope (Panz.) Wesm. - 우 ô - Eur. fere tota.

Vespa antilope Panzer, Faun. Insect. German. V. 1798 P. 53 T. 9.

Vespa antilope Panzer, Krit. Revis. II. 1806 p. 155.

Odynerus biglumis Spinola, Insect. Ligur. I. 1806 p. 89 n. 4 [nec auct.].

Vespa antilope Jurine, Nouv. méth. class. Hymén. 1807 p. 170, ㅇ.

Odynerus biglumis Spinola, Insect. Ligur. II. 1808 p. 186 n. 4, \& ô.

Odynerus antilope Wesmael, Monogr. Odynèr. Belgique 1433 p. 32 n. $5, \subseteq$ oै.

Odynerus antilope Westwood, Trans. Entom. Soc. London I. 2. 1835 p. $7 \$-\$ 0$.

Odynerus (Ancystrocerus antilope Tesmael. Bull. acad. se. Belgique III. 1536 p. 45.

Odỵnerus antilope Iepeletier, Hist. nat. Insect. Hymén. II. $14+1$ p. 649 n. 3.1. ऽ.

Odynerus antilope Smith, Trans. Fintom. Soc. Iondon IV. 1. 1545 Proc. p. CVIII.

Odynerus antilope Walker, Ann. \& Mag. Nat. Hist. XVII. 1sti p. (60-61.

Odynerus Ancistrocerus) antilope Saussure, litud. fam. Vespid. I. Eumén. 1852 p. 132 n. 13

Odynerus antilope Schenck, Jahrb. Ver. Naturk. Nassau IX. 18.53 p. 62 n. 21 , 5 3.

Odynerus antilope Saussure, litud. fam. Vespid. III. Masar. 1856 p. 203.

Odynerus Aneystrocerus) antilope Smith, Catal Brit. Fossor. Hymen. 1854 p. $21011.12 . \subseteq \widehat{o}$

Odynerus Ancystrocerus antilope Schenck. Jahrb. Yer. Naturk. Nassau XVI. 1 s(i1 p). As, si

[\& 62 n. 7, , 으.

Odynerus antilope Brischke, Schrift. phỵs. ökon. Ges. Königsberg II. 1562 p. $115 . \varsigma \hat{\jmath}$. Odynerus antilope 'laschenberg, Hymen. Deutschl. 1866 p. 248, \& oే.

Odynerus Ancystrocerus! antilope Morawitz. Horae soc. entom. Russ. IV. 1467 p. $115 \mathrm{n} .1$.

Odynerus antilope 'Thomson, Opuse. entom. P. 2. 1870 p. 88 n. 16, 오 of.

Ancistrocerus antilope Thomson, Hymen. Seandin. III. 1574 p. 73 n. $7 . \leq 5$.

Odynerus antilope Rudow, Arch. Ver. Fr. Naturg. Mecklenburg XXX. 1 s 76 p. 216 n. fi. $\subseteq$ j.

Odynerus Aneystrocerus, antilope E. Saunders, Trans. Entom. Soe. Iondon 154? p. 179n. 11.

Odỵnerus antilope Ed. André, Sppee. Hỵmén. Europe II. P. 21-23. 1854 p. 671 n.9. ธठૈ.

antueo Sauss. - of of $-\Delta \mathrm{m}$. : Chili.

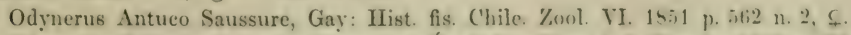

Odỵnerus Leionotus, Antueo Saussure. Kitud. fam. Vespiel. I. Eumén. 1452 p. 167 n. 55.

Odỵnerus Hypodỵnerus) Antucensis Saussure. Fitud. fam. Vespid. III. Masar. 14.5ti p. 228.

Odynerus IIypodynerus) Antucensis Saussure. Smithson. Miseell. C'ollect. XIV. P.2.14. 15 is

apicalis Cress. - $f$ f - Am. Cuba.

[p. 225 n. 61, Ф ठే.

Odynerus apicalis Cresson. Proc. Entom. Soc. Philadelphia IV. $16(i 5$ p. 161. \& J

Odỵnerus apicalis Saussure, Smithson. Miscell. Collect. X1Y. P. 254.1675 p. 279 n. 99. . S J. 
araueanus Sauss. - $0-$ Am.: Chili.

[n. 13, Q; T. 1 F. 8.

Odynerus (I.cionotus) Araucanus Saussure. Reise d. Novara. Zool. II. 1. 1567 Wespen p. 14 Odynerus Stenodynerus) Araucanus Saussure, Smithson. Miscell. Collect. XIV. P. 254. 1875

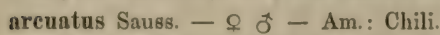

[p. $310 \mathrm{n} .113$, ..

Odynerus Jeionotus arcuatus Saussure, Ctud. fam. Vespid. I. Eumén. 1552 p. 160 n. $45, \rho \delta$. Odynerus Hypodynerus) arcuatus Saussure, Kitud. fam. Vespid. III. Masar. 1556 p. 226. Odỵerus (Hỵpodỵerus areuatus Saussure, Smithson. Miscell. Collect. XIV. P. 2:54. 1575 argentinus Sauss. - 0 oิ - Am.: Argentinia.

[p. 219 n. 48, 우 Odynerus (Protodynerus Argentinus Saussure, Rev. \& mag.zool. (2, XXII. 1871 p.56 n.6, o j. Odỵnerus Pachoḍ̣nerus/Argentinus Saussure, Smithson. Miscell.Collect. XIV.P.25 1. 1575

ip. 236 n. 69 , 우.

Odynerus Pachodynerus) Argentinus Berg, Stettin. entom.Zeitg. XIII. 1551 p. 70 n. $115, \subseteq \jmath$. Odynerus Argentinus Berg. Inform. Comic. Exped. Rio Negro. Zool. P. 1. 1851 p. 112 n. 11 . arista Sauss. - ot - Am. : Mexico.

Odynerus (Aneystrocerus) Arista Saussure, Rev. \& mag. zool. (2) IX. 1557 p. 274.

Odynerus (Ancystrocerus) Aristae Saussure, Smithson. Miscell. Collect. XIV. P. 25ł.1875

armatus Sauss. - 0 ơ - As.: Celebes.

[p. 189 n. 29, ठే.

Odynerus armatus Saussure. Stettin. entom. Zeitg. XXIII. 1562 p. 202 n. 35, $\subsetneq$ oै.

armeniacus Mor. - $ᄋ$ ô- As.: Turcestania.

Hoplomerus Armeniacus F. Morawitz, Horae soc. entom. Ross. XIX. 155.5 p. 157 n. 13. \& j.

Odynerus (Hoplomerus Armeniacus Ed. André, Spec. Hymén. Europe II. P. 24-26. 1856

aruanus Grib. - $ᄋ-$ As. : Sumatra.

[p. 886,807 .

() dynerus multipictus Smith, Journ. of Proc. Linn. Soc. Zool. III. 1558 p.165n.3, £ [non 1857 .

Odynerus Aruanus Gribodo, Bull. soc. entom. Ital. XXIII. 1891 p. 297 nota.

arvensis Sauss. - 우 ơ - Am.: Canada, United States.

Odỵnerus arrensis Saussure, Rer. \& mag. zool. (2) XXII. 1871 p. 59 n. $13, \subseteq \vec{\jmath}$.

Odỵnerus arvensis Saussure. Smithson. Miscell. Collect. XIV. P. 254.1575 p. 270 n. $92 . \subsetneq \sigma^{*}$;

'T. 3 F. $16 \& 16^{\mathrm{a}}$.

Odynerus arrensis Provancher. Natural. Canad. XIII. $1 \$ 82$ p. 142 n. $\$, \subseteq$ of.

Odynerus arvensis Provancher, Faun. entom. Canada. Hymén. 1853 p. 676 n. $8 . \varsigma$ o.

askhabadensis Rad. - $f$ ơ - As.: 'Transcaspia.

[p.45, 우우;'T.10 F.49.

Odynerus T.eionotus Askhabadensis Radoszkowski, Horae soc. entom. Ross. XX. $18 \varsigma 6$ aterrimus Sauss. $-\rho-$ As.: China.

Odynerus 'Ancistrocerus) aterrimus Saussure, Kitud. fam. Vespid. I. Eumén. 1552 p.125 n.7. S. atlanticns Kby. - 우 - Afr.: Cap verde, St. Vincent.

Odynerus Atlanticus W. F. Kirby, Ann. \& Mag. Nat. Hist. 5) XIII. 1 \&4. p. 4119 n. 31, ऽ. atrofasciatus (Mor.) D. T. - 오 ô - As.: China.

Lionotus atrofasciatus F. Morawitz. Horae soc. entom. Ross. XIX. 1845 p. 162 n. $15 . \subseteq \hat{\jmath}$. Odynerus atrofasciatus $\mathrm{m}$.

atropos Lep. - Q $\hat{o}-$ Afr.: Algeria.

()dynerus Atropos Jepeletier, Mist. nat. Insect. Iymén. II. Ist1 p. fitil n. 41. $f \hat{j}$ !

()dynerus Atropos Lueas. Explor. se. Algérie. Zool. III. 14t6 p. 2111 n. 194, S j.

Odynerus Aneistrocerus Atropos Saussure. Vitud. fam. Vespida. I. Fumén. 1452 p. 134 n. 15.

Gdynerus Atropos Jid. André, Spee. Hymén. Europe II. P. 21-23. 144 p. fi6s n. 3. 5 o .

augustus André - $ᄋ$ ơ - Eur.: Russia; As. : Caucasus.

[[nec Saussure].

Odyuerus Lcionotus angustus F. Morawitz. Horae soc. entom. Ross. IV. 1867 p. 122 n. 10.

Odynerus augustus Ed. André. Spee. Hỵmén. Europe II. P'.21-23. 1 s\$4 p. 693 n. 3, s j.

aurantiacus Mocs. $-\rho-$ Eur.: Hungaria.

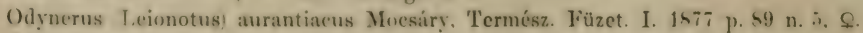

Oḍ̣nerus aurantiacus Jel. André. Sppee. IỴmén. Europe II. P. 21-23. 1844 p. 747 n. $17 . \Omega$. 
anreus Sauss. - $0-$ As.: Timor.

Odynerus aureus Saussure, Stettin. entom. Zeitg. XXIII. 1562 p. 197 n, 31.

austrinus Cress. - 우 ô - Am.: Texas.

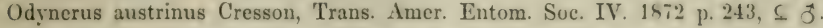

aztecus Sauss. - $\uparrow$ ô - Am.: Mexico.

Odynerus Aztecus Saussure, Rer. \& mag. zool. (2) IX. 1857 p. 275 . p.192n.30, 8 ơ.

Odỵnerus (Stenancystrocerus Aztecus Saussure, Smithson. Miscell.Collect. XIV.P. 254.1575

baeu Sauss. - 우 đَ-Am.: Cuba.

Odỹnerus Leionotus Bacu Saussure, Kitud. fam. Vespid. I. Eumén. 1552 p. 155 n. 40, ơ.

Odynerus Bacuensis Saussure, Litud. fam. Vespid. III. Masar. 1556 p. 232.

Odynerus Bacu Saussure, Sagra: Hist. fis. Cuba VII. 1556 p. 770, f); T. 19 F. 3.

Odynerus Bacuensis Cresson, Proc. Entom. Soc. Philadelphia IV. 16 ti5 p. 61.

Odynerus 'Stenodỵnerus) Bacuensis Saussure, Smithson. Miscell. Collect. XIV. P. 254. 1875

bairstowii Grib. - 우 - Afr.

[p.321 n.122, 우 Ô; T. 4 F. $28 \& 28^{a}$.

Odynerus Bairstowi Gribodo, Bull. soc. entom. Ital. XXIII. 1591 p. 292, s.

ballionii Mor. - $\subseteq$ $t$ - Eur.: Russia.

Odynerus Leionotus) Ballioni F. Morawitz, Horae soc. entom. Ross. IV. 1867 p. 129 n. 17 , \& 3

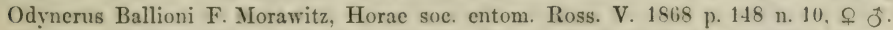

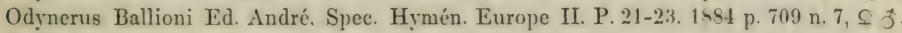

balyi Sauss. - 으-Austr.

Odynerus Balyi Saussure. Étud. fam. Vespid. III. Masar. 1656 p. 283 n. 167, §; T. 14 F. 6.

beckeri Mor. - $f \hat{\jmath}$ - Eur.: Russia.

Odỵnerus (Leionotus) Beckeri F. Morawitz, Horae soc. entom. Ross. IY. 1 S6 7 p p.1.27 n. 15. . $\hat{\jmath}$.

Odỵnerus Beckeri Ed. André, Spec. Hymén. Europe II. P. 21-23. 1544 p. 737 n. $3 . \subseteq .5$.

bellatulus Sauss. - o ô - Afr.: Senegal.

- Savigny, Deser. de l’Égypte. Hymén. 1812; T. 9 F. 14.

[T. 18 F. 10 .

Odynerus I Leionotus, bellatulus Saussure, Etud. fam. Yespid. I. Eumén. 15.52 p. 210 n.116. ô:

Odynerus bellatulus Saussure, litud. fam. Vespid. III. Masar. 1856 p. 243, \&.

bellulus Cress. - 으-Am.: Texas.

Odynerus bellulus Cresson. Trans. Amer. Entom. Soc. IV. 1872 p. 243, \&.

bicinctus (Fabr.) Lep. - $ᄋ$ ô - Afr.: Cap.

Vespa bicincta Fabricius, Spec. Inscet. I. 1781 p. 465 n. 39.

Vespa bicineta Fabricius, Mant. Insect. I. 1787 p. 291 n. 48.

Vespa bicincta Gmelin, Linné: Syst. nat. Ed. 13 a I. 5. 1790 p. 2757 n. 81 .

Vespa bicincta Olivier, Encycl. méthod. Insect. VI. 1791 p. 688 n. 94.

Vespa bicincta Fabricius, Entom. system. II. 1793 p. 271 n. 65.

.Vespa bicincta Fabricius, Syst. Piez. 1804 p. 265 n. 64.

Odynerus bizonatus Boisduval, Voy. Astrolabe. Zool. II. 6. 1533 p. fijs, ŝ; T. 12 F. 5.

Odỵnerus bicinetus Iepeletier, Hist. nat. Insect. IIymén. II. 1441 p. $644 \mathrm{n} .29$, ธ.

Odỵnerus Leionotus, bizonatus Saussure. Étud. fam. Vespid. I. Eumén. 1452 p. $156 \mathrm{n}, 40 . \varsigma j$.

Odỹnerus bicinctus Saussure, Étud. fam. Vespid. III. Masar. 1656 p. $224, \subseteq \hat{s}$.

Odynerus (Leionotus bizonatus Saussure, Reise d. Novara. Zool. II.1. 1467 Hymén. p. 9) n.4.

bicolor Sauss. - + ô - Austr.

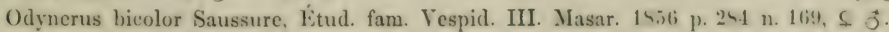

Odynerus bicolor H. I. Roth, Journ. Linn. Soe. London. Zool. XVIII. P. 1107. 156. p. 326.

bicoloratus Sauss. - - - Austr.

Odynerus bicoloratus Saussure. Etud. fam. Vespid. III. Masar. 1556 p. 241 n. 161. ๔.

bidens Sauss. - 0 ơ - Am.: Florida, T'ennessee.

Odynerus bidens Saussure, Rer. \& mag. zool. 2 XXII. 1571 p. $55 \mathrm{n.} 12$, s of.

Odynerus bidens Saussure. Smithson. Miscell. Collect. XIV. P. 25.4. 1875 p. 267 n. $90, \subseteq 3$;

['T. 3 F. $14 \& 14^{a}$. 
bidentatus Lep. - \& $\delta$ - Afr.: Algeria.

Odynerus bidentatus I,epeletier. Hist. nat. Inseet. Hymén. II. $14+1$ p. 123 n. $12 . \subseteq j$.

Odynerus bidentatus Iucas, Explor. se. Algéric. Zool. III. 1s tli p. 233 n. $184 . \varsigma$; T.11 F. 7. Odynerus (I,eionotus bidentatus Saussure. Litud. fam. Vespid. I. Eumén. 1852 p. $15 \varsigma$ n. $44, \subseteq \delta$. Odynerus bidentatus Saussure. Kitud. fam. Vespid. III. Ma ar. 1456 p. 233, \& 3 .

Odynerus bidentatus Ed. André. Spec. Ifymén. Europe II. P. $21-23.1554$ p. 702 n. $12, \subseteq 3$. bifaseiatus (L.) H.-Sch. -0 o - Eur. bor. \& centr.

Vespa bifasciata Linné, Fauna Suec. Ed. 2a 1761 p. 419 n. 1683.

Vespa bifasciata Linné, Syst. nat. Ed. $12^{\text {a }}$ I. 2.1767 p. 951 n. 14.

Vespa bifasciata Ph. L. Müller. Iinné: Vollst. Natursyst. V. 2. 1775 p. $4 \varsigma 3$ n. 14.

Vespa bifasciata Fabricius, Spec. Insect. I. 1781 p. 464 n. 38.

Vespa bifasciata Fabricius, Mant. Insect. I. 1787 p. 290 n. 45.

Vespa bifasciata Ström. Danske Vid. Selsk. Skrift. Nya Saml. III. 1785 p. 251 n. 61.

Vespa bifasciata Villers, C. Linnaci Entom. III. 1789 p. 270 \& 552 n. 11.

Vespa bifasciata Gmelin, Linné: Syst. nat. Ed. 13a I. 5.1790 p. 2757 n. 14.

Vespa bifasciata Christ, Naturg. d. Insect. 1791 p. 234.

Vespa bifasciata Olivier, Encycl. méthod. Insect. VI. 1791 p. 688 n. 91.

Vespa bifasciata Fabricius, Entom. system. II. 1793 p. 269 n. 60.

Vespa bifasciata Fabricius, Syst. Piez. 1804 p. 264 n. 59.

Vespa bifasciata Gravenhorst. Vergl. Übers. zool. System. $1 \varsigma_{07}$ p. 276 n. 3865.

Vespa bifasciata Jurine, Nouv. méth. class. Hymén. 1807 p. 170, ㅇ.

Odynerus bifasciatus Herrich-Schaeffer, Faun. Insect. German. 1535 P. 154 T. 16.

Odỵnerus bifasciatus Zetterstedt, Insect. Lappon. I. 1538 p. 457 n. $6, f \hat{\jmath}$.

Odynerus angustatus Zetterstedt, Insect. Lappon. I. 1836 p. 457 n. $7 . \subseteq$. n. $3 . \subsetneq 0^{*}$. Odynerus (Symmorphus; bifasciatus Saussure. Situd. fam. Vespid. I. Eumén. 185? p. 121 Symmorphus bifasciatus Schenck. Jahrb. Ver. Naturk. Nassau IX.1. 155.3 p. 74 n. 31.90 . Odynerus bifacciatus Saussure. Fitud. fam. Vespid. III. Masar. 1456 p. 193 n. $87, \subseteq 3$; T. 10 F. 5 . Odynerus Symmorphus' bifasciatus Schenck. Jahrb. Yer. Naturk. Nassau XVI. $1 \$ 61$ p. $3 \overline{7}$

$[38,39 \& 42$ n. 6, 요 0 .

Symmorphus bifasciatus 13rischke. Sehrift. phỵs. ökon. Ges. Königsberg II. 1562 p.116, $\subsetneq$ J (Odynerus I Protodynerus , bifasciatus F. Morawitz, Horae soc. entom. Ross. IV. $156-$ i p.112 n.2. Odynerus angustatus Thomson, Opusc. entom. P. 2. 1870 p. 89 n. 19, 오 oే.

Odynerus angustatus Thomson, Hymen. Scandin. III. 1874 p. 92 n. $6, \subseteq$ oै.

Odynerus (Symmorphus: bifasciatus Rudow, Arch. Ver. Fr. Naturg. Mecklenburg XXX.

[1876 p. 223 n. 8, 우

() dỵnerus bifasciatus Ed. André, Spec. Hymén. Lurope II. P. 21-23. 1854 p. 6.58 n. $5, \subseteq 0 \overrightarrow{0}$.

Odynerus bifasciatus Schulthess, Mymen. Helvet. 1)iplopt. 1547 p. 39 n. $6 . \Omega$ o.

Odỵerus bifasciatus Aurivillius, Öfvers. Srensk. Vet.-Akad. Förh. XLY.P. 10. 15ss p. 610.

bifllus (Mor.) André - 우 ôt As.: 'T'auria.

Microdynerus bifidus F. Morawitz. Horae soe. entom. Ross. XIX. 1955 p. 177 n. 25.5 . 5 . Odynerus Microdynerus bifidus Lid.André.Spec.Hymén.Furope II. P.24-26. 1486 p.474.S J. biphaleratus Sauss. - 으- Afr.: Aegyptus.

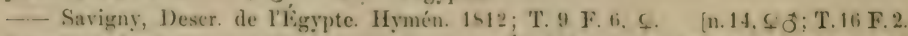
Odynerus Ancistrucerus biphaleratus Saussure, Eitud. fam. Vespid. I. Eumén. 1552 p. $1: 34$ ()dynerus biphaleratus Saussure. Etud. fam. Vespid. III. Masar. 1656 p. 204.

Odynerus biphaleratus Ed. André. Spee. $11 y m e ́ n$. Europe 11. P. $21-23.1594$ p. 67 i n. 14, bịpustulatus Sauss. -- $\& \tilde{f}-$ As.: China.

$?$ Vespa biguttata Fabricius \& auct. [s ô.

()

()dynerus bipustulatus Saussure, Etud. fam. Vespid. 111. Masar. 145ti p. 27i n. 159. $\$ 5$; Odynerus hipustulatus Saussure. Mém. soe. phỵs. d hist. nat. Geneve XVII. 1. 1 \$6 63 p. 226 
birenimacnlatus Sauss. - $§$ - Am: United States.

p. 135 n. 16, ㅇ․

Odỵerus Ancistrocerus; birenimaculatus Saussure. Kitud. fam. Vespid. I. Eumén. 1452 Odynerus (Aneystrocerus birenimaculatus Saussure, Smithson. Miscell. Collect. XIV.

bispinosus Lep. - 우 ठૈ - Afr.: Algeria.

[P. 254.1875 p. 175 n. 20 , \&.

Odynerus bispinosus Lepeletier. Hist. nat. Insect. IIymén. II. 1411 p. $622 \mathrm{n} .11 \subseteq 3$. Odynerus bispinosus Lucas, Explor. se. Algérie. Zool. III. 1446 p. 232 n. 157, S ô; T.11 F. 6. Odỵerus (Leionotus) bispinosus Saussure, Etud. fam. Vespid. I. Eumén. 1452 p. 206 n. 109 , Odỵnerus bispinosus Saussure, litud. fam. Vespid. III. Masar. 16.56 p. $241 . \quad$ Co

Odỵnerus bispinosus Ed. André. Spec. Hỵmén. Europe II. P. 21-23. 1 ş4 p. 714 n. 12.

bisstrigatus Spin.! - Eur.: Liguria.

Odynerus bisstrigatus Spinola, Insect. Ligur. II. 1808 p. 180 n. $5^{\mathrm{a}}$.

bisuturalis Sauss. - $\hat{\jmath}$ - Afr.: Senegal.

Odynerus (Ancistrocerus bisuturalis Saussure, litud. fam. Vespid. I. Fumén. 1952 p. 127

blackburnii Blackb. \& Kby. - 우 $\sigma^{\star}-$ Austr.: Kauai.

Odynerus rubritinctus Smith, Journ. Linn. Soc. Zool. XIV. 1879 p. 6i?), ş [excl. $\subsetneq$.

Odynerus rubritinctus Smith, Deser. New Spec. Hymen. 1879 p. 135 n. 3, oै [excl. ؟

Odynerus Blackburni Blackburn \& Kirby, Entom. M. Magaz. XVII. 1851 p. 57 11. 15.

Odrnerus Blackburni Blackburn \& Caméron, Mem. Manchester Lit. \& Philos. Soc. (3 X.

[1886 p. 215 n. 27, 워.

blanchardianus Sauss. $-\subseteq \hat{f}-$ Afr.: Algeria.

['T.12 F.8.

Odynerus Blanchardianus Saussure, Kitud. fam. Vespid. III. Masar. $1 \varsigma 56$ p. 276 n. $157, \subseteq \jmath$;

Odỵnerus Blanchardianus Ed. André, Spee. Hymén. Europe II. P. 21-23. 1 4 4 p p. 692 n. 13,

blandus Sauss. - Q - Am.: California.

[옹.

Odynerus Stenodynerus, blandus Saussure, Rer. \& mag. zool. (2) XXII. 1 s 11 p. 1145 n. $2 \bar{i}$. $\subseteq$.

Odynerus blandus Saussure, Smithson. Miscell. Collect. XIV. P. 254. 1575 p. 259 n. 104. S.

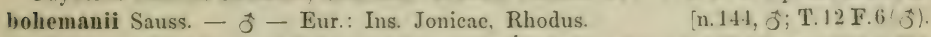

Odynerus Hypodynerus: Bohemani Saussure, Étud. fam. Vespid. IIl. Masar. 18.56 p. 264

Odynerus Bohemani Ed. André, Spec. Hymén. Europe II. P. 21-23. 1584 p. 659 n. 12, ô.

boscii I.ep. - 우 ô-Am.: United States.

Odynerus Boseii Lepeletier. Hist. nat. Insect. Hymén. II. $1 \$ 41$ p. 637 n. 22, ô

Odynerus Icionotus, Boscii Saussure, Ĺtud. fam. Vespid. I. Eumén. 1852 p. 177 n. 69, ô;

Odynerus Boseii Saussure, Kitud. fam. Vespid. III. Masar. 1856 p. 231, f. T. T7 F. 10.

Odynerus Boseii Saussure, Smithson. Miscell. C'ollect. XIV. P. 254.1575 p. 255 n. $4.3 .+5$.

var. castigatus Sauss. - 우 $\widehat{o}-$ Am.: Carolina.

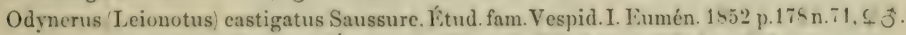

Odynerus castigatus Saussure. litud. fam. Vespid. III. Masar. 1556 p. 232.

Odynerus Boseii var. castigatus Saussure, Smithson. Miscell.Collect. XIV. P. 254. 1 \% 5 p. 254 .

bothriogaster Schlett. - ㅇ- Afr.: Congo.

Odynerus bothriogaster Schletterer. Ann. soc. entom. Belgique XXXY. 1491 p. 27, 5.

brachygaster Sauss. - 우 of - Am.: Brasilia.

[Q; T. 17 F.8.

Odynerus Leinotus brachygaster Saussure, Ktud. fam. Vespid. I. Jumén. 1452 p. 173 n.6.3.

Odỵnerus brachygaster Saussure. Sagra: Hist. fis. Cuba VII. 1956 p. 771; T. 19 F. 6.

Odynerus brachygaster Saussure, Rev. \& mag. zool. (2) X. 1858 p. 167 n. 5.

Odynerus Hypodynerus, brachygaster Saussure. Smithson. Miscell. Cullect. XIV. P. 25.

bravo Sauss. - $0-$ Am.: Mexico.

$[1875$ p. 238 n. 71, \&

Odynerus 'Ancistrocerus' Bravo Saussure. Rev. \& mag. zool. (2 IX. 145i p. 274.

Odymerus Stenancystrocerus Bravo Saussure, Smithson. Miscell. Collect. XIT. P. 251.

$[1875$ p. 205 n. 40, ?.

[Q [excl. tab.].

brevithorax Sauss. - 오 $\hat{o}-\mathrm{Am}$. mer.

1852 p. 172 n. 62 , Odynerus Teionotus; brevithorax Saussure. Etud. fam. Vespid. I. Tumen. 1852 p. 172 n.
Odynerus brevithorax Saussure. Etud. fam. Vespid. III. Masar. 18sit p. 231, f 3. 
Odynerus brevithorax Saussure. Rev. ( mag. zool. 2) X. 1555 p. 166 n.4. \{p. 231 n. $66, \varsigma$ ô. Odynerus Pachodynerus brevithorax Saussure, Smithson. Miscell.Collect. XIV P.254.1575 bulgaricus Moes. - o゙ - Eur.: Bulgaria.

Odynerus (Epipona Bulgaricus Mocsíry, Magy. Akad. Termész. Értek. XIII. P. 11. 15 \$3 Odỵnerus Bulgaricus Idd. André, Spec. Hymén. Europe II. P. 21-23. 1554 p. 755 n. 11, j̧. bustamente Sauss. - $q-$ Am.: Mexico.

Odynerus (Ancistrocerus) Bustamente Saussure. Rev. \& mag. zool. 2 IX. 1857 p. 273, \&. Odynerus (Anerstrocerus) Bustamenti Saussure, Smithson. Miseell. Collect. XIV P. 254. buyssonii Med. - 우 - Eur.: Hispania.

[1875 p. 172 n. 17, ㅇ․

Odynerus Buyssoni Medina, Anal. soc. Españ. hist. nat. XX. 1591 p. 106. S.

caelebs D. T. - $\widehat{o}-$ Eur. : Sicilia.

Odynerus similis Destefani. Natural. Sicil. VIII. $18 \varsigma 9$ p. 231. fే (nec Smith].

Odynerus caelebs $\mathrm{m}$.

ealabrensis D. T. - - ơ - Eur.: Italia.

Odỵnerus Calabricus Ed. André, Spee. Hymén. Europe II. P. $21-23.1554$ p. 750 \& 765 n. 4, Odynerus Calabrensis m.

calabricus Grib. - ô - Eur.: Italia.

[웅.

Odynerus (Leionotus) Calabricus Gribodo, Bull. soc. entom. Ital. XIII. $1 \leqslant 81$ p. 145 n. $106 . \jmath$. ealearatus (Mor.) André - 우 $\widehat{o}$ - As.: Transcaucasia.

Hoplomerus calcaratus F. Morawitz, Horae soc. entom. Ross. XIX. $1 \varsigma 55$ p. 153 n. $11, f \hat{\jmath}$. Odynerus Hoplomerus calcaratus Ed. André, Spec. Hṛmén. Europe II. P. 24-26. 1 \$86 ealedoniens Sauss. - $\widehat{o}$ - Austr.: New Caledonia.

[p. 884, 웋.

Odynerus Leionotus Caledonicus (Guérin) Saussure, Étud.fam.Vespid.I.1552 p. 205 n.10 ealiforniens Sauss. $-q-$ Am.: California.

Odynerus (Protodynerus) Californicus Saussure, Rev. \& mag. zool. (2) XXII. 1871 p.57 n.9. S. Odynerus (Pachodrnerus) Californicus Saussure, Smithson. Miscell. Collect. XIV. P. 254. [1875 p. 243 n. 79, 우; T. 3 F. $17 \& 17^{3}$.

eallosns Thoms. - 우 of - Eur.: Suecia, Germania, Gallia.

Odynerus callosus Thomson, Opusc. entom. P. 2. 1870 p. 87 n. 11 , 우 ఫิ.

Ancistrocerus callosus Thomson, Hymen. Seandin. III. 1874 p. 61 n. $1 . \subseteq \vec{j}$.

Odrnerus (Ancistrocerus) callosus E. Saunders, Trans. Entom. Soc. London $1 \leqslant 42$ p. 176 n. 6.

Odynerus callosus Fd. André, Spec. Hỵmén. Europe II. P. 21-23. 1854 p. 674 n. $17 . \varsigma$ oే.

Odynerus callosus Schulthess, Hymen. Helret. Diplopt. 1587 p. 44 n. $7, f \hat{\jmath}$.

eampestris Sauss. - 우 of - Am: Canada, United States.

[n. 18, ㅇ․

Odynerus (Ancistrocerus campestris Saussure, Etud. fam. Vespid. I. Eumén. 1852 p. 137

Odynerus campestris Saussure, litud. fam. Vespid. III. Masar. $1 \$ 560$ p. 204, $\widehat{0}$.

()dynerus Aneystrocerus campestris Saussure, Smithson. Miscell.Collect. XIV. P. 254.15i5

[p. 183 n. 25, 옹․

Odynerus eampestris Provancher, Natural. Canad. XIII. $1 \leqslant 52$ p. 141 n. $\vec{i} . f \vec{j}$.

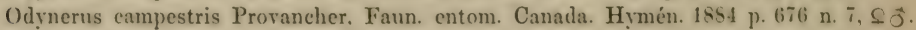
canadensis Sauss. - - oิ - Am.: Canada.

[n. 91, ㅇ.

Odynerus Symmorphus Canadensis Saussure, Etud. fam. Vespi\}. III. Masar. 1656 p. 196

Odynerus /Symmorphus, Canadensis S:uussure. Smithson. Miscell. Collect. XIV. P. 254. 1875 [p. 156 n. 7, ㅇ․

Odynerus Canadensis Provancher, Natural. Canad. XIII. 1542 p. 139 n. $2 . \Omega$ oิ.

Odynerus Canadensis Provancher. Faun. entom. Canada. Hymén. 1844 p. 673 n. 2.4 j. canaliculatus Sauss. - 으-As.: Arabia.

[n. 138, 우 T. 14 F. 4 .

Odynerus (Hypodynerus canalieulatus Saussure. Kitnd. fam. Vespid. III. Masar. 1656 p. 260 eapensis Sauss. - $\varrho^{3}-$ Afr: Cap.

Odỵnerus Anẹsstrocerus, Capensis Saussure, litud. fam. Yespid. III. Masar. 1 h5 6 p p. 214, $\subseteq$. 
capra Sauss $-\subseteq \breve{J}-A m:$ Canada. United States.

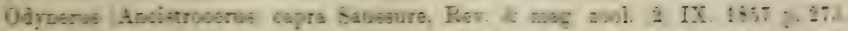

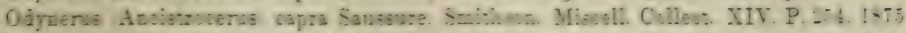

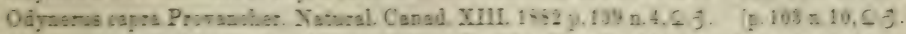

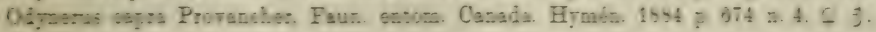

captirus Smith $-\subseteq-$ As: Japonia

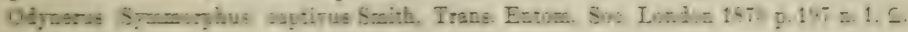
cardinalis Mor. - jo - As: Turcestania.

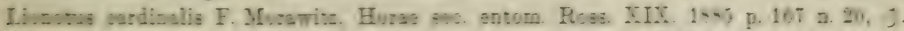

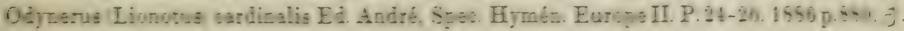

earinifer Kirsch - Am: Cavenne

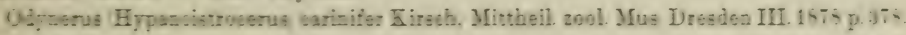
carinulatus Sauss. $-\subseteq-A f$. or.

[n. 137,$0 ;$ T. 14 F.3.

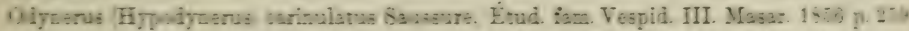

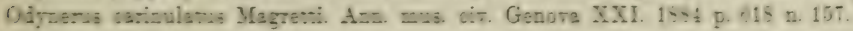

earoli Mor. - $\hat{o}$ - Afr.: Algeria

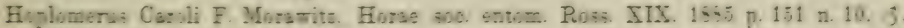

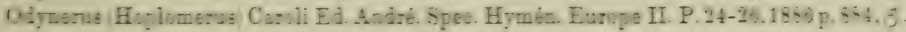
caspieus Mlor. $-\subseteq j-A s .:$ Reg. caspica.

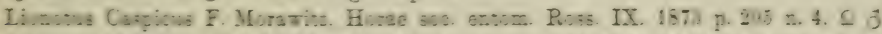

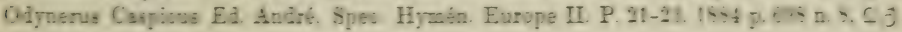

eatepetlensis Sauss. - $0 \overrightarrow{3}-\mathrm{Am}$ : Mexico.

[. $132,0 \hat{0}$

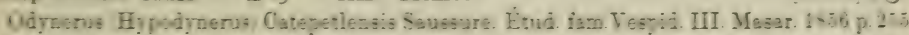

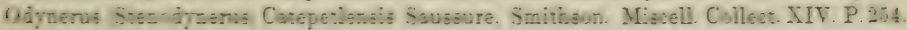

[1575 p. 339 n.136,, ơ.

eatskilii Sauss. $-\subseteq \vec{\jmath}-A m$ : United States.

[Q 0 [excl. tab.]

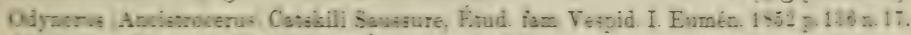

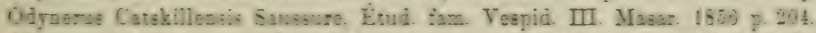

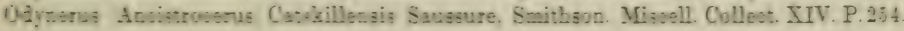

$[1575$ p. 165 n. 13, 오

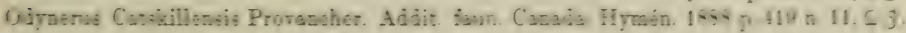

eavirentris hirsch $-\subseteq-$ Afr: Nrassa.

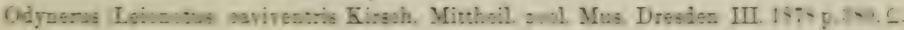

eeflonicus Sauss. - - oै - As: Ceylon.

n. 10,50 .

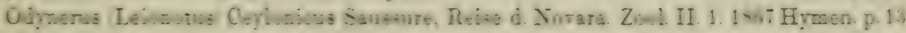
cherrieranus Bawas $-\subseteq \&-$ E.r. Gallia. Helvecis.

in 146.05 T.1:F.2.

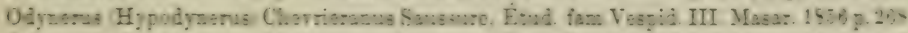

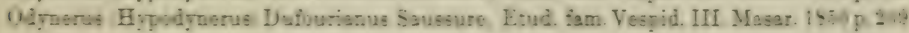

[a. 149, s.

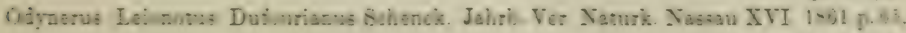

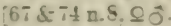

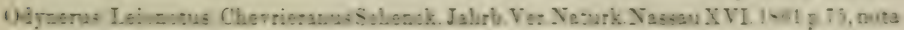

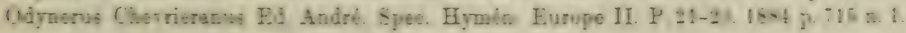

ehiehimecus Sauss. $-\subseteq-$ Am: Mexico.

Odynerus Chichimecus Saussure, Rer. \& mag. zool /2, LX. 1557 p. 250, S.

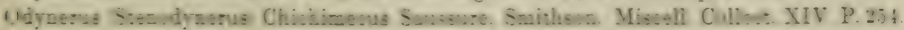

chiliotus Sense $-\subseteq \hat{\sigma}-$ Am: Chili.

$[1575$ p. 359 n. 153, ,

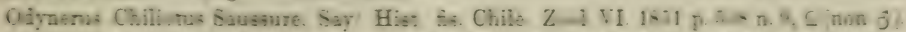

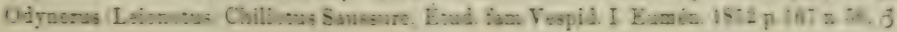


Odynerus Hypodynerus Chiliotus Saussure, Fitud. fam. Vespid. III. Masar. 1556 p. 229.

()dynerus Hypodynerus) (hiliotus Saussure, Smithsun. Miscell. Collect. XIV. P. 25ł. 1S75

chinensis Sauss. - $\subseteq-$ As.: China.

[p. $226 \mathrm{n} .62, .0 ี$.

Odynerus (Stenodynerus, (hinensis Saussure. Mém. soc. physs. \& hist. nat. Genère XVII. 1. chloroticus Spin. - 우 - Afr.: Aegyptus.

$[1863$ p. 230 n. 56, ․․

- Sarigny, Descr. de l'Egypte. Hymén. 1812 ; T. 10 F. 1.

Odynerus chloroticus Spinola. Ann. Soe. entom. France VII. 15.35 p. 500 n. XI.V. 5 .

Rhỵnchium chloroticus Spinola. Ann. soc. entom. France XI. 15.2 Bull. p. XXXYII n.12.

Odynerus (Leionotus, testaceus Saussure, Étud. fam. Tespid. I. Eumén. 1552 p. 195 n.94, . .

Odynerus chloroticus Saussure, Kitud. fam. Vespid. III. Masar. 15.56 p. 239.

Odynerus chloroticus L. r. Heyden, Berlin. entom. Zcitschr. XI. 1567 p. 395.

Odynerus chloroticus Magretti, Ann. mus. cir. Genova XXI. 1554 p. 616 n. 154.

Odynerus chloroticus Ed. André, Spec. Hymén. Europe II. P. 21-23. 1854 p. 657 n. 11". S. cingulatus Cress. $-\rho \widehat{o}-$ Am.: Cuba.

Odynerus cingulatus Cresson, Proe. Entom. Soc. Philadelphia IV. 1 s6i5 p. 16:. C.

Odynerus cingulatus Cresson. Trans. Amer. Entom. Soc. II. 1669 p. 2!4. ๖ృ.

Odỵnerus Aneystrocerus cingulatus Saussure. Smithson. Miscell. Collect. XIV. P. 251.

cingulifer Walk. - 우 - Afr.: ad Mare rubrum.

[1875 p. 179 n. 22, 옳.

Odynerus cingulifer Walker. List of Hymen. in Egypt 1871 p. 37 n. 181. Q.

circumspectus Smith - 우 - As.: Celebes.

[Suppl.p. 85 n. 3,8 .

(Odynerus Ancistrocerus! circumspectus Smith. Journ. of Proc. Linn. Soc. Zool. IV. 186 (it) eitrociuctus Sauss. - $\odot$ ơ - Austr.

[p. 10 n. 5, 옿 T. T. 1 F. 5.

Odynerus (Ieionotus, citrocinetus Saussure, Reise d. Norara. Zool. II. 1. 1567 Wespen claripemnis ('Thoms.) D. T. - 우 of - Eur.: Suecia.

Odynerus parictum var. Wesmael. Monogr. Odỵèr. Belgique 1833 p. 16 n. 4.

Odynerus parietum var. 7 Wesmael. Bull. acad. se. Belgique III. 1836 p. 16.

Ancistrocerus claripennis Thomson, Hymen. Seandin. III. 1471 p. 76 n. 4.55 .

Odynerus claripennis $\mathrm{m}$.

claxizianus Sauss. - 0 of - Am.: Brasilia, Argentinia.

[ڤठ․

()dynerus Ancistrocerus) Clarazianus Saussure, Rev. \& mag. zool. 2 XXII. 1571 p. 55 n. 3. Odynerus (Anevstrocerus' Clarazianus Saussure, Smithson. Miscell. Collect. XIV. P. 251.

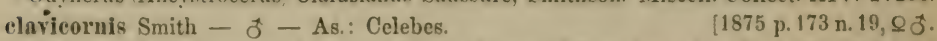
Odynerus (Ancistrocerus) clavicornis Smith, Journ. of Proe. Iinn. Soc. Zool. III. 1555 p. 21 cluniculus Sauss. - ఫ - Am.: Mexico.

Odynerus cluniculus Saussure, Rer. \& mag. zool. 2 XXII. $18 i 1$ p. 60 n. 18 , $\overrightarrow{0}$.

Odynerus cluniculus Saussure. Smithson. Miscell. Collect. XIV. P.254. 14.5 p.297 n.110. o. clusinus Cress. - $\subseteq$ 0 - Am.: Texas.

Odynerus Clusinus Cresson. Trans. Amer. Jintom. Soc. IV. 1572 p. $234 . \subseteq$ 3ै.

elypealis Thoms. - $\subseteq$ oิ - Eur.: Suecia.

Odynerus elypealis Thomson, Opuse. entom. P. 2. 1870 p. 85 n. 7, \& శే.

Leionotus elypealis Thomson, Hymen. Scandin. III. 1874 p. 53 n. 3 , \&

Odynerus clypealis Ed. André, Spec. Hymen. Europe II. P. $21-23$, I h4 p. 6 s5 n. 7.3 . elypentus Sauss. - $f$ of - Austr.: 'l'asmania.

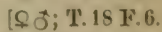

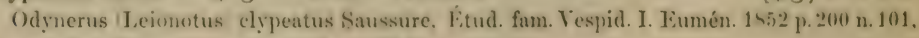
conrctatus Sauss. - \& $\sigma^{0}-$ Am.: Chile.

()dynerus coarctatus Saussure, Gay: Hist. fis. Chile. Zool. VI. 1 s.1 p. 565 n. $7, \leq 5$.

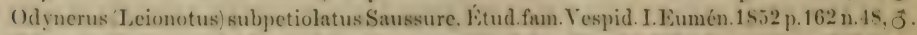

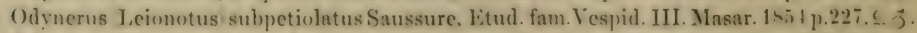
(Olỵerus Hypodỵerus subpetiolatus Saussure. Smithson. Miscell. Collect. XIV. P. 254.

$[1875$ p. 220 n. 50, \&. 
coguatus Walk. - o - As.: Arabia.

Odynerus cognatus Walker, List of Hymen. in Egypt 1571 p. 35 n. 1it6, ô.

collega Sauss. - ô-Am.: Connecticut.

Odynerus 'Stenodỵnerus! collega Saussure, Rev. \& mag. zool. i2 XXII. 1571 p. 61 n. 21, รૈ.

Odỵnerus (Stenodynerus) collega Saussure, Smithson. Miscell. Collect. XIV. P. 254. 1875

colon Cress. - 우 ox - Am.: Texas, Colorado.

[p.320 n.121, ठૈ.

Odynerus colon Cresson, Trans. Amer. Entom. Soc. IV. 1872 p. 241, 오 ఫే.

columbaris Sauss. - 우 - Am.: Columbia.

[우 T. 17 F. 3 .

Odynerus Leionotus; columbaris Saussure, Etud. fam. Vespid. I. Eumén. 1552 p. 15 \& n. 42.

Odynerus (Stenodynerus) columbaris Saussure, Smithson. Miscell. Collect. XIV. P. 254.

combustus Smith - ô-Afr.

$[1875$ p. 346 n. 142, ㅇ․

Odynerus combustus Smith, Catal. Hymen. Brit. Mus. V. 1857 p. 73 n. $151,3$.

concinuulus Walk. - ot - Afr.: ad Mare rubrum.

Odynerus concinnulus Walker, List of Hymen. in Egypt 1871 p. 39 n. 155, ô.

concolor Sauss. - 우 - Austr.: Tasmania.

[T.18 F. 7 .

Odynerus Leionotus, concolor Saussure, Étud. fam. Vespid. I. Eumén. 1852 p. 202 n. 104, 우

confluentus Smith - - As.: Sumatra.

Odỵnerus confluentus Smith, Catal. Hymen. Brit. Mus. V. 1857 p. 60 n. 62, $\subseteq$.

conformis Sauss. - $\&$ - Am. New York, Tennessee.

[n. 112, ㅇ..

Odynerus (Aneystrocerus) conformis Saussure, Étud. fam. Vespid. III. Masar. 1856 p. 219

Odynerus Stenodynerus; conformis Saussure, Smithson. Miscell. Collect. XIV. P. 254.1575

congener Mor. - 우 ot - Eur.: Graecia; As.: Syria.

[p. $315 \mathrm{n} .117$, 우.

Hoplomerus congener F. Morawitz, Horae soc. entom. Ross. XIX. 1555 p. 155 n. 12, $f \hat{\jmath}$.

Odỵnerus (Hoplomerus) congener Ed. André, Spec. Hymén. Europe II. P. 24-26. 1 \$ 4 p. 555 ,

congruas Smith - $\&$ ơ - Austr.: Honolulu.

[우오.

Odynerus congruus Smith, Descr. New Spec. Hymen. 1579 p. 136 n. $5, \subseteq \hat{o}$.

Odynerus congruus Smith, Journ. Jinn. Soc. Zool. XIV. 1579 p. $650, \neq \delta$.

Odynerus congruus Blackburn \& Kirby, Entom. M. Magaz. XVII. 1850 p. $\$ 7$.

consobriuus Duf. - 오 - Eur.: Gallia, Hispania; Afr.: Algeria.

Odynerus consobrinus Dufour, Ann. sc. nat. Zool. (2) XI. 1839 p. 91, ㅇ.

Odỵnerus notula Lepeletier, Hist. nat. Insect. Hỵmén. II. 1 s 41 p. 612 n. $4, \subseteq\{$ excl. $\vec{s}$ ?

Odỵnerus variolosus Lepeletier, Hist. nat. Insect. Hymén. II. 1841 p. 613 n. $5, \subseteq \hat{\jmath}$.

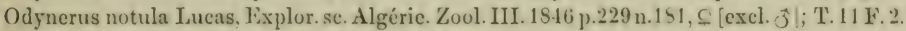

Odynerus rariolosus Lucas, Explor. sc. Algérie. Zool. III. 1846 p. 230 n. 152.

Odynerus (Oplopus) variolosus Saussure, Etud. fam. Vespid. I. Eumén. 1552 p. 220 n.127.

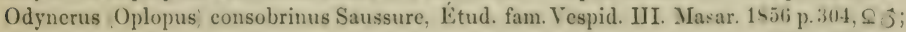

T. 15 F.8 (Q).

Odynerus consobrinus Ed. André, Spee. Hymén. Europe II. P. $21-23.1554$ p. 7.50 i j \$.774

consors Cress. - ô-Am.: Cuba.

[(ㅇ) n. 3 .

Odynerus consors Cresson, Proc. Entom. Soc. Philadelphia IV. 1stis p. 160, j

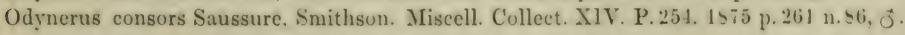

couspicuus Sauss. - $0-$ Am.: Mexico.

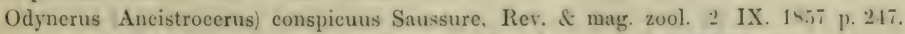

Odynerus Aneistrocerus conspicuus Saussure, Rev. d mag. zool. 2, XXII. 1 il p. 56 n. I.

Odynerus Ancystrocerus, conspicuus Saussure. Smithson. Miscell. Collect. XIV. P. 254.

corilorae Sauss. - $\Omega-$ Am.: Mexico.

$[1875 \mathrm{p}, 177 \mathrm{n}, 21$, ㅇ․

Odynerus Cordovae Saussure, Rev, de mag. zool. 2 XXII. 1571 p. is n. 10, s.

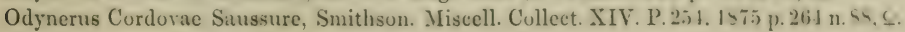


coyotus Sauss. - $\oint \hat{\jmath}-$ Am.: Mexico.

Odynerus (Stenodynerus, Coyotus Saussure. Rev. \& mag. zool. '2 XXII. 1571 p.106 n.30, f j. Odynerus (Stenodỵnerus Coyotus Saussure. Smithson. Miscell. Collect. XIV. P.254. 1875.

[p. 348 n. 144, ơ; T. 4 F. 26.

erassicornis (Panz.) Wesm. - 오

Vespa muraria Scopoli, Entom. Carn. 1763 p. 310 n. 828 [nee Linné].

Vespa crassicornis Panzer, Faun. Insect. German. V. 1798 P. 53 T. 8.

Vespa crassicornis Panzer, Krit. Revis. II. 1 S06 p. 155.

Vespa crassicornis Jurine, Nouv. méth. class. Hymén. 1807 p. 170, \&.

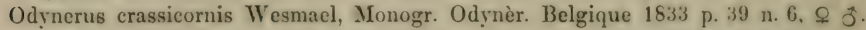

Odynerus (Symmorphus) crassicornis Wesmael. Bull. acad. sc. Belgique III. 1536 p. 45.

Odynerus alternans Zetterstedt, Insect. Lappon. I. 1838 p. 457 n. 8, ㅇ.

Odynerus erassicornis Blanchard, Hist. nat. Insect. III. 15.40 p. 393 n. 5.

Odynerus crassicornis Lepeletier, Hist. nat. Insect. Hymen. II. 1841 p. 663 n. $42 . \subseteq \jmath$.

Odynerus (Symmorphus) crassicornis Saussure, Étud. fam. Vespid. I. Eumén. 1552 p. 123

[n. 1, 옹 [p. p.].

Symmorphus crassicornis Schenck, Jahrb. Ver. Naturk. Nassau IX. 1.1553 p. 72 n. 29, $\subseteq$ j. Odynerus (Symmorphus, crassicornis Saussure, Etud. fam. Yespid. III. Masar. 1856 p. 15 i

[n. 79, 오; T. 10 F. 2 .

Odynerus Symmorphus arcticus Saussure, Étud. fam. Vespid. III. MIasar. 1556 p. 159 n. \$2. ऽ.

Odynerus Symmorphus) crassicornis Smith, Catal. Brit. Fossor. Hymen. 15.8 p. 200 n. 1 ,

[우 ô; T. 5 F. 4 (ㅇ).

Odynerus (Symmorphus) crassicornis Schenck, Jahrb. Ver. Naturk. Nassau XVI. 1861 p. 35,

[38 \& 39 n. ], \& ठ․

Sỵmmorphus crassicornis Brischke, Schrift. phỵs. ̈̈kon.Ges. Königsberg II. 1862 p.116. $\subsetneq \hat{\jmath}$.

Odynerus erassicornis Radoszkowski, Horae soc. entom. Ross. III. 186i5 Bull. p. XIII.

Symmorphus crassicornis Taschenberg, Hymen. Deutschl. 1566 p. $2 \cdot 15, \subseteq \hat{o}$.

Odynerus (Protodynerus) crassicornis F. Morawitz, Horae soc. entom. Ross. IV. 1567 p. 111

Odynerus alternans Thomson, Opusc. entom. P. 2. 1570 p. 90 n. $21 . \& \hat{j}$. n. 1.

Odynerus crassicornis Thomson, Hymen. Scandin. III. 1874 p. 54 n. $2, \subseteq j$.

Odynerus erassicornis Lichtenstein, Ann. soc. entom. France (5) IV. 157.4 Bull. p. I.XXXVI.

Odynerus crassicornis Rudow, Arch. Ver. Fr. Naturg. Mecklenb. XXX. $15 i 6$ p. 218 n. $\$, \subseteq \hat{\jmath}$.

Symmorphus crassicornis Fr. Lüw, Verh. zoul. bot. Ges. Wien XXIX. 1579 Sitzber. p. 33.

Odynerus (Symmorphus) crassicornis E. Saunders, Trans. Entom.Soc.London 1952 p. 1 s(1) n. 13.

Odynerus crassicornis Ed. André, Spec. Hymén. Europe II. P. 21-23. 1584 p. 660 n. $11 . \varsigma$ ô.

Odynerus crassicornis Schulthess, Hymen. Helret. Diplopt. 1557 p. 34 n. $2 . \subseteq \hat{\jmath}$.

Odynerus crassicornis Laneelerée, Bull. soc. sc. nat. Elbeuf 1889 p. 50.

Odynerus crassicornis Verhoeff, Entom. Nachr. XVI. 1590 p. 335.

crenatus Lep. - 우 ơ - Eur. mer.; Afr.: Algeria.

? Vespa aucta Fabicius \& auct.

Odynerus crenatus Lepeletier. Hist. nat. Insect. Hymén. II. 14.11 p. 629 n. 16.85.

()dynerus erenatus Iueas, Explor. se. Algérie. Zool. III. 14.46 p. 235 n. 190 . n.65, $\vec{j}$.

()dynerus Leionotus) rhynchiformis Saussure, Fitud. fam. Vespid. I. Fumén. 18.52 p. 174

Odynerus (I.eionotus) crenatus Saussure, Etud. fam. Vespid. I. Fumén. 1452 p. 191 n. \$9, 53 .

()dynerus crenatus Saussure. Etud. fam. Vespid. III. Masar. 1456 p. 236, $\subseteq$ b.

() dynerus . Leionotus, erenatus Sehenck, Jahrb. Ver. Naturk. Nassau XVI. 1861 p. 72 , nota.

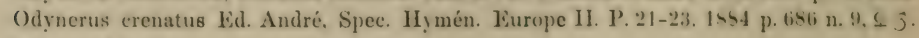

cribratus Mor. - 으 - As.: 'Transcaucasia.

I.ionotus eribratus F. Morawitz, Horae soe. entom. Ross. XIX. 1555 p. 171 n. 22 , s.

() dynerus (J.eionotust cribratus Jid. André. Spee. IIỴmén. Europe II. P. 24-26. 1 4sti p. S77. S. 
eristatus Sauss. $-\mathrm{Q}-\mathrm{Am}$. bor

Odynerus (Symmorphus) cristatus Saussure, litud. fam. Vespid. III. Masar. 15.31 p. 196 n.92, ᄃ. Odynerus (Symmorphus, cristatus Saussure. Smithson. Miscell. Collect. XIV. P. 254. 1875 ernentatus Brullé) D. T. $-f-$ Afr.: Insulae Canarienses.

p. $156 \mathrm{in.} 6,0$.

Eumenes cruentata Brullé, Barker-W ebb \& Berthelot: Hist. nat. iles Canar. II. 15.41 p.90 n.45.

Eumenes (Discoelius) cruentatus Brullé, Barker-Webb \& Berthelot : Hist. nat. iles Canar. II.

[1840; T. 3 F. 15.

Ieptochilus eruentatus Saussure, Étud. fam. Vespid. I. Eumén. 1 \$52 p. 235 n. 4, \&.

Odynerus cruentatus $m$.

eruentus Sauss. - 우 - Austr.

[n. 115 , ㅇ․

Odynerus (Ancistroceroides) cruentus Saussure, Etud. fam. Vespid. III. Masar. 1556 p. p. 221

Odỵnerus Parodynerus) cruentus Saussure, Etud. fam. Vespid. III. Masar. $1 \$ 56$ p. 321.

cruralis Sauss. - ơ - Afr.: Algeria.

[T. 15 F. 4 .

Odynerus (Oplopus) cruralis Saussure, Étud. fam. Vespid. III. Masar. 1856 p. 311 n. 195, ̧ै;

Odynerus cruralis Ed. André, Spee. Hymén. Europe II. P. $21-23.1554$ p. 761 n. 20, 0 .

crypticns Say - 오 ô - Am.: Arkansas.

Odynerus crypticus Say, West. Quart. Rep. Cincinnati II. 1823 p. 81.

Rhygchium erypticum Say, Boston Journ. Nat. Hist. I. 4.1837 p. 384 \& 765 n. 3.

Rhynchium erypticum Saussure, Étud. fam. Vespid. III. Masar. 1856 p. 184.

Odynerus crypticus Leconte, Writ. of Th. Say Entom. I. 1859 p. 168.

Rhynchium erypticum Leconte. Writ. of Th. Say Entom. II. $185 !$ p. 765 n. 3.

Odynerus crypticus Saussure, Smithson. Miscell. Collect. XIV. P. 254.1875 p. 276 n. 95.

cubensis Sauss. - 0 ơ - Am.: Cuba.

[오 ठี; T. 18 F. 8 (우).

Odynerus Leionotus Cubensis Saussure, Étud. fam. Vespid. I. Eumén. 1852 p. 181 n. 75 ,

Odỵnerus Cubensis Saussure. Sagra: Hist. fis. Cuba VII. 1856 p. 769, f 0 ; T. 19 F. 4.

Odynerus Cubensis Cresson, Trans. Amer. Entom. Soc. II. 1569 p. 294, $\jmath^{-}$. [p. 242 n. $78, \subseteq \jmath^{*}$.

Odynerus Pachodynerus, Cubensis Saussure, Smithson. Miscell. Collect. XIV. P. 254.1S75

cultus Cress. - ô-Am.: Texas.

Odynerus cultus Cresson, Trans. Amer. Entom. Soc. IV. 1872 p. 236, ð.

cylindricus Sauss. $-q-$ As.: Celebes.

[n. 27, 오.

Odynerus Ancystrocerus cylindricus Saussure, Stettin. entom. Zeitg. XXIII. 1862 p. 193

cyrtogaster Schlett. - 우 - Am.: Chili.

Odynerus eyrtogaster Schletterer, Entom. Nachr. XVII. 1891 p. s9, $f$.

dantici (Ross.) Spin. - 우 ơ - Eur. fere tota.

Vespa Dantici Rossi, Fauna Etrusea II. 1790 p. 89 n. 876 ; T. 6 F. 6.

Odynerus Dantici Spinola, Insect. Ligur. I. 1806 p. 88 n. 2.

Vespa Dantici Illiger. Rossi: Fauna Etrusea lid. 2 II. 1907 p. 1.16 n. 476 ; 'T. 6 F. 6.

Odynerus auctus Spinola, Inscet. Ligur. II. 1808 p. 185 , ڤે.

Odynerus Dantici Spinola, Insect. Ligur. II. 1808 p. 186 n. 2, §ో.

Lumenes Dantici Latreille, Gen. Crust. \& Insect. IV. 1809 p. 136.

Eumenes Dantici Klug, Germar: Reise n. Dalmat. II. 1817 p. 264 n. 367.

Odynerus Dantici Herrich-Schaeffer, Faun. Insect. German. 1439 P. 17.3 T. 23 ( 3 :.

Odynerus postscutellatus Lepeletier. Hist. nat. Insect. Hỵmén. II. $1 \$ 41$ p. 627 n. 15 . $f$ ș.

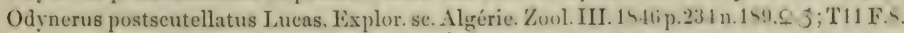

Odynerus (Leionotus) fastidiosus Saussure. Fitud. fam. Vespid. I. Eumén. 1452 p. 159 n. $5,5,5$.

Odynerus (Leionotus Dantici Saussure. Kitud. fam. Vespid. 1. Eumen. 1552 p. 192 n. 96.

Odynerus Dantici Saussure. Kitud. fam. Vespid. III. Masar. $18.1 \mathrm{p}$. 235. o o.

Odynerus Dantici Giraud, Verh. zool. bot. Ges. Wien VIII. k is p. 446 n. 4 . [n. $3, \subseteq 3$.

Odynerus Leionotus, ])antici Schenck. Jahrb. Ver. Naturk. Nassau XVI. 15611 p. 64, 67 d 70

Odỵnerus Leionotus, Dantici F. Morawitz. IFurae soc. entom. Russ. IV. 1567 p.127n.14. 3 . 
Odynerus Dantici Rudow, Arch. Ver. Fr. Naturg. Mecklenburg XXX. 1576 p. 225 n. $2 . \subseteq \vec{o}$. Odynerus I)antici Ed. André, Spec. Hymén. Europe II. P. 21-23. 1554 p.700 n. 10. \& ơ; T. 36 debilis Sauss. - 우 $\sigma^{\star}-$ Am.: Canada, United States.

[F. $13 \& 19$.

Odynerus Symmorphus debilis Saussure. Rev. \& mag. zool. 2 XXII. $15 i 1$ p. 55 n. 1. $\Omega$. Odynerus Symmorphus, debilis Saussure. Smithson. Miscell. Collect. XIV. P. 254. 15i5 Odynerus debilis Provancher. Natural. Canad. XIII. 1582 p. 139 n. $3 . \subseteq 3$. p.155 n.4,. . Odynerus debilis Provancher, Faun. entom. Canada. Hỵmén. 1554 p. 6773 n. $3, \subseteq$ j.

debilitatus Sauss. - 우 ô - Eur.: Germania, Suecia.

[Q⿻日木冖

Odynerus (Symmorphus; debilitatus Saussure. Etud. fam. Vespid. III. Masar. 1 S56 p. 194 n. 55 , Odynerus Symmorphus) debilitatus Schenck, Jahrb. Ver. Naturk. Nassau XVI. 1961 p. 37, 34 Odynerus debilitatus H. Müller, Befrucht. d. Blumen 1573 p. 466.

Odỵnerus debilitatus Thomson. Hymen. Scandin. III. 1574 p. $92 . \subseteq \hat{\jmath}$.

Odynerus debilitatus Rudow. Arch. Ver. Fr. Naturg. Mecklenburg XXX. 1876 p. 223 n.9, $\subseteq$. Odỵnerus debilitatus Ed. André. Spec. Hymén. Europe II. P. 21-23. 1854 p. 659 n. 6, ๔ 0 . decipieus Sauss. - 은 Austr.

[n. 8, 우 T. 1 F. 6.

Odynerus (Leionotus) decipiens Saussure, Reise d. Novara. Zool. II. 1. 1567 Hymen. p. 11 deflendus (Saund.) Sauss. - $f$ tิ - Eur.: Albania.

Ancystrocerus deflendus S. S. Saunders, Trans. Entom. Soc. London (2. II. 7. 1554 p. 141. Odynerus deflendus Saussure, Étud. fam. Vespid. III. Masar. 1556 p. 320 n. $207, \subseteq \hat{\jmath}$. dejeetus Cress. - 우 ơ - Am.: Cuba.

Odynerus dejectus Cresson, Proc. Entom. Soc. Philadelphia IV. 1565 p. 164, ᄃ.

Odynerus dejectus Cresson. Trans. Amer. Entom. Soc. II. 1569 p. 294, oే. p.20łn.39,.

Odynerus Stenancystrocerus dejectus Saussure, Smithson. Miscell.Collect. XIV.P.254.1575

delieatus Cress. - $9-$ Am.: Texas.

Odynerus delicatus Cresson, Trans. Amer. Entom. Soc. IV. 1872 p. 236. .

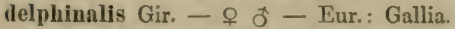

Odynerus (Leionotus) Delphinalis Giraud, Ann. soc. entom. France 1) VI. 1 s 66 p. 464 n. 14, f. Odynerus Delphinalis Ed. André, Spec. Hymén. Europe II. P. 21-23. $15 \varsigma 1$ p. 743 n. $5 . \varsigma j$.

denticulatus Sauss. - ơ - Am.: Mexico.

Leptochilus denticulatus Saussure, Rev. \& mag. zool. (2 VII. 1555 p. 373,3 .

Odynerus Antepiponus, denticulatus Saussure. Smithson. Miseell. Collect. XIV. P.254. 1575 designatus Cress. - - - Am.: Texas.

[p. 362 n. 155, ơ; T. 4 F. $30 \& 30^{\text {a }}$.

Odynerus designatus Cresson. Trans. Amer. Entom. Soc. IV. 1572 p. 235, \&.

destefauii André - 우 ơ - Eur.: Sicilia.

Odynerus Epipona Destefanii Ed. André, Natural. Sicil. II. 1553 p. 233, j̧.

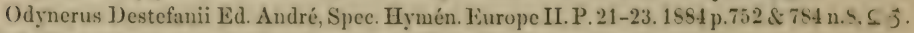

diabolieus Sauss. - 우 o - Am. mer., Brasilia, Venezuela.

Odynerus Leionotus) diabolieus Saussure, Étud. fam. Vespid. I. Eumén. 18.32 p. 171 n. 60. j.

Odynerus (Hypodynerus diabolieus Saussure, litud. fam. Vespid. III. Masar. Isit6 p. 230. C.

Odynerus diabolicus Saussure, Rev. \& mag. zeol. (2) X. 1555 p. 167 n. 6. \& 3.

Odynerus Pachodynerus diabolicus Saussure, Smithson. Miseell. Collect. XIV.P.254. 15i5

diemensis Sauss. - $\&$ - Austr.: Tasmania.

[p. $244 \mathrm{n} .80, \propto \sigma$.

Odynerus Leionotus, Diemensis Saussure, Étud. fam. Tespid. I. Eumén. 1 4ì2 p.201 n.103.S.

dietrichianus Sauss. $-\Omega-$ Austr.

Odynerus Dietrichianus Saussure, Stettin. entom. Zeitg. XXX. Isti9 p. 54 n. 3. S.

difflnis Sauss. $-f$ oิ - As.: India or.

Odynerus diffinis Saussure, Etud. fam. Tespid. III. Masar. 18 i6 p. 27.4 n. 155. S; T. 14 1.2.

Odynerus Leionotus diffinis Saussure, Reise d. Novara. Zoul. II. 1. 1 87 Hymen. p. 14 n. 12 , Aliformis Sauss. - ô- Afr.: Senegal.

- Savigny, Deser. de l'Égypte. Hymén. 1812; T. 8 F. 11.

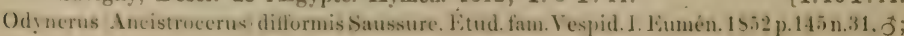


dilectus Sauss. - ô-Am. bor.

Odynerus Epipone dilectus Saussure, Rev. \& mag. Lool. 22 XXII. 14il p. 141 n. 34, oै.

Odynerus (Lipiponus dilectus Saussure, Smithson. Miscell. ('ollect. X1Y. P.2.34.1475 p. 363

dimidiatus Spin. - $€-$ Afr.: Aegyptus.

[n.15b, ठै; T. 4 F. $29 \& 29$.

Odynerus dimidiatus Spinola, Ann. soc. entom. France VII. 1838 p. 502.

Odynerus (IIypodynerus) dimidiatus Saussure. Etud. fum. Tespid.III.Masur. 1 -5 6 p p. 260 n. 13!).

Odynerus dimidiatus I: André. Spec. Hymén. Lurope II. P. 24-26. 1456 p. S67, s.

diseonotatus Lichtst. - 으 - Eur.: Gallia.

Odynerus disconotatus Lichtenstein, Ann. soc. entom. France 6) IV. 1444 Bull. p. L.

Odỵnerus disconotatus Ed. André, Spec. Hỵmén. Europe II. P. 21-23. Is 4 p. fis3 n. 4, 5 .

disjunctus Walk. - + - Afr.: Aegyptus.

Odynerus disjunctus Walker, List of Hymen. in Egrpt $15 i 1$ p. 39 n. 157, $\mathrm{f}$.

diversus Walk. - ot - Afr. Arabia.

Odynerus diversus Walker, List of Hymen. in Egypt 1571 p. 34 n. 17. 3

dorsalis (Fabr.) Sauss. - $\odot$ ơ - Am.: United States.

Vespa dorsalis Fabricius, Syst. entom. 1775 p. 367 n. 25.

Vespa dorsalis Fabricius, Spec. Insect. I. 1781 p. 463 n. 31.

Vespa dorsalis Fabricius, Mant. Insect. I. 1787 p. 289 n. 36.

Vespa dorsalis Gmelin, Linné: Syst. nat. Ed.13a I. 5. 1790 p. 2760 n. 91.

Vespa dorsalis Christ, Naturg. d. Insect. 1791 p. 238.

Vespa dorsalis Olivier, Encycl. méthod. Insect. VI. 1791 p. 685 n. 81.

Vespa dorsalis Fabricius, Entom. system. II. 1793 p. 265 n. 44.

Polistes dorsalis Fabricius, Syst. Piez. 1804 p. 273 n. 19.

Rhygchium balteatum Say, Boston Journ. Nat. Hist. I. 4.1837 p. 394 n. 1.

Monobia sylratica Saussure, Étud. fam. Vespid. III. Masar. 1856 p. 168.

Rhynchium dorsale Saussure, Étud. fam. Vespid. III. Masar. 1856 p. 171.

Rhynchium balteatum Leconte, Writ. of Th. Say Eintom. II. 1 S.99 p. 761 n. 1.

Rhynchium dorsale Cresson, Trans. Amer. Entom. Soc. IV. 1872 p. 233.

Rhynchium dorsale Saussure. Snithson. Miscell. Collect. XIV. P. 254. 1475 p. 143 n. 1.

Odỵnerus dorsalis Saussüre, Smitlıson. Miseell. Collect. XIV. P. 251. 1575 p. 257 n. S4. S.j.

var. Iouisianus (Sauss.) Cress. - 으 - Am.: Louisiana.

Rhygghium I.ouisianum Saussure, Étud. fam. Tespid. I. Eumén. 1 s 52 p.106 n. 7, S; T. 1:; F. 9.

Odỵnerus dorsalis var. Louisianus C'resson. Sỵnops. Hỵmen. Amer. 1Ss i p. 2 ss.

dotatus Walk. - ô-As.: Arabia.

Odynerus dotatus Walker, List of Hymen. in Egypt 1871 p. 35 n. 175, ơ.

doursii Sauss. - ô - Afr.: Algeria.

Odynerus Doursii Saussure. Kitud. fam. Vespid. III. Masar. 15sif p. 2is n. 160, j.

Odynerus Doursii lid. Andrí, Spec. IIymin. Europe II. P. $21-23$. Is-4 p. $70: 3$ n. 1, j.

drewsenii Sauss. $-\Omega-$ Austr.: Nova Hollandia.

Odynerus Drewseni Saussure, Ann. soc. entom. France (ii) V. 14.57 p. 314, f is??.

Aromedaxius Blackb. \& Cam. - $\subseteq-$ Austr.: Ins.: Hawaiac.

[p. $211 \mathrm{n} .211$, .

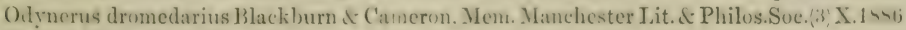

dubiosus Smith - đ - Austr.: Honolulu.

Odynerus dubiosus Smith, Journ. Linn. Soc. Zool. XIV. 1879 p. 651, ơ.

Odynerus dubiosus Blackburn of Kirby. Entomu. M. Maraz. XVII. 15々0 p. si.

dubius Sauss. - - of - Eur.: Gallia.

Odynerus Leionotus dubius Saussure. Litud. fam. Tespid. I. Eumén. 14.92 p. 193 n. 92, \&.

Odỵnerus dubius Saussure. Etud. fam. Vespid. III. Jiasar. 14.96 p. 23ī, $\leq \hat{\jmath}$.

Odynerus dubius Ed. André. Spece. Iymén. Europe II. 1'. $21-23.194$ p. 707 n. $9, \Omega 3$.

ductus Cress. - 0 ơ - Am.: Texas.

Odynerus ductus Cresson. Truns. Amer. Entom. Suc. IT. I4it p. 2:ls, $9 \hat{0}$.

Catulogus Iigmenopterorun. IS. 
duplicatus Klug! - $\subseteq$ - Eur.: Hispania.

Odynerus duplicatus Klug. Waltl: Reise d. Tirol ete. P. 2. 15:35 p. 101. ᄃ.

Odynerus duplicatus Saussure, litud. fam. Vespid. III. Masar. 14.66 p. 319 n. 206i. dyseherus Sauss. - - - As.: China.

Odỵnerus Leionotus dyseherus Saussure. Ftud. fam. Tespid. I. Eumén. 15.52 p. 175 n. wi.C. ebusianus Lichtst. - $\subseteq-$ Eur.: Ins. Baleares.

Odynerus Ebusianus Lichtenstein. Ann. soc. entom. France 6j. IV. 15-4 Bull. p. I.

Odynerus Ebusianus Ed. André. Spee. Hỵmén. Europe II. P. 21-23. $14 n$ p. 675 n. 10. . . egregius Herr.-Schacff. - ㅇํ ô - Eur. mer.; Caucasus.

Odynerus egregius Herrich-Schaeffer, Faun. Insect. German. $14+1$ P. 17 ; T. 2 ' $\subseteq$.

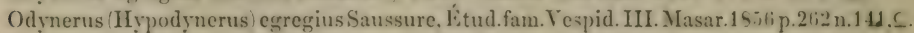

Odynerus egregrius Iid. André, Spec. Hymén. Europle II. P. 21-23. 14st p. fis n. 11 . f j.

electus Cress. - ô-Am.: 'T'exas.

Odynerus electus Cresson, Trans. Amer. Entom. Soc. IV. 1872 p. 243, ơ.

emeryanus Grib. - $\rho-$ Afr.: Sicrra Leone.

Odynerus Emeryanus Gribodo, Bull. soc. entom. Ital. XXIII. 1691 p. $293 . \subseteq$.

emortualis Sauss. - $q-$ Afr.: Algeria.

['T. 19 F. 9.

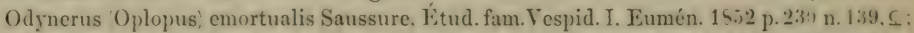

Odynerus emortualis Ed. André. Spec. Hrmén. Europe II. P. 21-23. $14 \$ 4$ p. is 1 n. 19. C. enyo Lep. - o ô - Am.: Cuba.

Odỵnerus linỵo Iepeletier. Hist. nat. Insect. Hỵmén. II. 1841 p. 64.5 n. 33. C.

Odynerus elegans Guérin, Ieonogr. rìgn. anim. VII. Insect. 1545 p. 446 ; T. 72 F. 5.

Odynerus (I.eionotus) Enyo Saussure. Étud. fam. Vespid. I. Eumén. 1552 p. 155 p. 41.

Odynerus Enyo Saussure, Sagra: Hist. fis. Cuba VII. 1856 p. 770.

Odỵnerus Enyo Cresson, Proc. Entom. Soc. Philadelphia IV. 14(;5) p. 165.

Odỵnerus Stenodynerus) Finyo Saussure. Smithson. Miscell Collect. XIY. P. 254. 14 i5 p. 313

ephippium Klug - $\subseteq \vec{\jmath}-$ Eur.: Dalmatia, Russia. Hispania.

in. 115.0 .5 .

Liumenes ephippium Klug. Germar: Reise n. J)almat. II. 1517 p. 264 n. 365.

Eumenes ephippium Germar, Faun. Insect. Europ. P. 3. 1S17; 'T'ab. 13.

Odynerus Leionotus difficilis F. Morawitz. Iorae soc. entom. Ross. IV. 1467 p. 132 n.19. S 5 .

Odynerus ephippium Ed. André, Spec. Iyymén. Jurope II. P. 21-23. 155.1 p. $7: 35$ n. $5 . \subseteq$ Jै.

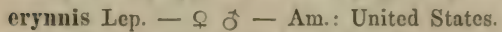

()dynerus Erymnis Lepeletier, Hist. nat. Insect. Hymén. II. 1441 p. 645 n. 30 . ᄃ.

Odynerus (I.cionotus) Erynnis Saussure. Etud. fam. Vespid. I. Eumén. 15.52 p. 17s n. 70. ᄃ.

Odynerus (Pachodynerus Erinnys Saussure, Smithson. Miscell. Collect. XIV. P. 254. 14i5

eumenoiles Smith - ô - Afr.: Port Natal.

[p. $245 \mathrm{n} .81, \subseteq \hat{\sigma}$.

Odynerus eumenoides Smith, Catal. Ilymen. Brit. Mus. V. $155 i$ p. il n. 134. j.

erectus Cress. - $\mathrm{f}-\mathrm{Am}$. : Texas.

Odynerus evectus Cresson, Trans. Amer. Entom. Soc. IV. 1872 p. 235, §.

eversmannii Rad. - $\odot-$ Afr.: Aegyptus.

- Sarigny, Descr. de l'Égypte. Hymén. 1812; T. 9 F. 8.

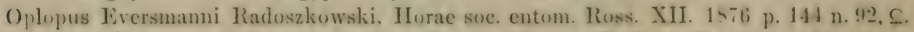

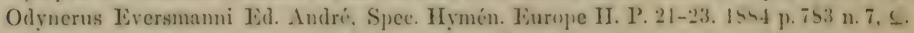

excipiendus (Spin.) Sauss. - $f$ ơ - Am.: Chili.

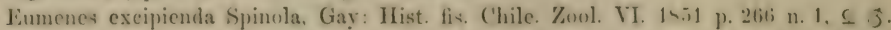

Odyneru; , Leionotus excipiendus Saussure, Fitud. fam. Vespid. I. Tumén. 14.52 p. 161 n. 47.

[ค ठี; T.17 F.4(ठ)

Odynerus illypolynerns exeipiendus Saussure. Smithson. Misecll. Collect. XIV.P. 25.4.1 45 var. colocolo Sauss. - 요 ơ - Am.: Chili.

[p. $220 \mathrm{n} .49, \subseteq \sigma^{\circ}$.

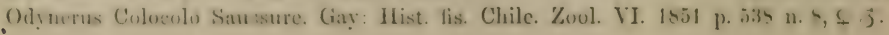


Odỵncrus I.eionotus Culocolo Saussure. Ėtud. fam. Vespid. I. Fumén. 15.52 p. 161 n. $46, \subseteq 3$.

Odỵerus excipiendus var. Colocolo Saussure, Kitud. fam. Vespid. III. Masar. 1556 p. 227.

excisus Thoms. - $\subseteq$ oै - Eur.: Suecia, Germania, Gallia.

Odynerus excisus Thomson, Opusc. entom. P. 2. 1870 p. 87 n. $12, \subseteq$ ô.

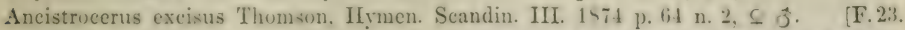

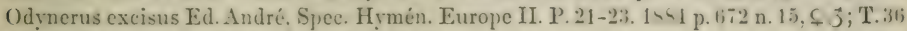

exiguus Sauss. - $+\widehat{f}-$ Afr.: Aegyptus.

- Sarigny, Descr. de l'Égypte. Hymén. 1812; T. 8 F. 11.

J.eptochilus exiguus Saussure. Étud. fam. Tespid. I. Eumén. 15.52 p. $2: 37 . \subseteq$ j.

Odynerus Parodynerus exiguus Saussure. Etud. fam. Vespid. III. Masar. 1556 p. 321. exilis Herr.-Schaeff. - 0 ô - Eur. centr.

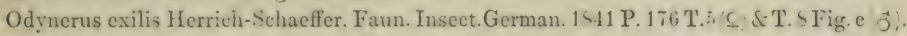

? Odỵneras bivittatus Lepeletier. Hist. nat. Insect. Hymén. II. 1541 p. 617 n. $\$$. 5 o.

Odynerus Leionotus; bivittatu: Saussure, Itud. fam. Tespid. I. Eumén. 1552 p. 211 n. 117.

[८ วิ; T.19 F.3.

Odynerus exilis Schenck, Jahrb. Ver. Naturk. Nassau IX. 1.1553 p. 70 n. $2 \mathfrak{7}, \mathbf{s}$.

Odynerus birittatus Saussure, Étud. fam. Vespid. III. Masar. 18.56 p. 243.

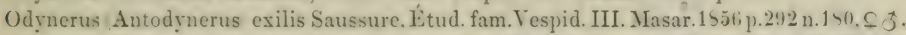

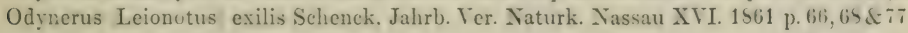

[n. 11, 용.

Odynerus exilis Brischke, Schrift. phỵs. ökon. Ges. Königrsberg II. 1462 p. 116. $\varsigma$ j̧.

Odynerus Leionotus exilis F. Morawitz. Horae soc. entom. Ross. IV. 1567 p. 13j n. 22.

Mierodynerus exilis Thomson, Hymen. Scandin. III. 1874 p. 59 n. 3, ㅇ.

Odynerus exilis Rudow. Arch. Ver. Fr. Jaturg. Mecklenburg XXX. 1 i iti p. 229 n. S, $\subseteq$ o .

Odỵerus Microdynerus exilis Ed. André. Spec. Hỵmén. Europe II. P. 21-23. 1454 p. 731

[n. 1, 오․

extranaeus Sauss. $-\Omega-$ Austr. : Tasmania.

[F. 2 [non H.-Sch.].

Odynerus Leionotus exilis Saussure, Etud. fam. Tespid. I. Eumén. 1552 p. 157 n. 41 . $\$$; T. 17

Odỵnerus extranacus Saussure. Etud. fam. Tespid. III. Masar. 15.56 p. 22.4, s.

exustus Walk. - $\underline{-}-$ As.: Arabia.

Odynerus exustus Walker, List of Hymen. in Egypt 1871 p. 35 n. 177, ㅇ․

facialis Smith - $\hat{o}-$ As. : Celebes.

$[\mathrm{n}, 2, \hat{\sigma}$.

Odynerus Ancistrucerus facialis Smith. Journ. of Proc. Linn. Soc. Zool. IV. 1 S6u Suppl. p. bn

fairmairi Sauss. - ô - Eur. : Hispania.

Odynerus Leionot Faimairi Saussure, Etud. fam. Tespid. I. Tumén. 14.2 p. 216 n. 124. 3.

Odymerus Faimairi Ed. André. Spee. Hymén. Europe II. P. 21-23. 1944 p. 75i n. 13. 3 .

fallax Sauss. $-\Omega \hat{o}-$ Am.?

I.eptochilus fallax Saussure. Fitud. fam. Tespid. I. Eumén. 15.52 p. 231 n. 2, 3: T. 20 F. 6. Odynerus Parodyneru: fallax Saussure. Etud. fam. Vespid. III. Masar. 1456 p. 321.

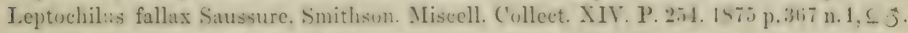

farias Sauss. - 우 ô - Am.: Mexico.

Odynerus Ancistrocerus, Farias Saussure, Rer. d mag. zoul. 2 IX. 16.5 p. 274.

() dynerus Stenancystroeerus Pariasi Sanssure. Smithsun. Miscell.Collect. XIV. P.2.4.14ij

fascieulatus Sauss. $-\propto-$ Am.: Cuba.

[p. $195 \mathrm{n} .32, £$ ô.

Odynerus Stenudynerus fasciculatus Saussure. Rev. \& mag zool. 2 XXII. I - il p. I 1 I n.26.C.

Odynerus Stenodynerus fascieulatus Saussure. Smithson, Miscell. ('ollect. XIV. P. 254. 14is

fastidiosissimus Sauss. $-\Omega$ ô - Eur. mer.; Afr.: Algeria.

[p. 330 n. 130, ㅇ․

Odynerus IIypodynerus, fastidiosissimus Saussure. Litud. fam. Vespid. III. Massar. 15.56

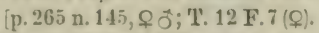

Odynerus lativentris Saussure, Litud. fam. Vespid. III. Masar. 14.66 p. 273 n. 136. S. 
Odynerus fastidiusissimus Saussure, Mém. soc. physs. of hist. nat. Genèc XVII. 1. 1563 p. 2.2.1 $[\mathrm{n} .50, \widehat{0}$.

Odyner:ıs fastidiosissimus Ed. André. Spec. IIymén. Eurepe II. P.21-23. $15-4$ p. 7.40 n.6. .5 . fastidiosusenlus Sauss. - - - Am.: Brasilia.

Odynerus (Ancistrocerus, fastidiususculus Saussure, Etud. fam. Vespid. I. 14.92 p. 137 n. 19.C. Odynerus (Aneystrocerus) fastidiosusculus Saussure, Smithson. Miscell.Collect. XIV. P.254.

$[1875$ p. 171 n. 15 , 우.

femoratns Sauss. - 우 ô - Eur.: Gallia.

[T.15 F.3 (Q) ).

Odynerus : Oplopus femoratus Saussure, litud. fam. Tespid. III. Masar. 14.56 p..310 n.194. \& J;

Odỵnerus Hoplopus; femoratus Sehenck. Jahrb. Ver. Naturk. Nassau XVI. 1 Sij1 p. 4.j nuta.

Odynerus femoratus Ed.André, Spec.Hymén. Europe II. P.21-23.15 4 p. 764 j 8772 - 11.24 .

ferrugineitarsis Dest. - 우 $\sigma-$ Eur.: Sicilia.

Odỵnerus ferrugineitarsis Destefani, Yatural. Sicil. VIII. 1499 p. $232, \leq \hat{\jmath}$.

ferruginosus Sauss. - - - Afr.: Cap.

[n. 188, ,

Odynerus Antepipone, ferruginosus Saussure, Etud. fam. Tespid. III. Masar. 1556 p. 2!99

fervidus Sauss. - Afr.

Odynerus (Antodynerus) forvidus Saussure, Etud. fam. Tespid. III. Masar. 1556 p. 255 n.174.

flgulns Sauss. - 우 - Am.: Guadeloupe.

Odynerus (Parodynerus figulus Saussure, Etud. fam. Vespid. III. Masar. 15.6 p. 247 n.120. .

Odynerus (Stenodynerus?) figulus Saussure. Smithson. Miscell. Collect. XIV. P. 254. 1575

flipalpis Sauss. - 우 - Afr.: Algeria.

[p. 331 n. 131, 우.

— Sarigny, Deser. de l'Égypte. Hymén. 1812; T. 9 F. 12.

Odynerus (Leionotus; filipalpis Saussure, Kitud. fam. Yespid. I. Eumén. 15.22 p. 197 n. 97, \&.

Odỵnerus filipalpis Saussure, Étud. fam. Vespid. III. Masar. 1856 p. 240.

Odỵnerus filipalpis Ed. André. Spec. Hymén. Europe II. P. 21-26. 15sti p. st6, 气.

firmus Cress. - 우 ơ - Am.: 'Texas.

Odynerus firmus Cresson, 'Trans. Amer. Entom. Soc. IV. 1872 p. 239, 오 oี.

fistulosus Sauss. - 우 - As.: Ceylon.

Odynerus (Leionotus) fistulosus Saussure, Reise d. Novara. Zool. II. 1. 14ti7 Hymen. p. 11

flammiger Sauss. - o - Austr.: Nouvelles Galles de Sud.

Odynerus flammiger Saussure, Étud. fam. Vespid. III. Masar. 1456 p. 252 n. 166, $\vec{\jmath}$.

flavipes (Fabr.) Walsh \& Ril. - Am. bor.

Vespa flavipes Fabricius, Syst. entom. 1775 p. 369 n. 34.

Vespa flavipes Fabricius, Spec. Insect. I. 1781 p. 466 n. 45.

Vespa flavipes Fabricius, Mant. Insect. I. 1787 p. 292 n. 56.

Vespa flavipes Gmelin, Limné: Syst. nat. Ed. 13 a I. 5. 1790 p. 2756 n. 69.

Vespa flavipes Christ, Naturg. d. Insect. 1791 p. 241.

Vespa flavipes Olivier, Encycl. méthod. Insect. VI. 1791 p. 690 n. 102.

Vespa flavipes Fabricius, Entom. system. II. 1793 p. 273 n. 72.

Vespa flaripes Fabricius, Syst. Piez. 1504 p. 268 n. 77.

? Vespa flavipes Gravenhorst, Yergl. Übers. zoul. System. 1sut p. 276 n. 34.

Odynerus flavipes Walsh \& Riley, Amer. Entomol. II. 1569 p. 10; Tig. 4.

flavocinctus Smith - ơ - Austr.

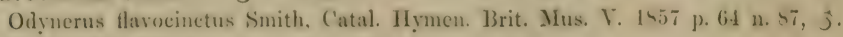

flarolineatus Smith $-\rho-$ As. : Java.

Odynerus flavolinestus Smith, Catal. Hỵmen. 13rit. Mus. V. 15.7 p. 60 n. 61, \&.

Odynerus flavolineatus Saussure, Stettin. entom. Zcitg. XXIII. 1 sti2 p. 197 n. 30,5 J.

Ilaropictus Sauss, - ㅇ - Am.: 'T'ennessee.

Odynerus flavopictus Saussure, Rev, \& mag. zool. (2) IX. 1557 p. 276.

Odynerus fiar (1pietus Saussure, Smithsun. Aiseell. Collect. XIV. P.251. 1575 p.293 n.107, s. 
flaras Walk. - ‡ - As.: Arabia.

Odynerus flarus Walker, List of Hymen. in Egypt 1871 p. 33 n. 16?, ô.

florieoln Sauss. - $f$ ô - Eur. mer.; Afr.: Algeria.

[T. 18 F.3. Odynerus (Lcionotus) floricola Saussure, Ritud. fam. Vespid. I. Eumén. 18.522 p. $196 \mathrm{j}$ n. 95 , $Q$; Odyncrus floricola Saussure, litud. fam. Vespid. III. Masar. 15it; p. 239. n.16,. Odynerus Leionotus floricola Saussure, Reise d. Novara. Zool. II. 1. 14 (j7 IIymen. p. 16

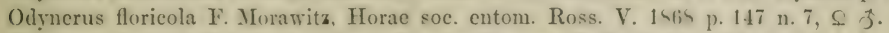
Odynerus floricola Ed. André. Spec. Hymén. Europe II. P. 21-23. 1444 p. 735 n. 2.

fluvialis Sauss. - $\hat{o}-$ Austr.

Odynerus (Ancystrocerus) fluvialis Saussure, Étud. fam. Vespid. III. Masar. 1556 p. 215 n. 106 ,

foraminatus Sauss. - 우 - Am.: United States.

Odỵnerus (Leionotus rugosus Saussure, Kitud. fam. Vespid. I. Fumén. $14 ., 2$ p. 179 n. 72, ô. Odỳnerus (Leionotus; foraminatus Saussure, litud. fam. Vespid. I. Eumén. 1452 p.180 n.73, ㅇ. Odynerus foraminatus Saussure. Étud. fam. Vespid. III. Masar. 1556 p. 232 . $\subseteq$ oే. Odynerus foraminatus Saussure, Smithson. Misecll. Collect. XIV. P. 25) 1. 1575 p. 255 n. 102,

formosus Sauss. - $Q-$ Am.: Mexico.

Odynerus formosus Saussure, Rev. \& mag. zool. (2) XXII. 1871 p. 59 n. 15.

Odynerus formosus Saussure, Smithson. Miscell. Collect. XIV. P. 254. 1875 p. 2 is n. 97, \&.

fragilis Smith - 우 ô - As.: Borneo.

Odynerus fragilis Smith, Catal. Hymen. Brit. Mus. V. 1857 p. 61 n. 64, ô.

Odynerus Parodrnerus fragilis Saussure, Stettin. entom. Zeitg.XXIII.1sli2 p.195n.29, $\subsetneq$ ơ.

frater D. T. - 千 0 - Austr.: Hawaiae.

[p. 219 n. 30, 우 ơ [nec Kirsch].

Odynerus pacifieus Blackburn \&. Cameron, Mem. Manchester Lit. \& Philos. Soc. (3) X. 1556 Odynerus frater $\mathrm{m}$.

frauenfeldii Sauss. - 우 - As.: China.

[n. 14, \&; T. 1 F. 9

Odynerus (Leionotus Frauenfeldi Saussure, Reise d. Novara. Zool. II. 1. 1567 Hymen. p. 15

frendens Grib. - $\odot-$ Afr. centr.

Odynerus frendens Gribodo. Bull. soc. entom. Ital. XXIII. 1591 p. 294, \&.

fulvipennis Smith - 오

Odynerus fulvipennis Smith. Journ. of Proc. Linn. Soc. Zool. III. 1555 p. 22 n. 4, ô. Odynerus fulvipennis Saussure, Stettin. entom. Zcitg. XXIII. 1562 p. $195 \mathrm{n}$. 32, $\subseteq$ j.

fulvipes Sauss. - 우 $\tilde{\sigma}-$ Am.: United States.

Odynerus flavipes I.epeletier, Hist. nat. Insect. Hymén. II. 1541 p. 659 n. 39 , f s [nec Fabr.] Odynerus (Ancistrocerus flavipes Saussure, Eitud. fam. Vespid. I. Jumén. 14.12 p. 142 n. 27 ,

[오후; T.16 F.3.

Odynerus fulvipes Saussure. Situd. fam. Vespid. III. Masar. 15.56 p. 205, $0 \hat{0}$.

Odỵerus Stenancystrocerus fulvipes Saussure. Smithson. Miseell.Collect. XIV. P.25.1.1475

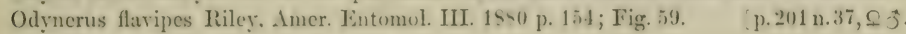

fumipennis Walk. - $\odot$ of - Afr.: Aegyptus.

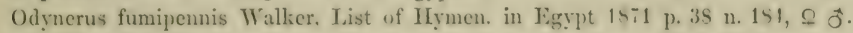

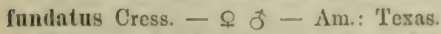

Odynerus fundatus Cresson. Trans. Amur. Entom. Suc. IV. 1572 p. $242, \subseteq j$.

funebris André $-Q-\Lambda$ s.: Sibiria.

Odynerus funcbris Ed. André. Spree. Hymén. Europe II. P. 21-23. 1554 p. 729 n. $20, \subseteq$.

fuscipes Ilerr.-Schacff. $-\&$ $f-$ Eur.: Succia, Germania.

Odynerus fuscipes Herrich-Schaeffer, Faum. Insect. German. 1435 P. 154 T. 15 '. Srmmorphus fuscipes Schenck, Jahrb. Yer. Naturk. Nassau IX. 1. 14.3. p. s., $\subseteq 3$.

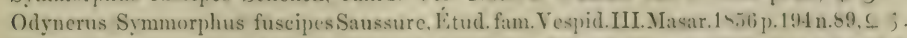
Odynerus Symmorphus) fuscipes Schenck. Jahrb. Ver. Yaturk. Nassau XVI. 1 \$1 1 p.37, 39 d Odynerus fuscipes 'Thomson, Iymen. Scandin. III. 1474 p. $94 \mathrm{n} .7, \subseteq \vec{J} . \quad$ 4: $\mathrm{n}, 4, \subseteq \hat{\jmath}$. 
Odynerus fuscipes Rudow. Arch. Ver. Fr. Nature. Mecklenburg XXX. $167 i$ p. $2 \cdot 22$ n. ti. $\subseteq j$. Odynerus fuscipes Ed. Indré. Spee. Iymén. Europe II. P. 21-23. 1544 p. 6i.je n. 10, 5 j. fuseus Cress. - $ᄋ$ ơ $-\Lambda \mathrm{m}$. : Texas.

Odynerus fuscus Cresson, Trans. Amer. Entom. Soc. IV. 1872 p. 238, 우 ơ.

gallieus Sauss. $-\rho \hat{\sigma}-$ Eur.: Gallia.

Odỵnerus (Hypodynerus Gallicus Saussure. Etud. fam. Vespid. III.Masar. 15.56 p. 273 n.154, 5 . Odynerus Gallicus Ed. André, Spec. Ifymén. Europe II. P. 21-23. 144.1 p. 720 n. 7,58 . gayi Spin. - ơ - Am.: Chili.

Odynerus Gayi Spinola, Gay: Hist. fis. Chile. Zool. VI. 1551 p. 21 it) n. 6. 3 . F. . Odỵnerus Leionotus! Gayi Saussure. Fitud. fam. Vespid. I. Eumén. 14.52 p. 170 n. 59 . Jै; T. 17 Odynerus Pachodynerus Gayi Saussure, Smithson. Misecll. Collect. XIV. P. 254.157.5 p.240 gazella (Panz.) Wesm. - $ᄋ$ $ో$ - Eur.: Germania. [n. 74, oิ.

Vespa gazella Panzer, Faun. Insect. German. V. 1798 P. 10 T. 10.

Vespa gazella Panzer, Krit. Rev. U. 1806 p. 155.

Odynerus gazella Wesmael, Bull. acad. sc. Belgique III. 1836 p. 50.

Odynerus gazella Herrich-Schaefer. Faun. Insect. German. IS41 P. $17 i ;$ T. 4 \& 14 (ö! Odynerus Ancistrocerus gazella Saussure. Étud. fam. Vespid. III. Masar. 1456 p. 249 n.97, $\vec{j}$. Odỵnerus gazella Brisehke, Schrift. phys. ökon. Ges. Königsberg II. 1St:2 p. 115, j. Ancistrocerus gazella Thomson, Hymen. Seandin. III. 1574 p. 50 n. $11, \subseteq \hat{\jmath}$.

Odynerus gazella Ed. André, Spec. Hymén. Europe II. P. 21-23. 1554 p. 676 n. $15, \varsigma \delta$. geminus Cress. - 우

Odynerus geminus Cresson, Trans. Amer. Entom. Soc. IV. 1872 p. 240 , \&.

germanieus Sauss. - 우 - Eur.: Germania, Gallia, Russia.

[n. 146, 우.

Odynerus (Hypodynerus) Germanicus Saussure, Étud. fam. Vespid. III. Masar. 15.56 p. 266 Odynerus Leionotus; Germanicus Schenck, Jahrb. Ver. Naturk. Nassau XVI. 1461 p. 65

[\& 74 n. 7, 우.

Odynerus (Leionotus; Germanicus F. Morawitz, IIorae soc. entom. Ross. IV. 156 i p. 133 n.21. Odynerus (Leionotus) Germanicus Rudow, Arch. Ver. Fr. Naturg. Mecklenburg XXX. 15 i6

[p. 228 n. 7, ㅇ.

Odỵnerus Germanicus Ed. André, Spec. Hymén. Europe II. P. $21-23.1854$ p. 729 n. 21, ऽ. gonioides Schlett. - 우 0 - Afr.: Congo.

Odỵnerus (Leionotus) gonioides Schletterer. Ann, soc. entom. Belgique XXXV. 1591 p. 25 , gracilis Brullé - Q ot - Eur. bor. \& centr.

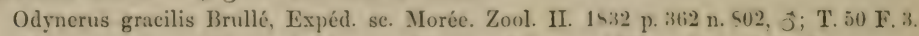
Odynerus elegans Wesmacl, Monogr. Odynèr. Belgique 15333 p. 43 n. $i, s, j$.

Odynerus (Symmorphus elegans Wesmael, Bull. acad. se. Belgique III. 1siat p. 45.

Odỵnerus elegrans Ierrich-Schaeffer, Faun. Insect. German. 1533 P. 154 T. 17.

Odỵnerus elegans Iepeletier. IIist. nat. Insect. IIymén. II. 1441 p. 664 n. $43 . \leq 3$.

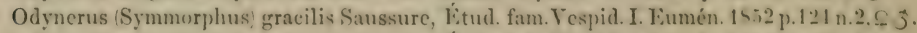
Odynerus Symmorphus, elegans Saussure, K.tud. fum. Vespid. I. Kumén. 14.22 p.125 n.1. 0 s. Symmorphus elegans Schenck, Jahrb. Ver. Naturk. Nassau IX. 1. 14.53 p. 74 n. 30. 0.5 . Odynerus iSymmorphus gracilis Smith. Catal. Brit. Fossor. Hymen. 15.is p. 202 n. 3 . f Jै. Odỵnerus (Sirmmorphus) elegans Sehenek. Jahrb. Ver. Naturk. Nassau XVI. 1 bti1 p. 3ti, z4

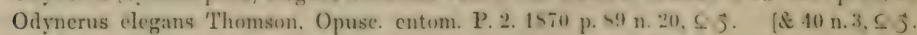
Odynerus elegans H. Muller, Befrucht. d. Blumen 1873 p. 466.

Odynerus elegans Thomson. Hymen. Seandin. III. 1 -74 p. 90 n. $5.5 \hat{j}$. p. 221 n. $4 . \subseteq \hat{j}$. () lynerus Symmorphus) eleuns Ruduw. Arch. Ver. Fr. Naturg. Mecklenburg XXX. 14is Odynerus Symmorphus gracilis Fi. Saunders. Trans. Fintom. Soc. Iondon $14 y 2$ p. 141 n. 14.

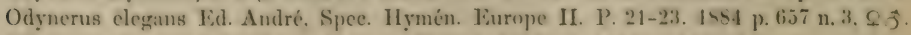
Odỵnerus clegans Sehulthess. Hỵmen. Helvet. 1)iplopt. 
grandidieri Sauss. - $\bigcirc-$ Afr.: Madagascar.

[T. 4 F. 16.

Odynerus Grandidieri Saussure, Grandidier: Hist. Maciagascar XX. P.1. 1591 p. 164 n.3. F; grandis Mor. - ô - As.: Persia.

Hoplomerus grandis F. Morawitz. Horae soc. entom. Ross. XIX. 1555 p. 159 n. 15. $\vec{j}$. Odynerus Hoplomerus grandis Ed. André, Spec. Hymén. Kurope II. P.2.4-21i. 155 li p.55i, J. graphicus Sauss. - 우 - Eur.: Gallia.

Odỵnerus Leionotus graphieus Saussure. 1tud. fum. Vespid. I. Eumén. 1452 p. 191 n. S4, f. Odỵnerus graphicus Saussure. Kitud. fam. Vespid. III. Masar. 1 h56 p. 234.

Odynerus graphicus Ed. André. Spec. Hỵmén. Europe II. P. 21-23. 1554 p. 736 n. 1, f. guadulpensis Sauss. - + ơ - Am. : Guatemala.

[n. 76, ㅇ․

Odynerus :Leionotus Guadulpensis Saussure, Étud. fam. Vespid. I. Fumén. 15.22 p. $1 \checkmark 2$ Odynerus Cubensis var. Guadulpensis Saussure, Etud. fan. Vespid. III. Masar. 1 -5ili p. 232. Odynerus Pachodỵnerus. Guadulpensis Saussure, Smithson. Misecll. Collect. XIV. P.254. gueriuii Sauss. - $\widehat{o}-$ Afr.

$[1875$ p. 238 n. 72 , 우

Odynerus (Leionotus Guérini Saussure, Kitud. fam. Vespid. I. Eumén. 1552 p. 176 n. 65, ̧ै guerrero Sauss. - $\odot$ ơ - Am.: Mexico.

Odynerus Guerrero Saussure, Rev. \& mag. zool. (2) IX. 1857 p. 277.

Odymerus Guerreri Saussure, Smithson. Miscell. Collect. XIV. P. 254. 1575 p. 294 n. 104 . guineensis Sauss. - 우 - Afr.: Guinea.

Odynerus Guineensis Saussure, Rev. \& mag. zool. (2) X. 1858 p. 167, \&.

Odỵnerus Guineensis Saussure. Mém. soc. physs. \& hist. nat. Genère XVII. 1563 p. 227 n.5 1, f. guttatus (Smith) D. T. - $f-$ As.: India or.

Ancistrocerus guttatus Smith, Ann. \& Mag. Nat. Hist. (2) IX. 1552 p. 49, f.

Odynerus guttatus m.

guttulosus Walk. - ㅇ - Afr.: Ad Mare rubrum.

Odynerus guttulosus Walker, List of Hymen. in Figypt $15 i 1$ p. $36 \mathrm{n} .150, \mathrm{f}$.

guzmanii Sauss. - ô-Am.: Mexico.

Odynerus Ancistrocerus) Guzmani Saussure, Rev. \& mag. zool. (2) IX. 1457 p. 275.

Odynerus (Stenancystrocerus) Guzmani Saussure, Smithson. Miscell. Collect. XIV. P.251.

haematodes Brullé - $ᄋ$ of - Afr.: Ins. Canarienses.

[1875 p. 206 n. 41, ఫ๋.

Odỵnerus haematodes Brullé, Barker-Webb of Berthelot: Hist. nat. iles Canar. II. 15.10 [p. 89 n. 43, \& §ิ; 'T. 3 F. 14.

Odynerus (Ancistrocerus, haematodes Saussure, Etud. fam. Vespid. I. Eumén. 1552 p. 149 in. $36, q \hat{\jmath}$.

Odynerus Aneistrocerus haematodes Saussure, Reise d. Novara. Zool. II. 1. 1567 Hymen. var. rubropietus Sauss. - $\mathrm{O}-\mathrm{Afr}$ : Ins. Canarienses.

[p. 9. n. 3, ठิ.

Odynerus (Ancistrocerus rubropictus Saussure, Kitud. fam. Vespid. I. Eumén. 1852 p. 150

haleakalae Blackb. \& Cam. - $\subsetneq$ ô-Austr.: Ins. Hawaiac.

[n. 37 , ㅇ․ Odynerus Haleakalac Blackburn it Cameron, Mem. Manchester Lit. S Philos. Soe. '3 X. I sali

hannibal Sauss. - 우 $\hat{\jmath}-A$ fr.: Algeria.

[p. 214 n. 23, ๑๐े.

Odynerus Antodynerus, Ifannibal Saussure, Ktud. fam. Vespid. III. Masar. $1<.5 t$ p. 291 n.179,

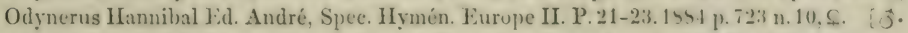

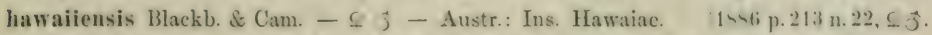
Odynerus Hawaiiensis Blackburn \& Cameron, Mem. Manchester Lit. \& Philus. Soc. 3) X. helretius Sauss. - o ơ - Eur.: Gallia, Alpes.

Odynerus parwulus Herrich-Schaeffer, Faun. Insect. German. 1 s.35 P'.154 T. 19 nec J.ep.). Odynerus Helvetius Saussure, Ktud. fam. Vespid. III. Masar. 1556 p. 245 n. 135 . 3 :

[T. 13 F.6(Q).

Odỵnerus :Leionotus Helvetius Schenck, Jahrb. Ver. Naturk. Nassau XVI. 1461 p. 6ti, is d

$\{78$ ก. 12, 웅. 
Microdynorus IIelvetieus 'Thomson. Ifymen. Seandin. III. Isit p. 59 n. 2,$5 ;$.

Odynerus (Leionutus; Helvetius Rudow. Arch. Ver. Fr. Naturg. Mecklenburg XXX. is if [p. 229 n. 9,90 .

Odynerus Mierodỵnerus, Helvetius Ed. André. Spec. Hỵmén. Europe II. P. 21-23. isst herrichianus Sauss. - $f$ f - Eur.: Germania.

[p. 732 n. $3,907$.

Odynerus Symmorphus Ierrichiamus Saussure, litud. fam. Vespid. III. Masar. 1 -.j6 p. 191

[n. 85, ठิ.

Odynerus Symmorphus) Iferrichiamus Schenck, Jahrb. Ver. Naturk. Nazsau XVI. 1sti1 [p. $36,38 \& 40$ n. 4, \& 0 .

Odrnerus Herrichianus Rudow, Arch.Ver.Fr.Naturg. Mecklenburg XXX. 1 s 66 p. 220 n.9. .5 .

Odynerus Herrichianus Jid. André. Spee. II ymén. Europe II. P. 21-23. 1554 p. 6661 n. 6.5 . 3.

herrichii Sauss. - 우 $\widehat{\sigma}$ - Eur. centr.; As.: Sibiria.

Odynerus rariegatus Herrich-Schaeffer, Faun.Insect.German.1 399 P.173 T.2u S [nee Fabr.]. Odynerus Pseudepipona) IIerrichii Saussure, Étud. fam. Vespid. III. Masar. 1 st p. 309 Odynerus basalis Smith, Catal. Hymen. Brit. Mus. V. 16.57 p. 55 n. 51 . . . [n. 193, 5 j. Odynerus (Leionotus, Merrichii Schenck, Jahrb. Ver. Naturk. Nassau XVI. 1561 p. 64, 66,

72\& 5 i n. $5, \subseteq$ ô.

Odynerus I.eionotus Herrichii F. Morawitz. Horae soc. entom. Ross. IV. 1467 p. 123 n. 11. Odynerus basalis Smith, Entomol. Annual f. 1869 p. 75; Fig. front. 1, ㅇ.

Odynerus Herrichii Rudow, Arch.Ver. Fr. Naturg. Mecklenburg XXX. 1 s 6 p. 230 n.10, $f$ J Odynerus basalis Dale, Entom. M. Magaz. XV. 1879 p. 257.

Odynerus Hoplopus: basalis F. Saunders, Trans. Entom. Soc. London 1552 p. 175 n. 5. Odynerus Herrichii Jid. André, Spec. Hymén. Europe II. P. $21-23.1554$ p. 697 n. $7, \subseteq 5$. Odynerus basalis Ed. André, Spec. Hỵmén. Europe II. P. 21-26. 1556 p. - 6 s, S.

Odynerus (Epipona Ierrichii Ritsema, Notes Ieyden Mus. VI. 154t p. 200.

heydenianus Sauss. - - - Afr.: Abyssinia. [XVII. 1. 1sti3 p. 211 n.39, §; T.2 F. 20. Odynerus (Hypancistrocerus Heydenianus Saussure, Mém. soc. phỵs. \& hist. nat. Genève hidalgo Sauss. - $ᄋ$ oิ - Am.: United States, Mexico.

Odynerus Hidalgo Saussure, Rev. \& mag. zool. (2) IX. 1857 p. 275.

Odynerus IIidalyi Saussure, Smithson. Miscell. C'olleet. XIV. P. 254. 1575 p. 252 n. S2. 53.

hildebrandtii Sauss. - + ô - Afr.: Madagasear.

[우후 T. 17 F.10.

Odynerus ILildebrandti Saussure, Grandidier: Hist. Madagasear XX. P. 1. 1591 p. 166 n 4 ,

histrio Lep. - f o - Am.: United States.

Odynerus histrio I.epeletier, Hist. nat. Insect. Iỵmén. II. $1 \$ 11$ p. 639 n. 23, \&.

Odynerus (Ancistrocerus) ammonia Saussure, Etud. fam. Tespid. I. Eumén. 15.52 p.144 n.30. . .

Odynerus (Ieionotus) histrio Saussure, litud. fam. Vespid. I. Eumén. 14.i2 p. 20s n. 112. C. Odynerus histrio Saussure, Etud. fam. Vespid. III. Masar. 1856 p. 242.

Odynerus Stenancystrocerus) histrio Saussure, Smithson. Miscell. Collect. XIY. P. 25I.

hospes Duf. \& Perr. - oૈ - Eur.: Gallia.

$[1575$ p. 199 n. $36,907$. Odynerus hospes 1)ufour \& Perris, Ann. soc. cutom. France IX. 1 - 10 p. 21 n. $9, \hat{j}$.

Odynerus hospes Ed. André, Spee. Hymén. Europe II. P. 21-23. $14-1$ p. 714 n. 12.5.

lottentottus Sauss. -

[[nec Herr.-Schacff.].

Odynerus (Teiomotus postieus Saussure, Etud. fam. Vespid. I. Eumén. 15.32 p. 214 n. 120.5

Odỵerus Antepipona IIottentotus Saussure, Litud. fam. Vespid. III. Masar. 15,56 p. $211 . \mathrm{C}$.

huastecus Sauss. - 우 ô - Am.: Mexico.

Odynerus IIuastecus Saussure, Rer. d mag. zoul. 2] IX. 1457 p. 27?. [p. 340 n. 134, C 3. Odynerus (Stenodynerus) Huastecus Saussure. Smithson. Miscell. ('ullect. XIV. P. 254. I is var. sobrinus Sauss. $-\vec{o}-$ Am.: Mexico.

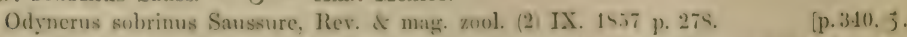
Odynerus IIuastecus var. sobrinus Saussure, Suithson. Mi*cell. Collect. XIV. P.254. 14i5 
humbel Rad. - $f-$ Afr.: Angola.

Odynerus Humbei Radoszkowski, Jorn. acad. se. Math. ctc. Iisboa VIII. 1551. p. 20.5 n.56, c.

humbertianus Sauss. - 우 ơ - As.: Ceylon.

[p. 13 n. 11, ᄋ ఠే.

Odynerus (Leionotus) Humbertianus Saussure, Reise d. Novara. Zool. II. 1. 1567 Hymen.

humeralis Hal. - 오 of - Am.: Chili, Columbia.

Odynerus humeralis IIaliday, Trans. Iinn. Soc. London XVII. :3. 1536 p. 324 n. 35, 5.

Odynerus Chilensis Lepeletier, Ilist. nat. Insect. Hymén. II. 1511 p. 613 n. 28 ,

Odynerus Chilensis Spinola, Gay: Hist. fis. Chile. Zool. VI. 15i1 p. 2.) n. 1, (.

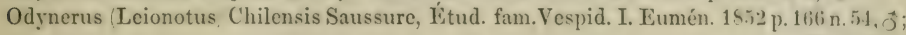

[T. 17 F.6.

Odynerus (Hypodynerus) humeralis Saussure, Gtud. fam. Vespid. III. Masar. 145ti p. 22 Odynerus (Hypodynerus, humeralis Saussure, Smithson. Misecll. Collect. XIV. P. 254. 1५

hungarieus André - $\&$ - Eur.: Hungaria.

[р. 223 n. 56, 웅ํำ. Odynerus Hungaricus Ed. André, Spee. Hymén. Europe II. P. 21-23. 1841 p. 777 n. 29, ธ.

hmo Sauss. - - - Am.: United States.

Odynerus (Antodynerus) Huro Saussure, Etud. fam. Vespid. III. Masar. 1556 p. 297 n. 15 i.

Odynerus Stenodynerus' Turo Saussure, Smithson. Miscell. Collect. XIV. P. 254. 14i5

hyacinthae Grib. - 우 ô- Afr.: Gabon.

[p. 35 I n. 147 , 우.

Odynerus Ifyacinthae Gribodo, 13ull. soc. entom. Ital. XXIII. 1 S91 p. 290, $\subseteq \overrightarrow{0}$.

hyalinipennis André - 우 - Eur.: Russia.

Odỵerus hyalinipennis Ed. André, Spee. Hymén. Europe II. P. 21-23. 185.1 p. 746 n. 15 . ऽ.

javanus Sauss. - - - As.: Java, Batavia.

[n. 9, \&; T. 1 F. 7 .

Odynerus Leionotus) Jaranus Saussure, Reise d. Norara. Zool. II. 1. 1567 Hymen. p. 12

iberieus Sauss. - $\mathrm{Q}-$ Eur.: Hispania.

[n. 17, o; T. 1 F. 11

Odynerus (Leionotus) Ibericus Saussure, Reise d. Norara. Zool. II. 1. 1 Sti7 Hymen. p. 1 i

Odỵnerus Ibericus Ed. André, Spee. Hymén. Europe II. P. 21-23. 1554 p. 742 n. 10, ๔.

ignobilis Smith - $\&-$ As.: Celcbes.

Odynerus ignobilis Smith, Journ. of Proc. Linn. Soc. Zool. IV. 1960 Suppl. p. $\varsigma 7$ n. 1, \&.

imbecillns Sauss. - $̋$ - Afr.: Sierra Leone.

[n. $5, \widehat{o}$.

Odynerus (Aneistroeerus) imbecillus Saussure, Kitud. fam. Vespid. I. Eumén. 1952 p. 12li

Odynerus 'Aneystrocerus) imbecillus Saussure, Etud. fam. Vespid. III. Masar. 15.66 p. 197.

impulsus Smith - 우 - As.: Morty.

Odynerus impulsus Smith, Journ. Linn. Soc. Zool. VIII. 1864 p. 88 n. 4, \&.

impunetatus Spin. - ơ - Afr.: Aegyptus.

—- Savigny, Descr. de l'Égypte. Hymén. 1812; T. 9 F. 5.

Odynerus impunctatus Spinola, Ann. soe. cntom. France VII. 1s.34 p. $503 \mathrm{n}$. XI.VII. $\vec{\jmath}$.

Odynerus Aneystrocerus impunetatus Saussure, Etud. fam. Vespid. III. Masar. 14.56 p. $21: 3$

[n. 103, ô.

Odỵerus Ancistrocerus impunctatus Saussure. Mém. soc. phys. of hist. nat. Genève XVII.

[1. 1863 p. $21.4 \mathrm{nn} .42$, ठ․

Odynerus impunctatus Fed. André, Spee. Hymén. Lurope II. P. $21-23.15-4$ p. 6 i 7 n 13, s.

inea Sauss. - 0 ơ - Am.: Peru.

Odynerus Stcnodynerus) Inea Saussure, Rer. A mag. zoul. 2) XXII. 1571 p. 10.5 n. 29 . S j.

Odynerus Stenodynerus Inea Saussure. Smithson. Miscell. Collect. XIV. P. 25. ISis

incommolus Sauss. - ô- Am.: Columbia, Cuba.

[p. 335 n. 134, 웅.

()dynerus ineommodus Saussure. Sagra: Ifist. fis. ('uba VII. IS.56 p. 76i9; T. 19 F. 2 iS .

Odynerus Aneistroecus incommodus Saussure. Ktud. fam. Yespid. I. Eumén. 1452 p. 1.13

11. 29,8 .

Odynerus (Stenancystrocerus' incommodus Saussurc, Smithson. Miscell. Collect. XIV.

[P. 254. 1575 p. 198 n. 34 . 
ineonstans Sauss. $-\subseteq-$ Afr.: Abyssinia. (1. 156.3 p. 217 n. 44, , ; T. 2 F. 24. Odỵnerus 'Stenancistrocerus ineonstans Saussure, Mém. soc. phys. \& hist. nat. Genère X VII. industrius Duf. \& Perr. - 9 - Eur.: Gallia.

Odynerus industrius Dufour \& Perris, Ann. soc. entom. France IX. 1540 p. 23 n. $\$, ~ \varsigma$. innumerabilis Sauss. - $ᄋ$ ô - Afr.: Algeria.

Odynerus (Leionotus) innumerabilis Saussure, Litud. fam. Vespid. I. Lumén. 1452 p. $1 \varsigma 9$ Odynerus innumerabilis Saussure, 1tud. fam. Vespid. III. Masar. 1556 p. 233, f. [n. 56 , 3. Odỵnerus innumerabilis Ed. André, Spec. Hỵmén. Europe II. P. $21-23.154$ p. fi96 n. 13, Ĵ. inoruatus Walk. - $\odot-$ Afr.: Aegyptus.

Odynerus inornatus Walker, I.ist of Hymen. in Egrpt 1571 p. 37 n. 152, s.

instabilis Smith - $\odot$ o - Afr.: Senegal.

Odynerus instabilis Smith, Catal. Hymen. Brit. Mus. V. 1557 p. 73 n. $152, \subseteq \delta$.

insularis Smith - o - As.: Celebes.

Odynerus (Leionotus; insularis Smith, Journ. of Proc. Iinn. Soc. Zool. III. 15.59 p. 21 n. 3, J.

insulicola D. T. - 우 ô - Eur.: Sicilia.

[[nec Smith].

Odynerus insularis Ed. André, Spee. Hymén. Europe II. P. 21-23. 1854 p. 718 n. 4. $\subseteq 3$ Odynerus insulicola m. [nee Blackburn \& Kirby].

intendens Walk. - - - As.: Ceylon.

Odynerus (Aneystrocerus) intendens Walker, Ann. \& Mag. Nat. Hist. 3) V. 1860 p. 304, f. interruptus (Brullé) Sauss. - 워 - Eur.: Graecia.

Polistes interrupta Brullé, Expéd. sc. Morée. Zool. II. 1832 p. 360 n. 799, f; T. 50 F. 1. Pterocheilus interruptus Herrich-Schaeffer, Faun. Insect. German. 1S:11. P. 176 T. $15(\vec{\jmath})$. Odvnerus (Antepipone) interruptus Saussure, Ėtud. fam. Vespid. III. Masar. 1856 p. 301 [n. 191, ᄋ. Odynerus interruptus Ed. André, Spec. Hymén.Europe II. P.21-23. 1554 p.751 \&. 784 n.6, S Oे. intrieatus Smith - ot - As.: India. Odynerus intricatus Smith, Catal. Hỵmen. Brit. Mus. V. 1857 p. 59 n. 60.

invisus D. T. $-f$ o - Afr. Aegyptus.

— Savigny, Deser. de l'Égypte. Hymén. 1812; T. 8 F. 14.

I.eptochilus parrulus Saussure, litud. fam. Vespid. I. Lumén. 15.22 p. $237, \subseteq$ of nec I.ep . . Odynerus invisus $\mathrm{m}$.

jocosus Gerst. - ô - Afr.

Odynerus jocosus Gerstaecker, Arch. f. Naturg. XXXVII. 1.1570 p. 351 n. $14 . \vec{\jmath}$.

Odynerus jocosus Gerstaeeker, v. d. Deeken: Reise in Ost-Afrika. Gliederthiere. 1873.

jonins Sauss. - 우 - Eur.: Gallia, Rhodus. [p.323 n.14, ช.

Odynerus (Hỵodỵnerus) Jonius Saussure, Etud. fam. Yespid. III. Masar. 1556 p. 263 n. 1 43. S. Odyuerus Jonius Ld. André, Spec. Ifymén. Europe II. P. 21-23. $15 \$ 4$ p. 7os n. 6. S.

iturbidi Sauss. $-\hat{o}-$ Am.: Mexico.

Odynerus Iturbidi Saussure, Rer. \& mag. zool. (2) IX. 1557 p. 27t, J. T. T. 3 F. 15 \& 153 . ()dynerus Iturbidi Saussure, Smithson. Viseell. Collect. XIV. P. 25. 1575 p. 265 n. $89, \hat{3}$; jueundus Moes. $-\subseteq \hat{s}$ - Eur.: Ilungaria; As. minor. [1553 p. 49 n. $63, \subseteq 3$. Odynerus Ancistrocerus) jucundus Mlocsary, Magy. Akad. 'Termész. Krtek. XIII. P. 11. Odỵnerus jucundus lid. André, Spee. Iỵmén. Europe Il. P. 21-23. 1551 p. 666 n. 6, s J. jurinei Sauss. - $q-$ Eur.: Gallia.

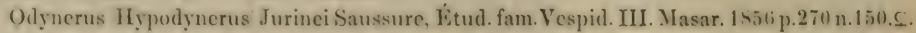
Odynerus Jurinei Ed. André, Spee. Hỵmén. Europe II. P. 21-23. 1 sht p. 717 n. 3. S. kalinowskii Rad. - $\odot-A s .:$ Corea.

(Odynerus Kalinewskii Radoszkowski. Horae soc. entom. Ross. XXIV. 1s!0 p. 231, S. 
kennicotianns Sauss. - 으 - Am. : Illinois.

Odynerus. Stenodynerus) Kennicottianus Saussure.Rer.(mag.zool. 2 XXII. 1 si 1 p. 104 n.23. Odynerus (Stenodynerus) Kennicotianus Saussure, Smithson. Miscell. Collect. XIV. P. 251.

kirbyi D. T. - \& - Austr.: Kauai.

[1875 p. $325 \mathrm{n} .125$, 우.

Odynerus extraneus Blackburn \& Kirby. Entom. MI. Magaz. XVII. 15-1) p. 86 n. 12, $f$ nec Odynerus Kirbyi Dalla Torre, Wien. entom. Zeitg. VIII. 1849 p. 125.

[Sauss.].

kokpetieus Rad. - + ơ - As.: 'Transcaspia.

[ใ ơ; T. 10 T. 50.

Odynerus (I.eionotus) Kokpeticus Radoszkowski, Horac soc. cntom. Ross. XX. 1886 p. 46 ,

komarowii Mor. - - - As.: Transcaspia.

Aneistrocerus Komarowi F. Morawitz, Horac soc. entom. Ross. XIX. 1S5.5 p. 175 n. 24, . . Odynerus Ancystrocerus, Komarowi Ed. André, Spec. Hỵmén. Europe II. P. 21-26. 1 hsti

labiatus Hal. - 우 $゚$ - Am.: Chili.

[p. 876, \&.

Odynerus labiatus Haliday, Trans. Linn. Soc. London XVII. 3. 1536 p. 321 n. 34, 5. Odynerus Lachesis Lepeletier, Hist. nat. Insect. Hymén. II. 1541 p. 667 n. 45 , S.

Odynerus marginicollis Spinola, Gay: Hist. fis. Chile. Zool. VI. 15501 p. 256 n. $2, f 0$. Odỵnerus (Leionotus) Lachesis Saussure, Étud. fam. Vespid. I. Eumén. 1 \$52 p. 164 n. 51 , [Q Оิ; T. 17 F. 5 (Q).

Odynerus (Hypodỵnerus) labiatus Saussure, Étud. fam. Vespid. III. Masar. 1856 p. 22. Odynerus Hypodynerus labiatus Saussurc, Smithson. Miscell. Collect. XIV. P. 25 1. 1875

laborans Costa - $\subsetneq$ ô - Eur.: Sardinia.

[p. 222 n. 54, 우

Odynerus (Ieionotus, laborans Costa, Atti accad. sc. fis. Napoli IX. P. 11.1552 p. 37 n.26, j.

Odynerus laborans Costa, Atti accad. sc. fis. Napoli (2) I. P. 2.1553 p. 91, o.

Odynerus laborans Ed. André, Spec. Hymén. Europe II. P. 21-23. 155.1 p. 716 n. 2, ô.

Iaboriosus Smith - ô - As.: Mysol.

Odynerus laboriosus Smith, Journ. of Proc. Linn. Soc. Zool. VII. 1863 p. 41 n. (i, ô laminiger Grib. - ô- As.: Borneo.

Odynerus laminiger Gribodo, Bull. soc. entom. Ital. XXIII. 1891 p. 299, ş.

laplatae Saussure - $\&$ of Am.: Argentinia.

Odynerus (Protodynerus) Laplatae Saussure, Rev. \& mag. zool. (2) XXII. 1 s 71 p. 57 n.7, f 3 . Odynerus Pachodynerus, Laplatae Saussure, Smithson. Niscell. Collect. XIV. 1.25.1. 1>75

latipenuis Smith - - As.: Borneo.

[p. 240 n. 76, ㅇ․

Odynerus Symmorphus, latipennis Smith, Journ. of Proc. Limn. Soc.Zool. II. 1 S5 i p.112n.6.

leucomelas Sauss. $-\propto$ oิ - Am.: Canada, United States.

[n. 131, \&

Odynerus (IYpodynerus leucomelas Saussure, Ktud. fam. Vespid. III. Masar. 15.6 p. 255 Odỵnerus leueomelas Saussure, Smithson. Miseell. Cullect. XIV. P. 251 . 1 55 p. 24 i n. 16:, [우우 'T. 3 F. $19 \& 19$ ‥

Odynerus leucomelas Provancher, Natural. Canad. XIII. 1442 p. 142 n. 9. \& 3.

Odỵnerus leucomelas Provancher, laun. entom. Canada. Hymén. 1844 p. 67 i n. 9. $f$ j.

levipes Shuck. - $+\hat{o}-$ Eur. centr. \& mer.; Afr.: Algeria.

Odynerus (Epipone) laevipes Shuckard, Magaz. of Nat. Hist. N. S. I. 15.37 p. $495 \mathrm{n}$ n. 2. \& j.

Pterochilus simplicipes Herrich-Schaeffer, Faun. Insect. German. 14.39 P. 173 T. Is ij.

Odyncrus cognatus Dufour, Ann. sc. nat. Zool. (2) XI. 1839 p. 92, f.

Odynerus rubicola Dufour, Ann. sc. nat. Zool. (2) XI. 1839 p. 102 ; T. 5 F. 1-4.

Odynerus cognatus Audouin, Ann. sc. nat. Zool. (2) XI. 1839 p. 104.

Odynerus rubicola Blanchard, Hist. nat. Insect. III. 1840 p. 391 n. 3.

Odynerus rubicola Dufour d Perris, Anm. soe. entom. France IX. 1840 p. 23 n. 7.

Odynerus reniformis I.epeletier, IList nat. Insect. IIymén. II. 1811 p. 6 it6 n. 1, 6 .

Epipone laevipes Blyuon, Zoologist I. 1813 p. 264.

[T. 20 F. 2 (ᄋ).

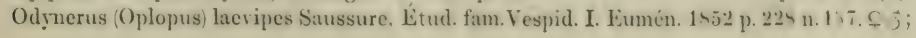


P'terocheilus simplicipes Schenek. Jahrb. Ver. Naturk. Nassau IX. 1.1 h.s3 p. 79 n. $1 . \subseteq j$. Odynerus laeripes Saussure, Étud. fam. Vespid. III. Masar. 1856 p. 308.

Odynerus (Oplopus laevipes Smith. Catal. J3rit. Fossor. Hymen. $15 . i 5$ p. 204 n. 5. C j. Odynerus (IIoplopus, laevipes Schenek, Jahrb. Ver. Naturk. Nassau XVI. 1 4it1 p. 42, 53 \& (-57 Odynerus rubicola Disconzi, Entom. Vicent. 1615.5 p. 126 ; T. 9 F. 139 \& 141 . n. 5. $0 \vec{o}$. ()dynerus laevipes Giraud, Ann. soc. entom. France (4/VI. 1566 p. $463 \mathrm{n}$ n. 12. 9 j.

Odyncrus laeripes Thomson, Opusc. cntom. P. 2.1870 p. 84 n. 4, \&.

Hoplumerus laevipes Thumson. Hymen. Seandin. III. 147.t p. 4 i n. 4 , 93 .

Odynerus rubicola Regimbart, Feuille jeun. natural. IV. 1874 p. 89-91.

Odynerus laevipes Rudow, Arch. Ver. Fr. Naturg. Mecklenburg XXX. 15 i6 p. 232 n. 3.85.

Odynerus (Hoplopus) lacvipes E. Saunders. Trans. Entom. Soc. London 1552 p. 171 n. ?. Odynerus rubicola Fabre, Nour. sour. entom. 1892 p. 234-236.

Odỵerus laevipes Ed. André. Spec. Hỵmén. Europe II. P. $21-23.14-4$ p. $756 \& 7$ \&5 n. $12 . \subseteq j$. Hoplomerus laevipes Borries, Entom. Meddel. 1888 p. 202-211.

Pterocheilus laevipes Verhoeff. Verh. naturh. Ter. preuss. Rheinl. XI.VIII. $14 ! 11$ p. 15-16 Hoplopus laevipes Verhoeff, Zool. Jahrb. Syst. II. 1592 p. $739 . \quad$ n. 13. Q 3; T. 2 T. 11-50. limbiferus Mor. - 오 주 - Eur.: Dalmatia.

[욱.

Odynerus Leionotus) limbiferus F. Morawitz, Horae soc. entom. Ross. IV. 14ti p. 136 nota. Odynerus limbiferus F. Morawitz. Horae soc. entom. Ross. V. 1864 p. 149 n. 13, $\subseteq j$. Odynerus limbiferus Ed. André. Spee. Hymén. Europe II. P. 21-23. 1 s4 p. 722 n. $10, \subseteq \delta$.

lobatus André - 우 đ̋ - Eur.: Sicilia, Graecia, Caucasus.

Odynerus (Ancistrocerus) lobatus Ed. André, Natural. Sicil. II. 155:3 p. 329, $\subseteq$.

Odynerus lobatus Ed. André, Spec. Hymén. Europe II. P. 21-23. $15 \varsigma 4$ p. 667 n. 7, $\subseteq$ j. Iongispinosus Sauss. - 0 - Afr. Algeria.

[n. 102, ठే.

Odynerus (Aneystrocerus) longispinosus Saussure, Étud. fam. Vespid. III. Masar. 1 s.jti p. $21:$ :

lueasius (Maindr.) D. T. - O - Austr.: New Guinea.

Ancistrucerus Lueasius Maindron, Amn. soc. cntom. France (fi) II. 1 s\$2 p. 2\$2, S; T. 3 T. 11. Odynerus Lucasius $\mathrm{m}$.

Inctuosus Sauss. - 우 ‡َ-Am. bor.?

Odynerus Pseudodynerus; lutuosus Saussurc, Etud. fam. Tespid. III. Masar. 155li p. 220

Odynerus Stenodynerus luctuosus Saussure. Smithson. Misecll. Collect. XIV. P. 254.1 15.5

Iuteolus Lep. - ồ - Afr.: Algeria.

[p. 311 n. $114, \Omega \Im^{7}$.

? Vespa labiata Fabricius \& auct.

Odynerus luteolus Jepeletier, Hist. nat. Insect. Hỵmén. II. 1541 p. $616 \mathrm{n} . \vec{\imath}, \hat{o}$.

Odynerus luteolus Lueas, Explor. se. Aléréc. Zool. III. 14.46 p. 231 n. 1 st, j̧; T. 11 F. 4.

()dynerus (I.eionotus) luteolus Saussure, Etud. fam. Tespid. I. Eumén. 1452 p. 216 n. 123.

Odỵerus (Antepipuna: luteolus Saussure, Etud. fam. Vespicl. III. Masar. 1856 p. 245.

Odynerus luteolus Fil. André. Spee. Hỵmén. Europe II. 1'. 21-23. 155 p. 7.15 n. 1. 3.

macilentus Sauss. - $\subseteq-$ Austr.

Odyneru- (I.cionotus, macilentus Saussure, Reise d. Novara. Zoul. II. 1. 1 sti IIỴmen. p. 16

mactre Lep. - - 우 $\widehat{o}-$ Afr.: Algeria.

(n. $15, \$ ;$ T. 1 F. 10.

Odynerus Mactate Iejeletier, Hist. nat. Inscet. Ifymén. II. 1411 p. 639 n. $24 . \hat{\jmath}$.

Odynerus Mactae Lucas, Jixplor. se. Algérie. Zool. III. 14.16 p. 2:31; n. 192, 3.

Odynerus Ideionotus Mactae Saussure. Litud. fam. Vespid. I. Jumén. 1<52 p. 159 n. 43.

Odynerus Mactae Sau-sure, Etud. fam. Vespid. III. Masar. Lis p. 225, S J; T. 10 F. 7 .

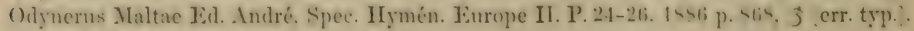

maculipenuis Smith - 으 - As.: Borneo.

Odynerus maculipennis Smith. Joum. of Proe. Linn. Soe. Zoul. II. hasi p. 111 n. 4. C. malerae Sauss. - \&- Afr.: Madeirn.

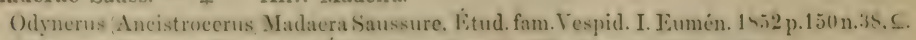
Oilynerus Maderae Saussure, Eitud. fam. Vespid. III. Masar. 14.t6 p. 20 i. 
magnifieus Mor. - - Eur.: Russia.

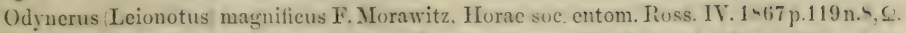

Odynerus magnifieus Ed. André, Spee. Iymén. Europe II. P. $21-23.1541$ p. 69.4 n. 4, S. magrettii Grib. - 오

Odynerus Magrettii Gribodo, Am. mus. cir. Genora XXI. 1551 p. 290 nota, $\subseteq \hat{\jmath}$.

Odynerus Magrettii Magretti, Ann. mus. civ. Genova XXI. 1941 p. 619 n. 155.

maindronii D. T. - 우 ơ - As.: Neu Guinea.

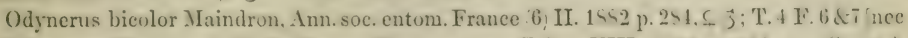

Odynerus Maindronii 1)alla T'orre. Wien. entom. Zeitg. YIII. $15 \varsigma 9$ p. 124. Sauss. .

mammillatus Mor, - 우 - As.: Persia.

Hoplomerus mammillatus F. Morawitz, Horac soc. entom. Ross. XIX. 144.5 p. 14!9 n. 9.

Odynerus (Hoplomerus) mamillatus Ed. André. Spee. Hymén. Europe II. P. 24-26. 1s‘ij

mandibularis Mor. - $\widehat{o}-$ As.: Transcaspia.

[p. 883, ㅇ.

Hoplomerus mandibularis F. Morawitz, Horae soc. entom. Ross. XIX. 164.) j. 15s n. 14, of.

Odynerus (Ioplomerus) mandibularis Ed. André, Spee. Hymén. Europe II. P. 24-26. 15nt;

manifestus Smith - ơ - As.: Borneo, Java.

[p. 886, ô.

Odynerus manifestus Smith, Journ. of Proc. Limn. Soc. Zool. II. $1 \varsigma 57$ p. 110 n. 2, ŝ.

maroceanus D. T. - 우 ô - Eur.: Sicilia; Afr.: Marocco.

Odynerus Leionotus; insularis Ed. André, Natural. Sicil. II. 1553 p. 232, \& $\hat{u}$ [nee Smith? Odynerus Maroccanus m.

massanensis Sauss. - 으-Afr.: Abyssinia.

[n. 41, ᄋ; 'T. 2 F. 22.

Odỵnerus Massauensis Saussure, Mém. soc. phỵs. \& hist. nat. Genève XVII. 1. 1 14i3 p. 213

Odynerus Hypancistrocerus) Massauensis Magretti, Anr. mus. cir. Genora XXI. 144

mauritanieus (Lep.) Sauss. - $ᄋ$ of - Afr.: Mauritius.

[p. $621 \mathrm{n} .162$.

Pterochilus Mauritanicus I.epeleticr, Hist. nat. Insect. Hỵmén. II. 14.41 p. 675 n. $4, \subseteq \hat{j}$.

Pterochilus Mauritanicus Iucas. Explor. se. Algéric. Zool. III. 1811 i p. 242 n. 201 ; T. 12 F. 1.

Ieptochilus Mauritanicus Saussure. Étud. fam. Vespid. I. Eumén. 1852 p. 233 n. 1.

Odynerus (Parodyneru', Mauritanieus Saussure, Etud. fam. Vespid. I. Eumén. 1532 p. 321.

Odỵnerus Mauritanicus Ed. André, Spec. Hymén. Europe II. P. 21-23. 1 s4. p. 725 n. 13.

maya Sauss. - 우 하- Am.: Mexico.

[ᄋ ठ; T. 43 F.3.

()dynerus Maya Saussure, Rev. \& mag. zool. 2) IX. 1457 p. 279. \{p.3334n.137. @ j.

Odynerus Stenodynerus Mayus Sausure, Smithson. Miscell. Collect. XIV. P. 254. 1575

maypinus Sauss. $-\rho \cdot \tilde{\jmath}-\Lambda \mathrm{m}$ : Chili.

Odynerus Maypinus Sau-sure. Gay: Irist. fis. (hile. Zool. VI. IS5̆ p. ilit n. 5. $\vec{j}$.

Odynerus Leionotus, Maipinus Sausure, Litud. fam. Vespid. I. Lumén. 14.j2 p. 169 n. (iৎ. j. Odynerus Hypodynerus Maipinus Saussure. Gitud. fam. Vespid. III. Ma-ar. 14.76 p. 229. Odynerus (IIypodynerus) Maypinus Saussure, Smithson. Mi cell. Collect. XIV. P. 251. 1 - is

medanae Grib. - $Q-A f r:$ Tunis.

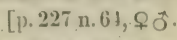

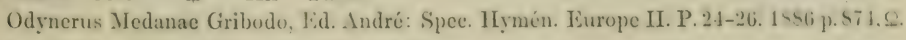

mégaera Lep. $-q \hat{\sigma}-A$ m.: United States.

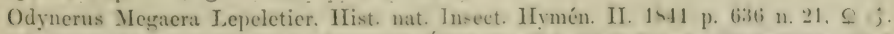

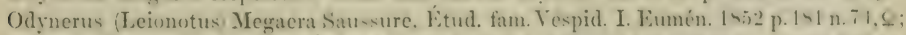

Odỵnerus Megaera Saussure, Ktud. fum. Tespid. III. Masar, 14jti p. 232 . T. 17 F.11.

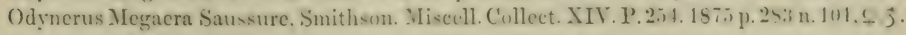

melanocephalus (Gmel.) Wesm. - $Q$ ô - Lur. fere tota.

Vespa spec. Zschach, Mus. Leskean. 1788 p. 75 n. 420.

Vispa melanocephala Gmelin. Iinné: Sist. nat. Ed. $13^{2}$ I. 5.1790 p. 2760 n. 96.

Vespa albofasciata Rossi, Fauna Etrusca II. 1790 p. 87 n. 870 , ㅇ.

Vespa spinipes Olivier, Encyel. méthod. Insect. VI. 1791 p. lisi n, s7, $\vec{\jmath}$. 
Vespa melanocephala Olivier, Encycl, méthod. Insect. VI. 1791 p. 696 n. 13.

Odynerus spinipes Spinola, Insect. Ligur. II. 1806 p. 89 n. 3, 우 ơ.

Vespa allofasciata Illiger, Rossi: Fauna Etrusea Ed. 2 II. 1807 p. 143 n. 570.5 .

Odynerus spinipes Spinola, Insect. Ligur. II. 1808 p. 186 ń. 3.

Odynerus melanocephalus Wesmael, Monogr. Odyner. Belgique 1633 p. 12 n. $3, \subseteq \hat{o}$. (Odynerus (Oplopus melanocephalus Wesmael, Bull. acad. se. Belgique III. 1536 p. 45. Odynerus melanocephalus I.epeletier. Hist. nat. Insect. IIymén. II. 1511 p. 610 n. $5 . \subseteq$ j. Pterocheilus dentipes Herrich-Schaeffer, Faun. Insect. German. $1 \sim 41$ P. 1 i6; T. 16 Fig. a ' $\vec{j}$. Odỵerus Oplopus melanocephalus Saussure, Étud. fam. Vespid. I. Eumén. 1552 p. 224 [n. 132, 웧 T T. 20 F. $3($ ㅇ) .

Pterocheilus dentipes Schenck, Jahrb. Ver. Naturk. Nassau IX. 1. 1 hi3 p. 54 n. 15, C J. Odynerus melanocephalus Saussure. Etud. fam. Vespid. III. Masar. 1556 p. $305, \subseteq 3$. Odỵnerus Oplopus, melanoecpholus Smith, Catal. Brit. Fossor. Hymen. 1与54 p. 205 n. 6,

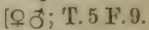

Odynerus Hoplopus melanocephalus Schenck, Jahrb. Ver. Naturk. Nassau XVI. $1 \uparrow 61$ p. \$1. Pterocheilus serripes Taschenberg, Hymen. Deutschl. 1566 p. $24 i, \leq j$. S3. 5.5 n. $3 . \subseteq j$. Odynerus Fpipone melanocephalus F. Morawitz, Horae soc. entom. Ross. IV. Isti p. $1: 3 i$ Odynerus melanocephalus Thomson. Opusc. entom. P. 2.1570 p. 84 n. $3, \subseteq j$. n. 24. Hoplomerus melanocephalus Thomson, Hymen. Scandin. III. 1574 p. 46 n. $3 . \subseteq \hat{\jmath}$. Odynerus melanocephalus Rudow, Arch. Ver. Fr. Naturg. Mecklenburg XXX. 1576 p. $2: 31$ Hoplopus melanocephalus H. Müller, Alpenblumen $1 S \$ 1$ p. 592 n. 606 . n. $1 . \subseteq j$. Odynerus 'Hoplopus) melanocephalus E. Saunders. Trans. Entom. Soc.London 1452 p. 17 in. 2. Odynerus melanocephalus Ed. André, Spec. Hymén. Europe II. P. $21-23.15 \$ 4$ p. 764 \& 776 Hoplopus melanocephalus Rudow, Soc. entom. I. 1557 p.16.4. [n. 25, S 5ే; T.36 F.15. melanocerus D. T. - ô-As. centr.

Ancistrocerus nigricornis F. Morawitz, Horae soc. entom. Ross. XXIII. 1859 p. 161 n. $\hookrightarrow \cdot \vec{\jmath}$ Odynerus melanocerus $\mathrm{m}$.

melanurus (Mor.) D. T. - ô - As.: Mongolia.

[[nec Morawitz 1885].

Ancistrocerus melanurus F. Morawitz, Horac soc. entom. Ross. XXIII. 1 ss? p. 162 n. 11. j. Odynerus melanurus $\mathrm{m}$.

melauns Sauss. - ㅇ- Afr.: Madagascar.

[T. 18 F. 9.

Odynerus Leionotus, melanus Sauss. İtud. fam. Vespid. I. Fumén. 1452 p. 159 n. 44. \&; Odynerus melanus Saussure, Grandidier: Hist. Madagascar XX. 1. 1.1591 p. 172 n. i, $\subseteq$. membranacens Mor. - 우 - Eur.: Russia; As.: Turcestania. [n. 23, ㅇ. Odynerus 'Leionotus, membranaceus F. Morawitz. Horae soe. entom. Ross. IV. $1>67$ p. 135 Odỵerus membranaceus Ed. André, Spec. Hỵmén. Jurope II. P.21-23. 1 s 4 p. 726 n. 16 , ᄃ. meridionalis Sauss. - $\hat{o}-$ Afr.: Cap.

[n. 38,0 .

Odynerus (Antepipone) meridionalis Saussure, Stettin. entom. Zeitg. XXIII. 1562 p. 205 mervensis (Rad.) D. 'T'. $-Q-$ As. : Korea.

Jeptochilus Mervensis Radoszlowski. IIorae soc. entom. Ross. XXI. 1557 p. 100. (. . Odynerus Mervensis m.

metathoracieus Sauss. - - - Austr.

Odynerus metathoracicus Saussure. Btud. fam. Vespid. III. Masar. Liti p. 2̧ti n. 171. S. metemmensis Magr. - ఫ - Afr. orient.

Odynerus Metemmensis Magretti, Ann. mus. cir. Genova XXI. 1s41 p. 617 n. 15ti, $\vec{j}$. mieado Kirsch - \& - As. : Japonia.

Odynerus (Leionotus! Micado Kirsch. Mittheil. zool. Mus. J)resden III. 15is p. 340. S. mierodynerus 1). 'T'. $-\subseteq-$ As.: 'Transcaspia.

[[nec Sauss.].

Mierodynerus alastoroides F. Morawitz, Horae soc. entom. Ross. XIX. 1445 p. $179 \mathrm{n}$. 26. . Odynerus Microdỵnerus, alasturoides Ed. André. Spee. Jrmén. Europe II. P. 24-26. 1s46 Odynerus Microdynerus ])alla Torre. Wien. entom. Zeitschr. VIII. 1859 p.124. [p.sig.L. 
miniatus Sauss. - $Q-$ As.: India.

[n. 124, \&; T. 11 F. 6.

Odynerus Parodynerus; miniatus Saussure, Eitud. fam. Vespid. III. Masar. 1556 p. 24!

minutus (Fabr.) Lep. - 오 $\widehat{o}$ - Eur. fere tota.

Vespa bifasciata Rossi, Fauna Etrusca II. 1790 p. 86 n. 869 [nec Linné].

Vespa minuta Fabricius, Suppl. entom. system. 1798 p. 262 n. 65-66.

Vespa minuta Fabricius, Syst. Piez. 1804 p. 268 n. 78.

Vespa minuta Jurine, Nouv. méth. class. Hymén. 1807 p. 170, ㅇ․

Vespa bifasciata Illiger, Rossi: Fauna Etrusea Ed. $2^{\text {a }}$ II. 1507 p. 143 n. .69.

Odynerus minutus Lepeletier, Hist. nat. insect. Hymén. II. 1411 p. $6: 32$ n. $14, f$ j.

Odynerus pictus Herrich-Schaeffer, Faun. Insect. German. Ist1 P. 176. 'T. 3 (S \& 'T. A

[Fig. d $(\widehat{o})$.

Odynerus (Leionotus) minutus Saussure, Fitud. fam. Tespid. I. Eumén. 1852 p. 2107 n. 110, C. Odỵnerus pictus Schenck, Jahrb. Ver. Naturk. Nassau IX. 1.14 .53 p. 69 n. $26, f \jmath$.

Odynerus minutus Schenck, Jahrb. Ver. Naturk. Nassau IX. 15.53 p. $\$ 4$ n. 6, s. $o^{*}$.

Odynerus minutus Saussure, Étud. fam. Vespid. III. Masar. 1856 p. 241 . [n. $147, f \mathfrak{f}$.

Odynerus (Hypodynerus) minutus Saussure. Eitud. fam. Vespid. III. Masar. 1556 p. 267

Odỵnerus (Leionotusi minutus Schenck, Jahrb. Ver. Naturk. Nassau XVI. 1561 p. 65,67 \& if;

[n. 9, 우우.

Odynerus pictus Brischke, Schrift. phỵs. ökon. Ges. Königsberg II. 1862 p. $115, f$ ఫे.

Odynerus (Leionotus) minutus F. Morawitz, Horae soc. entom. Ross. IV. 1567 p. 133 n. 20.

Odynerus dentisquama Thomson, Opuse. entom. P. 2. 1870 p. 86 n. 10 , 오

Leionotus dentisquama Thomson, Hrmen. Scandin. III. 1574 p. 55 n. 5.

Odynerus minutus Rudow, Arch. Ver. Fr. Naturg. Mecklenburg XXX. 1 s 6 p. 228 n. 6 , f o .

Odynerus minutus Ed. André, Spec. Hỵmén. Europe II. P.21-23. 1594 p. 719 n. 5; \& ơ; T.36 F.2

moesaryi André - 우 - Eur.: Russia.

[\& T. 43 F. 6.

Odỵnerus Moesárỵi Ed. André, Spec. Hymén. Europe II. P. 21-23. 1884 p. 741 n. 9, S. modestus Sauss. - 우 ơ - Afr.: Algeria.

Leptochilus modestus Saussure, Kitud. fam. Vespid. I. Eumén. 1552 p. 234 n. 3, \&; 'T. 20 F. 5.

Leptochilus modestus Saussure, Étud. fam. Vespid. III. Masar. 1456 p. 321 ,

Odynerus (Parodynerus, modestus Saussure, Etud. fam. Vespid. III. Masar. 1456 p. 321

Odỵnerus modestus Ed. Audré, Spec. Hymén. Europe II. P. 21-23. 1554 p. 735 n. 4, S oే.

molicanus Sauss. - $\widehat{o}-$ Am.: New York.

[n. 186, ơ.

Odynerus (Antodynerus) Mohicanus Saussure, Gitud. fam. Vespid. III. Masar. 1S54 p. 297

Odỵnerus Stenodỵnerus) Mohicanus Saussure, Smithson. Miscell. Collect. XIY. P. 2.5 I.

molestus Sauss. - 우 ơ - Am.: T'ennessee.

[1875 p. 352 n. 148, ơ.

Odynerus molestus Saussure, Rer. \& mag. zool. (2) XXII. 1871 p. 61 n. 19 , of excl. S.

Odynerus molestus Saussure, Smithson. Misecll. Collect. XIY. P. 25.1. 1575 p. 2!o n. 10.5,

molinae Sauss. - 우 - Am.: Chili.

$[0 ึ[$ excl. $\odot]$.

Odynerus Molinae Saussure, Gay: Hist. fis. Chile. Zool. VI. 1451 p. 562 n. 3.. .

Odỵnerus Hypodynerus .II linius Saussure, Etud. fam. Vespid. III. Masar. 14.i6 p. 257 n.126.C.

Odynerus (Hypodynerus Molinae Saussure. Smithson. Misecll. Collect. XIY. P. 254. 14i5

montauus Smith - $ᄋ \hat{o}-$ Austr.: Honolulu.

[p. 222 n. 53, .

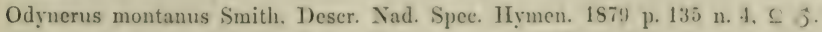

Odynerus montanus Smith, Journ. Linn. soc. zool. XIV. 1879 p. 650 , \& o

Odynerus montanus Blackburn \& Kirby, Entom. M. Magaz. XVII. 1s40 p. 4i.

morawitzii André - ô - Eur.: Russia.

Odỵnerus Morawitzi Ed. André: Spee. Hỵmén. Europ. II. P. $21-23$. lnht p. 69.5 n. 5 , 3.

morelius Saussure - $\delta$ - Am.: Mexico.

Odynerus Morelius Saussure, Rev. \& mag. zool. (2) IX. 1857 p. 276.

Odỵnerus Morelii Saussure, Smithson. Miscell. Collect. XIV. P. $25+1$ is p p. 299 n. 111. oै. 
mucronatus Sauss. - ô - Afr.: Guinea.

Odynerus mucronatus Saussure, Stettin. entom. Zeitg. XXIII. 1462 p. 203 n. 36, jै. multicolor Sauss. - 우 - Afr.: Senegal.

Odynerus (Leionotus) multicolor Saussure, Gitud. fam. Vespid. I. Lumén. 1552 p. 209 n. 113 , multipictus Smith - 으-As.: Borneo.

Odynerus multipictus Smithson, Journ. of Proc. Linn. Soc. Zool. II. 1 4; 7 p. 112 n. 5, C. Odynerus guttulatus Saussure, Stettin. entom. Zeitg. XXIII. 1462 p. 200 n. 34..

Odynerus multipictus Gribodo, Bull. soc. entom. Ital. XXIII. 14!91 p. 297, c.

murarius (L.) Westw. - $9 \hat{0}-$ Eur.: Suecia, Germania.

— Frisch, Beschr. Insect. Teutschl. IX. 1730 p. 23; T. 12 F. 8 \& 9.

- Linné, Fauna Suec. 1746 p. ? n. 991.

Vespa muraria Linné, Syst. nat. Ed. $10^{a}$ I. 1758 p. 573 n. 5.

Vespa muraria Linné, Fauna Succ. Ed. 2a 1761 p. 416 n. 1674.

Vespa muraria Poda, Insect. mus. Graec. 1761 p. 109 n. 4.

Vespa muraria O. F. Müller, Fauna Insect. Fridrichsdal 176 t p. 73 n. 6:34.

Vespa II Schaeffer, Icon. Insect. Ratisbon. I. 1. 1766; T. 24 F. 3.

Vespa muraria Linné, Syst. nat. Ed. $12^{a}$ I. 2.1767 p. 950 n. 8.

Vespa muraria Fabricius, Syst. entom. 1775 p. 365 n. 27.

Vespa muraria Ch. I. Müller, Linné: Vollst. Natursyst. V. 2.1775 p. $\$ \$ 2$ n. $\$$.

Vespa muraria O. F. Müller, Zool. Dan. prodr. 1776 p. 163 n. 1590.

Vespa muraria Fabricius, Spec. Insect. I. 1781 p. 464 n. 35.

Vespa muraria Schrank, Enum. Insect. Austr. 1781 p. 393 n. 793.

Vespa muraria Fabricius, Mant. Insect. I. 1757 p. 290 n. 40.

Vespa muraria Razoumowski, Hist. nat. Jorat I. 1. 1789 p. 218 n. 25 S.

Vespa muraria Villers, C. Linnaei Entom. III. 1789 p. 267 n. 6.

Vespa muraria Gmelin, Linné: Syst. nat. Ed. $13^{\text {a }}$ I. 5.1790 p. 2751 n. 8.

Vespa muraria Rossi, Fauna Etrusca II. 1790 p. 85 n. 866.

Vespa muraria Oliver, Encycl. méthod. Insect. VI. 1791 p. 687 n. 85.

Vespa muraria Fabricius, Entom. system. II. 1793 p. 267 n. 50.

Vespa muraria Cederhjelm, Faun. Ingric. prodr. 1798 p. 169 n. 523.

Vespa muraria Schrökenstein, Verz. d. Halbkäfer etc. 1802 p. 31 n. 19.

Vespa muraria Walckenaer, Fauna Paris. II. 1802.p. 89.n. 7.

Vespa muraria Fabricius, Syst. Piez. 1804.p. 262 .n. 49.

Vespa muraria Illiger, Rossi: Fauna Etrusca Ed. 2a II. 1807 p. 141 n. 866.

Vespa muraria Jurine, Nouv. méth. class. Hymén. 1807 p. 170, 우.

Eumenes muraria Lamarck, Hist. nat: anim. s. vert. IV. 1817 p. 83 n. 3.

Eumenes muraria Lamarck. Hist. nat. anim. s. vert. Fid. $2 *$ IV. 1535 p. $310 \mathrm{n.} 3$.

Vespa muraria Wesmael. Bull. acad. se. Belgique IV. 15:37 p. 3s!3-391; Tab. Fig. 1-3.

Odynerus murarius Westwood. Introd. mod. Classif. Inseet. II. 14t0 Synops. p. 43.

Odynerus murarius Herrich-Sehaefler. Faun. Insect. Cicrman. 1 s.11 1'. 176 'T. 12 s.

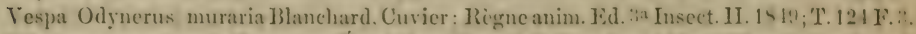

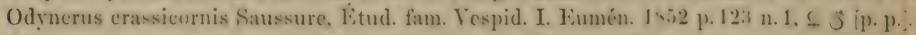

Odỵnerus murarius Schenck, Jahrb. Ver. Naturk. Nassau IX. 1. 1 -5.3 p. 8.3 n. 5,6 ;.

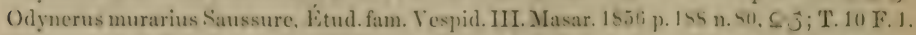

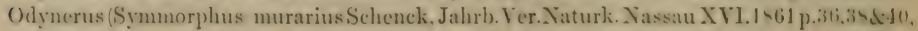

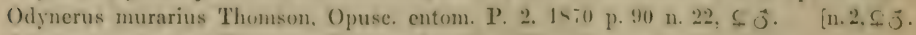

Odynerus murarius Thomson, Hymen. Scaridin. III. 1874 .p. 81 n. 1 , $f$ ot.

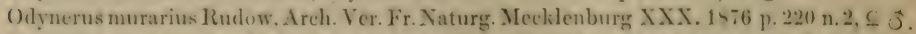

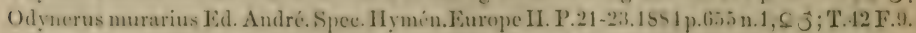

Odynerus murarius Aurivillius, Bihang Siensk. Vet.-Akad. Ifandl. XII. P. 4. 5. 1586 p. 6.

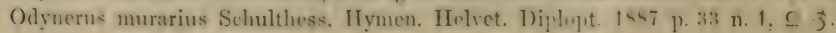


Odynerus murarius Aurivillius, Oefvers. Svensk. Vet.-Akad. Förh. XI,V. P. 10. 1558 p. 609. Odynerus murarius Alfken, Entom. Nachr. XVIII. 1892 p. 210.

Odynerus murarius King, Sc. Goss. 1892 p. 196-198; Fig.

Odynerus murarius Alfken, Entom. Nachr. XVIII. 1892 p. 210 n. 2.

mutabilis Sauss. - $\widehat{\jmath}$ - Afr.: Aegyptus, Abyssinia.

— Sarigny, Deser. de l'Égypt. 1512; T. 9 F. 13 \& $15 . \quad$ p. 231 n. 57 , 亏ै; T. 2 F. 26.

Odynerus (Antodỵnerus) mutabilis Saussure, Mém. soc. phỵs. \& hist.nat.Genève XVII.1.1563

mutaus Sauss. - $ᄋ-$ Afr.: Senegal.

[Q; T.13 F. 7.

Odynerus (Antodynerus) mutans Śaussure, Étud. fam. Vespid. III. Masar. 1556 p. 255 n. 175.

muticus Zett. - 우 - Eur. Lapponia.

Odynerus muticus Zetterstedt, Insect. Lappon. I. 1839 p. 456 n. 2, ㅇ․

Odynerus muticus Saussure, Étud. fam. Vespid. III. Masar. 1456 p. 319 n. 214.

mysolicus D. T. - $\uparrow-$ As.: Mysol.

Odynerus conspicuus Smith, Journ. of Proc. Linn. Soc. Zool. VII. 1563 p. 40 n. 4 , $f$ [nec

Odynerus Mýsolicus Dalla Torre, Wien. entom. Zeitg. WIII. 185! p. 124. Sauss. .

mystecus Sauss. - 우 ô - Am.: Mexico.

Odynerus Mỵstecus Saussure, Rev. \& mag. zool. (2) IX. 1857 p. 279 . (p.336n. 135, . 5 .

Odynerus (Stenodynerus) Mystecus Saussure, Smithson: Miscell. Collect. XIV. P. 254. 1575 nahuns Sauss. - \& - Am.: Mexico.

Odyncrus (Stenodynerus) Nahuus Saussure, Rer.\&mag. zool. (2) XXII. 1871 p. 141) n. 32, f. Odynerus Stenodynerus Nahuus Saussure, Smithson. Miscell. Collect. XIV. P. 254. 1575

[p. 355 n. 151, 오.

nasidens Iatr. $-\&$ $f-$ Am. Mexico, Columbia, Venezucla, Brasilia. [T.15 F.1-2.

Odynerus nasidens Latreille, Humboldt \& Bonpland. Voy, rég. équin. Zool. II. 1812 p. 112 ;

Odynerus (Leionotus) nasidens Saussure, Étud. fam. Vespid. I. Eumén. 14522 1) 171 n. 61 , $\subseteq$ oే.

Odynerus (Leionotus) brevithorax Saussure, Étud. fam. Vespid. I. Eumén. 1552, \&; T. 17 F. 9.

Odynerus nasidens Saussure, Étud. fam. Vespid. III. Masar. 18.56 p. 230, $\subseteq \vec{f}$.

Odynerus nasidens Saussure, Rev. \& mag. zool. (2) X. 1559 p. 165 n. 1, \&.

Odynerus auratus Saussure, Rev. \& mag. zool. (2) X. 1858 p. 166 n. 3, 우.

Odynerus nasidens Smith, th Rep. Peabody Acad. Sc. 1869 p. $60, \subseteq$ of.

Odynerus Pachodynerus nasidens Saussure, Smithson. Miscell. Collect. XIV. P. 254. 1575

nataleusis Sauss. - đే - Afr.: Cap, Madagascar.

[p. 232 n. 67, 우․

[n. 140, ơ.

Odynerus Hypodynerus) Natalensis Saussure, Ktud. fam. Vespid. III. Masar. $1 \$ 56$ p. 261

Odynerus Natalensis Saussure, Grandidier: Hist. Madagascar XX. P. 1. 1५90) p. 175 , n. 4. $\hat{0}$.

nantarum Sauss. - \& ฮึ-Austr.

Odynerus(Antodynerus) nautarum Saussure, Etud.fam. Vespid. III. Masar.1556p.259n.176. oే.

Odynerus insulicola Blackburn \& Cameron, Mem. Manchester Lit. \& Philos. Soc. :) X. 1S56

neglectus Sauss. - 우 - Austr.

[p. 223 n. 35, 우 ô [nec Dalla Torre].

Odynerus (Parodynerus, neglectus Saussure, Etud.fam. Vespid. III. Masar. 14.56 p.2.15n. 11 s, c.

nidulator Sauss. - ㅇ - Eur. centr. \& mer.

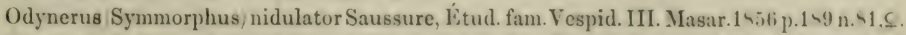

Odynerus Symmorphus) nidulator Sehenck, Jahrb. Ver. Naturk. NassauXVI. 1561 p). 40, nota

[\& p. 45, nota 2 , ᄋ.

Odỵnerus nidulatorEd.André, Spec.Hymén.Europe II. P.21-23.154.1 p.656n.2. ; T.42 F.T\&-s.

Odynerus murarius var. nidulator Schulthess, Hymen. Helvet. Diplopt. 1557 p. 34.

Odynerus nidulator Fabre, Souv. entom. IV. 1891 p. ?

nigrieoruis Mor. - $f \hat{\sigma}-$ As.: Tauria.

Lionotus nigricornis F. Morawitz. Horae soc. entom. Ross. XIX. 1545 p. 160 n. 16. s f

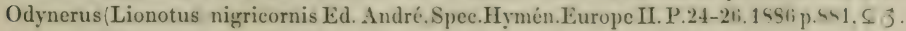

Catalogus Hymenopterorum. IX. 
nigrieulas Berg. - ठี - Am.: Argentina.

Odỵnerus (Pachyodynerus; nigriculus Berg, Stettin. entom. Zeitg. XIII. 1551 p. 70 n. 119 , J. Odymerus nigriculus Berg, Inform. Comic. Exped. Rio Negro Zool. P. 1. 1541 p. 112 n.115. nigrifrons Smith - Q - As.: China.

Odyuerus nigrifrons Smith, Catal. Hymen. Brit. Mus. V. 1457 p. 62 n. 65, \&. nigripennis (Holmgr.) D. T. - 오 $\precsim$ - Austr.: Honolulu.

Rhynchium nigripenne Holmgren. Eugenies Resa. Insect. 1565 p. 441 n. 10:3, c.

Odynerus Maurus Smith. Descr. New Spee. Hymen. 1579 p. 135 n. 2, $\subseteq$ j.

Odynerus Maurus Smith, Journ. Linn. Soc. Zool. XIV. 1879 p. 679, 요 of.

Odynerus Maurus Blackburn \& Kirby, Entom. M. Magaz. XVII. 1850 p. 56.

Odynerus nigripennis m.

nigrocinetus Sauss. - o - Austr.: Tasmania.

Odynerus (Leionotus, nigrocinctus Saussure, Étud.fam. Vespid. I. Eumén. 1552 p. 201 n. 1102, J.

Odynerus nigrocinctus Saussure, Étud. fam. Vespid. III. Masar. 1556 p. 240.

nobilis Sauss. - 우 ô - Eur. : Hispania.

Odynerus Oplopus, nobilis Saussure, Étud. fam. Vespid. III. Masar. 1556 p. 313 n. 197, S.

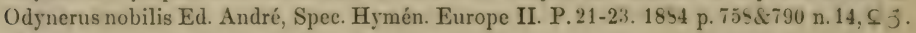

nortoniauus Sauss. - $\mathrm{Q}-$ Am.: Connecticut.

Odynerus (Stenodynerus Nortonianus Saussure, Rer. \& mag. zool. 2 XXII. 1 S71 p.105̃ n. $28, \subseteq$.

Odynerus Stenodynerus) Nortonianus Saussure, Smithson. Miscell. Collect. XIV. P.254.1575

notabilis Walk. - ô - Afr.: Ad mare rubrum.

[p. 333 n. 132, 우.

Odynerus notabilis Walker, List of Hymen. in Egrpt 1571 p. 35 n. 156. 3.

notatus (Jur.) Spin. - ㅇ - Eur.: Helvetia.

Vespa notata Jurine, Nouv. méth. class. Hymén. 1807 p. 170, ㅇ; T. 9 F. 15.

Odynerus notatus Spinola, Insect. Ligur. II. 1808 p. 179 n. 5.

Eumenes notata Latreille, Gen. Crust. \& Insect. IV. 1809 p. 136.

Odynerus (Hy podynerus ) notatus Saussure, Étud. fam. Vespid. III. Masar. 1856 p.262 n.142, ᄃ.

Odynerus notatus Ed. André, Spec. Hymén. Europe II. P. 21-23. 1554 p. 652 n. 3, S.

notula Lep. - 우 ô - Afr.: Algeria.

Odynerus notula Lepeletier, Hist. nat. Insect. Hỵmén. II. 1441 p. 612 n. 4, j.

Odynerus notula Lucas, Explor. se. Algéric Zool. III. 1 \$6 p. 229 n. 141, 3 excl. s]; T. 11 F. 2.

Odynerus (Oplopus) notula Saussure, Fitud. fam. Vespid. I. Eumén. 1552 p. 219 n. 126 , ؟.

Odynerus Oplopus) notula Saussure, litud. fam. Vespid. III. 1856 p. $303 . \hat{o}$.

()dynerus notula Ed. André, Spec. Hymén. Europe II. P. 21-23. 1584 p. 760 n. 1s, 3.

nudus (Mor.) D. T. - 우 $\widehat{0}-$ As. centr.

Jionotus mudus Morawitz. Horae soc. entom. Ross. XXIII. 1659 p. 164 n. 11 , \& $\hat{\jmath}$.

Odynerus nudus $\mathrm{m}$.

nugdunensis Sauss. - 우 of - Eur. mer.

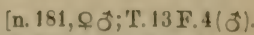

Odynerus Antodynerus, Nugdunensis Saussure. Étud. fam. Vespid. III. Masar. 1556 p. 293

Odynerus (Leionotus, Nugdunensis Schenck, Jahrb. Ver. Naturk. Nassau XV1. 1861 p. 6it, lis

[\&. $78 \mathrm{n} .13,907$.

Odynerus Microdynerus Xugdunensis Lid. André, Spee. IIymén. Europe II. P. 21-23. 15\$4

[p. 733 n. $4, \subseteq \hat{\jmath}$.

oahensis D. 'T. - 오 - Austr.: Oahu.

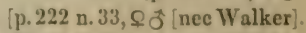

Odynerus diversus Blackburn \& ('amerom. Mem. Manchester Lit. \& Philos. Soc. 3 X. 14s6

Odynerus Oahensis Dalla Torre, Wien. entom. Zeitg. VIII. 1889 p. 124.

oblicuus Cress. - \& $\hat{f}-$ Am.: Cuba.

Odynerus obliquus C'resson, Proc. Entom. Soc. Philadelphia IV. 1\$65 p. 163. $\subseteq, \hat{\jmath}$.

Odynerus Stenaneystrocerus! obliquus Saussure. Smithson. Miscell. Collect. XIV. P. 254

[1875 p. 197 n. 33 , 우 రో. 
obseurepunctatus Blackb. \& Cam. - f ô - Austr.: Ins. Hawaiae.

Odynerus obscurepunetatus Blackburn \& Cameron. Mem. Manchester Lit. \& Philos. Soe. (3) obseuripennis Spin. - 우 - Am.: Chili.

[X. 1886 p. 221 n. 32, 오우.

Odynerus obscuripennis Spinola, Gay: Hist. fis. ('hile. Zool. VI. 1551 p. 259 n. 4, s.

Odỵnerus Coquimbensis Saussure. Gay: Hist. fis. Chile. Zool. VI. 14501 p. 561 n. 1. $\subseteq$.

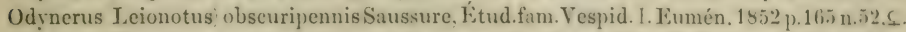
Odỵnerus (Hypodỵnerus; obscuripennis Saussure, Smithson. Miscell. Collect. XIV. P. 25.

obseurus Rad. - ơ - Afr.: Angola.

[1875 p. 225 n. 60

Odynerus obscurus Radoszkowski, Jorn. aead. se. Math. ete. Lisboa VIII. 1 4 1 p. 204 n.55. 3 .

occidentalis Sauss. - tิ - Am.: Mexico.

Odynerus Ancistrocerus) occidentalis Saussure, Rev. \& mag. zool. 2) IX. 1457 p. 27.4.

Odynerus Stenancystrocerus, oceidentalis Saussure. Smithson. Miscell.Collect. XIV. P.2.54.

olmecus Sauss. - ô- Am. : Mexico.

$[1875$ p. 207 ก. 42, ઠ

Odynerus Olmecus Saussure, Rer. \& mag. zool. 2) XXII. 1571 p. 140 n. 33, ş.

Odynerus Stenodynerus Olmecus Saussure. Smith opacus Mor. - 우 ơ - Eur.: Russia.

[р. $356 \mathrm{n} .152$, đ.

Odynerus Leionotus opaeus F. Morawitz. Horae soc. entom. Ross. IV. 1567 p. 124 n. 13 . C 3 .

Odynerus opacus F. Morawitz, Horae soc. entom. Ross. V. 1569 p. 1.45 n. 8, ว.

Odynerus opacus Ed. André, Spec. Hỵmén. Europe II. P. 21-23. 1554 p. 705 n. 3, $\subseteq \hat{\jmath}$. oraniensis Lep. - 우 - Afr.: Algeria.

Odynenus Oraniensis Iepeletier. Hist. nat. Insect. Hỵmén. II. 14.11 p. 6.10 n. 25, S.

Odynerus Oraniensis Lucas, Explor. se. Algérie Zool. III. 1546 p. 237 n. 193, \&; T. 11 F. 10 \&.

I.eptochilus Oraniensis Saussure. Étud. fam. Vespid. I. Eumén. 1552 p. 236 n. 5.

Odynerus Parodynerus) Oraniensis Saussure, Étud. fam. Vespid. III. Masar. 1856 p. 321.

Ody̧nerus Oraniensis Ed. André, Spec. Hymén. Europe II. P. 21-23. 1 sh4 1). 726 n. 14. $\Omega$.

orbitalis (Thoms.) D. T. - 으 ơ - Eur.: Germania.

Lionotus orbitalis Thomson, Hymen. Scandin. III. 1874 p. 57 n. 1, 오

Odynerus orbitalis $\mathrm{m}$.

orenburgensis André - $ᄋ-$ Eur.: Russia.

Odynerus Orenburgensis Ed. André, Spee. Hymén. Europ. II. P. 21-23. 154.4 p. 719 n.6. S. orientalis D. T. $-\widehat{\jmath}-$ As.: India.

Ancystrocerus ornatus Smith. Ann. \& Mag. Nat. Hist. (2, IX. 1552 p. 4!1, oै.

Odynerus orientalis Dalla Torre. Wien. entom. Zeitg. VIII. $185 !$ p. 125.

otomitus Sauss. - $ᄋ$ ô- Am.: Mexico.

Odynerus Otomitus Saussure, Rev. \& mag. zool. (2) IX. 1857 p. 278.

Odynerus Stenodynerus Otomitus Saussure. Smithson. Miseell. Collect. XIY. P.254. Isis ovalis Sauss. - 우 fo As.: India.

[p. $342 \mathrm{n}, 139$, \& ơ;'T. 4 F. 21.

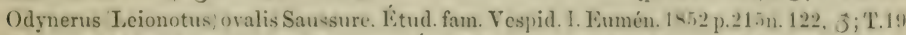

Odynerus Antepipona) ovalis Saussure, Etud. fam. Tespid. III. Mavar. 1556 p. 215. (F.4.

Odynerus ovalis Desmarest. Chenu: Eneycl. hist. nat. Annelles 1stil p. 1.43 F. 124.

Odynerus ovalis Saussure. Mém. soe. phỵs. \& hist. nat. Genève XVII. 1. 14ti3 p. 227 n. 53. S. oriventris Wesm. - 0 ô - Eur. bor. \& centr.

Odynerus oriventris Wesmael, Bull. acad. se. Belgrique III. 1. 16.36 p. 4. n. 4. 5 3 .

Odynerus (Ancistroecrus) ovicentris Wesmael. Bull. acad. se. Belgique III. 1h.3ti p. 45.

Odynerus ovirentris Lepeletier. Hist. nat. Insect. Hymén. II. 1841 p. 611 n. $85, \subseteq j$.

Odỵnerus Aneistrocerus oviventris Saussure. Kitud. fam. Vespid. I. Eumén. 1452 p. 132 n.12.

Odynerus ovirentris Schenek. Jahrb. Yer. Naturk. Nassau IX. 1. 1453 p. 61 n. 1, s 3

Odỵnerus ovirentris Saussure, Étud. fam. Vespid. III. Masar. 15iti p. 202.

Odynerus Ancystrocerus oviventris Schenek, Jahrb. Ver. Naturk. Nassau XVI. 1 słi p. 49,

[50\&61 n. 5, 옹․ 
Odynerus Aneystrocerus) oviventris F. Morawitz, Horae soc. entom. Ross. IV. 1867 p. 116

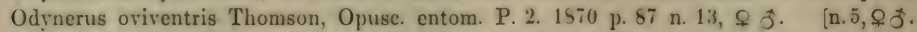
Ancistrocerus oviventris Thomson, Hymen. Scandin. III. $157 \downarrow$ p. 65 n. $3, \subseteq j$. Odynerus oviventris Rudow, Areh. Ver. Fr. Naturg. Mecklenburg XXX. 1576 p. 215 n.4, $\Omega$ J. Odynerus oviventris H. Müller, Alpenblumen 1881 p. 592 n. 607.

Ancistrocerus oviventris Aurivillius, Bih. Svensk. Vet.-Akad. Handl. XII. P. 4. 5. 1856 p. 12. Odynerus oviventris Schulthess, Hymen. Helret. Diplopt. IS57 p. 48 n. 9, $\odot$ jे.

paciflens Kirsch - - - Austr.

[Blackb.\& Cam.].

Odynerus Leionotus) pacificus Kirseh, Mittheil. zool. Mus. Dresden III. 1578 p.379, \& [nec paraensis Sauss. - ơ - Am.: Brasilia.

[n. 95, ô.

Odynerus 'Subancistrocerus Paraensis Saussure, Étud. fam. Vespid. III. Masar. 1856 p. 207

Odynerus 'Stenancystrocerus' Paraensis Saussure, Smithson. Miscell. Collect. XIV. P. 254.

parietum (L.) Latr. - 우 of - Eur. tota; As.: Caucasus; Afr.: Algeria.

Frisch, Beschr. Insect. Teutschl. IX. 1730 p. 23; T. 12 F. 1 \& seq.

Linné, Fauna Suec. 1746 p. ? n. 990.

Vespa parietum Linné, Syst. nat. Ed. $10^{\text {a }}$ I. 1758 p. 572 n. 4.

Vespa parietum Brünnich, Prodr. insectol. Siaelland. 1761 p. 18.

Vespa parictum Linné, Fauna Suec. Ed. 2a 1761 p. 416 n. 1673.

Vespa parietina Linné, Fauna Suec. Ed. $2^{\text {a }} 1761$ p. 418 n. 1679.

Vespa parietina Geoffroy, Hist. abr. Insect. U. 1762 p. 376 n. 9.

Vespa parietum O. F. Müller, Fauna Insect. Fridrichsdal 1764 p. 73 n. 637.

Vespa parietina O. F. Müller, Fauna Insect. Fridrichsdal 1764 p. 73 n. 641.

Vespa I Schaeffer, Icon. Insect. Ratisbon. I. 1. 1766; T. 24 F. 2.

Vespa III Schaeffer, Icon. Insect. Ratisbon. I. 1. 1766; T. 24 F. 4.

Vespa parictum Linné, Syst. nat. Ed. 12a I. 2. 1767 p. 949 n. 6.

Vespa XV Schaeffer, Icon. Insect. Ratisbon. I. 2. 1767; T. 93 F. 8.

Vespa parietum Fabricius, Syst. entom. 1775 p. 368 n. 26.

Vespa parietum Ph. L. Müller, Linné: Vollst. Natursyst. V. 2. 1775 p. 851 n. 6.

Vespa parietum O. F. Müller, Zool. Dan. prodr. 1776 p. 162 n. 1889.

Vespa parietum Fabricius, Spec. Insect. I. 1781 p. 463 n. 32.

Vespa parietum Schrank, Enum. Insect. Austr. 1781 p. 392 n. 792.

Vespa parictum Fourcroy, Entom. Paris. II. 1785 p. 435 n. 9.

Vespa parietum Fabricius, Mant. Insect. I. 1787 p. 289 n. 37.

Vespa parietina Ström, Danske Vid. Selsk. Skrift. Nya Saml. III. 1785 p. 2 n. 56.

Vespa parietina Ström, Danske Vid. Selsk. Skrift. Nya Saml. III. 17 ss p. 291 n. 60.

Vespa parietum Razoumowski, Hist. nat. Jorat. I. 1. 1789 p. 217 n. 257.

Vespa parietum Villers, C. Linnaei Entom. III. 1789 p. 265 n. 4.

Vespa parietina Villers, C. Linnaei Entom. III. 1789 p. 272 n. 16.

Vespa parictum Gmelin, Linné: Syst. nat. Ed. 13a I. 5. 1790 p. 2751 n. 6.

Vespa parietum Rossi, Fauna Etrusca II. 1790 p. 85 n. 865

Vespa nimpha Christ, Naturg. d. Insect. 1791 p. 232 ; T. 21 F. 2.

Vespa parietum Christ, Naturg. d. Insect. 1791 p. 237.

Vespa sexpunctata Christ, Naturg. d. Insect. 1791 p. 241 ; 'T. 22 F. 6.

Vespa aeneipennis Christ, Naturg. d. Insect. 1791 p. 244 ; 'I. 23 F. 3.

Vespa parietum J. B. Fiseher, Verh. Naturg. Lieslund Ed. 2* 1791 p. 344 n. 759.

Vespa parictum Olivier, Encyel. méthod. Insect. VI. 1791 p. 686 n. 82.

Vespa parietum Fabricius, Entom. system. II. 1793 p. 265 n. 45.

Vespa parietina Fabricius, Entom. system. II. 1793 p. 268 n. 53.

Vespa parietum Cederhjelm, Faun. Ingric. prodr. 1798 p. 168 n. 522.

Vespa parictum Panzer, Faun. Insect. German. V. 1798 P. 49 T. 23 F. 45. 
Vespa parietina Panzer, Faun. Insect. German. V. 1798 P. 49 T. 24 F. 53.

Vespa parietum Schrank, Fauna Boica U. 2. 1802 p. 353 n. 2208.

Vespa parietina Schrank, Fauna Boica II. 2. 1802 p. 353 n. 2209.

Vespa parietum Walckenaer, Fauna Paris. II. 1802 p. 89 n. 6.

Vespa parietum Fabricius, Syst. Piez. 1 S04 p. 261 n. 44.

Vespa parietina Fabricius, Syst. Piez. 1804 p. 263 n. 52.

Odynerus parietum Latreille, Hist. nat. Insect. XIII. 1805 p. 3$\lrcorner 7$.

Odynerus parietinus Latreille, Hist. nat. Insect. XIII. 1805 p. 347.

Vespa parietum Panzer, Krit. Revis. II. 1806 p. 155.

Vespa parietina Panzer, Krit. Revis. II. 1806 p. 155.

Vespa parietum Illiger, Rossi: Fauna Etrusca Ed. 2a II. 1807 p. 141 n. 565.

Vespa parietum Jurine, Nouv. méth. class. Hymén. 1807 p. 169, 우 §̋.

Vespa parietina Jurine, Nour. méth. class. Hymén. 1807 p. 170, ㅇ.

Vespa parietum Klug, Magaz. Ges. naturf. Fr. Berlin II. 1807 p. 51 n. 56.

Vespa parietina Klug, Magaz. Ges. naturf. Fr. Berlin II. 1807 p. 51 n. 56.

Odynerus parietum Spinola, Insect. Ligur. II. 1808 p. 180 n. 6.

Odynerus Geoffroyanus Spinola, Insect. Ligur. II. 1808 p. 183 n. 7.

Eumenes parietum Latreille, Gen. Crust. \& Insect. IV. 1809 p. 136.

Odynerus similis Curtis, Brit. Entom. III. 1826 p. 137 n. 7.

Odynerus parietinus Curtis, Brit. Entom. III. 1826 p. 137 n. 9, $;$; T. 137 ( ;.

Odynerus parietum Wesmacl, Monogr. Odynèr. Belgique 1833 p. 16 n. 4 , $\subseteq$ sै.

Vespa parietum Bouché, Naturg. d. Insect. 1834 p. 178 n. 76.

Odynerus Aneystrocerus parietum Wesmael, Bull. acad. se. Belgique III. 1836 p. 45

Odynerus parietum Wesmael, Bull. acad. se. Belgique III. 1836 p. 46 n. 5 , 우

Odynerus parietum Zetterstedt, Insect. Lappon. I. 1838 p. 455 n. 1, 우 oึ.

Odynerus quadrifasciatus Zetterstedt, Insect. Lappon. I. $183 \mathrm{~S}$ p. $456 \mathrm{n.} 3$, oै.

Odynerus affinis Herrich-Schaeffer, Faun. Insect. German. 1839 P. 173 'T. $24(f)$.

Odynerus parietum Blanchard, Hist. nat. Insect. 1840 p. 392 n. 4. [F.87. 1; Synops. p.84.

Ancystrocerus parietinus Westwood, Introd. mod. Classif. Insect. II. 1840 Synops. p. 249 ;

Odynerus parietum Lepeletier, Hist. nat. Insect. Hymén. II. 1541 p. $656 \mathrm{n}$. 35. $\subseteq$ o

Odỵnerus parietum Wesmael, Bull. acad. sc. Belgique VIII. 2. 1 s 11 p. $34-35$.

Vespa Odynerus) parietum Ratzeburg. Forstinsect. III. 15.4 p. 52 n. 4.

Odynerus parietum Lucas, Explor. se. Algérie. Zool. III. 1446 p. 239 n. 197.

Odynerus /Ancistrocerus parietum Saussure, Étud. fam. Vespid. I. Eumén. 1552 p. 130 n. 10,

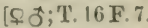

Odỵnerus parietum Schenck; Jahrb. Ver. Naturk. Nassau IX. 1.1553 p. 56 n. $20, \varsigma \hat{o}$.

Odynerus parietum Sehenck. Jahrb. Ver. Naturk. Nassau IX. 1. 1853 p. 61 n. 2, $f$ of.

Odynerus parietum Smith. Trans. Entom. soc. London 2) III. 1. 1554 p. 43.

Odynerus parietum Saussure, litud. fam. Yespid. III. Masar. 1856 p. 201.

Odynerus parietum \& X Cemonus unicolor ơ Bouillon, Ann. soc. entom. Belge II. 1 s. is p. 2 I9.

Odynerus Anevstrocerus parietum Smith. Catal. Brit. Fossor. Hymen. 1858 p. 206 n. 7. $\subseteq 3$;

['T. 5 F. 5 (우) \&6(ठ).

Odynerus 'Aneystrocerus! parietum Schenek, Jahrb. Ver. Naturk. Nassau XVI. 1861 p. th. $^{\text {h }}$

$[50 \& 61 \mathrm{n} \cdot 2, \subsetneq$ ठี.

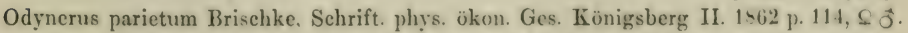

Odynerus parietum Taschenberg, Hymen. Deutschl. 18666 p. 248, $\varsigma$ j.

Odynerus (Ancystrocerus parictum F. Morawitz, Horae soc. entom. Ross. IV. 1867 p. 117

Odynerus parietina Thomson, Opuse. entom. P. 2. 1570 p. 85 n. 14. S oే. n. 6.50 .

Odynerus parictum Thomson, Opusc. entom. P. 2. 1870 p. 89 n. 17, 우 ơ.

Odynerus parietum H. Müller, Befrucht. d. Blumen 1873 p. 466.

Ancistrocerus parietinus Thomson, Hymen. Scandin. III. 1874 p. 7 () n. $6, s \hat{\jmath}$ 
Aneistroecrus parietum Thumsun, IIymen. Seandin. III. 1474 p. if n. $5 . \subseteq 3$.

Odynerus parietum Brischke, Schrift. naturf. Ges. 1)anzig. N. F. III. 4. 157.5 Art. 5 p. 29. Odynerus parietum Rudow. Arch. Ver. Fr. Naturg. Mecklenburg XXX. 1576 p.214n.2, fठ . Odynerus parietinus E. A. Fitch, Intomologist XIII. 1880 p. 19; Fig.

Odynerus parietum E. A. Fitch, Entomologist XIII. 1880 p. 20 ; Fig.

Odynerus /Ancystrocerus/ parietum E. Saunders. Trans. Entom. Soc. London 1552 p. 177 n. 7. Odyuerus (Ancystrocerus) parictinus E. Saunders. Trans. Entom. Sue.London 1542 p. 179 n.12. Odyncrus parietum İd. André. Sppec. Hỵmén.Furope II. P. 21-23. 1854 p. 679 n. 20, 8 ร; T. 36

[F. 11, 12, 18; T. 43 F. 1.

Aneistrocerus parictum Aurivillius. Bih. Srensk. Vet.-Akad. Handl.XII.P.4. 5.1686 p. 12. Odynerus parietum Verhoeff, Entom. Nachr. XVI. 1890 p. 334.

Odynerus parictum Verhoeff, Verh. naturh. Ver. preuss. Rheinl. XI,VIII. 1\$91; T. 3 F.5.4. var. auctus (Fabr.) D. T. - Q - Eur.

Vespa aucta Fabricius, Entom. system. II. 1793 p. 272 n. 69.

Vespa aucta Fabricius, Syst. Piez. 1804 p. 267 n. 74.

Odynerus auctus Spinola, Insect. Ligur. I. 1. 1806 p. 88 n. 1, ㅇ.

Vespa aucta Jurine, Nouv. méth. class. Hymén. 1807 p. 170, ㅇ.

Eumenes aucta Latreille, Gen. Crust. \& Insect. IV. 1809 p. 136.

Vespa aucta Sehummel, Übers. Arbeit. schles. Ges. f. vaterl. Cultur i. J. 1\$24. 1829 p. 75.

Odynerus parietum var auctus $\mathrm{m}$.

var. emarginatus (Fabr.) D. T. - ô - Eur.

Vespa emarginata Fabricius, Entom. system. II. 1793 p. $26 \overline{\text { n. }} 51$.

Vespa emarginata Walckenaer, Fauna Paris. II. 1802 p. 89 n. 8.

Vespa emarginata Fabricius, Syst. Piez. 1804 p. 263 n. 50.

Vespa cmarginata Jurine, Nouv. méth. class. Hymén. 1807 p. 170, đ̃.

Odynerus parietum var. emarginatus $\mathrm{m}$.

var. incisoides Verh. - Eur.: Germania.

Odynerus parietum var. incisoides Verhoeff, Entom. Nachr. XVI. 1 \&90 p. 335.

var. ineisns Verh. - Eur.: Germania.

Odynerus parictum var. incisus Verhoeff. Entom. Naehr. XVI. $1 \$ 90$ p. 335.

var. parietoides Verh. - Eur.: Germania.

Odynerus parietum var. parietoides Verhoeff, Entom. Nachr. XVI. 1890 p. 335.

var. quadricinctus (Fabr.) Wesm. - $\odot$ of - Eur.

Vespa quadricincta Fabricius, Entom. system. II. 1793 p. 266 n. 47.

Vespa quadricincta Fabricius, Syst. Piez. 1804 p. 262 n. 46.

Vespa quadricineta Jurine, Nouv. méth. class. Hymén. 1807 p. 170, ᄋ.

()drnerus quadricinctus Wesmacl. Bull. acad. se. Belgique III. 1 s36 p. 51 .

() dynerus quadricinctus Schenck, Jahrb. Ver. Naturk. Nassau IX. 1 s5i3 p. 66 n. $24, \subseteq$ ô.

()druerus quadricinctus Brischle, Selırift. phrs. ökon. Ges. Künigsberg II. $1 \leqslant 62$ p. 115. S.

Odynerus parictum var. quadricintus $\mathrm{m}$.

var. renimacula Lep. - of 0 - Afr.: Algeria.

— Savigny, Deser. de l'Égypte. Hymén. 1812; T. 9 F. 2 (ठ̋) \& 3 (\$).

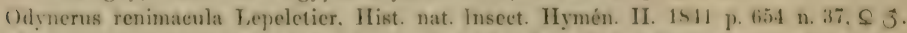
() lynerus renimacula Jacas, Explor. se. Algéric. Zool. III. 144ti p. 237 n. $196, \Omega$ of.

Odynerus (Ancistrocerus) renimacula Saussure, Eitud. fam. Vespid. I. Eumén. 1552 p. 12 s

[n. $8,80 \%$.

()dynerus Ancistrocerus, ochlerus Saussure, Butud. fam. Vespid. I. Fumen. 14.52 p.131 n.11, S. Odynerus Aneystrocerus renimaculata var, ochlerus Saussure, Ltud. fam. Vespid.III. Mnsar. Odynerus renimatcula Saussure. Étud. fam. Vespid. III. Masar. 1456 p. 199. [14.51 p. 203. Odynerus Aneistrocerus, renimacula Schenck. Jahrb. Ver. Naturk. Nassau XVI. 146i1 p. 46. [50 \& $51 \mathrm{n} .1$, 우 
Odynerus Ancystrocerus renimaculatus Saussure, Reise d. Novara. Zool. II. 1. $1867 \mathrm{Hymen}$. [p. 9 n. 2, \&

Odynerus (Ancistrocerus) renimacula Rudow, Arch. Ver. Fr. Naturg. Mecklenburg XXX. Odynerus parietum var. renimacula $\mathrm{m}$. var. sexfasciatus (Fabr.) D. T. - 으 - Eur. fere tota. $[1876$ p. 213 n. 1, \& 0 .

Vespa sexfasciata Fabricius, Spec. Insect. I. 1781 p. 465 n. 42.

Vespa sexfasciata Fabricius, Mant. Insect. I. 1787 p. 291 n. 51.

Vespa sexfasciata Villers, C. Linnaci Entom. III. 1789 p. 276 n. 23.

Vespa sexfasciata Gmelin, Linné: Syst. nat. Ed. $13^{\text {a }}$ I. 5.1790 p. 2755 n. (i5.

Vespa sexfasciata Rossi, Fauna Etrusea II. 1790 p. 87 n. 871.

Vespa sexfasciata Olivier, Encycl. méthod. Insect. VI. 1791 p. 689 n. 97.

Vespa sexfasciata Fabricius, Entom. system. II. 1793 p. 268 n. 56.

Vespa sexfasciata Schrank, Fauna Boica II. 2. 1802 p. 358 n. 2216.

Vespa sexfasciata Fabricius, Syst. Piez. 1804 p. 263 n. 55.

Vespa sexfasciata Illiger, Rossi: Fauna Etrusea Ed. $2^{\mathrm{a}}$ II. 1807 p. 144 n. \$i1.

Vespa sexfasciata Jurine, Nouv. méth. class. Hymén. 1807 p. 170, ㅇ․

Odynerus parietum var. sexfasciatus $\mathrm{m}$.

var. trifaseiatus (Fabr.) D. T. - \& ô - Eur. fere tota.

Vespa trifasciata Fabricius, Mant. Insect. I. 1787 p. 290 n. 46.

Vespa trifasciata Villers, C. Linnaei Entom. III. 1789 p. 279 n. 32.

Vespa trifasciata Gmelin, Linné: Syst. nat. Ed. $13^{a}$ I. 5. 1790 p. 2757 n. 78.

Vespa trifasciata Olivier, Encycl. méthod. Insect. VI. 1791 p. 688 n. 92.

Vespa trifasciata Fabricius, Entom. system. II. 1793 p. 270 n. 61.

Vespa trifasciata Fabricius, Syst. Piez. 1804 p. 264 n. 60.

()dynerus Ancystrocerus) trifasciatus Wesmael. Bull. acad. sc. Belgique III. $18: 36$ p. 4.).

Odynerus trifasciatus Wesmael, Bull. acad. sc. Belgique III. 1536 p. 50 n. 6, \& of.

Odynerus tricinetus Herrich-Schaeffer, Faun. Insect. German. 1841 P. 176 T. 10 (S).

Odynerus trifasciatus Lueas, Explor. sc. Algéric. Zool. III. 1546 p. 237 n. 195.

Odynerus trifasciatus Sehenck, Jahrb. Ver. Naturk. Nassau IX. 1. 1853 p. 62 n. 3. f j

Odynerus tricinctus Schenck. Jahrb. Ver. Naturk. Nassau IX. 1. 1553 p. 64 n. $22 . \subseteq$ ô

Odỵnerus Ancystrocerus) trifasciatus Saussure, Ltud. fam. Tespid. III. Masar. 1856 p. 211

[n. 100, 웅.

Odynerus (Aneystrocerus) trifasciatus Smith, Catal. Brit. Fossor. Iymen. 1454 p. 209 n.9.5. $\vec{j}$.

Odynerus Aneystrocerus trifasciatus Schenck, Jahrb. Ver. Naturk. Nassau XVI. $1 \backsim(i 1$ p. 47.

[51 \& 59 n. $3, \Omega$ ठૈ.

Odynerus tricinetus Brischke, Schrift. phys. ökon. Ges. Königsberg II. 1 s62 p. 115, ऽ. Odynerus tricinctus Brischke, Sehrift. physs. ökon. Ges. Königsberg V. 196.4 p. $210 . \& \mathrm{j}$. Odynerus trifasciatus H. Müller, Befrucht. d. Blumen 1873 p. 466.

Ancistrocerus trifasciatus Thomson, Hymen. Scandin. III. 1574 p. 6 is n. 5. \& 3

Odynerus trifasciatus Rudow. Areh.Ver. Fr. Naturg. Mecklenburg XXX. 1476 p. 214 n. 3.5 .3$.

Odynerus trifasciatus H. Müller, Alpenblumen 1881 p. 592 n. 608.

Odynerus Ancystrocerus trifasciatus I: Saunderk. Trans. Entom. Soe. Iondon 1s\$2 p. 1 in

Odynerus parictum var. trifasciatus $\mathrm{m}$.

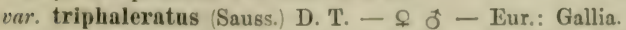

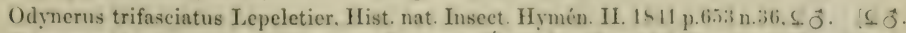
Odỵnerus (Incistrocerus trifasciatus Saussure. Kitud. fam. Vespid. I. Eumén. 1552 p p. 129 n.9. Odynerus renimacula var. triphaleratus Saussure, Etud fam. Vespid. III. Masar. 14.56 p. 2611. Odynerus parietum var. triphaleratus $\mathrm{m}$.

parisiensis Sauss. - 우 of - Eur.: Gallia.

Odynerus Hypodrnerus, Parisiensis Saussure. Kitud. fam. Vespid. III. Masar. 1556 p. 271

Odynerus Parisiensis Ed. André, Spec. Iymén. Europe II. P. 21-23.1454 p. 72 n n. 15 . S. S. 
parredes Sauss. - 0 - Am.: Mexico.

Odynerus Ancistrocerus Parredes Saussure, Rev. \& mag. zool. 2, IX. 1557 p. 273.

Odynerus Aneystrocerus Parredesi Saussure. Smithson. Miscell. Collect. XIV. P. 254.1875 parrulus Lep. - \& ơ - Regio mediterranea.

[p. 180 n. 23, ㅇ․

Odỵnerus parvulus Iepeletier. Hist. nat. Inseet. Hymén. II. 1441 p. 631 n. 17 . $\subseteq j$.

Odynerus parvulus Lucas, Explor. se. Algérie. Zoul. III. 1446 p. 236 n. $191 . \subseteq j$; T. 11 F.9. Odỵnerus Leionotus; parvulus Saussure, Ktud fam. Vespid. I. Fumén. 1452 p. 193 n $91, \subseteq$ Jै. Odynerus parrulus Schenck, Jahrb. Ver. Naturk. Nassau IX. 1. 1653 p. $\$ 4$ n. $7 . \varsigma$ Ј Odỵnerus parrulus Saussure. Etud. fam. Vespid. III. Masar. $1554 ;$ p. 237, 8 j.

Odynerus Ancistrocerus parrulus Saussure. Gitud. fam. Vespid. III. Masar. 1856 p. 321. Odynerus parrulus Saussure, Mém. soc. phys. \& hist. nat. Genève XVII. 1.1863 p.2.25 n.51. j. Odỵnerus Leionotus! parvulus Schenck. Jahrb. Ver. Naturk. Nassau XVI. $1 \$ 61$ p. 64.67 \&

[71 n. 4, 오웅.

Odynerus parrulus Rudow, Arch. Ver. Fr. Naturg. Mecklenburg XXX. 1476 p. 224 n.1,. f.

Odynerus parrulus Ed. André, Spec. Hymén. Europe II. P. 21-23.1 554 p. 711 n. $10 . \varsigma$ วే; T. 36 var. orbitalis Herr.-Schaeff. - $\widehat{\jmath}-$ Eur.: Germania.

[F.14.

Odynerus orbitalis Herrieh-Schaeffer. Faun. Insect. German. 1841 P. 173 T. 15.

Odynerus parrulus var. orbitalis Schenck. Jahrb. Ver. Naturk. Nassau IX. 1.15 .33 p. 81 n. $1, \hat{\jmath}$.

Odỵnerus orbitalis Saussure, Étud. fam. Vespid. III. Masar. 1856 p. 277 n. 155.37.

var. ruthenicus Mor. - 우 oే - Eur.: Russia.

[n. 18, \& 0 .

Odỵnerus parrulus var. Ruthenicus F. Morawitz, Horae soc. entom. Ross. IV. 1567 p. 131 paulii Med. - 우 of - Eur.: Hispania.

Odỵnerus Pauli Medina, Act. soc. Españ. hist. nat. XX. 1592 p. 142, $\subseteq \vec{\jmath}$. perlestris Sauss. - $\subseteq>-$ Am.: Connecticut, New York. : non Herr.-Schaeff. . Odṛnerus (Ancistrocerus) fuscipes Saussure. Kitud. fam. Vespid. I. Eumén. 1452 p. 143 n.2§, j Odynerus pedestris Saussure, Étud. fam. Vespid. III. Masar. 1856 p. 206.

Odynerus Ancistrocerus proximus Saussure, Rev. \& mag. zool. (2) IX. 1957 p. 27.4.

Odynerus Stenodynerus; pedestris Saussure, Smithson. Miscell. Collect. XIV. P. 254. 1575 [p. 322 n. 123, 우 ठ๋; T. 4 F. $22 \& 22$ 3.

penusylranieus Sauss. $-\subseteq 3$ - Am.: Canada. United States.

[n. $134, \mathrm{~s}$.

Odynerus Hypodynerus, Pennsylyanicus Saussure, litud. fam. Tespid. III. Masar.1 55 fi p. 257

Odỵnerus 'Stenodỵnerus Pennsylvanicus Saussure. Smithson. Miscell. Collect. XIV.P. 254. $[1875$ p. 327 n. 127, ㅇํ.

Odynerus Stenodỵnerus) Pennsylvanicus Provancher, Natural. Canad. XIII. 1852 p. 143 (n. 10, \& 0 .

Odynerus 'Stenodynerus) Pennsylvanicus Provancher, Faun. entom. Canada. Hỵmén. 1ss4 perennis Sauss. - 으-Am. Tennessec, New York. [p.677n. 10, 우.

Odynerus perennis Saussure, Rev. \& mag. zool. (2) IX. 1857 p. 277.

()dynerus Stenodynerus, peremnis Saussure, Smithson. Miseell. Collect. XIV. P. 25 I. 1875) persa Mor. - $\rho-$ As.: Persia.

[p. 328 n. $128, \Omega$ ơ; T. 4 F. 25.

Hoplomerus Persa F. Morawitz. Horae soc. entom. Ross. XIX. l445 p. Its n. S, S.

Odynerus /Hoplomerus Persa Ed. André. Spee. Hymén. Europe II. P. 2.1-26. 1446 p. 442, \&. peruensis Sauss. - \& ơ-Am. : Peru.

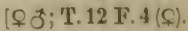

Odynerus Hypodynerus. Peruensis Saussure, litud fam. Tespil. III. Masar. 16.5tip. 253 n. 129,

Odynerus P'achodynerus Peruensis Saussure. Smithson. Miscell. ('ollect. XIV. P. 25 I. 1575

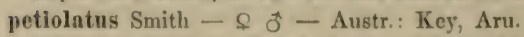

[p. 237 n. 70,00 .

Odynerus petiolatus Smith. Journ. of Proc. Linn. Soc. Zool. III. L Lis p. 1ti.4 n. 1, C.

Odynerus petiolatus Smith. Journ. of Prue. Linn. Soe. Zool. IV. 1s(io Suppl. p. 129 n. 1, 3 .

petulans Smith $-\rho-\Lambda \varepsilon$ : Celebes.

Odynerus petulans Smith, Journ. of Proe. I.inn. Soc. Zool. IV. 1560 Suppl. p. 5! n. 4. S. 
peyrotii Sauss. - 우 ơ - Am.: Mexico.

Odynerus Peyroti Saussure, Rev. \& mag. zool. (2) IX. 1857 p. 278.

Odynerus (Stenodynerus) Peyroti Saussure, Smithson. Miscell. Collect. XIV. P. 251. 1875

pharao Sauss. - - o

$\left[\mathrm{p} .345 \mathrm{n} .141, \AA \sigma^{\star}\right.$.

—- Savigny, Descr. de l'Égypte. Hymén. 1812; T. 18 F. 14 (ठ઼) A (ᄋ).

Odỵnerus Hỵpaneistrocerus, Pharao Saussure, Mém. soc. phys. \& hist. nat. Genève XVII. 1.

[1 863 p. 212 n. 40 , 우 ô;'T'. 2 F. 21

Odynerus Pharao Ed. André, Spec. Hymén. Europe II. P. 21-23. 1594 p. 669 n. 5. $\varsigma \jmath$.

philadelphine Sauss. - 우 $\tilde{-}-$ Am.: Canada, United States.

Odynerus (Protodyuerus) Philadelphiae Saussure, Rer. \& mag. zool. (2) IX. 1557 p. 272. Odynerus Srmmorphus; Philadelphiae Saussure, Smithson. Miscell. Collect. XIV. P. 251.

picticrus (Thoms). D. T. - 오 ૈ- Eur.: Suecia.

[1875 p. 153 n. 2 , 오

Odynerus dentisquama rar. Thomson, Opuse. entom. P. 2.1870 p. 86 n. 10 , S $\jmath$.

Leionotus picticrus Thomson, Hymen. Scandin. III. $187-1$ p. 57 n. 6 , 오 0 .

Odynerus picticrus $\mathrm{m}$ :

pietipes (Thoms.) D. T. - \& 0 - Eur. bor.

Odyuerus parietum var. 5 Wesmael, Monogr. Odynèr. Belgique 1833 p. 16 n. 4.

Odynerus parietum Wesmael, Bull. acad. sc. Belgique III. 1836 p. 46.

Ancistrocerus pictipes Thomson, Hymen. Scandin. III. 1574 p. 78 n. $10, \subseteq f$

Odynerus pictipes $\mathrm{m}$.

pictus Curt. - 오

Odynerus pictus Curtis, Brit. Entom. III. 1826; T. 138.

Odynerus constans Herrich-Schaeffer. Faun. Insect. German. 1841 P. 176 T. 11 S'.

Odynerus constans Schenek, Jahrb. Ver. Naturk. Nassau IX. 1.1853 p. $\$ 3$ n. 4, f.

Odynerus (Anevstrocerus) pictus Saussure, Étud. fam. Vespid. III. Masar. 1856 p. 21 " n. 99.

[Q Oे; T. 10 F. 10 .

Odynerus (Ancystrocerus) pictus Smith. Catal. Brit. Fossor. Hymen. 1855 p. 209 n. 11, \& ơ.

Odynerus Anerstrocerus' pictus Schenek, Jahrb. Ver. Naturk. Nassau XVI. 1561 p. 49 \& 61

[n. 6, 요․

Odynerus pictus Rudow, Arch. Ver. Fr. Naturg. Mecklenburg XXX. 1876 p. 216 n. 5, $\subseteq$ Ð

Odynerus pictus Bignell, Entomologist. XIV. 1881 p. 188-189.

Odynerus pictus Bignell, Entomologist. XV. 1882 p. 164.

Odynerus Ancystrocerus) pictus E. Saunders, Trans. Entem. Soc. I.ondon 1882 p. 177 n. 8.

Odynerus pictus Ed. André, Spec. Hymén. Europe II. P. 21-23. 1sst p. 67s n. 19. S j.

pilosus Sauss. - of ơ - Am.: Peru.

[S

Odynerus (Aneystrocerus) pilosus Saussure, Ḱtud. fam. Vespid. III. Masar. $16.56 \mathrm{p}$. 215 n. 111.

Odynerus Aneystrocerus) pilosus Saussure, Smithson. Miscell. Collect. XIV. P. 254. 1575

pocillum Sauss. - $\vec{f}-$ As.: Timor.

[p. 18 i n. 28, 우

Odynerus (Antepipone) pocillum Saussure, Stettin. entom. Zeitg. XXIII. $141 ; 2$ p. 2114 n.37. $\overrightarrow{.}$.

poecilus Sauss. - $\Omega$ - Eur.: Gallia.

T. 15 F. 6.

Odynerus Oplopus poecilus Saussure, Fitud. fam. Vespid. III. Masar. 1556 p. 314 n. 199, ૬; Odrnerus poecilus Ed. André, Spec. IIvmén. Europe II. P. 21-23. In-4 p. $771 \mathrm{n}$. 24, S.

polyphemus Kby. - Austr.: Christmas Island.

Odynerus Polyphemus W. F. Kirby, Proc. Zool. Soc. I.ondon 1658 p. 551.

pontebae Sauss. - 9 - Afr.: Algeria.

Odynerus Pontebae Saussure, Etud. fam. Yespid. III. Masar. 1456 p. 279 n. 16il. $\subseteq$.

Odỵnerus Pontebae Ed. André, Spee. Hỵmén. Europe II. P. $21-23.1544$ p. 704 n. 2. S.

ponticerianus Maindr. - $\rho$ o - As.: Pondichery.

Odynerus Ponticerianus Maindron, Ann. suc. entom. France bi) IJ. 1542 I3ull. p. XV, $₫ 5$. 
postieus Herr.-Sehaeff. - - - Eur.: Germania.

Odynerus posticus Herrich-Sehaeffer, Faun. Insect. German. 14 11 P. 176 T. 9 (S:

praecox Sauss. - 우 ô - Am.: Brasilia, Uruguay.

[; T.11 F.9.

()dynerus (Hypodynerus: praeeox Saussure, F́tud. fam. Yespid. III. Masar. 1556 j). 254 n. 130.

Odynerus Pachodynerus, praceox Saussure. Smithson. Miscell. Collect. XIV. P. 254. 1575

pratensis Sauss. $-\subseteq \vec{g}-A m$.: California, New Mexico.

[p. 210 n. $75 . \subseteq \jmath$.

Odynerus pratensis Saussure, Rev. \& mag. zool. 2) XXII. 1571 p. 61 n. $20, \subseteq j$.

Odynerus pratensis Saussure, Smithson. Miscell. C'ollect. XIV.P.2.54. 1575 p.292 n. 106.5 j.

primus D. T. - 우 oే - Am.: Texas.

Odynerus manifestus C'resson, Trans. Amer. Entom. Soc. IV. 1472 p. $240, \delta$ o inee Smith?.

Odynerus primus Dalla Torre, Wien. entom. Zeitg. VIII. 1889 p. 125.

privatus Walk. - ฮ゙-As.: Arabia.

Odynerus privatus Walker, List of Hymen. in Egypt 1571 p. 34 n. 173, oै.

proctus Cress. - 으 - Am.: C'uba.

Odynerus proctus ('resson, Proc. Entom. Soc. Philadelphia IV. 1s(i.5 p. 159. ऽ.

Odynerus proctus Saussure, Smithson. Miscell. Collect. XIV. P. 254.157 .5 p. 2 tit n. 55.5. productus Smith - ô - Am.: Panama.

Odynerus produetus Smith. Trans. Entom. Soc. London (3) I. 1.1862 p. 37. ठ․

Odynerus productus Saussure, Smithson. Miscell. Collect. XIV. P. 254.1575 p. 365 n. 2, วิ. propinquus Sauss. - - - Am.: Mexico.

Odynerus (Stenodynerus) propinquus Saussure, Rev. \& mag. zool. (2) XXII. 157 ) p. 104 n. 24,5 .

()dynerus (Stenodynerus) propinquus Saussure, Smithson. Miscell.Collect. XIV.P.254.1575 proximns Mor. - $ᄋ-$ Eur.: Russia.

[p. 326 n. 126, ㅇ.

Odynerus (Leionotus) proximus F. Morawitz, Horae soc. entom. Ross. IV. 1 st; 7 p. 124 n. 16 . S.

Odỵnerus proximus Ed. André. Spee. Hỵmén. Europe II. P. 21-23. $15 \varsigma 4$ p. 706 n. 4, $\varsigma$. pruinosus Smith - 우 - Am.: San Domingo.

Odynerus pruinosus Smith, Catal. Hymen. Brit. Mus. V. 1457 p. 79 n. 200. $\varsigma$.

Odỵnerus Stenodỵnerusj pruinosus Saussure, Smithson. Miscell. Collect. XIV. P. 254. 1875

przewalskyi (Mor.) D. T. - ô - As.: China.

[p. $351 \mathrm{n} .146$, ㅇ․

I.ionotus Przewalskyi F. Morawitz. Horae soc. entom. Ross. XIX. 185. p. 161 n. 17. j.

Odynerus Przewalskyi m.

psendodynerus D. T. $-€$ ô-As.: Java.

[우 of [nec Sauss. 1852].

Odynerus Pseudodynerus exiguus Saussure, Stettin. entom. Zeitg. XXII. 1 \&62 p. 194 n. 29.

Odỵnerus Pseudodynerus J)alla Torre, Wien. entom. Zeitg. VIII. I\$S! p. 125.

psilothorax Schlett. $-q-$ Am.: Chili.

Odynerus psilothorax Schletterer, Entom. Nachr. XVII. 1891 p. $\$ 7$, \&.

pubescens Thoms. - 우 ơ - Eur.: Succia, Russia, Caucasus.

Odynerus pubescens Thomson, Opusc. entom. P. 2.1870 p. 85 n. 5 , \& oै.

Leionotus pubescens Thomson, Hymen. Scandin. III. 1874 p. 49 n. 1, 우 7 .

Odynerus pubescens Ed. André. Spee. Hymén. Europe II. P. 21-24. 1n-1 p. 6int n. 5, S ô.

I.ionotus pubescens Aurivillius, Bih. Svensk. Vet.-Akad. Handl. XII. P. 4.5. 1586 p. 3.

Lionotus pubescens Aurivillius. Öfrers. Svensk. Vet.-Akat. Förh. XLY. P. 10.1 sse p. (607.

var. ungularis (Thoms.) D. 'T. $-\subseteq \hat{f}-$ Eur.: Succia.

Odynerus ungularis Thomson, Opuse. entom. P. 2.1870 p. 85 n. 6, $\&$ f.

lionotus pubescens var. ungularis Thomson. Hymen. Scandin. III. 1874 p. 50 n. 1.

Odynerus (Lionotus) pubescens $\mathrm{m}$.

pumilus Sauss. - $\&$ ơ - Am.: Peru, Cayenne.

C)lynerus Symmorphus pumilus Saussure, Ktud. fam. Yespid. III. Masar. 1s.3t p.197n.93. j.

Odynerus isymmorphus pumilus Saussure. Smithson. Miscell. Collect. XIV. P. 254. 1si5

[p. $156 \mathrm{n} .5$, 우 
punctatipennis Sauss. $-q-$ As.: India.

Odynerus (Leionotus) punctatipennis Saussure, Kitud. fam. Vespid. I. Eumén. 1552 p. 210

punctifrons (Thoms.) D. T. - \& oิ - Eur.: Helvetia.

[n. 115,ㅇ.

Lionotus punctifrons Thomson, Hymen. Sandin. III. 1874 p. 57 n. 2. 4.3.

Odynerus punctifrons $\mathrm{m}$.

punctum Sauss. - $\&$ - As.: Philippinae, Bengal.

T.19F.2.

Odynerus .I.cionotus) punctum Saussure, Ltud. fam. Vespid. I. Kumén. 15.52 p. 209 n. 114, $;$;

Odynerus punctum Saussure, litud. fam. Vespid. III. Masar. 155ti p. 243.

Odynerus punctum Horne, Trans. Zool. Soc. London VII. P. 3. 1870 p. 167, §; T. 20 F. 7 \& $7^{\mathrm{a}}$.

puniens Grib. - o. - Afr. bor.

Odynerus Punicus Gribodo, Ed. André: Spec. Hymén. Europe II. P. 21-26. 1586 p. 874,3.

pasillus Sauss. - $\odot-$ Austr.

Odynerus pusillus Saussure, Étud. fam. Vespid. III. Masar. 1556 p. 257 n. 172, f.

quadratus (Panz.) Kenn.1 - 우 ơ - Eur.

Vespa quadrata Panzer, Faun. Insect. German. VI. 1799 P. 63 T. 3.

Vespa quadrata Panzer, Krit. Revis. II. 1806 p. 156.

Vespa quadrata Jurine, Nouv. méth. class. Hymén. 1807 p. 170, ㅇ․

Odynerus quadratus Kennedy, Philos. Magaz. (3) XII. 1838 p. 17 n. 14.

Odynerus /Aneystrocerus; quadratus Smith, Catal. Brit. Fossor. Hymen. 1855 p. 207 n. 4.5 oै.

Odynerus quadratus Smith. Trans. Entom. Soc. London (3) V. 1867 Proc. p. XC.

quadricolor Mor. - 우 $\hat{o}-$ As.: Transeaspia.

Hoplomerus quadricolor F. Morawitz. Horae soc. entom. Ross. XIX. 1555 p. 146 n. 7, f.

Odynerus (Hoplomerus' quadricolor Ed. André, Spec. IYymén. Europe II. P. 24-26. 1 s86 p.68:2,

quadrifasciatus (Fabr.) Herr.-Schacff. - ot - Eur. fere tota.

[오 ㅎ․

Vespa quadrifasciata Fabricius, Entom. system. II. 1793 p. 266 n. 48.

Vespa simplex Fabricius, Entom. system. II. 1793 p. 267 n. 52.

Vespa quadrifasciata Fabricius, Syst. Piez. 1804 p. 262 n. 47.

Vespa simplex Fabricius, Syst. Piez. 1804 p. 263 n. 51.

Vespa quadrifasciata Gravenhorst, Vergl. Übers. zool. System. $1 \varsigma 07$ p. 276 n. 3862.

Odynerus trifasciatus Spinola, Insect. Ligur. II. 1808 p. 184 n. 8.

Odynerus quadrifasciatus Herrich-Schaeffer, Faun. Insect. German. 1839 P. 173 T. 19.

Odynerus nigripes Herrich-Schaeffer, Faun. Insect. German. 1839 P. 173 T. 21 if \& 22.

Odỵnerus Liudenii Lepeletier, Hist. nat. Insect. Hỵmén. II. $18+1$ p. $62.4 \mathrm{n} .13 . \subseteq \vec{\jmath}$.

Odynerus maculatus Lepeletier. Hist. nat. Insect. Hỵmén. II. 1411 p. 626 n. 14. $\subseteq$ oै.

Odỵnerus nigripes Ilerrich-Schaeffer. Faun. Insect. German. 1811 1'. 176 T. 1 ' is

Odynerus Ieionotus nigripes Saussure, Ftud. fam. Vespid. I. Fumén. 14.2 p. 1930 n. 57.

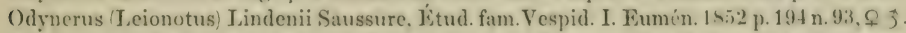

Odynerus quadrifasciatus Sehenck. Jahrb. Ver. Naturk. Nassau IX. 1. 14.53 p. 65 n. 23.50

Odynerus rigripes Schenck. Jahrb. Ver. Naturk. Nassau IX. 1. 1453 p. 82 n. 3. $\subseteq$ j.

Odynerus nigripes Saussure, Etud. fam. Vespid. III. Masar. 1451 p. $234, \subseteq \vec{\jmath}$.

Odynerus simplex Saussure. Btud. fam. Vespid. III. Masar. 1456 p. 236 \& 3.

Odynerus Leionotus, simplex Sehenek, Jahrb. Ver. Xaturk. Nassau XVI. 1961 p. fil, fi d f tis

[n. $1,90^{\circ}$

Odynerus (Leionotus nigripes Sehenek, Jahrb. Ver. Naturk. Nassau XVI. $1 \$ 661$ p. 64,67 d

699 n. $2, \propto 07$.

Odynerus quadrifasciatus Brischke, Schrift. phys. ökon. (ies. Königsberg II. 1\$62 $\}, 115, \S \delta$.

Odynerus L.eionotus simplex F. Morawitz. Iorae soc. entom. Ross. IV. 1 - 67 p. 12.4 n. 12.

Odynerus simplex F. Morawitz, Horae soc. entom. Ross. V. $1 \$ 65$ p. $146 \mathrm{n}$. 6. C.

Odynerus quadrifasciatus Thomson, Opuse. entom. P. 2. 1570 p. \& n. 15, \& . 3 .

Odynerus simplex H. Müller, Befrucht. d. Blumen 1873 p. 466.

Odỵnerus simplex Rudow, Arch. Ver. Fr. Naturg. Mecklenburg XXX. 1476 p. 225 n. 3. 5 o 
Odynerus nigripes Rudow. Arch. Ver. Fr. Naturg. Mecklenburg XXX. $1 \varsigma ; 6$ p. 226 n. $4, \Omega \delta$. Odỵnerus simplex Ed. André. Spee. Hỵmén. Europle II. P. $21-23.1644$ p. $687 \mathrm{n} .5, \subseteq$ Jै; T. 43 quadrimaenlatus André - $\subseteq-$ Eur.: Russia.

[F. $7,8 \& 13$.

Odỵnerus qquadrimaculatus Ed. André, Sp̣ec. Hỵmén. Europe II. P. 21 -23. 145.4 p. 699 n. 9.5. quadrisectus Say - of of - Am.: United States.

Odynerus quadrisectus Say, Boston Journ. Nat. Hist. I. 4. $14: 37$ p. 345 n. 1.

Odỵnerus Bellone Lepeletier. Hist. nat. Insect. Hỵmén. II. 1911 p. 6 i6io n. 40 , I gै.

Odỵnerus Ancistrocerus Bellone Saussure, F́tud. fam. Vespid. I. Eumén. 1 \$. 2 p.116 n.32. \&; Odỵnerus quadrisectus Saussure, Étud. fam. Vespid. III. Masar. 1856 p. 206. (T.16 F.10. Odỵnerus quadrisectus Leeonte. Writ. of Th. Say Entom. II. 1559 p. 765 n. 1.

Odynerus Stenaneystrocerus, quadrisectus Saussure, Smithson. Misecll.Collect.XIV.P.254. quadrituberculatus Smith $-\vec{\jmath}-A f r$ : Port Natal.

1975 p. 193 n. 31 , ๔ ว.

Odỵnerus quadritubereulatus Smith, Catal. Hymen. Brit. Mus. V. 1557 p. 70 n. 133, Jै. quartinae Grib. - Q - Afr. or.

Odṛnerus Quartinae Gribodo, Ann. mus. cir. Genova XXI. $1 \$ 54$ p. 290 n. $22 . \Omega$.

quixensis (Spin.) Osc.! - Am. : Brasilia.

Odynerus Quixensis Spinola Osculati, Explor. reg. equat. 1454 p. 203 n. 15 s. deser.]. radoszkowskii André - - - As.: Turcestania.

Odynerus Radoschowskii Fd. André, Spec. Hỵmén. Europe II. P. 21-23. 1554 p. i27 n. 17. C. radula (Fabr.) D. T. - 9 ot - Austr.: Ins. Sandwichenses.

Vespa radula Fabricius, Mant. Insect. I. 1787 p. 290 n. 44.

Vespa radula Gmelin, Linné: Syst. nat. Ed. $13^{3}$ I. 5. 1790 p. 2757 n. 82.

Vespa radula Olivier, Encycl. méthod. Insect. VI. 1791 p. 687 n. 90.

Vespa radula Fabricius, Entom. system. II. 1793 p. 269 n. 58.

Vespa radula Fabricius, Syst. Piez. 1804 p. 264 n. 57.

Odynerus localis Smith, Journ. Linn. Soc. Zool. XIV. 1879 p. 678 , 요 fే.

Odynerus localis Smith, Descr. New Spec. Hymen. 1879 p. 134 n. 1, \& o.

Odynerus loealis Blackburn \& Kirby, Entom. M. Magaz. XVII. 1850 p. 6.

Odynerus radula $\mathrm{m}$.

rafhrayi Rad. - ô - Afr.: Abyssinia.

Odynerus Raffrayi Radoszkowski, Horae soc. entom. Ross. XII. 1 s76 p. $145 \mathrm{n} .93 . \hat{\jmath}$. rectus 1). T. $-Q-$ Eur.: Hispania.

Odynerus Epipona albicinetus Mocsáry, Magy. Akad. Termész. Ḱrtek. XIII. P.11.1\$\$3 p.51

Odỵnerus albieinetus Ed. André. Spec. Hymén. Europe II. P. 21-23. 1 s\$4 p. 769 n. 24. ऽ.

Odynerus rectus Dalla T'orre, Wien. entom. Zeitg. VIII. 1889 p. 124.

reflexus Brullé - - ơ - Afr.: Ins. Canarienses.

()dynerus reflexus Brullé, Barker-Webb \& Berthelot : Hist. nat. ilesCanar. II. 15.10 p. 49 n.42.

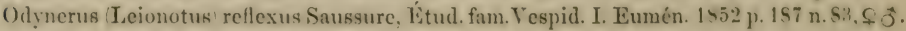

regulus Sauss. - $\subsetneq$ $ో-\Lambda$ fr.: Algeria.

[Q ठै; T. 11 F. 5 .

Odynerus (Parodynerus regulus Saussure, Ktud. fam. Vespid. III. Masar. 1 siti p. 247 n. 121.

Odynerus regulus Ed. André, Spec. Hymén. Europe II. P. 21-23. 1 that p. 691 n. 2,5 s.

reniformis (Gmel.) Wesm. - $\subseteq$ oै - Eur. centr. \& mer.

Vespa XVIII Schaeffer, Icon. Insect. Ratisbon. II. 1. 1765; T. 117 F. 3.

Vespa XXIV Schaeffer, Icon. Insect. Ratisbon. III. 1779; T. 233 F. 6.

Vespa spec. Zschach, Mus. Leskcan. 1788 p. 74 n. 418 \& 449.

Vespa reniformis Gmelin. Linné: Syst. nat. Ed. $13+$ I. 5. 17990 p. 2760 n. 94. $f$.

Tespa melanochra Gmelin. Tinné: Syst. nat. Ed. 133 I. 5.1790 p. 2760 n. 95, j.

Vespa reniformis Olivier, Eneycl. méthod. Insect. VI. 1791 p. 695 n. 10.

Vespa melanochra Olivier, Eneycl. méthod. Insect. VI. 1791 p. 695 n. 11.

Vespa aucta Panzer, Faun. Insect. German. VU. 1801 P. 81 T. 17. 
Vespa aucta Panzer, Krit. Revis. II. 1806 p. 156.

Odynerus reniformis Wesmael, Monogr. Odynèr. Belgique 1533 p. 1 n. 1, 5 o .

Odynerus (Oplopus) reniformis Wesmael, Bull. acad. sc. Belgique III. 1536 p. 45.

Odynerus spec. Spinola, Ann. soc. entom. France VII. 1838 p. 504.

Pterochilus coxalis Herrich-Schaeffer, Faun. Insect. German. 1539 P. 173 T. $17(\hat{\jmath})$.

Odynerus Reaumuri Dufour, Ann. sc. nat. Zool. (2) XI. 1839 p. 90, 오

Odynerus coxalis Spinola, Ann. soc. entom. France VIII. 1539 Bull. p. XL.

Odynerus Reaumurii Blanchard, Hist. nat. Insect. IU. 1810 p. 390 n. 2.

Odynerus reniformis Lepeletier, Hist. nat. Insect. Hymén. II. 1 s 11 p. 6016 n. 1, ơ (excl. S).

Odynerus Dufourii Lepeletier, Hist. nat. Insect. Hymén. II. 1541 p. 642 n. $27, s$.

Pterocheilus coxalis Herrich-Schaeffer, Faun. Insect. German. 1841 P. $171 ;$ T. 19 if.

Odynerus |Oplopus) Reaumurii Saussure, Étud. fam. Vespid. I. Eumén. 18.2 p. 2222 n.1311. ठ .

Odynerus (Oplopus) reniformis Saussure, Étud. fam. Vespid. I. Eumén. 1852 p. 226 n. 135.

[우 ठ; T. 20 F. 1 (ㅇ).

Odynerus (Oplopus) relox Saussure, Étud. fam. Vespid. I. Eumén. 18.52 p. 228 n. $136, \varsigma$.

Pterocheilus reniformis Schenck. Jahrb. Ver. Naturk. Nassau IX. 1.1853 p. 54 n. $19, \varsigma \widehat{o}$.

Pterocheilus coxalis Schenck, Jahrb. Ver. Naturk. Nassau IX. 1. 1853 p. $\$ 1$ n. 4, o j

Odỵnerus Reaumurii Saussure, Étud. fam. Vespid. III. Masar. 1856 p. 304.

Odynerus reniformis Saussure, Litud. fam. Vespid. III. Masar. 1556 p. 307, $\subsetneq$ ș; T. 15) F. $9(\subseteq)$.

Odỵnerus relox Saussure, litud. fam. Vespid. III. Masar. 1456 p. $308 . \quad$ n. $4, \subseteq \hat{\jmath}$.

Odỵnerus Hoplopus) reniformis Schenek, Jahrb. Ver. Naturk. Nassau XVI. 1561 p. $52, \$ 3$ \& 86

Pterocheilus coxalis Brischke, Schrift. physs. ökon. Ges. Königsberg II. 1862 p. 114. ؟.

Pterocheilus reniformis Brischke, Schrift. phys. ökon. Ges. Königsberg II. 1862 p.114, $\Omega \jmath$.

Pterocheilus reniformis Taschenberg, Hymen. Jeutschl. 1866 p. 247, $f \hat{o}$.

Odynerus reniformis Thomson, Opusc. entom. P. 2. 1870 p. 81 n. 1, 우 đ.

Hoplomerus reniformis Thomson, Hymen. Scandin. III. 1874 p. 43 n. $1, \subseteq \hat{o}$.

Odynerus reniformis E. Saunders. Entom. M. Magaz. XIII. 1876 p. 114 nota.

Odynerus reniformis Rudow, Arch.Ver. Fr. Naturg. Mecklenburg XXX. $18 ; 6$ p. $2: 31$ n. $2, \subseteq \jmath$

Odynerus (Hoplopus) ruficornis Rudow, Arch.Ver. Fr. Naturg. Necklenburg XXX. 1s itjp. 234

Odynerus reniformis Smith, Entomologist X. 1877 p. 67.

[n. 6 .

Odynerus Hoplopus, reniformis E. Saunders, Trans. Entom. Soc. London 1882 p. 17in n.4.

Odỵnerus (Epipona) depressus Ed. André, Natural. Sicil. II. 10. 1543 p. 232, 5.

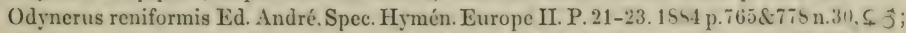

Odynerus reniformis Billups, Entom. M. Magaz. XXI. 15st p. 6\$-69.

T. 43 F. 11.

Odynerus reniformis I. Saunders, Entom. M. Magaz. XXIV. 1557 p. 65.

Pterocheilus reniformis Verhoeff, Verh. naturh. Ver. preuss. Rheinl. XLVIII. 1s91 ; T. 3 F. is.

republicanus D. T. - 우 - Am.: Carolina.

[Smith, nec Sauss.].

Leptochilus ornatus Saussure, Kitud. fam. Vespid. I. Eumén. 1552 p. 231 n n. 6, ऽ; T. 20 I . 4 [nec

Leptochilus urnatus Saussure. Smithson. Miscell. Collect. XIV. P. 254.1575 p. 367 n. 2, . .

Odynerus republicanus 1)alla 'ourre, Wien. entom. Zeitg. VIII. 15s!) p. 125.

rhachiphorus Schlett. - ô-Am.: Chili.

Odynerus rhachiphorus Sehletterer, Entom. Nachr. XVII. 1s!1 p. $55, \hat{\jmath}$.

rhodensis Sauss. - 0 - Eur.: Rhodus.

[n. 96, ㅇ; 'T. 10 F.7.

Odynerus (Subaneỵstrocerus, Rhodensis Saussure, Etud. fam. Vespid. III. Masar. 1 a ati p. 201 -

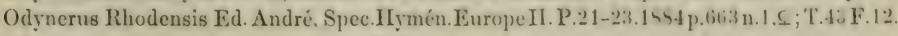

rhodopterus Schlett. - $\mathrm{Q}-\mathrm{Am}$.: Chili.

Odynerus rhodopterus Sehletterer, Entom. Nachr. XVII. 15!1 p. 92, s.

rhombiferus Duf. - $q-$ Afr.: Algeria.

Odynerus rhombiferus Dufour, Aun. soe. entom. France 3.15 .53 p. 341 n. 10 , s.

rhynehoides Sauss. - ô - Afr.: Senegal.

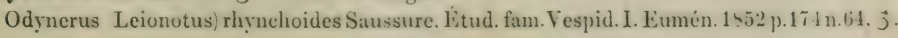


Odỵnerus rhynchoides Saussure, Étud. fam. Vespid. III. Masar. 1456 p. 231.

Odỵnerus rhynchoides Magretti, Ann. mus. cir. Genova XXI. 1654 p. 619 n. 159. roccellne Dest. - - - Eur.: Sicilia.

Odynerus Rocellae Destefani, Natural. Sicil. VIII. 1859 p. 230. \&; T. 3 F. 5-6. romandinns Sauss. - 0 ơ - Am.: Venezuela, Columbia.

()dynerus Leionotus' Romandinus Saussure, Étud. fam. Vespid. I. Eumén. 15.52 p. 1 4 n. i9.

Odỵnerus Romandinus Saussure, Smithson. Miscell. Collect. XIV. P. 254. 1575 p. 246 [n. 109, 웅.

Odynerus Leionotus) Romandinus Kirsch, Mittheil. zool. Mus. Dresden III. 1878 p. 379. rossii Lep. - 워 - 0 Eur. centr. \& mer.

Odynerus Rossii Lepeletier, Hist. nat. Insect. Hymén. II. 1841 p. 633 n. 19. ๔ j.

Vespa Odynerus ichneumonidea Ratzeburg. Forstinsect. III. 1444 p. 52 n. 5 , Jै; T. 4 F. .

Odynerus Leionotus Rossii Saussure, Étud. fam. Vespid. I. Eumén. 1552 p. 207 n. 111. Odỵnerus Rossii Saussure, Ėtud. fam. Vespid. III. Masar. $1856 \mathrm{j}$ p. $211, \subseteq \hat{\sigma}$.

Odynerus I.eionotus Rossii Schenck, Jahrb. Ver. Naturk. Nassau XVI. 1 sij1 p. 70 nota. S. Leionotus Rossii Thomson, Hymen. Scandin. III. 1874 p. 54, \& ơ.

Odỵnerus Rossii Ed. André, Spec. Hymén. Europe II. P. 21-23. 1854 p. 740 n. 7, S ふै. rotundutus Walk. - ơ - Afr.: Aegyptus.

Odynerus rotundatus Walker, List of Hymen. in Egypt $15 i 1$ p. 37 n. 183. j.

rotundigaster Sauss. - $ᄋ \hat{f}-$ Afr.: Algeria.

['I' 19 F. 8.

Odynerus (Oplopus rotundigaster Saussure, Étud. fam.Vespid.I. Jumén. 15.52 p. 221 n.125.S;

Odynerus rotundiventris Saussure, Étud. fam. Vespid. III. Masar. 1856 p. 304 . [\& 3 .

Odynerus rotundiventris Ed. André, Spec. Hymén. Europ̣e II. P.21-23. 1554 p. 763 \& 769 n. 22.

var. tunetanus Grib. - Afr.: Tunisia.

[26.1586 p.875.

Odynerus rotundiventris var.Tunetanus (Gribodo) Ed. André, Spec.Hymén. Europe II. P.24-

rubiginosus André - $\&-$ As.: Caucasus.

Odynerus rubiginosus Ed. André, Spec. Hymén. Europe II. P. 21-23. 1854 p. 7 ti n. 16. S. rubripes André - 우 - Eur.: Russia.

Odynerus rubripes Ed. André, Spee. Hymén. Europe II. P. 21 23. 1554 p. 706 n. 5. S. rubropustulatus 13lackb. \& Cam. - $\vec{\jmath}-$ Austr.: Ins. Hawaiae. $[1 \leqslant 46$ p. 219 n. $31 . \hat{\jmath}$. Odynerus rubroplustulatus Blackburn \& Cameron, Mem. Manchester Lit. \& Philos.Soc. 3 X. rubrosignatus André - - - As.: Turcestania.

Odynerus rubrosignatus Ed. André, Spee. Hymén. Europe II. P. 21-23. 1854 p. 742 n. 11. \&. rudolphi D. T. - $f \hat{\sigma}-$ Austr.: Ins. Hawaiae.

Odynerus cardinalis 13lackburn \& Cameron, Mem. Manchester I.it. \& Philos. Soc. '3!X. 1456 [p.218n.29, \& ठ̛ [nec Blackb. \& Cam., nec Mor.].

Odynerus Rudolphi Dalla Torre. Wien. entom. Zeitg. VIII. 1559 p. 124.

rufescens Smith - 오 - As.: Ceram.

Odynerus rufesecus Smith, ('atal. Ifymen. Brit. Mus. V. 1557 p. 61 n. 6i3. C.

Odynerus rufescens Saussure. Stettin. entom. Zeitg. XXIII. 1562 p. 199 n. 33.5.

rufleollis Spin. - $\odot \hat{o}-$ Am.: Chili.

Odynerus ruficollis Spinola. Gay: Hist. fis. ('hile. Zool. VI. 1 s) p. 259 n. $5 . \subseteq$.

Odỵerus Leionotus ruficollis Saussure, litud. fam. Vespid. I. Eumen. 1852 p. $16 \mathrm{in}$ n. 57,5 . Odynerus Hypodynerus; ruficollis Saussure. Smithson. Miseell. Colleet. XIV. P. 254. 1475 rufldulus Lep. $-Q-$ Afr.: Algeria.

[p. $226 \mathrm{n} .63,9$ ఠิ

Odynerus rufidulus I.epeletier. Hist. nat. Inscet. IYymén. II. $15+1$ p. 611 n. 26. 5.

Odynerus rufidulus Lucas. Explor. se. Algérie. Zool. III. 14.16 p. 237 n. 194, ऽ; T. 11 F. 11.

Odynerus (Oplopus rufidulus Saussure, Etud. fam. Vespid. I. Eumén. 1452 p. 222 n. 129.

()dynerus rufidulus Ed. André, Spee. Hỵmén. Europe II. P. 21-23. 1554 p. 773 n. 2. S. 
ruflnodus Cress. - Q - Am.: New Mexico.

Odynerus rufinodus Cresson, Trans. Amer. Entom. Suc. I. Ialis p. 341 n. 22, c.

Odynerus rufinodus Saussure, Smithson. Miscell. Collect. XIV. P. 254. 1575 p. 365 n. 1, $\subseteq$. saecnlaris Sauss. $-\propto$ oิ - Am.: United States.

Odynerus /Ancistrocerus' saeeularis Saussure, Kitud. fam. Vespid. I. Eumén. 1852 p. 142 n.26.

Odỵerus 'Stenaneystrocerus) saecularis Saussure, Smithson. Miscell. C'ollect. XIV. P.254.

[1875 p. 202 n. 38 , 웅.

sakalarus Sauss. - - - Afr.: Madagascar.

[T. 22 F. 4 .

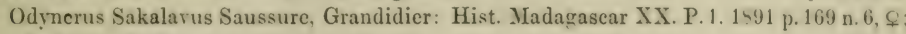

sandwichensis Sauss. - 우 ô - Austr.

[n. 177, ơ.

Odynerus 'Antodynerus) Sandwichensis Saussure, Étud. fam. Vespid. III. Masar. 1856 p. 259

Odynerus rubritinetus Smith, Journ. Linn. Soc. Zool. XIV. 1879 p. (77!). \& excl. of].

Odynerus rubritinctus Smith. 1)eser. New Spec. Hymen. 1879 p. 1:55 n. 3. $\subseteq$ excl. $\hat{\jmath}$.

sanguinolentus Sauss. - $\hat{o}-$ Patria?

[p. $221 \mathrm{n} .116$, ơ.

Odynerus Ancistroceroides; sanguinolentus Saussure, Étud. fam. Vespid. III. Masar. 1456 santa-annae Sauss. - 0 to - Am.: Mexico.

Odynerus Ancistrocerus, Santa-Anna Saussure. Rev. \& mag. zool. 22) IX. 15.5 i p. 273.

Odyneru: Ancystrocerus, Santa-Amnae, Saus*ure, Smithson. Miscell. Collect. XIV. P. 254

sareptanns André - Q -- Eur.: Russia.

$[1875$ p. $171 \mathrm{n} .16$, ๑

Odynerus Sareptanus Ed. Audré, Spec. Hymén. Europe II. P. 21-23. 1954 p. 772 n. 27. 5

saucius Sauss. - 으- Austr.

Odynerus saucius Saussure, Étud. fam. Tespid. III. Masar. 1856 p. 280 n. 163, $\subsetneq$.

saussurei André - $q-$ Afr.: Aegyptus.

- Sarignt, Deser. de l'Égpyte. Hymén. 1s12; T. 9 F. 17, ؟. n. 4i, $\subseteq[$ nec Brullé].

Odynerus interruptus Saussure, Mém. soc. phỵs. d hist. nat. Genère XVII. $1.18: 3$ p. 221

Odỵnerus Saussurei Ed. André, Spec. Hymén. Europe II. P. 21-23. 185.4 p. (152 n. 2. S.

Odỵnerus Saus:urei Magretti, Ann. mus. civ. Genova XXI. 1584 p. 620 n. 161.

sarignyi Sauss. - 우 - As.: Arabia.

Odynerus (Oplopus Sarignỵi Saus-ure, Étud. fam. Vespid. I. Eumén. 15.22 p. 226 n. 134, ؟

Odynerus Sarignyi Saussure, Étud. fam. Vespid. III. Masar. 1456 p. 307.

seabriuseulus Spin. - 오

Odynerus scabriusculus Spinola, Gay: Hist. fis. Chile. Zool. VI. 1551 p. 262 n. $\triangleleft, \subseteq 5$

()dynerus |Ancistrocerus| scabriusculus Saussure, Ėtud. fam. Vespid. I. Eumén. 1552 p. 140

[n. $24,907$.

Odỵnerus / Ancistrocerus, scabriusculus Saussure, Eitud. fam. Vespid. III. Masar. 1คว4; T. 11

$[\mathrm{F} .1(\widehat{o})$.

Odrnerus Stenaneystrocerus seabriusculus Saussure, Smitlıson. Miscell. Colleet. XIV scandinarus Sauss. - ô - Eur.: Seandinavia.

(P. 254. 1875 p. 198 n. 35, ơ.

Odynerus Oplopus) Scandinavus Saussure, Etud. fam. Vespid. III. Masar. Lhifi p. 31.4

[n. 198, ơ; 'T'. 15 F. 10.

Odynerus Seandinavus Ed. André, Spee. Hymén. Europe II. 1. $21-23.1484$ p. 755 n. 1., 3.

sehlettereri D. I' - 우 - Am.: Chili.

Odynerus Leionotus, Fairmairei Schletterer. Entom. Nachr. XVII. 1 s91 p.43, \& nee Sauss. i.

Odynerus Schlettereri m.

scutellaris Sauss. - $\hat{o}-$ Am.: California.

Odynerus seutellaris Saussure. Rev. d mag. zool. (2) XXII. 14il p. 54 n. 11, j.

Odynerus seutellaris Saussure, Smithsen. Miscell. Cullect. XIV. P. 251.1575 p. 262 n. 7 , j.

seenndus D. T. $-\subseteq-$ As.: Gilolo.

Odynerus fallax Smith. Journ. of Proe. I.imn. Soc. Zool. VI. 14 (tif p. 35 n. 2. S [nec Sauss. .

Odynerus secundus Dalla Torre. Wien. entom. Zeitg. VIII. 1ss! p. 125. 
selectus Walk. - $\mathrm{Q}-$ As.: Arabia.

Odynerus selectus Walker, List of Hymen. in Egypt 1571 p. 34 n. 172.5 .

sellutus Mor. - \& ơ - As.

Lionotus sellatus F. Morawitz, Horae soc. entom. Ross. XIX. 1555 p. 172 n. 23, $\subseteq j$.

Odynerus (Leionotus' sellatus Ed. André, Spec. Hỵmén. Europe II. P. 24-26.1556 p. $577, \mp j$.

semisaecularis D. T. - 우 - As.: Turcestania.

[Haliday].

Odynerus humeralis Ed. André, Spee. Hymén. Europe II. P. 21-23. 1554 p. 701 n. 11. ๔ [nee

Odynerus semisaecularis Dalla Torre. Wien. entom. Zeitg. VIII. 1559 p. 125.

senegalensis Sauss. - 우 - Afr.: Senegal.

[8; T. 19 F. 7 .

Odynerus (Oplopus' Senegalensis Saussure, lítud. fam.Vespid. I. Eumén. 1852 p. 219 n. 125. septemfasciatus Smith - 우 - As.: Borneo.

Odynerus septemfasciatus Smith, Journ. of Proc. Linn. Soc. Zool. II. 1857 p. 111 n. 3. j.

Odynerus septemfasciatus Gribodo, Bull. soc. entom. Ital. XXIII. 1891 p. $295, \mathrm{~S}$.

serripes Mor. - 0 t - Eur.: Russia.

Odynerus (Epipona serripes F. Morawitz, Horac soc. entom. Ross. IV. 1867 p. 137 n. 2. . $\hat{\jmath}$.

Odynerus serripes Ed. André, Spec. Hymén. Europe II. P.21-23.1994 p.763 วิ| \& 775 \& s. n.23.

sesquicinetus Sauss. - 우 - Afr.: Cap.

[n. 189, ㅇ․

Odynerus Antepipone) sesquicinctus Saus:ure, Étud. fam. Vespid. III. Masar. 1556 p. 299 sessilis Sauss. - 우 - Eur.: Hispania.

Odynerus Leionotus, sessilis Saussure, Étud. fam. Vespid. I. Eumén. 1852 p. 197 n. 98, C.

Odynerus sessilis Ed. André, Spec. Hymén. Europe II. P. 24-26. 1556 p. 8titi, $\subseteq$.

sibiricus Mocs. - $\subseteq-$ As.: Sibiria.

[p. 54 n. 68 , 오.

Odynerus Epipona, Sibiricus Mocsary, Magy. Akad. Termész. Értek. XIII. P. 11. 1s43

Odynerus Sibiricus Ed. André, Spec. Hymén. Europe II. P. 21-23. 1854 p. 745 n. 5. S.

sichelii Sauss. - - ơ-As.: India. [n. 94, \&; T.10 F.6.

Odynerus (Subancistrocerus, Sichelii Saussure, Étud. fam. Vespid. III. Masar. 15.16 p. 206

Odynerus (Ancystrocerus) Sichelii Saussure, Reise d. Novara. Zool. II. 1. 1567 Hymen. p. 4 siculus Dest. - 우 - Eur.: Sicilia.

Odynerus Leptochilus Siculus Destefani, Natural. Sicil. II. 15533 p. 5.5, S.

Odynerus Siculus Ed. André, Spec. Hymén. Europe II. P. 21-23. 1 ş4 p. 721 n. 8, ธ. signaticollis (Mor.) D. T' - 우 - Eur.

Hoplomerus signaticollis F. Morawitz, Horae soc. entom. Ross. XXII. 18s5 p. 262, ؟. Odynerus signaticollis $\mathrm{m}$.

signatus Sauss. - - - Afr. mer.

[n. 136, ㅇ.

Odynerus Hypodynerus) signatus Saussure, Étud. fam. Vespid. III. Masar. 1556 p. 255

silaos Sauss. - Q ô - Afr.: Cap, Mauritius.

[T. 19 F. $6($ Q).

Odynerus (Leionotus) Silaos Saussure, Kitud. fam. Vespid. I. Eumén. 15.52 p. 213 n. 119 . \ 3:

Odynerus (Antepipona) Silaensis Saussure. Kitud. fam. Vespid. LI. Masar. 14, 6 p. 24.

(Odynerus (Antepipona) Silaensis Saussure. Mém. soc. phys. \& hist. nat. Genère XVII. 1.

[1863 p. 233 n. 58, ơ.

Odynerus Silaensis Saussure, Grandidier: Iist. Madagasear XX. P.1. 1491 p. 172 n. S. $\subseteq$ S.

simillimus Mor. - \& ô - Eur.: Russia.

Odynerus lipipone simillimus F. Morawitz, Horae soc. entom. Ross. IV. 1467 p. 138 n.26, \& j

Odynerus simillimus Ed. André, Spee. Hymén. Europe II. I. 21-23. 1 s 4.4 p. $76 f$ d 776 n.32.

similis Smith - ㅇ - Am.: Mexico.

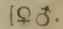

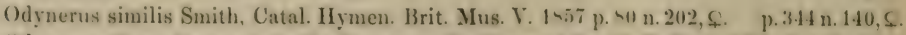

()dynerus Stenodynerus, similis Saussure. Smithson. Miseell. Collect. XIV. P. 25. 16is

simplicicornis Sauss. - $ᄋ$ ơ-Am.: Cuba.

[n. 128, ô.

Odynerus Hypodynerus) simplieicornis Saussure, Etud. fam. Vespid. III. Masar. 15.56 p. 253

Odynerus simplicicornis Saussure, Sagra: Hist. fis. Cuba V11. 1556 p. 7i1; T. 19 F. 5. 
Odynerus simplicicornis Saussure, liev. dt mag. zool. (2) X. 15.45 p. 166 n. 2.

()dynerus simplicicornis C'resson, Proc. Entom. Sue. Philadelphia IV. 1 slia p. 165, o 5 .

() lynerus Pachodynerus) simplicicornis Saussure, Smithson. Miscell. Collect. XIY. P.251.

simuatofaseiatus Costa $-\propto \hat{o}-$ Eur.: Sardinia.

$[1875$ p. 235 n. 68, 웅.

Odynerus sinuatofasciatus Costa. Rend. acead. se. fis. Napoli 2 II. 145s p. 10.4.

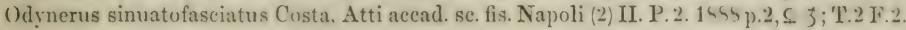

sinuatus (Fabr.) Spin. $-\propto$ oै - Eur. centr.

Vespa sinuata Fabricius, Entom. system. II. 1793 p. 270 n. 62.

Vespa sinuata Fabricius, Syst. Piez. 1804 p. 264 n. 61.

Odynerus bifasciatus Spinola, Insect. Ligur. II. 1808 p. 184 n. 9.

Odynerus bifasciatus Wesmael, Monogr. (Odynèr. Belgique 1533 p. $45 \mathrm{n} .8$, .

Odynerus Symmorphus bifasciatus Wesmacl. Bull. acad. se. Belgique III. 15:36 p. 4., S. Odynerus bifasciatus Jeppeletier, Hist. nat. Insect. IYymén. II. $14+1$ p. 665 n. $14, \subseteq j$. (Odynerus sinuatus Saussure, Fitud. fam. Vespid. III. Masar. $15.51 \mathrm{p}$ p. 192 n. 56.03. Odynerus Symmorphus sinuatus Smith. Catal. 13rit. Fossor. Ifymen. 1455 p. 201 n. $2.6 \hat{\jmath}$. Odynerus Symmorphus sinuatus Sehenek, Jahrb. Ver. Naturk. Nassau XVI. $1 \checkmark 61$ p. 37, Odynerus sinuatus H. Müller. Befrucht. d. Blumen $1573 \mathrm{p} .466 . \quad 38,39 \& 41 \mathrm{n} .5 . \mathrm{s}$. Odỵnerus sinuatus Rudow. Arch. Ver. Fr. Naturg. Mecklenburg XXX. 157 i p p. 222 n. $\bar{i}, \subseteq \vec{s}$. ()dyerus (Srmmorphus) sinuatus E. Saunders, Trans. Jintom. Soc. London $1 \$ 52$ p. 150 n. 15. Odynerus sinuatus Ed. André. Sprec. Hymén. Europe II. P. 21-23. I44 p. 6.j7 n. 4, ธ. Odỵerus sinuatus Schulthess. Hymen. Helvet. Jiplepte 1557 p. 39 n. 5,0 의.

smithii D. T. - 우 ô - Austr.: Ins. Hawaiac.

()dynerus agilis Smith. Journ. Linn. Soe. Zool. XIV. 1579 p. 651, of nee Smith 155 . Odynerus agilis Smith, Deser. New Spec. Hymen. 1879 p. 137 n. 6, J.

Odrnerus agilis Blackburn it Kirbr; Entom. M. Magaz. XVII. 1sse p. ST.

Odynerus agilis Blackburn of Cameron. Mem. Manchester Lit. \& Philos. Soe. 3) X. 1956 p.22:

Odrunerus Smithii Dalla Torre, Wien. entom. Zcitgr. VIII. Iss!! p. 124. [n. 34, fơ.

sobrinus Smith - $\mathrm{Q}-$ As.: Ceram.

Odynerus sobrinus Smith, Journ. of Proc. Lium. Soc. Zool. VII. 1 Sti3 p. 10 n. 5, f.

solstitialis Sauss. - 우 ơ - Afr.: Cap.

[n. 187, oิ.

Odynerus Antepipona solstitialis Saussure, Kitud. fam. Vespid. III. Masar. 1556 p. 294

Odynerus solstitialis Magretti. Ann. mus. eir. Genova XXI. $15 \$ 4$ p. $620 \mathrm{n} .160 . \subseteq$ s .

spectabilis Sauss. - 우 - Am.: Cuba.

Odynerus speetabilis Saussure, Rev. \& mag. zool. (2) XXII. 1471 p. 60 n. 16.

()dynerus spectabilis Saussure. Smithson. Miscell. ('ollect. XIV. P. 251. 1475 p. 27 s n. $95, f$.

spinifer Sauss. - 우 - Am.: Brasilia.

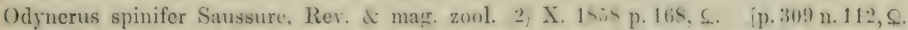

Odrnerus Stenodynerus, spinifer Saussure. Smithson. Miseell. ('ollect. XIV. 1'.254. 1-75

spinipes (L.) Spin. - $f$ t - Eur. tota.

Vespa spinipes Linné, Syst. nat. Ed. $10^{3}$ I. 1758 p. 573 n. 7.

Vespa spinipes Linné, Fauna Suce. Ed. 2a 1761 p. 419 n. 1682.

Vespa spinipes Linné, Sỵst. nat. Ed. $12^{a}$ I. 2.1767 p. 950 n. 10.

Vespa spinipes Fabricius, Syst. entom. 1775 p. 368 n. 28.

Vespa spinipes Ph. T. Müller. Limé: Vollst. Natursyst. V. 2. 1775 p. 4५2 n. 10.

Vespa spinipes O. F. Müller, Zool. Dan. Prodrom. 1776 p. 163 n. 1891.

Vespa spinipes Fabricius, Spec. Insect. I. 1781 p. 464 n. 36.

Vespa spinipes Fabricius, Mant. Insect I. 1787 p. 290 n. 42.

Vespa spinipes Villers, C. Linnaei entom. III. 1789 p. 267 n. 7.

Vespa spinipes Gmelin, Linné: Syst. nat. Ed. $13^{\text {a }}$ I. 5. 1759 p. 2752 n. 10.

Vespa spinipes Rossi, Fauna Etrusea II. 1790 p. 86 n. 867 .

Catalogus Hymenopteroram. IX. 
Vespa spinipes Christ, Naturg. d. Insect. 1791 p. 233.

Vespa muraria Christ, Naturg. d. Insect. 1791 p. 233.

Vespa quinquefasciata Fabricius, Entom. system. U. 1793 p. 267 n. 49.

Vespa spinipes Fabricius, Entom. 6ystem. II. 1793 p. 268 n. 55.

Vespa spinipes Panzer, Faun. Insect. German. II. 1794 P. 17 T. 18.

Odynerus murarius Latreille, Hist. nat. Crust. \& Insect. III. 1802 p. 362.

Vespa quinquefasciata Fabricius, Syst. Piez. 1804 p. 262 n. 48.

Vespa spinipes Fabricius, Syst. Piez. 1804 p. 263 n. 54.

Odynerus murarius Latreille. Hist. nat. C'rust. \& Insect. XIII. 1805 p. 347.

Vespa spinipes Panzer, Krit. Revis. II. 1806 p. 156.

Vespa spinipes Illigrer, Rossi: Fauna Etrusea Ed. 2a II. 14117 p. 142 n. blis.

Vespa spinipes Jurine, Nouv. méth. class. Hymén. 1807 p. 170, đ.

Vespa spinipes Klug, Magaz. Ges. naturf. Fr. Berlin II. 1807 p. 51 n. 56.

Eumenes (Odynerus' spinipes Latreille, Gen. C'rust. \& Insect. IV. 1 so9 p. 140.

Odynerus spinipes Wesmael. Monogr. Odynèr. Belgique $15: 3: 3$ p. $6 \mathrm{n}$. 2. 5 .

Odỵnerus Oplopus spinipes Wesmael. Bull. acad. se. Belgique III. 1536 p. 45.

Fpipone spinipes Westwood. Trans. Entom. Soc. London I. 3. 1836 p. 200.

Epipone spinipes Hewitson, Entom. Magaz. V. 1837 p. 77.

Odynerus spinipes Audouin, Ann. se. nat. Zool. (2, XI. 1539 p. 104; T. 5 F. 5-7.

Odynerus spinipes Blanchard, Hist. nat. Insect. III. 1840 p. 389 n. 1.

Oplomerus spinipes Westwood, Introd. mod. Classif. Insect. II. 1510 Synops. p. 54.

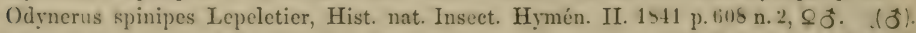

Pterocheilus spinipes Herrich-Schaefer. Faun. Insect. German. $15+1$ P. 176 T.16 (\$) Fig. b

Odynerus Oplopusi spinipes Saussure, Kitud. fam. Yespid. I. Eumén. 1552 p. 223 n. 131, \& J.

Pterocheilus spinijes Schenck, Jahrb. Ver. Naturk. Nassau IX. 1.1553 p. 53 n. 17, \& j.

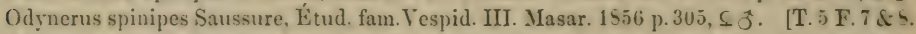

Odynerus Oplopus) spinipes Smith, Catal. Brit. Fossor. Hymen. 155̄s p. 203 n. 4, Q

Odynerus (Hoplopus) spinipes Schenck, Jahrb. Ver. Naturk. Nassau XVI. 1 S61 p. S2, S3

[\$. $\$ 4$ n. $2, \subseteq 3$.

Pterocheilus spinipes Brischke, Schrift. phỵs. ökon. Ges. Königsberg II. 1562 p.114, $\Omega$ ฮ̃.

Pterocheilus spinipes Taschenberg, Hymen. Deutschl. 1५66 p. 247, $\subseteq$ oै.

Odynerus spinipes Thomson, Opusc. entom. P. 2, 1870 p. 84 n. 2, 우 ठొ.

Odynerus spinipes Chapman, Entom. M. Magaz. VI. 1870 p. 214.

Odynerus spinosus H. Müller, Befrucht. d. Blumen 1873 p. 466.

Odỵerus quinqquefisciatu: H. Müller, Befrucht. d. Blumen $1<73$ p. 466.

Hojilomerus spinipes Themson, IIymen. Seandin. III. I474 p. 45 n. 2 , $\subseteq$ of.

()dynerus spinipes Rulow, Arch. Ter. Fr. Naturg. Mecklenburg XXX. 1576 p. 233 n. 4. $\subsetneq 0\}$

()dymerus Iloplopus spinipes E. Saunders. Trans. Fntom. Soc. London 1552 p. 173 n. 1.

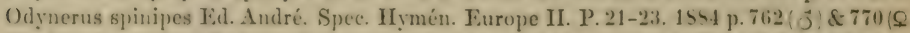

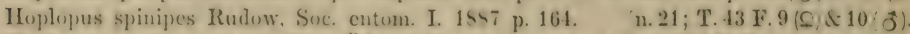

IIoplonerus spinipes Aurivillius, Öfvers. Srensk. Vet.-Akad. Förh. XI.V. P. 10. 1 ss p. 606.

Odynerus spinipe: (arpentier. Mém. sue. I.imn. Nord France VII. 14s9 p. 6i-69.

()dynerus spinipes Carpentier, 13ull. soc. I,imn. Nord de la France X. 1592 p. 326.

J'terocheilus spinipes Verhoeff, Verh. naturh. Ver. preuss. Rheinl. XI,VIII. 1 \$91 p.40-57 n.1,

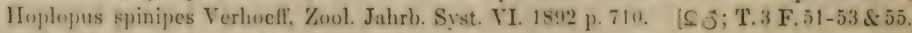
spinolae Sauss. - $\subsetneq-A m$.: United States.

Odynerus Ancyst ruecrus, Spinolae Saussure, Fitud. fam. Vespid. III. Masar. 1856 p. 216 n.107.

(Blynerus Aneystrocerus Spinolae Saussure, Smithson. Miscell. Collect. XIV. P. 254. 1475

spirieoruis Spin. - $\subsetneq$ ơ - Eur. mer.

Odynerus spiricornis Spinola, Insect. Ligur. II. 1 S0S p. 257 n. 92.

[p. $165 \mathrm{n} .11$, \&.

l'terocheilus spiricornis Herrich-Schaefier, Faun. Insect. German. 14 41 P. 176 T. $17(o ̂)$. 
Odynerus (Oplopus) discoidalis Saussure, Etud. fam. Yesp)id. III. Masar. 1 5 56 p. 31.5 n. 200 ,

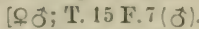
Odynerus Oplopus) spiricornis Saussure, Kitud. fam. Vespid. III. Masar. 15.51 p. 316 n. 2n1, Jै. Odynerus Epipona! spiricornis Giraud, Yerh. zool. bot. Ges. Wien XIII. 1 sti4 p. 25.

Odynerus Hoplomerus, rugulosus Rudow, Arch. Ver. Fr. Naturg. Meeklenburg XXX. $15 ; 6$

[p. 234 n. 5 .

Odỵnerus spiricornis Ed. André, Spec.Hymén. Europe II. P.21-23. 158.4 p. $760 \& 779$ n.17, ç ô.

stigma Sauss. - ơ - Afr.: Acgyptus, Abyssinia.

Odỵnerus stigma Saussure, Mém. soe. phỵs. \& hist. nat. Genève XVVII. 1. $146: 3$ p. $21 ! 1$ n. 41 i. 3 . Odynerus stigma Ed. André, Spee. Hymén. Europe II. P. 21-23. $15 \$ 4$ p. 651 n. 1, ô.

stipatus Walk. - ô- As.: Aegyptus.

Odynerus stipatus Walker, List of Hymen. in Egypt 1571 p. 36 n. $175, \vec{g}$.

stramineus André - 우 - As.: Turcestania.

(T. 43 F. 4.

Odỵnerus stramineus Ed. André, Spec. Hỵmén. Europe II. P. $21-23.154 \frac{1}{1}$ p. 74.5 n. 14, S; subalaris Sauss - 으- Austr.

Odynerus subalaris Saussure, F.tud. fam. Tespid. III. Masar. $1 \triangleleft 56$ p. 240 n. 162, С; T. 14 F. 5. Odỵnerus subalaris Saussure, Kitud. fam. Vespid. III. Masar. 1556 p. 210.

snecinctus Sauss. - - - Austr.

Odỵnerus 'Leionotus, succinctus Saussure, F́tud. fam. Vespid. I. Eumén. 1552 P. 204 n. 107, ऽ.

suecicus Sauss. - 우 fే Eur.: Suecia.

[; T. 10 F.3.

Odynerus Symmorphus) Suecicus Saussure, Étud. fam. Tespid. III. Masar. 15.51 j p. 190 n. S:;, Odynerus 'Symmorphus, Suecicus Schenck, Jahrb. Ver. Naturk. Nassau XVI. 1661 p. 15 , Odynerus laevirentris Thomson, Hymen. Scandin. III. is it p. $\$ 6 \mathrm{n}, 3, \subseteq \vec{o}$. inota 1. Odỵnerus Suecicus Ed. André, Spec. Hymén. Europe II. P. 21-23. 15 st p. 661 n. $7, \subseteq 0 ే$.

suleatus Ed. André - ô - Eur.: Sicilia.

Odynerus Aneistrocerus suleatus Ed. André, Natural. Sicil. II. 155.3 p. 230,0 ô.

Odỵnerus sulcatus Eid. André, Spec. Hymén. Europ̣e II. P. 21-23. 1554 p. 673 n. 16, ơ. sulphureus Sauss. $-\Omega-$ Am.: California.

Odynerus sulphureus Saussure. Rev. \& mag. zool. (2, X. 15is p. 170, S. [T.3 F. 15 \& 15 ? Odyncrus sulfureus Saussure, Smithson. Miscell. Collect. XIV. P. 254. 15ij p. 2iti n.96, S:

sulphnripes Mor. - ot - As.: Transcaspia.

Lionotus sulfuripes F. Morawitz. Horae soc. entom. Ross. XIX. $184 ;$ p. 169 n. 21 . of. Odynerus J ionotus sulfuripes Ed. André. Spee. Ilymén. Europe II. P.24-26. 1 s46 p. 479. J゙.

sumichrastii Sauss. $-\Omega-$ Am.: Mexico.

Odynerus (Ancistrucerus Sumichrasti Saussure. Rer. of mag. zool. 2, IX. 1857 p. 275. Odynerus Stenaneystrocerus Sumichrasti Saussure, Smithson. Miscell. Collect. XIV. P.2.i4.

superbus Mor. - 오 - ํ - Eur.: Russia.

1875 p. 209 n. 44, ㅇ․

Odynerus (J.eionotus, superbus F. Morawitz, Horae soc. entom. Ross. IV. 1567 p.121 n.9. S. 5 .

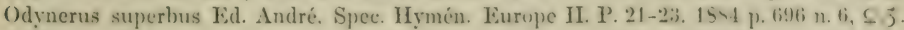

sutteriauns Sauss. $-\subsetneq \hat{\sigma}-$ Am.: California.

[1875 p. $156 \mathrm{n} .27$, \& 0 .

Odynerus Ancystrocerus Sutteriunus Saussure. Smithaon. Miscell. Cinllect. XIV. P. 254.

sylreirae Sauss. - 우 - Am.: Brasilia.

[n. 110, s; T. 10 F. 8.

Odynerus Aneystrocerus Sylveirae Sususure. Etud. fam. Vespid. III. Masar. 16.56 p. 217 Odvnerus Anerstrocerus) Silveirae Saussure, Smithson. Misecll. Collect. XIV. P. 2 it.

[1575 p. 173 n. 18.

synagroides Sauss. - 우 ô - Afr.: Senegal.

[n. 99, \&; T. 18 F. 2.

Odynerus Leionotus, synagroides Saussure. Fitud. fam. Vespid. I. Lumén. 15:2 p. 194

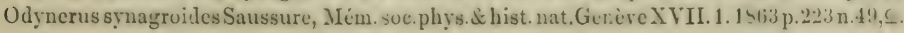


Odynerus synagroides Gribodo, Ann. mus. civ. Genova XXI. 1554 p. 25!3 n. 21, ᄃ. [T.17 F.9.

Odynerus ventralis Saussure. Grandidier: Hist. Madagasear XX. P. 1. 1 \$11 p. 161 n. 1,, 0 ;

tachkensis André - $ᄋ-$ As.: Turcestania.

[nec Sauss.].

Odynerus ornatus Ėd. André. Spec. Hṛmen. Europle II. P. $21-23.154$ p. 713 n. $11 . \subseteq$ nee Smith.

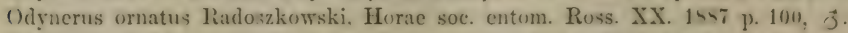

Odynerus Tachkensis Dalla 'Torre. Wien. entom. Zeitg. VIII. 1:5!9 p. 125.

tacubayae Sauss. - ô- Am.: Mexico.

Odynerus Tacubayae Saussure, Rer. \& mag. zool. (2) IX. 1857 p. 279, ô.

Odỵerus Stenodỵnerus, Tacubajae Saussure. Snithson. Miscell. Collect. XIV. P. 254.

tamariuus Sauss. $-\leq \hat{\jmath}-$ Austr.: Tasmania.

1475 ?. 3.49 n. 145.3 ; T. 4 F. 27.

Odynerus (Leionotus) 'Tamarinus Saussure. Étud. fam. Vespid. I. Eumén. 15.52 p. 203 n. 105.

Odynerus Tamarinus Saussure. Kitud. fam. Vespid. III. Masar. 15.56 p. 240.

[s. J.

taos Cress. - 오

Odynerus Toas Cresson, Trans. Amer. Entom. Soc. I. 1565 p. 351 n. 24. \& f crr. typogr.].

Odynerus (Stenodynerus) Toas Saussure. Smithson. Miseell. Collect. XIV. P. 254.1575 p. 319

tapiensis Sauss. - $f \hat{o}-$ Am.: La Plata, Equador.

[n. 120,우.

Odynerus Hypodynerus Tapiensis Saussure, Rer. \& mag. zool. 2) XXII. 1571 p. 56 n. $5 . \subseteq$ oే.

Odynerus (Hypodynerus) Tapiensis Saussure, Smithson. Miscell. Collect. XIV. P. 254. 1575

[p. 218 n. 47, ठ.

tarabncensis Sauss. - ô - Am.: Bolivia.

[n. 125, ठే.

Odynerus IIypodynerus 'Tarabueensis Saussure. Étud. fam. Tespid. III. Masar. 1856 p. 25 ॥

Odỵnerus 'Hypodynerus' Tarabucensis Saussure, Smithson. Miscell.C'ollect. XIV.P.254. 1 575

[p. $224 \mathrm{n} .57,0$ oี.

tarsatus Sauss, - $ᄋ$ ơ - Eur.: Helvetia, Italia, Alpes.

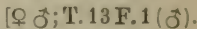

Odynerus (Antodynerus) tarsatus Saussure, Etud. fam. Tespid. III. Masar. 1556 p. $290 \mathrm{n} .175$.

Odynerus tarsatus Ed. André, Spec. Hymén. Europ. II. P. $21-23.1454$ p. 722 n. 9. 5 j.

tasmaniensis Sauss. - $\widehat{o}-$ Austr.: 'Tasmania.

$[\Xi ี ;$ T. $18 \mathrm{~F} .4$.

Odynerus (Leionotus, Ta:maniensis Saussure, Titud. Fam. Tespid. I. Eumén. 1552 p.199 n.10n.

teetus (Fabr.) Sauss. - Afr.

Vespa tecta Fabricius, Spec. Insect. I. 1781 p. 466 n. 48 !

Vespa tecta Fabricius, Mant. Insect. I. 1787 p. 292 n. 60.

Vespa tecta Gmelin, Linné: Syst. nat. Ed. 13 a I. 5.1790 p. 2756 n. 73.

Vespa tecta Olivier, Encycl. méthod. Insect. VI. 1791 p. 690 n. 107.

Vespa tecta Fabricius, Entom. system. II. 1793 p. 274 n. 76.

Vespa tecta Fabricius, Syst. Piez. 1804 p. 261 n. 40.

Odynerus Hypodynerus) tectus Saussure, Ktud. fam. Vespid. III. Masar. 1456 p. 255 n. 135.

tegularis Mor. - 우 ô - As.: Transcaspia.

Lionotus tegularis F. Morawitz, Horac soc. entom. Ross. XIX. 1545 p. 165 n. 19 , f j.

(Odynerus (Leionotus) tegularis Tid. André, Spee. Iymén. Lurope II. P. 24-26. 1546 p. 850 . . .

tepanecus Sauss. - ot - Am.: Mexico.

Odynerus Tepanecus Saussure. Rex. \& mag. zool. 2) IX. 1457 p. 200. p. 359 n. 154. Jै.

Odynerus Stenodynerus Tepaneeus Saussure, Smithson. Miscell. Collect. XIV.P.254. 1875

terricola Mocs. $-\rho$ o - Eur: Hungaria.

[n. $66, \subseteq \sigma^{-0}$.

Odynerus (Epipona terricula Moesary, Magy. Akad. T'ermésy. Lirtek. XIII. P. 11.1543 p. 52

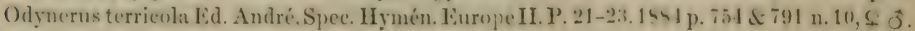

tertius 1D. T. - - - As.: Mysol.

Odynerus fallax Smith. Journ. of Proe. Jimn. Soe. Zool. VII. 1 sti3 p. 40 n. 3 , S nee Sauss.!

Odynerus tertius Dalla 'Torre, Wien. entom. Zcitg. VIU. 1889 p. 125.

texanus Cress. $-Q-A m$. : Texas.

Odynerus 'Texanus Cresson, 'Trans. Amer. Entom. Soc. IV. 1872 p. 234 , ᄋ. 
texensis Sauss. - $\hat{o}$ - Am.: Texas.

Odynerus Stenod! nerus Texensis Sumssure, Rev.d mag. zool. 12, XXII. 1871 \}, 104 n. 25, j.

Odynerus Stenodynerus; Texensis Saussure, Smithsun. Miscell. Cullect. XIV. P. 254. 1875

thalassaretos 1). T. $-\subseteq-$ As.: Aru.

[р. 329 n. 129, ठี.

Odynerus modestus Smith, Journ. of Proc. I,imn. Soc. Zool. IIl. 1s.5 p. $16.5 \mathrm{n}, 4,5$ nec

Odynerus thalassarctos Dalla Torre, Wien. entom. Zeitg. VIII. 15s!? p. 12.5. Sauss.].

tibialis Sauss. - $f$ of - Am.: Venezuela, Haiti.

Odynerus Ieionotus) tibialis Saussure, Fitud. fam. Vespid. I. Eumén. 14.52 p. $15: 3$ n. $74, \subseteq \hat{\jmath}$.

Odynerus Pachodynerus, tibialis Saussure. Smithson. Niscell. Collect. XIV. P. 25.1.1575.

tigris Sauss. - $ᄋ$ $\widehat{\sigma}-$ Am.: Canada, United States.

[p. $241 \mathrm{n} .77$, 우

Odynerus Dacdaleus Harris, Rep. Geol. ete. Massachusetts $18: 3.3$ p. 549 s. deser.].

?Odynerus (Aneystrocerus) pertimax Saussure, Etud.fam. Vespid. III. Masar. 1454, p. 216 n.105,

Odynerus Ancistrocerus) tigris Saussure, Rer. \& mag. zool. 2 IX. 1857 p. 273. I3.

Odynerus Ancistrocerus cerrus Saussure, Rer. \& mag. zool. (2; X. 18. is p. 16.).

Odynerus Aneistrocerus) tigris Saussure, Smithson. Miscell. Collect. XIV. P. 25.4. 1875 p. 160

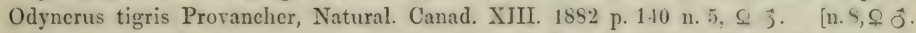

Odynerus tigris Provancher, Faun. entom. Canada. Hymén. 1583 p. 671 n. 5 , f of.

timidus Sauss. - $\subseteq-$ Eur.: Gallia.

Odyncrus Antodynerus) timidus Saussure, Étud. fam. Yespid. III. Masar. 1856 p. 296 n.184, \&. Odynerus (Leionotus) timidus Schenck, Jahrb.Ver. Naturk. Nassau XVI. 1 S61 p.66 \& 79 n.1.1, Odynerus timidus Giraud. Ann. soc. entom. France (1) VI. 1866 p. 464 n. 13, f. [f $\delta$. Odynerus Microdynerus) timidus Ed.André.Spec.Hymén.EuropeII.P.21-23.1884 p. 731 n.2, 5 .

tiuetipennis Walk. - 우 - As.: Ceylon.

Odynerus Ancystrocerus) tinctipennis Walker, Ann. \& Mag. Nat. Hist. (3) V. 1860 p. 304, \&. tinctus Walk. - $\subseteq-$ As.: Arabica.

Odyncrus tinctus Walker, List. of Hymen. in Egypt 1571 p. 34 n. 171, S:

tinnieus (Scop.) Sauss. - 오 하 - Eur.: Carnia.

Vespa tinniens Scopoli, Entom. Carn. 1763 p. 311 n. 829.

Vespa tinniens Christ, Naturg. d. Insect. 1791 p. 240 ; 'T. 22 F. 5.

Pterochilus tinniens Herrich-Sehaefer, Faun. Insect. German. 1539 P. 173 T. 16 (f)

Pterocheilus timniens Schenck, Jahrb. Ver. Naturk. Nassau IX. 1. 1453 p. 80 n. 2. $\subseteq$ ơ. Odynerus Oplopus) tinniens Saussure, Jitud. fam. Vespid. III. Masar. 1556 p. $317 \mathrm{n} .202, \mathrm{f}$ oै.

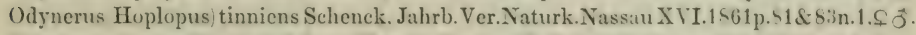
Odynerus tinniens Ed. André, Spec. Iymén. Europe II. P. 21-23. 1854 p. 759 \& 7 ss n. 16, ऽ ô.

tisiphone Lep. - 우 - Am.?

Odynerus Tisiphone I.epeletier, Hist. nat. Insect. Hỵmén. II. 1841 p. 6.16 n. 31, S.

Odynerus (Leionotus) Tisiphone Saussure, Kitud. fam. Vespid. I. Eumén. 1552 p. 153 n. 77.

Odynerus Tisiphone Saussure, Smithson. Miscell. Collect. XIV. P. 254. 1575 p. 366 n. 3.

toltecus Sauss. - ô-Am.: Mexico.

Odynerus Toltecus Saussure, Rer. \& mag. zool. 2) IX. 1557 p. 277. [p.316n.118, ơ. Odynerus Stenodynerus, Toltecus Saussure, Smithson. Miseell. Collect. XIV. P. 25. 1575

tomentosus Thoms. - 우 of - Eur.: Suecia, Fennia, Italia; As.: Sibiria.

Odynerus tomentosus Thomson, Opusc. entom. P. 2. 1870 p. 86 n. 8, \& of.

Leionotus tomentosus Thomson, Hymen. Scandin. III. 187.1 p. $31 \mathrm{n} .2, f \mathrm{f}$.

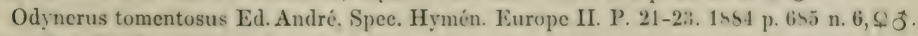

torridus Walk. - 으-As.: Arabia.

Odynerus torridus Walker, List of Hymen. in Egypt 1 ini p. 33 n. 170, f.

totonacus Sauss. - $\subseteq$ of - Am.: Mexico.

Odynerus 'Totonacus Saussure, Rev. \& mag. zool. (2) LX. 1857 p. 278.

Odỵnerus :Stenodynerus Totonacus Saussure. Smithson. Miscell. Collect. XIV. P. 254. 1875

[p. 347 n. 143,90 ; 'T. 4 F. 23. 


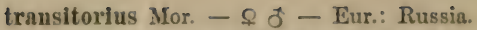
[n. 7, 웅.

Odynerus Ancystrocerus transitorius F. Morawitz. Horac soc. entom. Ross. IV. 1 sfi p. 117

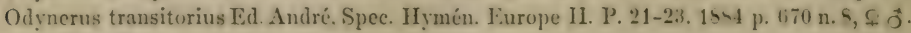
triangulum Sauss. - $\vec{\sigma}-$ Austr.

Odynerus triangulum Saussure, Kitud. fam. Vespid. III. Masar. 1956 p. 26.4 n. 171 . 3 ; T. 14 F.S. trilobus (Fabr.) Lep. - $f$ of - Afr.: Madagascar, Cap. Mauritius.

Vespa triloba Fabricius, Mant. Insect. I. 1787 p. 290 n. 41.

Vespa triloba Gmelin, Linné: Syst. nat. Ed. 13 a I. 5. 1790 p. 2752 n. 44.

Vespa triloba Olivier, Ėneycl. méthod. Insect. VI. 1791 p. 687 n. 86.

Vespa triloba Fabricius, Entom. system. II. 1793 p. 268 n. 54.

Vespa triloba Fabricius, Syst. Piez. 1804 p. 263 n. 53.

Odynerus trilobus Lepeletier, Hist. nat. Insect. Hymén. II. 1 s41 p. 635 n. $20, \rho$ o

Odynerus Leionotus) trilobus Saussure, Etud. fam. Vespid. I. Eumén. 1 52 p. 1Su n. \$3. Odynerus trilobus Saussure, Etud. fam. Vespid. III. Masar. 15.26 p. 232.

Odynerus Leionotus) trilobus Saussure, Reise d. Novara. Zool. II.1.1 6 6 i Hymen. p.10 n.6, $\subseteq$. Odynerus trilobus Saussure, Grandidier: Hist. Madagascar XX. P. 1.1591 p. 164 n. 2, trimarginatus Zett. - $ᄋ \hat{o}-$ Eur.: Suecia, Germania.

iㅇ ฮิ; T. 4 F. 15.

Odynerus trimarginatus Zetterstedt, Insect. Iappon. I. 1534 p. $4: 6$ n. 4. 5 o.

Odynerus quadricinetus Herrich-Schaeffer, Faun. Insect. German. 1541 P. 176 T. 15 \$! Odynerus Odynerus, trimarginatus Saussure, Etud.fam. Vespid.III.Masar. 1556 p. 212 n.101, [Q웅'T.10 F. 9.

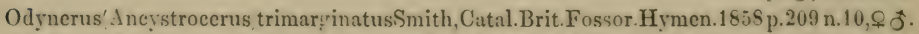
Odynerus (Anệstrocerus; trimarginatus Schenck, Jahrb. Ver. Naturk. Nassau XVI. 1861 p.49, [51 \& $59 \mathrm{n} \cdot 4$, 우 ఫิ. Odynerus'Aneystrocerus trimaryinatus F. Morawitz, Horae soc. entom. Ross. IV. 1867 p. 113 [n. $3, \subseteq$ ฮี.

Ancistrocerus trimarginatus Thomson, Hymen. Scandin. III. $1 \$ 74$ p. $67 \mathrm{n} .4, \subseteq \hat{\jmath}$. if 0 . Odynerus trimarginatus Ruduw. Arch. Ver. Fr. Naturg. Mecklenburg XXX. 1876 p. 217 n. 7 , Odynerus (Ancystrocerus trimarginatus L. Saumders, Trans. Entom. Soc.London $15 s 2$ p.175n.9. Odynerus trimarginatus Eid. André. Spee. Hymén. Furope II. P. 21 -23. $15 \$ 4$ p. 676 n. $12, \varsigma$ j. Odynerus trimarginatus Schulthess. Hymen. Helvet. Diplopt. $18 s i$ p. $46 \mathrm{n} . \&$. $\subseteq \hat{\jmath}$.

trinacriae André - 으 - Eur.: Sicilia.

Odynerus Leionotus) Trinacriac Ed. André, Vatural. Sicil. II. 14\$3 p. 2:31, \&.

Odỵnerus Trinacriae Ed. André, Spec. Hỵmén. Lurope II. P. 21-23. 1584 p. 724 n. 12, s. tripunctatus (Fabr.) Lep. - -0 - Afr.: Acgyptus, Algeria.

Vespa tripunctata Fabricius, Mant. Insect. I. 1787 p. 290 n. 47.

Vespa tripunctata Gmelin, Linné: Syst. nat. Ed. 13 a I. 5.1790 p. 2757 n. 79.

Vespa tripunctata Olivier, Encycl. méthod. Insect. VI. 1791 p. 688 n. 93.

Vespa tripunctata Fabricius, Entom. system. U. 1793 p. 270 n. 63.

Vespa tripunctata Fabricius, Syst. Piez. 1804 p. 264 n. 62.

Rhynchium ? tripunctata Spinola, Insect. Ligur. II. 3. 1808 p. 189, nota.

- Savigny, Descr. de l'ligypte. Hymén. 1812; 'T. 9 F. 11.

Odynerus tripunetatus Lepeletier. Hist. nat. Insect. Hymén. II. 1411 p. ti2n n. 10, \& $\delta^{f}$. Odynerus trijunctatus Jueas. Expler. se. Algéric. Zool. III. 14.46 p. 2:32 n. 181 .

Odynerns Jeionotus tripunctatus Sausture. Ftud. fam. Vespid. I. Fumén. 1552 p. 196 n.96. Odyerus tripunetatus Saussure. Fitud. fam. Vespid. III. Masar. 14i6 p. 240, 3.

Odynerus tripunctatus Ed. André. Speec. Iỵmén. Europe II. P. 21-26. 1\$4t p. 492.

tristis Thoms. - \& o - Eur.: Succia.

Odyncrus tristis Thomson, Opusc. entom. P. 2.1870 p. 8 ti n. 9, \& ơ.

Leionotus tristis Thomson, Hymen. Seand. III. $18 i 4$ p. $53 \mathrm{n}, 4$, 우 ơ.

()dỵnerus tristis Ed. André, Spee. Iỵmén. Europe II. P. 21-23. 1584 p. 745 n. 13, j. 
troglodytes Sauss. - ơ - Afr.: Senegal.

Odynerus(Parodynerus troglodytes Saussure, Ltud.fam. Yespid.III.Masar. 15.56 p."24?n.123.j.

tropicalis Sauss. - $ᄋ$ of - Afr.: Abyssinia.

['T.19 F. 5(Q):

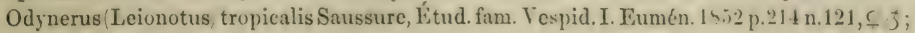

truneatus Sauss. - 우 oิ - Afr.: Senegal.

Odynerus (Leionotus) truncatus Saussure, Étud. fam. Vespid. I. Eumén, 1552 p. 175 n. $67, \subseteq 3$.

tuberenlatus Sauss. - 우 ô - Am.: Chili.

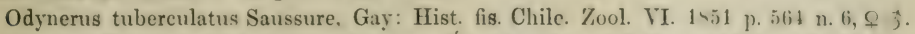

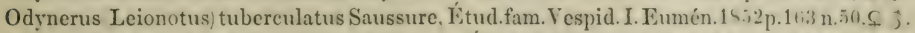
Odynerus (Leionotus tuberculatus Saussure, Vitud. fam. Vespid. III. Masar. 15.56 p. 22.2S. Odynerus (IIypodynerus tuberculatus Saussure. Smithson. Miseell. Collect. XIV. 1.25 !. 1575

tubereuliventris Spin. - 오 $\widehat{\sigma}-$ Am.: Chili.

[p. 221 n. 51, 우 0 .

Eumenes tuberculiventris Spinola, Gay: Hist. fis. Chile. Zool. VI. 1551 p. 267 n. $2, f \vec{\jmath}$

Odynerus(I,eionotus) tuberculirentris Saussure, Kutud. fam. Tespid.I.Eumén. 1s.52p.162n.49, C.

Odynerus . Hypodynerus) tuberculiventris Saussure, Etud. fam. Tespid. III. Masar. 1556 p.2227.

Odynerus (Hypodynerus) tuberculiventris Saussure, Smithson. Miscell. Collect. XIV.P. 254.

tuberenlocephalus Sauss. $-\subsetneq \hat{\jmath}-$ Am. : Mexico.

$[1575$ p. $221 \mathrm{n} .52,05$

Odynerus Ancistrocerus tuberculocephalus Saussure, Etud. fam. Vespid. I. Eumén. 1852 [p. 139 n. 22 , \& ; T. T. 16 F. 9.

Odynerus tuberculoceps Saussure, Étud. fam. Vespid. III. Masar. 1556 p. 205.

Odynerus (Ancystrocerus) tuberculiceps Saussure, Smithson. Miscell. Collect. XIV.P. 251.

turea Sauss. - - - As.: Tureia?

$[1875 \mathrm{p} .184 \mathrm{n} .26$, 웅.

Odynerus(Stenancistrocerus) Turca Saussure, Mém.soc.phys. \& hist. nat. Genève X VII. 1.1 163

[p. $218 \mathrm{n} .45$, , ․

Odynerus Turea Ed. André, Spec. Hymén. Europe IJ. P. 21-23. 1854 p. 669 n. 4, f.

turpis Sauss, - 우 - Am.: United States.

Odynerus turpis Saussure, Rev. \& mag. zool. (2) XXII. 1871 p. 60 n. 17, \&.

Odynerus turpis Saussure. Smithson. Miscell. Collect. XIV. P. 254.1875 p. 281 n. 100, f.

uncinatus Say - 우 ô - Am.: United States.

Odynerus uncinata Say, Boston Journ. Nat. Hist. I. 4. $1 \$ 37$ p. $3 \$ 8$ n. 4, f [non Fabr.]. Odynerus 'Ancistrocerus) unifasciatus Saussure, ḱtud. fam. Vespid. I. Eumén. 1852 p. 135 Odynerus unifasciatus Saussure, Etud. fam. Tespid. III. Masar. 15.56 p. $205 . \quad$ [n.21, . Odynerus uncinatus Leconte, Writ. of Th. Say Entom. II. 1859 p. 766 n. 4. $\subseteq$.

Odynerus (Aneystrocerus: unifasciatus Saussure, Smithson. Miscell. Collect. XIV. P. 254.

[1875 p.181 n. 24, ○ðึ.

vagabundus $\mathrm{D}$. T. $-\subseteq \widehat{0}-$ As: : Transeaspia.

[T. 10 F. 51 [nee Saussure?

Odynerus (T,eionotus; vagus Radoszkowski, Horae soc. entom. Ross. XX. 1856 p. 4S, \& j;

Odynerus vagabundus Dalla Torre, Wien. entom. Zeitg. VIII. 18S!) p. 125.

vagus Sauss. - 우 - Am.: United States.

Odynerus ragus Saussure, Rev. \& mag. zool. (2) IX. 14.;7 p. 277, f. [p.314n.111i,. .

Odynerus Stenodỵnerus' vagus Saussure, Smithson. Miseell. Collect. XIV. P. 254. 1575

variegatus (Fabr.) Sauss. - 우 ô - Afr.: Algeria.

Vespa variegata Fabricius, Entom. system. II. 1793 p. 269 n. 59.

Vespa variegata Fabricius, Syst. Piez. 1804 p. 264 n. 58.

- Sarigny;, Deser. de l'Égypte. Hymén. 1812; T' 8 F. 9 (ơ).

Odỵnerus flarus Lepeleticr, Hist. nat. insect. Hỵmén. II. 1441 p. 61 is n. $6, \subseteq$ ô.

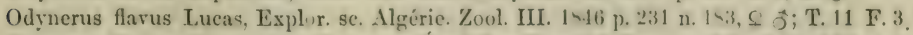

Odynerus Oplopus) variegatus Saussure, litud. fam. Vespiıl. I. Fumén. 15.22 p.2.29 n.134, .00

Odynerus variegatus Schenck, Jahrb. Ver. Naturk. Nassau IX. 1. 185.3 1. $\$ 2$ n. ㄱ, $\leq 5$. 
Odynerus variegratus Saussure. Kitud. fam. Vespid. III. Masar. 1sali p. 3us.

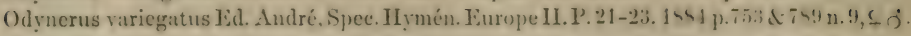

vernalis Sauss. - $f$ of - Austr.: T'asmania.

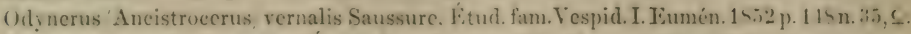

Oḍnerus vernalis Saussure, E.tud. fam. Vespid. III. Masar. 155ti p. 2015, 5ु.

verus Cress. - 0 o 0 - Am.: 'Texas.

Odynerus verus Cresson, Trans. Amer. Entom. Soc. IV. 1872 p. 237, $f$ oิ.

respiformis $\mathrm{Hal}$. - 우 $o^{\star}-\mathrm{Am}$. : Chili.

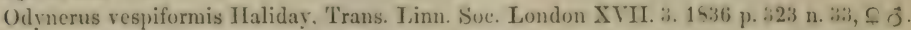
Odynerus hirsutulus Spinola, Gay: Hist. fis. ('hile. Zool. VI. 1 s.1 p. 257 n. $3, \subseteq$ j.

Odynerus |I.cionotus) hirsutulus Saussure, litud. fam. Vespid. I. Eumén. 1552 p. 212 n. 11 ×.

[옿;'I'.19 F.1 ().

Odynerus vespiformis Saussure. Kitud. fam. Yespid. ilI. Masar. 15.ti p. 241.

Odynerus (IIypodynerus) vespiformis Saussure, Smithson. Aliscell.Collect. XIV. P. 251.15i5

restitus Sauss. - 0 f

[p. 227 n. 65, \& ठี.

Odynerus Hypodynerus restitus Saussure. Etud. fam. Vespid. III. Masar. 1 G jtip.252 n.127,..

Odynerus (IIypodynerus) restitus Saussure, Smithson. Miseell. Collect. XIV. P. 25\%. 1575)

victoria Sauss. - $\odot \widehat{o}-$ Am.: Mexico.

[p. 223 n. 55, 우 ठૈ.

Odynerus Victoria Saussure, Rev. \& mag. zool. (2) IX. 1857 p. 279, ㅇ.

()dynerus (Stenodynerus) Victoria Saussure, Smitlıson. Miscell. Collect. XIV. P. 254. 1575

viduns Herr.-Schaeff. - $\vec{o}$ - Eur.: Germania, Gallia. Russia. \{p. $331 \mathrm{n}$. $133, \subseteq \hat{\jmath}$.

()dynerus viduus Herrich-Schacffer. Faun. Insect. German. 14t1 P. 176 T. 13 (ठ઼).

Odynerus viduus Schenek, Jahrb. Ver. Naturk. Nassau IX. 1. 1853 p. 65 n. 25, ô.

Odynerus (Ancystrocerus) viduus Saussure, Kitud. fam. Vespid. III. Masar. 1856 p.209 n.95, ठే.

Odỵnerus viluus Ed. André, Spec. Hymén. Europe II. P. 21-23. 185 1 p. 675 n. 11, fơ.

villosus Sauss. - 우 - Am.: Chili.

Odynerus villosus Saussure. Gay: Hist. fis. Chile. Zool. VI. 1551 p. 5633 n. 4, $f$.

()dynerus Leionotus' villosus Saussure. Kitud. fam. Vespid. 1. Eumén. 1852 p. 165 n. 53.5 .

()dynerus IIypodynerus) villosus Saussure, Smithson. Miscell. Collect. XIV. P. 25. 1575

[p. 225 n. 59, ㅇ․

rulcanus Blackb. \& Cam. - $\subseteq-$ Austr.: Ins. Hawaiae.

[p. $212 \mathrm{n} .21$, \&.

()dynerus Vulcanus Blackburn \& Cameron, Mcm. Manchester Lit. \& Philos. Soc. 3) X. 1546

vulneratus Sauss. - 오

[우.

Odynerus (Parodynerus; vulneratus Saussure, Litud.fam. Vespid. III. Masar. $1556 \mathrm{i}$ p.245n.122,

พalshianus Sauss. - $\Omega-$ Am.: Illinois.

Odynerus Srmmorphus, Walshianus Saussure. Rev. \& mag. zool, 2 XXII. 1571 p. 55 n. 2. f. () dynerus Symmorplus! Walshianus Saussure. Smithson. Mise ell. Colleet. XIV. P. 25. Isis

[p. 152 n. 1, ㅇ.

()dynerus Walshiamus Provancher. Natural. Canad. XIII. 1ss2 p. 134 n. 1. C.

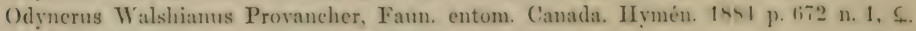
xanthinnus Sauss. $-\Omega-$ Am.: California.

Odynerus (Stenodynerus Xanthiams Saussure. Rev. d mag. zool. 2/ XXII. 1 sil p. 10:3 n. 2.2. Odynerus (Stenodymerus Xanthianu': Saussure, Smithson. Miseell. Collect. XIV. 1'. 254.1

[p. 324 n. 124, s.

xanthomelas Herr.-Schaell. $-\subseteq 3$ - liur. Germania, Gallia.

Fig. a ( 3$)$.

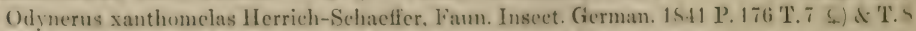
() Hynerus xanthumelas Sehenck, Jahrb. Ver. Naturk. Nassau IX. 1. 14,3is p. 71 n. 24, S j. Odynerus IIypodỵerus) xanthomelas Saussure, litud. fam. Vespid. III. Masar. 1 siti p. 271

[n. 152, \& 
Odynerns (Icionotus' xanthomelas Schenck, Jahrb. Ver. Naturk. Nassau XVI. 1501 p. 65,

[67 \& 73 n. 6, 웅.

Odynerus xanthomelas Brischke, Sehrift. physs. ökon. Ges. Könimsherg II. 14ti2 p. 116, 6$\}$. Lionotus xanthomelas Thomson, Hymen. Seandin. III. 1874 p. $5 \mathrm{~S}$ n. 3.

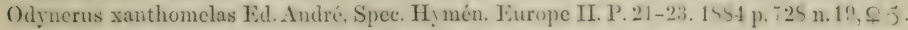
xanthosoma Schlett. - ô - Afr.: Congo.

[p. 24, ơ.

Odynerus /Ancistrocerus xanthosoma Schletterer, Ann. soc. entom. Belgique XXXY. 1 s91 zebra Sauss. - $\subseteq$ ô - Afr.: Abyssinia.

[T. 2 F. 23 .

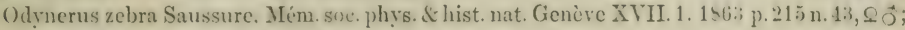
zendulus Sauss. - 0 - Am. : Mexico.

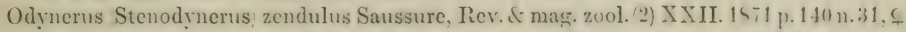
()dynerus Stenodynerus zendulus Saussure, Smithson. Miscell. Collect. XIV. P. 25.1. 15i. zonatus Sauss. - $\odot$ ơ - Am.: Cayenne.

[p. 354 n. 150, \&; T. 1 F. $24 \& 212$

Odynerus (Protodynerus, zonatus Saussure, Rev. \& mag. zool. (2) XXII. $15-1$ p. 57 n. 4. 3 Odynerus Pachodynerus, zonatus Saussure, Smithson. Misecll. Collect. XIV. I'.2.1. 15i.) [p. 239 n.73, 우.

\section{PTEROCHEILUS}

Klug, Beitr. z. Naturk. I. 1805 p. 143 [Pterochilus].

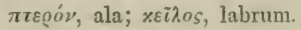

Synon.: Eumenes Latreille.

Odynerus Lepeletier, Lucas, Saussure, Morawitz.

Rhynchium Spinola, Say.

Vespa Panzer, Jurine, Blanchard.

aberrans Mor. - 우 - As.: Transcaucasia.

Pterochilus aberrans F. Morawitz. Horae soc. entom. Ross. XIX. 1SS5 p. 145 n. 6, C. Pterochilus aberrans Ld. André, Spee. Hymén. Europe II. P. 21-26. 1896 p. SS9, f.

albofasciatus Smith - As.: Turcestania.

Pterochilus albofasciatus Smith, Scient. Res. 24 Yarkand Miss. 157 , p. 17 n. 15 ; 'Tab. F. 12.

albopictus Kriechb. $-\uparrow-$ Eur.: Syria.

Pterochilus albopictus Kricchbaumer, Verh. zool. bot. Ges. Wien XIX. 1569 p. 599, $\subseteq$.

Pterochilus albopictus Ed. André, Spec. Hỵmén. Europe II. I’. 24-26. 1556 p. \$91. f.

atrohirtus Mor. - $ᄋ$ o - Eur.: Gracia; As.: Syria.

Pterochilus atrohirtu: F. Morawitz. Horae soc. entom. Ross. XIX. 1495 p. 112 n. 4 , f j.

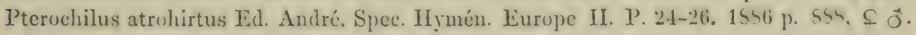

aztecus Sauss. - 으 - Am. bor.

Pterochilus Aztecus Saussure, Rev. \& mag. zool. (2) XXII. 1 Si1 p. 141 n. 35. ᄃ.

Pterochilıs Aztecus Saussure. Smithson. Miscell. Collect. XIV. P. 254. 1575 p, ito n. 3. .

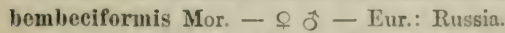

[n. $27, \subseteq 0$.

Odynerus Pterochilus) bembeciformis F. Murawitz. Horaesoc. entom. Ross. IV. Is6 - p. 13!

Pterocheilus bembeciformis Ed. André, Spee. Hymén. Europe II. P. $21-2 \%$. 1s 1 p. 4(1)

bezzii Cob. - 으- Eur.: Italia.

[n. 8, \& 0 .

Pterochilus Bezzii Cobeli, Verh. zool. bot. Ges. Wien XIJI. 1592 p. 67, s.

biglumis Sauss. - 우 - Afr.: Cap.

Pterochilus biglumis Saussure, Fitud, fam. Vespid. I. Eumén. 1 52 p. 224:3 n. 7, - ; T. 21 F.3.

biplagiatus Cress. $-\subseteq$ ơ - Am.: California.

Pterochilus biplagiatus Cresson. Trans. Amer. Entum. Sue. VII. 1 $\$$ i! P'roce p. XIX, \& j.

eapensis Sauss. - $f-$ Afr.: Cap.

Pterochilus C'apensis Saussure, Ltud. fau. Vespid.III. Musar. $1 \$ 56$ p. 323 n. 2119, 5; T. 15 F. 11. 
ehevrieranus Sauss. - $ో$ - Eur.: Helvetia.

Pterochilus Chevricranus Saussure, Etud. fam. Vespid. III. Masar. 15.56 p. 222 n. 205 , 3.

P'terochilus Chevricranus Ed. André, Spec. Hymén. Europe II. P. 24-26. 1456 p. 591, o . coecineus André - $\&-$ Afr.: Algeria.

P'terocheilus coecineus Ed.André, Spec.Hymén.Europe II. P.21-23.1 s54 p.502 n.2,5;'T.44 F.4.

comptus Cress. - 우 - Am.: Nevada.

Pterochilus eomptus Cresson, Trans. Amer. Entom. Soc. VII. 1679 Proc. p. XYIII, S. crabroniformis Mor. - o - Eur.: Russia.

[n. 28, ठే.

Odynerus Pterochilus crabroniformis F. Morawitz. Horae soc. entom. Ross. IV. 186 i p. 141

Pterocheilus crabroniformis Ed. André, Spec. Hymén. Europe II. P. 21-23. 1554 p. 797 n. 7 , Jै.

decorus Cress. - ㅇ - Am.: Nevada.

Pterochilus decorus Cresson. Trans. Amer. Entom. Soc. VII. 1 s79 Proc. p. XVIII, S.

dives Rad. - $\&$ - Afr.: Aegyptus.

Pterocheilus dives Radoszkowski, Horae soc. entom. Ross. XII. 1576 p. 145 n. 97, S.

Pterocheilus dires Ed. André, Spec. IIymén. Europe II. P. 21-23. 1854 p. $\$ 10$ n. 11, S.

ecklonii Mor. - $ᄋ$ oิ - As.: China.

Pterochilus Eckloni F. Morawitz. Horae soc. entom. Ross. XIX. 1545 p. 139 n. 3, \& ô.

eximius Smith - $\hat{o}-$ Austr.: New Guynea.

Pterochilus eximius Smith, Journ. Linn. Soc. Zool. VIII. 18 fi p. 59 n. 1, ฮే; T. 4 F. 4.

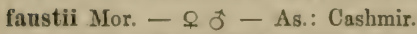

Pterochilus Fausti F. Morawitz, Horae soc. entom. Ross. IX. 1573 p. 296 n. 5 , f o

Pterocheilus Fausti Ed. André, Spec. Hymén. Europe II. P. 21-23. 1554 p. $\$ 05$ n. 10, § ơ.

formosus Friv. - 우

[p. 357, ㅇ.

Pterochilus formosus Frivaldszki, Magy. 'Tud. Akad. Math. és Termész. Közlem. XIII. 1S76

Ptcrochilus phaleratus var. formosus Moesary, Magy. Tud. Akad. Math. és 'Termész. Közlem.

(XVI. 1879 p. 6, ๑

Pterochilus formosus Ed. André, Spec. Hymén. Europe II. P. 24-26. 1456 p. \$:12.

gigas Rad. - - - As.: Transcaspia.

Pteroehilus gigas Radoszkowski, Horae soc. entom. Ross. XX. 1856 p. 45, \&.

grandis Lep. - 우 - Afr.: Algeria.

Pterochilus grandis I.epeletier. Hist. nat. Insect. Hrmén. II. $1 \$ 41$ p. 671 n. 1, ๔.

Pterochilus grandis Lucas, Ixplor. se. Algérie. Zool. III. 1846 p. 241 n. 194. S; T. 12 F.1.

Pterochilus grandis Saussure, Étud. fam. Vespid. I. Eumén. 1452 p. 246 n. 10. S.

Pterocheilus grandis Ed. André, Spec. Hymén. Europe II. P. 21-23. 1554 p. 806 n. 9. $\subseteq$.

helleniens Mor. - $-\widehat{f}-$ Eur.: Graecia; As.: Syria, Rhodus.

Pterochilus Hellenicus F. Morawitz, Horae soc. entom. Ross. XIX. 1555 p. 137 n. 2. s j.

Pterochilus Ifellenicus Ed. André, Spee. Hymén. Europe II. P. 24-26. $16-6$ p. \$5i, S.

hispanicus Med. - \& ฮึ - Eur.: Hispania.

P'terocheilus Hispanieus Medina, Aet. soc. Jispañ. hist. nat. XXI. 1592 p. 144. S jo.

insignis Sauss. - $\subseteq \hat{\jmath}-$ Afr.: Cap.

Pterochilus insignis Saussure. Fitud. fam. Vespid. I1I. Masar. 1 4.jli p. 324 n. 210, S 3ै; T.15 F.12

interrnptus Klug - ô - Eur.: Germania; Afr.: Algeria.

Pterochcilus interruptus Klug, Beitr. z. Naturk. I. 1505 p. 154 n. 2.

Pterochilus interruptus Saussure, Fitud. fam. Vespid. I. Eumén. 1952 p. 246 n. 11, ơ.

Pterochilus interruptus Saussure. Etud. fam. Vespid. III. Masar. 1856 p. 323.

Pterocheilus interruptus Ed. André. Spee. Hymén. Furope II. P. 21-23. 1584 p. 409 n. 13, כै.

laticeps Cress. $-\& \vec{f}-\mathrm{Am}$ : : Texas.

l'terocheilus laticeps Cresson, Trans. Amer. Entom. Suc. IV. 1572 p. 24., \& o 
lewisii Cress. - 으-Am.: New Mexico.

Pterochilus Lewisii Cresson. Trans. Amer. Fntom. Soc. I. 1sis p, is.2 n. 26, $\subseteq$.

Pterochilus I, wisii Saussure. Smithson. Miseell. Colleet. XIY. P. 25'. 147.5 p. 3 its n. 1, C. major Sauss. - 우 - Afr.: Cap.

Pterochilus major Saussure, Kitud. fam. Vespid. I. Eumén. 14.j2 p, $2 ! 4$ n. S. \&; 'T. 21 F. 1. mandibularis Mor. - $\rho-A s$.

Pterochilus mandibularis F. Murawitz, Horac soc. entom. Ross. XXIII. 1-६! p. 165 n. 17, $\mathrm{S}$. meridionalis Costa - 으 - Eur.: Italia.

Pterochilus meridionalis Costa, Rend. acead. sc. fis. Napoli 1857 p. 243.

Pterochilus meridionalis Costa, Atti acead. se. fis. Napoli (2) I. P. 10. $15 \varsigma 7$ p. 5 , f; T. 1 F. 4. mexicanus Sauss. - o - Am: Mexico.

Pterochilus Mexicanus Saussure. Rer. \& mag. zool. (2 XXII. 1 Si1 p. 141 n. 31, S.

Pterochilus Mexicanus Saussure. Smithson. Miscell. Collect. XIV. P. 254.1575 p. 369 n. 2. S.

mirandus Cress. - $ᄋ$ of - Am. Nevada.

[T. 4 F. $31 \& 31 \mathrm{a}$.

Pterochilus mirandus C'resson. Trans. Amer. Entom. Soc. VII. ! morrisonii Cress. - $f$ ô - Am.: Nerada.

Pterochilus Morrisoni Cresson, Trans. Amer. Entom. Soc. VII. 1679 Proc. p. XIX, s j. numida J $_{\text {ep. }}-\mathrm{Q}-$ Afr.: Algeria.

Pterochilus Numida Lepeletier. Hist. nat. Insect. Hymén. II. 1 \11 p. 672 n. 2 , $\subsetneq$.

Pterochilus Numidicus Jucas, Explor. sc. Algéric. Zool. III. 1446 p. 241 n. 200 . 5; T. 12 F.5.

Pterochilus Numida Saussure. Fitud. fam. Tespid. I. Eumén. 1552 p. 245 n. 9, S.

Pterochilus Numida Saussure. Eitud. fam. Yespid. III. Masar. 15.56 p. 322.

Pterocheilus Numida Ed. André. Spec. Hymén. Europe II. P. 21-23. is\$4 p. 510 n. 12, s. oruatus Lep. - ot - Afr.: Algeria.

Pterochilus ornatus Iepeletier. Hist. nat. Insect. Hỵmén. II. 1541 p. 677 n. 6. 3.

Pterochilus ornatus Iuear, Expler. se. Algérie. Zool. III. 1446 p. 243 n. 203. gૈ; T.12 F.4.

Pterochilus ornatus Saussure, Étud. fam. Ve-pid. 1. Eumén. 1552 p. 242 n. 5.

Pterocheilus ornatus Ed. André, Spee. Hymén. Furope II. P. 21-23. 1-b1 p. 196 n. 1, 今े.

pallasii Klug - 오 - Eur.: Russia, Italia; As. min.

Pterocheilus Pallasii Klug, Beitr. z. Naturk. I. 1505 p. 150 n. 1; T. 3 F. 3.

Pterochilus latipalpis Iepeletier, Ifist. nat. Insect. Hrmén. II. 1411 p. 6 is n. 7 , s.

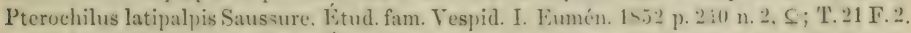

Pterochilus Pallasii Saussure, Litud. fam. Ve pill. I. Eumén. 14.2 p. 211 n. 3, s.

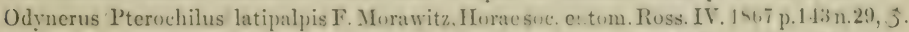

Odynerus latipalpis F. Morawitz, Horac soc. entom. Ross. V. 1868 p. 149 n. 16.

Pterocheilus Pallasii Ed. Andre. Spee. II mén. Europe II. P. $21-23$. L4h p. 7a! n. li, S.

phaleratus (Panz.) Klug - $f \widehat{o}-$ Eur. fere tota.

Vespa phicerata Panzer, Faun. Insect. German. IV. 1797 P. 4 i T. 21.

Pterocheilus phaleratus Klug, Beitr. z. Naturk. I. 1805 p. 154 n. 3.

Pterocheilus phaleratus Panzer, Krit. Revis. II. 1806 p. 148.

Vespa phalerata Jurine, Nouv. méth. class. Hymén. 1507 p. 170, ô.

Pterochilus phaleratus Illiger, Magaz. f. Insectenk. VI. 1507 p. 196.

Pterocheilus Klugii Panzer, Faun. Insect. German. IX. 1809 P. 101 T. 18.

Eumenes Klugii Latreille, Gen. Crust. \& Insect. IV. 1809 p. 131 .

Eumenes Pterocheilus phaleratus Iatreille. Gen. Crust. \& Insect. IV. 1so9 p. 136.

Pterochilus phalerata Blanchard, Hist. nat. Insect. III. 1840 p. 389.

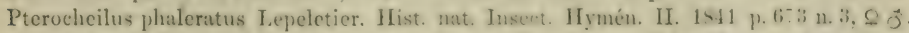

Vespa Pterochilus, phaleratus Blanchard. C'u iør Rime anim. El. a Juseet. 1 49; T. 121

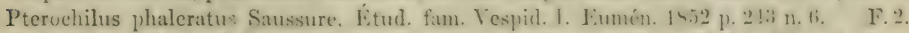

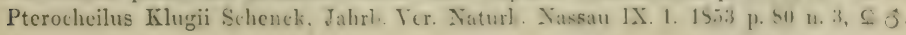




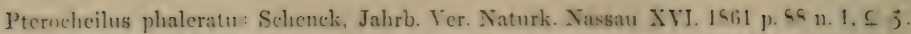
Oilyerus Pterochilus phaleratus F. Morawitz. Iforae soc. entom. Russ. Y. 1464 p. $14 ! 1$ n. 15 , $\subseteq$. P'terochilus phaleratus 'Thomson, Opusc. entom. P. 2. 1570 p. 83 n. $1, \subseteq \delta$.

Ptcrochcilus phaleratus H. Müller, Befrucht. d. Blumen 1873 p. 466.

Ptcrochilus phaleratus Thomson, Hymen. Seandin. III. 1874 p. 39 n. $1, \&$ of.

Pterocheilus phaleratus Rudew. Arch. Ver. Fr. Naturg. Mccklenburg XXX. 1576 p. 235, fij.

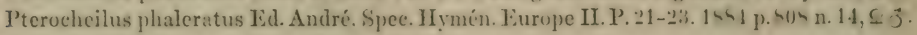
pulchellus Smith $-\subseteq \hat{\sigma}-\Lambda$ s.: India bor.

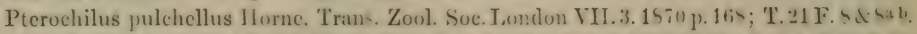

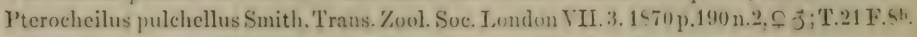
punctiventris Mor. $-\subseteq-$ As.: Persia.

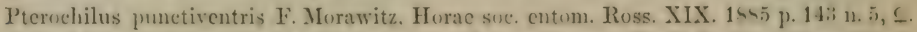
P'terochilus punctiventris Ed. Andrí, Spec. IIymén. Europe II. 1'. 24-26. 1456 p. s4 ,, . punicus André $-\subseteq-$ Afr.: Tunis.

P’terochilus P’unieus Gribodo Ed. André. Spee. Ifymén. Europe II. P. 21-23.15\$4 p. \$01, S. quincquefaseiatus Say $-\subseteq-$ Am.: Missouri, 'I'exas.

Pterochilus quinquefasciatus Say, Keating's Narrat. Exped. II. 1524 App. p. 347.

Rhygchium quinquefasciatum Say, Boston Journ. Yat. Ilist. I. 4.1637 p. 35.1 n. 2.

Pterochilus quinquefasciatus Saussure, Fitud. fam. Vespid. I. Eumén. 15.52 p. 245 n. 13, ᄃ. Pterochilus quinquefasciatus Leconte, Writ. of Th. Say Entom. II. 18.59 p. 234.

Rhygchium quinquefasciatum Leconte, Writ. of Th. Say Entom. II. 1559 p. 765 n. 2. Pterochilus quinquefasciatus Saussure, Smithson. Miscell. Collect. XIV. P. 254 . 1 S75 p. 371 sibirieus Mor. $-\subseteq-$ As.: Sibiria.

$[\mathrm{n} .4,9$.

Odynerus Pterochilus) Sibirieus F. Morawitz. Iforae soc. entom. Ross. IV. $1 \varsigma 6 ; 7$ p. 144 nota, S.

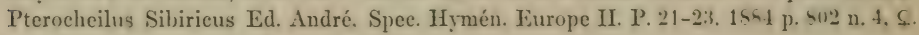
texauus Cress. - $\mathrm{q}-$ Am.: Texas.

Pterochilus T'exanus Cresson, Trans. Amer. Entom. Soc. IV. 1472 p. $211, \subseteq$.

unipunetatus Lep. - - - Afr.: Algeria.

Pterochilus unipunetatus I.epeleticr, Hist. nat. Insect. Hỵmén. II. 1541 p. 676 n. 5.5. Pterochilus unipunctatus Lueas. Explor. se. Algéric. Zool. III. 1446 p. 243 n. 502; T. 12 F. 3. Pterochilus unipunctatus Saussure, Kitud. fam. Vespid. I. Eumén. 1952 p. 242 n. 4.

P'terochilus unipunctatus Saussure, Ktud. fam. Vespid. III. Masar. 16.56 p. 322.

P'terocheilus unipunctatus IEd. André, Spec. Hymén. Europe II. P. 21-23. 1 S54 p.796 n. 5, ૬. zonatus Cress. - ô- Am.: Nevada.

Pterochilus zonatus C'resson. Trans. Amer. Fintom. Soc. VII. 1879 Proc. p. XVIII. j̧.

\section{CTENOCHILUS}

Saussure, Étud. fam. Vespid. III. Masar. 1556 p. 323.

Saussure, Smithson. Miscell. Collect. XIV. P. 254.1575 p. 371.

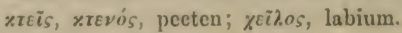

Synon.: Epipona Spinola.

Pterochilus Saussure.

pilipalpis Spin. - $9-$ Am.: Chili.

Epipona pilipalpa Spinola, Gay: Hist. fis. Chile. Zool. VI. 1851 p. 252 (n. 3: f. 1'terochilus pilipalpus Saussure, Litud. fam. Vespid. I. Eumen. 1552 p. 247 n. 12.5 ; T. 20 F.s. P'terochilus (Ctenochilus pilipalpus Saussure, Fitud. fam. Vespid. III. Masar. 1856 p. 323. Ctenochilus pilipalpus Saussure. Smithson. Miscell. Collect. XIV. P. 251.1575 p. 372, S. 


\section{PSEUDOCHILUS}

Saussure, Étud. fam. Vespid. III. Masar. 1856 p. 321.

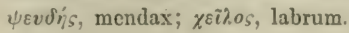

Synon.: Pterochilus Saussure.

glabripalpis Sauss. - 우 - Afr.: Senegal.

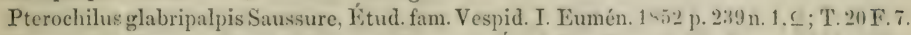

Pterochilus Pseudochilus glabripalpis Saussure, Ktud. fam. Vespid. III. Masar. 1 s 5 ti p. 321.

\section{NORTONIA}

Saussure, Stettin. entom. Zeitg. XXX. 1869 p. 53.

Norton, nom. propr. Hymen.

Synon.: Leionotus Saussure.

Odynerus Saussure, Ed. André.

Parodynerus Saussure.

amaliae Sauss. - 0 of - Austr.

Fortonia Amaliac Saussure. Stettin. cntom. Zeitg. XXX. 1<69 p. 53 n. 2, ธ ๑.

intermedia Sauss. - $ᄋ \widehat{o}-$ Eur. Gallia, Graecia; Afr.: Algeria. [T.17 F.1.

Odynerus Leionotus, intermedius Saussure, Etud. fam. Vespid. I. Eumén. 14.52 p. 155 n.39. .;

Odynerus intermedius Saussure. Ftud. fam. Vespid. III. Masar. 14,56 p. 221, 3.

Nortonia intermedia Saussure, Stettin. entom. Zeitg. XXX. 1869 p. 53.

Nortonia intermedia Saussure, Smithson. Miseell. Collect. XIV. P. 254.1575 p. 139 n. 1. .

Odynerus intermedius Ed. André, Spec. Hymén. Europe II. P. 2t-26. 1556 p. $665, \Omega .3$.

sehulthessiana Sauss. - ô - Afr.: Madagascar.

(T. 17 F. 8 .

Tortonia Schulthessiana Saussure. Grandidier: Hist. Madagasear XX. P. 1. 1591 p. 15i, 3; ;

symmorpha Sauss. $-€ \hat{\jmath}-$ Am.: Florida, New York, Connecticut. [n.119, $\subseteq \vec{s}$.

Odynerus (Parodynerus) simmorphus Saussure, Étud. fam. Vespid. III. Masar. 1556 p. 216

Nortonia symmorpha Saussure. Smithson. Miscell. Collect. XIV. P. 254.1575 p. 139 n. $2,+\hat{j}$.

tolteca Sauss. - 우 ô - Am.: Mexico.

[T. 1 F. 13 \& 133 .

Nortonia Tolteca Saussure. Smithson. Miscell. Collect. XIV. P. 254.1575 p. 140 n. 3,5 3;

\section{ALASTOR}

Lepeletier, Hist. nat. Insect. Hymen. II. 1841 p. 668.

Alastor, nom. propr. Hymen.

Synon.: Odynerus Guérin, Spinola.

Vespa Fabricius \& auct. ant.

Subg.: Alastoroides Saussure. Eitud. fam. Vespid. III. Masar. 1656 p. 327.

Alastor, nom. propr. Hymen.; eifos, forma.

Antalastor Saussure, Étud. fam. Vespid. III. Masar. 1856 p. 328.

'éví, contra; Alastor, nom. propr. Hymen.

Antal ast oroides Saussure. Eitud. fam. Vespid. III. Masar. 14.96 p. 324.

Antalastor, nom. propr. Hymen.; eî́os, visus.

Hypalastor Saussure, Etud. fam. Vespid. UI. Masar. 1856 p. 325.

ขंনó, sub; Alastor, nom. propr. Hymen.

Hypalast oroides Saussure. Kitud. fam. Vespid. III. Masar. 14,56 p. 325.

Hypalastor, nom. propr. Hymen.; Eiઈos, forma. 
Sulul. : P a ralastor Saussure. Litud. fam. Yespid. III. Masar. 15.56 p. 325. $\pi$ «ẹ́, ad; Alastor, nom. propr. Hymen.

Paralastoroides Saussure, Lttud. fam. Yespid. III. Masar. 1 nijo p. 323.

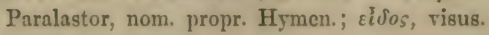

albifrous (Fabr.) Sauss. - ot - Austr.

Yespa albifrons Fabricius, Syst. cntom. 1775 p. 366 n. 20.

Vespa albifrons Fabricius, Spee. Insect. I. 1181 p. 462 n. 25.

Vespa albifrons Fabricius, Mant. Insect. I. 1787 p. 289 n. 30.

Vespa albifrons Gmelin, Linné: Syst. nat. Ed. 1:3 I. 5.1790 p. 2759 n. 86.

Vespa albifrons Christ, Naturg. d. Insect. 1791 p. 242.

Vespa albifrons Olivier, Encycl. méthod. Insect. VI. 1791 p. 684 n. 75.

Vespa albifrons Fabricius, Entom. system. II. 1793 p. 263 n. 37.

Vespa albifrons Fabricius, Syst. Piez. 1804 p. 259 n. 29.

Alastor Paralastor) albifrons Saussure. Etud. fam. Vespid. III. Masar. 1556 p. 335 n. 221, J .

albocinetus Smith - ô - Austr.: Tasmania.

Alastor albocinctus Smith. C'atal. Hymen. Brit. Mus. Y. 1577 p. 91 n. 20, 0 .

angulieollis (Spin.) Sauss. $-\rho$ of - Am.: Chili.

Odynerus angulieollis Spinola, Gay: Hist. fis. Chile. Zool. VI. 15.51 p. 261 n. 7 , ô.

Alastor angulicollis Saussure. Gay: Hist. fis. (hile. Zool. VI. 1551 p. 569 n. 1.

Alastor angulicollis Saussure, Litud. fam. Tespid. I. Eumén. 15.52 p. 2:58 n. 14, f fే; T. 21 F.i. Alastor (Hrpala tor' angulicollis Saussure. Kitud. fam. Vespid. III. Masar. 1556 p. 328. Alastor iHypalastor angulicollis Saussure, Smithson. Miseell. Collect. XIV. P. 254. 1575 apieatus Smith - ô - As. : Aru.

[p. $376 \mathrm{n} .3$, 우 $九$.

Alastor apieatus Smith. Journ. of Proc. Linn. Soc. Zool. III. 1 sis p. 166 n. 2, ô. argentifrons Smith $-q-A$ ustr.

Alastor argentifrons Smith, Catal. Hymen. Brit. Mus. V. 1 s.i p. 90 n. 12 , $\varsigma$. atropes Lep. - 우 ô - Eur. mediterr.

—- Savigny, Descr. de l'Égypte. Hymén. 1812; T. 9 F. 16.

Alastor Atropos Lepeletier, Hist. nat. Insect. Hymén. II. 1541 p. 669 n. 2.

Alastor Atropos Saussure, Étud. fam. Vespid. I. Eumén. 1852 p. 257 n. 12.

Alastor Atropos Saussure, Kitud. fam. Vespid. III. Masar. 15.66 p. $32 i, \underline{f} \vec{j}$.

Alastor Antalastor: Atropos Saussure. Étud. fam. Vespid. III. Masar. 1456 p. 325.

Alastor Atropess Ed. André. Spec. H! mén. Jurope II. P. $21-23.1954$ p. 793 n.1, \& đ; T. 44 F.1.

aureocinctus (Guér.) Sauss. - $\mathrm{Q}-$ Austr.

Odyuerus aureocinetus Guérin, Duperry: Vor Coqquille. Zool. II. 2. 1530 p. 266, \&; T.9 F.4.

Alastor aurencinetus Saussure, lítud. fam. Vespid. I. Kumén. 1452 p. 252 n. 5.

Alastor (Paralastor aurencinctus Situssure, Eitud. fam. Vespid. III. Masar. $18.56 \mathrm{p}$ p. 330. australis' Sauss. $-\subseteq-$ Austr.

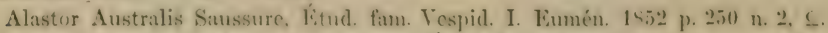

Alastor Paralaster. Australis Saussure, Retud. fam. Yespid. III. Masar. IS:6 p. 3330.

brasiliensis Sauss. - ot - Am.: Brasilia.

[đ;'T.16 F.1.

Alastor Alastoroides Tirasiliensis Saussure, Litud. fam. Vespid. III. Masar. 15.5ti p.329 n.211.

Alastor HYyalastoroides Brasiliensis Saussure. Smithson. Aiseell.Collect. XIV.P.254.14i5

bruneus Sauss. $-\rho-$ Austr.

[p. $376 \mathrm{n} .2$.

Alastor Paralastor brumeus Saussure, litud. fam. Vespid. III. Masar. 1 s.56 p.337 n. 224 , 4 . bueida Sauss. $-Q-\Lambda$ fr.: Cap.

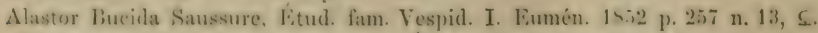

Alastur Autalastor Bucida Saussure, Bitud. fam. Yespid. III. Masar. 15.56 p. 324.

Alastor Bucida Saussure, Stettin. entum. Zeitg. XXIII. 1562 p. 206 n. 39. 
carinatus Smith - - - Austr.

Alastor carinatus Smith, Catal. Hymen. Brit. Mus. V. 1857 p. 90 n. 13, f.

elotho Lep. - Austr.

Alastor Clotho Lepeletier, Hist. nat. Insect. Hymén. II. 1811 p. 665 n. 1.

Alastor Clotho Saussure, Eitud. fam. Vespid. I. Eumén. 1552 p. 250 n. 1.

Alastor (Paralastoroides Clotho Saussure, Étud. fam. Vespid. III. Masar. 15.60 p. 324. coguatus Smith - oี - Austr.: New Guinea.

Alastor cognatus Smith, Journ. of Proc. Liun. Soc. Zool. IV. 1 Stio Suppl. p. 129 n. 1, ô.

cruentatus Sauss. - ㅇ - Austr.

T.1F.12.

Alastor (Paralastor) cruentatus Saussure, Reise d.Norara. Zool. II.1. 156 i Hỵmen.p.15n.2, ;

emarginatus Sauss. - $\subset-$ Austr.: Tasmania.

Alastor emargrinatus Saussure, Étud. fam. Vespid. I. Eumén. 1552 p. 254 n. 8, f.

Alastor (Paralastor) emarginatus Saussure, Étud. fam. Vespid. III. Masar. 1856 p. 3331.

eriurgus Sauss. - 0 - Austr.

Alastor Eriurgus Saussure, Étud. fam. Vespid. I. Eumén. 1852 p. 251 n. 4, \&; T. 21 F. 4.

Alastor (Paralastor) Eriurgus Saussure. Etud. fam. Vespid. III. Masar. 1556 p. 330.

flariceps Sauss. - 우 - Austr.

Alastor (Paralastor) flaviceps Saussure, Étud. fam. Vespid. III. Masar. 1556 p. 336 n. 222, f.

frateruus Sauss. - $\&-$ Austr.

Alastor Paralastor fraternus Saussure, Étud. fam. Vespid. III. Masar. 1856 p. 330 n. 213, f.

graeffei Sauss. - $\&-$ Austr.: Ovalau.

Alastor Graeffei Saussure, Stettin. entom. Zeitg. XXX. 1869 p. 55 n. 4, S.

inferualis Sauss. - Austr.

Alastor (Paralastor' infernalis Saussure, '́tud. fam. Vespid. III. Masar. 1 S56 p. 332 n. 215.

insularis Sauss. - 으- Austr.

[T. 16 F. 3.

Alastor (Paralastor) insularis Saussure, Étud. fam. Vespid. III. Masar. 1456 p. 334 n. 219, \&;

lachesis Sauss. - 우 - Austr.: Tasmania.

Alastor Iachesis Saussure, Étud. fam. Vespid. I. Eumén. 1852 p. 251 n. 3, ऽ; T. 21 F. 5. Alastor (Paralastor Lachesis Saussure, Etud. fam. Vespid. III. Masar. 1450 p. 330.

lateritius Sauss. - $Q$ - Austr.

Alastor (Paralastor lateritius Saussure, Reise d. Norara. Zool. II. 1. 186 ; Hymen. p.17 n.1, $f$.

maculiventris Sauss. - - - Austr.

[Q; T. 16 F. 2.

Alastor Paralastor) maculiventris Saussure, Kitud. fam. Vespid. III. Masar. 18.56 p.33 i n.223,

melanosoma Sauss. - 으- Am.: Brasilia.

Alastor melanosoma Saussure, Etud. fam. Tespid. I. Eumén. 1552 p. 259 n. 15 , \&; T. 21 F. ^. Alastor Hypalastor) melanosoma Saussure. Etud. fam. Vespid. III. Masar. 14.56 p. 324. Alastor Hypalastor melanosoma Saussure. Smithson. Miscell. Collect. XIV. P. 254. 15i5

mexicanus Sauss. - $f \hat{f}-$ Am. : Mexico.

[p. 376 n. 4 , Q.

Alastor Alastoroides Mexicanus Saussure. Rev. \& mag. zool. 2) XXII. 1471 p. 111 n. $37 . \subseteq \hat{\jmath}$.

Alastor IIypalastoroide Mexicanus Saussure. Smithson. Miscell.Collect. XIV.P. 254.1575

nautarum Sauss. - of - Austr.

[p. $374 \mathrm{n} \cdot 1$, \& ఝे.

Alastor Paralastor nautarum Saussure, Kitud. fam. Vespid. III. Masar. 1456 p. 3311 n. 212, J.

parea Sauss. - o of - Austr.

Alastor Parca Saussure, Ktud. fam. Vespid. I. Eumén. 15.52 p. 254 n. 9,03 .

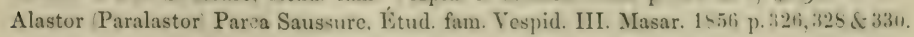

pictetii Sauss. $-f$ of - Austr.: Tasmania.

Alastor Pieteti Saussure, Etud. fam. Tespid. I. Eumén. 1552 p. 2 ic n. 12, 3 .

Alastor (Paralastor) Picteti Saussure, Etud. fam. Vespid. III. Masar. 1556 p. 327 \& 330 . \& 
punctulatns Sauss. - 우 - Austr.: Tasmania.

Alastor punctulatus Saussure, Étud. fam. Vespid. I. 1852 p. 255 n. 10, \&.

Alustur 'Paralastor punctulatus Sauszure. Ctud. fam. Vespid. III. Masar. 1 5.56 p.327 de 330.

pusillus Sauss. - $\subseteq-$ Austr.

[T. 16 F. 5 .

Alastor Paralastor' pusillus Saussure. Titud. fam. Tespid. III. Masar. 4.71 p. 332 n. $216, \subseteq$;

sanguineus Sauss. - Austr.

Alistor Paralastor sanmineus Saussure. Citud. fam. Vespid. III. Masar. 1`ił p. 331 n. 211.

similis Sauss. - 으 - Austr.

Alastor similis Saussure, Étud. fam. Vespid. I. Jumén. 1452 p. $256 \mathrm{n}$ n. $11 . \mathrm{C}$.

Alastor Paralastor: similis Saussure. Eitud. fam. Vespid. III. Masar. 14.56 p. 327 \& 3311. singularis Sauss. - - - Am. : Brasilia.

Alastor singularis Saussure, Etud. fam. Vespid. I. Eumén. 1652 p. 259 n. 16, C; T. 21 F. 9. Alastor Ifypalastor, singularis Saussure. Etud. fam. Vespid. III. Masar. $15_{56}$ p. 325.

Alastor Hypalastor singularis Saussure, Smithson: Miseell. Collect. XIV. P. 254. 15i5

smithii Sauss. - 요- Austr. [p.377 n. 5 ,우.

Alastor:Paralastor; Smithii Saussure. S'tud. fam. Vespid. III. Masar. 1556 p. 333 n. 217, $\subseteq$; tasmaniensis Sauss. - Austr.: Tasmania.

[T. 16 F. 4 .

Alastur Tasmaniensis Saussure. Etud. fam. Vespid. I. Eumén. 1552 p. 253 n. 6.

Alastor Paralastor Tasmaniensis Sanssure. Kitud. fam. Yespid. III. Masar. 1956 p. 324. tuberetlatus Sauss. - 오 of Tasmania.

Alastor tuberenlatus Saussure, Fitud. fam. Tespid. I. Eumén. 1552 p. 253 n.7, ᄃ J゙; T. 21 F. 6. Alastor tuberculatus Saussure. Étud. fam. Vespid. III. Masar. 1456 p. 326.

Alastor Paralastor tuberculatus Saussure, Étud. fam. Vespid. III. Masar. 1556 p. 325. unifasciatus Smith - 우 - As.: Aru.

Alastor unifasciatus Smith, Journ. of Proc. Linn. Soc. Znol. III. 15.5s p. 16.5 n. 1. ᄃ.

vulneratus Sauss. - - Austr.

[T. 16 F. 7 .

Alastor Paralastor, vulneratus Saussure. Étud. fam. Vespid. III. Masar. 1 -56 p. 334 n. 21 \&, $\subseteq$ :

vulpinns Sauss. $-\underline{Q}-$ Austr.

[T. 16 F. 6.

Alastor Paralastor) rulpinus Saussurc, Etud. fam. Tespid. III. Masar. 15.56 p. 335 n. 220. (;

GENERA DUBIA.

\section{CATILOSTENUS}

Meunicr, Natural. Sicil. VII. 1888 p. 150.

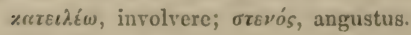

nigroviolaceus Meun. - $\rho-$ Afr.: Banana.

Catilostenus nigroviolacea Meunier, Natural. Sicil. VII. 1858 p. 151, @.

\section{EUMENESTIFERUS}

Meunicr, Natural. Sicil. VII. 1 s8s p. 300.

Eumenes, nom. propr. Hymen.; 'jẹ́̂, porto.

brasiliensis Mem. - $\Omega-$ Am. : Brasilia.

Eumcnestiferus Brasiliensis Meunier. Xatural. Sicil. VII. 1444 p. 3100. ‥ 


\section{Subfam. Vespinae}

(Stephens) Smith, Catal. Hymen. Brit. Mus. V. 1857 p. 92.

\section{ISCHNOGASTER}

Guérin, Duperry: Voy. Coquille. Zool. II. 2. 1830 p. 268.

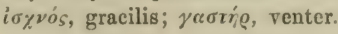

agilis Smith - of - As.: Makassar.

Ischnogaster agilis Smith, Journ. of Proc. Linn. Soc. Zool. IV. 1560 Suppl. p. 5!) n. 2. j. aurifrons Smith $-\rho-$ As.: Celebes.

Ischnogaster aurifrons Smith, Journ. of Proc. Linn. Soc. Zool. VI. 1 s61 p. 54 n. 1, f. cilipennis Smith - ơ - As.: Malacea.

Ischnogaster cilipennis Smith, Catal. Hymen. Brit. Mus. V. 1457 p. 92 n. 4. 3 .

drewsenii Sauss. - $\widehat{o}$ - As.: Archipelus Indicus.

Ischnogaster 1)rewseni Saussure, Ann. soc. entom. France (3) V. 1857 p. 315, ô.

fulgidipennis Guér. - 9 - Austr. New Guinea.

Ischnogaster fulgipennis Guérin, Duperry: Voy. Coquille. Zool. II. 2.1530 p. 269 ; T. 9 F. 9.

Ischnogaster fulgipennis Sauss:ıre, Ann. soc. entom. France 2 X. 1452 p. 23 n. 1, $\subseteq$ 9

Ischnogaster fulgipennis Saussure, Etud. fam. Vespid. II. Vesp. 15.53 p. 7 n. 1, ( 9 .

iridipennis Smith - ot - As.: Aru.

Ischnogaster iridipennis Smith, Journ. of Proc. Linn. Soc. Zool. III. 19.5s p. $16 t i$ n. 1. j.

mellyi Sauss. - - As.: Java.

Ischnogaster Mellyi Saussure, Ann. soc. entom. France (2) X. 1852 p. 25 n. 3, $\subseteq$; T.21 F.1-4 \& 5

Ischnogaster Mellyi Saussure, Étud. fam. Vespid. II. Vesp. 1853 p. 9 n. 3; T. 2 F. 1-4.

micans Sauss. - $Q-$ As.: Java.

Ischnogaster micans Saussure, Amm. soc. entom. France (2) X. 1852 p. 24 n. 2 , ๑.

Ischnogaster micans Saussure, Étud. fam. Vespid. II. Vesp. 1953 p. 8 n. 2.

nigrifrons Smith - 으-As.: Borneo.

Ischnogaster nigrifrons Smith, Journ. of Proc. Linn. Soc. Zool. II. 1557 p. 113 n. 3, f. nitidipenuis Sauss. - ot - Am.: Cayenne.

Ischnogaster nitidipennis Saussure, Étud. fam. Vespid. II. Vesp. $1 \varsigma^{3} 3$ p. 10 n. $1 . \hat{\text { Jे }}$; T. 34 F. 4 . picta Smith - \& - As.: Makassar.

Ischnogaster pictus Smith, Journ. of Proc. Linn. Soc. Zool. IV. 1560 Suppl. p. 59 11. 1. ᄃ. unicolor Smith - ô - As.: Mysol.

Ischnogaster unicolor Smith. Journ. of Proc. Linn. Soc. Zool. VII. 18633 p. 41 n. 2. 3.

\section{ISCHNOGASTEROIDES}

Magretti, Ann. mus. eiv. Genova XXI. 1884 p. 603.

Ischnogaster, nom. propr. Hymen.; عỉosos, forma.

flavus Magr. - $q-$ Afr.: Abyssinia.

Ischnogasteroides flavus Magretti, Bull. soe. entom. Ital. XV. Ihs4 p. 242; Fig. 3.

Ischnogasteroides flarus Magretti, Ann. mus. civ. Genova XXI. 1544 p. 606 n. 139. \&; Fig.

\section{SIBYLLINA}

Westwood, 'Trans. Entom. Soc. London 1868 p. 329 [Sybillina].

Sibylla, nom. propr. Hymen.

Synon.: Sybillina Westwood, Trans. Entom. Suc. London 1slis p. 329.

aenigmatiea Westw. - Am. : Haiti.

Sybillina aenigmatica Westwood. Trans. Entom. Soc. London 1S6 p p. 329.

Catalogus Hymenopterorum. IX. 


\section{BELONOGASTER}

Saussure, Étud. fam. Vespid. II. Vesp. 1853 p. 235.

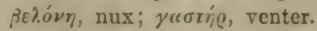

Symon.: Rh a p hig a ster Saussure. Kitud. fam. Vespid. II. Vesp. 1553 p.12; T. 2 nec Lep.1533]. gerpis, acus; yecorị, venter.

Eumenes Fabricius.

Polistes Latreille.

Sphex Degeer, Retzius, Christ.

Vespa Fabricius \& auct. ant.

Zethus Fabricius.

agilis Kohl - $Q$ ơ - Afr.: Angola.

Belonogaster agilis Kohl. Jahrb. Hamburg. wiss. Anstalt. X. 1593 p. $2 . \varsigma$ ว.

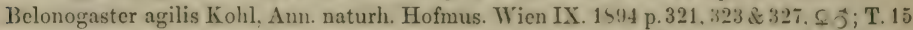

bilentata Kby. - 9 - Austr.

[F. $73 \& 85$, T. 16 F. $95 \&$ T. 17 F. $134 \& 155$.

Belonogaster bidentatus W. F. Kirby, Ann. \& Mag. Nat. Hist. 15, XIII. 1554 p. 410 n. 35. S. brachycera Kohl - ㅇ - Afr.: Port Natal, Cap.

[F. $82 \&$ T. 17 F. 138.

Belonogaster brachỵcerus Kohl, Ann. naturh. Hofmus. Wien IX. 1594 p. 322 \& 331 , \&; T. 15

brachystoma Kohl - of $\hat{o}-$ Afr. orient.

[T. 15 F. $78 \&$ T. 17 F. 136.

Belonogaster brachỵstomus Kohl. Ann. naturf. Hofmus. Wien LX. 1594 p. 322 \& $326, \subseteq \jmath$;

braunsii Kohl - $Q-$ Afr. mer.

Belonogaster Braunsii Kohl, Ann. naturh. Hofmus. Wien IX. 1 \$4 p. 323 \& 331, \&.

brevipetiolata Sauss. - o o 0 - Madagascar.

[n. 7, \& oै; T. 4 F. 1.

Belonogaster brevipetiolatus Saussure, Grandidier: Hist. Madagascar XX. P. 1. 1591 p. 94

Tolonogaster brevipetiolatus Kohl, Ann. naturh. Hofmus. Wien IX. 1 s94 p. 321 \& 323.

brunea Rits. - $\mathrm{Q}-$ Austr.: New Guinea.

Bclonogaster brunneus Ritsema, Tijdschr. r. Entom. XVII. 1574 p. 202 n. 21, S; T.11 F.1. clypeata Kohl - 우 of - Afr.: Mossambique, Madagasear.

[F. 88 \& 91 .

Belonogaster clypeatu٪ Kohl. Ann. naturh. IIofmus. Wien IX. 1594 p. 321 \& $325, \subseteq 3$; T. 15 colonialis Kohl - of of - Afr.

[F. $72 \& 84 \&$ T. 17 F. 132.

Belonogaster colonialis Kiohl, Ann. naturh. Ilofmus. Wien IX. 1 S9.4 p. 320 \& 323, ᄃ 3; T. 16 distinguenda Kohl - 오 $\hat{o}-\Lambda \mathrm{fr}$. occid.

(T. 15 F. 79 \& 94.

Belonogaster distinguendus Kohl, Ann. naturh. Hofmus. Wien IX. 1594 p. 322.323 \& $32 s$. f j

dubia Kolll - 9 ô - Afr.

F. 80 \& T. 16 F. 100.

Belonogaster dubius Kohl, Amn. naturf. IIofmus. Wien IX. 1894 p. 322, 323 \& 329. 5 3 : T. 1 s

elegans Gerst. - ९ - Afr.: Mossambique.

Belonogaster elegans Gerstaecker, Monatsber. Akad. Wiss. Berlin 1457 p. 463 n. 12 , $Q$.

13elonogaster elegans Gerstaecker. P'eters: Reise n. Moseambique. Zool. V. 1462 p. 46 is. $Q$;

Belongaster elegans Kohl, Ann. naturh. Hofmus. Wien IX. 1594 p. 321 \& $323 . \quad$ T. 30 F.s.

eumenoides Sauss. - $ᄋ-$ Afr.: Madagascar.

Belomogaster eumenoides Saussure. Grandidier: Ifist. Madagasear XX. P. 1. 1591 p. 94

flliformis Sauss. $-\rho-\Lambda$ s.: Arabia.

[n. 3, \&; T. 17 F. 12.

Rhaphigaster filifurmis Saussure, litud. fam. Vespid. II. Vesp. 14.33 p. 18 n. 7 . S; T. 2 F. 4. Belonegaster filiformis Kohl, Ann. naturh. Hofmus. Wien IX. 1594 p. 335 n. 14.

nliventris Sauss. - $P-\Delta f r$ : Senegal.

Rhathigaster filirentris Saussure. 1.tud. fam. Yespid. II. Vesp. 1453 p. 16 n. 1. Q; T. 2 F. 5. Belenegaster filiventris Smitl, Catal. IYymen. Brit. Mus. V. 1457 p. 9.4 n. 5. 


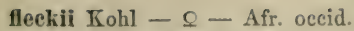

Belonogaster Fleckii Kohl. Ann. naturh. Hofmus. Wien IX. 1594 p. 322 \& 332 , s.

fulripennis Sauss. - Afr.: Madagascar.

Belonogaster fulvipennis 1)egeer, Saussure, Grandidier: Hist. Madagascar XX. P. 1. 1691

grisea (Fabr.) Sauss. - Afr.: Senegal, Sierra Leone.

Vespa grisea Fabricius, Syst. entom. 1775 p. 372 n. 43.

Vespa grisea Fabricius, Spec. Insect. I. 1781 p. 468 n. 59.

Vespa macilenta Fabricius, Spec. Insect. I. 1781 p. 468 n. 61.

Vespa grisea Fabricius, Mant. Insect. I. 1757 p. 293 n. 72.

Vespa macilenta Fabricius, Mant. Insect. I. 1787 p. 293 n. 74.

Vespa grisea Gmelin, Linné: Syst. nat. Ed. 13a I. 5.1790 p. 2753 n. 55.

Vespa macilenta Gmelin, Linné: Syst. nat. Ed. $13^{\text {a }}$ I. 5.1790 p. 2754 n. 57.

Sphex grisea Christ, Naturg. d. Insect. 1791 p. 313.

? Sphex dimidiata Christ, Naturg. d. Insect. 1791 p. 313 ; T. 31 F. 4.

Vespa grisea Olivier, Encycl. méthod. Insect. VI. 1791 p. 672 n. 13.

Vespa macilenta Olivier, Eneycl. méthod. Insect. VI. 1791 p. 673 n. 15.

Vespa grisea Fabricius, Entom. system. II. 1793 p. 279 n. 91.

Vespa macilenta Fabricius, Entom. system. II. 1793 p. 280 n. 93.

Zethus macilentus Fabricius, Syst. Piez. 1804 p. 293 n. 4.

Eumenes grisea Fabricius, Syst. Piez. 1804 p. 286 n. 6.

[excl. Synon.].

Rhaphigaster rufipemis Saussure. Étud. fam. Vespid. II. Vesp. 1553 p. 15 n. 2, S; T. 2 F.6

Belonogaster rufipennis Saussure. Kitud. fam. Vespid. II. Vesp. 1553 p. 235.

Belonogaster griseus Smith, Catal. Hymen. Brit. Mus. V. 1857 p. 94 n. 3.

guérinii Sauss. - $ᄋ$ Q

Rhaphicraster Guérini Saussure. Ėtud. fam. Vespid. II. Vesp. 15.53 p. 17 n. 5, SQ ; T. 2 F. 3 . \&).

Belonogaster Guérini Smith, Catal. Hymen. Brit. Mus. V. 1857 p. 94 n. 6.

Belonogaster Guérini Saussure, Grandidier: Hist. Madagascar XX. P. 1.1491 p. 91 n. 1, 8 J.

hildebrandtii Sauss. - 우 ơ - Afr.: Madagascar.

[n. 4, 우 ठ; T. 17 F. 11 .

Belonogaster Hildebrandti Saussure, Grandidier: Hist. Madagascar XX. P. 1. 1 \$ 91 p. 95

indica Sauss. - 우 - Afr.

Rhaphigaster Indicus Saussure, F́tud. fam. Vespid. II. Vesp. $1 S 43$ p. 17 n. 6. $\subseteq$.

Belonogaster Indicus Smith, Catal. Hymen. Brit. Mus. V. 1857 p. 94 n. 7.

juncea Fabr. Gerst. - $\subseteq j \bigcirc-$ Afr.: Congo, Senegal, Abyssinia, Mossambique.

Vespa juncea Fabricius, Spec. Insect. I. 1781 p. 468 n. 60.

Vespa juncea Fabricius, Mant. Insect. I. 1787 p. 293 n. 73.

Vespa juncea Gmelin, Linné: Syst. nat. Ed. 13a I. 5. 1790 p. 2754 n. 56.

Vespa juncea Christ, Naturg. d. Insect. 1791 p. 245; T'. 23 F. 8.

Vespa juncea Olivier, Encycl. méthod. Insect. VI. 1791 p. 673 n. 14.

Vespa Guineensis Fabricius, Entom. system. II. 1793 p. 277 n. 85.

Vespa cinerea Fabricius, Entom. systcm. II. 1793 p. 279 n. 92.

Zethus Guineensis Fabricius, Syst. Piez. 1804 p. 283 n. 2.

Zethus cinereus Fabricius, Syst. Piez. 1804 p. 283 n. 3.

Vespa cinereus Jurine, Nouv. méth. class. Hymén. 1807 p. 172, f ơ.

Polistes cinerea Latreille, Gen. Crust. \& Insect. IV. 1809 p. 142.

Rhaphigaster junceus Saussure. Ftud. fam. Vespid. II. Vesp. 14.3 .3 p. 14 n. I. $\subseteq$ 3ै; 'T. 2 F. 2 S'.

Rhaphigaster Guineensis Smith. Trans. Jintom. Soe. London 2 III. 15.66 Proe. p. 129 n. 4.

Belonogaster juneeus Gerstaecker, Peters: Reise n. Mossambique. Zeol. Y. 1 s6i2 p. 46 S.

Belonogaster junceus Gerstaccker, v. d. Decken: Reise in (Ost-Afrika. Glicderthiere. 18 i3

[p.324 n. 15, ㅇ․

Belonogaster junceus Ritsema. Tijdschr. v. Entom. XVIII. 1474 p. 202 n. 20 , S.

Belonogaster junceus Magretti, Amn. mus. cir. Genova XXII. 1554 p. 599 n. 137. 
lateritia Gerst. - $Q$ - Afr.: Mosambique. [[recte 11], Q. Belonogaster lateritius Gerstaecker. Monatsber. Akad. Wiss. Berlin 1557 p. 463 n. 10 Belonogaster lateritius Gerstaecker, Peters: Reise n. Mossambique. Zool. V. $14 t ; 2$ p. 4 fis, Q; linearis (Oliv.) Kohl'1) - $Q-$ Afr.

[T. 30 F. 7.

Vespa linearis Olivier, Encycl. méthod. Insect. VI. 1791 p. 673 n. 16.

Belonogaster linearis Kohl, Ann. naturh. Hofmus. Wien IX. 1594 p. 336 n. 24.

longistyla Sauss. - 우 - Madagascar.

? Belonogaster filirentris Saussure, Etud. fam. Vespid. II. Vesp. 1653 p. 16 n. 4 ; T. 2 F. 5.

Belonograster longistylus Saussure, Grandidier: Hist. Madagascar XX. P. 1. 1591 p. 97 n.5, f; madecassa Sauss. - $\odot Q$ - Afr.: Madagascar.

[T. 17 F. 13.

Rhaphigaster Madecassus Saussure, 1́tud. fam. Tespid. II. Vesp. 15.53 p. 16 n. 3, Q; T. 2 F. 7.

Belonogaster Madecassa Smith, Catal. Hymen. Brit. Mus. V. 1557 p. 94 n. 4.

Bclonogaster Madecassus Saussure, Grandidier: Hist. Madagasear XX. P.1. 1891 p. 95 n.6, \&. menelikii Grib. - o o - Afr.

Belonogaster Meneliki Gribodo, Ann. mus. civ. Genova XIV. 1879 p. 342 n. 3 , $\subseteq$.

Belonogaster Menelikii Gribodo, Ann. mus. eir. Genora XVI. 1851 p. 235 n. 1, $\subseteq$ of. Belonogaster Menelikii Gribodo, Aun. mus. cir. Genora XX. 1584 p. 394 n. 7 , $\subsetneq$.

Belonogaster Menelikii Gribodo, Ann. mus. cir. Genova XXI. 1584 p. 285 n. 19, $\subseteq ~ §$. Belonogaster Menelikii Kohl, Ann. naturh. Hofmus. Wien IX. 1894 p. 320 \& 323.

petiolata (Deg.) Kohl!2) - Patria?

Vespa petiolata Degeer, Mém. hist. Insect. VII. 1778 p. 610 n. S; T. 45 F. 10.

Vespa petiolata Güze, Degeer: Abh. Gesch. Insect. VII. 1753 p. 217 n. 8; T. 45 F. 10.

Vespa petiolata Retzius, Gen. \& spec: Insect. 1783 p. 64 n. 242.

? Vespa linearis Olivier, Encycl. méthod. Insect. VI. 1791 p. 673 n. 16.

? Polistes linearis Latreille, Gen. Crust. \& Insect. IV. 1809 p. 142.

Polistes petiolata Lepeletier, Encycl. méthod. Insect. X. 1525 p. 173 n. 16.

Belonogaster petiolata Kohl, Ann. naturh. Hofmus. Wien IX. 1594 p. 337 n. 25.

picta Kohl - $ᄋ$ ô - Afr. occid.

Belonogaster pietus Kohl, Ann. naturh. Hofmus. Wien IX. 1894 p. $320,323 \& 324$. ऽ Jै; T. 16

prasina Sauss. - 0 ơ - Afr.: Madagascar.

[T. 19 F. 5.

Belonogaster prasinus Saussure, Grandidier: Hist. Madagascar XX. P.1. 1891 p. 92 n. 2. \& Jै; pusilla Kohl - $ᄋ$ ô-Afr.

[T. 15 F. 75 \& 77 \& T. 16 F. 116.

Belonogaster pusillus Kohl, Ann. naturh. Hofmus. Wien IX. 1894 p. 320,323 \& 325, , § ; saeva Sauss. - 우 - Afr. tropica.

Belonogaster saevus Saussure, Grandidier: Hist. Madagasear XX. P.1. 1591 p. 92 nota, \&. s̊ussureii Kby. - Afr.: Socotra.

Belonogaster Saussurei W. F. Kirby, Proc. Zool. Soc. London 1551 p. 649 n. 2.

tarsata Kohl $-\& \cdot \vec{j}-$ Afr. orient. T.15F.73.55 \& 4 6 \& T.17F.134, 150 \& 154. Belonogaster tarsatus Kohl. Ann. naturh. Hofmus. Wien IX. 1594 p. 321, 323 \& 333 , \& 3;

tricolor Taschbg, - $\odot-$ Afr: : Socotra.

Belonogaster tricolor O. Taschenberg, Zeitschr. f. Naturw. L.VI. 1553 p. $175, \propto$.

turbulenta Kohl - $\&$ - Afr.: Sierra Leone.

[F. 87 \& T. 17 F. 147. Belonogaster turbulentus Kohl, Am. naturh. Hofmus. Wien IX. 1494 p. 3233 \& 3330, f; T. 15

turgidu Kohl $-\rho-\Lambda$ fr. : Fernando-Po.

[T.16 F.114.

Belonogaster turgidus Kohl, Amm. naturh. Hofmus. Wien IX. 1594 p.322 \& 333, s; T.15 F. i4 \&

1) $=$ ? 13. petiolata (Deg.) Kohl.

2) $=$ ? 13. elegans Gerst., an 13. brachycerus Kohl. 


\section{MISCHOCYTTARUS}

Saussure, Etud. fam. Vespid. II. Vesp. 1853 p. 19; F. 3.

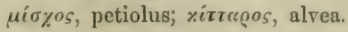

Synon.: Zethus Fabricius.

drewsenii Sauss. - ot - Am.: Brasilia.

Misehocyttarus Drewseni Saussure, Ann. soc. entom. France (3) V. 1457 p. 317. $\hat{\jmath}$.

labiatus (Fabr.) Sauss. - 우 ơ - Am. mer.

Zethus labiatus Fabricius, Syst. Piez. 1804 p. 284 n. 6.

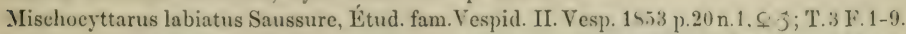

Mischocyttarus labiatus Smith, Trans. Entom. Soc. London (2. III. 1556 Proc. p. 129 n 5.

smithii Sauss. - ô- Am. : Brasilia.

Mischoeyttarus Smithii Saussure, Étud. fam. Vespid. II. Vesp. 18.53 p. 21 n. 2, ơ.

\section{PARAMISCHOCYTTARUS}

Magretti, Ann. mus. civ. Genova XXI. 1884 p. 600.

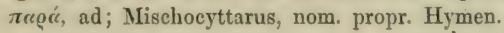

subtilis Magr. - ơ - Afr.: Kassala.

Paramischocyttarus subtilis Magretti, Ann. mus. civ. Genova XXI. 1854 p. 602 n. 138 , ơ; Fig.

Paramischocyttarus subtilis Magretti. Bull. soc. entom. Ital. XV. 1583 p. 251, 3̂.

\section{ICARIA.}

Saussure, Étud. fam. Vespid. II. Vesp. 1853 p. 22 ; T. 4.

Icarus, nom. propr. Hymen.

Synon.: Rhopalidia Guérin, Duperry: Voy. Coquille. Zool. II. 2. 1830 p. 265 [nec Lep.].

Epipona Lepeletier, Smith.

Polistes Fabricius, Guillou.

Vespa Fabricius.

aberrans Grib. - $\Omega-$ As.: India.

Icaria aberrans Gribodo, Bull. soc. entom. Ital. XXU. 1591 p. 246, \&; Fig.

anarehiea Sauss. - 9 - Afr.: Madagascar.

Icaria anarchica Saussure, Etud. fam. Vespid. II. Vesp. 1553 p. 34 n. 12, P; T. 5 F. 4.

Icaria anarchica Saussure, Grandidier: Hist. Madagasear XX. P. 1. 1 S91 p. 121 n. 5, Q.

antenuata Sauss. - 우 ô - Mossambique.

Iearia antennata Saussure, Grandidier: Hist. Madagascar XX. P. 1. 1891 p. 136 nota, $\subseteq \hat{\jmath}$. aristocratica Sauss. $-9-A$ s.: India or.

Iearia aristocratica Saussure, Citud. fam. Vespid. II. Vesp. 1553 p. 37 n. 15, Q.

artifex Sauss. - $Q-$ Am.: New Cambria.

? Polistes bioculata Fabricius, Syst. Piez. 1804 p. 278 n. 41.

Icaria variegata Saussure, Etud. fam. Vespid. II. Yesp. 1553 p. 25 n. 3. Q; T. 4 F. 3.

Icaria artifex Saussure, litud. fam. Vespid. II. Vesp. 1853 p. 236; T. 4 F. 3.

atra Sauss. - \& - Afr.: Madagascar.

Iearia atra Saussure, Grandidier: Hist. Madagasear XX. P. 1. 1<91 p. 119 n. 3, f. australis Sauss. - $f 9-$ Austr.: New Guinea.

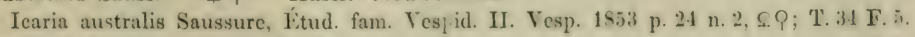
bieincta Sauss. - $\mathrm{P}$ - Afr.: Madagascar.

Iearia bieincta Saussure, Grandidier: Hist. Madagasear. XX. P. 1.1591 p. 136 n. 15, Q; T. 4 bicolor Smith - - Afr.: New Guinea.

Icaria bicolor Smith, Journ. Linn. Soc. Zool. VIII. 1864 p. 90 n. 7, ㅇ. 
brunea Smith - $Q-$ As.: Aru.

Icaria brunnea Smith. Journ. of Pros. Iinn. Sne. Zool. III. $15: 5$ p. 167 n. 4, $S$.

cabetii Sauss. - 우 9 - Austr.: 'T'asmania.

Icaria C'abeti Saussure, Etud. fam. Vespid. II. Vesp. 14.53 p. 26 n. 4, $\subseteq$ Q; T. 5 F. 2 ธ. eapensis Sauss. - $\mathrm{P}-$ Afr.: Cap.

Iearia Capensis Saussure. Stettin. entom. Zeitgr. XXIII. 1462 p. 139 n. 11, Q.

carinata Sauss. - $ᄋ$ ơ - Afr.: Madagascar.

Icaria carinata Saussure, Grandidier: Hist. Madagasear XX. P. 1. 1591 p. 122 n. i. S.j. cincta (Lep.) Sauss. - 우 9 ô- Afr. fere tota.

Eppipona cineta Lepeletier. Hist. nat. Insect. Hymén. I. 1436; p. 541 n. 2, $Q$.

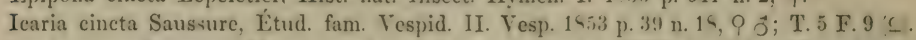

Iearia cincta Saussure, Mém. soc. phỵs. \& hist. nat. Genève XVII. 1. 1 (6.3 p. 234 n. 59, 3 .

Iearia cineta Gerstaecker, v. d. Decken : Reise in Ost-Afrika. Gliederthiere. 1573 p. 324 n. 16.

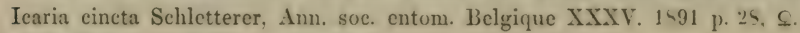

clarata Sauss. - 우 9 - Afr.: Cap.

Iearia clavata Saussure. Kitud. fam. Vespid. II. Vesp. 1453 p. 40 n. 20, ऽ?.

couservator Smith - $\odot-$ As.: Dory.

Icaria conservator Smith, Journ. of Proc. Linn. Soc. Zool. IV. $15 t j 0$ Suppl. p. 130 n. 1, ᄃ. constitutionalis Sauss. - $Q-$ Afr.: Madagascar.

Icaria constitutionalis Saussure, Gitud. fam. Vespid. II. Vesp. 1553 p. 30 n. s, Q; T.4 F. 4.

Icaria constitutionalis Saussure, Grandidier: Hist. Madagasear XX. P.1.15:11 p. 125 n. 12, 9 .

copiaria Sauss. - ô-As.: Java.

Icaria copiaria Saussure, Stettin. entom. Zeitg. XXIII. 1562 p. 135 n. 5, oै.

deceptor Smith - 우 - As.: Mysol.

Icaria deceptor Smith, Journ. of Proc. Linn. Soc. Zool. VII. $1 \varsigma_{63}$ p. 42 n. 8, S.

democratica Sauss. - $\& 9$ - Afr.: Madagascar.

Icaria democratica Saussure, Citud. fam. Vespid. II. Vesp. 1553 p. 33 n. 11, $\subseteq$ P.

Icaria democratica Er. André, Naturaliste 1889 p. 189.

Icaria democratica Saussure, Grandidier: Hist. Madagasear XX. P.1. 15!11 p. 142 n. 22, $\subseteq(Q$.

distigma Gerst. - $Q$ - Afr.: Mossambique.

Iearia distigma Gerstaecker, Monatsber. Akad. Wiss. Berlin $155 \mathrm{p}$ p. $161 \mathrm{n} .16$, Q.

Icaria distigma Gerstaceker, Peters: Reise n. Mossambique. Zool. V. 1 62 p. 471, Q.

dubia Sauss. - $\& 9-$ Afr.: Madagascar.

Icaria dubia Saussure, Étud. fam. Vespid. II. Vesp. 1853 p. 33 n. 10, 우

Icaria dubia Saussure, Grandidier: Hist. Madagascar XX.P.1. 1 S!1 p.141 n.21, O; T.15 F.19.

fasciata Smith - - -. As.: Aru.

Icaria fasciata Smith, Journ. of Proc. Linn. Soc. Zool. III. 1455 p. 167 n. 3, S?.

ferruginea (Fabr.) Sauss. - $\odot Q-$ As.: India.

Vespa ferruginea Fabricius, Entom. system. II. 1793 p. 280 n. 95.

Polistes ferruginea Fabricius, Syst. Piez. 1804 p. 277 n. 39.

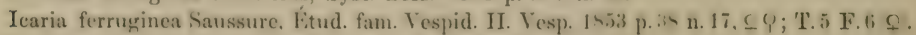

Icaria ferruginea Saussure, ĺtud. fam. Vespid. II. Vesp. 1853 p. 237.

Icaria ferruginea Horne, Trans. Zool. Soc. London VII. 3. 1870 p. 169.

Icaria ferruginea Ėr. André, Naturaliste 1859 p. 189.

festina Smith - $\mathrm{Q}-$ Austr.: New Guiner.

Icaria festina Smith, Journ. Linn. Soc. Zool. VII. 1 S64 p. 90 n. 6, \&.

flavopicta Smith $-\rho-$ As.: Borneo.

Icaria flavopicta Smith, Catal. Hymen. Brit. Mus. V. 1857 p. 99 n. 29 ,. .

Icaria flavopicta Saussure. Stettin. entom. Zeitg. XXIII. 1sti2 p. 135 n. 4.

formosa Sauss. - $\Omega-$ As.: India or.

Iearia formosa Saussure, Étud. fam. Vespid. II. Vesp. 1853 p. 37 n. 16, ㅇ․ 
fulvipennis Grib. - 우 $(Q)$ - As.: Sumatra.

Icaria fulripennis Gribodo, Bull. soc. entom. Ital. XXIII. 1891 p. 245.. .

galimatia Sauss. - 우 9 - Afr.: Madagascar.

Icaria galimatia Saussure, Étud. fam. Vespid. II. Vesp. 185.3 p. $36 \mathrm{n} .11 . \hat{\jmath} p$.

Icaria Madecassa Saussure, Grandidier: Hist. Madagasear XX. P. 1. 14!11 p. 125 n. 111, C ( ;

gracilis Smith - $Q-$ As.: Key.

T. 18 F. 16.

Icaria gracilis Smith, Journ. of Proc. Linn. Soc. Zool. III. 1858 p. 167 n. 5, \&.

grandidieri Sauss. - 우 ô - Afr.: Madagascar.

T. 4 F.5.

Icaria Grandidieri Saussure, Grandidier: Hist. Madagascar XX. P. $1.1-91$ p. 1211 11. 1. \& j;

gregaria Sauss. - Austr.

Icaria gregaria Sauseure, Étud. fam. Vespid. II. Vesp. 1853 p. 236.

Iearia gregaria Saussure, Stettin. entom. Zeitg. XXIII. 1862 p. 137 n. 7.

guttatipennis Sauss. - 9 - Afr.: Senegal.

Icaria guttatipennis Saussure, Étud. fam. Vespid. II. Vesp. $155: 3$ p. 40 n. 19, ९; T. j T. ५.

Iearia guttatipennis Smith. Trans. Entom. Soc. London (2) IIl. 155 i Proc. p. 129 n. 6.

hongkongensis Sauss. - 우 $P-$ As.: China.

Icaria Honghongensis Saussure, Étud. fam. Vespid. II. Vesp. 18.53 p. 239, \& $Q$.

hova Sauss. - 오 ô $9-$ Afr.: Madagascar.

[n. 15, 웅; T. 4 F. 2.

Icaria Hova Saussure, Grandidier: Hist. Madagascar XX. P. 1. 1891 p. 104, Fig. A \& p. 131

iguobilis Sauss. - $\odot \hat{\jmath}$ - Afr.: Madagascar.

F.7.

Icaria ignobilis Saussure, Grandidicr: Hist. Madagascar XX. P. 1.1891 p. 127 n.11, \& j3; T. 4

impetuosa Smith - $\&-$ As.: Batchian.

Icaria impetuosa Smith, Juurn, of Proc. Linn. Soc. Zool. IV. 1860 Suppl. p. 131 n. 2, f.

irritata Smith - $q-$ As.: Mysol.

Icaria irritata Smith, Journ. of Proc. Linn. Soc. Zool. VII. 1863 p. 42 n. 6, Q.

lefebrrei (Guill.) Sauss. - 우 - Austr.: New Guinea.

Polistes Lefebrrei Guillou, Ann. soc. entom. France X. 1811 p. 322 n. 26, ㅇ. .

Polistes Lefebvrei Guillou, Rer. zool. 1841 p. 325, \&.

Icaria ? Lefebvrei Saussure, Étud. fam. Vespid. II. Vesp. 1853 p. 241, ㅇ․

lngubris Smith - $\mathrm{O}-\mathrm{As}$ : Borneo.

Iearia lugubris Smith, Journ. of Proc. Linn. Soc. Zool. II. 1857 p. 115 n. 4, \&.

Icaria lugubris Saussure, Stettin. entom. Zeitg. XXIII. 1862 p. 134 n. 3.

maculata Rad. - ô - Afr.: Angola.

Iearia maculata Raduszkowski. Jorn. acad. se. Math. ete. Lisboa VIII. 1 s-1 p. 202 n. 53. 3ૈ.

maenliventris (Guér.) Sauss. - $Q 9-$ Austr.

Rhopalidia maculiventris Guérin. Duperry: Voy. Coquille. Zool. II. 2. 1 s.31) p. 266 ; T. 9 F. 8.

Icaria maculiventris Saussure, Ėtud. fam. Vespid. I. Vesp. 1453 p. 23 n. 1, ऽ Q .

Icaria maculiventris Smith, Journ. of Proc. Limn. Soc. Zool. III. 1 his p. 167 n. 1.

marangensis Grib. - $\subsetneq-$ As.: Sumatra, Malacea.

Iearia Marangensis Gribodo, Bull. soc. entom. Ital. XXII. 1891 p. 243, \&.

marginata (Lep.) Sauss. - + Q of - As.: India.

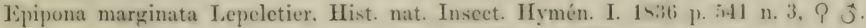

Epipona marginata Smith, Ann. \& Mag. Nat. Hist. (2) IX. 1852 p. 47.

Icaria marginata Saussure, Étud. fam. Vespid. II. Vesp. 1853 p. 237, Q.

Icaria marginata Saussure, Stettin. entom. Zeitg. XXIII. 1562 p. 139 n. 9.

Iearia marginata Gribodo, Ann. mus. civ. Genora XXI. 1884 p. 355 n. 11, \&.

mollesta Smith - - As.: Borneo, Malacca.

Icaria modesta Smith, Journ. of Proc. Linn. Soc. Zool. II. 1857 p. 115 n. 5, ㅇ.

morosa Smith - Q - As.

Icaria morosa Smith, Journ. of Proc. Linn. Soc. Zool. VII. 1463 p. 42 n. 5 , S. 


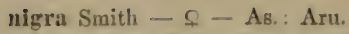

Iearia nigra Smith, Journ. of Proc. Iinn. Soc. Zool. III. 1855 p. $16 \mathrm{it}$ n. 2, s.

nitidula Sauss. - $Q$ - Afr.: Madagasear.

[F. 17.

Icaria nitidula Saussure. Grandidier: Hist. Madagasear XX. P. 1. 1591 p. 122 n. 6. Q; T. 15 nobilis Gerst. - Q - Afr.: Mossambique.

Iearia nobilis Gerstaecker, Monatsber. Akad. Wiss. Berlin 1557 p. 464 n. 15, ?.

Icaria nobilis Gerstaceker. Peters: Reise n. Mossambique. Zool. V. 1462 p. 470, Q; T. 30 F. 9. Icaria nobilis Saussure, Grandidier: Hist. Madagascar XX. P. 1. 1591 p. 133 nota, Q. opulenta Smith - ㅇ - As.: Borneo.

Icaria opulenta Smith, Catal. Hymen. Brit. Mus. V. 1857 p. 99 n. 28, \&.

Iearia opulenta Smith, Journ. of Proc. Linn. Zool. II. 1857 p. 115 n. 1.

Icaria opulenta Saussure, Stettin. entom. Zeitg. XXIII. 1862 p. 133 n. 1.

pendula Smith - $ᄋ$ Q - As.: India, Java.

Icaria pendula Smith, Catal. Hymen. Brit. Mus. V. 1857 p. 98 n. 23, \&.

Icaria pendula Saussure, Reise d. Novara. Zool. II. 1. 156 i Hymen. p. 22 n. 1, Q.

phalansteriea Sauss. - \& 0 9 - Afr.: Madagascar.

Icaria phalansterica Saussure, Etud. fam. Vespid. II. Vesp. 15.53 p. 35 n.13, § Q; T. 4 F. 5 s; \& Icaria phalansterica Er. André, Naturaliste 1889 p. 189.

Icaria phalansterica Saussure, Grandidier : Hist. Madagascar XX. P. 1.1591 p.140 n.20, \& $\widehat{o} Q$. philippinensis Sauss. - ㅇ - As.: Philippines.

Icaria Philippinensis Saussure, Ėtud. fam. Vespid. II. Vesp. 1853 p. 240, S.

pieta Sauss. - $q 9-$ As.: Bengal.

Icaria picta Saussure, Étud. fam. Vespid. II. Vesp. 1853 p. 238, $q$ Q.

pilosa Smith - of - As.: Celebes.

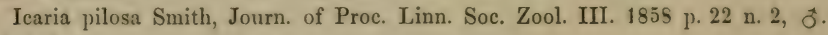

plebeja Sauss. - Q - Austr.

Icaria plebeja Saussure, Stettin. entom. Zeitg. XXIII. 1862 p. 135 n.. .

Iearia plebeja Saussure, Ném. soe. phỵs. \& hist. nat. Genère XVII. 1. 1863 p. 235 n. 60, s.

politiea Sauss. - of 0 - Afr.: Senegal.

Icaria politica Saussure, Etud. fam. Vespid. II. Vesp. 1553 p. $240, \subseteq \hat{\jmath}$.

pomicolor Sauss. - \& - Afr. : Madagasear.

Icaria pomicolor Saussure, Etud. fam. Vespid. II. Vesp. 1553 p. 32 n. 9, ૬; T. 5 F. 3. Iearia pomicolor Saussure, Grandidier: Hist. Madagasear XX. P. 1. 1891 p. 117 n. 1, S. ranavalii Sauss. - of $\hat{o}-$ Afr.: Madagasear.

[T. 18 F. 18.

Icaria Ranavali Saussure, Grandidier: Hist. Madagasear XX. P. 1. 1891 p. 135 n. 19, f ô; renctionalis Sauss. $-Q-$ Austr.: New Guinea.

Icaria reactionalis Saussure, Ettud. fam. Yespid. II. Vesp. 1553 p. 28 n. 6, Q.

revolutionalis Sauss. $-\subseteq 9-$ Austr.

Iearia revelutionalis Saussure, Ktud. fam. Vespid. II. Yesp. 1853 p. 29 n. 7, ऽ ?; T. 5 F. 7 ऽ Iearia revolutionalis Saussure, Mém. soc. phỵs. \& hist. nat.Genère XVII. 1. 1463 p.235 n.61. romandii (Guill.) Sauss. - $\odot-$ Austr.

Polistes Romandi Guillou, Ann. soc. entom. France X. 1541 p. 3322 n. 27 , S.

Polistes Romandi Guillou, Rev. zool. 1541 p. 325, ᄋ.

Iearia ? Romandi Saussure, Étud. fam. Vespid. II. Vesp. 1853 p. 41.

schulthessii Sauss. $-f-$ Afr.: Madagascar.

[T. 18 F. 14.

Iearia Schulthessi Saussure, Grandidier: Hist. Madagasear XX. P. 1. 1 s91 p. 117 n. 2. \&; seottiana Sauss. - \& - Afr.: Madagasear.

Iearia Scottiana Saussure, Grandidier: Hist. Madarnascar XX. P. 1. 1591 p. 124 n. \&, S. socialis Sauss. $-\varsigma-$ As.: India.

Icaria secialis Saussure, Stettin. entom. Zeitg. XXill. 1 \$62 p. 136 n. 6, 5. 
socialistica Sauss. - $q 9-$ Austr.: Tasmania.

Icaria socialistica Saussure, Etud. fam. Vespid II. Vesp. 155.3 p. 27 n. 5, $\subseteq$ ?; T. 4 F. 6 ()). speciosa Sauss. - ơ - As.: Sumatra, Malacea, Borneo.

Icaria speciosa Saussure, Rev. \& mag. zool. (2) VII. 1855 p. 374, of.

Icaria speciosa Smith, Catal. Hymen. Brit. Mus. V. 1857 p. 98 n. 27.

Icaria speciosa Saussurc, Stettin, entom. Zeitg. XXII. 1862 p. 134 n. 2.

subelarata Sauss. - $ᄋ$ ơ $Q$ - Afr.: Madagascar.

[T. 4 F. 6.

Icaria subclavata Saussure, Grandidier: Hist. Madagasear XX. P. 1. 1591 p. $13+1 \mathrm{n} .16, \subseteq 5$ Q

tomentosa Gerst. - $Q$ - Afr.: Mossambique.

Iearia tomentosa Gerstaecker, Monatsber. Akad. Wiss. Berlin 14.57 p. 464 n. 17, ?.

Icaria tomentosa Gerstaceker. Peters : Reise n. Mossambique. Zool. V. 1\$62 1. 471. Q; T. 30

torrida Smith - $\subseteq-$ As.: Ceram.

[F.10,

Icaria torrida Smith, Journ. of Proc. Linn. Soc. Zool. VII. 1863 p. 42 n. 7, ㅇ.

nuicolor Smith - + - As.: Key.

Icaria unicolor Smith, Journ. of Proc. I,inn. Soc. Zool. III. 1558 p. 168 n. 6, \&.

rariabilis Sauss. - 우 Ø゚ - Afr.: Madagasear.

[T. 18 F. 15 .

Iearia variabilis Saussure, Grandidier: Hist. Madagascar XX. P. 1.1591 p. 1355 n. 17, $\subseteq 3$;

rariegata (Smith) Sauss. - 으 - As.: India or.

Epipona variegata Smith, Ann. \& Mag. Nat. Hist. (2) IX. 1852 p. 48, \&.

Icaria variegata Saussure, Étud. fam. Vespid. II. Vesp. 1853 p. 237, ‥

Icaria variegata Horne, Trans. Zool. Soc. London VII. 3.1670 p. 169, \&; T. 20 F. \&, Sa \& 9. relutina Sauss. - $\subsetneq-$ Afr.: Madagascar.

Icaria velutina Saussure, Grandidier: Hist. Madagascar XX.P.1.1591 p.129 n.13, \&; T.4F.4.

ritripennis Sauss. - $Q$ - Afr.: Madagascar.

Icaria vitripennis Saussure, Grandidier: Hist. Madagascar XX. P. 1. 1591 p. 130 n. 14, Q. xanthura Sauss. - - - Afr.: Madagascar.

Icaria xanthura Saussure, Étud. fam. Vespid. II. Vesp. 1853 p. 236, ㅇ.

Icaria xanthura Magretti, Ann. mus. civ. Genora XXI. 1884 p. 606 n. 140.

Icaria xanthura Saussure, Grandidier: Hist. Madagascar XX. P. 1. 1591 p. 125 n. 9, ᄃ.

\section{PARAICARIA.}

Gribodo, Bull. soc. entom. Ital. XXIII. 1891 p. 248.

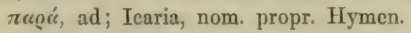

bicolor Grib. - $ᄋ(Q)$ - As.: Birmania.

Paraicaria bicolor Gribodo, Bull. soc. entom. Ital. XXIII. $1 \$ 91$ p. $249, \subseteq \uparrow ?$.

\section{ANTHRENEIDA.}

White, Ann. \& Mag. Nat. Hist. VII. 1841 p. 321 nota.

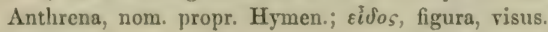

Synon.: Icaria Saussure.

Polistes Fabricius.

Vespa Weber, Illiger.

sumatrae (Web.) Sauss. - 으 - As.: Sumatra.

Vespa Sumatrae Weber, Observ. entom. 1801 p. 103 n. 7.

Vespa mutillata Illiger, Magaz. f. Insectenk. I. 1802 p. 189 n. 19.

Polistes pubescens Fabricius, Syst. Piez. 1804 p. 279 n. 49.

Anthreneida coronata White, Ann. \& Mag. Nat. Hist. VII. 1 h.11 p. 321 nota.

Iearia Sumatrae Saussure, Étud. fam. Vespid. II. Vesp. 1853 p. 211.

Anthrencida coronata Saussure, Ftud. fam. Vespid. II. Vesp. 1453 p. 246, S. 


\section{POLISTES}

Latreille, Hist. nat. Crust. \& Insect. III. 1802 p. 363 [em.].

$\pi 0 \lambda i \zeta \omega$, urbem condo.

Synon.: Cyelostoma Kirby \& Spenee, Introd. Entom. Ed. $5^{a}$ III. 1 s 35 p. 631.

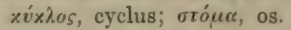

Gyrostoma Kirby \& Spence, Introd. Entom. Ed. 5a III. 1838 p. 633.

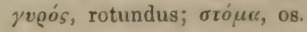

Odynerus Spinola.

Rhopalidia Spinola.

Vespa Linné \& auct. ant.

actaeon Hal. - ㅇ 9 - Am.: Columbia, Cayenne.

Polistes Actaeon Lepeletier, Encycl, méthod. Insect. X. 1825 p. 171.

Polistes Actaeon Haliday, Trans. Linn. Soc. London XVII. 3. 1436 p. 323 n. 32, ᄃ.

Polistes Actaeon Saussure, Étud. fam. Vespid. II. Vesp. 1853 p. 98 n. 60, 9; T. 11 F. 2.

albieinctus Sauss. - $\subseteq 9-$ Afr.: Madagascar.

Poli-tes albicinctus Saussure, Grandidier: Hist. Madagascar XX. P. 1. 1591 p. 14 n n. 5, . ;

albimaeula Fabr.! - Am. mer.

[T. 18 F. 21 .

Polistes albimacula Fabricius, Syst. Piez. 1804 p. 277 n. 36.

anaheimensis Prov. - 오 $\delta$ - Am.: California.

Polistes Anaheimensis Provancher. Addit. faun. Canada. Hymén. $1 \varsigma 5 \varsigma$ p. 423 n. $2, \subseteq \vec{\jmath}$. analis Fabr. - ô - Am.: Cayenne.

Vespa analis Fabricius, Suppl. entom. system. 1798 p. 261 n. 40-41.

Polistes analis Fabricius, Syst. Piez. 1804 p. 272 n. 15.

Vespa analis Jurine, Nouv, méth. class. Hymén. 1807 p. 169, đૅ.

Polistes analis Saussure, Étud. fam. Vespid. II. Vesp. 1853 p. 80 n. 42.

aunularis (L.) Fabr. - 우 $9-$ Am. bor. \& centr.

Vespa annularis Linné, Centur. Insect. rar. 1763 p. 31 n. 93.

Vespa annularis Linné, Amoen. acad. VI. 1763 p. 413 n. 93.

Vespa annularis Linné, Syst. nat. Ed. 12 a I. 2.1767 p. 950 n. 9.

Vespa Virginiensis Drury, Illustr. Nat. Hist. I. 1770 p. 98; T. 43 F. 6.

Vespa annularis Degeer, Mém. hist. Insect. III. 1773 p. 583 n. 7; T. 29 F. 11.

Vespa annularis Fabricius, Syst. entom. 1775 p. 366 n. 16.

Vespa annularis Ph. I. Müller, Linné: Vollst. Natursyst. V. 2.1775 p. 582 n. 9.

Vespa annularis Ph. F. Gmelin, Onomatol. hist. nat. VII. 1777 p. 704.

Vespa annularis Göze, Degneer: Abh. Geseh. Insect. III. 1750 p. 377 n. 7; T. 29 F. 11.

Vespa annularis Fabricius, Spec. Insect. I. 1781 p: 461 n. 19.

Vespa annularis Retzius, Gen. \& spec. Insect 1783 p. 64 n. 238.

Vespa annularis Fabricius, Mant. Insect. I. 1787 p. 298 n. 22.

Vespa annularis Gmelin, Linné: Syst. nat. Ed. $13^{2}$ I. 5.1790 p. 2752 n. 9.

Vespa annularis Olivier, Encycl. méthod. Insect. VI. 1791 p. 682 n. 61.

Vespa annularis Fabricius, Entom. system. Il. 1793 p. 260 n. 26.

Polistes annularis Fabricius, Syst. Piez. 1804 p. 270 n. 3.

Vespa annularis Jurine, Nouv. méth. class. Hymén. 1807 p. 168,. .

Polistes annularis Lamarek, Hist. nat. anim. s. vert. IV. 1817 p. 90 n. 4.

Polistes annularis Lepeletier, Encycl. méthod. Insect. X. 1825 p. 171.

Polistes annularis Iamarck, Hist. nat. anim. s. vert. Ed. 2a IV. 1535 p. $306 \mathrm{n.} 4$.

Polistes cincta Lepeletier, Hist. nnt. Insect. Hymén. I. 1836 p. 522 n. 6, Q.

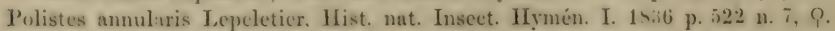

Polistes annularis Westwood, Urury: Illustr. Nat. Hist. Ed. 2 I. 1837 p. 95; T. 43 F. 6. 
Polistes annularis Saussure, Étud. fam. Vespid. II. Vesp. 1853 p. 79 n. 41, ᄃ; T. s F. 4. Polistes annulata Möbius, Arch. f. Naturg. XXII. 1. 14.56 p. 330; T. 12 F. 2.

Polistes annularis Saussure, Ann. soc. entom. France (3) V. 1857 p. 313 n. 2.

apachus Sauss. - Am.: New Mexico.

Polistes Apachus Saussure, Ann. soc. entom. France (3) V. 1857 p. 314 n. 11.

apiealis Sauss. - 우 $\hat{\sigma}-$ Am.: Guyana.

Polistes apicalis Saussure, Rev. \& mag. zool. (2) X. 1858 p. 260 , 우 ơ.

aterrimus Sauss. - $\mathrm{O}-\mathrm{Am}$.: Brasilia.

Polistes aterrimus Saussure, Ktud. fam. Vespid. II. Vesp. 1553 p. 73 n. 33, ?.

atrophiea Fabr.! - As.: India or.

Vespa atrophica Fabricius, Suppl. entom. system. 1798 p. 264 n. 101-102.

Polistes atrophica Fabricius, Syst. Piez. 1804 p. 280 n. 52.

aurifer Sauss. - $\&-$ Am.: Californien; Austr.: Honolulu.

Polistes aurifer Saussure, Itud. fam. Vespid. II. Vesp. 1853 p. 78 n. 40, ㅇ.

Polistes aurifer Saussure, Ann. soc. entom. France (3) V. 1857 p. 313 n. 6.

Polistes aurifer W. F. Kirby, Ann. \& Mag. Nat. Hist. (5) XIII. 1554 p. 410 n. 37.

badius Gerst. - $ᄋ-$ Afr.: Wanga.

Polistes badia Gerstacker, Arch. f. Naturg. XXVII. 1. 1870 p. 351 n. 18 , 오.

Polistes badia Gerstaecker. r. d. Decken: Reise in Ost-Afrika. Gliederthiere. 1873 p. 325

balder Kby. - Austr.: Christmas Ins.

[n. 19, \&;T.14 F.1.

Polistes balder W. F. Kirby, Proc. Zool. Soc. London 1888 p. 552.

bellicosus Cress. - 0 ô - Am.: Texas.

Polistes bellicosus Cresson, Trans. Amer. Entom. Soc. IV. 1572 p. $247, f o$.

bengalensis Fabr. - As.: Bengal.

Polistes Bengalensis Fabricius, Syst. Piez. 1801 p. 277 n. 38.

beruardii Guill. — $q$ - Austr. bor.

Polistes Bernardii Guillou, Ann. soc. entom. France X. 1841 p. 321 n. 25, 오.

Polistes Bernardii Guillou, Rev. zool. 1841 p. 325, 우.

Polistes Bernardii Saussure, Etud. fam. Vespid. II. Vesp. 18.53 p. 70 n. 29 , $f$.

Polistes Bernardii Roth, Journ. Linn. Soc. London. Zool. XVIII. 107. 1555 p. 326.

bicolor Lep. - $\mathrm{P}-\mathrm{Am}$. : Cayenne.

Polistes bicolor Lepeletier, Hist. nat. Insect. Hỵmén. I. 1536 p. 521 n. 4. Q.

Polistes bicolor Saussure, Étud. fam. Vespid. II. Vesp. 1853 p. 76 n. 37, Q.

biglumis (L.) Panz. - 우 9 ơ - Eur. fere tota.

— Réaumur, Mem. hist. Insect. VI. 1742 p. ?; 'T. 17 F. 7 \& 8 \& T. 25 F. 3 \& 4.

Linné, Fauna Suec. 1746 p. ? n. 1011.

? Vespa rupestris Linné, Syst. nat. Ed. $10^{2}$ I. 1758 p. 573 n. 8.

Vespa biglumis Linné, Syst. nat. Ed. $10^{\mathrm{a}}$ I. 1758 p. 573 n. 11.

Vespa biglumis Linné, Fauna Suec. Ed. 2a 1761 p. 418 n. 1680.

Vespa parietum var. 2 \& 3 Scopoli, Entom. Carn. 1763 p. 309 n. 827.

Vespa biglumis Linné, Syst. nat. Ed. 12a I. 2. 1767 p. 951 n. 17.

Vespa X Schaeffer, Icon. Insect. Ratisbon. I. 2. 1767; T. 70 F. 3,4 \& 5.

Vespa biglumis Fabricius, Syst. entom. 1775 p. 373 n. 48.

Vespa biglumis Ph. I. Müller, Linné: Vollst. Natursyst. V. 2. 177.5 p. ş.4 n. 17.

Vespa biglumis Fabricius, Spec. Insect. I. 1781 p. 469 n. 67.

Vespa biglumis Fabricius, Mant. Insect. I. 1787 p. 294 n. 81.

Vespa biglumis Villers, C. Linnaei Entom. III. 1789 p. 271 n. 13.

Vespa bighumis var. rupestris Gmelin. Iinné: Syst. nat. Ed. 13 I. 5.1790 p. 27. is n. 17.

Vespa biglumis Christ, Naturg. d. Insect. 1791 p. 245.

Vespa biglumis Olivier, Encycl. méthod. Insect. VI. 1791 p. 692 n. 115.

Vespa biglumis Fabricius, Entom. system. II. 1793 p. 271 n. 64. 
Vespa biglumis Panzer, Faun. Insect. German. V. 1798 P. 53 T. 7.

Vespa diadema Iatrcille, Ann. mus. hist. nat. I. $1+12$ p. 292 n. 5 ; T. 21 F. 4-6.

Vespa biglumis Fabricius, Syst. Piez. 1804 p. 264 n. 63.

Polistes diadema Latreille, Hist. nat. Crust. \& Insect. XIII. 1605 p. 349 n. 2.

Vespa (Polistes) biglumis Panzer, Krit. Rev. II. 1806 p. 157.

Odynerus biglumis Spinola, Insect. Ligur. I. 1806 p. $\$ 9$ n. 4.

Vespa biglumis Jurine, Nouv. méth. class. Hymén. 1807 p. 169, $\subsetneq$ ơ.

Vespa diadema Jurine, Nouv. méth. class. Hymén. 1807 p. 169, 우 đิ.

Polistes Gallicus var. biglumis Klug. Magraz. Ges. naturf. Fr. Berlin II. 1507 p. 52 n. 57.

Odynerus biglumis Spinola, Insect. Ligur. II. $180 \mathrm{~S}$ p. 186 n. 4, 오 రิ.

Polistes diadema Klug, Germar: Reise n. Dalmat. II. 1817 p. 264 n. 369.

Polistes diadema Lamarck, Hist. nat. anim. s. vert. IV. 1817 p. 90 n. 2.

Polistes diadema I,epeletier, Encycl. méthod. Inseet. X. 1425 p. 173 n. 7 , 5.

Polistes diadem Lamarck, Hist. nat. anim. s. vert. Ed. 2 IV. 1435 p. 306 n. 2.

Polistes diadema Lepeletier, Hist. nat. Insect. Hymén. I. 1636 p. 525 n. 16,0 of 9 .

Polistes diadema Erersmann, Bull. physs. math. acad. sc. St. Pétersbourg II. 1544 p. 125.

Polistes biglumis Saussure, Étud. fam. Yespid. II. Vesp. 1553 p. 46 n. 1, \& 9 j.

Polistes biglumis Saussure, Étud. fam. Vespid. II. Vesp. 1853 p. 242.

Polistes diadema Saussure, Étud. fam. Vespid. II. Vesp. 1853 p. 243.

Polistes bighumis Sichcl, Ann. soc. entom. France (3) II. 15.4 Bull. p. XII-XIII.

Polistes diadema Schenck, Jahrb. Ver. Naturk. Nassau XVI. $1 \varsigma 1 ; 1$ p. 27 n. $1, \subseteq 9 \vec{j}$.

Polistes biglumis e biglumis Radoszkowski, Horae soc. cntom. Ross. III. 1565 Bull. p. IV.

Polistes bighumis $\beta$ diadema Radoszkowski, Horae soc. entom. Ross. III. 1865 Bull. p. IV.

Polistes biglumis 'Thomson, Opusc. entom. P. 1. 1869 p. 82 n. 1, 우 9 ơ.

Polistes diadema Siebold, Beitr. z. Parthenog. 1871 p. 1-101.

Polistes diadema Rouget, Mém. acad. sc. Dijon (3) I. 1873 Sc. p. 194.

Polistes diadema Rouget, Pet. nouv. entom. I. 5. 1873 p. 269.

Polistes biglumis Thomson, Hymen. Seandin. III. 1 sit p. 28 n. 1, $\subseteq$ ? j.

Vespa diadema Rudow, Arch. Ver. Fr. Naturg. Mecklenburg XXX. 1676 p. 209 n. $2, \subseteq 90$.

Polistes biglumis H. Müller, Alpenblumen 1881 p. 592 n. 610.

Polistes diadema H. Müller, Alpenblumen $188 \mathrm{l}$ p. 592 n. 611.

Polistes biglumis Destefani, Natural. Sicil. II. 1882 p. 55-58.

Polistes Gallicus var. biglumis Schulthess, Hymen. Helret. Diplopt. 185i p. 21.

Polistes Gallicus var. biglumis Marchal, Feuill. jeun. natural. XIX. 1559 p. 157.

Polistes diadema Bertkau, Verh. naturh. Ver. preuss. Rheinl. XXXIII. 1si6 Sitzber. p.106.

Polistes diadema Hürter, Ber. oberhess. Ges. Natur- u. Heilk. XXVI. 1549 p. 94.

var. chinensis Fabr. - \& $9-$ As.: China, Sibiria, Persia; Afr.

Vespa Chinensis Fabricius, Entom. system. II. 1793 p. 261 n. 29.

Polistes Chinensis Fabricius, Syst. Piez. 1804 p. 270 n. 6.

Vespa Chinensis Jurine, Nouv. méth. class. Hymén. 1807 p. 168, ㅇ․

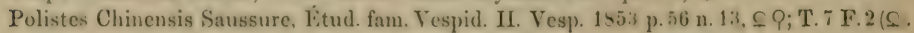

Polistes Chinensis Saussure, Reise d. Novara. Zool. II. 1. 1 S6i IImen. p. 1! n. 1, \&?.

Polistes biglumis var. Chinensis Smith, Trans. Entom. Soe I.ondon 1573 p. 197.

Polistes Chinensis Smith, Scient. Res. 2d Yarkand Miss. 1878 p. 17.

biguttatus Hal. - +- Am. mer.

Polistes biguttatus Haliday, Trans. Linn. Soc. London XVII. 1536 p. 323 n. 30, $\mathrm{s}$.

Polistes biguttatus Saussure, litud. fam. Vespid. II. Vesp. 1453 p. sti n. 49, $\mathrm{f}$.

Polistes? biguttatus Smith, Entomol. Annual f. 1868 p. 87 \& 96.

Polistes ? versicolor Smith, Entomol. Annual f. 1868 p. 87 \& 96.

Vespu higuttatus Smith. Trans. Intom. Soe. Iondon (3: V. 1s64 Proe. p). CVIII.

Polistes biguttatus Smith, Entomol. Annual f. 1569 p. 65. 
binotatus Sauss. - $\subsetneq-$ Am.: Brasilia.

Polistes binotatus Saussure, Jitud. fam. Vespid. II. Vesp. 1553 p. 47 n. 50, \&; T. 7 F. 6. bipustulatus Smith - Eur.: Anglia (imp.).

Polistes bipustulatus Saussure) Smith, Entom. M. Magaz. XII. 1575 p. 156-157 [s. descr.].

bucharensis Er. - 우 -

[n. 986, 우.

Polistes Bucharensis Frichson, Mém. acad. sc. St. Pétersbourg (6, VI. 1 s.49 Nat. sc. p. 307 callimorphus Sauss. - 9 P ơ - As.: Timor.

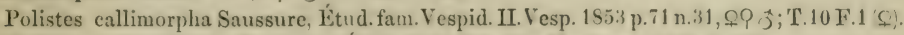

Polistes callimorphus Saussure, Étud. fam. Vespid. II. Vesp. 185.3 p. 255.

canadensis (L.) Sauss. $-\subseteq 9 \hat{o}-$ Am. bor.

- Réaumur, Mém. hist. Insect. VI. 1742 p. 169; T. 17 F. 4.

Vespa Canadensis Linné, Syst. nat. Ed. $10^{a}$ I. 1758 p. 574 n. 15.

Vespa Canadensis Sulz, Kennz. d. Insect. 1761 p. 51; T. 19 F. 122 a.

Vespa Canadensis Linné, Mus. Ludov. Ulric. 1764 p. 411 n. 3.

Vespa Canadensis Linné, Syst. nat. Ed. $12^{\text {a }}$ I. 2.1767 p. 952 n. 25.

Vespa Canadensis Degeer, Mém. hist. Insect. III. 1773 p. 580 n. 3; T. 29 F. 7.

Tespa nigripennis Degeer, Mém. hist. Insect. III. 1773 p. 552 n. 6; T. 29 F. 10.

Vespa lanio Fabricius, Syst. entom. 1775 p. 365 n. 15.

Vđspa Canadensis Ph. L. Müller, Linné: Vollst. Natursyst. V. 2. 1775 p. 856 n. 25.

Vespa Canadensis Ph. F. Gmelin, Onomatol. hist. nat. VII. 1777 p. 706.

Vespa Canadensis Göze, Degeer: Abh. Gesch. Insect. III. 1780 p. 375 n. 3; T. 29 F. 7.

Vespa nigripennis Göze, Degeer: Abh. Gesch. Insect. III. 1750 p. 377 n. 6; T. 29 F. 10.

Vespa lanio Fabricius, Spec. Insect. I. 1781 p. 461 n. 17.

Vespa Canadensis Retzius, Gen. \& spec. Insect. 1783 p. 63 n. 234.

Vespa nigripennis Retzius, Gen. \& spec. Insect. 1783 p. 63 n. 237.

Vespa lanio Fabricius, Mant. Insect. I. 1787 p. 288 n. 20.

Vespa Canadensis Gmelin, Linné: Syst. nat. Ed. 1:3a I. 5.1790 p. 2759 n. 25.

Vespa lanio Gmelin, Linné: Syst. nat. Ed. 13 a I. 5. 1790 p. 2749 n. 38.

Vespa marribous Christ, Naturg. d. Insect. 1791 p. 217.

Vespa Canadensis Christ, Naturg. d. Insect. 1791 p. 225.

Vespa lanio Christ, Naturg. d. Insect. 1791 p. 238.

Vespa lanio Olivier, Encycl. méthod. Insect. VI. 1791 p. 682 n. 59.

Vespa nigripennis Olivier, Encycl. méthod. Insect. VI. 1791 p. 654 n. 73.

Vespa Canadensis Olivier, Eneycl. méthod. Insect. VI. 1791 p. 684 n. 74.

Vespa lanio Fabricius, Entom. system. II. 1793 p. 260 n. 24.

Polistes lanio Fabricius, Syst. Piez. 1804 p. 269 n. 1.

Vespa lanio Jurine, Nouv. méth. class. Hymén. 1807 p. 168, \& đే.

Vespa nigripennis Jurine, Nouv. méth. class. Hymén. 1807 p. 171, ㅇ.

Polistes lanio Lamarck, Hist. nat. anim. s. vert. IV. 1817 p. 90 n. 3.

Polistes lanio Lepeletier, Encycl. métlıod. Insect. X. 1825 p. 171.

Polistes lanio Lamarck, Hist. nat. anim. s. vert. Ed. 2a IV. 1835 p. 306 n. 3.

Polistes infuscata Lepeleticr, Hist. nat. Insect. Hỵmén. I. $15: 36$ p. 520 n. 2, Q.

Polistes unicolor Lepeletier, Hist. nat. Insect. Hymén. I. 1534 p. 521) n. 3, ?.

Polistes lanio Smith, Trans. Entom. Soc. I.ondon 2) I. 6. 1551 p. 176-17s; T. 16 F. 1 d:3.

Polistes Canadensis Saussure, Kitud. fam. Vespid. II. Vesp. 1553 p. 72. n. 32.8 Jै; T. 9 F. 1.

Polistes Canadensis Möbius, Ablı. naturw. Ver. Hamburg III. 1856 p. 16 tiт p. 50 ; T. 14 F. .

Polistes Canadensis Saussure, Ann. soc. entom. France :3 V. 1557 p. $31: 3$ n. 1.

Polistes Canadensis Lucas, Ann. soc. entom. France (5) III. 1873 p. 106 ; T. 3 F. 1 (na \& h.

carnifex Fabr. - $\&$ Q ơ - Am. m., Mexico, San Domingo.

Vespa carnifex Fabricius, Syst. entom. 1775 p. 365 n. 14.

Vespa carnifex Fabricius, Spec. Insect. I. 1781 p. 461 n. 16. 
Vespa carnifex Fabricius, Mant. Insect. I. 1787 p. 288 n. 19.

Vespa carnifex Gmelin, Linné: Syst. nat. Ed. $13^{3}$ I. 5. 1790 p. 2749 n. 37.

Vespa carnifex Christ, Naturg. d. Insect. 1794 p. 239.

Vespa carnifex Olivicr, Encycl, méthod. Insect. VI. 1791 p. 681 n. 58.

Vespa carnifex Fabricius, Entom. system. II. 1793 p. 260 n. 23.

Polistes carnifex Fabricius, Syst. Piez. 1804 p. 272 n. 13.

Polistes major Palisut-Beaurais, Insect. Afr. \& Amér. 1505 p. 206 Hymén.; T. S F. 1.

Polistes chlorostoma I.epeletier. Hist. nat. Insect. Hỵmén. I. 14.36 p. 521 n. 5. Q.

Polistes onerata I.epeleticr, Ifist. nat. Insect. Hỵmén. I. 1536 p. 52.4 n. 11, j.

Polistes valida Say, Boston Journ. Nat. Hist. I. 4. 1837 p. 389 n. 3, ㅇ.

Polistes earnifex Saussure, Sagra: Hist. fis. Cuba VII. 14il; p. 772. $\subseteq$ 9.

[n. 1.5 .

Polistes Rhyopalidia transwersesignata Spinola. Mem. accad. se. Torino 2) XIII.15.j1 p.is

Polistes carnifex Saussure, Etud. fam. Vespid. II. Vesp. 14.33 p. 90 n. $53 . \subseteq$ \&; T. 10 F. 5 ค.

Polistes carnifex Saussurc, Ann. soc. entom. France (3) V. 1857 p. 313 n. 5.

Polistes valida Leconte, Writ. of Th. Say Entom. II. 1859 p. 769 n. 3, \&.

Polistes carnifex W. F. Kirby. Am. \& Mag. Nat. IIist. 5, XIII. 1 sht p. 411 n. 41.

carapyta Sauss. - 우 ô - Am.: Argentinia.

Polistes carapyta Saussure, Étud. fam. Vespid. II. Vesp. 1553 p. Si n. 51. \& S; T. 11 F. S ' S.

Polistes cavapyta Saussure, Ann. soc. entom. France (3) V. 1857 p. 313 n. 3.

colonicus Smith - ô-As.: Amboina.

Polistes colonicus Smith, Journ. of Proc. Limn. Soc. Zool. IV. 1560 Suppl. p. 129 n. 3, j.

comanchus Sauss. - Am.: New Mexico.

Polistes Comanchus Saussure, Ann. soc. entom. France 3) V. 1557 p. 314 n. $\bar{\imath}$

confusus Smith - As.: India, China.

Polistes orientalis I.ppeletier. Hist. nat. Insect. Hỵmén. I. 1531 p. 519 n. 1, \& nec Kirby:

Polistes orientalis Saussure, Étud. fam. Vespid. II. Vesp. 1853 p. 251.

Polistes confusus Smith, Catal. Hymen. Brit. Mus, V. 1857 p. 102 n. 7.

consobrinus Sauss. - 오 ô - Am.: Brasilia.

Polistes consobrinus Saussure, Rev. \& mag. zool. (2) X. 1858 p. 259, 우 ô.

crinitus (Felt.) Smith - 우 $\mathrm{Q}-\mathrm{Am}$. fere tota.

Vespa crinita Felton, Philos. Trans. Roy. Soc. LIV. 1764 p. 53-57.

Vespa tricolor Fabricius, Syst. entom. 1775 p. 369 n. 32.

Vespa Americana Fabricius, Syst. entom. 1775 p. 370 n. 38.

Vespa tricolor Fabricius, Spec. Insect. I. 1781 p. 465 n. 43.

Vespa Americana Fabricius, Spec. Insect. I. 1781 p. 467 n. 52.

Vespa tricolor Fabricius, Mant. Insect. I. 1787 p. 291 n. 52.

Vespa Americana Fabricius, Mant. Insect. I. 1787 p. 292 n. 64.

Vespa tricolor Gmelin, Linné: Syst. nat. Ed. 13a I. 5. 1790 p. 2755 n. 67.

Vespa Americana Gmelin, Linné: Syst. nat. Id. $13^{\text {a }}$ I. 5.1790 p. 2756 n. 77.

Vespa crinita Christ, Naturg. d. Insect. 1791 p. 225.

Vespa tricolor Christ, Naturg. d. Insect. 1791 p. 238.

Vespa Americana Christ, Naturg. d. Insect. 1791 p. 243.

Vespa tricolor Olivier, Encycl. méthod. Insect. VI. 1791 p. 689 n. 95.

Vespa Americana Olivier, Encyel. méthod. Insect. VI. 1791 p. 691 n. 111.

Vespa multicolor Olivier, Eneycl. méthod. Insect. VI. 1791 p. 691 n. 113.

Vespa tricolor Fabricius, Entom. system. II. 1793 p. 271 n. 67.

Vespa Americana Fabricius, Entom. system. II. 1793 p. 276 n. 81.

Polistes Americana Fabricius, Syst. Piez. 1804 p. 275 n. 29.

Vespa tricolor Fabricius, Syst. Piez. 1804 p. 266 n. 70.

Pulistes Amerieanus Saussure, Litud. fam. Vespid. II. Vesp. 14.33 p. 93 n. 55, S; T. 11 F. 4 \&. 5. 
Polistes crinita Smith, Catal. Hymen. Brit. Mus. V. 1857 p. 108 n. 51.

Polistes Americanus Saussure. Ann. soc. entom. France (3. V. 1557 p. 313 n. 4.

Polistes Americana Lebert, Zeitschr. f. wissenseh. Zool. IX. 14is p. 449-4i2; 'T. 15 F. 15-26.

Polistes Americana Walsh (i Riley, Amer. Entomol. I. 1469) p. 141; Fig. 112.

Polistes Americanus Saussure, Sagra: Hist. fis. Cuba VII. 15.56 p. 772, S. P.

var. billardieri Sauss. - Am. mer.

Polistes Billardieri Fabricius, Syst. Piez. 1804 p. 274 n. 26.

Polistes Americanus var. Billardierii Saussure, Ann. soe. entom. France (3) V. 1557 p. 313. var. instabilis Sauss. - Am.

Polistes Americanus var. instabilis Saussure, Ann. soc. entom. France (3) V. 1557 p. 313. var. lineatus Fabr. - 우 $P$ - Am. mer.

Vespa lineata Fabricius, Syst. entom. 1775 p. 365 n. 13.

V'espa lineata Fabricius, Spec. Insect. I. 1781 p. 461 n. 15.

Vespa lineata Fabricius, Mant. Insect. I. 1787 p. 288 n. 17.

Vespa lineata Gmelin, Linné: Syst. nat. Ed. 13 I. 5.1790 p. 2749 n. 35.

Vespa lineata Christ, Naturg. d. Insect. 1791 p. 239.

Vespa lineata Olivier, Encycl. méthod. Insect. VI. 1791 p. 681 n. 56.

Vespa lineata Fabricius, Entom. system. II. 1793 p. 259 n. 20.

Polistes lineata Fabricius, Syst. Piez. 1804 p. 271 n. 9.

Polistes Cubensis Lepeletier, Hist. nat. Insect. Hymén. I. 1536 p. 526 n. 13, Q.

Polistes lineatus Saussure, Etud. fam. Vespid. II. Vesp. 1953 p. 95 n. 56, s; T. 11 F. 6.

Polistes Americanus var. lineatus Saussure, Ann. soc. entom. France 3) V. 1557 p. 313.

defectivus Gerst. - $Q$ - Afr.: Uru.

Polistes defectiva Gerstacelier, Areh. f. Naturg. XXXVII. 1. 1 \$70 p. 351 n. $20, \Omega$.

Polistes defectiva Gerstaecker, r. d. Jecken: Reise n. Ost-Afrika. Gliederthiere. 1573 p. 326

diaboliens Sauss. - $\subseteq 9-$ As.: Java, Timor.

[n. 21, ㅇ.

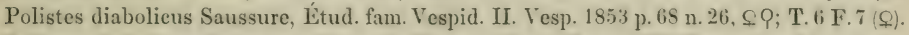

Polistes diabolicus Saussure, Reise d. Novara. Zool. II. 1. 1567 Hymen. p. 21 n. 5, $\Omega$.

Polistes diabolicus W. F. Kirby, Amn. \& Mag. Nat. Hist. (5) XIII. 1 sst p. 412 n. 43.

dorsatus Fabr. - Am. mer.

Polistes dorsata Fabricius, Syst. Piez. 1804 p. 281 n. 57.

dubius Sauss. - 9 ơ - As.: Manilla.

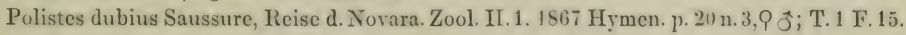
elegans Smith - $Q-$ As.: Aru.

Polistes elegans Smith. Journ. of Proe. I,imn. Soe. Zool. III. 1 \55 p. 169 n. 5 , C.

Polistes elegans W. F. Kirby. Ann. \& Mag. Nat. Hist. (j) XIII. 1554 p. 411 n. 42.

erytlirinus Holmgr. - O - Austr.

Polistes erythrinus Holmgren, Eugenies Resa. Insect. 1465 p. 440 n. 100, S.

extraneus Kby. - As.: Timor.

Polistes extrancus W. F. Kirby, Proc. Zool. Soc. London 1883 p. 344.

facilis Sauss. - Austr.

Polistes facilis Saussure, Étud. fam. Vespid. II. Vesp. 1853 p. 53 n. 9.

fastiliosus Sauss. - ô - Afr.: Senegal.

Polistes fastidiosus Saussure, Ftud. fam. Vespid. II. Vesp. 14.33 p. 60 n. 14, 3.

Polistes fastidiosus Gerstaecker. Peters: Reise n. Mossambique. Zool. Y. 1562 p. 470).

Polistes fastidiosus Magretti. Ann. mus. eir. Genova XXI. 14ht p. ben n. 141.

ferreri Sauss. - $9-A m$. mer.

Polistes Ferreri Saussure, Étud. fam. Vespid. II. Vespl. 1453 p. 77 n. 39, ?.

flaripennis Sauss. - $P$ - Afr.: Cap.

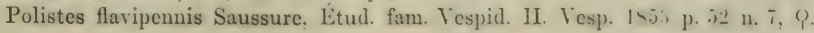


flarus Cress. - $\mathrm{Q}-$ Am.: New Mexico.

Polistes flarus Cresson, Trans. Amer. Entom. Soc. I. 1868 p. 383 n. 25, \&.

fortunatus Kby. $-\subseteq-$ Afr. Caput viride, San Jago.

Polistes fortunatus W. F. Kirby. Ann. \& Mag. Nat. Hist. (5; XIII. 1554 p. 410 n. 39. S.

fusens Fabr. - Am.: New Cambria.

Polistes fusea Fabricius, Syst. Piez. 1804 p. 274 n. 25.

Polistes fuscus Packard, Amer. Natural. XIV. 1850 p. 600.

fuseatus Fabr. - Am. mer.

Vespa fuscata Fabricius, Entom. system. II. 1793 p. 260 n. 27.

Polistes fuscata Fabricius, Syst. Piez. 1804 p. 270 n. 4.

Polistes fuscatus Saussure, Ann. soc. entom. France (3) V. 1857 p. 314 n. 9.

Polistes fuscata L. v. Heyden, Berlin. entom. Zeitschr. XI. 1867 p. 398.

var. cinerascens Sauss. - $\mathrm{Q}-$ Am. : Brasilia.

Polistes cineraseens Saussure, Kitud. fam. Vespid. II. Vesp. 1553 p. 99 n. 61, ᄃ; T. 10 F. 4.

Polistes fuscatus var. eineraseens Saussure, Ann. soc. entom. France (3) V. 1857 p. 314. var. exilis Sauss. - $\widehat{o}-$ Am. bor.

Polistes exilis Saussure, Étud. fam. Vespid. II. Vesp. 1553 p. $\$ 5$ n. 47, Jै; T. 12 F. 5.

Polistes fuscatus rar. exilis Saussure, Amn. soc. entom. France (3. V. 1557 p. 314.

var. instabilis Sauss. - 으 - Am.: United States, Mexico. [T.11 F.1.

Polistes instabilis Saussure, Étud. fam. Vespid. II. Vesp. 1553 p. 91 n. 54 , . P; T. 10 F. 2 ( ) \&

Polistes fuscatus rar. instabilis Saussure, Ann. soc. entom. France (3) V. 1557 p. 314. var. pacifleus Fabr. $-9-$ Am. : Brasilia.

Polistes pacifica Fabricius, Syst. Piez. 1804 p. 274 n. 28.

Polistes pacificus Saussure, Etud. fam. Vespid. II. Vesp. 1853 p. \$4 n. 46, Q.

Polistes fuscatus var. pacificus Saussure, Ann. soc. entom. France (3) V. 1557 p. 314.

gallica (L.) Latr. - 우 9 ô - Eur. fere tota; Afr. bor.; As. occ.; Am. bor.

— Réaumur, Mém. hist. Insect. VI. 1742 p. ?; T. 25 F. 6.

- Roesel, Insectenbelustigungen II. 1749 Wespen p. ?; T. 7 F. 8.

? Vespa parietum Poda, Insect. mus. Graec. 1761 p. 108 n. 3.

? Vespa parietum var. 1 Scopoli, Entom. Carn. 1763 p. 309 n. 827.

Vespa IV Schaeffer, Icon. Insect. Ratisbon. I. 1. 1766; I. 24 F. 5.

Vespa VI Schaeffer, Icon. Insect. Ratisbon. I. 1. 1766; 'I. 35 F. 5.

Vespa Gallica Linué, Syst. nat. Ed. $12^{2}$ I. 2.1767 p. 949 n. 7.

Vespa XI Schaefler, Icon. Insect. Ratisbon. I. 2. 1767; T. 71 F. 2.

Vespa XIV Sehacfer, Icon. Insect. Ratisbon. I. 2. 1767; T. 91 F. 5.

Vespa Gallica Ph. I. Müller, Linné: Vollst. Natursyst. V. 2. 1775 p. 581 n. 7.

Vespa Gallica Fabricius, Spec. Insect. I. 1781 p. 460 n. 10.

Vespa Galliea Schrank, Enum. Insect. Austr. 1781 p. 391 n. 789.

Vespa Gallica Fabricius, Mant. Insect. I. 1787 p. 287 n. 11.

Vespa Gallica Razoumowski, Hist. nat. Jorat. I. 1789 p. 300.

Vespa Gallica Villers, C. Linnaei Entom. III. 1769 p. 266 \& 552 n. 5.

Vespa Gallica Gmelin, Linné: Syst. nat. Ed. 13 a I. 5. 1790 p. 2751 n. 7.

Vespa Galliea Rossi, Fauna Etrusca II. 1790 p. 83 n. 862.

Vespa dominula Christ, Naturg. d. Insect. 1791 p. 229 , 우 ठే; T. 21 F. 1.

Vespa Gallica Christ, Naturg. d. Insect. 1791 p. 233.

Vespa Gallica Olivier, Encycl. méthod. Insect. VI. 1791 p. 680 n. 50.

Vespa Gallica Thunberg, Nov. acta soc. sc. Upsal. V. 1792 p. 90 n. 7.

Vespa Gallica Fabricius, Entom. system. II. 1793 p. 257 n. 13.

Vespa Gallica Panzer, Faun. Insect. German. V. 1798 P. 49 'T. 22.

Polistes Gallica Latreille, Hist. nat. Insect. III. 1802 p. 363.

Vespa Gallica Schrank, Fauna Boica II. 2. 1802 p. 352 n. 2207. 
Vespa Gallica Walckenaer, Fauna Paris. II. 1802 p. 88 n. 5.

Vespa Gallica Latreille, Ann. mus. hist. nat. I. 1802 p. 291 n. 4.

Polistes Gallica Fabricius, Syst. Piez. 1804 p. 271 n. 8.

Polistes Gallieus Latreille, Hist. nat. Crust. \& Insect. XIII. 1 SUj p. 345 n. 1.

Vespa (Polistes) Gallica Panzer, Krit. Revis. II. 1806 p. 157.

Vespa Gallica Illiger, Rossi: Fauna Etrusca Ed. 2a II. 1507 p. 136 n. 862.

Vespa Gallica Jurine, Nouv. méth. class. Hymén. 1807 p. 169, 우 ô.

Polistes Gallica Klug. Magaz. Ges. naturf. Fr. Berlin II. 1507 p. 52 n. 57.

- Savigny, Descr. de l'Égypte. Hymén. 1812; T. 8 F. 2.

Vespa Gallica Disderi, Mém. acad. roy. sc. Torino XXII. P. 3. 1816 p.1-19.

Polistes Gallica Lamarck, Hist. nat. anim. s. vert. IV. 1817 p. 89 n. 1.

Polistes Gallica Lepeletier, Eneycl. méthod. Insect. X. 1825 p. 171 n. 6, $\subseteq$ of.

Polistes Gallica Brullé, Exped. sc. Morée. Zool. II. 1832 p. 360 n. 798.

Polistes Gallica Lamarck, Hist. nat. anim. s. rert. Ed. 2a IV. $19: 35$ p. 306 n. 1.

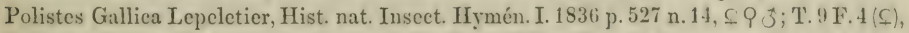

Polistes Gallica Dufour, Amm. se. nat. Zool. (2; VII. 1537 p. 1 .

$[5(\hat{\jmath}), 8+6)$.

Polistes Gallica Blanchard. Hist. nat. Insect. III. 1540 p. 397 n. 1; T. 6 F. 2.

Polistes Gallica Westrood, Trans. Entom. Soc. London IV. 2. 15.5 p. 136.

Polistes Gallica Lucas, Explor. sc. Algérie. Zool. III. 1846 p. 224 n. 174.

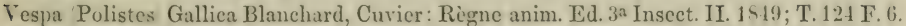

Polistes Gallica Saussure, Eitud. fum. Vespid. II. Vesp. 1853 p.48 n.2, f P J ; T.6 F.1. T. ‘ F. 1 \& 2

Polistes Gallicus Saussure, Étud. fam. Vespid. If. Vesp. 1s.53 p. $243 . \quad$ [ET.9 F.2 \&3.

Polistes Gallica Schenck, Jahrb. Ver. Naturk. Nassau IX. 1. 15:3 p. 41 n. 13, o 9 j̧.

Polistes Gallica Schenck, Progr. Gymnas. Weilburg 1853 p. 16, 우 9 of.

Polistes Galliea Desmarest, Chenu: Eneyel. hist. nat. Annelles $1 \checkmark 60$ p. 140 n. 121, f o ; T. 11

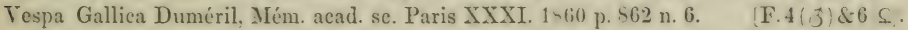

Polistes Gallica Schenck, Jahrb. Ver. Naturk. Nassau XVI. 1561 p. 24 n. 2, \& ? j.

Polistes Gallica Disconzi, Entom. Vicent. 1865 p. 124; T. 9 F. 135 \& 136.

Polistes Gallica Radoszkowski, Horae soc. entom. Ross. III. 1stij Bull. p. IV.

Polistes Gallica Köppen, Horae soc. entom. Ross. III. 1865 p. 289.

Polistes Gallica Taschenberg, Hymen. Deutschl. 1866 p. 247.

Polistes Gallica Goureau, Bull. soc. sc. hist. nat. Yonne 1866 p. ?

Polistes Gallica Iueas. Ann. soc. entom. Franee (4) VIII. 1 6 is 13ull. p. XXY \& XXYI.

Polistes Gallica Giraud, Ann. soc. entom. France (i) VIII. 1Stis Mull. p. XXVII.

Polistes Gallica Siebold, Tagbl. 43. Naturf. Vers. 1869 p. 71-72.

Polistes Gallica Breyer, Amn. sue. entom. Belgique XIII. 14io (R. p. XII.

Polistes Gallica Siebold, Ann. sc. nat. Zool. (5) XIII. 1870 Art. 13 p. 11-13.

Polistes Galliea Mikklin, Öfrers. Finsk. Vet. Soc. Fürh. XII. 15i0 p. 112-11 h.

Polistes Galliea Kraatz, Berlin. entom. Zeitschr. XIV. 1870 p. 47-48.

Polistes Gallica Siebold, Zeitschr. f. wissensch. Zoul. XX. 1570 p). 236-242.

Polistes Gallica Siebold, Beitr. z. Parthenog. 1871 p. 1-101.

Polistes Gallica H. Müller, Verh. naturh. Ver. preuss. Rheinl. XXIX. 1s72 p. 31, \&; T.1 F.2.

Polistes Gallica H. Müller, Befrucht. d. Blumen 1873 p. 466.

Polistes Gallica Rouget, Mém. acad. sc. Dijon (3) I. 1873 Sc. p. 194.

Polistes Gallicus Rouget, Pet. nouv. entom. I. 5.1873 p. 269.

Polistes Galliea S. Saunders, Trans. Entum. Soe. Jondon 1575 Prue. p. II.

Polistes Gallica Rudow. Arch. Ver. Fr. Naturg. Mecklenburg XXX. i 76 p. 2u!! n. 1. $\subseteq$ P.j.

Polistes Gallica Fettig, Bull. soc. hist. nat. Colmar XV./XVI. 1876 p. 125

Polistes Gallica Bertkau, Verh. naturh. Jer. preuss. Rheinl. XXXIII. 1 iti Sitzber. p. 106.

Polistes Gallica H. Müller, Alpenblumen 1891 p. 592 n. 612.

Polistes Gallica Canestrini \& Berlese, Atti soe. Venet.-Trentin. VII. 1. 1451 p.73; 'T.7 F.12. 
Polistes Gallica Magretti, Bull. soc. entom. Ital. XIII. 1851 p. 215 n. 103.

Polistes Gallica Destefani, Natural. Sicil. II. 1882 p. 55-58.

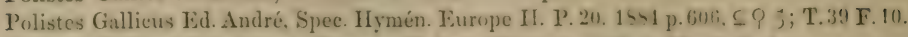

P'olistes Gallicus Schulthess, Hymen. Ilelvet. 1)iplupt. 14ai p. 20, $\subseteq$ ? 3.

Polistes Gallicus Marchal, Feuill. jeun. natural. XIX. 1859 p. 157.

Polistes Gallica Graber, Menkschr. Akad. Wiss. Wien IV. 1459 p. 144-146; T. 7 F. 46.

? Polistes maculatus Rudow, Soc. entom. III. 1889 p. 171.

Polistes Gallieus Magretti. Ann. mus. eir. Genova XXIX. 1890 p. 536 n. 35.

Polistes Gallicus Medina, Act. soc. Españ. XXI. 1892 p. 177.

var. bimaculatus (Fourcr.) D. T. - 오 9 o - Eur. fere tota.

Vespa spec. Geoffroy, Hist. abr. Insect. II. 1762 p. 374 n. 3.

Vespa bimaculata Fourcroy, Entom. Paris. II. 1785 p. 433 n. 5.

Pulistes Geoffroyi Lepeletier, Enercl. méthod. Insect. X. 1425 p. $171 \mathrm{n} .8, \subseteq$.

Polistes Geoffroyi Iepeletier, Hist. nat. Insect. Hymin. I. 1436 p. 527 n. $15, \leq 9$ Oే.

Polistes Galliea var. Geoffroyi Sehulthess. Hymen. Helvet. Diplopt. 1

Polistes Gallicus var. Geoffroyi Marchal, Feuill. jeun. natural. XIX. 1549 p. 157.

Polistes Gallica var. bimaculata m.

var. lefelovrei Guér. - Patria?

Polistes gallica var. I.efebrrei Guérin, Iconogr. règn. anim. VII. Insect. 1545 p. 447 ; T.72 F.t. var. pectoralis Herr.-Schaeff. - 9 Q of - Eur.: Germania.

Polistes pectoralis Megerle) Herrich-Schacfier, Faun. Insect. German. 1841 P. 179 T. 6 j J.

Polistes pectorulis Schenck, Jahrb. Ver. Naturk. Nassau IX. 1. 14.53 p. 43 n.14, C.S.5.

Polistes pectoralis Schenck, Progr. Gymnas. Weilburg 15.33 p. 16, $\subseteq$ P $\vec{j}$. .

Polistes Gallica var. pectoralis $\mathrm{m}$.

generosus Cress. - $ぇ-$ Am.: Texas.

Polistes generosus Cresson, Trans. Amer. Fntom. Soc. IV. 1872 p. 246, ô.

hebraeus Fabr. - $ᄋ 9$ ơ - As.: China, India, Persia, Mauritius.

Vespa hebraca Fabricius, Mant. Insect. I. 1787 p. 292 n. 58.

Vespa hebraea Gmelin, Linné: Syst. nat. Ed. $13^{23}$ I. 5.1790 p. 2756 n. 71.

Vespa undata Olivier, Encycl. méthod. Insect. VI. 1791 p. 694 n. 72.

Vespa hebraea Olivier, Encycl. méthod. Insect. VI. 1791 p. 690 n. 105.

Vespa hebraca Fabricius, Entom. system. II. 1793 p. 274 n. 74.

Vespa hebraea Fabricius, Syst. Piez. 1804 p. 273 n. 21.

Vespa hebraca Jurine, Nour. méth. class. Hymén. 1807 p. 169, 우.

Polistes hebraea Lamarck, Hist. nat. anim. 8. rert. IV. 1817 p. 90 n. 5.

Polistes hebraea Lepeletier, Encycl. méthod. Insect. X. 1825 p. 171.

Polistes hebraea Lamarck, Hist. nat. anim. s. vert. Ed. $2^{\text {a }}$ IV. 1835 p. 306 n. 5.

Polistes helraea Lepeletier, Hist. nat. Insect. Iymén. I. 1536 p. 525 n. 12, Q.

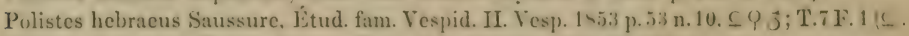

Polistes hebraeus Horne, Trans. Zool. Soc. I.ondon VII. 3. 1870 p. 170.

Polistes hebraea (ierstaecker, r. d. 1)ecken : leise in Ost-Afrika. Gliederthierc. 15:3 p. 321

Polistes hebracus Smith, Trans. Intom. Soc. London 1873 p. 197 n. $2 . \quad$ [n.17.

Polistes hebracus Rothney, Lintom. M. Magaz. XIV. 1877 p. 92.

Polistes hebraeus Mate Lachlan, Trans. Entom. Soe. Iondon Las Proe. p. III.

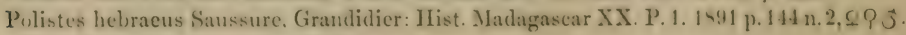

hoplites Sauss. $-\subsetneq-$ As.: India or.

Polistes hoplitus Saussure, Citud. fam. Vespicl. II. Vesp. 1453 p. 53 n. 11 , S.

Polistes hoplites Saussure, Étud. fam. Vespid. II. Vesp). 1853 p. 255.

humilis Fabr. - $P-$ Austr.: 'Tasmania.

Vespra humilis Fabricius, Spec. Insect. I. 1781 p. 161 n. 20.

Vespa humilis Fabricius, Mant. Insect. I. 1787 p. 258 n. 29. 
Vespa humilis Gmelin, Linné: Syst. nat. Ed. $13^{a}$ I. 5.1790 p. 2752 n. 45.

Vespa humilis Olivier, Encyel. méthod. Insect. VI. 1791 p. 682 p. 62.

Vespa humilis Fabricius, Entom. system. II. 1793 p. 261 n. 28.

Polistes humilis Fabricius, Syst. Piez. 1804 p. 270 n. 5.

Polistes humilis Saussurc, Étud. fam. Vesp. II. Vesp. 1853 p. 52 n. 8, Q.

japonicus Sauss. - +- As.: Japonia.

Polistes Japonicus Saussure, Rev. \& mag. zool. (2) X. 1858 p. 260, ㅇ.

incertus Cress. - ơ - Am.: Cuba.

Polistes incertus Cresson, Proc. Entom. Soc. Philadelphia Ir. 1565 p. 1lici, of.

inornatus Rits. - $Q$ - Afr.: Guinea.

Polistes inornatus Ritsema. Tijdschr. v. Entom. XTII. 157.4 p. 203 n. 22, ?; T. 11 F. s. jokahamae Rad. - 우 - As.: Japonia.

Polistes Jokahamae Radoszkowski, Horac soc. entom. Ross. XXI. 1557 p. 135, S.

lateritius Smith - 우 $\widehat{o}-$ As.: Ceram.

Pulistes lateritius Smith, Catal. Hymen. Brit. Mus. V. 1557 p. $10: 3$ n. $15, \subseteq j$.

liliaceuseulus Sauss. - $\mathrm{Q}-$ Am.: Brasilia.

Polistes liliaceusculus Saussure, Etud. fam. Vespid. II. Vesp). 1553 p. 95 n. 5 !), .

liliaciosus Sauss. - 으 - Am.

Polistes liliaciosus Saussure, Ktud. fam. Vespid. II. Vesp. 15.53 p. 97 n. 5 S, \&; T. 11 F. 7. macaensis Fabr. - 으-As.: India.

Vespa Macaensis Fabricius, Entom. system. II. 1793 p. 259 n. 22.

Vespa Macaensis Donovan, Epit. Nat. Hist. Insects India 1800; Tab. 57.

Polistes Macaensis Fabricius, Syst. Piez. 1804 p. 272 n. 12.

Vespa Macaensis Jurine, Nouv. méth. class. Hymén. 1807 p. 169, \&.

Polistes Maeaensis Cameron. Proc. \& Trans. Nat. Hist. Soc. Glasgow 2, I. 3. 155.5 4 4 p). 26.3. maculipennis Sauss. - $f-$ As.: Jara.

Polistes maculipennis Saussure. Etud. fam. Vespid. II. Vesp. 1853 p. 61 n. 19, f; T. (; F. 4. madecassus Sauss. $-q-$ Afr. : Madagascar.

Polistes Madecassus Saussure. Kitud. fam. Vespid. II. Vesp. $14 . ; 3$ p. 51 n. 6, @.

Polistes Madecassus Saussure, Grandidier: Hist. Madagascar XX. P. 1. 1 \91 p. 144 n.1, ᄃ : madocii Kby. - 으 - Am.: St. Thomas.

['T. 4 F.8

Polistes Madoci W. F. Kirby, Ann. \& Mag. Nat. Hist. (5. XIII. 145.t p. 111 n. 40. f. mandarinus Sauss. - $\mathrm{Q}-$ As.: China.

Polistes Mandarinus Saussure. Titud. fam. Vespid. II. Vesp. 1553 p. 54 n. 15, ᄃ.

Polistes Mandarinus Saussure. Reise d. Novara. Zool. II. 1. 1 sti I Iymen. p. 20 n. 4, \&. manillensis Sauss. - of - As.: Philippines.

Polistes Manillensis Samssure. Étud. fum. Tespid. II. Vesp. 14,53 p. 72 n. 30, $\hat{\jmath}$.

marginalis Fabr. $-f Q \vec{o}-$ Afr.: Sierra Lcone.

Vespa marginalis Fabricius, Syst. entom. 1775 p. 367 n. 24.

Vespa marginalis Fabricius, Spec. Insect. I. 1781 p. 463 n. 29.

Vespa marginalis Fabricius, Mant. Insect. I. 1757 p. 289 n. 34.

Vespa marginalis Gmelin, Linné: Syst. nat. Ed. $13^{a}$ I. 5.1790 p. 2759 n. S9.

Vespa marginalis Christ, Naturg. d. Insect. 1791 p. 210.

Vespa marginalis Olivier, Encyel. méthod. Insect. VI. 1791 p. 655 n. 79.

Vespa marginalis Fabricius, Entom. system. II. 1793 p. 264 n. 42.

Polistes marginalis Fabricius, Syst. Piez. 1504 p. 272 n. 17.

Polistes media Palisot-Beaurais. Insect. Afr. \& Amér. 1-0.; p. 207 Irrmén.; T. \& F. 2.

Vespa marginalis Jurine, Nouv. méth. class. Hymén. 18ñ p. 169, ơ.

Polistes Africana Palisot-Beaurais, Insect. Afr. A Amér. 1-21 p. 207 Irmen.; T. 5 F. 4.

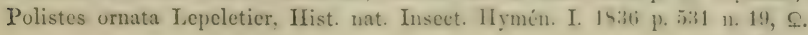




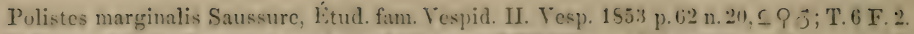
Polistes plebeja Gerstaecker, Arch. f. Naturg. XXXVII. 1. 14i0 p. 351 n. 1:1. ᄃ. [n.1s. Polistes marginalis Gerstaecker, v. d.J)ecken. Reise in ()st-Afrika. Gliederthiere. 1573 p.325 Pulistes plebeja Gerstaecker, v.d. Decken: Reise in Ost-Afrika. Gliederthiere. 1573 p.325

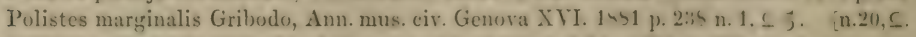
Polistes marginalis Gribodo, Ann. mus. civ. Genova XXI. $184+$ p. $2-6$ n. 17. ?.

var. stigma (Fabr.) Sauss. $-q-$ As.: India, Ceram, Celebes, Aru.

Vespa stigma Fabricius, Entom. system. II. 1793 p. 275 n. 78.

Vespa tamula Fabricius, Suppl. entom. system. 1798 p. 263 n. is \& 79.

Vespa stigma Fabricius, Syst. Picz. 1804 p. 261 n. 41.

Polistes tamula Fabricius. Syst. Picz. 1804 p. 274 n. 27.

Vespa tamula Jurine, Nouv. méth. class. Hymén. 1807 p. 169, 卫.

Polistes stigma Saussure, Étud. fam. Vespid. II. Vesp. 1553 j). 64 n. 21, 5; T. 6 F. .

Polistes stigma Smith, Journ. of Proc. Linn. Soc. Zool. III. 1858 p. 168 n. 3.

Polistes stigma Saussure, Stettin. entom. Zeitg. XXII. 1562 p. 140 n. 11.

Polistes stigma Horne, 'I'rans. Zool. Soc. London VII. 3. 1870 p. 172.

Polistes marginalis var. stigma Magretti, Ann. mus. eiv. Genova XXI. 1544 p. 607 n. 142. melanosoma Sauss. - ô - Am.: Brasilia.

Polistes melanosoma Saussure, Etud. fam. Vespid. II. Yesp. 1553 p. 76 n. $36, \vec{\jmath}$. metricus Say - 우 - Am.: United States.

Polistes metrica Say, Boston Journ. Nat. Hist. I. 4. 1837 p. 388 n. 1.

Polistes metricus Saussure, Étud. fam. Vespid. II. Vesp. 1553 p. 75 n. 35, , ; T. 7 F. 4. Polistes metrica Lecontc, Writh of 'Th. Say Entom. II. 1859 p. 768 n. 1.

minor Pal.-Beaur. - ㅇ 9 ơ - Am.: Texas, Cuba.

Polistes minor Palisot-Beauvais, Insect. Afr. (A Amer. 1505 p. 207 Iỵmén.; T. 5 F. 3.

Polistes Pocyi Lepeletier, Hist. nat. Insect. Hymén. I. 1836 p. 532 n. 20.

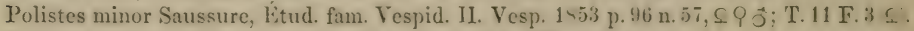

Polistes minor Saussure, Sagra: Hist. fis. Cuba VII. 1856 p. 773, Q.

Polistes minor Saussure, Ann. soc. entom. France (3) V. 1857 p. 314 n. 10.

Polistes Poeyi Marshall, Entom. M. Magaz. XVI. 1879 p. 19.

minutissimus Spin. - 우 - Am.: Brasilia.

Polistes minutissima Spinola, Mem. accad. se. Torino (2) XIII. 15.51 p. is n. 59, S.

molestus Smith - 우 - Am.: Panama.

Polistus modestus Smith, Tans. Entom. Soc. London (3) I. 1. 1562 p. 35 (n. 4) 4.

multipictus Smith - $\subsetneq-$ As.: Amboina.

Polistes multipictus Smith, Journ. of Proc. Limn. Soc. Zool. IV. 1560 Suppl. p. 1:30 n. 5, f. nestor Fabr. - Am. bor.

Vespa Nestor Fabricius, Suppl. entom. system. 1798 p. 262 n. 42-43.

Polistes Nestor Fabricius, Syst. Piez. 1804 p. 272 n. 18.

nigrifrons Smith - +- As.: Aru.

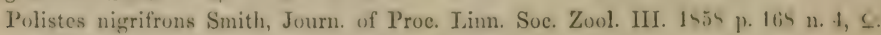
nigripennis Fabr. - Am. mer.

Polistes nigripennis Tabricius, Syst. Picz. 1501 p. 272 n. 14.

novajoe Cress. - $q-\Lambda \mathrm{m}$.: New Mexico, Arizone.

Polistes Norajoc Cresson, Trans. Amer. Entom. Soc. I. 1868 p. 383 n. 27, \&.

norarae Sauss. - $f$ o $\widehat{o}-$ Austr.: Nicobares.

[F.13\&14(O).

I'ulistes Novarae Saussure. Reise d. Novara. Zoul. II. 1. 1 hli Hymen. p. 19 n. 2, Q Q j; T. 1

Polistes Novarac Smith, Trans. Entom. Soc. London 1873 p. 198 n. 3.

obseurus Sauss. - - - Am.: Brasilia.

Polistes obsenrus Saussure, Mém. soe phys. \& hist. nat. Geneve XVII.1.1563 p. 236 n. 63, o. 
oculatus Smith - oే - Am.: Mexico.

Polistes oculatus Smith, Catal. Hymen. Brit. Mus. V. 15.57 p. 111 n. 6ï, ơ. olivaceus (Deg.) Sauss. - Am. mer.?

Vespa olivacea Degreer, Mém. serr. hist. Insect. III. 1773 p. 5 ๖ n. 5 ; 'T. 29 F. 9.

Vespa olivacea Gözc, Decreer: Abh. Gesch. Insect. III. 1740 n. 377 n. 5; T. 29 F. 9.

Vespa olivacea Retzius, Gen. \& spec. Insect. 1783 p. 63 n. 236.

Vespa olivacea Olivier, Encycl. méthod. Insect. VI. 1791 p. 684 n. 71.

Polistes olivaceus Saussure, Étud. fam. Vespid. II. Vesp. 1853 p. 102 n. 3.

opalinus Sauss. - $ᄋ-$ Am.: Brasilia.

Polistes opalinus Saussure, Etud. fam. Vespid. II. Vesp. 1553 p.59 n. 52 , f; T. 12 T. 6. orientalis (Kby.) Sauss. - 우 $\widehat{\sigma}-$ As.: India, China.

Cyclostoma orientalis W. Kirby, Introd. Entom. III. Ed. 1a $1 \leqslant 26$ p. 633 , oै.

Gyrostoma orientalis W. Kirby; Introd. Entom. III. Ed. 5 a 1535 p. 631, ơ.

Polistes Gyrostoma grrostoma Saussure. litud. fam. Vespid. II. Vesp. 1853 p. 104, fơ.

Polistes orientalis Smith, Catal. Hymen. Brit. Mus. V. 1557 p. 102 n. 6.

pallidipes Lep. $-Q-$ Am. bor. \& centr.

Polistes pallipes I.epeletier, Hist. nat. Insect. Hymén. I. 1536 p. 530 n. 15, 9.

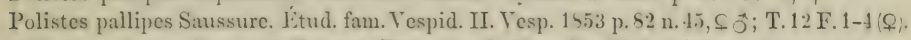

Polistes pallipes Coues \& Yarrow. Proc. Acad. Nat. Se. Philadelphia 157 s p. 314.

Polistes pallipes Provancher, Natural. Canad. XIII. 1882 p. 168 n. 1, 우 ơ.

Polistes pallipes Provancher, Faun. cntom. Canada. Hỵmén. 1 s 1 p. $656 \mathrm{k}$ n. 1 , f $\hat{\jmath}$.

panamensis Holmgr. - Am.: Panama.

Polistes Panamensis Holmgren, Lugenies Resa. Insect. 1565 p. 439 n. 98.

perplexus Cress. - ô - Am.: 'Texas, Bermudas.

Polistes perplexus Cresson, Trans. Amer. Entom. Soc. IV. 1872 p. 245, ô.

Polistes perplexus W. F. Kirby, Ann. \& Mag. Nat. Hist. (5. XIII. 1 s 54 p. 410 n. 36.

philipinensis Sauss. - - - As.: Philippines.

Polistes Philippinensis Saussure, Kitud. fam. Vespid. II. Tesp. 15.13 p. is n. 14, $\Omega$.

pictetii Sauss. - $\&-$ Austr.

Polistes Picteti Saussure, Etud. fam. Vespid. II. Vesp. 15.53 p. 69 n. 28 , f; T. 6 F.S. puncticollis Mor. - As.: Sibiria.

Polistes puncticollis F. Morawitz, Horae soe. entom. Ross. XXVI. 1 \&y2 p. 150.

rubidus Lep. - ơ - Afr.: Cap.

Polistes rubida Iepeletier, Hist. nat. Insect. IỴmén. I. 14336 p. $523 \mathrm{n} .4, \overrightarrow{0}$.

Polistes rubidus Saussure, Etud. fam. Vespid. II. Vesp. 1853 p. 50 n. 4.

rubiginosus Lep. - $\widehat{\sigma}-$ Am.: United States.

Polistes rubiginosa I.ppeletier, Hist. nat. Insect. Hỵmén. I. 1536 p. 524 n. 10, ô.

Polistes rubiginosus Saussure. Etud. fam. Vespid. II. Vesp. 15.53 p. it n. 31.

Polistes rubiginosus Saussure, Ann. soc. entom. France (3) V. 1857 p. 314 n. 8.

Polistes rubiginosa likley, 5th Ann. Rep. Insect. Missouri 1673 p. 51 ; Jig. 25.

Polistes rubiginosus W. F. Kirby, Ann. \& Mag. Nat. IIist. . XIII. 1544 p. 410 n. 35.

Polistes rubiginosus Nehrling, Zool. Garten XXV. 1884 p. 264.

ruficornis Sauss. - 우 - Am. mer.

Polistes ruficornis Saussure, Ftud. fam. Vespid. II. Vesp. 1553 p. 5. n. 4 , \&; T. 10 F. 3

rufldens Sauss. - $\mathrm{Q}-\mathrm{Am}$.: Venezuela.

Polistes rufidens Saussure, litud. fam. Vespiel. II. Vesp. 14.53 p. 77 n. 35,

rnflna Er. - Am.: Guiana.

Polistes rufina Illiger Erichson. Schomburgk: Reisen in Guiana III. 14.15 p. 594 .

sagittarius Sauss. - 우 - As.: China, India.

Polistes sarittarius Saussure, litud. fam. Tespied. II. Tesp. $15_{3}$ p. 56 n. 12, §. 
Polistes sagittarius Smith, Catal. Hymen. Brit. Mus. V. 1857 p. 102 n. 10.

Polistes sacrittarius Smith, Journ. of Proce. Iimn. Suc. Zool. II. 15.7 p. $11:$.

saussurei D. 'T. - $Q$ - Afr.: Madagascar.

Polistes incertus Saussure, Grandidier : Hist. Madagascar XX. P.1. 1591 p.115 n.3, f;T. 1 F.4

Polistes Saussurei m.

[[non C'resson].

sehach Fabr. - 우 $9 \pm-$ Austr.

Vespa schach Fabricius, Spec. Insect. I. 1781 p. 461 n. 18.

Vespa schach Fabricius, Mant. Insect. I. 1787 p. 258 n. 21.

Vespa schach Gmelin, Linné: Syst. nat. Ed. 13a I. 5.1790 p. 2750 n. 39.

Vespa schach Olivier, Encycl. méthod. Insect. VI. 1791 p. 682 n. 60.

Vespa schach Fabricius, Entom. system. II. 1793 p. 260 n. 25.

Polistes schach Fabricius, Syst. Piez. 1804 p. 270 n. 2.

Vespa schach Jurine, Nouv, méth. class. Hymén. 1807 p. 168, ㅇ.

Polistes schatch Saussure, Kitud. fam. Vespid. II. Vesp. 14.53 p. 50 n. $3, \subseteq$ ? 3 .

semenowii Mor. - 오 ठే - As.: Transcaspia.

Polystes Semenowii F. Morawitz. Horae soc. entom. Ross. XXIII. 1559 p. 552 n. 2, 50 .

semillarus Holmgr. - 우 ơ - Am.: Guatemalà.

Polistes semiflavus Holmgreen, Fugenies Resa. Insect. 1 slis p. 439 n. 99, $\subseteq$ Jै.

simulatus Smith - o ô-As.

Polistes simulatus Smith, Journ. of Proe. Linn. Soc. Zool. IV. 1860 Suppl. p. 130 n. 4, S 3. smithii Sauss. - 우 0 - Afr.: Senegal.

Polistes Smithii Saussure, Kitud. fam. Vespid. II. Yesp. 1553 p. lit n. 17, S; T. 7 F. 3.

Polistes Smithii Gerstaceker, Peters: Reise n. Mossambique. Zool. V. 1562 p. 170, ?.

Polistes Smithii Gribodo, Ann. mus. civ. Genova XXI. 1884 p. 287 n. 18, Q.

Polistes Smithii Saussure, Grandidier: Hist. Madagasear XX. P. 1. $18: 1$ p. 146 n. $4, \Omega \delta$.

snellenii Sauss. - $9-$ As.: Japonia.

Polistes Snelleni Saussure. Stettin. entom. Zeitg. XXIII. 1Sti2 p. 140 n. 12, Q.

spilophorus Schlett. - $\subsetneq-$ Afr.: Congo.

Polistes spilophora Schletterer, Ann. soc. entom. Belgique XXXVI. 1991 p. 29, ᄃ.

spinolae Sauss. - $9-$ Am.: Brasilia.

Polistes Spinolac Saussure, 1.tud. fam. Tespid. II. Vesp. 1853 p. 80 n. 43, ?.

sfuamosus Drury - Patria?

Vespa squamosa Drury, Illustr. Nat. Hist. I. 1770 p. 95 ; T. 43 F. 7.

Polistes sqquamosa Westwood, Drury: Illustr. Nat. IList. I.d. 23 I. 1537 p. 96 ; T. 43 F. 7.

striatus Fabr.! - Am.: Cayenne.

Vespa striata Fabricius, Mant. Insect. I. 1787 p. 258 n. 18.

Vespa striata Gmelin, Linné: Syst. nat. Ed. 13 I. 5.1790 p. 27.19 n. 36.

Vespa striata Olivier, Encycl. méthod. Insect. VI. 1791 p. 651 n. 57.

Vespa striata Fabricius, Entom. system. II. 1793 p. 259 n. 21.

Polistes striata Fabricius, Syst. Piez. 1804 p. 271 n. 11.

subsericeus Sauss. - $f-A$ m.: Brasilia.

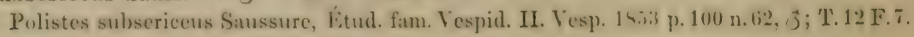

sulentus Smith - 우 §ึ-As.: China.

Polistes sulcatus Smith, 'I'rans. Entom. Soc. London (2) U. 2. 1552 p. 35, f o.

Polistus sulcatus Saussure, Etud. fam. Vespid. II. Vesp. 1853 p. 59 n. 16, \& ơ.

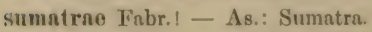

Polistes Sumatrae Fabricius, Syst. Piez. 180.4 p. 273 n. 23.

synoecus Sauss, - 우 ô- Austr.

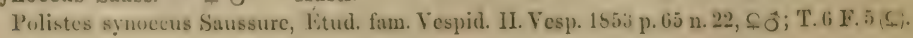


tasmaniensis Sauss. - 오 0 - Austr.

[\&T.8 F.3.

Polistes Tasmaniensis Saussure, Kitud. fam. Yespid. II. Yesp. 1553 p. 66 n. 23, \& ơ; T. 6 F.6 (\$)

P’olistes Tasmaniensis Saussure, Mém. soc. phys. \& hist. nat. Geneve XVII.1. 15633 p. 236 r.62.

tenebricosns Lep. - $9-$ As.: Java.

Polistes tenebricosa (de Haan Lepeletier, Hist. nat. Insect. II mén. I. $19: 36$ p. 529 n. 17, ?.

Polistes tenebricosus Saussure, litud. fam. Vespid. II. Yesp. 1 \$53 p. $51 \mathrm{n}$. 5.

tepidus Fabr. - $f-$ Austr.: New Guinea.

Vespa tepida Fabricius, Syst. entom. 1775 p. 366 n. 17.

Vespa tepida Fabricius, Spec. Insect. I. 1781 p. 462 n. 21.

Vespa tepida Fabricius, Mant. Insect. I. 1787 p. 288 n. 25.

Vespa tepida Gmelin, Linné: Syst. nat. Ed. $13^{a}$ I. 5. 1790 p. 2752 n. 47.

Vespa tepida Christ, Naturg. d. Insect. 1791 p. 242.

Vespa tepida Olivier, Encycl. méthod. Insect. VI. 1791 p. 682 n. 64.

Vespa tepida Fabricius, Entom. system. II. 1793 p. 262 n. 31.

Vespa tepida Donovan, Epit. Nat. Hist. Insects India 1800; Tab. 57.

Polistes tepida Fabricius, Syst. Piez. 1804 p. 271 n. 7.

Polistes tepidus Saussure, Etud. fam. Vespid. II. Vesp. 15.53 p. lis n. 27, \&; T. 8 F. 1.

Pulistes tepidus Mocbius, Abh. naturw. Ver. Hamburg UI. $1 \$ 56$ p. 167 (p. 51 ); T. 15 F. 7 .

texanus Cress. - 우 - Am.: Texas.

Polistes Texanus Cresson, Trans. Amer. Entom. Soc. IV. 1S72 p. $246, \propto f \hat{o}$.

translucidus Spin. - 우 - Am.: Brasilia.

Polistes (Rhopalidia) translucida Spinola, Mem. accad. sc. 'Torino 2) XIII. 1451 p. 79 n. 3, \&.

tricolor Sauss. - $\subseteq-$ Austr.

Polistes tricolor Saussure, Etud. fam. Vespid. II. Vesp. 1853 p. 67 n. 25 , \&.

ureeolicus Klug - Am.: Guiana.

Polistes urecolica Klug. Schomburgk: Reisen in Guiana III. 1515 p. 590.

varius Fabr.! - As.: China.

Vespa varia Fabricius, Mant. Insect. I. 1787 p. 293 n. 79.

Vespa varia Gmelin, Linné: Syst. nat. Ed. $13^{a}$ I. 5.1790 p. 2754 n. 62.

Vespa varia Olivier, Encycl. méthod. Insect. V1. 1791 p. 674 n. 23.

Vespa raria Fabricius, Entom. system. II. 1793 p. 282 n. 100.

Polistes varia Fabricius, Syst. Piez. 1804 p. 279 n. 48.

rariabilis Fabr. - Austr.

Vespa rariabilis Fabricius, Spec. Insect. I. 1781 p. 466 n. 46.

Vespa variabilis Fabricius, Mant. Insect. I. 1787 p. 292 n. 57.

Vespa variabilis Gmelin, Linné: Syst. nat. Ed. $13^{a}$ I. 5. 1790 p. 2756 n. 70.

Vespa variabilis Olivier, Encyel. méthod. Insect. VI. 1791 p. 690 n. 104.

Polistes variabilis Fabricius, Syst. Piez. 1804 p. 273 n. 20.

Polistes variabilis Saussure, Litud. fam. Vespid. II. Vesp. 14.33 p. 66 n. 24.

rariatus Cress. - $Q$ ô - Am.: T'exas.

Polistes variatus Cresson, 'Trans. Amer. Entom. Soc. IV. 1872 p. 247, ᄋ ఠี.

variegatus Lep. - ô - Am.: Cayenne.

Polistes variegata Lepeletier. IIist. nat. Insect. Ifymén. I. 1836 p. 523 n. 9, j̧.

Polistes variegata Saussure, ḱtud. fam. Vespid. II. Vesp. 1853 p. 101 n. 1.

rersicolor (Oliv.) Sauss. - $\subseteq-$ Am.: Cayenne, Brasilia, San Domingo.

Vespa versicolor Olivier, Encycl. méthod. Insect. VI. 1791 p. 692 n. 114.

Vespa myops Fabricius, Suppl. cntom. system. 1798 p. 261 n. 40-11.

Polistes myops Fabricius, Syst. Piez. 1804 p. 272 n. 16.

Polistes versicolor Saussure, Etud. fam. Vespid. II. Vesp. 1453 p.41 n.41, .; T.7 F. 5 \& T. \$ F.6.

Polistes versicolor Möbius, Abh. naturw. Ver. Ilamburg III. 1556 p. 167 p. 51!; T. 15 F. 6. 
Polistes rersicolor Mïbius, Arch. f. Naturg. XXII. 1. 1556 p. 330; T. 12 F. 3. Polistes versicolor Smith, Catal. Hymen. Brit. Mus. V. 1857 p. 108 n. 50. Vespa versiculor Smith, Trans. Entom. Soc. London (3. V. 1565 Proc. p). CVIII.

\section{APOICA.}

Lepeletier, Hist. nat. Insect. Hymén. I. 1836 p. 536 n. 5. a้สoเxos, colonus.

Synon.: Agelaia Blanchard. Polistes Fabricius. Vespa Olivier, Jurine.

arborea Sauss. - 오 - Am. mer.

Apoica arborea Saussure, Kitud. fam. Vespid. II. Vesp. $155: 3$ p. 104 n. 3, f; T. 26 F. 1. cubitalis Sauss. - $\rho-$ Am.: Brasilia.

Apoica cubitalis Saussure, litud. fam. Vespid. II. Vesp. 1553 p. 109 n. 4, f; T. 18 F. 3. pallida (Oliv.) Sauss. - 우 $9-$ Am. mer.

Vespa pallida Olivier, Encycl. méthod. Insect. VI. 1791 p. 675 n. 26.

Polistes pallens Fabricius, Syst. Piez. 1804 p. 276 n. 35.

Vespa pallens Jurine, Nouv. méth. class. Hymén. 1807 p. 171, \&.

Apoica pallida I.epeletier. Hist. nat. Inseet. Hỵmén. I. 1536 p. 535 n. 2, Q. [F.1-2. Apoica pallida Saussure, Etud. fam. Vespid. II. Vesp. 1553 p.107 n.1.9; T. 15 F. 1 S \& \& T.2S Apoica pallida Möbius, Abh. naturw. Ver. Hamburg III. 14.56 p. 167 (p. 50 ; ; T. 1 S F. 1-5. Apoica pallida Möbius, Arch. f. Naturg. XXII. 1. 1856 p. 330; T. 12 F. 1.

virginen (Fabr.) Sauss. - Q - Am. mer.

Polistes Virginea Fabricius, Syst. Piez. 1804 p. 277 n. 37.

Vespa Virginea Jurine, Nouv. méth. class. Hymén. 1807 p. 171, ㅇ․

Apoica lineolata Lepeletier, Hist. nat. Insect. Hymén. I. 1536 p. 537 n. 1, ᄃ. Agelaia (Apoica) lineolata Blanchard, Ifist. nat. Insect. III. 14.40 p. $396 \mathrm{n.} 2$.

Apoica Virginea Saussure, Étud. fam. Vespid. II. Vesp. 1553 p. 107 n. 2, ᄃ; T. 1s F. 2. Apoica Virginea Saussure, İtud. fam. Vesp. II. Vesp. 1853 p. 244.

Apioica Virginea Desmarest, Chenu: Eneycl. hist. nat. Annelles 1s60; T. 11 F. 3.

\section{VESPA}

Linné, Syst. nat. (Ed. 1a 1735) Ed. $10^{a}$ I. 1758 p. 343 n. 216 \& p. 573 n. 217. Vespa, nom. propr. Hymen.

Syrron.: Bembex Riley.

Odynerus Kennedy.

Rhynchium Dewitz.

Sphex Sulzer.

Sulyg.: Pseudorespa Schmiedekncelit, Fntom. Nachr. VII. 1451 p. 313-31s.

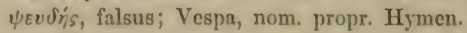

Vespula Thomson, Opusc. entom. P. 1. 1869 p. 79. Vespa, demin.

affinis Fabr. - 9 P

Vespa affinis Fabricius, Mant. Insect. I. 1787 p. 287 n. 2.

Vespa affinis Gmelin, Linné: Syst. nat. Ed. $13^{\text {a }}$ I. 5.1790 p. 27.18 n. 29.

Vespa affinis Olivier, Eneycl. méthod. Insect. VI. 1791 p. 677 n. 38.

Vespa affinis Fabricius, Entom. system. II. 1793 p. 253 n. 2.

Vespa affinis Fabricius, Syst. Piez. 1504 p. 254 n. 2.

Vespa affinis Latrcille, Gen. Crust. \& Insect. IV. 1506 p. 142. 
Vespa affinis Jurine, Nouv. méth. class. Hymén. 1807 p. 168, 요.

Vespa affinis Leneletier, Hist. nat. Insect. Hymén. I. 1536 p. $506 \mathrm{n}$. 2, 9.

Vespa affinis Saussure, Ktud. fam. Vespid. II. Vesp. 1553 p. 154 n. 39.

Vespa affinis Gribodo, Ann. mus. cir. Genova XXI. 155.1 p. 355 n. 10, Q.

analis Fabr. - 우 ô - As.: India, China.

Vespa analis Fabricius, Syst. entom. 1775 p. 363 n. 5.

Vespa analis Fabricius, Spec. Insect. I. 1781 p. 459 n. 5.

Vespa analis Fabricius, Mant. Insect. I. 1787 p. 287 n. 6.

Vespa analis Gmelin, Linné: Syst. nat. Ed, 13 a I. 5. 1790 p. 2749 n. 33.

Vespa Crabro Sphinx Christ, Naturg. d. Insect. 1791 p. 217 ; T. 18 F. 5.

Vespa Crabro analis Christ, Naturg. d. Insect. 1791 p. 218.

Vespa analis Olivier, Encycl. méthod. Insect. VI. 1791 p. 677 n. 43.

Vespa analis Fabricius, Entom. system. II. 1793 p. 254 n. 6.

Vespa analis Fabricius, Syst. Piez. 1804 p. 254 n. 6.

Vespa analis Jurine, Nouv. méth. class. Hymén. 1807 p. 168, \&.

Yespa analis Lepeletier, Hist. nat. Insect. Hymén. I. 1836 p. 50 S n. 6, S.

Vespa analis Saussure, Étud. fam. Vespid. II. Vesp. 1553 p. 152 n. 36, ô.

anmulata Smith - 9 - As.: Malacca, Borneo.

Vespa annulata Smith, Journ. of Proc. Linn. Soc. Zool. II. 15.77 p. 116 n. 6, Q.

anomala Sauss. - 우 - As.: India or., Archipelagus Malayensis.

Vespa anomala Saussure, Étud. fam. Vespid. II. Vesp. 1853 p. 112 n. 1, f; T. 14 F. 2.

Vespa dorylloides Saussure, Etud. fam. Vespid. II. Vesp. 1453 p. 256 , ᄃ; T. 14 F. 2.

Vespa doryloides Gribodo, Ann. mus. cir. Genora XXI. 1554 p. 354 n. s, \&.

apilinguaria Christ! $\left.{ }^{1}\right)$ - Patria?

Vespa apilinguaria Christ, Naturg. d. Insect. 1791 p. 224; T. 19 F. 8.

arenaria Fabr. - 우 $P$ - Am. bor.

Vespa arenaria Fabricius, Syst. entom. 1775 p. 365 n. 12.

Vespa arenaria Fabricius, Spec. Insect. I. 1781 p. 461 n. 14.

Vespa arenaria Fabricius, Mant. Insect. I. 1787 p. 288 n. 16.

Vespa arenaria Gmelin, Linné: Syst. nat. Ed. $13^{a}$ I. 5.1790 p. 2749 n. 34.

Vespa arenaria Olivier, Encycl. méthod. Insect. VI. 1791 p. 681 n. 55.

Vespa arenaria Fabricius, Entom. system. II. 1793 p. 258 n. 19.

Vespa arenaria Fabricius, Syst. Piez. 1804 p. 258 n. 20.

Vespa marginata W. F. Kirby, Fauna Bor-Amer. IV. 1537 p. 265 n. 366, Q; T. 6 F. 2.

Vespa arenaria Saussure, Etud. fam. Vespid. II. Vesp. 14,33 p. 134 n. 14, Q.

Vespa consobrina Saussure, Eitud. fum. Vespid. II. Vesp. 1553 p. 141 n. 21, Q.

Vespa arenaria Saussure, Stettin. entom. Zeitg. XVIII. 1857 p. 116, \&.

Vespa marginata Saussure, Stettin. entom. Zeitg. XVIII. 14.57 p. 116, ऽ.

Vespa consobrina Provancher, Natural. Canad. XIII. 1882 p. 165 n. 2, ㅇ.

Vespa consobrina Provancher, Faun. entom. Canada. Iỵmén. 1s5:3 p. tis:3 n. 2, S.

atavina Heer - Eur.: Carinthia.

Vespa atarina Heer, Denkschr. schweiz. Ges. Naturw. XI. 1550 j. 101 n. ↔; T. 7 F. S.

Vespa attavina Giebel, Deutschl. Petrefact. 1852 p. 645.

Vespa attavina Giebel, Faủna d. Vorwelt II. 1. 1856 p. 160.

atrata Fabr.! - Am. mer.

Vespa atrata Fabricius, Suppl. entom. system. 1798 p. 262 n. 44-45.

Vespa atrata Fabricius, Syst. Picz. 1804 p. 260 n. 36.

? Rhynchium atratum Dewitz, Berlin. entum. Zeitschr. XXV. 1 sul p. 2001.

1) $=$ ? Bembex spec. 
auraria Smith - 으

Vespa auraria Smith, Trans. Entom. Soc. Tondon 2, II. 2. 1552 p. 16, S: T. \& T. 4. Vespa auraria Saussure. Citud. fam. Tespid. II. Vesp. 145;3 p. 147 n. 29 \& 3 .

Vespa simillima Smith, Entom. M. Magaz. IV. 1568 p. 250.

anrata Fabr.! - Afr.: Sierra Leone.

Vespa aurata Fabricius, Mant. Insect. I. 1787 p. 289 n. 28.

Vespa aurata Gmelin, Linné: Syst. nat. Ed. $13^{a}$ I. 5.1790 p. 2759 n. 81.

Vespa aurata Olivier, Encycl. méthod. Insect. VI. 1791 p. 653 n. 67.

Vespa aurata Fabricius, Entom. system. II. 1793 p. 263 n. 35.

Vespa aurata Fabricius, Syst. Piez. 1804 p. 259 n. 27.

anstriaca Panz. - 0 oิ - Eur.

Vespa Austriaca Panzer, Faun. Insect. German. VI. 1799 P. 63 T. 2.

Vespa Austriaca Panzer, Krit. Revis. II. 1806 p. 154.

Vespa Austriaca Gravenhorst, Vergl. C̈bers. zuol. System. 15117 p. 275 n. 35511.

Vespa Austriaca Jurine, Nouv. méth. class. Hymén. 1807 p. 168, \&.

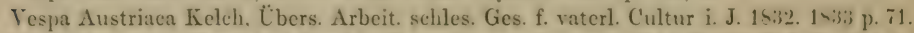

Vespa Austriaca IIerrich-Schaeffer, Faun. Insect. German. 1441 P. 17!) T. 3 Fig. b J J.

Vespa borealis Smith, Zoologist I. 1513 p. 170 n. 6, ᄃ; Fig. inec Kirby, nec Zetterstedt.

Vespa arborea Ross, Zoologist III. 1845 p. 1156.

Vespa arborea Smith, Zoologist VII. 1849 App. p. LX.

Vespa arborea Saussure, Kitud. fam. Vespid. II. Vesp. 1553 p. 122 n. 5, S; T. 14 F. s.

Vesja rufa var. Austriaca Schenck. Progr. Gymnas. Weilburg $155 ; 3$ p. $16, \subseteq \widehat{o}$.

Vespa arborea Smith, Catal. Brit. Fossor. Hymen. 1855 p. 215 n. 4, ᄃ; T. 5 F. 11 \& 12.

Vespa arborea Bold, Zoologist XVII. 1859 p. 6792.

Vespa arborca Schenck, Jahrb. Ver. Naturk. Nassau XVI. 1861 p. 27 nota.

Vespa Austriaca Giraud, Verh. zool. bot. Ges. Wien XIII. 1863 p. 27, ฮิ.

Vespa Austriaca F. Morawitz, Bull. soc. natural. Moscou XXXVII. 4. 1>64 p. 439, 5 J.

Vespa arborea Ormerod, Brit. Soc. Wasps 1868 p. 42 ; T. 3 F. 1.

Vespa (Vespula) Austriaca Thomson, Opuse. entom. P. 1. 1569 p. S. n. S, S $\vec{o}$.

Vespa Austriaca Puton, Pet. nouv. entom. I. 5.1873 p. 344 n. 9.

Vespa (Vespula, Austriaca Thomson, Hymen. Scandin. III. 15it p. 2.2 n. $\mathbf{7}, \subseteq j$.

Vespa Austriaca Rudow, Arch. Ter. naturf. Fr. Mecklenburg XXX. 1876 p. 207 n. $6, \subseteq 9 \hat{\jmath}$.

Pseudorespa Austriaca Schmiedeknecht. Entom. Nachr. VII. $15>1$ p. 31:3,317 c d $31 \mathrm{~s} / 3$ :

Vespa arborea E. Saunders, Trans. Entom. Soc. London 1582 p. 171 n. 5 , \&.

Vespa Austriaca Holmgren, Entom. Tidschr. IV. 1583 p. 60 \& 115.

Vespa Austriaca Ed. André, Spec. Hymén. Europe II. P. 20. 1884 p. 597, \& ō.

Vespa arborea E. Saunders, Entom. M. Magaz. XX. 1584 p. 271 n. 4.

Vespa Austriaca Schulthess, Hymen. Helvet. Diplopt. $18 s 7$ p. 18 n. $8, \subseteq$ ô.

Vespa Austriaca Marchal, Feuill. jeun. natural. XIX. 1859 p. 155.

Vespa Austriaça Konow, Soc. entom. V. 1890 p. 159.

basalis Smith $-\varrho-$ As.: India bor.

Vespa basalis Smith, Trans. Entom. Soc. London (2) II. 2. 1852 p. 46, \&.

Vespa basalis Saussure, Litud. fam. Vespid. II. Vesp. 1853 p. 148 n. 31, \&.

bellicosa Sauss. - $\subseteq-$ As.: Java.

Vespa belliensa Saussure. Litud. fam. Vespid. II. Vesp. 15.73 p. $146 \mathrm{n}$. 24,5 ; T. 14 F. 10. belloun Smith $-\subseteq 9-$ As.: China.

Vespa Bellona Smith, Proc. Zool. Soe. London 1471 p. 245 n. 1, C P; T. Is F. 6.

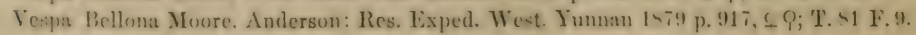

bicincta Grav.! - Patria?

Vespa bicincta Grawenhorst. Vergl. Übers. zool. System. 1507 p. 277 n. 3566. 
bicolor Fabr. - $\subseteq-$ As.: India, China.

Vespa bicolor Fabricius, Mant. Insect. I. 1787 p. 288 n. 14.

Vespa bicolor Gmelin, Linné: Syst. nat. Ed. 133 I. 5.1790 p. 2750 n. 40.

Vespa bicolor Olivier, Encyel. méthod. Insect. VI. 1791 p. 650 n. 53.

Vespa bicolor Fabricius, Entom. system. II. 1793 p. 258 n. 17.

Vespa bicolor Fabricius, Syst. Piez. $180-1$ p. 257 n. 15.

Vespa bicolor Lepeletier, Hist. nat. Insect. Hymén. I. 1836 p. 512 n. 10.

Vespa bicolor Saussure, Étud. fam. Vespid. II. Vesp. 1853 p. 143 n. 25, ᄋ.

bidens L. [1) - Eur.

Vespa bidens Linné, Syst. nat. Ed. $12^{a}$ I. 2.1767 p. 951 n. 16.

Vespa bidens Fabricius, Syst. entom. 1775 p. 368 n. 29.

Vespa bidens Ph. L. Müller, Linné: Vollst. Natursyst. V. 2. 1775 p. 884 n. 16.

Vespa bidens Fabricius, Spec. Insect. I. 1781 p. 464 n. 37.

Vespa bidens Fabricius, Mant. Insect. I. 1787 p. 290 n. 43.

Vespa bidens Villers, C. Linnaei Entom. III. 1789 p. 271 n. 12.

Vespa bidens Gmelin, Linné: Syst. nat. Ed. 13a I. 5. 1790 p. 2757 n. 16.

Vespa bidens Christ, Naturg. d. Insect. 1791 p. 245.

Vespa bidens Olivier, Encyel. méthod. Insect. VI. 1791 p. 687 n. 89.

Vespa bidens Fabricius, Entom. system. II. 1793 p. 269 n. 57.

Vespa bidens Schranck, Fauna Boica II. 2. 1802 p. 354 n. 2210.

Vespa bidens Fabricius, Syst. Piez. 1804 p. 263 n. 56.

? Odynerus bidens Kennedy, Philos. Magaz. (3) XII. 1838 p. 17 n. 15.

biguttata Fabr.! - As.: China.

Vespa biguttata Fabricius, Mant. Insect. I. 1787 p. 291 n. 53.

Vespa biguttata Gmelin, Linné: Syst. nat. Ed. 13 I. 5.1790 p. 2755 n. 67.

Vespa biguttata Olivier, Encycl. méthod. Insect. VI. 1791 p. 689 n. 99.

Vespa biguttata Fabricius, Entom. system. II. 1793 p. 272 n. 68.

Vespa biguttata Fabricius, Syst. Piez. 1804 p. 267 n. 72.

biloba Schill.! - Eur.: Silesia.

Vespa biloba Schilling, 24. Jahresher. schles, Ges. f. vaterl.Cultur i.J.1550. 1851 1.Abth. p. 7 .

bimaculata Oliv.! - As.

Vespa bimaculata Olivier, Encycl. méthod. Insect. VI. 1791 p. 678 n. 46.

bimaculata Guér. - ô - As.: Jara.

Vespa bimaculata Guérin, 1)uperry: Voy. Coquille. Zoul. II. 2. 1s;31 p. 24i!; T. !) F. (5.

Vespa Alduini Guérin, Duperry: Voy. Coquille. Zool. II. 2. 1830; 'I. 9 F. 6.

Vespa Alduini Saussure, Êtud. fam. Vespid. II. Vesp. 1853 p. 154 n. 38, ô.

Vespa Alduini Smith, Journ. of Proc. Linn. Soc. Zool. VII. 1863 p. 43 n. 2.

binotata Fabr. I - Am. mer.

Vespa binotata Fabricius, Syst. Picz. 1804 p. 266 n. 71.

bipnstulata Vill.! - Eur.: Gallia.

Vespa bipustulata Villers, C. Linnaci Entom. III. 1789 p. 280 n. 36.

bistriata Mac Farl. - Am.

Vespa bistriata Mac Farland. Trans. Amer. Entom. Soe. XV. 1445 p. 2!9S.

bistriata Fabr. I - Am. mer.

Polistes bistriata Fabricius, Syst. Yicz. 1804 p. 281 n. 56.

borealis Kby. - Am. bor.

Vespa borealis W. F. Kirby, Fauna Bor.-Amer. IV. 1837 p. 264 n. 365.

Vespa borealis Saussure, Etud. fam. Vesp. II. Vesp. 1853 p. 140 n. 20.

1) $=$ ? Nysson spinosus auct. 
bruneipes Fabr.! - As.: Sumatra.

Vespa brunnipes Fabricius, Syst. Piez. 1804 p. 203 n. 66.

eampanaria Forrl. - Patria?

Vespa campanaria Forler, Magaz. of Nat. Hist. VI. 1833 p. 538-539.

capitata Fourcr.! - Eur.: Gallia.

Vespa capitata Fourcroy, Entom. Paris. II. 1785 p. 440 n. 23.

earolina L. - $\mathrm{Q}-\mathrm{Am}$. bor.

Vespa Carolina Linné, Syst. nat. Ed. $12^{\text {a } ~ I . ~} 2.1767$ p. 948 n. 1.

Vespa Carolina Fabricius, Syst. entom. 1775 p. 363 n. 6.

Vespa Carolina Ph. L. Müller, Linné: Vollst. Natursyst. V. 2.1775 p. 879 n. 1; T. 27 J. 1.

Vespa Carolina Fabricius, Spec. Insect. I. 1781 p. 459 n. 6.

Vespa Carolina Fabricius, Mant. Insect. I. 1787 p. 287 n. 7.

Vespa Carolina Gmelin, Linné: Syst. nat. Ed. 13a I. 5. 1790 p. 2719 n. 1.

Vespa Carolina Christ, Naturg. d. Insect. 1791 p. 217.

Vespa Carolina Olivier, Encycl. méthod. Insect. VI. 1791 p. 678 n. 44.

Vespa Carolina Fabricius, Entom. system. II. 1793 p. 255 n. 7.

Vespa Carolina Fabricius, Syst. Piez. 1804 p. 255 n. 7.

Vespa Carolina Lepeletier, Hist. nat. Inseet. Hymén. I. 1836 p. 513 n. 11.

Polistes C'arolinus Saussure, Étud. fam. Vespid. II. Vesp. 1 53 p. 102 n. 2.

Vespa Carolina Saussure, Etud. fam. Vespid. II. Vesp. 1853 p. 142 n. 23.

Vespa Carolina Saussure, Stettin. entom. Zeitg. XVIUI. 1857 p. 116, ㅇ.

eayana Oliv.! - Am.: Cayenne.

Vespa Cayana Olivier, Act. soc. hist. nat. Paris I. 1792 p. 122 n. 67.

Vespa Cayana Reiche, Magaz. d. Thierr. I. 1793 p. 131 n. 1.

chrysoptera Vill.! - Eur.: Gallia.

Vespa chrysoptera Villers, C. Linnaei Entom. III. 1789 p. 284 n. 46.

chrysoptera-armata Vill.! - Eur.: Gallia.

Vespa chrysoptera-armata Villers, C. Linnaci Entom. III. 1759 p. 254 n. 4 i.

cincta Fabr. - 우 9 - As.: China, India, Java, Sumatra.

Vespa cincta Fabricius, Syst. entom. 1775 p. 362 n. 1.

Sphex tropica Sulzer, Abgek. Gesch. Insect. I. 1776 p. 192; T. 27 F. 5.

Vespa cincta Fabricius, Spec. Insect. I. 1781 p. 458 n. 1.

Vespa cincta Fabricius, Mant. Insect. I. 1787 p. 287 n. 1.

Vespa cincta Römer, Gen. Insect. 1789 p. 60 n. 124; T. 27 F. 5.

Vespa cincta Gmelin, Linné: Syst. nat. Ed. $13^{\mathrm{a}}$ I. 5.1790 p. 2715 n. 28.

Vespa tenebrionis Christ, Naturg. d. Insect. 1791 p. 216 ; 'T. 18 F. 4.

Vespa cincta Christ, Naturg. d. Insect. 1791 p. 219.

Vespa cincta Olivier, Encycl. méthod. Insect. VI. 1791 p. 676 n. 37.

Vespa unifasciata Olivier, Encycl. méthod. Insect. VI. 1791 p. 677 n. 39.

Vespa cincta Fabricius, Entom. system. II. 1793 p. 253 n. 1.

Vespa cincta Donovan, Epit. Nat. Hist. Insects India 1800 ; Tab. 57.

Vespa cincta Fabricius, Syst. Piez. 1804 p. 253 n. 1.

Vespa cincta Jurine, Nouv. méth. class. Hymén. 1807 p. 168, \&.

Vespa cincta Latreille, Gen. Crust. \& Insect. IV. 1809 p. 142.

Vespa cincta Lamarck, Hist. nat. anim. s. vert. IV. 1817 p. 88 n. 5.

Vespa cincta Lamarck, Hist. nat. anim. 8. vert. Ed. 2 a IV. 1835 p. 305 n. 5.

Vespa cincta Lepeletier, Hist. nat. Insect. Hymén. I. 1836 p. 505 n. 1, Q.

Vespa cineta Blanchard, Hist. nat. Insect. III. 1810 p. 397 n. 1; 'T. 6 F. 6.

Vespa cincta Saussure, Étud. fam. Vespid. II. Vesp. 1853 p. 152 n. 37, Q.

Vespa cincta Horne, Traus. Zool. Soc. London VII. 3. 1870 p. 172.

Yespa cincta Rothney, Entom. M. Magaz. XIII. 1877 p. 254. 
Vespa cincta Rothney, Entom. M. Magaz. XIV. 1877 p. 92.

Vespa cincta Gribodo, Ann. mus. cir. Genova XXI. 1884 p. 354 n. 9, ㅇ․

Vespa cincta Lucas, Ann. soc. entom. France 1891 Bull. p. LXVIII.

cinerascens Fabr. - Am.

Vespa cinerascens Fabricius, Syst. entom. 1775 p. 369 n. 35.

Vespa cinerascens Fabricius, Spec. Insect. I. 1781 p. 466 n. 47.

Vespa cinerascens Fabricius, Mant. Insect. I. 1787 p. 292 n. 59.

Vespa cinerascens Gmelin, Linné: Syst. nat. Ed. $13^{a}$ I. 5.1790 p. 2756 n. 72.

Vespa cinerascens Christ, Naturg. d. Insect. 1791 p. 241.

Vespa cinerascens Olivier, Encycl. méthod. Insect. VI. 1791 p. 690 n. 106.

Vespa cinerascens Fabricius, Entom. system. II. 1793 p. 274 n. 75.

Vespa cinerascens Fabricius, Syst. Piez. 1804 p. 260 n. 37.

clypeata Christ!') - 우 of - Patria?

Vespa clipeata C'hrist. Naturg. d. Insect. 1791 p. 221, \& j; T. 19 F. $3(\hat{\jmath}) \& 4(\subseteq$.

communis Schrk! - Eur.: Bararia.

Vespa communis Schrank, Neu. Magaz. Tiebh. Entom. II. 17 5.5 p. 32 s. 100.

Vespa communis Schrank, Fauna Boica II. 2. 1802 p. 351 n. 2206.

Vespa communis C. Huber, Vollst. Naturg. d. Bau- u. Baumhölzer II. 1i. 1507 p. 302.

communis Sauss. - $\mathrm{Q}-\mathrm{Am}$. bor.

Vespa communis Saussure, Stettin. entom. Zeitg. XVIII. 1857 p. 117, ㅇ․

conoidea Fabr.1 - As.: China.

Vespa conoidea Fabricius, Mant. Insect. I. 1787 p. 293 n. 69.

Vespa conoidea Gmelin, Linné: Syst. nat. Ed. $13^{a}$ I. 5.1790 p. 2753 n. 52.

crabro L. - 우 9 ơ - Eur.

Aldrovandi, De anim. Insect. 1602 p. 225.

Schwenckfeld, Theriotr. Siles. 1603 p. 525.

Moufet, Insect. 8. minim. anim. theatr. 1634 Cap. IX; Fig.

Merrett, Pinax rer. natur. Brit. 1667 p. 196.

Anonym, Neu. Schaupl. d. Nat. IV. 1688 p. 119.

— Rajus, Hist. Insect. 1710 p. 250.

- Frisch, Beschr. Insect. Teutschl. IX. 1730 p. 20; T. 11 F. 1-7.

— Réaumur, Mém. hist. Insect. IV. 1738; T. 10 F. 9.

— Réaumur, Mém. hist. Insect. VI. 1i42; I'. 18 F. 1.

— Linné, Fauna Suec. 1746 p. ? n. 988.

Ph. F. Gmelin, Onomatol, hist. nat. I. 1758 p. 537.

Vespa Crabro Linné, Syst. nat. Ed. $10^{3}$ I. 1758 p. 572 n. 1.

Vespa Crabro Brünich, Prodr. insectol. Siaelland 1761 p. 18.

Vespa Crabro Linné, Fauna Succ. Ed. 2a 1761 p. 415 n. 1670.

Vespa Crabro Poda, Insect. mus. Graec. 1761 p. 108 n. 1.

Vespa Crabro Sulzer, Kennz. d. Insect. 1761 p. 50; T. 19 F. 122.

Vespa spec. Geoffroy, Hist. abr. Insect. II. 1762 p. 368 n. 1.

Vespa Crabro Scopoli, Entom. Carn. 1763 p. 308 n. 824.

Vespa Crabro O. F. Müller, Faun. Insect. Fridrichsdal. 1764 p. 73 n. 634.

? Vespa Crabro Fermin, Beschr. v. Surinam II. 1765 p. 282.

Vespa Crabro Linné, Syst. nat. Ed. $12^{a}$ I. 2.1767 p. 948 n. 3.

Vespa VIII Schaeffer, Icon. Insect. Ratisbon. I. 2. 1767; 'T. 53 F. 7.

Vespa Crabro Eberhard, Vers. c. neu. Thiergesch. 1768 p. 214 n. 216.

Vespa XXII Schaeffer, Icon. Insect. Ratisbon. II. 1. 1768; T. 136 F. 3.

1) $=$ ? Bembex mediterranea auct. 
Veppa ('rabro Berkenhout. ()utlin. Nat. Hist. Gr. Britain I. 1769 p. 167 n. 1.

Yespa Crabro 1)egeer, Mém. serv. hist. Insect. II. 1. 1771 p. s(1) ; T. 27 F. 9-10. Vespa ('rabro J. Sehmidt. Gemeinn. Arbeit. oberlaus. Bienenges. I. 177.3 p. 5!-115.

Vespa Crabro Fabricius, Syst. entom, 1775 p. 364 n. 8.

Vespa C'rabro Ph. I. Müller. Linné: Vollst. Vatursyst. V. 2.177 p. 540 n. 3; T. 2 i F. 2.

Vespa Crabro O. F. Müller, Zool. Dan. prodr. 1776 p. 162 n. 1886.

Vespa Crabro Ph. F. Gmelin, Onomatol. hist. nat. VII. 1777 p. 708.

Vespa Crabro Fischer, Naturg. v. Licvl. 1778 p. 159 n. 396.

Vespa ('rubro Gütze. Degeer: Abh. Gesch. Insect. II. 1. 1779 p. 132 ; T. 27 F. 9-10.

Vespa Crabro Sander, Vaterl. Bemerk. Naturg. 1780 p. ?

Vespa Crabro Fabricius, Spec. Insect. I. 1781 p. 459 n. 8.

Vespa Crabro Schrank, Enum. Insect. Austr. $17 \$ 1$ p. 389 n. 786.

Vespa Crabro major Retzius, Gen. \& spec. Insect. 1783 p. 63 n. 231.

Vespa Crabro Fourcroy, Entom. Paris. II. 1785 p. 430 n. 1.

Vespa Crabro Petagna, Specim. Insect. ult. Calabr. 1786 p. 32 n. 162.

Vespa Crabro Fabricius, Mant. Insect. 1. 1787 p. 287 n. 9.

Vespa Crabro Razoumowski, Hist. nat. Jorat. I. 1789 p. 217 n. 255.

Vespa Crabro Villers, C. Linnaei Entom. III. 1789 p. 262 n. 1.

Vespa Crabro Gmelin, Linné: Syst. nat. Ed. 13a I. 5.1790 p. 2750 n. 3.

Vespa Crabro Rossi, Fauna Etrusea II. 1790 p. 83 n. 860.

Vespa Crabro Germana Christ. Naturg. d. Insect. $17 ! 11$ p. 212 ; T. I F T.1 \& p. $215 ;$ T. 15 F.:3.

Vespa Crabro J. 13. Fischer. Vers. Naturg. Lierland ISd. $2^{a} 17 ! 11$ p. 3411 n. 758.

Vespa Crabro Olivier, Encycl. méthod. Insect. VI. 1791 p. 677 n. 47.

Vespa Crabro Fabricius, Entom. system. II. 1793 p. 255 n. 9.

Vespa Crabro Cederhjelm, Faun. Ingric. prodr. 1798 p. 168 n. 520.

Vespa Crabro Spitzner, Ökon. Hefte XIII. 1799 p. 19j-219; Tab.

Vespa Crabro Lamarck, Syst. anim. s. vert. 1801 p. 271 n. 131.

Vespa Crabro Schrank, Fauna Boica II. 2. 1802 p. 347 n. 2202.

Vespa Crabro Walckenaer, Fauna Paris. II. 1802 p. 87 n. 3.

Vespa Crabro Fabricius, Syst. Piez. 1801 p. 255 n. 8.

Vespa C'rabro C. Huber. Vollst. Naturg. d. Bau- u. Baumhölzer I. 1. 150.5 p. 32.

Vespa Crabro Latreille, Hist. nat. Insect. XIII. 1805 p. 350 n. 1.

Vespa Crabro Gravenhorst, Vergl. Übers. zool. System. 1407 p. 274 n. 3542-14.

Vespa Crabro Illiger, Rossi: Fauna Etrusea Ed. $2^{\text {a }}$ II. 1807 p. 135 n. 860.

Vespa Crabro Jurine, Nouv. méth. class. Hymén. 1807 p. 168, 우 ఫ̋.

Vespar Crabro Malinowsky, Magaz. Ges. naturf. Fr. Berlin II. 1s(1) p. 151-15ti.

Vespa Crabro Latreille, Gen. Crust. \& Insect. IV. 1809 p. 142.

Vespa Crabro Lamarck, Hist. nat. anim. s. vert. IV. 1817 p. 87 n. 1.

Vespa Crabro Bechstein, Forstinsectol. 1818 p. 147 n. 311 \& p. 505 n. 25.

Vespa Crabro Ph. W. Müller, Magaz. f. Entom. III. 1818 p. 56-68.

Vespa (irabro Sehummel. C̈bers. Arbeit. schles. (ies. f. raterl. Cultur i. J. 1524. 1429 p. 75.

Vespa Crabro Strauss-1)ürkheim. 13ull. uniw. sc. nat. \& géol. Seet. 2. XXI1. 15:10 p. 347-34!.

Vespa Crabro Brullé, Expéd. sc. Morée. Zool. II. 1832 p. 360 n. 797.

Vesp̧a Crabro Hartig, Forstl. Conversationslex. 1834 p. 201.

Vespa Crabro Dale, Magaz. of Nat. Hist. VII. 1834 p. 529-530; Tig. 65.

Vespa Crabro Lamarck, Hist. nat. anim. 6. vert. Ed. 2a IV. 1835 p. 304 n. 1.

Vespa Crabro Imhof \& Labram, Insect. d. Schweiz I. 1836; 'T. 39.

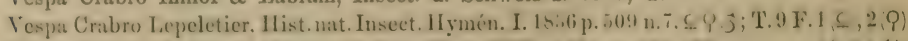

Vespa Crabos Anonym, Magaz. of Nat. llist. VIII. 1436 p. $1,2-7-1,25 . \quad$ de $3(0)$

Vespa Crabro Zetterstedt, Insect. Lappon. I. 1838 p. 453 n. 1, \& 9 ơ.

Vespa Crabro Blanchard, Iist. nat. Insect. III. 1810 p. 397 n. 2. 
Vespa Crabro IIerrich-Sehaeffer, Faun. Insect. German. $14+1$ P. 179 T. 1 \& j:

Vespa Crabro Gené, Atti $3^{a}$ riun. scient. Ital. 1841 p. 20.

Vespa Crabro Brants, Tijdselır. r. natuurl. Geschied. VIII. 1511; T. 1 F. 1-7 \& 9.

Vespa Crabro Newport, Trans. Entom. Soc. London III. 3. 1812 p. 183.

Vespa Crabro Dufour, Ann. sc. nat. Zool. (2) XIX. 1843 p. 145; 'T. 7 F. 12 \& 13.

Vespa Crabro Siebold, Zeitschr. f. Entom. IV. 1843 p. 388 ; T. 2 F. 2.

Vespa Crabro Smith, Zoologist I. 1843 p. 161 n. 1.

Vespa Crabro Ratzeburg, Forstinsect. III. 1844 p. 51 n. 1; T. 4 F. 6 \& T. 5 F. 16.

Vespa Crabro Gené, Mom. mat. \& fis. soc. Ital. sc. Modena XXIII. 1844 P. fis. p. 49.

Vespa Crabro Letris, Zoologist II. 1844 p. 748.

Vespa Crabro Lacaze-Duthiers, Ann. sc. nat. Zool. (3) XII. 1849; T. 12 F. 1-9.

Vespa Crabro Grube, Arch. f. Anat. Physiol etc. 1849 p. 47.

Vespa Crabro H. W. Newman, Trans. Entom. Soc. London (2) I. 14.51 Proc. p. 72-733.

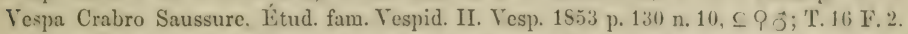

Vespa Crabro Schenck, Jahrb. Ver. Naturk. Nassau IX. 1. 1553 p. 21 n. 1, @ Q j.

Vespa Crabro Schenck, Progr. Gymnas. Weilburg 1853 p. 13.

Vespa Crabro Nördlinger, D. kleinen Feinde 1855 p. 421.

Vespa Crabro Möbius, Abh. naturw. Ver. Hamburg III. 1556 p. 165 (p. 51; ; T. 19 F. 2.

Vespa Crabro Smith, Catal. Brit. Fossor. IIymen. 15.55 p. 221 n. 7. ऽ Q j; T. 5 T. 10.

Vespa Crabro Stone, Entom. Weekly Intellig. VIII. 1860 p. 188.

Vespa Crabro Stone, Entom. Weekly Intellig. IX. 1860 p. 37-40.

Vespa Crabro Duméril, Mém. acad. sc. Paris XXXI. 1860 p. 359 n. 1.

Vespa Crabro Stone, Zoologist XVIII. 1860 p. 7291.

Vespa Crabro Schenck, Jahrb. Ver. Naturk. Nassau XVI. 1861 p.16.19,21 ( 24 n. 1, §? j.

Vespa Crabro Rathke, Schrift. phys. ükon. Ges. Künigsberg I. 2.1561 p. 150; I'. S F. 5.

Vespa Crabro Stone, Zoologist XIX. 1861 p. 7449-7451.

Vespa Crabro Radoszkowski, Horae soc. entom. Ross. II. 1863 p. 127 n. 7.

Vespa Crabro Schmidt, Zool. Garten V. 1864 p. 161.

Vespa Crabro Sichel, Ann. soc. entom. France (4) V. 1865 Bull. p. XXV.

Vespa Crabro Goureau, Bull. soc. sc. hist. nat. Yonne 1566 p. ?

Vespa Crabro Dohrn, Stettin. entom. Zeitg. XXVII. 1866 p. 462.

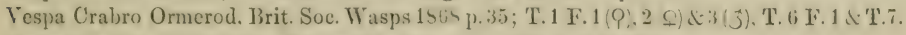

Vespa Crabro Ratzeburg, Waldverderbniss II. 1868 p. 276-283.

Vespa Crabro Thomson, Opusc. entom. P. 1.1869 p. 79 n. 1, \& Q ô.

Vespa Crabro Nichols, Amer. Entomol. I. 1869 p. 20 .

Vespa Crabro Anonym, Proc. Essex Soc. 1870 p. 38.

Vespa Crabro Smith, Entomol. Annual f. 1871 p. 65.

Vespa Crabro Jäekel, Corresplbl, zoul.-min. Ver. Regensburg XXT. 19il p. 107.

Vespa Crabro Smith, 'Irans. Entom. Soc. London 1571 Proc. p. VII.

Vespa Crabro Riley, 4 $4^{\text {th }}$ Ann. Rep. Insect. Missouri 1872 p. 22.

Vespa Crabro Smith, Trans. Entom. Soc. London 1572 Proc. p. X.

Vespa Crabro Rouget, Móm. acad. sc. Dijon (3) I. 1873 Sc. p. 184.

Vespa Crabro Puton, Pet. nouv. entom. I. 5.1873 p. 344 n. 1.

Vespa Crabro Thomson, Hymen. Scandin. III. 1874 p. 9 n. 1 , \& 9 f̊.

Vespa Crabro Newman, Entomologist VII. 1874 p. 209-210.

Vespa Crabro Smith, Entomologist VII. 1874 p. 257-259.

Vespa Crabro Altum, Forstzoologic III. 2. 1875 p. 225 ; Fig. 16.

Vespa ('rabro Rudow, Arch. Ver. Fr. Naturg. Mecklenburg XXX. 1siti p. 203 n. 1, $\leq$ PJ.

Vespa Crabro Fettig, Bull. soc. hist. nat. Colmar XV./XVI. 1876 p. 125.

Vespa Crabro Chapman, Entom. MI. Magaz. XIU. 1876 p. 88.

Vespa Crabro Coues d Yarrow. Proc. Aead. Nat. Sc. Philadelphia 15 is p. 3. 
Vespa Crabro Prinsac, Feuille jeun. natural. IX. 1879 p. 143.

Vespa Crabro Standon, Entomologist XIV. 1881 p. 23.

Vespa Crabro Saunders, Entomologist XIV. 1881 p. 159-190.

Vespa Crabro Schmiedeknecht, Entom. Nachr. VII. 1591 p. 316 s 9 \& 17 ioj:

Vespa Crabro Verrall, Trans. Entom. Soc. London 1881 p. XXXVII.

Vespa Crabro Paragallo, Insect. nuisibles etc. II. 1882.

Vespa ('rabro E. Saunders, Trans. Entom. Soc. Iondon 1582 p. 165 n. 1.

Vespa C'rabro Lucas, Ann. soc. entom. France (6) III. 1553 Bull. p. XXIX.

Vespa Crabro Ed. André, Spec. Hymén. Europe II. P. 20.1554 p. $555, \subseteq 93$; T. 39 F. 4.

Vespa Crabro Humbert, Bull. Insect. agric. IX. 1884 p. 9-12; Fig. 1-2.

Vespa Crabro Lesueur, Bull. Insect. agric. IX. 1884 p. 63-64 \& 92-94.

Vespa Crabro Bütschli, Biol. Centralbl. IV. 1884 p. 113; Fig.

Vespa Crabro Hess, Entom. Nachr. XI. 1885 p. 218-219.

- Vespa Crabro Dahl, Arch. mikr. Anat. XXV. 1886 p. 255; T. 12 F. 18-20.

Yespa Crabro Schulthess, Hymen. Helvet. Diplopt. 1 s

Vespa Crabro Delaby; Bull. soc. Iinn. Nord de la France VII. 185i p. 201-202.

Vespa Crabro Viallanes, Compt. rend. assoc. sc. France $2, \mathrm{XT} .155 \mathrm{i}$ p. 608.

Vespa Crabro Viallanes, Ann. sc. nat. Zool. (i) II. 1887 p. 1; T. 1 F. 6.

Vespa Crabro Marchal, Feuill. jeun. natural. XIX. 1559 p. 1 ¿̌s.

Vespa Crabro Konow, Soc. entom. IV. 1890 p. 175 n. 1.

Vespa Crabro Anonym, Entom. Tidskr. XI. 1890 p. 18.

Vespa Crabro Grill, Entom. Tidskr. XII. 1891 p. 18.

var. anglica Grib. - 우 - Eur.: Anglia.

Vespa Crabro var. Anglica Gribodo, Bull. soc. entom. Ital. XXIII. 1891 p. 242, S. var. borealis Rad. - Eur.: Russia.

Vespra Crabro var. borealis Radoszkowski. Horae soc. entom. Ross. II. 1563 p.125; T.16 F.15.

Vespa Crabro var. borealis Ed. André, Spec. Hymén. Europe II. P. 20. $15 \$ 4$ p. 5 și. var. cornuta Christ - As.: India.

Vespa Crabro var. cornuta Christ, Naturg. d. Insect. 1791 p. 214; T. 15 F. 2. var. immaculata Mor. - As. centr.

Vespa Crabro var. immaculata Morawitz, Horae soc. entom. Ross. XXIII. $1 \$ \varsigma 9$ p. 161 n. 1. crabroniformis Heer - Eur. Radoboj.

Vespa crabroniformis Heer, Denksehr. schweiz. Ges. Naturw. XXII. 1867 p. 6 n. 1; T. 3 F.15. crabroniformis Smith - 우 ô - As.: China.

Yespa crabroniformis Smith, Trans. Fintom. Soc. London (2) II. 2.1452 p. $40 . \subseteq \overrightarrow{3}$.

Vespa crabroniformis Saussure, Eitud. fam. Vespid. II. Vesp. 1553 p. $145 \mathrm{n} .27, \subseteq \hat{\jmath}$. crassipes Christ! - Patria?

Vespa crassipes Christ, Naturg. d. Insect. 1791 p. 246; T. 23 F. 10.

eribriformis Christ! - Eur.: Germania.

Vespa cribriformis Christ, Naturg. d. Insect. 1791 p. 237; T. 22 F. 4.

cuneatr Fabr. - $\subseteq \widehat{o}-$ Am. : Mexico, United States.

Vespa cuneata Fabricius, Syst. Piez, 1804 j). 258 n. 21.

Vespa cuneata Jurine, Nouv. méth. class. Hymén. 1807 p. 165, \&.

Vespa cuneata T.epeletier. Hist. nat. Inseet. Hymén. I. $1 \$ 31$ p. 513 n. 12. \& j.

Vespa cuneata Saussure, Étud. fam. Vespid. II. Vesp. 1853 p. 135 n. 15, ô.

Vespa cuneata Saussure, Stettin. entom. Zeitg. XVIII. 1857 p. 116, \&.

curvipes Christ1 - Patria?

Vespa curvipes Christ, Naturg. d. Insect. 1791 p. 246 ; 'T. 23 F. 9.

daedalea Web.1 - Am.

Vespa daedalea Lichtenstein, Catal. Mus. Holth. 1796 p. 203 n. 173. 
Vespa daedalea Weber, Observ. Entom. 1801 p. 103 n. 8.

Tespa daedala Saussure, Smithson. Miscell. Collect. XIV. P. 254.1575 p. 379 n. 7.

dentata Oliv.1 - Am. mer.

Vespa dentata Olivier, Encycl. méthod. Insect. VI. 1791 p. 675 n. 25.

densta Lep. - of $\sigma$ - As.: Philippines.

Vespa deusta Lepeletier, Hist. nat. Insect. Hymén. I. 1836 p. 506 n. 3.

Vespa deusta Saussure, Étud. fam. Vespid. II. Vesp. 1853 p. 149 n. 33, 오.

Vespa deusta Kirseh, Mittheil. zool. Mus. Dresden III. 1875 p. 381 , s. 3 .

diabolica Sauss. $-\subseteq$ $P-$ Am. bor.

Vespa diabolica Saussure. F́tud. fam. Vespid. II. Vesp. 1853 p. 135 n. 15, @ P.

Vespa diabolica Saussure, Stettin. entom. Zeitg. XVIII. 1857 g. 116, f.

Vespa diabolica Prorancher, Natural. Canad, X̃III. 1882 p. 166 n. 4, ᄃ.

Vespa diabolica Prorancher, Faun. entom. Canada. Hymén. 1884 p. 684 n. 4, \&.

diadema Christ! - Eur.

Vespa diadema Christ, Naturg. d. Insect. 1791 p. 223; T. 19 F. 7.

ducalis Smith - $99 \hat{\sigma}-A_{s}$ : China, India.

Vespa ducalis Smith, Trans. Entom. Soc. London (2) II. 2. 1852 p. $39, \&$ \& oิ.

Vespa ducalis Saussure, Étud. fam. Vespid. II. Vesp. 1853 p. 151 n. 35, $f$ Q ô. dybowskii André - $\mathrm{Q}-$ As.: Sibiria.

Vespa Dybowskii (Radoszkowski) Ed. André, Spec. Hymén. Europ̣e II. P.20.1884 p. 582, Q.

emarginata L. - Am. bor.

Vespa emarginata Linné, Syst. nat. Ed. $10^{a} 1.1758$ p. 574 n. 16.

Vespa emarginata Linné, Mus. Ludov. Ulric. 1764 p. 412 n. 4.

Vespa emarginata Linné, Syst. nat. Ed. $12^{\text {a }}$ I. 2. 1767 p. 952 n. 26.

Vespa emarginata Ph. L. Müller, Linné: Vollst. Natursyst. V. 2. 1775 p. 886 n. 26.

Vespa emarginata Ph. F. Gmelin, Onomatol, hist. nat. VII. 1777 p. 708.

Vespa emarginata Gmelin, Linné: Syst. nat. Ed. $13^{\text {a }}$ I. 3.1790 p. 2759 n. 26.

Vespa emarginata Christ, Naturg. d. Insect. 1791 p. 225.

Vespa marginata Olivier, Encyel. méthod. Insect. VI. 1791 p. 674 n. 24.

erythrocephala Gmel.! - Patria?

Vespa spec. Zschach, Mus. Leskean. 1788 p. 75 n. 421.

Vespa erythrocephala Gmelin, Linné: Syst. nat. Ed. 13 3. I. 5.1790 p. 2760 n. 97.

Vespa erỵthrocephala Olirier, Encycl. méthod. Insect. VI. 1791 p. 695 n. 12.

exotiea Gmel.! - Patria?

Vespa spec. Zschach, Mus. Leskean. 1788 p. 74 n. 412.

Vespa exotica Gmelin, Linné: Syst. nat. Ed. 13 a I. 5.1790 p. 2760 n. 93.

Vespa exotica Olivier, Encycl. méthod. Insect. VI. 1791 p. 695 n. 9.

ferruginea Oliv.! - Am. mer.

Vespa ferruginea Olivier. Eneycel. méthod. Insect. VI. 1791 p. 653 n. 70.

ferrida Smith - 9 Q - As.: Celebes.

Vespa fervida Smith. Journ. of Proc. Linn. Soc. Zool. III. 1858 p. 23 n. 2, f Q.

flarescens Fabr. - 우 - As.: India.

Vespa flavescens Fabricius, Syst. entom. 1775 p. 370 n. 36.

Vespa flarescens Fabricius, Spec. Insect. I. 1781 p. 466 n. 50.

Vespa flarescens Fabricius, Mant. Insect. I. 1787 p. 292 n. 62.

Vespa flavescens Gmelin, Linné: Syst. nat. Ed. $13^{3}$ I. 5. 1790 p. 2756 n. 75.

Vespa flavescens Christ, Naturg. d. Insect. 1791 p. 243.

Vespa flavescens Olivier, Encycl. méthod. Insect. VI. 1791 p. 691 n. 109.

Vespa flavescens Fabricius, Entom. system. II. 1793 p. 275 n. 79.

Vespa flarescens Fabricius, Syst. Piez. 1804 p. 261 n. 42.

Vespa flavescens Jurine, Nouv. méth. class. Hymén. 1807 p. 170, 오.

Catalogus Hymenopterorum. IX. 
flaviceps Smith $-Q 9-\Lambda s .:$ India.

$[\& 11(9)$.

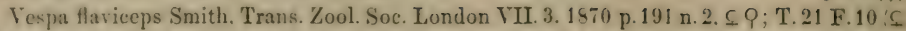

Vespa flariecps Horne, Trans. Zuol. Soc. London VII. 3. 1870 p. 174; T. 21 F. 10 \& 11.

flaricincta Grav.! - Patria?

Vespa flavicinta Gravenhorst, Vergl. Ü̉bers. zool. Sỵstem. 1807 p. 276 n. 3563.

florisequa major Christ! - Patria?

Vespa florisequa major Christ, Naturg. d. Insect. 1791 p. 244; T. 23 F. 4.

florisequa minor Christ! - Patria?

Vespa florisequa minor Christ, Naturg. d. Insect. 1791 p. 244 ; T. 23 F. 5.

fulva Gmel.! - Eur.: Russia.

- Lepechin, Reise d. russ. Reich I. 1774 p. ?; T. 19 F. 4.

Vespa fulva Gmelin, Linné: Syst. nat. Ed. $13^{\text {a }}$ 1. 5. 1790 p. 2757 n. 80.

Vespa fulva Olivier, Encycl. méthod. Insect. VI. 1791 p. 694 n. 7.

fulvipes Fourcr.! - Eur.: Gallia.

Vespa fulvipes Fourcroy, Entom. Paris. II. 1785 p. 439 n. 22.

fusus major Christ! - Patria?

Vespa fusus major Christ, Naturg. d. Insect. 1791 p. 242; T. 23 F. 1.

fusns minor Christ! - Patria?

Vespa fusus minor Christ, Naturg. d. Insect. 1791 p. 243; T. 23 F. 2.

galbula Pall. - 오 0 - Eur.: Russia.

Vespa galbula Pallas. Reise n, d. rersch. Prov. russ. Reich. I. 1771 p. 473 n. 73.

Vespa galbula Ph. L. Müller, Linné: Vollst. Natursyst. Suppl. 1776 p. 325 n. 30,5 9 j̧. genieulata Grav.1 - Eur.

Vespa geniculata Gravenhorst. Vergl. Übers. zool. System. 1807 p. 276 n. 3861.

germanica Fabr. - $ᄋ$ $Q$ - Eur.

? Vespa maculata Scopoli, Entom. Carn. 1763 p. 312 n. 831.

Vespa XX Schaeffer, Icon. Insect. Ratisbon. II. 1. 1768; T. 127 F. 2.

? Vespa macularis Olivier, Eneycl. méthod. Insect. VI. 1791 p. 695 n. 8.

Vespa Germanica Fabricius, Entom. system. II. 1793 p. 256 n. 11.

Vespa Germanica Panzer, Faun. Insect. German. V. 1798 P. 49 T. 20.

Vespa Germanica Fabricius, Syst. Piez. 1804 p. 256 n. 10.

Vespa Germanica Panzer, Krit. Revis. II. 1806 p. 154.

Tespa Germanica Gravenhorst. Vergl. Z̈bers. zool. System. 1507 p. 275 n. 3949.

Vespa Germanica Jurine, Nouv. méth. class. Hymén. 1807 p. 168, 요 ơ.

Vespa Germanica Latreille, Gen. Crust. \& Insect. IV. 1809 p. 142.

Vespa Germanica Klug, Germar: Reise n. Dalmat. II. 1817 p. 264 n. 370.

Vespa Germanica Lepeletier. Hist. nat. Insect. Hỵmén. I. 15:36 p. 315 n. 13, ऽ 9 j.

Vespa vulgaris Blanchard, Hist. nat. Insect. III. 1840 p. 397 n. 3.

Vespa Germanica Herrich-Schaeffer. Faun. Insect. German. 1541 P. 179 T. 2 ?.

Vespa rulgaris Smith. Zoologist I. 1543 p. 162 n. 2, $\subseteq 9$ j; Fig. a.b(j).c.d $\subseteq$ \& e. $f(S$.

Vespa Germanica Ineas, Iixplor. se. Algéric. Zool. III. 1846 p. 224 n. 173.

Vespa Germanica Smith, Zoologist IX. 1851 App. p. CLXXVII; Fig.

Vespa Germanica Smith, Zoologist X. 1852 p. 3699; Fig. 1.

(T.16 F.1.

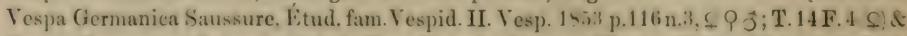
Vespa Germanica Schenck, Jahrb. Ver. Naturk. Nassau IX. 1. 15.33 p. 29 n. 7. \& 9 j.

Vespa Ciermanica Schenck, P'rogr. Gymnas. Weilburg 15.53 p. 14, \& Q jo .

Vespa Germanica Smith, Catal. Brit. Fossor. Hymen. 1 sis p. 216 n. 2, $\subseteq$ Q $f$.

Vespa Germanica Duméril, Mém, acad. sc. Paris XXXI. 1860 p. 862 n. 3.

Vespa Germaniea Schenek. Jahrb.Ver. Naturk. Nassau XVI.1661 p. 18, 21, 24 \& 26 v. $5, \subseteq 93$.

Vespa Germanica Brischke, Schrift. plı̣s. ökon. Ges. Königsberg II. 1562 p. 113. s $9 \hat{J}$.

Vespa Gicruanica J,estuck, Ann. d Mag. Nat. Hist. 3) XUI. Is64 p. 166. 
Yespa Germanica Brischke. Schrift. phỵs. ükon. Ges. Künigsberg V. 1561 p. 210. S.

Vespa Germanica Stone. Trans. Entom. Soc. London 3, II. 1561 Proe. p. 33.

Vespa Germanica Smith. Trans. Entom. Soc. Lordon (3) II. 1464 Proc. p. 57.

Vespa Germanica Goureau, Bull. soc. sc. hist. nat. Yonne 1866 p. ?

Vespa Germanica Dohrn, Stettin. entom. Zeitg. XXVII. 1866 p. 463.

Vespa Germanica Ormerod. Brit. Soc. Wasps 1 Slis p. 43 ; T. 3 F. 2 \& T. 9.

Vespa Vespula Germanica Thomson, Opuse. entom. P. 1. 1869 p. $\$ 2$ n. 7 . $\leq .9 .3$.

Vespa Germanica Ankum, Arch. Néerland sc. ex. nat. V. 1870 p. 443-446.

Vespa Germanica Couper, Canad. Entomol. II. 1870 p. 49.

Vespa Germanica H. Müller, Befrucht. d. Blumen 1873 p. 466.

Vespa Germanica Rouget, Mém. acad. sc. Dijon (3) I. 1873 Sc. p. 190.

Vespa Germanica Rouget, Pet. nouv, entom. I. 5. 1873 p. 335.

Vespa Germanica Puton, Pet. nouv. entom. I. 5.1873 p. 344 n. 7.

Vespa Vespula: Germanica Thomson, Hymen. Scandin. III. 1574 p. 20 n. $6 . \subseteq 9$ J.

Vespa Germanica Weyenbergh. Tijdschr. r. Entom. v. XVII. 1574 p. 163.

Vespa Germanica Kuwert. Stettin. entom. Zeitg. XXXVI. 1875 p. 221-2.2.4.

Vespa Germanica Rudow. Arch.Ver. Fr. Naturg. Mecklenburg XXX. 1876 p. 207 n. 7.993

Vespa Germanica Corbin, Entomologist X. 1877 p. 144.

Vespa Germanica Haury, Pet. nouv. entom. II. 9. 1877 p. 178.

Vespa Germanica Smith, Sec. Yarkand Miss. 1878 p. ?

Vespa Germanica Cornelius, Entom. Nachr. V. 1879 p. 249.

Vespa Germanica Schmiedeknecht. Entom. Nachr. VII. 1881 p. 317 ic 9$)$ d $315(\hat{j})$.

Tespa Germanica Prorancher. Natural. Canad. XIII. 1582 p. 165 n. 3. s.

Vespa Germanica Billups. Trans. Fintom. Soc. London 1552 Proc. p. III.

Vespa Germanica Billups, Entom. M. Magaz. XVIII. 1882 p. 234.

Tespa Germanica E. Saunders. Trans. Entom. Soc. London 1642 p. 16!! n. 3.

Vespa Germanica Provancher. Faun. entom. ('anada. Hymén. 1584 p. 683 n. 3. S.

Vespa Germanica Ed. André. Spec. Hỵmén. Europe II. P. 20. 1854 p. 595, ऽ ? 3ै.

Vespa Germanica Dowker, Trans. E. Kent Soc. (2) 1886 p. 54-66.

Vespa Germanica Dittrich, Zeitschr. f. Entom. XI. 1886 p. XXI.

Vespa Germanica Schulthess. Hymen. Helvet. Diplopt. 1557 p. 15 n. $5 . \subseteq 9$ j.

Vespa Germanica Marchal, Feuill. jeun. natural. XIX. 1889 p. 159.

Vespa Germanica Konow, Soc. entom. IV. 1890 p. 175 n. 5.

Tespa Germanica Marlatt, Proc. Fintom. Soc. Washington II. $15 \$ 1$ p. $\$ 0-\$ 3$.

guttata Fourcr.! - Eur.: Gallia.

Vespa spec. Geoffroy, Hist. abr. Insect. U. 1762 p. 379 n. 13.

Vespa guttata Fourcroy, Entom. Paris. II. 1785 p. 436 n. 13.

Vespa quadrum Olivier, Encycl. méthod. Insect. VI. 1791 p. 694 n. 2.

hirsutissima Vill.! - Eur.: Germania.

Vespa hirsutissima Villers, C. Linnaci Entom. III. 1879 p. 284 n. 45.

horticula Müll.! - Eur.

Vespa horticula O. F. Müller, Mélang. soc. roy. Turin III. 1766 p. 196.

japoniea Sauss. - $\mathrm{Q}-$ As.: Japonia.

Vespa Japonica Saussure, Rev. \& mag. zool. (2) X. 1858 p. 261, ‥

infernalis Sauss. $-\subseteq Q-A m$. bor.

Vespa infernalis Saussure, Fitud. fam. Vespid. II. Vesp. 1553 p. 139 n. 19. Q.

Vespa infernalis Saussure. Stettin. entom. Zeitg. XVIII. 14.5 p. $116 . \subseteq$.

insularis D. T. - o - As. : Japonia.

Vespa Japonica Smith. Entom. M. Magaz. IV. 1564 p. 279. 3 [nec Sauss.|.

Vespa Japonica Smith, Trans. Entom. Soc. London 1873 p. 198 n. 4.

Vespa insularis $\mathrm{m}$. 
intersecta Fourcr.! - Eur.: Gallia.

Vespa intersecta Fourcroy, Entom. Paris. II. 1785 p. 439 n. 20.

italica Christ! - Eur.: Italia.

Vespa Italica Christ, Naturg. d. Insect. 1791 p. 221.

koreensis Rad. - $ᄋ$ oิ - As.: Korea.

Vespa Koreensis Radoszkowski, Horae soc. entom. Ross. XXI. 1557 p. 432. $\subseteq$ j.

labiata Fabr. - As.: Tranquebar.

Vespa labiata Fabricius, Suppl. entom. system. 1798 p. 260 n. 1819.

Vespa labiata Fabricius, Syst. Piez. 1804 p. 257 n. 18.

ligata Müll.! - Eur.: Dania.

Vespa ligata O. F. Müller, Fauna Inseet. Fridrichsdal 1764 p. 74 n. 642.

Vespa ligata O. F. Müller, Zool. Dan. prodr. 1776 p. 163 n. 1893.

Vespa ligata Villers, C. Linnaei Entom. III. 1789 p. 250 n. 34.

longicornis Fourcr.! - Eur.: Gallia.

Vespa spec. Geoffroy, Hist. abr. Insect. II. 1762 p. 374 n. 6.

Vespa longicornis Fourcroy, Entom. Paris. II. 1785 p. 433 n. 6.

Vespa longicornis Olivier, Encycl. méthod. Insect. VI. 1791 p. 693 n. 119.

luetuosa Sauss. - of - As.: Philippines.

Vespa luctuosa Saussure, Etud. fam. Vespid. II. Vesp. 1553 p. 143 n. 24 , ô.

Intea Coq.! - As.: India.

Vespa lutea Coquebert, Illustr. iconogr. Insect. III. 1804 p. 94; T. 21 F. 10.

macrocephala Christ! - Patria?

Vespa macrocephala Christ, Naturg. d: Insect. 1791 p. 245; T. 23 F. 7.

maculata $L$. - $\odot$ Q $\overparen{o}-$ Am. bor.

Vespa maculata Linné, Centur. Insect. rar.:1763 p. 30 n. 91.

Vespa maculata Linné, Amoen. acad. VI. 1763 p. 412 n. 91.

Vespa maculata Linné, Syst. nat. Ed. $12^{2}$ I. 2. 1767 p. 948 n. 2.

Vespa maculata Degeer, Mém. serv. hist. Insect. III. 1773 p. 544 n. 9; T. 29 F. 13.

Vespa maculata Fabricius, Syst. entom. 1775 p. 364 n. 11.

Vespa maculata Ph. I. Müller. Linné: Vollst. Natursỵst. V. 2.1775 p. $\$ 80$ n. 2.

Vespa maculata Ph. F. Gmelin, Onomatol, hist. nat. VII. 1777 p. 709.

Vespa maculata Göze, Degeer: Abh. Gesch. Insect. III. 1750 p. 375 n. 9; T. 29 F. 13.

Vespa maculata Fabricius, Spec. Insect. I. 1781 p. 460 n. 13.

Vespa maculata Retzius, Gen. \& spec. Insect. 1783 p. 64 n. 240.

Vespa maculata Fabricius, Mant. Insect. I. 1787 p. 288 n. 15.

Vespa maculata Villers, C. Linnaei Entom. III. 1789 p. 274 n. 19.

Vespa maculata Gmelin, Linné: Syst. nat. Ed. 13n I. 5. 1790 p. 2749 n. 2.

Vespa maculata Christ, Naturg. d. Insect. 1791 p. 217 \& 240.

Vespa maculata americana Christ, Naturg. d. Insect. 1791 p. 239.

Vespa maculata Olivier, Encycl. méthod. Insect. VI. 1791 p. 681 n. 54.

Vespa maculata Fabricius, Entom. system. II. 1793 p. 258 n. 18.

Vespa maculata Fabricius, Syst. Piez. 1804 p. 257 n. 17.

Vespa maculata Jurine, Nouv. méth. class. Hymén. 1807 p. 168, ㅇ.

Vespa maculata Lepeletier, Hist. nat. Insect. Hymén. I. 1836 p. 512 n. 9.

Vespa maculata W. F. Kirby, Fauna Bor.-Amer. IV. 1837 p. 266 n. 367, Q.

Vespa maculata Saussure, Étud. fam. Vespid. II. Vesp. 1853 p. 141 n. 22.

Vespa maculata Saussure, Stettin. entom. Zeitg. XVIII. 1857 p. 116, \&.

Vespa maculata Walsh \& Riley, Amer. Entomol. I. 1869 p. 138; Fig. 111.

Vespa maculata Gillman. Amer. Entomol. (S Botan. II. 1470 p. 16i7; Fig. 106.

Vespa maculata Couper, Canad. Entom. II. 1870 p. 49. 
Bember fasciata Riley, $5^{\text {th }}$ Ann. Rep. Insect. Missouri 1873 p. 9.

Vespa maculata Riley, 5th Ann. Rep. Insect. Missouri 1873; Fig. 2.

Vespa maculata Mechan, Proc. Acad. Nat. Sc. Philadelphia 1878 p. 15.

Vespa maculata Riley. General-Index Rep. Insect. Missouri 1581 p. 4 .

Tespa maculata Provancher. Natural. Canad. XIII. 1442 p. 164 n. 1. $\leq ? \hat{\jmath}$.

Vespa maculata Provancher, Faun. entom. Canada. Hymén, 155.4 p. lis: n. 1. $\subseteq$ ? $\vec{j}$. magniflea Smith $-9 Q-$ As.: India.

Yespa magnifica Smith. Trans. Entom. Soc. I.ondon (2) II. 2. 15.52 p. 4.5, $\subseteq$ ?.

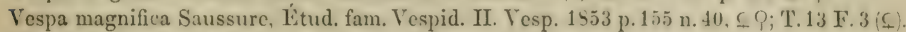

Vespa magnifica Hewett, Journ. Bombay Nat. Hist. Soc. IV. 1559 p. 312.

mandarina Smith - 우 $9-$ As.: China.

Vespa mandarina Smith. Trans. Entom. Soc. London (2) II. 2. 1552 p. 39. S. P; T. 5 F. 1.

Vespa mandarina Saussure, Etud. fam. Vespid. II. Vesp. 15.53 p. 150 n. 34, $\subseteq$ ?.

Vespa Japonica (Radoszkowsky Motschulsky, Étud. entom. VI. 1 s.57 p. 10 [non Smith].

Vespa mandarina Smith, Trans. Entom. Soc. London 1873 p. 197 n. 1.

media Retz. - $ᄋ$ 9 ô - Eur.

—- Degeer, Mém. hist. Insect. II. 1. 1771 p. 791; T. 27 F. 1-8.

Göze, Degeer: Abh. Gesch. Insect. II. 2. 1779 p. 125; T. 27 F. 1-8.

Vespa Crabro media Retzius, Gen. \& spec. Insect. 1783 p. 63 n. 230.

Vespa Crabro media Villers, C. Linnaci Entom: IV. 1789 p. 273 n. 17.

Vespa media Olivier, Encycl. méthod. Insect. VI. 1791 p. 679 n. 48.

Vespa media Latreille, Hist. nat. Insect. XIII. 1805 p. 351 n. 2.

Vespa media Schummel. Übers. Arbeit. schles. Ges. f. vaterl.Cultur i. J. 1528.1829 p.75. . 9.3.

Vespa Geerii Lepeletier. Hist. nat. Insect. Hymén. I. 1536 p. 510 n. S, \& ? 引े.

Vespa media Saussure. Fitud. fam. Vespid. II. Vesp. 1553 p. 129 n. 9. C; T. 14 F. 9.

Vespa Geerii Schenck, Jahrb. Ver. Naturk. Nassau IX. 1. 15.53 p. 22 n. 2, ธ Q j.

Vespa Geerii Schenck, Progr. Gymnas. Weilburg 1853 p. 13, \& 9 ot.

Vespa media Schenck. Jahrb. Ver. Naturk. Nassau XVI. 1861 p. 16, 20,22 \& 21 n. 2 . $\subseteq$ P j.

Vespa media Radoszkowski. IIorac soe. entom. Ross. II. 1563 p. 129 n. 8 ; T. 17 F. 1-4.

Vespa (Vespula) media Thomson. Opusc. entom. P. 1. 1569 p. 79 n. 2, S ? j.

Vespa media H. Müller, Befrucht. d. Blumen 1873 p. 467.

Vespa media Rouget, Mém. acad. sc. Dijon (3) I. 1873 Sc. p. 188.

Vespa media Puton, Pet. nouv. entom. I. 5. 1873 p. 344 n. 2.

Vespa Vespula media Thomson. Hymen. Scandin. III. 1574 p. 10 n. $2 . \subseteq 9.3$

Vespa media Sehmiedeknecht. Entom. Nachr. VII. 1851 p. 316 c Q $1317 / \vec{j}$.

Vespa media Provancher, Natural. Canad. XIII. 1542 p. 166 n. 5. $\leq$ Q 3 .

Vespa media Provancher, Faun. entom. Canada. Iymén. 1584 p. lin.t 11. 5. S ? 3 .

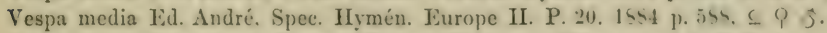

Vespa media Schulthess. Hymen. Helvet. Diplopt. 1557 p. 11 n. 2. $\subseteq$ 9 3 .

Vespa media Marchal, Feuill. jeun. natural. XIX. 1889 p. 158.

Vespa media Konow, Soc. entom. IV. 1890 p. 191 n. 9.

var. erassa Herr.-Schaeff. - ô - Ėur.: Germania.

Vespa crassa Herrich-Schaeffer. Faun. Insect. German. 144l P. 179 T. 4 s.

Vespa crassa Schenck, Jahrb. Ver. Naturk. Nassau IX. 1. 1853 p. 26 n. 4, ô.

Vespa crassa Schenck, Progr. Gymnas. Weilburg 1858 p. 14, §ิ.

Vespa media var. crassa Schenck, Jahrb. Ver. Naturk. Nassau XVI. 15 til p. 24.

var: flavieineta Schck. $-9 \vec{\jmath}-$ Eur.: Germania.

Vespa flaricincta Schenck. Jahrb. Ver. Naturk. Nassau IX. 1. 1453 p. 27 n. 5, อ 9.

Vespa flavicincta Schenck, Progr. Gymnas. Weilburg 1553 p. 14, 9 đ̃.

Vespa flaricincta Brischke. Schrift. phys. ökon. Ges. Königsberg II. 1562 p. 113. 9.

Vespa media var.? flavicincta Schenck, Jahrb. Ver. Naturk. Nassau XVI. 1Giil p. 21. 
vur. rufosentellata Schek, - $\odot$ ơ - Eur.: Germania.

Vespa rufoseutellata Sehenek, Jahrb. Ver. Naturk. Nassau IX. 1. 1453 p. 24 n. 6. j.

Vespa rufoscutellata Schenck. Progr. Gymnas. Weilburg 1853 p. 13. j.

Vespa rufoseutellata Brisehke. Schrift. phys. ükon. Ges. Königsberg II. 1<62 p. 113. ᄃ.

Vespa media var. rufoseutellata Schenek. Jahrb. Ver. Naturk. Nassau XVI. 1661 p. 21. rar. similis Schck, - 우 9 ơ - Eur.: Germania.

Vespa similis Schenck, Jahrb. Ver. Naturk. Nassau IX. 1. 145.3 p. 24 n. 3. S ? j.

Vespa similis Schenck, Progr. Gymnas. Weilburg 1853 p. 13, \& 9 ơ.

Vespa media var. similis Schenck. Jahrb. Ver. Naturk. Nassau XVI. 1561 p. 24.

minima Poda! - Patria?

Vespa minima Poda, Insect. mus. Graec. 1761 p. 109 n. 6.

mongolica André - 9 f - As.: Sibiria.

Vespa Mongolica Ed. André, Ann. soc. entom. France (6) IV. 1684 p. $\$ 1$ n. 1. ? j.

Vespa Mongolica Ed. André. Ann. soc. entom. France 6) IV. 1454 Bull. p. LIX. ? 3.

mncronata Schrk.! - Eur.: Bavaria.

Vespa mucronata Schrank, Fauna Boica II. 2. 1802 p. 355 n. 2211.

nigxa Fourcr.! - Eur.: Gallia.

Vespa spec. Geoffroy, Hist. abr. Insect. II. 1762 p. 381 n. 17.

Vespa nigra Fourcroy, Entom. Paris. II. 1785 p. 438 n. 17.

Vespa nigra Olivier, Encyel. méthod. Insect. VI. 1791 p. 694 n. 4.

nigripennis Sauss. - As.: Philippines.

Vespa nigripennis Saussure, Ĺtud. fam. Vespid. II. Vesp. 1553 p. 146 n. 41. norregica Fabr. - 우 9 ð - Eur.

Vespa Norwegica Fabricius, Spec. Insect. I. 1781 p. 460 n. 12.

Vespa Norwegica Fabricius, Mant. Insect. I. 1787 p. 288 n. 13.

Vespa Norwegica Villers, C. Linnaei Entom. IV. 1789 p. 275 n. 20.

Vespa Norwegica Gmelin. I,inné: Syst. nat. Ed. 13a I. 5. 1790 p. 2751 11. 41.

Vespa Norwegica Olivier, Encṛcl. méthod. Insect. VI. 1791 p. tis0 n. 52.

Vespa Norwegica Fabricius, Entom. System. II. 1793 p. 258 n. 16.

Vespa Norwegica Panzer, Faun. Insect. German. VII. 1801 P. 81 T. 16.

Vespa Norwegica Fabricius, Syst. Piez. 1804 p. 256 n. 14.

Vespa Norwegica Panzer, Krit. Revis. II. 1806 p. 154.

Vespa Norwegica Jurine, Nouv. méth. class. Hymén. 1807 p. 168, ㅇ․

Vespa Norwegica Latreille, Gen. Crust. \& Insect. IV. 1809 p. 142.

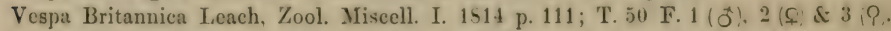

Vespa Britannica Dale, Magaz. of Nat. Hist. VI. 1833 p. 5:5-535; Fig. 65-71.

Vespa Britannica Strickland. Magaz. of Nat. Hist. VII. 1534 p. 264-265.

Vespa Britannica Anonym, Magaz. of Nat. Hist. VIII. 1835 p. 626.

Vespa Norwegica Zetterstedt, Insect. Lappon. I. 1838 p. 454 n. 5, 우 đే.

Yespa Britannica Shuckard, Magaz. of Nat. Hist. N. S. III. 1539 p. 45 S.

Vespa Britannica G. Newman, Entomologist 1811 p. 106.

Vespa Britannica Yewport. Trans. Entom. Soc. I.ondon III. 3. 1 s42 p. 190.

Vespa Britannica Bladon, Zoologist I. 1843 p. 32.

Vespa Britannica Smith, Zoologist I. 1513 p. 169 n. 5 ; Fig. n ( $\hat{\sigma}, 0$ \& \& p 9 .

Vespa Britannica Hogg. Trans. Entom. Soc. London IV. 1. 1545 Proc. p. IXXXII.

Vespa Britannica Smith. Trans. Entom. Soc. Iondon (2) 1I. 2. 1852 Proc. p. 34.

Vespa Norwegica Smith, Zoologist X. 1852 p. 3699; Fig. 5.

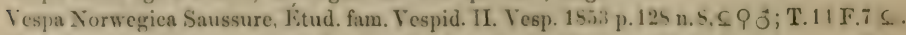

Vespa Nurmegrica Sehenck. Jahrb. Ver. Naturk. Nassau IX. 1. 1553 p. 36 n. 10 \& s6. $\subseteq$ S

Vespa Norwegica Sehenck. Progr. Gymnas. Weilburg 14.i3 p. 15. $\leq$ ( 3 .

Vespa Norwegica Smith, Catal. Hymen. Brit. Mus. V. 1857 p. 115 n. 7. 
Vespa Norregica Smith. Catal. Brit. Fossor. Hymen. 18 is p. 220 n. 6, \& ? ô.

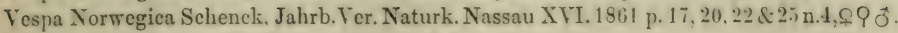

Vespa Norvegica Smith, Entomol. Annual f. 1863 p. 53.

Yespa Norwegica Radoszkowski. Horae soc. entom. Ross. II. 1563 p.121 n.5; T.16 F.11 \& 12.

Vespa Britannica Lestock, Ann. \& Mag. Nat. Hist. (3) XIII. 1564 p. 172.

Vespa Norvegica Harding, Entomologist II. 1865 p. 319-320.

Vespa Britannica Ormerod, Brit. Soc. Wasps 1865 p. 3!) ; T. 2 F. 1. T. 5 F. 2 \& ' T. 11,13 \& 14.

Vespa (Vespula) Norvegica Thomson, Opuse. entom. P. 1. 1569 p. 81 n. 5. S. ? j.

Vespa Nortregica Puton, Pet. nouv, entom. I. 1873 p. 344 n. 3.

Vespa (Tespula) Saxonica Thomson, Hymen. Scandin. III. 1874 p. 13 n. 3, $\subseteq$ ?

Vespa Britannica Corbin, Entomologist VII. 1874 p. 70.

Vespa Norwegica Rudow, Arch. Ver. naturf. Fr. Mecklenburg XXX. 1576 p. 201 n. 2 , ક 9 ठే.

Vespa Norwegica H. Müller, Alpenblumen 1881 j. 592 n. 613.

Vespa Norvegica Fowler, Entomologist XIV. 1881 p. 71.

Vespa Norregica Howe, Entomologist XIV. 1881 p. 300.

Vespa Norvegica E. A. Fitch, Entomologist XIV. 1881 p. 300.

Vespa Norvegica Fowler, Entom. M. Magaz. XVII. 1881 p. 237.

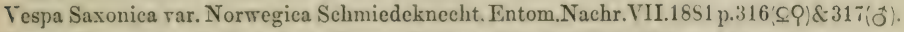

Vespa Norwegica E. Saunders, Trans. Entom. Soc. London 1852 p. 171 n. 7.

Vespa Norvegica Cole, Trans. Entom. Soc. London 1885 Proc. p. II.

Vespa Norwegica Rudow, Soc. entom. I. 1886 p. 83.

Vespa Saxonica rar. Norregica Schulthess, Hymen. Helret. Diplopt. 1887 p. 14.

Vespa Saxonica rar. Normegica Marchal, Feuill. jeun. natural. XIX. 1889 p. $15 S$.

Vespa Norvegica Konow, Soc. entom. IV. 1890 p. 191 n. 8.

obliterata Smith - 으- As.: India.

Vespa obliterata Smith. Trans. Entom. Soc. London (2) II. 2. 1852 p. 47, f.

Vespa obliterata Saussurc. Kitud. fam. Vespid. II. Vesp. 1853 p. 149 n. 32.

occidentalis Cress. - \& 9 ơ - Am.: New Mexico.

Vespa occidentalis Cresson, Trans. Amer. Ertom. Soc. V. 1874 p. 100, \& o

Vespa oecidentalis Cresson, Rep. geogr. \& geol.Explor. \& Surr. $100^{\text {th }}$ Merid. 1875 V.p. 719 ochreata Web.! - As.: India.

$[\mathrm{n} .92$, 우; T. 34 F. $1 \& 2$.

— Lichtenstein, Catal. Mus. Holth. 1796 p. 202 n. 166.

Vespa ochreata Weber, Observ. entom. 1801 p. 101 n. 2.

ordinata Fourer.1 - Eur.: Gallia.

Vespa spec. Geoffroy, Hist. abr. Insect. II. 1762 p. 378 n. 11.

Vespa ordinata Fourcroy, Entom. Paris. II. 1785 p. 435 n. 11.

Vespa seriata Olivier, Encyel. méthod. Insect. VI. 1791 p. 693 n. 120.

orientalis $L$. - 우 9 o $-A_{8}$ : : India.

Vespa orientalis Linné, Mant. plant. II. 1771 p. 540.

Vespa Turcica Drury, Illustr. Nat. Hist. II. 1773 p. 74; T. 39 F. 1.

Vespa orientalis Fabricius, Syst. entom. 1775 p. 363 n. 3.

Vespa orientalis Ph. L. Müller, Iinné: Vollst. Natursyst. Supl̨l. 1776 p. 325 n. 29.

Vespa orientalis Fabricius, Spec. Insect. I. 1781 p. 458 n. 3.

Vespa orientalis Fabricius, Mant. Insect. 1787 p. 257 n. 4.

Vespa orientalis Gmelin, Linné: Syst. nat. Ed. $13^{a}$ I. 5. 1790 p. 2748 n. 31.

Vespa fusca Christ, Naturg. d. Insect. 1791 p. 216.

Vespa orientalis Christ, Naturg. d. Insect. 1791 p. 237.

Vespa orientalis Olivier, Encycl. méthod. Insect. VI. 1791 p. 677 n. 41.

Vespa orientalis Fabricius, Entom. system. U. 1793 p. 254 n. 4.

Vespa orientalis Fabricius, Syst. Piez. $180+2$ p. 254 n. 4.

Vespa orientalis Jurine, Nouv. méth. class. Hymén. 1807 p. 168, \&. 
Vespa orientalis Latreille, Gen. Crust. \& Insect. IV. 1809 p. 142.

Vespa orientalis Brullé, Expéd. sc. Morée. Zool. II. 1832 p. 360 n. 796.

Vespa orientalis I.epeletier, Hist. nat. Insect. Hymén. I. 16.36 p. 507 n. $5 . \subseteq 9.3$.

Tespa orientalis Westwood, Drury: Illustr. Nat. Hist. Ed. 2a II. 1537 p. 52; T. 39 F. 1.

Tespa orientalis Saussure, Etud. fam. Vespid. II. Vesp. 1553 p. 132 n. 11, ऽ? S̈; T. 1.1 F. 1.

Vespa orientalis Horne, Trans. Zool. Soc. London VII. 3. 1870 p. 172.

Vespa orientalis Smith, Trans. Entom. Soc. London 1872 Proc. p. X.

Vespa oricntalis Goodman, Trans. Entom. Soe. Iondon 1477 Proe. p. XXXIII.

Vespa orientalis Destefani. Natural. Sicil. II. 1. 1552 p. 17-20; 2. p. 42-44.

Vespa orientalis Ed. André, Spec. Hymén. Europe II. P. 20. 1554 p. $553, \subsetneq 9$ J̈; T. 39 F. 3.

Vespa orientalis Rudow, Soc. entom. I. 1886 p. 83.

Vespa orientalis Mik, Wien. entom. Zeitg. V. 1856 p. 101-102.

Vespa orientalis Konow, Soc. entom. IV. 1890 p. 175 n. 2.

var. aegyptiaca André - Afr.: Aegyptus.

Vespa orientalis var. Aegyptiaca Ed. André, Spee. Hymén. Europe II. P. 20. 1584 p. 552. var. jurinei Sauss. - Eur.: Albania.

Vespa Jurinei Saussure, Étud. fam. Vespid. II. Vesp. 1853 p. 133 n. 12.

Vespa orientalis var. Jurinei Ed. André, Spec. Hymén. Europe II. P. 20. 1854 p. 552. pacicephala Scop.! - Eur.: Hungaria.

Vespa pacicephala Scopoli, Annus hist.-nat. V. 1772 p. 122 n. 147.

parallela André - 9 - As.: Sibiria.

Vespa parallela Ed. André, Bull. soc. entom. France (6. IV. 1584 p. 52 n. 2, 9.

Vespa parallela Iid. André, Ann. soc. entom. France (6) IV. 1554 Bull. p. I.XI, S.

pennsylyaniea Sauss. - - - Am.: Pennsylvania.

Vespa Pennsylranica Saussure, Stettin. entom. Zeitg. XVIII. 1957 p. 117, ᄋ.

peruana Sauss. - 우 - Am.: Peru.

Vespa Peruana Saussure, Reise d. Novara. Zool. II. P. 1 A. 1565 Hymen. p. 1S, s.

petiolata Schrk. - Eur.: Bararia.

Vespa petiolata Schrank, Fauna Boica II. 2.1802 p. 358 n. 2217.

philippinensis Sauss. - $\hat{o}-$ As.: Philippines.

Vespa Philippinensis Saussure, Étud. fam. Vespid. Ul. Hymén. 1553 p. 145 n. 30 , ô. picipes Oliv.! - Am.: Cayenne.

Vespa picipes Olivier, Act. soc. hist. nat. Paris I. 1792 p. 122 n. 68.

Vespa picipes Reiche, Magaz. d. Thierr. I. 1793 p. 131 n. 2.

pilosella Grav.1 - Patria?

Vespa pilosella Gravenhorst, Vergl. Übers. zool. System. 1507 p. 275 n. 3\$54-3\$55.

pilosella Costa! - 우 ठే - Eur.: Italia.

Vespa pilosella C'osta, Ric. entom. monti Partenii 1855 p. 20 \& 28 n. 3t, $\subseteq$ oै.

pteropoda Scop.! - Eur.: Hungaria.

Vespa pteropoda Scopoli, Annus hist.-nat. V. 1772 p. 122 n. 148.

punctum (Fabr.) Jur.! - $\hat{o}-$ Am.: Nova Cambria.

Polistes punctum Fabricius, Syst. Piez. 1804 p. 273 n. 24.

Vespa punctum Jurine, Nouv. méth. class. Hymén. 1807 p. 169, ơ.

quadrimaculata Christ! - Am.: Jamaica.

Vespa quadrimaculata Christ, Naturg. d. Insect. 1791 p. 216.

quadripunetata Fourcr.! - Eur.: Gallia.

Vespa spec. Geoffroy, Hist. abr. Insect. II. 1762 p. 379 n. 12.

Vespa quadripunctata Fourcroy, Entom. Paris. II. 1785 p. 436 n. 12.

Vespa quadriguttata Olivier. Eineycl. méthod. Insect. VI. 1791 p. 6993 n. 1.

quadripunetata Forsk.! - Afr.: Aegytus.

Vespa quadripunctata Forskal, Deser. anim. 1775 p. 84 n. 19. 
quadripunctata Fabr. - As: India.

Vespa quadripunctata Fabricius, Mant. Insect. I. 1787 p. 291 ฉ. 55.

Vespa quadripunctata Gmelin, Linné: Syst. nat. Ed. $13^{a}$ I. 5.1790 p. 2755 n. 68.

Vespa quadripunctata Olivier, Encycl. méthod. Insect. VI. 1791 p. 690 n. 101.

Vespa quadripunctata Fabricius, Entom. system. II. 1793 p. 273 n. 71.

Vespa quadripunctata Fabricius, Syst. Piez. 1804 p. 268 n. 76.

quinquefasciata Müll. - Eür.

Vespa quinquefasciata O. F. Müller, Mélang. soc. roy. Turin III. 1766 p. 196.

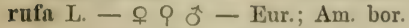

Linné, Fauna Suec. 1746 p. ? n. 998.

Vespa rufa Linné, Syst. nat. Ed. $10^{a}$ I. 1758 p. 572 n. 3.

Vespa rufa Brünnich, Prodr. insectol. Siaelland 1761 p. 18.

Vespa rufa Linné, Fauna Suec. Ed. 2a 1761 p. 416 n. 1672.

Vespa rufa O. F. Müller, Fauna Insect. Fridrichsdal 1764 p. 73 n. 636.

Vespa rufa Linné, Syst, nat. Ed. $12^{\text {a }}$ I. 2. 1767 p. 948 n. 5.

Vespa XIII Schaeffer, Icon. Insect. Ratisbon. I. 2. 1767; T. 81 F. 6.

Vespa rufa Fabricius, Syst. entom. 1775 p. 364 n. 10.

Vespa rufa Ph. L. Müller, Linné: Vollst. Natursyst. V. 2.1775 p. 881 n. 5.

Vespa rufa O. F. Müller, Zool. Dan. prodr. 1776 p. 162 n. 1888.

Vespa rufa Sulzer, Abgek. Gesch. Insect. I. 1776 p. 196; T. 27 F. 13.

Vespa rufa Fabricius, Spec. Insect. I. 1781 p. 460 n. 11.

Vespa rufa Schrank, Enum. Insect. Austr. 1781 p. 390 n. 788.

Vespa rufa Fabricius, Mant. Insect. I. 1787 p. 288 n. 12.

Vespa rufa Ström, Danske Vid. Selsk. Skrift. Nya Saml. III. 1788 p. ? n. 55.

Vespa rufa Römer, Gen. Insect. 1789 p. 60; T. 27 F. 13.

Vespa rufa Villers, C. Linnaei Entom. III. 1789 p. 264 n. 3.

Vespa rufa Gmelin, Linné: Syst. nat. Ed. $13^{a}$ I. 5.1790 p. 2751 n. 5.

Vespa rufa Christ, Naturg. d. Insect. 1791 p. 236 ; T. 22 F. 3.

Vespa rufa Olivier, Encycl. méthod. Insect. VI. 1791 p. 680 n. 51.

Vespa rufa Fabricius, Entom. system. II. 1793 p. 258 n. 15.

Vespa rufa Schrank, Fauna Boica II. 2, 1802 p. 350 n. 2204.

Vespa rufa Schrökenstein, Verz, d. Halbkäfer etc. 1802 p. 31 n. 116.

Vespa rufa Fabricius, Syst. Piez. 1804 p. 256 n. 13.

Vespa rufa Latreille, Hist. nat. Insect. XIII. 1805 p. 352 n. 5.

Vespa rufa Gravenhorst, Vergl. Übers. zool. System. 1807 p. 276 n. 3856-3857.

Vespa rufa Jurine, Nouv. méth. class. Hymén. 1807 p. 168, 우 ఫే.

Vespa rufa Latreille, Gen. Crust. \& Insect. IV. 1809 p. 142.

Vespa rufa Lamarck, Hist. nat. anim. s. vert. IV. 1817 p. 88 n. 4.

Vespa rufa Lamarck, Hist. nat. anim. 8. rert. Ed. 2a IV. 1835 p. 305 n. 4.

Vespa rufa Lepeletier, Hist. nat. Insect. Hymén. I. 1836 p. 517 n. 15, \& 9 .

Vespa rufa Zetterstedt, Insect. Lappon. I. 1838 p. 454 n. 4, \& 9 ơ.

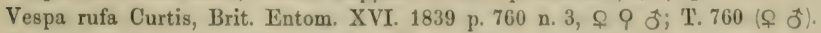

Vespa rufa Sicbold, Arch. f. Naturg. V. 1. 1839 p. 107-108.

Vespa rufa Herrich-Schaeffer, Faun. Insect. German. 1841 P. 179 T. 5 (@ $Q$ ơ).

Vespa rufa Smith, Zoologist I. 1843 p. 167 n. 3, 우 Q ơ; Fig. g ( ̋ૈ), h (ㅇ) \& i (Q).

Vespa rufa Smith, Zoologist X. 1852 p. 3699 ; Fig. 3.

Vespa rufa Saussure, Étud. fam. Vespid. II. Vesp. 1553 p. 120 n. 4. . Q Jे; T. 14 F. 5 S.

Vespa rufa Schenck, Jahrb. Ver. Naturk. Nassau IX. 1. 1853 p. 33 n. 9, ㅇ Q ô.

Vespa rufa Schenck, Progr. Gymmas. Weilburg 1853 p. 15, 우 9 .

Vespa rufa Smith, Zoologist XIV. 1856 p. 5169-5174.

Vespa rufa Smith, Catal. Brit. Fossor. IIynen. 18.55 p. 217 n. 3, S. Q J̈; T. 5 F. 23. 
Vespa rufa Desmarest. Chenu: Encycl. hist. nat. Annelles 1560 p. 125, of; Fig. 105.

Vespa rufa Duméril, Mém. acad. sc. Paris XXXI. 1860 p. 862 n. 4.

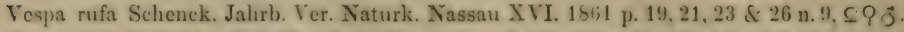

Vespa rufa lirischke. Schrift. phỵs. ökon. Ges. Königsberg II. 1562 p. 113. $\subseteq$ ? J.

Vespa rufa Radoszkowski, Horae soc. entom. Ross. II. 186.3 p. 125 n. 6; T. 16 F. 13 of 14.

Vespa rufa Lestock, Ann. \& Mag. Nat. Hist. (3) XIII. 1864 p. 472.

Vespa rufa Disconzi, Entom. Vicent. 1865 p. 124; T. 9 F. 138.

Vespa rufa 'T'aschenberg, Hymen. Deutschl. 1866 p. 247.

Vespa rufa Ormerod, Brit. Soc. Wasps 1868 p. 47; T. \& F. 2 \& T. 10.

Vespa (Tespula rufa Thomson. Opuse. entom. P. 1.1569 p. 82 n. $9 . \subseteq$ ? วै.

Vespa rufa $H$. Müller, Befrucht. d. Blumen 1873 p. 467.

Vespa rufa Rouget, Mém. acad. sc. Dijon (3) I. 1873 Sc. p. 190.

Vespa rufa Puton, Pet. nouv. entom. I. 5.1873 p. 344 n. 8.

Vespa Vespula rufa Thomson. Hymen. Scandin. III. 1574 p. 25 n. S. ऽ ? j.

Vespa rufa Rudow, Arch. Ver. naturf. Fr. Mecklenburg XXX. 1876 p. 206 n. $5 . \subseteq 9\}$.

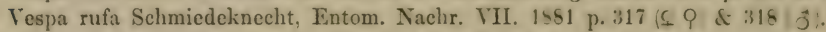

Vespa rufa Provancher, Natural. Canad. XIII. 1882 p. 167 n. 6, Q.

Vespa rufa E. Saunders, Trans. Entom. Soc. London 1882 p. 170 n. 4.

Vespa rufa Ed. André. Spee. Hymén. Europe II. P. 20. 1561 p. 602. \& 9 วิ; T. 39 F. 9.

Vespa rufa Provancher. Faun. entom. Canada. Hymén. 1554 p. 655 n. 6, ?.

Vespa rufa Sehulthess. Hymen. Helvet. Diplopt. 1857 p. 17 n. $7, \subseteq 9 \jmath$ J

Vespa rufa Marchal, Feuill. jeun. natural. XIX. 1889 p. 158.

Vespa rufa Konow, Soc. entom. IV. 1890 p. 175 n. 3.

ruflcornis Forst.! - Eur. : Hispania.

Vespa ruficornis Forster, Nov. spee. Insect. 1771 p. 90 n. 90.

Vespa ruficornis Villers, C. Linnaei Entom. III. 1789 p. 278 n. 31.

ruspatrix L.1 - Afr.

Vespa ruspatrix Linné, Syst. nat. Ed. $12^{a}$ I. 2.1767 p. 951 n. 19.

Vespa ruspatris Ph. L. Müller, Linné: Vollst. Natursyst. V. 2.1775 p. 884 n. 19.

Tespa ruspatrix Gmelin, Linné: Syst. nat. Ed. $13^{\text {n }}$ I. 5.1790 p. 2755 n. 19.

Vespa ruspatrix Christ, Naturg. d. Insect. 1791 p. 241.

Vespa ruspatrix Olivier, Encycl. méthod. Insect. VI. 1791 p. 691 n. 112.

samsauliea Rad. - - - As.: Transeaspia.

Vespa Samsaulica Radoszkowski, Horae soc. entom. Ross. XXI. Iss7 p. 100, S. saxonica Fabr. - Q 9 ơ - Eur.

Vespa XII Schaeffer, Icon. Insect. Ratisbon. I. 2. 1767; T. 74 F. 5 \& 6.

Vespa Saxonica Fabricius, Entom. system. II. 1793 p. 256 n. 12.

Vespa Saxonica Panzer, Faun. Insect. German, V. 1798 P. 49 T. 21.

Vespa Bavarica Schrank, Fauna Boica II. 2. 1802 p. 350 n. 2205.

Vespa Saxonica Tabricius, Syst. Piez. 1804 p. 256 n. 11.

Vespa Saxonica Panzer, Krit. Revis. II. 1806 p. 154.

Vespa Saxonica Jurine, Nouv. méth. class. Hymén. 1807 p. 168, \&.

Vespa Saxonica Latreille, Gen. Crust. \& Insect. IV. 1809 p. 142.

Vespa borealis Zetterstedt, Insect. Lappon. I. 1838 p. 454 n. 3, \&.

Vespa Saxonica Saussure, Eitud. fam. Vespid. II. Vesp. 15033 p. 126 n. $7, \subseteq$, , j.

Vespa borealis Saussure. Ktud. fam. Vespid. II. Vesp. 1453 p. 133 n. $13,4$.

Vespa Saxonica Sehenck. Jahrb. Ver. Naturk. Nassau IX. 1. 1553 p. 37 n. 11. \& 9 j.

Vespa Saxonica Duméril, Mém. acad. sc. Paris XXXI. 1860 p. 862 n. 5.

Vespa Saxonica Schenck. Jahrb. Ver. Naturk. Nassau XVI. 14ti1 p. 14, 21,23 \& 25 n. 5. . 9 J.

Vespa Saxonica Brischke, Schrift. physs. ükon. Ges. Künigsberg II. 1562 p.113, \& 9 đ.

Vespa Vespula Saxonica Thomson, Opusc. Entom. P. 1. 1560 p. 80 n. $3, \subsetneq 9$ oै. 
Vespa Saxonica H. Müller, Befrucht. d. Blumen 1873 p. 467.

Vespa Saxonica Puton, Pet. nour. entom. I. 1873 p. 344 n. 4.

Vespa Tespula) Saxonica Thomson, Hymen. Scandin. III. 1574 p. 13 n. . . S 9 ;

Vespa Saxonica Rudow. Arch. Ver, naturf. Fr. Mecklenburg XXX. 14 if p. 205 n.3.

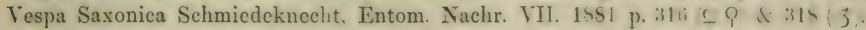

Tespa Saxonica Ed. André, Spee. Hỵmén. Europe II. P. 20. 155.1 p. 591. s ? 3.

Tespa Saxonicu Schulthess. IIymen. Ifulvet. Diplopt. 1457 p. 13 n. $3, \leq$ Q j.

Vespa Saxonica Marchal, Feuill. jeun. natural. XIX. 1889 p. 158.

Vespa Saxonica Konow, Soc. entom. IV. 1590 p. 191 n. 7.

var. tridens Schek. - 우 9 ơ - Eur.: Germania.

Vespa tridens Schenck. Jahrb. Ver. Naturk. Nassau IX. 1. 1553 p. 38 n. 12. $\subseteq$ ? .

Vespa tridens Schenck, Progr. Gymnas. Weilburg 1853 p. 15, 우 우 ㅎ․

Vespa tridens Brischke, Sehrift. physs. ökon. Ges. Königsberg II. 2. 1562 p. 113. ( ?.

Vespa Saxonica var. tridens Schenck, Jahrb. Ver. Naturk. Nassau XVI. 1 Stil p. 136.

scelesta Mac Farl. - Am. bor.

Vespa scelesta Mac Farland. Trans. Amer. Entom. Soc. XV. 1855 p. 2!S.

schrenckii Rad. - 0 Q - As.: Amuria.

Tespa Schrenckii Radoszkowski, Horae soc. entom. Ross. I. 1861 p. 81; 'T. 2 F. 4 \& 5.

Vespa Schrencki Ed. André, Spec. Hymén. Europe II. P. $20.15 \$ 4$ p. 600, ऽ 9.

sentellaris Fabr.! - Am. mer.

Vespa scutellaris Fabricius, Syst. Piez. $180 \pm$ p. 265 n. 67.

sentellata Web.! - As.: India or.

— Lichtenstein, Catal. mus. Holth. 1796 p. 203 n. 176.

Vespa scutellata Weber, Observ. entom. 1801 p. 103 n. 11.

serripes Fabr.! - Am. bor.

Vespa serripes Fabricius, Spec. Insect. I. 1781 p. 464 n. 34.

Vespa serripes Fabricius, Mant. Insect. I. 1787 p. 289 n. 39.

Vespa serripes Gmelin, Linné: Syst. nat. Ed. 13a I. 5.1790 p. 2751 n. 43.

Vespa serripes Olivier, Encycl. méthod. Insect. VI. 1791 p. 686 n. 84.

Vespa serripes Fabricius, Entom. system. II. 1793 p. 264 n. 46.

Vespa serripes Fabricius, Syst. Piez. 1804 p. 262 n. 45.

sesfuicineta Web.l - As.: India.

- Lichtenstein, Catal. mus. Holth. 1796 p. 202 n. 163.

Vespa sesquicincta Weber, Observ. entom. 1801 p. 101 n. 4.

sessilis Oliv.! - Am. mer.

Vespa sessilis Olivier, Encycl. méthod. Insect. VI. 1791 p. 683 n. 69.

sexeineta Vill.! - Eur.: Gallia.

Vespa sexcincta Villers, C. Linnaei Entom. III. 1789 p. 278 n. 29.

sexeinct Schrk.! - Eur.: Bavaria.

Vespa sexcincta Schrank, Fauna Boica II. 2. 1802 p. 356 n. 2213.

sexcincta Panz. - $ᄋ$ ơ - Eur.: Germania.

Vespa sexcincta Panzer, Faun. Insect. German. VI. 1799 P. 63 T. 1.

Vespa sexcincta Panzer, Krit. Revis. II. 1806 p. 154.

Vespa sexcincta Jurine, Nouv. méth. class. Hymén. 1807 p. 168, \& ơ.

sexmaenlata Müll.! - Eur.

Vespa sexmaculata O. F. Müller. Mélang. soc. roy. Turin. III. 17fiti p. 196.

sexpustulata Vill.! - Eur.: Gallia.

Vespa sexpustulata Villers, C. Linnaci Entom. III. 1789 p. 281 n. 39.

sibirica André - $Q$ Q ơ - As.: Sibiria.

Vespa Sibirica İd. André, Spec. Hymén. Europe II. P. 20. 154 p. 599. \& 9 Jे. 
silrestris Scop. - 우 $9 \hat{\sigma}$ - Eur.

Vespa silvestris Scopoli, Entom. Carn. 1763 p. 309 n. 826.

Vespa silvestris Villers, C. Linnaei Entom. III. 1789 p. 274 n. 18.

Vespa silvestris Christ, Naturg. d. Insect. 1791 p. 235 ; T. 22 F. 1.

Vespa Holsatica Fabricius, Entom. system. II. 1793 p. 257 ฉ. 14.

Vespa Holsatica Latreille, Ann. mus. hist. nat. 1. 1802 p. 289 n. 2.

Vespa frontalis Latreille, Ann. mus, hist. nat. I. 1802 p. 290 n. 3.

Vespa Holsatica Fabricius, Syst. Piez. 1804 p. 256 n. 12.

Vespa Holsatica Latreille, Hist. nat. Insect. XIII. 1805 p. 352 n. 4.

Vespa Holsatica Latreille, Gen. Crust. \& Insect. IV. 1809 p. 142.

Vespa Holsatica Klug, Germar: Reise n. Dalmat. II. 1817 p. 264 n. 371.

Vespa Holsatica Lamarck, Hist. nat. anim. s. vert. IV. 1817 p. 88 n. 3.

Vespa IIolsatica Schummel. C̈bers. Arbeit. schles. Ges. f. vaterl. Cultur i.J. 1529.1530 p. 54.

Vespa Holsatica Schummel, Übers. Arbeit. schles. Ges. f. vaterl. Cultur i. J. 1530.1531 p. 92.

Vespa Holsatica Lamarek, Hist. nat. anim. s. vert. Ed. 2 IV. 1835 p. 304 n. 3.

Vespa Holsatica Anonym, Magaz. of Nat. Hist. VIII. 1835 p. 627.

Vespa Holsatica Shuckard, Mag. of Nat. Hist. N. S. III. 1839 p. 459.

Vespa Holsatica Smith, Zoologist I. 1543 p. 165 n. 4; Fig. k (J., l S \& m P).

Vespa Holsatica Gould. Trans. Entom. Soc. London IV. 1. 1545 Proc. p. LII \& LXXXII.

Vespa sy-lvestris (Holsatica Smith, Zoologist X. 1852 p. 3699; Fig. 4.

[T. 17 F. 1.

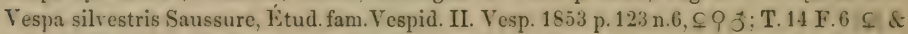

Vespa sylvestris Smith, Catal. Brit. Fossor. Hymen. 1855 p. 219 n. 4, ऽ Q J; T. 5 F. 22.

Vespa Holsatica Schenck, Jahrb. Ver. Naturk. Nassau XVI. 1461 p. $18,21,23$ \& 26 n. $6, \subseteq 9$ J.

Vespa silvestris Radoszkowski, Horae soc. entom. Ross. II. 1563 p. 122 n. 3: T. 16 F. 5 -5.

Vespa Holsatica Radoszkowski, Horae soc. entom. Ross. II. 1563 p. 123 n. 4 ; T. 16 F. 9 \& 10.

Vespa silvestris Lestock, Ann. \& Mag. Nat. Hist. (3) XIII. 1864 p. 473.

Vespa sylrestris Sichel, Ann. soc. entom. France 4, V. 1465 Bull. p. XXY.

? Vespa Alsatica Erber, Yerh. zool. bot. Ges. Wien XVII. 1567 Sitzber. p. 107.

Vespa silvestris Ormerod, Brit. Soe. Wasps 1565 p. 41 ; T. 2 \& 6 F. 2 \& 'T. 12.

Vespa (Vespula) Holsatica Thomson, Opuse. entom. P. 1.1569 p. 50 n. $4 . \subseteq 9 \vec{\jmath}$.

Vespa Holsatica Siebold, Beitr. z. Parthenog. 1871 p. 102-105.

Vespa Holsatica Taschenberg, Zeitschr. f. d. ges. Naturw. XXXVII. $1 \$ 71$ p. 97-95.

Vespa Holsatica H. Müller, Befrucht. d. Blumen 1873 p. 467.

Vespa silvestris Rouget, Mém. acad. sc. Dijon (3) I. 1873 Sc. p. 189.

Vespa silvestris Rouget, Pet. nouv. entom. I. 5.1873 p. 335.

Vespa silvestris Puton, Pet. nouv. entom. I. 5.1873 p. 344 n. 5.

Vespa (Vespula) Holsatica Thomson, Hymen. Seandin. III. 1474 p. 16 n. 4 , 9 o

Vespa IIolsatica Rudow, Arch. Ver. naturf. Fr. Mecklenburg XXX. 1476 p. 206 n. 4. $\subseteq$ P. 3.

Vespa Holsatica Katter, Entom. Nachr. IV. 1878 p. 23.

Vespa silvestris H. Muller, Alpenblumen 1881 p. 592 n. 614.

Vespa IIolsatica Schmiedeknecht, Intom. Nachr. VII. 1 sh p. 317 (\& P) d $318(\vec{\jmath})$.

Vespa sylvestris E. Saunders, Trans. Entom. Soe. London 1452 p. 171 n. 6.

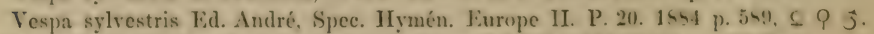

Vespa IIolsatica Wulp. Tijdschr. v. Fintom. XXVII. 14S4 Versl. p. XVII.

Vespa silvestris Cole, Trans. Entom. Soc. London 1585 Proc. p. II.

Vespa silvestris Schulthess. Hymen. Helvet. Diplopt. 1547 p. 11 n. 4 , f 9 j.

Vespa sylvestris Marchal, Feuill. jeun. natural. XIX. 1859 p. 158.

Vespa silvestris Konow, Soc. entom. IV. 1890 p. 176 n. 6.

sinuata Fourcr.1 - Eur.: Gallia.

Vespa sinuata Fourcroy, Entom. Paris. II. 1785 p. 438 n. 19. 
squamigera Fabr.! - Afr.: Guinea.

Vespa squamigera Fabricius, Syst. Piez. 1804 p. 267 n. 73.

structor Smith $-9-$ As.: India.

Vespa structor Smith, Trans. Zool. Soc. London VII. 3. 1570 p. 191 n. 3, Q; T. 21 F. 12.

sulphurea Sauss. - - - Am.: California.

Vespa sulphurea Saussure, Étud. fam. Vespid. II. Tesp. 1853 p. 137 n. 17, S.

Vespa sulphurea Saussure, Stettin. entom. Zeitg. XVIII. 1857 p. 116, ㅇ․

tahitensis Web.! - Austr.: Tahiti.

Lichtenstein, Catal. mus. Holth. 1796 p. 201 n. 142.

Vespa Tahitensis Weber, Observ. entom, 1801 p. 100 n. 1.

tesserazona Schrk.! - Eur.: Bavaria.

Vespa tesserazona Schrank, Fauna Boica II. 2. 1802 p. 357 n. 2215.

tibialis Oliv.! - Am.: Georgia.

Vespa tibialis Olivier, Encycl. méthod. Insect. VI. 1791 p. 690 n. 103.

tricolor Pall.! - Eur.: Russia.

Vespa tricolor Pallas, Reisen d. rersch. Pror. russ. Reich. I. 1771 p. 474 n. 74.

Vespa tricolor Ph. L. Müller, Linné: Vollst. Natursyst. Suppl. 1776 p. 326 n. 31.

trifasciata Müll.! - Eur.: Dania.

Vespa trifasciata O. F. Müller, Zool. Dan. prodr. 1776 p. 163 n. 1894.

tripunctata Schck.! - 우 - Eur.: Bavaria.

Vespa tripunctata Schenck, Jahrb. Yer. Naturk. Nassau XVI. 1561 p. 17 \& 25 n. 3, \&. tristrigata Fabr.! - Patria?

Vespa tristrigata Fabricius, Entom. system. IV. 1794 p. 459 n. 71-72.

tyrauniea Smith - 오 9 - As.: Singapore.

Vespa tyrannica Smith, Catal. Hymen. Brit. Mus. V. 1557 p. 119 n. 21, S Q.

unicolor Smith - $Q-$ As.: Bouru.

Vespa unicolor Smith, Journ. of Proc. Linn. Soc. Zool. VII. 1563 p. 44 n. 4, f.

relox Christ! - Patria?

Vespa velox Christ, Naturg. d. Insect. 1791 p. 245 ; T. 23 F. 6.

velutina Lep. - - - As.: Java.

Vespa velutina (Haan) Lepeletier, IIist. nat. Insect. Hymén. I. 1836 p. 507 n. 4. S.

Vespa relutina Saussure, Etud. fam. Vespid. II. Vesp. 1853 p. 144 n. 26 , S.

Vespa velutina Horne, Trans. Zool. Soc. London VII. 3. 1870 p. 172.

vidua Sauss. - $Q 9$ - Am.: Carolina.

Vespa vidua Saussure, Étud. fam. Vespid. II. Vesp. 1853 p. 136 n. 16, 9.

Vespa vidua Saussure, Stettin. entom. Zeitg. XVII. 1857 p. 117, ㅇ.

vivax Smith $-9-$ As.: India.

Vespa vivax Smith. Trans. Zool. Soc. London VII. 3. 1570 p. 190 n. 1, Q; T. 21 F. 9. vulgaris $\mathrm{L}$. - 우 9 ơ - Eur.

- Plinius, Hist. nat. I. 9. c. 21.

- Aldrovandi, De anim. Insect. 1602 p. 195.

- Moufet, Insect. s. minim. anim. theatr. 1634 Cap. VII; Fig.

Hollar, Muscar. ete. variae Figurae 1646; T. 7 F. 3.

Merrett, Pinax rer. natur. Brit. 1667 p. 196.

Rajus, Hist. Insect. 1710 p. 250.

- Frisch, Beschr. Insect. Teutschl. IX. 1730 p. 22; T. 12 F. 2.

Linné, Fauna Suec. 1746 p. "? n. 989.

Vespa vulgaris Linné, Syst. nat. Ed. $10^{3}$ I. 1758 p. 572 n. 2.

Vespa rulgaris Brünnich, Prodr. insectol. Siaclland. 1761 p. 18.

Vespa vulgaris Linné, Fauna Suec. Ed. $2^{3} 1761$ p. 415 n. 1671.

Vespa vulgaris Poda, Insect. mus. Graec. 1761 p. 108 ฉ. 2. 
Vespa spec. Geoffroy, Hist. abr. Insect. II. 1762 p. 369 n. 2.

Vespa vulgaris Gronow, Acta Helvet. V. 1762 p. 353 n. 401.

Vespa vulgaris Scopoli, Entom. Carn, 1763 p. 308 n. 825.

Vespa vulgaris O. F. Müller, Fauna Insect. Fridrichsdal 1764 p. 73 n. 635.

Vespa V Schaeffer, Icon. Insect. Ratisbon. I. 1. 1766; T. 35 F. 4.

Vespa vulgaris Linné, Syst. nat. Ed. $12^{2}$ I. 2.1767 p. 949 n. 4.

Vespa vulgaris Degeer, Mém. hist. Insect. II. 1. 1771 p. 766; T. 26 F. 1-17.

Vespa vulgaris Fabricius, Syst. entom. 1775 p. 364 n. 9.

Vespa vulgaris Ph. L. Müller. Limné: Vullst. Natursyst. V. 2. 1775 p. 850 n. 4; T. 27 F. 3.

Vespa rulgaris O. F. Müller, Zool. Dan. prodr. 1776 p. 162 n. 1887.

Vespa vulgaris Ph. F. Gmelin, Onomatol. hist. nat. VII. 1777 p. 712.

Vespa vulgaris Fischer, Naturg. v. Lievl. 1778 p. 159.

Vespa vulgaris Göze, Degeer: Abh. Gesch. Insect. II. 2. 1779 p. 111; T. 26 F. 1-17.

Vespa XXV Schaeffer, Icon. Insect. Ratisbon. III. 1779; T. 238 F. 7.

Vespa vulgaris Fabricius, Spec. Insect. I. 1781 p. 460 n. 9.

Vespa rulgaris Schrank, Enum. Inseet. Austr. 1781 p. 390 n. 787.

Vespa rulgaris Retzius, Gen. \& spec. Insect. 1783 p. 63 n. 229.

Vespa vulgaris Fourcroy, Entom. Paris. II. 1785 p. 430 n. 2.

Vespa vulgaris Fabricius, Mant. Insect. I. 1787 p. 287 n. 10.

Vespa rulqaris Ström. Danske Vid. Selsk. Skrift. Nya Saml. III. 1755 p. 279 n. 54.

Vespa vulgaris Razoumowski, Hist. nat. Jorat. I. 1789 p. 217 n. 256.

Vespa vulgaris Villers, C. Linnaei Entom. III. 1789 p. 263 \& 552 n. 2.

Vespa rulgaris Gmelin, Linné: Syst. nat. Ed. $13^{\text {a }}$ L 5. 1790 p. 2750 n. 4.

Vespa vulgaris Rossi, Fauna Etrusca II. 1790 p. 83 n. 861.

Vespa vulgaris Christ, Naturg. d. Insect. 1791 p. 235; T. 22 F. 2.

Vespa rulgaris J. B. Fischer, Vers. Naturg. Lievland Ed. 2a 1791 p. 343 n. 787.

Vespa vulgaris Olivier, Encycl. méthod. Insect. VI. 1791 p. 679 n. 49.

Vespa vulgaris Fabricius, Entom. system. II. 1793 p. 256 n. 10.

Vespa vulgaris Cederhjelm, Faun. Ingric. prodr. 1795 p. 168 n. 521.

Vespa vulgaris Panzer, Faun. Insect. German. 1798 P. 49 T. 19.

Vespa vulgaris Latreille, Hist. nat. Insect. III. 1802 p. 364.

Vespa vulgaris Latreille, Ann. mus. hist. nat. I. 1802 p. 288 n. 1.

Vespa vulgaris Schrank, Fauna Boica II. 2. 1802 p. 348 n. 2203.

Vespa vulgaris Walckenaer, Fauna Paris. II. 1802 p. 88 n. 4.

Vespa vulgaris Fabricius, Syst. Piez. 1804 p. 255 n. 9.

Vespa vulgaris Latreille, Hist. nat. Crust. \& Inseet. XIII. 1505 p. 351 n. 3.

Vespa rulgaris Panzer, Krit. Revis. II. 1806 p. 153.

Vespa rulgaris Gravenhorst. Vergl. C̈bers. zool. System. 1 sot p. 275 n. $3445-35.15$.

Vespa vulgaris C. IIuber. Vollst. Naturg. d. Bau- u. Baumbülzer II. 6. 1507 p. 262.

Vespa vulgaris Illiger, Rossi: Fauna Etrusea Ed. $2^{2}$ II. 1507 p. 135 n. 861.

Vespa vulgaris Jurine, Nouv. méth. class. Hymén. 1807 p. 165, $\Omega$ ơ.

Vespa vulgaris Latreille, Gen. Crust. \& Insect. IV. 1809 p. 142.

Vespa vulgaris Lamarck, Hist. nat. anim. s. vert. IV. 1817 p. 88 n. 2.

Vespa vulgaris 13echstein, Forstinsectol. 1818 p. 147 n. 310 \& p. 503 n. 24.

Vespa vulgaris Schummel. ('bers. Arbeit. schles. Ges. f. vaterl. ('ultur i. J. 1529. 1530 p. 54.

Yespa rulgaris Sehummel. Übers. Arbeit. schles. Ges. f. vaterl. ('ultur i. J. 1530. 1531 p. 92.

Vespa vulgaris Davis, Entom. Magaz. I. 1832 p. 90.

Vespa vulgaris Anonym, Magaz. of Nat. Hist. VII. 1834 p. 265.

Vespa vulgaris Lamarck, Hist. nat. anim. s. vert. Ed. 2 IV. 1835 p. 304 n. 2.

Vespa vulgaris Lepeletier, Hist. nat. Insect. Hymén. I. 1836 p. 516 n. 14, \& 9 ơ.

Vespa vulgaris Newport, Trans. Soc. Entom. London I. 3. 1836 p. 228-229. 
Vespa vulgaris W. F. Kirby, Fauna Bor-Amer. IV. 1837 p. 263 n. 364, 9.

Vespa vulgaris Dufour, Ann. sc. nat. Zool. (2) VII. 1837 p. 18.

Vespa vulgaris Bond, Entom. Magaz. IV. 1837 p. 221.

Vespa vulgaris Zetterstedt, Insect. Lappon. I. 1838 p. 453 n. 2, 우 9 đ..

Vespa vulgaris Westwood. Introd.mod. Classif. Insect. II. 1840 p. 214 ; F. \& s; Synops. p. b.1.

Vespa vulgaris Herrich-Schaeffer, Faun. Insect. German. 1541 P. 179; T. 3 Fig. a \& :

Vespa vulgaris Ratzeburg, Forstinsect. III. 1814 p. 51 n. 2.

Vespa rulgaris Blanchard, Cuvier: Règne anim. Ed. 3a Insect. II. 1419; T. 124 F. 5.

Vespa vulgaris Grube, Arch. f. Anat. Physiol. etc. 1849 p. 47.

Vespa vulgaris Imhoff. Ber. ü. d. Verh. naturf. Ges. Basel VIII. 1 s 4 ! Sitzber. 1 s 18 p. 41.

Vespa vulgaris Hogg, Proc. Linn. Soc. London II. 1849 p. 33-34.

Vespa rulgaris Smith, Zoologist IX. 1851 App. p. CLXXVI; Fig.

Vespa vulgaris Smith, Zoologist X. 1852 p. 3699; Fig. 2.

Vespa vulgaris Saussure. Étud. fam. Vespid. II. Vesp. 1853 p. 113 n. $2 . \subseteq$ P j; T. 14 F. $3 \subseteq$.

Vespa rulgaris Schenck. Jahrb. Ver. Naturk. Nassau IX. 1. $1 \$ 53$ p. 31 n. 8, S. O J

Vespa vulgaris Schenck, Progr. Gymnas. Weilburg 1853 p. 14, \& 9 of.

Vespa rulgaris Mac Intosh. Naturalist V. 1855 p. 32-34 \& 139-141 \& VI. 1856 p. 30-31.

Vespa vulgaris Nördlinger, D. kleinen Feinde 1855 p. 413 ; Fig.

Vespa vulgaris Möbius. Abh. naturw. Yer. Hamburg III. 1856 p. 165 (p. 51 ); T. 19 F. 3.

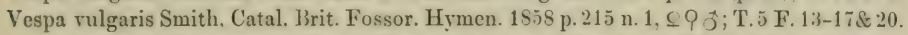

Vespa rulgaris Desmarest. Chenu: Encycl. hist. nat. Annelles 1860 p. 151; Fig. 151.

Vespa vulgaris Duméril, Mém, acad. sc. Paris 1860 p. 861 n. 2.

Vespa vulgaris Schlegel. Jahresber. naturf. Ges. Graubünden Y. 1860 p. 10!1-110.

Vespa vulgaris Wailes, Trans. Entom. Soc. London (2) V. 1860 Proc. p. 109-110.

Vespa vulgaris Hunter, Essays \& Observ. on Nat. Hist. II. 1861 p. 456-459.

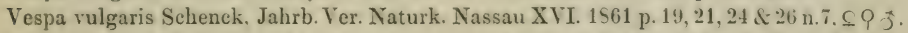

Vespa vulgaris Radoszkowski. Horae soc. entom. Ross. II. 1563 p. 119 n. 1 ; T. 16 F. 1 \& 2.

Vespa vulgaris William, Trans. Entom. Soc. London (3) I. 1863 Proc. p. 182.

Vespa vulgaris Lestock, Ann. \& Mag. Nat. Hist. (3) XIII. 1864 p. 467.

Vespa rulgaris Stone, Trans. Entom. Soc. London (3) II. 1864 Proc. p. 34.

Vespa vulgaris Smith, Trans. Entom. Soc. London (3) II. 1864 Proc. p. 57.

Vespa rulgaris Disconzi, Entom. Vicent. 1865 p. 124; T. 9 F. 137.

Vespa vulgaris Köppen, Horae soc. entom. Ross. III. 1865 p. 289.

Vespa vulgaris Goureau, Bull. soc. sc. hist. nat. Yonne 1866 p. ?

Vespa vulgaris Ormerod, Brit. Soc. Wasps 1868 p. $45 ;$ T. 4 F. 1 \& T. 8.

Vespa (Vespula) vulgaris Thomson, Opusc. entom. P. 1. 1869 p. 81 n. 6, 우 우 ơ.

Vespa vulgaris Rathron, Amer. Entomo]. I. 1869 p. 200.

Vespa vulgaris Smith, Entomol. Annual f. 1870 p. 30.

Vespa vulgaris Smith, Entomol. Annual f. 1871 p. 65.

Vespa vulgaris H. Müller, Befrucht. d. Blumen 1873 p. 467.

Vespa vulgaris Rouget, Mém. acad. sc. Dijon (3) I. 1873 Sc. p. 192.

Vespa vulgaris Rouget, Pet. nouv. cntom. I. 5.1873 p. 335.

Vespa vulgaris Puton, Pet. nouv. entom. I. 5.1873 p. 344 n. 6.

Vespa Vespula rulgaris Thomson. Hymen. Seandin. III. 15i4 p. 15 n. $5,59 \overrightarrow{3}$.

Vespa rulgaris Riley, 6th Ann. Rep. Insect. Missouri 1874 p. 125.

Vespa vulgaris Rudow, Arch. Ver, naturf. Fr. Mecklenburg XXX. 1576 p. 205 n. s, S 9.3.

Vespa rulgaris Schmiedeknecht, Entom. Nachr. VII. 1851 p. 316 s ?

Vespa rulgaris J. P. Stein, Berlin. entom. Zeitschr. XXV. 1881 p. 224.

Vespa vulgaris E. Saunders, Trans. Entom. Soc. London 1882 p. 168 n. 2.

Vespa vulgaris Gadeau, Ann. soc. entom. France (6) III. 1883 p. 187.

Vespa vulgaris E. Saunders, Entom. M. Magaz. XX. 1883 p. 42-43. 
Vespa vulgaris Ed. Andrú. Spec. Hymén. Europe II. P. 20. 1454 p. 593. S 93.

Vespa vulgaris Forel, Bull, soc. Vaud, se. nat. (2) XX. P.91. 1844 p. 379. Q: T. 11 F. 10-13.

Vespa vulgaris Landois. Jahresber. Westphizl. Provinz. Ter. f. 1564 XIII. 1555 p. 21-22.

Vespa vulgaris Anonym, Trans. Norw. Soc. IV. 1885 p. 50.

Vespa vulgaris Dowker, Trans. E. Kent Soc. (2) 1886 p. 54-66.

Vespa rulgaris Schulthess. Hymen. Helvet. Diplopt. 1557 p. 16 n. 6, \& ? j.

Vespa vulgaris Viallanes, Compt. rend. assoc. sc. France (2: XY. 2. 1557 p. 6un.

Vespa vulgaris Viallanes, Ann, sc. nat. Zool. (i) II. 1887 p. 1; T. 1-6.

Vespa rulgaris Marchal, Feuill, jeun. natural. XIX. 1889 p. 158.

Vespa vulgaris Konow, Soc. entom. IV. 1890 p. 175 n. 4, ơ.

xanthocera Ill. - Afr.: Sierra Leone.

Vespa xanthoceros Illiger, Magaz. f. Insectenk. I. 1802 p. 193 n. 22.

\section{PARAVESPA}

Radoszkowski, Horac soc. entom. Ross. XX. 1856 p. 44 ; 'T. 10 F. 48.

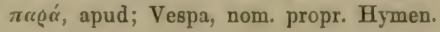

komaromiil Rad. - of-As.

Paravespa Komarowii Radoszkowski, Horae soc. entom. Ross. XX. 1856 p.45, ơ; T. 10 F. 45.

\section{SYNOECA}

Saussure, Ann. soc. entom. France (2) X. 1852 p. 549.

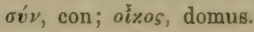

Synon.: Epipona Haliday.

Polistes Fabricius.

Vespa Linné \& auct. ant.

Zethus Blanchard.

azurea Sauss. - $9-$ Am. : Brasilia.

Synocea azurea Saussure, Ann. soc. entom. France (2) X. 1552 p. 554 n. 3. 9.

Synoeca azurea Saussure. Fitud. fam. Vespid. II. Vesp. 1553 p. 160 n. 3, Q.

chalybaea Sauss. - $P$ - Am.: Cayenne.

Synoeca chalybaca Saussure, Ann. soc. entom. France (2 X. 1552 p. 556 n. 6..

Synocea chalybea Saussure, Etud. fam. Vespid. II. Vesp. $1 \$ 53$ p. 161 n. 6, P; 'T. 18 F. 5. cyanea (Fabr.) Sauss. - 9 Q ơ - Am. : Cayenne.

Vespa cyanea Fabricius, Syst. entom. 1775 p. 372 n. 45.

Vespa cyanea Fabricius, Spec. Insect. I. 1781 p. 469 n. 61.

Vespa cyanea Fabricius, Mant. Insect. I. 1787 p. 293 n. 78.

Vespa cyanea Gmelin, Linné: Syst. nat. Ed. $13^{a}$ I. 5.1790 p. 2754 n. 61.

Vespa cyanea Christ, Naturg. d. Insect. 1791 p. 227.

Vespa cyanea Olivier, Encycl, méthod. Insect. VI. 1791 p. 674 n. 22.

Vespa cyanea Fabricius, Entom. system. II. 1793 p. 252 n. 99.

Polistes cyanea Fabricius, Syst. Piez. 1804 p. 279 n. 47.

Vespa cyanea Jurine, Nouv. méth. class. Hymén. 1807 p. 172, \&.

Polistes cyanea Lepeletier. Eneyel. méthod. Insect. X. 1525 p. 171 n. 3. ᄃ . j.

Tipipona cyonea Haliday. Trans. I.inn. Soc. Jondon XVIl. $18: 5$ p. 322 n. 27.

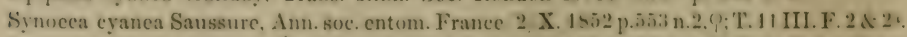

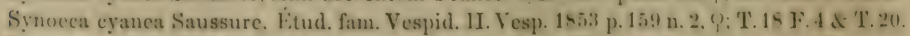

Synoeca eyanea Mobius, Abh. naturw. Ver. Hamburg III. 1 S.56 p. 122 (p. a); T. 1.

Surinama $(\dot{\mathrm{L}}$.) Sauss. $-\rho Q P-\Lambda \mathrm{m}$.: Cayenne.

Vespa Surinama Linné, Syst. nat. Ed. 12a I. 2.1767 p. 952 n. 23. 
Vespa Surinama Ph. L. Müller, Linné: Vollst. Natursyst. V. 2. 1775 p. 886 n. 23.

Vespa Surinama Gmelin, Linné: Syst. nat. Ed. 13a I. 5. 1790 p. 2759 n. 23.

Vespa Surinamensis Christ, Naturg. d. Insect. 1791 p. 215.

Vespa nigricornis Olivier, Encycl. méthod. Insect. VI. 1791 p. 675 n. 27.

Vespa Surinama Olivier, Encycl. méthod. Insect. VI. 1791 p. 676 n. 34.

Polistes coerulea Fabricius, Syst. Piez. 1804 p. 279 n. 46.

Vespa coerulea Jurine, Nouv. méth. class. Hymén. 1807 p. 171, ㅇ.

Polistes coerulea Latreille, Gen. Crust. \& Insect. IV. 1809 p. 142.

Zethus cocruleus Blanchard, Hist. nat. Insect. III. 1840 p. 394; T. 5 F. 9.

Srnoeca coerulea Saussure. Ann. soc. entom. France $(2$, X. 1852 p. 552 n. 1, Q.

Synocea Surinama Saussure, Litud. fam. Vespid. II. Vesp. 1853 p. 158 n. 1, Q.

testacen Sauss. - $Q-$ Am.: Brasilia.

Synoeca testacea Saussure, Étud. fam. Vespid. II. Vesp. 1853 p. 162 n. 7, Q.

ultramarina Sauss. - -9 ơ - Am.: Brasilia.

[F. 1-1b.

Synoeea ultramarina Saussure. Ann. soc. entom. France (2; X. 1852 p. 554 n.4. c. ; T. 11 III.

Srnocea ultramarina Saussure. Étud. fam. Vespid. II. Yesp. 1853 p. 160 n. 4 , $Q$ f

violacea Sauss. $-9-$ Am.: Brasilia.

Synoeca violacea Saussure. Ann. soc. entom. France (2, X. 1952 p. 555 n. 5. Q.

Sỵoeca violacea Saussure. Kitud. fam. Vespid. II. Vesp. $1 \$ 53$ p. 161 n. 5. 9.

\section{LEIPOMELES}

Möbius, Abh. naturw. Ver. Hamburg III. 1856 p. 145 [p. 25]. $\lambda \varepsilon i \pi \omega$, linquo; $\mu \varepsilon \dot{\lambda} o s$, artus.

\section{lamellaria Möb. - $9-$}

I.eipomeles lamellaria Möbius, Abh. naturw. Ver. Hamburg III. 18.56 p. 145 p. 25). \&; T.17.

Leipomeles lamellaria Möbius. Arch. f. Naturg. XXII. 1. 1s5ti p. 330: T'. 12 F. 9.

\section{POLYBIA}

Lepeletier, Hist. nat. Insect. Hymén. I. 1836 p. 533 n. 3. $\pi o \jmath v ́ \beta \iota ⿻ s$, vividus.

Synon.: Agelaia Lepeletier, Hist. nat. Insect. Hymén. I. 1836 p. 535 n. 4.

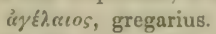

Alpha Saussure. Mém. soe. phỵs. \& hist. nat. Genòve XVII. 1. 1563 p. 239. ¿ littera.

Jo ta Saussure, Mém. soc. phys. \& hist. nat. Genève XVlI. 1. 1863 p. 240. $i$ littera.

Subg.: Cly pearia Saussure, Étud. fam. Vespid. II. Vesp. 1853 p. 165. clypeus.

Myrapetra White, Ann. \& Mag. Nat. Hist. VII. 1841 p. 320.

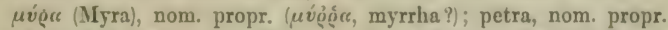

Pse u du poly bi a Saussure, Mém. soe physs. d hist. nat. Genève XVII. 1.18635 p.237.

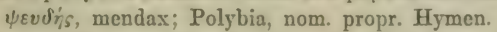

Rhopalidia Lepeletier, Hist. nat. Insect. Hymén. I. 1836 p. 538 n. 6. ¡órสch.ov, pistillum.

Chartergus Mübius.

Polistes Fabricius \& auct. plur. ,

Vespa Deeger, Retzius, Olivier \& auct. ant.

albopicta Smith - $\subsetneq-$ Am.: Brasilia.

Polybia albopicta Smith, Catal. Hymen. Brit. Mus. V. 1857 p. 131 n. 54, ᄋ.

Catalogus Hymenopteroram. IX. 
ampullaria Möb. - $9-$ Am.: Brasilia.

Polỵbia ampullaria Mübius. Abh. naturw. Yer. Hamburg III. 1556 p. 130 ip. 1:3 . Q; T. 7.

Polỵbia ampullaren Möbius. Arch. f. Naturg. XXII. 1. 145ł; p. 330; T. 12 F. 9.

anceps Sauss. - Am.: Brasilia.

Polybia aneeps Saussure. Citud. fam. Vespid. II. Vesp. 1553 p. 190 n. 31 .

augulata (Fabr.) Sauss. - 9 - Am.: Brasilia.

Polistes angulata Fabricius, Syst. Piez. 1804 p. 275 n. 32.

Polybia angulata Saussure, Etud. fam. Vespid. II. Yesp. $145 . ;$ p. 185 n. 24. ?.

angulicollis (Spin.) Sauss. - + - Am.: Brasilia.

Polistes angulicollis Spinola, Yem. acead. se. Torino :2. XIII. 15.51 p. 7 n. 55, c.

Polybia angulicollis Saussure. Citud. fam. Vespid. II. Vesp. 15.53 p. 19.4 n. 23. S; T. 23 F.3.

apicipennis (Spin.) Sauss. - 우 - Am.: Brasilia.

Polistes apicipennis Spinola. Mem. accad. se. Torino 2) XIII. 15.11 p. 75 n. 55, C.

Polybia (Clypearia, apicipennis Saussure, Etud, fam. Vespid. II. Vesp. 1 -53 p. 166 n. 1. ᄃ; T. 14

areata (Say) Sauss. - Am.: Mexico.

Polistes areata Say, Boston Journ. Nat. Hist. I. 4. 1837 p. 388 n. 2.

Polybia areata Saussure, Étud. fam. Vespid. II. Vesp. 1853 p. 210 n. 2.

Polistes areata Leconte, Writ. of Th. Say Entom. II. 1859 p. 768 n. 2.

argentina Berg - 우 0 - Am.: Patagonia.

[T. 2 F. 19 ,

Polybia Argentina Berg. Inform. Comie. Exped. Rio Negro. Zool. P. 1.1841 p. 111 n.115;

Polybia Argentina Berg. Stettin. entom. Zeitg. XLII. 1-81 p. fis n. 116, $\subseteq 3$.

artifex Smith - 우 - As.: Celebes.

Polybia artifex Smith, Journ. of Proc. Iinn. Soc. Zool. IV. 1 s6 Suppl. p. 90 n. 1. S. atra (Oliv.) Sauss. - 9 o 0 - Am.: Cayenne.

Vespa atra Olivier, Encycl. méthod. Insect. VI. 1791 p. 674 n. 20.

Polistes ignubilis Haliday. Trans. I.inn. Soc. London XVII. 3. 15336 p. 322 n. 25.8.

Polybia atra Saussure. Vitud. fam. Vespid. II. Vesp. 1553 p. 151 n. 20. 9 3; T. 24 F. 5. auriehaleea Sauss. $-9-$ Am.: Brasilia.

Polýbia aurichalcea Saussure. Fitud. fam. Vespid. II. Vesp. 15.53 p. 175 n. 15.9.

bicolor Smith - 요 Am.: Brasilia.

Polybia bicolor Smith, Catal. Hymen. Brit. Mus. V. 1857 p. 131 n. 55, 여.

bifasciata Sauss. - - - Am.: Brasilia.

Polybia bifasciata Saussure. Etud. fam. Tespid. II. Vesp. 15.53 p. 172 n. 7. ․ : T. 22 F. 3.

bohemaunii Holmgx. - - - Ins. St. Joseph.

Polybia Bohemanni Holmgren. Eugenies Resa. Insect. 1564 p. 411 n. 102. S.

brunea (Curt.) Sauss. - $Q-$ Am.: Brasilia.

Myrapetra brunnea Curtis, Trans. Lim. Soe. London XIX. 1. 1 \$4 p. 25ti n. 4 : T. 31 F. s.t. 4.

Polybia brumea Saussure, Etud. fam. Vespid. II. Vesp. 1 sis p. 211 n. 3, ?.

earbonaria Sauss. - 으 - Am. : Brasilia.

Polybia carbonaria Saussure. Etud. fam. Vespid. II. Vesp. 1 53 p. 194 n. 40. L: T. 26 F. 5. entillifex Müb. - Am.: Brasilia.

Polybia catillifex Mobius, Abh. naturw. Ver. Hamburg III. 15:36 p. 13:3 p. 16: : T. s.

elrysothorax (Web.) Sauss. - $q 9$ - Am.: Cayenne, Brasilia.

Vespa chrysothorax Lichtenstein, Catal. Mus. Zool. III. 1796 p. $20 ;$ n. 174.

Vespa chrysothorax Weber, Observ. entom. 1801 p. 103 n. 9.

Polistes aurulenta Fabricius, Syst. Piez. 1804 p. 275 n. 31.

Vespa aurulenta Jurine, Nouv. méth. class. Hymén. 1S07 p. 171, \&.

Pulplia chrysothoras Saussure, Citud. fam. Vespid. II. Inij3 p. 17s n. 16. 9: T. 24 F. 3. constructor Sauss. $-9-$ Am. mer.

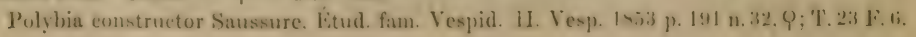


cordata Smith - ․ - Am.: Brasilia.

Polybia cordata Smith, Catal. Hymen. Brit. Mus. V. 1857 p. 129 n. 41, ㅇ.

cubensis Sauss. - 9 Q o - Am.: Cuba, Antillae.

$[\mathbb{R} 6(3)$

Polybia Cubensis Saussure. litud. fam. Tespid. II. Vesp. 14.33 p. 202 n.46. c ;: T. 2.5 F. . S S

Polybia Cubensis Saussure, Sagra: Hist. fis. Cuba 1856 p. 774, 9.

Polybia Cubensis Fox, Entom. News I. 1890 p. 93.

decorata Smith - \&-As.: Borneo.

Polybia decorata Smith. Journ. of P'roc. Limn. Soc. Zool. II. 14.77 p. 114 11. 4. $\leq$.

diligens Smith - 으-Am.: Brasilia.

Polybia diligens Smith, Catal. Hymen. Brit. Mus. V. 1857 p. 132 n. 56, ㅇ.

dimidiata (Oliv.) Sauss. - $Q-$ Am.: Cayenne.

Vespa dimidiata Olirier, Encycl. méthod. Insect. VI. 1791 p. 675 n. 28.

Polybia dimidiata Saussure. litnd. fam. Vespid. II. Vesp. 1553.3 p. 177 n. 1\%. Q.

emaciata Luc. - Am.: Brasilia.

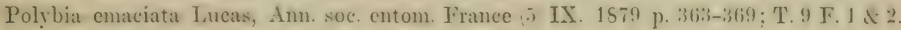
enxius Smith - Q - Am.: Brasilia.

Polỵbia Enxius Smith. Trans. Entom. Soe. Iondon (3) I. 6. 1463 p. 511 n. 5. P.

exigua Saus. - $9-$ Am. : Brasilia.

Polybia exigua Saussure. Fitud. fam. Vespid. II. Tesp. 1553 p. 173 n. 9, : '1. 22 F. 1.

fasciata Lep. $-\Omega Q-$ Am. : Panama.

Polistes fasciata Lepeletier, Encycl. méthod. Insect. X. 1825 p. 171 n. 4.

Polybia fasciata Lepeletier, Hist. nat. Insect. Hymén. I. 1836 p. $53 \pm$ n. 2, Q.

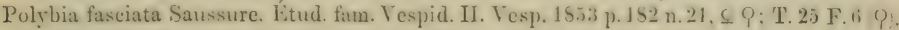

fastilliosnscula Sauss. - $ᄋ-$ Am. : Brasilia.

Polỵbia fastidiosuscula Saussure. Etud. fam. Vespid. II. Tesp. 1533 p. 197 n. 39. C : T. 25 T: 4.

filiformis Sauss. - 으- Am.: Brasilia.

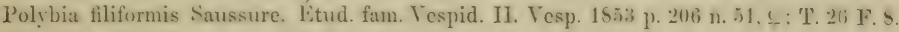

flaricans (Fabr.) Sauss. - $Q 9 \neq-$ Am. mer.

Polistes flaricans Fabricius, Syst. Picz. 1804 p. 276 n. 33.

Polistes testacea Fabricius, Syst. Piez. 1804 p. 276 n. 34.

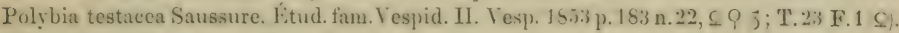

flavifrons Smith $-\Omega-A m$. Venezucla.

Polybia flavifrons Smith, Catal. Hymen. Brit. Mus. V. 1857 p. 152 n. 57, \&.

flaritarsis Sauss, - 요 - Am.: California.

Polybia flaritarsis Saussure. Litud. fam. Vespid. II. Vesp). 1453 1) 199 n. 42. C.

fulvofasciata (1)ge.) D. T. - ㅇ O - Am.: Surinam, Brasilia, St. Thomas.

Réaumur, Mém. hist. Insect. VI. 1742 p. 207 ; T. 14 F. 8.

Vespa fulvofasciata Degeer. Mim. hist. Insect. 1II. 1775 p. is n 4 : T. 29 F. S.

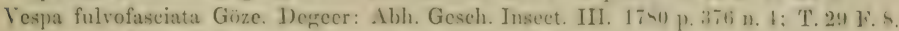

Vespa fulvofasciata Retzius, Gen. \& spec. Insect. 1783 p. 63 n. 235.

Vespa fasciata Olivier, Encycl. méthod. Insect. VI. 1791 p. 676 n. 35.

Vespa phthisica Fabricius, Entom. system. II. 1793 p. 281 n. 97.

Vespa spec. Lichtenstein, Catal. mus. Zool. III. 1796 p. 203 n. 175.

Vespa Cajennensis Fabricius, Suppl. entom. system. 1798 p. 265 n. 103.

Vespa C'ajennensis ('oupuebert. Illustr. iconogr. Insect. II. In01 p. 62: T. 15 F. 3.

Vespa ochrosticta Weber, Observ. entom. 1801 p. 103 n. 10.

Polistes phthisica Fabricius, Syst. Piez. 1804 p. 278 n. 42.

Polistes hectica Fabricius, Syst. Piez. 1804 p. 278 n. 43.

Polistes Cajennensis Fabricius, Syst. Piez. 1804 p. 280 n. 54.

Vespa Cajennensis Jurine, Nouv. méth. class. Hymén. 1807 p. 172, 오. 
Polistes fulvofasciatus Latreille. Gen. Crust. \& Insect. IV. 1 su9 p. 142.

Polistes fulvofasciatus Lepeletier. Hist. nat. Insect. Hymén. I. 1536 p. 5.34 n. 2 (p. p. . Polybia phthisica Saussure. Ktud. fam. Yespid. II. Vesp. 1553 p. $156 \mathrm{n}$ n. 26. . $0:$ T.23 F.7-5 is Polybia Cajennensis Möbius, Abh. naturw. Ver. Hamburg III. 1555; p. 124 p. 11 ; 'T. 5 \& 6. Polybia fulvofasciata $\mathrm{m}$.

fuscicoruis (Lep.) Sauss. - $9-$ Patria?

Agelaia fuscicornis Lepeletier. Hist. nat. Insect. Hymén. I. 1536 p. 336 n. 1. Q.

Agelaia fuscicornis Blanchard, Hist. nat. Insect. III. 1840 p. 396 n. 3.

Polybia fuscicornis Saussure, Étud. fam. Vespid. II. Vesp. 1553 p. 210 n. 1.

heydeniana Sauss. - - - Am.: Brasilia.

[n. 67, \&.

Polybia Jota Heydeniana Saussure. Mém. soc. physs. de hist. nat. Genère XVII. 1.156 .3 p. 241

indeterminabilis Sauss. - Am. mer.

Polybia indeterminabilis Saussure. Etud. fam. Vespid. II. Vesp. 1553 p. 201 n. 45.

indiea Sauss. - 으. As.: China.

Polybia Indica Saussure, Etud. fam. Vespid. II. Vesp. 1453 p. 207 n. 52 . ᄃ; T. 26 F. 3. infernalis Sauss. - 으. Am.: Brasilia.

Polybia infernalis Saussure. Étud. fam. Vespid. II. Vesp. 1853 p. 195 n. 36, S; T. 25 F. 3. injuenuda Sauss. - 우 - Am.: Brasilia.

Polybia injucunda Saussure, Etud. fam. Vespid. II. Vesp. 1553 p. 200 n. 43, ᄃ; T. 25 F. ๖.

irina (Spin.) Sauss. - 우 - Am.: Brasilia.

Polistes irina Spinola, Mem. accad. sc. Torino (2) XIII. 1551 p. 75 n. 56 , S.

Polybia irina Saussure, Étud. fam. Vespid. II. Vesp. 1853 p. 212 n. 4.

jurinei Sauss. - 우 - Am.: Brasilia.

Polybia Jurinei Saussure, Étud. fam. Vespid. II. Vesp. 1853 p. 176 n. 12, \&: T. 22 F. 6.

Iaboriosa Sauss. - 9 - Am.: Mexico.

Polybia laljoriosa Saussure, Etud. fam. Vespid. II. Vesp. 15.53 p. 171 n. 5, Q.

liliacea (Fabr.) Lep. - 우 9 - Am.: Cayenne.

Polistes liliacea Fabricius, Syst. Piez. 1804 p. 271 n. 10.

Vespa liliacea Jurine, Nouv. méth. class. Hymén. 1807 p. 171, ㅇ․

Polybia liliacea Lepeletier. Hist. nat. Insect. Hymén. I. $1 \$ 36$ p. 533 n. 1, \%.

Polybia liliacea Blanchard, Hist. nat. Insect. III. 1840 p. 396 n. 1.

Polỵbia liliacea Saussure, Etud. fam. Vespid. II. Vesp. 1553 p. 174 n. 10. ธ: T. 22 F. 7.

Polybia (I'ulistes liliacea Lucas, Ann. soc. entom. France 4 ; VII. 1867 p. 369-370; 'I' 9.

limatnla Smith - - - As.: Mysol.

Polybia limatula Smith, Journ. of Proc. Linn. Soe. Zool. VII. 1563 p. 43 n. 1, S.

Inetuosa Smith - 오 - As.: Borneo.

Polybia luctuosa Smith. Journ. of Proc. Iinn. Soc. Zool. II. 1557 p. 114 n. 3. S.

Iugubris Sauss. - $P-$ Am.: Guyana.

Polybia lugubris Saussure, Vitud. fam. Vespid. II. Vesp. 15.73 p. 140 n. 1s, (; T. 24 F. 2. mathematica Smith - 요 $-\Lambda$ s. : Colobes.

Polybia mathematica Smith. Journ. of Proe. Limn. Soc. Zool. IV. 1stio Suppl. p. 9(0 n. 2. . . metathoracien Sauss. - $\mathrm{Q}-$ Am.: Cayenne.

Polybia metathoracica Saussure. Citud. fam. Vespid. II. Vesp. I 4.33 p. 195 11. 41. S; T. 25 F. 1. mexieana Sauss. - Q - Am.: Mexico.

Polybia Mexieana Saussure, Litud. fam. Vespid. II. Vesp. 1853 p. 203 n. 47. ?: T. 26 F. 4. minutissima (Spin.) D. 'T. - Am.: Brasilia.

Rhopalidia minutissima Spinola, Osculati: Esplor. reg. equat. 1454 p. 2113 n. 13 [s. deser. . Polybia minutissima m.

minutissima Sauss. - $Q-A m$. mer.

Polybia minutissima Saussure. Vitud. fam. Vespid. II. Yesp) 1453 p. 170 n. 4. Q. 
multipicta (Hal.) Sauss. - + - Am.: Brasilia.

Polites multipictus Haliday. Trans. I,inn. Soe. London XVII. 3. 1836 p. 322 n. 29, \&.

Polybia multipicta Saussure, Kitud. fam. Vespid. II. Vesp. 1853 p. 1 ss n. $27, \mathrm{~s}$.

nana Sauss. - O - Am.: Brasilia.

[n. 66, \&; T. 2 F. 68.

Polỵbia Alpha nana Saussure. Mém. soe. physs. \& hist. nat. Genère XVII. 1. 1863 p. 240 occidentalis (Oliv.) Sauss. - $\subseteq$ Q ô - Am.: Brasilia.

Vespa occidentalis Olivier, Encycl. méthod. Insect. VI. 1791 p. 675 n. 31.

Myrapetra elegans Curtis. Trans. I.inn. Soc. London XIX. 1. 1544 p. 259 n. 5, Q; T. 31 F.9.

Polybia occidentalis Saussure. Etud. fam. Vespid. II. Vesp. 1553 p. 194 n. 35. s 9 of.

Polybia occidentalis W. F. Kirby. Ann. \& Mag. Nat. Hist. (5) XIII. 1584 p. 412 n. 44.

oecodoma Sauss. - $P-$ Am. : Brasilia.

Polybia oecodoma Saussurc, kitud. fam. Vespid. II. Vesp. 1853 p. 195 n. 37. O; T. 25 F. 7.

orientalis Sauss. - $\subseteq-$ As.: China.

Polybia orientalis Saussure. Etud. fam. Vespid. II. Vesp. 1853 p. 208 n. 53, \&; T. 26 F. 2. pallidipecta Smith - ô-Am.: Mexico.

Polybia pallidipectus Smith. Catal. Hymen. Brit. Mus. V. 1857 p. 125 n. 36. f .

pallidipes (Oliv.) Sauss. - 우 9 - Am.: Cayenne, Brasilia.

Vespa pallipes Olivier, Encycl. méthod. Insect. VI. 1791 p. 675 n. 30.

Rhopalidia pallens Lepeletier. Hist. nat. Insect. Hymén. I. 1836 p. 539 n. 2. Q.

Polistes Rhopalidia; pallens Spinola, Mem. acead. sc. Torino (2, XIII. 15.51 p. 79 n. 2.

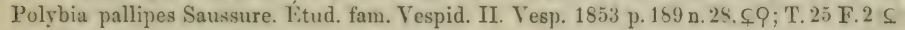

paraensis (Spin.) Sauss. - 우 - Am.: Brasilia.

Polistes Paraensis Spinola. Mem. acead. sc. Torino (2) XIII. 1551 p. 76 n. 57.5 .

Polỵbia Paraensis Saussure, Etud. fam. Vespid. UI. Vesp. 1853 p. 185 n. 25 . S; T. 23 F. 2. parvula (Fabr.) Sauss. - 우 ô - Am.: Mexico.

Polistes parvula Fabricius, Syst. Piez. 1804 p. 280 n. 55.

Polybia parrula Saussure, Kitud. fam. Vespid. II. Vesp. 1853 p. 196 n. $35 . \subseteq$ o .

pediculata Sauss. - 우 - Am.: Brasilia.

Polybia pediculata Saussure. Etud. fam. Vespid. II. Vesp. 1853 p. 205 n. 50. \&; T. 26 F. T.

Polybia pediculata Möbius. Abh. naturw. Ver. Hamburg III. 1856 p. $1: 36$ (p. 19; T. 11.

Polybia pediculata Möbius. Arch. f. Naturg. XXII. 1. 1856 p. 330; T. 12 F. 6.

pietetii Sauss. - 으-Am.: Columbia.

Polybia Picteti Saussure. Jitud. fam. Vespid. II. Vesp. 1553 p. 16 s n. 2 , S; T. 21 F. 2. plebeja Sauss. - 우 $\widehat{o}-$ Am.: Mexico.

Polybia plebeja Saussure. Reise d. Novara. Zool. II. 1. 1867 Hymen. p. 21 n. 1. $\subseteq \widehat{o}$.

pumila Sauss. - $\&-$ Am.: Brasilia.

[n. 65, $\Omega$.

Polybia (Alpha pumila Suussure. Mém. soe. phys. \& hist nat. Genère XVII. 1. 1 S63 p. 239

pygmaen (Fabr.) Sauss, - 우 9 ô - Am.: Cayenne.

Vespa pygmaea Fabricius, Entom. system. II. 1793 p. 283 n. 102.

Polistes pygmaea Fabricius, Syst. Piez. 1804 p. 280 n. 53.

Vespa pygmaea Jurine, Nouv. méth. class. Hymén. 1807 p. 172, ㅇ․

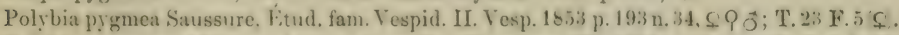

Polybia prgmaca Möbius. Abh. naturw. Ver. Hamburg III. 1 s.5t p. 134 p. 17 ; T. 9 \& 10.

Polybia pygmaea Smith. Trans. Entom. Soc. London 3, V. 4. 1566 p. 324,

quadrieineta Sauss. - \& $-\Lambda \mathrm{m}$. mer.

Polybia quatricincta Saussure, Etud. fam. Vespid. II. Vesp. 1553 p. 173 n. 8 , \&.

raphigastra Sauss. $-9-$ Am.

Polybia raphigastra Saussure. Etud. fam. Vespid. II. Vesp. 1853 p. 204 n. 49, P.

rejecta (Fabr.) Sauss. - $99-$ Am.: Cayenne.

lespa rejecta Fabricius, Suppl. entom. system. 1795 p. 264 n. 100-101. 
Polistes rejecta Fabricius, Syst. Picz. 1804 p. 280 n. 51.

[F. 4-6.

Polybia rejecta Saussure. Etud. fam. Vespid. II. Vesp. 15.53 p. 1 s n n. 19.5 P: 'T.24 F.5 \& T.29 Polybia rejecta Möbius. Abh. naturw. Ver. Hamburg IIl. 14.;fi p. 127 p. 16!; T. 4.

Polybia rcjecta Möbius, Arch. f. Naturg. XXU. 1. 1856 p. 330; T. 12 F. 5.

rufldeus Sauss. - 9 - Am.: Cayenne.

Polỵbia rufidens Saussure. Kitud. fam. Vespid. II. Vesp. 1653 p. 204 n. 45 . ?.

sanssurei Holmgr. - 오 $\not{0}-$ Am. : California.

Polybia Saussurei Holmgren, Eugenies Resa. Insect. 1465 p. 440 n. 111. \& 3.

scutellaris (White) Sauss. - \& 9 ô - Am.: Brasilia.

Myrapetra seutellaris White, Ann. \& Mag. Nat. Hist. VII. 1411 p. 322; T. \& F. 4-7.

Myrapetra scutellaris White. Ann. \& Mag. Nat. Hist. XII. 18.13 p. 264-270 \& 322; T. 4 F.4-7.

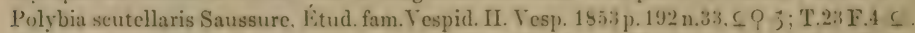

Chartergus scutellaris Möbius. Abh. naturw. Ver. Hamburg III. $15.51 j$ p. 140 (p. 23 ; : T. 15.

Polybia scutellaris Smith, Trans. Entom. Soe. I.ondon (3, I. 1 stil Proc. p. 29.

Polybia Mrrapetra) scutcllaris Lucas. Ann. soc. entom. France (1) VII. 1467 p. 365-364. Myraptera scutellaris I.ucas. Ann. soc. entom. France (6; $\mathrm{V}$. 154.; Bull. p. I.IV err. typogr.

Myrapetra scutellaris Duns. Proc. Phys. Soc. Edinbourgh X. 1549 p. 70-71.

sedula Sauss. - 09 - Am.: Brasilia.

Polybia sedula Saussure, F.tud. fam. Vespid. II. Vesp. 1853 p. 169 n. 3. Q ?; T. 21 F. 3-1i.

Polybia sedula Möbius. Abh. naturw. Ver. Hamburg III. 1556 p. 123 p. ti; T. 2.

Polybia sedula Möbius, Arch. f. Naturg. XXII. 1. 1856 p. 330; T. 12 F. 4.

Polybia sedula Desmarest. Chenu: Encycl. hist. nat. Annelles 1660 p. 140; Fig. 120.

sericea (Oliv.) Sauss. - 9 - Am.: Cayenne, Brasilia.

Vespa sericea Olivier, Encycl. méthod. Insect. VI. 1791 p. 675 n. 29.

Rhopalidia rufithorax Iepeletier. Hist. nat. Insect. Hymén. I. $14: 36$ p. $5: 39$ n. 1. ?.

Agelaia (Rhopalidia rufithorax Blanchard. Hist. nat. Insect. III. 14.40 p. $395 \mathrm{n} .1$.

Polybia sericea Saussure. litud. fam. Tespid. II. Tesp. 1 5.3 p.179 n.17.(P) 'T'. 21 F.1.'T.24 F.4 d.

Polybia serieca Möbius, Abh. naturw. Ter. Hamburg III. 1 s5tjp.125 p.8 : T.3. [T.29 F.1-i. simillima Smith - 으 - Am.: Panama.

Polybia simillima Smith. Trans. Fintom. Soc. I.ondon 3) I. 1. 146 fi2 p. 39 n. $6 . \varsigma$.

socialis Sauss. $-9-$ Am.: Brasilia.

Polỵbia socialis Saussure. litud. fam. Vespid. II. Vesp. 1553 p. 177 и. 14. P; T. 24 F. 1.

- stigma Smith - ơ - As.: Borneo.

Polybia stigma Smith, Journ. of Proc. Iinn. Soe. Zool. If. 1 557 p. 11.1 n. 2. 3.

sulenta Sauss. - $0-$ Am.: Brasilia.

Polybia sulcata Saussure. Jitud. fam. Vespid. II. Vesp. 1653 p. 175 n. 11. S; T. 22 F. 4. sumatrensis Sauss. - of - As.: Sumatra.

Polybia Sumatrensis Saussure, Rev. \& mag. zool. (2) VII. 1855 p. 374, đ.

snrinamensis Sauss. - 우 o ơ - Am.: Surinam, Brasilia.

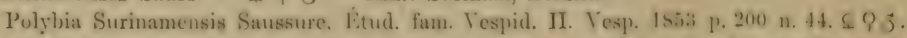

Polybia Surinamensis Saussure, Reise d. Nurara. Zoul. II.1.18tit II ymen.p. 21 n.2.5; T.1 F.16.

syeophanta Grib. - 으. Am.: Brasilia.

Polybia sycophanta Gribodo. Bull. soc. entom. Ital. XXIII. 14!11 p. 25: ؟.

sylveibne Sauss. - $99-$ Am. : Brasilia.

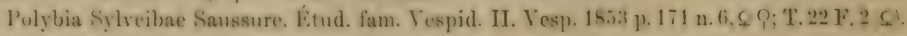

tabida (Fabr.) Sauss. $-\Omega-\Lambda$ fr.

Vespa tabida Fabricius, Spec. Insect. I. 1781 p. 468 n. 62.

Vespa tabida Fabricius, Mant. Insect. I. 1787 p. 293 n. 76.

Vespa tabida Gmelin, Linné: Syst, nat. Ed. $13^{3}$ I. 5. 1790 p. 2754 n. 59.

Vespa tabida Olivier, Encyel. méthod. Insect. VI. 1791 p. 673 n. 18.

Vespa tabida Fabricius, Lintóm, system. U. 1793 p. 282 n. 96. 
Polistes tabida Fabricius Syst. Piez. 1804 p. 278 n. 40.

I'olybia tabida Saussure, Litud. fam. Vespid. II. Vesp. $155: 3$ p. 209 n. 54, C; T. 26 F. 4. vespiceps Sauss. - 우 - Am.: Brasilia.

[1863 p.237 n.61,0; T. 2 F. 27.

Polybia Pseudopolybia vespiceps Saussure, Mém. soc. phys. \& hist. nat. Genève XVII. 1.

vieina Sauss. - $Q$ - Am. : Brasilia.

Polybia vicina Saussure, Etud. fam. Vespid. II. Yesp. 1553 p. 159 n. 29, Q; T. 24 F. 7.

xanthopus Sauss. - $9-$ Am. : Mexico.

Polỵbia xanthopus Saussure, Étud. fam. Vespid. II. Vesp. 1853 p. 190 n. 31, ?.

\section{TATUA}

Saussure, Etud. fam. Vespid. II. Vesp. 1853 p. 213; T. 21 F. 1.

Nom. propr.

Synon.: Fpipona Latreille, Hist. nat. Crust. \& Insect. III. 1502 p. 363.

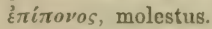

Polistes Fabricius, Latreille, Lepeletier, M. Edwards.

Vespa Cuvier, Fabricius, Jurine.

guérinii Sauss. - 9 - Am.: Mexico.

Tatua Guérini Saussure. Étud. fam. Vespid. II. Vesp. 1953 p. 215 n. 2, S.

morio (Fabr.) Sauss. - 우 9 ơ - Am.: Brasilia.

Vespa tatua Cuvier, Bull. soc. philom. Paris I. 1797 p. 57 n. 2; T. 1 F. 1.

Vespa morio Fabricius, Suppl. entom. system. 1798 p. 264 n. 98-99.

Epipona tatua Latreille, Hist. nat. Crust. \& Insect. III. 1802 p. 364.

Polistes morio Fabricius, Syst. Piez. 1804 p. 279 n. 45.

Epipona tatua Latreille, Hist. nat. Insect. XIII. 1805 p. 350 n. 2.

Epipona tatua Latreille, Gen. Crust. \& Insect. I. 1806; T. 14 F. 5.

Vespa morio Jurine, Nouv. méth. class. Hymén. 1807 p. 171, ㅇ.

Epipona morio Illiger, Magaz. f. Insectenk. VI. 1807 p. 197.

Polistes morio Latreille, Gen. Crust. \& Insect. IV. 1809 p. 142.

Polistes tatua Lamarck, Hist. nat. anim. s. vert. IV. 1817 p. 91 n. 7.

Polistes morio Lepeletier. Encỵcl. méthod. Insect. X. 1825 p. 171 n. 2. S J .

Epipona tatua Latreille, Cuvier: Règne anim. V. 1829 p. 339.

Polistes tatua Lamarck, Hist. nat. anim. s. vert. Ed. 2a IV. 1835 p. 306 n. 7.

Epipona tatua Lepeletier. Hist. nat. Insect. Hymén. I. 1536 p. 510 n. 1. ? ja.

Epipona morio Blanchard, Hist. wat. Insect. III. 1840 p. 395.

Polistes morio M. Edwards, Ann. soe. entom. France (2) I. 1443 Bull. p. XXXIV-XXXY.

Polistes morio Curtis, Trans. Linn. Soc. London XIX. 1844 p. 258 n. 6.

Vespa (Epipona morio Blanchard. Cuvier: Regne anim. Ed.3a Insect. II. 14.19; T. 124 F. 7.

Tatua morio Saussure, Eitud. fam. Vesplid. II. Vesp. 1453 p. 214 n. 1, f; T. 31 F. 1 \& 32.

Vespa Tpipona) morio Duméril. Mém. acad. se. Paris XXXI. 1460 p. ati3 n. 9.

quadritubereulata Grib. - $q-$ As.: Saryaku.

Tatua quadrituberculata Gribodo. Bull. sece. entom. Ital. XXIII. 14!1 p. 251, S.

\section{CHARTERGUS}

Lepeletier, Hist. nat. Insect. Hymén. I. 1836 p. 542 n. 8.

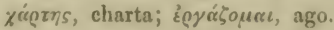

Synon, : Epipona Latreille, Illiger.

Polistes Latreille \& auct. plur.

Vespa Christ, Olivier \& auct. ant.

apicalis Fabr. Sauss. - $s$ ? - Am. : Mexico. Cayenne, Brasilia. Bolivia.

Vespa apicalis Fabricius, Syst. Picz. 1804 p. 260 n. 38. 
Vespa apicalis Jurine, Nouv. méth. class. Hymén. 1807 p. 170, 2.

Polistes apicalis G. R. Gray, Griffith: Anim. Kingdom XV. 1832 p. 575 ; T. 3 F. 3.

Chartergus ater Iepeletier. Hist. nat. Inseet. Hymén. I. 1536 p. 546 n. 1, Q.

Chartergus apicalis Saussure, Étud. fam. Vespid. II. Vesp. $14: 33$ p. 217 n. 1. ऽ; T. 31 F. .

Chatergus apiealis Möbius. Abh. naturw. Ver. Hamburg III. 1956 p. $1: 34$ p. 21 ; T. 13.

Chartergus apicalis Mübius, Arch. f. Naturg. XXII. 1.1556 p. 3:31; T. 12 'T. 7.

Chatergus apicalis Smith, Trans. Entom. Soc. London 1868 p. 135.

ater Sauss. - Am.: Brasilia.

Chartergus ater Saussure, Etud. fam. Vespid. II. Vesp. 14.53 p. 222 n. 7.

chartarius Oliv.) Sauss. $-\subseteq 9-$ Am.: Surinam. Mrasilia, Mexico, Cayenne, Guiana.

- Réaumur, Mém. hist. Insect. VI. 1742 p. ?; T. 20 F. 1-4, T. 21 F. 1 \& T. 22-24.

Vespa artifex Surinamensis Christ, Naturg. d. Insect. 1791 p. 226 ; T. 21 F. 1.

Vespa chartaria Olivier, Encycl. méthod. Insect. VI. 1791 p. 687 n. 88.

Vespa nidulans Fabricius, Entom. system. II. 1793 p. 271 n. 66.

Vespa nidulans Cuvier, Bull. soc. philom. Paris I. 1797 p. 58 n. 1; T. 1 F. 2.

Vespa nidulans Coquebert. Illustr. iconogr. Insect. I. 1799 p. 24; T. 6 F. 3.

Epipona nidulans Latreille, Hist. nat. Insect. III. 1802 p. 364.

Vespa chartifex Vallot, Concord. system. 1802 p. 171.

Vespa nidulans Fabricius, Syst. Piez. 1804 p. 266 n. 68.

Epipona chartaria Latreille, Hist. nat. Crust. \& Insect. XIII. 1805 p..349 n. 1; T. 102 F. 6 \& . .

Epipona chartaria Latreille, Gen. Crust. \& Insect. I. 1806; T. 14 F. 6.

Vespa nidulans Jurine, Nouv. méth. class. Hymén. 1807 p. 170, \&.

Epipona nidulans Illiger, Magaz. f. Insectenk. VI. 1807 p. 197.

Polistes nidulans Latreille, Gen. Crust. \& Insect. IV. 1809 p. 141.

Polistes chartaria Lamarck, Hist. nat. anim. s. vert. IV. 1817 p. 90 n. 6.

Polistes nidulans Lepeletier, Eneyel. méthod. Insect. X. 1525 p. 171 n. 5.

Epipona nidulans Latreille, Cuvier: Règne anim. V. 1829 p. 339.

Polistes chartaria Iamarek. Hist. nat. anim. s. vert. Ed. 2 a 1535 p. 306 n. 6.

Chartergus nidulans Lepeletier. Hist. nat. Insect. Hỵmén. I. 1536 p. 516 n. 2, ?.

Chartergus nidulans Blanchard, Hist. nat. Insect. UII. 1840 p. 395.

Epipona chartaria Curtis, Trans. Linn. Soc. London XIX. 14.4 p. 25 s n. 4.

Polistes nidulans Guérin, Iconogr. règn. anim. VII. Insect. 1545 p. 417; T. 72 F.7. T.33.

Chartergus chartarius Saussure, Etud. fam. Vespid. II. Vesp. 15.33 p. 220 n. $5, \subseteq$ P;T.31 F. $4 \subseteq$ \&

Chartergus chartarius Desmarest, Chenu: Eneycl. hist. nat. Annelles $1660 ;$ T. 11 F.1 \& . S) 5.

Vespa nidulans Duméril. Mém. acad. sc. Paris XXXI. 15 sio p. $\$ 63$ n. 10.

colobopterus (Web.) Sauss. - 으 - Am.: Surinam, Columbia.

- Seba, Descript. Curios. nat. IV. 1765 ; T. 98 \& 99.

- Lichtenstein, Catal. mus. Zool. III. 1796 p. 202 n. 159.

Vespa coloboptera Weber, Observ, entom. 1801 p. 102 n. 5.

Chartergus colobopteru* Saussure. Kitud. fam. Vespid. II. Vesp. 1853 p. 215 n. 3. \&; T. 31 F.2.

Chartergus colohopterus Mübius, Abh. naturw. Ver. Hamburg III. 15.56 p. 137 p. 20 ; T. 12.

compressus Sauss. $-q-$ Am.: Brasilia.

Chartergus compressus Saussure, Litud. fam. Vespid. II. Vesp. 1 - 33 p. 22.3 n. 9, f; 'T. 31 F. t.

concolor Grib. - - - Am.: Venezuela.

('hatergus conenlor Gribodo. Bull. soe. entom. Ital. XXIII. 1894 p. 25i, S.

emortualis Sauss. - Am.: Brasilin.

Chartergus emortualis Saussure, Rev. \& mag. zool. (2) VII. 1855 p. 374.

Praternus Grib. - $\rho-$ Am.: Brasilia, Cayenne.

Chatergus fraternus Gribodo. Bull. soc. entum. Ital. XXIII. 1591 p. 255, 5. 
frontalis (Fabr.) Möb. - Am. mer.

Vespa frontalis Fabricius, Syst. Piez. 1804 p. 265 n. 65.

Clhatergus frontalis Mübius, Mbh. naturw. Ver. Hamburg III. 15:23 p. $11: 3$, p. 23; ;' 1 I.

fulgidipennis Sauss. $-\propto-$ Am. : Brasilia.

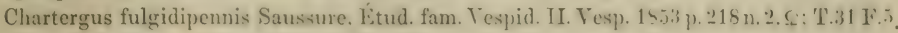

globiventris Sauss. - 우 - Am. : Brasilia.

Chartergus glubirentris Siussure. Jitud. fam. Vespid. II. Vesp. 1553 p. 221 n.fi, . ; T. 31 F. 3

Chatergus globiventris Gribodo, Bull. soc. entom. Ital. XXIII. I4!!1 p. 2:is.

Iuctuosus Smith — 우 - Am.: Brasilia.

Chartergus luetuosus Smith. Catel. Hymen. Brit. Mus. Y. 15.57 p. 135 n. 11 . ᄃ.

Chatergus luctuosus Gribodo, Bull. soc. entom. Ital. XXIII. 1891 p. 257, ㅇ.

sericeus (Fabr.) Möb. - Am. mer.

Vespa sericea Fabricius, Syst. Piez. $180 \pm$ p. 266 n. 69.

Chatergus sericeus Möbius, Abh. naturw. Ver. Hamburg III. 1S5if p. 141 p. 2.4; 'T. 16. smithii Sauss. - ô - Am.: Brasilia.

('hartergus Smithii Saussure. Ėtud. fam. Vespid. II. Tesp. 1553 p. 219 n. 4. ô.

zonatus Spin. - 으 - Am.: Brasilia.

Chartergus zonatus Spinola. Mem. accad. sc. Torino (2) XIII. 1551 p. 73 n. $5: 3.4$.

(Chartergus zonatus Saussure. Étud. fam. Vespid. II. Vesp. 1853 p. 222 n. \&.

\section{NECTARINIA}

Shuckard, Cab. Cyclop. Hist. Insect. 1840 p. 181 [Nectarina].

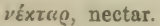

Synon.: Brachygastra Perty. Delect. anim. artic. Brasil. 1533 p. 146 [nec Brachygaster

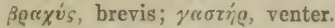

Polistes Latreille.

Vespa Saint Hilaire.

analis (Perty) Shuck. - $9-$ Am.: Brasilia.

Brachygastra analis Perty. Delect. anim. artic. I3rasil. 1833 p. 146; 'T. 28 F. 6.

Nectarina analis Shuckard, Cab. Cyclop. Hist. Insect. 1840 p. 183.

Nectarinia analis Guérin. Iconogr. règn. anim. VII. Insect. 1845 p. 144: T. 25 F. 6.

Nectarinia analis Saussure. E.tud. fam. Vespid. II. Vesp. 1853 p. 230 n. 6, $Q$.

angusti Sauss. - 9 - Am.: Mexico.

Nectarinia Augusti Saussure. Kitud. fam. Vespid. II. Vesp. 1 s.i3 p. 233 n. 11. 9 .

Nectarina Augusti Smith, Catal. Hymén. Brit. Mus. V. 1857 p. 137 n. 10.

Nectarina Augusti Gribodo, 33ull. soe. entom. Ital. XXIII. 1591 p. 254. 9.

aurulenta (Er.) Smith - Am.: British Guyana.

Brachygastra aurulenta Rrichson. Schomburgk: Reisen in Guiana UI. 14k p. 590.

Nectarina aurulenta Smith, Catal. Hymen. Brit. Mus. V. 1857 p. 137 n. 11.

azteca Sauss. - $\mathrm{Q}-\mathrm{Am}$. : Mexico.

Nectarinia Azteca Saussure, Rev. \& mag. zool. (2) IX. 1857 p. 280.

Yectarinia Azteca Saussure. Reise d. Novara. Zool. II. 1. Isti7 Hrmen. p. 23 n. 2. ᄃ.

bilineolata (Spin.) Sauss. - \& 9 - Am.: Cayenne.

Brachygastra bilineolata Spinola. Ann. soc. entom. France X. 1811 p. 126 n. 76. $\subsetneq$.

Nectarinia bilineolata Saussure. Etud. fam. Vespid. II. Vesp. 155.3 p. 22 n n. 3, (8; T. 34 F. 2.

Nectarina bilineolata Smith, Catal. Hymen. Brit. Mus. V. 1457 p. 156 n. 3.

Nectarinia bilineolata 1)esmarest. (henu: Eneyel. hist. nat. Annelles 18601 p. 1411 ; Fig. 122.

binotata Sauss. - ơ - Am.: Cayenne.

Nectarinia binotata Saussure, litud. fam. Vespid. II. Vesp. 1553 p. 230 n. .. 3.

Nectarina binotata Smith, Catal. Hymen. Brit. Mus. V. 1857 p. 136 n. 5. 
chartergoides Grib. - $0-$ Am.: Cayenne.

Nectarina chartergoides Gribodo, Bull. soe. entom. Ital. XXIII. $14 ! 1 !$ p. 25:3 ᄃ. leeheguana (Latr.) Sauss. - \& $9-$ Am. : Brasilia.

Polistes Lecheguana Jatreille, Mém. mus. list. nat. XI. 1524 p. 313-315 \&. XII. T. 12 Fig. B.

Polistes Lecheguana Lassaigne, Mém. mus. hist. nat. XI. 1524 p. 319-3210.

Polistes Lecheguana Latreille, Ann. sc. nat. IV. 1825 p. 335.

Vespa Lecheguana Saint Hilaire. Mém. mus. hist. nat. XII. 1525 p. 293-345.

Polistes Brachygastra: Iecheguana White, Ann. \& . Mag. Nat. Hist. VII. 1541 p. 319 nota.

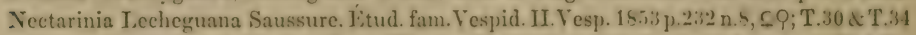

Nectarina Lecheguana Smith. Catal. Hymen. Brit. Mus. Y. 1457 p. 136 n. s. F. 3 Q. melliflea (Say) Sauss. - 우 o - Am. : Mexico.

Polistes mellifica Say. Boston Journ. Nat. Hist. I. 4. $15: 37$ p. 390 n. $4 . \subseteq 5$ J.

Nectarinia mellifica Saussure, Etud. fam. Vespid. II. Yesp. 18.73 p. 233 n. $4 . \subseteq$ g.

Nectarina mellifica Smith, Catal. Hymen. Brit. Mus. V. 1857 p. 137 n. 9.

Polistes mellifica Leconte, Writ. of Th. Say Entom. II. 1859 p. 769 n. 4.

moehiana Sauss. - \& - Am.: Surinam.

Nectarinia Mübiana Saussure, Reise d. Norara. Zool. II. 1. $156 ; 7$ Hymen. p. 22 n. 1, S. rufiventris Sauss. - $0-$ Am.: Brasilia.

Nectarinia rufiventris Saussure, Etud. fam. Vespid. II. Vesp. 1453 p. 226 n. 1, S.

Nectarina rufiventris Smith. Catal. Hymen. Brit. Mus. V. 1857 p. 136 n. 1.

seutellata (Spin.) Sauss. - 우 $9-$ Am.: Brasilia.

Brachygastra scutellata Spinola. Mem. accad. sc. Torino (2) XIII. 1851 p. 74 n. 54, S. Nectarinia scutellata Saussure, Etud. fam. Yespid. II. Yesp. 1553 p. 227 n. 2 , 9.

Nectarina scutellata Smith, Catal. Hymen. Brit. Mus. V. 1857 p. 136 n. 2.

Nectarinia scutellata Gribodo. Bull. soc. entom. Ital. XXIII. 1591 p. 254. S.

smithii Sauss. - - - Am.: Brasilia.

Nectarinin Smithii Saussure, Ettud. fam. Tespid. II. Vesp. 1453 p. 229 n. 4. \&; T. 31 F. ५.

Nectarina Smithii Smith, Catal. Hymen. Brit. Mus. V. 1857 p. 136 n. 4.

velntina (Spin.) Sauss. - $09-$ Am. : Cayenne, Brasilia.

Brachygastra velutina Spinola. Aun. soc, entom. France X. 1541 p. 126 n. $77 . \mathrm{S}$.

Xectarinia velutina Saussure. Etud. fam. Vespid. II. Vesp. 1553 p. 231 n. i. P.

Nectarina velutina Smith, Catal. Hymen. Brit. Mus. V. 1857 p. 136 n. 7. 


\section{REGISTER.}

Abbreviata 5

abbreviatus 5, 6 . abdelkader 50 . abdominale 42.

abdominalis 16,17 , 34,42 .

aberrans 105, 117.

Abispa 41.

abyssinica 34 .

abyssinicum 43 .

acolhuus 50 .

actaeon 122 .

acuminata 17.

acuminatus 17 .

acutus 50 .

adialbatus 50 .

advena 50.

aegyptiaca 152 .

aegyptiacus 50 .

aeneipennis 84 .

aenigmatica 113 .

aequatorialis 34 .

aequinoctialis 50 .

aestimandus 50 .

aestuans $34,43,50$.

aethiopica 17,34 .

acthiopicus 50.

afer 6.

affinis $34,85,136,137$.

affinissima 30 .

africana $46,131$.

africanum 46.

Agelaia 136, 161.

agilis $17,30,50,97$,

$113,114$.

agillima 17 .

alariformis 50 . alaris 50.

Alastor 109.

alastoripennis 50 .

Alastoroides 109.

alastoroidẹs 51,78 .

albicinctus 92, 122.

albifer 51 .

albifrons 110 .

albimacula 122 .

albocinctus 51, 110 .

albofasciata $7 \pi, 78$.

albofasciatus 105 .

albomarginatus 51 .

albophaleratus 51 .

albopicta 161 .

albopictus 9, 51, 105 .

albotrinctus 51 .

aldrichii 51 .

alduini 139 .

alecto 43 .

alexandrinus 51 .

alienus 51 .

allobrogus 51 .

alpestris 51, 52 .

Alpha 16, 38, 161.

alsatica 156 .

alternans 62 .

alvarado 52 .

amadanensis 52 .

amaliac 109.

ambiguus 52.

amedei 17,33 .

americana 5, 17, 126 ,

127.

americanus 17,126 , 127.

ammonia 72. amplissima 34 .

ampullaria 162 .

anaheimensis 122 .

analis $34,38,122,137$, 169.

anarchica 117.

anceps $38,43,162$.

Ancistroceroides 49 .

Ancistrocerus 42, 49 .

andeus 38 .

andreanus 52 .

andrei 17,52 .

anglica 144 .

angolensis 52.

angulata 162.

angulatus 52 .

angulicollis 110, 162 .

angulosa 40 .

angustatus 56 .

angustus 52 .

annectens 52 .

annularis $29,122,123$.

annulata 123, 137.

annulatum 52.

annulatus 52 .

annuliferum 43 .

anomala 40, 137.

anormis 52,53 .

Antagris 34 .

Antalastor 109.

Antalastoroides 109.

antennata 117.

Antepipona 49.

Anterhynchium 42.

Antezumia 38 ?

Anthrencida 121.

antillarum 53 . antilope 53.

Antodynerus 49.

antucensis 53 .

antuco 53 .

apachus 123.

apicalipennis 40 .

apicalis $53,123,167$.

apicatus 110.

apicipennis 162.

apiformis 6.

apilinguaria 137.

Apis 9, 34.

Apoica 136.

araucanus 54 .

arborea 136, 138.

arbustorum 17, 18,

26.

architecta 30 .

architectus 30 .

arcticus 62 .

arcuata 18.

arcuatus 54 .

ardens 43,46 .

areata 162 .

arenaria 137.

argentata 43.

argentatum 43.

argentifrons 110 .

argentina 162.

argentinus 54.

arietis 9.

arista 54

aristae 54

aristocratica 117.

armata 14.

armatus 54

armeniacus 54 . 
artifex $18,117,102$, 168.

aruanus 54.

arrensis 54 .

aสina 18.

askhabadensis 54 .

atavina 137.

ater $18,168$.

aterrimus $54,123$.

atlanticus 54.

atra $15,11 i, 162$.

atrata 18,137 .

atricornis $18,19,20$,

21.

atrofasciatus 5. .

atrohirtus 105 .

atrophica 123.

atropos 54, 110.

atrum 44.

attavina 137.

attenuata 16.

aucta $62,86,92,93$.

auctus 63,86 .

augusti 169.

augustus 54 .

nurantiacus 54 .

auraria 138.

aurata 19, 138.

auratus 19,81 .

aureocinctus 110 .

aureus 55.

aurichalcen 162.

murifer 123.

aurifrons 113.

auromaculatum 13.

auropilosa 19.

aumlens 9.

aurulenta 162, 169.

aurulentus 9.

australis $1,8,41,110$,

117.

austriacn 1:38.

austrinus 55.

avicula 19 .

azteca $19,38,169$.

aztecus 9, 19, 55,

105.

azurea (60).

azureipennis 40.

szurescens :38.
Bacu 55.

bacuensis 55 .

badia 123 .

badius 123 .

baeri 17.

bairdii 52 .

bairstowii 55 .

balder 123 .

ballionii 55 .

balteatum 65 .

balyi 55 .

basalis 72,138 .

bavarica 154 .

beckeri 55 .

belfragii 19 .

bellatulus 55 .

bellicosa 35,138 .

bellicosus 123 .

bellona 138 .

bellone 92 .

bellulus 55 .

Belonogaster 114.

bembeciformis 105 .

Bembex 136.

bengalense 43 .

bengalensis 123 .

bernardii 123 .

Beta 16, 38.

bezzii 105 .

biangulata 40 .

bicincta $19,55,117$, 138.

bicinctus 55 .

bicolor 1, 14, 40, 55, $77,117,121,123$, $139,162$.

bicoloratus 55 .

bicoloripenne 43 .

bidens 1, 55, 139.

bidentata 114 .

bidentatus 56, 114.

bifasciata $56,79,162$.

bifasciatus $51,56,97$.

bifidus 56 .

biglumis $9,53,105$, $123,124$.

biguttata 56,139 .

biguttatus 124 .

bilineolatn 169.

billardieri $12 \pi$.

biloba 139. bimaculata 21, 130 , 139.

bimaculatus 21 .

binodis 9, 10, 13.

binotata 139, 169 .

binotatus 125 .

bioculata 117 .

bioculatum 43 .

biphalcratus 56 .

biplagiatus 105 .

bipunctis 19 .

bipustulata 139 .

bipustulatus 56, 125 .

birenimaculatus 57 .

bisignata 19 .

bisignatus 19 .

bispinosa 19.

bispinosus 19, 57 .

bisstrigatus 57 .

bistriata 139 .

bisuturalis $5 \pi$.

bivittatus $50,6 \tilde{\imath}$.

bizonatus 55 .

blackburnii 57 .

blanchardianus 57 .

blanchardii 18 .

blanda 30 .

blandus 30,57 .

bohemanni 57,162 .

bollii 19 .

borealis $138,139,144$, 154.

boscii 57 .

bothriogaster 57 .

brachyeera 114 .

brachyeerus 114.

brachygaster 57 .

Brachygastra 169.

brachystoma 114.

brachystomus 114 .

brasilana 19 .

brasilanus 19.

brasiliensis 10,38 , $110,112$.

braunsii 114 .

bravo 57 .

brevipetiolata 114 .

brevipetiolatus 114.

brevirostrata 19.

brevirostratus 19.

brerithorax $57,58,81$. britannica 150, 151.

brunea $38,114,118$, 162.

bruncipes 140 .

bruneus 110 .

brunnea $38,45,118$; 162.

brunneum 45 .

brunneus $45,110,114$.

brunnipes 140 .

bucharensis 125 .

bucida 110 .

bulgaricus 58 .

bustamente $5 \mathrm{~S}$.

bustamenti 58 .

bustillos 52 .

bustillosi 52 .

buyssonii 58 .

Cabetii 118.

caelebs 58 .

caeruleopennis 10 .

caffer 2.

caffra 19.

cajennensis 163,164 .

calnbrensis 58 .

calabricus 58 .

calcaratus $5 \mathrm{~S}$.

caledonicus 58 .

calida 1, 35 .

californica 40.

californicus 58 .

Calligaster 9 .

callimorpha 19, 125 .

callimorphus 19,125.

callosus 58 .

campanaria 140 .

campaniformis 23.

campanulata 19.

campestris 58 .

canadensis 58, 125.

canaliculata 20.

canaliculatus 25,58 .

capensis $2,35,58,105$,

I1s.

capitata 35, 140.

capra 59.

captivus 59.

carbonaria 35,162 .

carbonarium 43.

carbonarius 10. 
cardinalis 59, 94 . earinata $35,118$. carinatus 10,111 . carinifer 59 . carinulata 38. carinulatus 59. earnatica 45. carnaticum 45. carnifex 125, 126. caroli 59 .

carolina 140 . caspicus 59 . castigatus 57. catepetlensis 59 . catillifex 162 .

Catilostenus 112. catskilii 59 . catskillensis 59 . cancasicus 2. cavapyta 126. cavifrons 40. eaviventris 59 . cayana 140.

Celonites 5. ceperoi 43.

Ceramioides 2.

Ceramius 2, 4. cerceriformis 2 . cervus 101. ceylonicus 10,59 . chalicodomac 20 . chalybaea 60 . chalybea 38,60 . chalybeus 10 . chartaria 168. chartarius 168 . chartergoides 170 . Chartergus 161, 167 . cherrieranus 59,106. chichimecus 59 . chiconteneatl 10. chilensis 15, 73. chiliotus 59, 60 . chinense 43. chinensis 60,124 . chlorostoma 126. chlorotica 5 . chloroticus 60 . Chrysis 5. chrysomallum 43. ebrysoptera 140 . chrysoptera - armata 140.

chrysopterus 10 . chrysothorax 20,162 . cilipennis 113 .

Cimbex 5 . cincta $20,118,122$, $140,141$. cincta-nigra 41 . cinctus 20. cinerascens 10,128 . 141.

cinerea 115 .

cinereus 115 . cingulata 20 . cingulatus 20,26, 60 . cingulifer 60 . circinalis 20 . circumspectus 60 . citrocinctus 60 . clarazianus 60 . claripennis 60 . clavata 118. clavicornis 60 . clipeata 141. clotho 111. cluniculus 60 . clusinus 60 . clypealis 60 . Clypearia 161. clypearis 10 . clypeata 11., 141. clypeatus $60,114$. coangustata 32,33 . coangustatus 33 . coarctata $20,21,23$, 29, 32.

coarctatus $21,2 \bar{\imath}, 30$, (ii).

coccineus 106.

coerulea 38, 161. coeruleopennis 10 . coeruleus 161. cognatus $61,75,111$. collega 61 .

coloboptera 168. colobopterus 165. colocolo 66,67 . colon 61 . colona 16. colonialis 111 . colonicus 126. coloradensis 21. columbaris 61 . comachus 126. combusta 35 . combustus 61 . communis 141 . compressa 21. compressus 21, 168. comptus 106.

concinna 1, 22. concinnulus 61 . concinnus 22 . concolor $61,168$. confluentus 61 . conformis 22,61 . confusus 126. congener 61 . congruus 61 . conica 22, 32. conoidea 141. conservator 118 . consobrina $22,137$. consobrinus $3,22,61$, 126.

consors 61 .

conspicuus 61,81 . constans 89. constitutionalis 118 . constructor 162 . copiaria 118. coquimbensis 83 . cordata 163. cordavae 61. cornuta $35,36,144$. coronata 30,121 . cortesia 39 . cortesiana 39. costac 50 . coxalis 93. coyotus 62. crabro $141,142,143$, 144.

crabro-analis 137 . crabro-media 149. crabroniformis 106 , 1.14.

crabro sphinx 137. crassa 149. crassicornis $62, \mathrm{~s}$. crassipes $36,144$. cratiata 19.

crenatus 62 .

crenulatus 6 .

cressoniana 22 .

cressonianus 22 .

cribratus 62 .

cribriformis 144

crinita 126.

crinitus 126.

cristatus 63 .

cruciata 19.

crucifera 22.

cruentata 63 .

cruentatus $63,111$. cruentus 63. cruralis 63 . crypticum 63. crypticus 63.

Ctenochilus 108.

cubensis 22, 63, 127, 163.

cubitalis 136.

cultus 63 .

cuneata 14.

cursor 32 .

curvata 22 .

curvatus 22.

curvipes 144.

cyanea 60.

cyanipennis $12,40$.

cyanoptera 10.

cyanopterum 43,44 .

cyanopterus 10 .

cyathiformis 22 .

Cyclostoma 122.

cylindricus 63 .

cyprius $\mathbf{6}$.

eyrtogaster 63 .

Dacdala 145 .

dacdalea 14t, 145 .

daedaleus 101.

dantici 63,64 .

deceptor 1, 118

decipiens 164 .

decorata 22.

decoratum 4.4 .

decoratus 22, 163.

decorus 106.

debilis 64 .

debilitatus (i.4. 
defectiva 127.

defectivus 127.

deflendus 64.

dejectus 64 .

delicatus 64 .

delphinalis 64 .

democratica 118

dentata 36, 145.

denticulata 64 .

dentipes 78 .

dentisquama 79,89 .

depressa 22.

depressus 22, 93.

designatus 64 .

destefanii 64 .

deusta 145 .

diabolica 145.

diabolicus 64, 127.

diadema 20,124, 145 .

dichotomum 42 .

dichroa 22.

dichrous 22.

dicomboda 10 .

didymogaster 11.

Didymogastra 9.

diemensis 64 .

dietrichianus 64 .

difficilis 66 .

diffinis 64 .

difformis 64 .

dilecta 7 .

dilectula 22 .

dilectus 65 .

diligens 22, 163.

dimidiata $17,39,115$,

163.

dimidiatipennis 22 .

dimidiatum 44.

dimidiatus $17,18,44$, 65.

diseoclioides 11 .

Discoelius 15, 49 .

discoidalis 99.

disconotatus 65 .

disjunctus 65 .

dispar 5.

distigma 115.

distinctn 22 .

distinctus 22.

distinguenda 114 .

distinguendus 11.1. diversus 65,82 .

dives 106.

dominula 128.

dorsale 65.

dorsalis 65 .

dorsata 127.

dorsatus 127 .

doryeus 23.

dorylloides 137 .

doryloides 137 .

dotatus 65 .

doursii 3,65 .

drewsenii65,113,117.

dromedarius 65 .

dubia 5, 6, 23, 36,

114, 118.

dubiosus 65 .

dubius $11,23,65$,

114, 127.

duealis 145 .

ductus 65 .

dufourianus 59 .

dufourii 15, 93 .

dumetorum 23, 33.

duplicatus 66 .

dybowskii 145 .

dyschera 23.

dyscherus 66 .

Ebusianus 66.

ecklonii 106.

edwardsii 4, 23.

egregia 41 .

egregius 66 .

electus 66 .

elegans 23, 66, 70,

114, $127,165$.

clegantula 23.

Elimus 8.

elongatus 11, 15.

emaciata 163 .

emarginata 36,86 ,

145.

emarginatus 86,111 .

emeryanus 66.

emortualis 66, 168.

enxius 163 .

cnyo 60.

cphippium 15,42, 66 .

Epipona 9) 15, 49, (ii) $109,117,167$.
Epiponus 49.

Epsilon 49.

erinnys 66.

eriurgus 111.

Erynnis 5.

erynnis 66.

erythrinum 44.

erythrinus $12 \bar{i}$.

erythrocephala 145 .

esuriens 23.

Eumenes 9, 15, 16, $38,49,105,114$.

Eumenestiferus 112.

Eumenidinae 7 .

eumenoides $7,8,66$, 114.

Euparagia 2.

europacum 47.

Eurynnis 4.

evectus 66 .

eversmannii 66 .

excellens 1.

excipienda $66,67$.

excipiendus 66.

excisus 67.

exigua 24, 163.

exiguus $24,67,90$.

exilis $67,128$.

eximius 24, 106.

exotica 145.

extensa 16.

extranaeus 67 .

extraneus $75,127$.

exustus 67.

Facialis 67 .

fncilis 127 .

fairmnirci 95.

fairmairi 67 .

fallnx 44, 67, 95, 100 .

farias $6 \pi$.

fariasi 67 .

fascinta $24,118,149$. 113.3.

fasciculatus 67 .

fastidiosissimus 67 . (i8.

fastidiosus $63,127$.

fastidiosuscula 163.

fastidiosuseulus 69 .

faustii 106. farillaceus 11.

femoratus 68 .

fenestralis 24 .

ferox 36.

ferreri 127.

ferruginea $24,39,118$, 145.

ferrugineitarsis 68 .

ferrugineum 44 .

ferrugineus $11,24$.

ferruginosus 68 .

fervens $25,44$.

fervida $36,145$.

fervidus 68 .

festina 118 .

figulus 68 .

filiformis $7,24,114$, 163.

filipalpis 68 .

filiventris 114, 116.

firmus 69.

fischeri 6.

fistulosus 68 .

flammiger 68.8

flareseens 145.

flaricans 163 .

flariceps 111, 146.

flaricincta 146, 149.

flaricornis 24 .

flarifrons 163.

flavipennis 127 .

flaripes $65,69$.

flavitarsis 163.

flarocinctus 65 .

flavolineatus 68 .

flavomarginatum 44 .

flavopectus 24.

flavopicta 18, 118.

flaropictus 18, 65 .

flaropunctatum 44.

flaropunctatus 44 .

flarus 69, 103, 113, 127.

fleckii 115 .

floralis 30 .

floricola $\mathrm{s}, 69$.

florisequa major 146.

florisequa minor 146.

fluctuans 24.

flurialis 69 .

fonseolombei 3. 
fonscolombii 3 .

foraminatus 69 .

formicaria 24.

formosa $24,26,118$.

formosus 69, 106.

fortunatus 129 .

fragilis 69 .

frater 69 .

fratercula 24.

fraterna 24.

fraternus 11, 24, 111, 168.

frauenfeldii 69 .

frendens 69.

frivaldskyi 17.

frontalis 156, 169.

fulgidipennis 113 , 169.

fulgipennis 113 .

fulva $25,146$.

fulvipennis 18,69 , $115,119$.

fulvipes $69,146$.

fulvofasciata 163 , 164.

fulvofasciatus 164 .

fulvus 25 .

fumipennis 69 .

fundatus 69 .

funcbris 41, 69 .

furax 44 .

fusca $11,128,151$.

fuscata 128.

fuseatus 128 .

fuscicornis 164 .

fuscipes $69,70,88$.

fuscus $11,70,128$.

fusus major 146 .

fusus minor 146 .

(Xalbula 146.

galimatia 119.

gallica 128, 129, 130 .

gallicus $70,124,128$, 130.

Gayella 8 .

gayi 70 .

gazella 70 .

geerii 149 .

geminus 70 .

generosus 130 . geniculata 11, 146. | hebraea 130.

geniculatus 11 .

geoffroyanus 85 .

geoffroyi 130 .

germainii 25.

germanica 146, 147.

germanicus 70 .

gestroi 44 .

ghilianii 25,39 .

gianellii 44 .

gigas $11,106$.

glabripalpis 109 .

globirentris 169 .

globulicollis 25 .

globulosa 29.

globulosus 25.

Gnatho 2.

gonioides 70 .

gracilis 11, 23, 24, $70,119$.

graeffei 111.

grandidieri 71, 119.

grandis 71, 106.

graphicus 71 .

grayi 44.

gregaria 119.

grisea 115.

griseus 115.

guadulpensis 71 .

guatemotzin 13.

guerinii $25,71,115$, 167.

guerreri 71 .

guerrero 71 .

guineensis $71,115$.

guttata 149 .

guttatipennis 119.

guttatus 71 .

guttulatus 80 .

guttulosus 71 .

guzmanii 71 .

Gyrostoma 122.

gyrostoma 133.

Haematodes 71.

haemorrhoidale 44, 45.

haemorrhoidalis 44 .

haleakalae 71 .

hannibal $\mathbf{7 1}$.

hawaiiensis 71 .

Hoplopus 49. hebraeus 130 .

hectica 163.

heerii 27.

hellenicus 106.

helveticus 72 .

helvetius 71,72 .

hero 11.

Heros 9.

herrichinnus 72 .

herrichii 72 .

hesperus 28 .

heydeniana 36, 164.

heydenianus 72 .

heydenii 11 .

hidalgi 72 .

hidalgo 72 .

hilarianus 11 .

hildebrandtii 72,115 .

hirsutissima 147.

hirsutulus 104.

hispanicus 106.

histrio 29,72 .

histrionicum 46 .

holosericeum 46 .

holsatica 156.

hongkongensis 119 .

hoplites 130 .

hoplitus 130.

Hoplomerus 49.

horticula 147 .

hospes 72 .

hottentotta 25 .

hottentottus 72 .

hova 119.

huasteca 39.

huastecus 72 .

hubertii 33,36 .

humbei 73 .

humbertiana 25.

humbertianus $8,73$.

humeralis 73,96 .

humilis $130,131$.

hungaricus 6,73 .

huro 73.

hyacinthae 73.

hyalinipennis 73 .

hylaciformis 4 .

Hypagris 34.

Hypalastor 109 .
Hypalastoroides 109.

Hypancistrocerus 49.

Hypodynerus 49 .

Japonica 25, 147, 149.

japonicum 46.

japonicus 25, 131.

javanum 45 .

javanus 11, 73.

ibericus 73 .

Icaria 117, 121.

ichneumonidea 94 .

ignobilis $73,119,162$.

imbecillus 73 .

imitator 12.

immaculata 144.

imperatrix 25.

impetuosa 119.

impulsus 73.

impuncta 25.

impunctatus 73 .

impunctus 25.

inca 12,73 .

incerta 25 .

incertus 25, 131, 134 .

incincta 41 .

incisoides 86 .

incisus 86 .

incommodus 73 .

inconspicua 30 .

inconstans 74 .

indeterminabilis 164 .

indiana 25.

indianus 25 .

indica $39,115,164$.

indieus 115 .

industrius 74 .

infernalis 25, 39, 111 , $147,164$.

inflexa 25.

inflexus 25.

infundibuliformis 29,

$32,33,39$.

infuseata 125 .

injucunda 16.1 .

innumerabilis 74 .

inornatus 74, 131.

insignis 15, 106.

insolens 26.

instabilis 74, 127, 128. 
insularis $26,74,77$, $111,147$.

insulicola $7.4,81$.

intendens 74 .

intermedia 109.

intermedius 109.

interrupta 74 .

interruptus 74, 95, 106.

intersecta 148.

intricatus 74 .

invisus 74 .

jocosus 74 .

jokahamae 131.

jonius 74 .

Jota 161.

iridipenne 46.

iridipennis 113.

irina 164.

irritata 119 .

Ischnogaster 113.

Ischnogasteroides

113.

italica 148.

iturbide 26.

iturbidi 74 .

jucundus 74 .

Jugurthia 5.

Jugurtia 5.

juncea 115.

junceus 115.

jurinei 12, 74, 152.

164.

Kalinowskii 74.

kennicotianus 75.

kirbyi 75 .

klugii 107.

kohlii 26.

kokpetieus 75 .

komarowii 60, 75 .

koreensis 3, 148.

künckelii 46.

Labiata $76 ; 148$.

labiatus $75,117$.

laborans 75 .

laboriosa 26, 164.

laboriosus 75 .

Iabus 8 .

lachesis $75,111$. lnevinodis 12.

lacripes 75,76 .

laeviventris 99.

lamellaria 161.

laminata 26.

laminatum 46 .

laminiger 75 .

lanio 125 .

lapicida 32.

laplatae 75.

laterale 46.

lateralis 46 .

lateritia 116 .

lateritius 111,116 , 131.

laticeps 106.

latipalpis 107 .

latipennis 75 .

lativentris 67 .

latreillei 26.

latreillii 26.

lecheguana 170.

lefebvrei 47, 119, 130 .

Leionotus 49, 109.

Lcipomeles 161.

lepeletieri 26.

lepricuri 39.

Leptochilus 49.

leptogaster 26.

leptogastra 26.

leucomelas 75 .

levinodus 12.

levipes 75 .

levis 26.

lewisii 107.

lichtensteinii :3.

ligata 148.

liliacea 39, 164.

liliaceusculus 131.

liliaciosa 39 .

lilinciosus 131.

limatula 164.

limbatum 46.

limbiferus 76.

lindenii 91.

linearis $3,116$.

lineata 127.

lineatus 127.

lineolata 136.

lobatulus 12.

lobitus $7 t$. localis 92.

longicornis 145 .

longirostris 26 .

longispinosus 76 .

longistyla 116.

lonisianum 65 .

louisianus 65.

lueasia 26.

lucasius 76 .

luctuosa 145, 164.

luctuosum 46.

luctuosus 76, 164.

lugubris 12,119, 164 .

lunulata 29.

lusitanicus 3.

lutea 148.

luteolus 76 .

Macaensis 131.

macilenta 115 .

macilentus 76 .

macrocephala 26, 39 , 148.

macrocephalus 3 .

macrops 25.

mactae 76 .

macularis 146 .

maculata 119, 146, 148, 149.

maculatus 91, 130.

maculipennis 76,131 .

maculiventris 111 ,

119.

madaern 76 .

madecassa $116,119$.

madecasss.e 46 .

madecasse 46.

madecassum 46 .

madecassus 116,131 .

maderae $i 6$.

madocii 131.

magnifica 149.

magnificum 46 .

magnificus $7 \pi$.

magnus 10.

magrettii 77 .

maindronii 77 .

mainpuriensis 26.

major $107,126$.

maipinus 77 .

maltac 76 . mamillatu 37 . :

mammillatus 77 .

mandariria 149.

mandarineum 46 .

mandarinus 131 .

mandiluularis $36,7 \tau$, 107.

manifestus 77,90 .

manillensis 131 .

marangensis 119 .

marginalis 4, 131, 132.

marginata 29, 119, 137.

marginella 21, 46 .

marginicollis 75 .

maroccanus 77 .

marribous 125.

marthae 39.

Masaridinae 1.

Masaris 2, 4, 5 .

massauensis 77 .

mathematica 164 .

matzicatzin 12 .

mauritanicus 77 .

maurus 52 .

maxillosa 26, 36 .

maxillosus 27 .

maya 77.

maypinus 77 .

mayus 77.

medanac 77 .

media $131,149$.

mediann 27.

medianus 27 .

mediterranea 27.

mediterraneus 27 .

medium 45.

megnera 77.

melanocephala 77,78 .

melanocephalus 77 ,

78.

melanocerus 78 .

melanochra 92.

melanosoma 27, 111,

132.

melanurus 78 .

melanus 78 .

mellifien 170.

membranaceus 78 .

mellyi $16,113$. 
menelikii 116.

mephisto 46 .

meridionalis 78,107 .

merula 15 .

mervensis 78 .

metallicum 43.

metathoracica 16.4 .

metathoracicus 78 .

Metazumia 38.

metemmensis 78 .

metrica 132.

metricus 132.

mexicana $12,27,39$,

164.

mexicanus $12,2 i$,

107, 111.

micado 78 .

micans 113.

micelii 37.

Micragris 34.

Microdynerus 49.

microdynerus 78 .

microgaster 13 .

microrrhoea 35 .

microseopica 27.

microscopicus 27.

miles $2 \pi$.

mina-palumboi 41 .

miniatus 13,79 .

minima 150 .

minor 132 .

minuta $25,27,37,79$.

minutissima 132,164.

minutissimus 132.

minutus $27,51,79$.

mirabile 46.

mirabilis 37 .

mirandus 107.

Mischocyttarus 117.

miscogaster 13.

mocsaryi 79.

modesta 119.

modestus 79,101 , 132.

moebiana 170 .

mohicanus 79 .

molestus 7 !).

molinae 79 .

molinius 79 .

Monerebia 11.

mongolica 21, 150. nigriculus 82.

mongolicus 7 .

Monobia 40, 49.

montanus 79 .

montezuma 13.

Montezumia 38, 40.

morawitzii 79 .

morelii 79 .

morelius 7 ?.

morio 167.

morosa 1, 39, 119.

morrisonii 107 .

mortuorum 40.

mucronata 150 .

mucronatus 80 .

multicolor 80,126 .

multipicta 27, 165.

multipictus $2 \bar{\imath}, 54$,

s0, 132, 165.

multispinosum 47.

muraria $62,80,98$.

murarius $80,81,98$.

mutabilis 47,81 .

mutans 81 .

muticus 81 .

mutillata 121.

mutilloides 8 .

myops 135 .

Myrapetra 161.

mysolicus 81 .

mysticus 81 .

Nahuus 81.

nana 27,165 .

nanus $2 \overline{7}$.

nasidens 81 .

nasuta 1.

natalensis $\tau, 8,81$.

nautarum 81, 111.

Nectarina 169.

Nectarinia 169.

neesii 2 i.

neglectus 81 .

nestor 132 .

nidulans 168 .

nidulator 81 .

niger 10.

nigra 27, 120, 150.

nigriceps 39.

nigricornis $13,78,81$, 161.

Catalogue Hymenopterorum. IX. nigrifrons \$2, 113, 132.

nigripenne 82 .

nigripennis $3,41,82$,

$125,132,150$.

nigripes 91,92 .

nigrocinctus 8:.

nigroviolacea 112.

nigroviolaceus 112 .

niloticum 47.

nimpha 84 .

nitidipennis 113 .

nitidula 47, 120.

nitidulum 47.

nobilis 82, 120 .

Nortonia 109.

nortoniana 27.

nortonianus 27,82 .

norvegica $150,151$.

normegica 150, 151.

notabilis 82 .

notata 82 .

notatus 82 .

notula 61,82 .

novajae 132.

novarae 27, 132.

nudus 82 .

nugdunensis 82 .

numida 5, 107.

numidicus 107 .

nyassae 47 .

Oahensis 82.

obliterata 151.

obliquus S2.

obseura 28.

obscurepunctatus 53.

obscuripennis 83 .

obscurum 47.

obscurus $17,28,83$, $1: 32$.

occidentalis 4, S3,

151, 165.

ochlerus 86.

ochrenta 151.

ochrosticta 165.

oculata 47 .

oculatum 47.

oculatus 52, 53, 133.

odontophora 37.

odyneroides $1, \mathrm{~S}$.
Odynerus 38, 40, 41 , 42, 49, 105, 109, $122,136$.

oecodoma 165 .

olivacea 28, 133.

olivaceus 28, 133.

olivieri 33 .

olmecus 13, 28, 83 .

Omicron 16.

Oncorrhinus 41 .

oncrata 126 .

opacus 83.

opalinus 133.

Oplomerus 49.

Oplopus 49.

opulenta 120.

oraniensis $3,5,83$.

orbignyi 28 .

orbitalis 83,88 .

ordinata 151 .

orenburgensis 83 .

orientalis $40,83,126$,

133, 151, 152, 165.

ornata 17,131 .

ornatum 47 .

ornatus $17,83,93$, $100,107$.

osseus 7.

otomitus 13,83 .

ovalauensis 28 .

ovalis 53 .

ovirentris 83,84 .

Pachodynerus 49 .

Pachymenes 16.

pacicephala 152.

pacifica 128.

pacificus $69,84,125$.

pallasii 107 .

pallens 136, 165.

pallida 136 .

pallidipeetus 165 .

pallidipes 28, 133, 165.

pallichus 13 .

pallipes $28,133,165$.

pamparum 13, 28.

panamensis 133.

papillaria 29.

Paraceramius 2.

paraensis 81, 165. 
Paragia 1.

Paragris 34.

Paraicaria 121.

Paralastor 110.

Paralastoroides 110. parallela 152 .

Paramischocyttarus 117.

Paravespa 60.

Parazumia 38.

parea 111.

parentissimum 45 .

Pareumenes 16.

parietina 84,85 .

parietinus 85,86 .

parietoides 86 .

parietum $60,84,85$,

$86,89,123,128$.

parisiensis 87 .

Parodynerus 49, 109. parredes 88 .

parredesi 88 .

Parrhynchium 42.

parrula 28, 165.

parvulus $13,28,71$,

74,88 .

paulii 88 .

pectoralis 130 .

pedestris 88 .

pediculata 23,165 .

pedunculata 30 .

pelagica 39.

pendula 120.

pennsylvanica 14,28 ,

152.

pennsylvanicus 88 .

pensilis 28.

pentameria 37.

perennis 88 .

perplexa 28.

perplexuв 28, 133.

persa 88.

parsezutor 53.

pertinax 101.

peruana 152 .

peruensis 88 .

petiolata $28,40,116$,

152.

petiolatus'28, 88, 116 .

petulans 88 .

peyrotii 89. phalansterica 120.

phalerata 107.

phaleratus 106, 107, 108.

pharao 89.

philadelphiae 89.

philantes 28.

philippinensis 120 .

$133,152$.

phthisica $28,163,164$.

picipes 152.

picta $17,113,116$, 120.

pictetii $28,111,133$,

165.

picticrus 89.

pictifrons 1, 29.

pictipes 89 .

pictus $79,89,113,116$.

pilipalpa 108.

pilipalpis 108 .

pilipalpus 10 S.

pilosa 120 .

pilosella 152.

pilosus 89 .

piriformis $13,29,30$.

placida 29.

placidus 29.

platensis 40 .

platinia 40.

plebcja 120,132, 165.

pocillum 89 .

poecilus 89 .

poeyi $13,132$.

Polistes 9, 16, 42, 49,

$60,114,117,121$,

$122,136,161,167$,

169.

polita 31.

politica 120.

politus 31 .

Polybia 161.

polyphemus 89 .

pomicolor 120 .

pomiformis 17,29 , $30,31$.

pontebae 89 .

ponticerianus 89.

posticus $72,90$.

postscutellatus 63 .

praecox 90. pracdator 2 .

prasina 116.

prasinus 116.

praslinia 18.

pratensis 90 .

primus 90.

privatus 90 .

proctus 90.

productus 90 .

propinquus 90 .

Prorhynchium 42.

proserpina 37 .

Protodynerus 49.

proximus $88,90$.

pruinosus 90 .

przewalskyi 90 .

Pseudepipona 49.

Pseudochilus 109.

Pseudodynerus 49.

pseudodynerus 90.

Pseudopolybia 161.

Pseudovespa 136.

Pseudozumia 38.

Psiloglossa 8.

psilothorax 90 .

Pterocheilus 105.

Pterochilus 49, 105.

108, 109.

pteropoda 152.

pubescens 13, 90,121.

pulchella 8 .

pulchellum 47 .

pulchellus 15, 108.

pullata 31 .

pullatus 31 .

pumila 165.

pumilus 90 .

punctata 31 .

punctatipennis 91 .

puncticollis 133.

punctifrons 91 .

punctirentris 108.

punctulatus 112.

punctum 91, 152.

punicus 91, 108.

pusilla $31,116$.

pusillus $31,91,112$,

116.

pusio 31.

pygmaea 165.

pyriformis 30 .
Quadrata 31, 91.

quadratus $31,91$.

quadricincta $86,165$.

quadricinctus 86 ,

102.

quadricolor 91.

quadridens 41 .

quadrifasciata 91 .

quadrifasciatus 85 ,

91.

quadriguttata 152.

quadrimaculata 152 .

quadrimaculatus 92.

quadripunctata 152 ,

153.

quadrisectus 92.

quadrispinosa 31 .

quadrispinosus 31 .

quadrituberculata

167.

quadrituberculatus

92.

quadrum $14 i$.

quartinae 92.

Quartinia 7.

quinquecincta 45 .

quinquecinctum 45 .

quinquefasciata $9 S$,

153.

quinquefasciatum

108.

quinquefasciatus 98 .

108.

quixensis 92.

Radiale 47 .

radoschowskii 92.

radoszkowskii 92 .

radula 92

raffrayi 92.

rannvalii 120.

renctionalis 120 .

reaumuri 93.

rectus 92.

recurvirostra 12 .

recurvirostris 12 .

reflexus 92.

regina 31 .

regulus $31,92$.

rejecta $165,166$.

reniformis $75,92,93$. 
renimacula S6, 57. rufinoda 13. renimaculatus 87 . republicanus 93 . rethoides 31 . rerolutionalis $121 \%$. rex 3 .

rhachiphorus 93 . Rhaphidoglossa 7 . Rhaphigaster 114 . rhaphigastra 165. Rhaphiglossa $7, \mathrm{~S}$. rhodensis 93. rhodopterus 93. rhombiferus 93. Rhopalidia 117, 122 . 161.

\section{rhynchiformis 62 .}

Rhynchium 42,49 , 105,136 .

rhynchoides $93,94$. rocellac 94 .

romandii 4,120 .

romandinus 13,94 .

rossii 94.

rothii 48.

rotundatus 94.

rotundigaster 94. rotundiventris 9.4 rubens 8 .

rubicola 75,76 . rubicunda 28. rubida 133. rubidus 133. rubiginosa 133. rubiginosus 94, 133. rubripes 94 .

rubritarsis 38 . rubritinctus 57, 95 . rubropictum 45 . rubropictus 71 . rubropustulatus 94 . rubrosignatus 94. rudolphi 94 . rufa $138,153,154$. rufescens 94 . ruficollis 94 . ruficornis 133, 154. rufidens $133,166$. rufidentata 40. rufidulus 94 . rufina 133. rufinodis 13 .

rufinodus $13,95$.

rufipennis 115 . rufipes $38,4 \mathrm{~S}$. rufithorax 166 . rufiventre 48 . rufiventris 170 . rufoscutellata 150 . rugosus 69 . rugulosus 99 . rupestris 123 . ruppelliana 37 . ruspatrix 154. ruthenicus 58 .

Sabulosum 48. saccularis 95 . saeva 116 . saevus 116 . sagittarius 133,134 . sakalavus 95 .

samsaulica 154 .

sandwichensis 95 . sanguineum 45 . sanguineus 112 . sanguinolenta 31 . sanguinolentus 95 . santa-anna $31,95$. santa-annae 95 .

santanna 31.

sareptana 31. sareptanus $31,95$. saucius 95 .

saundersii 31 .

saussurei $2,40,95$, $116,134,166$. savignyi $7,26,27$. 95.

saxonica 154, 155. seabriusculus 95. scandinavus 95. seclesta 155 . schach 134. schlettereri 95. schrenkii 155 . schulthessiana 10!9. schulthessii 120 .

Scolia 42.

scottiann 120.

sculpturalis 14 . scutellaris 2, 95, 155 $16 i 6$.

seutellata $155,170$. secundus 95.

sedula 166.

selectus 96 .

sellatus 96 .

semenowii 134.

semiflavus 134.

semisacularis 96 .

senegalensis 96.

septemfasciatus 96 .

sepulchralis 40 .

seriata 1 . 1 .

sericea $31,37,160$, 169.

sericeus $31,169$.

serripes $78,96,155$.

sesquicincta 155 .

sesquicinctus 96.

sessilis 96,155 .

seulii 48 .

sexcincta 155 .

sexfasciata 87.

sexfasciatus 87 .

sexmaculata 155 .

sexpunctata 84 .

sexpustulata 155.

sibirica 155.

sibiricus 96, 108.

Sibyllina 113.

sicheliana 37 .

sichelianus $\mathrm{S}$.

sichelii $31,48,96$.

siculus 96 .

signaticollis 96 .

signaticornis 32 .

signatus 96 .

silaensis 96 .

silnos 96 .

silvatica 41 .

silvestris 156 .

similis 55, 55, 96, 112 ,

150.

simillima 135, 166.

simillimus $9 i$.

simplex 91, 92 .

simplicicornis 96,97 simplicipes 75,76 .

simulans 32 .

simulatus 134 . sinensis 32 .

singularis 31,112 .

sinuata 97,156 .

sinuatofasciatus 97.

sinuatus 97.

slossonac 14.

Smithia 8 .

smithii 2, 14, 32, 48,

$97,112,117,134$.

169, 170 .

sncllenii 45, 134.

sobrina 2.

sobrinus 72,97 .

socialis 120,166 .

socialistica 121.

solstitialis 97 .

speciosa 121.

spectabilis 97 .

Sphex 16, 114, 136.

spilophora 134.

spilophorus 134 .

spinifer 97 .

spiniger 8 .

spinipes $14,77,78$,

97, 98 .

spiniventris 35 .

spinolac 4, 15, 37, 39 ,

$98,134$.

spinosa 32.

spinosus 14, 15.

spinosuscula 37 .

spiricornis 3, 98, 99 .

splendida 42.

splendidus 42.

squamigera 157 .

squamosa 134.

squamosus 134.

Stenancistrocerus50.

Stenodynerus 50 .

Stenoglossa 7 .

stigma 99, 132, 166.

stipatus 99.

stramineus 99.

striata 134.

striatus 134 .

strigosus 1.1.

Stroudia 14

structor 157 .

subalaris 99 .

Subancistrocerus 50 .

subclavata 121. 
sublnevis 32 .

sublevis 32 .

subpetiolatus 60 .

subsericeus 134.

substricta 14 .

substrictus 14 .

subtilis 117.

succinctus 99.

succicus 99.

sulcata 166.

sulcatus 99, 134.

sulcigastra 32.

sulfurens 99 .

sulfuripes 99 .

sulphurea 157.

sulphureus 99.

sulphuripes 99.

sumatrae 121, 134.

sumatrensis 166 .

sumichrastii 32, 99.

superbum 48 .

superbus 99.

surinama $160,161$.

surinamensis $12,16 \mathrm{I}$ $16(i)$.

sutterianus 99.

Sybillina 113.

sycophanta 166 .

sylvatica 65.

sylveibae 166 .

sylveirae 99.

sylvestris 156 .

symmorpha 7, 109.

Symmorphus 50 .

symmorphus 109.

Synagris 34.

synagroides 48, 99, 101).

Synoeca 60.

synoecus 134.

Tabida 32, 166, 167. tabidus 32.

tachkendensis 100.

tacubnyac 100.

tahitense 48.

tahitensis 157.

tamarinus 100 .

tamula $1: 32$.

tans 111).

tapiensis 100. tarabucensis 100 .

tarsalis 37.

tarsata 111 .

tarsatus 100, 116.

tasmaniensis 100 ,

$112,135$.

'Iatua 167.

tatua 167 .

taurica 18.

tauricus 18 .

tecta 100 .

tectus 100.

tegularis 100 .

tenebrieosa 135.

tenebricosus 135 .

tenebrionis 140 .

tenuis 32 .

tepanecus 10 (1)

tepida 135 .

tepidus 135 .

terricola 100 .

tertius 100 .

tesserazona 157 .

testacen 161, 163.

testaceus 60 .

tetrameria 37 .

texanus 4, 100, 108,

135.

texensis 101 .

thalassarctos 101 .

thoracica 28, 32.

thoracicus 32 .

tibialis 101,157 .

tigris 101 .

timidus 101.

tinctipennis 101 .

tinctor $26,27$.

tinctorius 27.

tinctus 101.

tinniens 101.

tisiphone 101.

toas 100 .

tolteca 109 .

toltecus 14, 101.

tomentosa 121.

tomentosus 101.

torrida 121.

torridus 101.

totonacus 32, 101.

trankitorius 102 .

translucida 135. translucidus 135. transversa 42 . transversesignatus 126.

transversum 42.

triangulum 102.

tricinctus 87.

tricolor 2, 32, 116, $126,135,157$.

tridens 155 .

trifasciata 87,157 .

trifasciatus 87,91 .

triloba 102.

trilobus 102.

trimarginatus 102.

Trimeria 5.

trinacriae 102.

triphaleratus 87 .

tripunctata 32,102 , 157.

tripunctatus $32,102$.

tristis 102 .

tristrigata 157.

troglodytes 103.

tropica 140.

tropicalis $32,103$.

tropidia 37.

truncatus 103.

tuberculatus 103,112.

tuberculifer 3.

tubereuliventris 103.

tuberculocephalus 103.

tuberculoceps 103.

tubulifer 14.

tunetanus 94.

turbulenta 116.

turbulentus 116.

turea 103.

turcica 151.

turgida 116.

turgidus 116.

turpis 103.

turrimarius 28.

tyrannica 157.

Ultramarina 161.

uncinata 41, 103.

uncinatus 103.

undinta 130.

unguicula 33. unguiculata 32 .

unguiculus 33 .

ungularis 90 .

unicolor 113, 121,

$125,157$.

unifasciata 140 .

unifasciatus $103,112$.

unipunctatus 105.

urceolica 135.

urceolicus 135 .

uruguyensis 33 .

urrillei 24.

Vagabundus 103.

vagus 103.

valida 126 .

varia 135 .

variabilis $41,121,135$.

variatus 135 .

variegata 103, 117,

121, 135.

variegatus $14,72,103$,

104, 135.

variolosus 61 .

varius 135 .

velox $93,157$.

velutina 121,157 ,

170.

ventralis 100 .

ventricosa 33 .

rentricosus 33 .

venusta 2, 32 .

vernalis 104 .

rerreauxii 15.

versicolor 17, 124,

$135,136$.

verticalis 33 .

verus 104.

Vespa 5, 9, 15, 16, 34,

$40,41,42,49,60$,

$105,109,114,117$,

$121,122,136,161$,

$167,169$.

vespiceps 167 .

vespiformis $2,3,4$,

$5,6,104$.

Vespinac 113.

vespoides 4 .

Vespula 136.

vestitus 104.

viaticn 29. 
vicina 167.

victoria 104.

vidua 157.

viduus 104 .

villosus 104 .

vindex 31 .

violacea 161.

virginea 136.

virginiensis 122 .

viridis 33 .

vitripennis 121.

vivax 157. volatilis 33 .

vollenhovenii 45 .

vulcanus 104.

vulgaris 157,158 , $159,160$.

vulneratus 104, 112.

vulpinus 112 .

Wagae 33.

wagneriana 33.

wagnerianus 33 .

walkeri 33. walshianus 104 .

westwoodii 14.

Xanthianus 104.

xanthocera 160.

xanthomelas $104,105$.

xanthopus 167.

xanthosoma 105.

ranthospilos 42.

xanthospilus 42.

xanthura $33,37,121$.

xanthurum 48 .
Zebra 105.

zendulus 14, 105 .

Zeta 16.

zethoides 7 .

Zethus 9, 15, 16, 114, $117,160$.

Zethusculus 9.

zonale 48.

zonalis $5,15,16$.

zonata 15.

zonatum 48.

zonatus $105,108,169$. 




PRESERVATION REVIEW

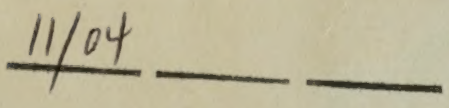


UNIVERSITY OF ILLNOIS-UABANA 